\title{
Management of frozen shoulder: a systematic review and cost-effectiveness analysis
}

E Maund, D Craig, S Suekarran, AR Neilson, K Wright, S Brealey, L Dennis, L Goodchild, N Hanchard, A Rangan, G Richardson, J Robertson and C McDaid

Health Technology Assessment NIHR HTA programme www.hta.ac.uk 


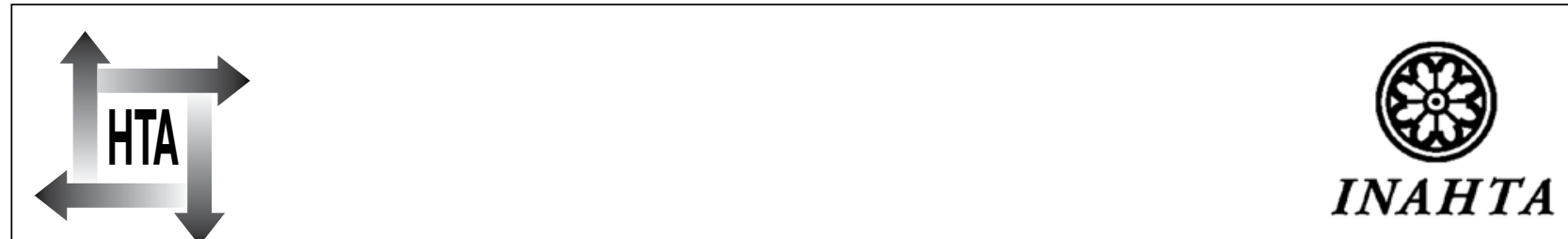

\section{How to obtain copies of this and other HTA programme reports}

An electronic version of this title, in Adobe Acrobat format, is available for downloading free of charge for personal use from the HTA website (www.hta.ac.uk). A fully searchable DVD is also available (see below).

Printed copies of HTA journal series issues cost $£ 20$ each (post and packing free in the UK) to both public and private sector purchasers from our despatch agents.

Non-UK purchasers will have to pay a small fee for post and packing. For European countries the cost is $£ 2$ per issue and for the rest of the world £3 per issue.

How to order:

- fax (with credit card details)

- post (with credit card details or cheque)

- phone during office hours (credit card only).

Additionally the HTA website allows you to either print out your order or download a blank order form.

\section{Contact details are as follows:}

Synergie UK (HTA Department)

Digital House, The Loddon Centre

Wade Road

Basingstoke

Hants RG24 8QW

\section{Email: orders@hta.ac.uk}

Tel: 08458124000 - ask for 'HTA Payment Services'

(out-of-hours answer-phone service)

Fax: 08458124001 - put 'HTA Order' on the fax header

\section{Payment methods}

Paying by cheque

If you pay by cheque, the cheque must be in pounds sterling, made payable to University of Southampton and drawn on a bank with a UK address.

Paying by credit card

You can order using your credit card by phone, fax or post.

\section{Subscriptions}

NHS libraries can subscribe free of charge. Public libraries can subscribe at a reduced cost of $£ 100$ for each volume (normally comprising 40-50 titles). The commercial subscription rate is $£ 400$ per volume (addresses within the UK) and $£ 600$ per volume (addresses outside the UK). Please see our website for details. Subscriptions can be purchased only for the current or forthcoming volume.

\section{How do I get a copy of HTA on DVD?}

Please use the form on the HTA website (www.hta.ac.uk/htacd/index.shtml). HTA on DVD is currently free of charge worldwide.

The website also provides information about the HTA programme and lists the membership of the various committees. 


\title{
Management of frozen shoulder: a systematic review and cost-effectiveness analysis
}

\author{
E Maund, ${ }^{1}$ D Craig,,${ }^{1}$ S Suekarran, ${ }^{1}$ AR Neilson, ${ }^{1}$ \\ K Wright, ${ }^{1}$ S Brealey, ${ }^{2}$ L Dennis, ${ }^{3}$ L Goodchild, ${ }^{4}$ \\ $\mathrm{N}$ Hanchard, ${ }^{3}$ A Rangan, ${ }^{5} \mathrm{G}$ Richardson, ${ }^{6} \mathrm{~J}$ Robertson ${ }^{7}$ \\ and C McDaid ${ }^{1 *}$
}

${ }^{1}$ Centre for Reviews and Dissemination, University of York, York, UK ${ }^{2}$ Department of Health Sciences, University of York, York, UK ${ }^{3}$ School of Health \& Social Care, Teesside University, Middlesbrough, UK ${ }^{4}$ Department of Rehabilitation, The James Cook University Hospital, Middlesbrough, UK

${ }^{5}$ The James Cook University Hospital, Middlesbrough, UK ${ }^{6}$ Centre for Health Economics, University of York, York, UK ${ }^{7}$ Cambridge Medical Group, Middlesbrough, UK

${ }^{*}$ Corresponding author

Declared competing interests of authors: Amar Rangan has received consultancy fees from DePuy International relating to shoulder replacement prostheses. A division of DePuy is involved in marketing products for surgical treatment of the frozen shoulder. The other authors report no conflicts of interest.

Published March 2012

DOI: 10.3310/hta16110

This report should be referenced as follows:

Maund E, Craig D, Suekarran S, Neilson AR, Wright K, Brealey S, et al. Management of frozen shoulder: a systematic review and cost-effectiveness analysis. Health Technol Assess 2012;16(11).

Health Technology Assessment is indexed and abstracted in Index Medicus/MEDLINE, Excerpta Medica/EMBASE, Science Citation Index Expanded $\left(\right.$ SciSearch $\left.^{\circledR}\right)$ and Current Contents ${ }^{\circledast} /$ Clinical Medicine. 
The Health Technology Assessment (HTA) programme, part of the National Institute for Health Research (NIHR), was set up in 1993. It produces high-quality research information on the effectiveness, costs and broader impact of health technologies for those who use, manage and provide care in the NHS. 'Health technologies' are broadly defined as all interventions used to promote health, prevent and treat disease, and improve rehabilitation and long-term care.

The research findings from the HTA programme directly influence decision-making bodies such as the National Institute for Health and Clinical Excellence (NICE) and the National Screening Committee (NSC). HTA findings also help to improve the quality of clinical practice in the NHS indirectly in that they form a key component of the 'National Knowledge Service'.

The HTA programme is needs led in that it fills gaps in the evidence needed by the NHS. There are three routes to the start of projects.

First is the commissioned route. Suggestions for research are actively sought from people working in the NHS, from the public and consumer groups and from professional bodies such as royal colleges and NHS trusts. These suggestions are carefully prioritised by panels of independent experts (including NHS service users). The HTA programme then commissions the research by competitive tender.

Second, the HTA programme provides grants for clinical trials for researchers who identify research questions. These are assessed for importance to patients and the NHS, and scientific rigour.

Third, through its Technology Assessment Report (TAR) call-off contract, the HTA programme commissions bespoke reports, principally for NICE, but also for other policy-makers. TARs bring together evidence on the value of specific technologies.

Some HTA research projects, including TARs, may take only months, others need several years. They can cost from as little as $£ 40,000$ to over $£ 1$ million, and may involve synthesising existing evidence, undertaking a trial, or other research collecting new data to answer a research problem.

The final reports from HTA projects are peer reviewed by a number of independent expert referees before publication in the widely read journal series Health Technology Assessment.

Criteria for inclusion in the HTA journal series

Reports are published in the HTA journal series if (1) they have resulted from work for the HTA programme, and (2) they are of a sufficiently high scientific quality as assessed by the referees and editors.

Reviews in Health Technology Assessment are termed 'systematic' when the account of the search, appraisal and synthesis methods (to minimise biases and random errors) would, in theory, permit the replication of the review by others.

The research reported in this issue of the journal was commissioned by the HTA programme as project number 09/13/02. The contractual start date was in March 2010. The draft report began editorial review in March 2011 and was accepted for publication in September 2011. As the funder, by devising a commissioning brief, the HTA programme specified the research question and study design. The authors have been wholly responsible for all data collection, analysis and interpretation, and for writing up their work. The HTA editors and publisher have tried to ensure the accuracy of the authors' report and would like to thank the referees for their constructive comments on the draft document. However, they do not accept liability for damages or losses arising from material published in this report.

The views expressed in this publication are those of the authors and not necessarily those of the HTA programme or the Department of Health.

Editor-in-Chief:

Series Editors:

Professor Tom Walley CBE

Dr Martin Ashton-Key, Professor Aileen Clarke, Dr Tom Marshall, Professor John Powell, Dr Rob Riemsma and Professor Ken Stein

Associate Editor:

Dr Peter Davidson

Editorial Contact: edit@southampton.ac.uk

ISSN 1366-5278 (Print)

ISSN 2046-4924 (Online)

ISSN 2046-4932 (DVD)

(c) Queen's Printer and Controller of HMSO 2012. This work was produced by Maund et al. under the terms of a commissioning contract issued by the Secretary of State for Health.

This journal is a member of and subscribes to the principles of the Committee on Publication Ethics (COPE) (http://www. publicationethics.org/).

This journal may be freely reproduced for the purposes of private research and study and may be included in professional journals provided that suitable acknowledgement is made and the reproduction is not associated with any form of advertising. Applications for commercial reproduction should be addressed to: NETSCC, Health Technology Assessment, Alpha House, University of Southampton Science Park, Southampton SO16 7NS, UK.

Published by Prepress Projects Ltd, Perth, Scotland (www.prepress-projects.co.uk), on behalf of NETSCC, HTA.

Printed on acid-free paper in the UK by the Charlesworth Group. 


\title{
Abstract
}

\section{Management of frozen shoulder: a systematic review and cost-effectiveness analysis}

\author{
E Maund, ${ }^{1}$ D Craig, ${ }^{1}$ S Suekarran, ${ }^{1}$ AR Neilson, ${ }^{1} \mathrm{~K}$ Wright, ${ }^{1}$ S Brealey, ${ }^{2}$ \\ L Dennis, ${ }^{3} \mathrm{~L}$ Goodchild, ${ }^{4} \mathrm{~N}$ Hanchard, ${ }^{3} \mathrm{~A}$ Rangan, ${ }^{5} \mathrm{G}$ Richardson, ${ }^{6}$ \\ J Robertson ${ }^{7}$ and C McDaid, ${ }^{1 *}$
}

${ }^{1}$ Centre for Reviews and Dissemination, University of York, York, UK

${ }^{2}$ Department of Health Sciences, University of York, York, UK

${ }^{3}$ School of Health \& Social Care, Teesside University, Middlesbrough, UK

${ }^{4}$ Department of Rehabilitation, The James Cook University Hospital, Middlesbrough, UK

${ }^{5}$ The James Cook University Hospital, Middlesbrough, UK

${ }^{6}$ Centre for Health Economics, University of York, York, UK

${ }^{7}$ Cambridge Medical Group, Middlesbrough, UK

${ }^{*}$ Corresponding author

Background: Frozen shoulder is condition in which movement of the shoulder becomes restricted. It can be described as either primary (idiopathic) whereby the aetiology is unknown, or secondary, when it can be attributed to another cause. It is commonly a selflimiting condition, of approximately 1 to 3 years' duration, though incomplete resolution can occur.

Objectives: To evaluate the clinical effectiveness and cost-effectiveness of treatments for primary frozen shoulder, identify the most appropriate intervention by stage of condition and highlight any gaps in the evidence.

Data sources: A systematic review was conducted. Nineteen databases and other sources including the Cumulative Index to Nursing and Allied Health (CINAHL), Science Citation Index, BIOSIS Previews and Database of Abstracts of Reviews of Effects (DARE) were searched up to March 2010 and EMBASE and MEDLINE up to January 2011, without language restrictions. MEDLINE, CINAHL and PsycINFO were searched in June 2010 for studies of patients' views about treatment.

Review methods: Randomised controlled trials (RCTs) evaluating physical therapies, arthrographic distension, steroid injection, sodium hyaluronate injection, manipulation under anaesthesia, capsular release or watchful waiting, alone or in combination were eligible for inclusion. Patients with primary frozen shoulder (with or without diabetes) were included. Quasi-experimental studies were included in the absence of RCTs and case series for manipulation under anaesthesia (MUA) and capsular release only. Full economic evaluations meeting the intervention and population inclusion criteria of the clinical review were included. Two researchers independently screened studies for relevance based on the inclusion criteria. One reviewer extracted data and assessed study quality; this was checked by a second reviewer. The main outcomes of interest were pain, range of movement, function and disability, quality of life and adverse events. The analysis comprised a narrative synthesis and pair-wise meta-analysis. A mixed-treatment comparison (MTC) was also undertaken. An economic decision model was intended, but 
was found to be implausible because of a lack of available evidence. Resource use was estimated from clinical advisors and combined with quality-adjusted life-years obtained through mapping to present tentative cost-effectiveness results.

Results: Thirty-one clinical effectiveness studies and one economic evaluation were included. The clinical effectiveness studies evaluated steroid injection, sodium hyaluronate, supervised neglect, physical therapy (mainly physiotherapy), acupuncture, MUA, distension and capsular release. Many of the studies identified were at high risk of bias. Because of variation in the interventions and comparators few studies could be pooled in a metaanalysis. Based on single RCTs, and for some outcomes only, short-wave diathermy may be more effective than home exercise. High-grade mobilisation may be more effective than low-grade mobilisation in a population in which most patients have already had treatment. Data from two RCTs showed that there may be benefit from adding a single intra-articular steroid injection to home exercise in patients with frozen shoulder of $<6$ months' duration. The same two trials showed that there may be benefit from adding physiotherapy (including mobilisation) to a single steroid injection. Based on a network of nine studies the MTC found that steroid combined with physiotherapy was the only treatment showing a statistically and clinically significant beneficial treatment effect compared with placebo for short-term pain (standardised mean difference $-1.58,95 \%$ credible interval -2.96 to -0.42 ). This analysis was based on only a subset of the evidence, which may explain why the findings are only partly supportive of the main analysis. No studies of patients' views about the treatments were identified. Average costs ranged from £36.16 for unguided steroid injections to $£ 2204$ for capsular release. The findings of the mapping suggest a positive relationship between outcome and European Quality of Life-5 Dimensions (EQ-5D) score: a decreasing visual analogue scale score (less pain) was accompanied by an increasing (better) EQ-5D score. The one published economic evaluation suggested that low-grade mobilisation may be more cost-effective than high-grade mobilisation. Our tentative costeffectiveness analysis suggested that steroid alone may be more cost-effective than steroid plus physiotherapy or physiotherapy alone. These results are very uncertain. Limitations: The key limitation was the lack of data available. It was not possible to undertake the planned synthesis exploring the influence of stage of frozen shoulder or the presence of diabetes on treatment effect. As a result of study diversity and poor reporting of outcome data there were few instances where the planned quantitative synthesis was possible or appropriate. Most of the included studies had a small number of participants and may have been underpowered. The lack of available data made the development of a decision-analytic model implausible. We found little evidence on treatment related to stage of condition, treatment pathways, the impact on quality of life, associated resource use and no information on utilities. Without making a number of questionable assumptions modelling was not possible.

Conclusions: There was limited clinical evidence on the effectiveness of treatments for primary frozen shoulder. The economic evidence was so limited that no conclusions can be made about the cost-effectiveness of the different treatments. High-quality primary research is required.

Funding: The National Institute for Health Research Health Technology

Assessment programme. 


\section{Contents}

Glossary vii

$\begin{array}{ll}\text { List of abbreviations } & \text { ix }\end{array}$

Executive summary

1. Background 1

The decision problem 1

Frozen shoulder 1

Diagnosis and management 3

Previous systematic reviews 4

Focus of the synthesis 5

2. Methods $\quad 7$

Overview $\quad 7$

Review of clinical effectiveness and cost-effectiveness

Literature searches $\quad 7$

Inclusion and exclusion criteria 8

Screening and study selection 13

$\begin{array}{ll}\text { Data extraction } & 13\end{array}$

$\begin{array}{ll}\text { Assessment of risk of bias } & 14\end{array}$

Synthesis 14

Systematic review of patients' views of interventions for frozen shoulder 18

$\begin{array}{ll}\text { Assessment of cost-effectiveness } & 18\end{array}$

3. Results 21

Assessment of clinical effectiveness 21

$\begin{array}{ll}\text { Mixed-treatment comparison results } & 91\end{array}$

Patients' views of interventions for frozen shoulder $\quad 96$

$\begin{array}{lr}\text { Economic analyses } & 97\end{array}$

Decision model $\quad 98$

4. Discussion 117

$\begin{array}{lr}\text { Principal findings } & 117\end{array}$

Strengths and limitations of the review $\quad 125$

$\begin{array}{ll}\text { Improving the evidence on the effectiveness and cost-effectiveness } & 127\end{array}$

5. Conclusions 131

Implications for service provision $\quad 131$

Suggested research priorities 131

$\begin{array}{ll}\text { Acknowledgements } & 133\end{array}$

$\begin{array}{ll}\text { References } & 135\end{array}$ 
Appendix 1 Search strategies for effectiveness review

Appendix 2 Search strategy for review of views of people with frozen shoulder

Appendix 3 R code for standard deviation

Health Technology Assessment programme

Appendix 4 Quality assessment checklist

Appendix 5 List of excluded studies

Appendix 6 Study details

Appendix 7 Data extraction tables

Appendix 8 Study quality

Appendix 9 WinBUGS code

Appendix 10 Mixed-treatment comparison

Appendix 11 Economic evaluation study quality checklist

Appendix 12 Economic evaluation data extraction/summary 385

Appendix 13 Resource-use table

Appendix 14 Exploratory mapping analysis 389

Appendix 15 Protocol 


\section{Glossary}

Case series A group of case reports of patients who were given similar treatments. There is no control group involved.

Cost-effectiveness analysis An economic analysis that expresses the effects or consequences of interventions on a single dimension. This would normally be expressed in 'natural' units (e.g. cases cured, life-years gained, additional strokes prevented). The difference between interventions in terms of costs and effects is typically expressed as an incremental cost-effectiveness ratio (e.g. the incremental cost per life-year gained).

Cost-utility analysis The same as a cost-effectiveness analysis but the effects or consequences of interventions are expressed in generic units of health gain, usually quality-adjusted life-years.

Exercise therapy Gentle rhythmic active exercises, function-based exercises [to maintain/restore the range or quality (co-ordination and/or control) of movement] or both.

Incremental cost-effectiveness ratio An expression of the additional cost of health gain associated with an intervention relative to an appropriate comparator. Expressed as the difference in mean costs (relative to the comparator) divided by the difference in mean health gain.

Intention-to-treat analysis Analysis that compares participants in the groups to which they were originally assigned.

Mixed-treatment comparison This is an extension of a traditional meta-analysis. Whereas a traditional meta-analysis includes only trials making direct comparisons between an intervention and a comparator, a mixed-treatment comparison also includes indirect evidence. This approach overcomes the limitations of the traditional approach in cases in which there are no or limited trials making the relevant head-to-head comparison.

Mobilisations Therapist-applied passive movements of joints or other structures performed in such a way that they are always within the control of the patient. They may be performed by various techniques and may be combined with active movement on the part of the patient.

Physical therapy This term is used to refer to the broad range of physical interventions such as physiotherapy (including mobilisation or other modalities such as electrotherapy) and chiropractic and osteopathic interventions.

Quality-adjusted life-year An index of health gain in which survival duration is weighted or adjusted by the patient's quality of life during the survival period. Quality-adjusted life-years have the advantage of incorporating changes in both quantity (mortality) and quality (morbidity) of life.

Randomised controlled trial A trial in which the participants are randomly allocated to the control or treatment groups.

WinBUGS Statistical software for Bayesian analysis using Markov chain Monte Carlo methods. 



\section{List of abbreviations}

\begin{tabular}{|c|c|}
\hline $15 \mathrm{D}$ & 15-dimension utility measure \\
\hline ASES & American Shoulder and Elbow Surgeons \\
\hline $\mathrm{CI}$ & confidence interval \\
\hline CLAD & censored least absolute deviations \\
\hline CRD & Centre for Reviews and Dissemination \\
\hline CrI & credible interval \\
\hline DASH & Disabilities of the Arm, Shoulder and Hand \\
\hline EPPI & Evidence for Policy and Practice Information \\
\hline EQ-5D & European Quality of Life-5 Dimensions \\
\hline ERM & end-range mobilisation \\
\hline FLEX-SF & Flexilevel Scale of Shoulder Function \\
\hline GP & general practitioner \\
\hline HAQ & Health Assessment Questionnaire \\
\hline HGMT & high-grade mobilisation technique \\
\hline HRQoL & health-related quality of life \\
\hline HUI & Health Utilities Index \\
\hline ICER & incremental cost-effectiveness ratio \\
\hline IQR & interquartile range \\
\hline ITT & intention to treat \\
\hline LGMT & low-grade mobilisation technique \\
\hline MAE & mean absolute error \\
\hline MCID & minimum clinically important difference \\
\hline MCMC & Markov chain Monte Carlo \\
\hline MCS & mental component summary \\
\hline MD & mean difference \\
\hline $\mathrm{ME}$ & mean error \\
\hline MRM & mid-range mobilisation \\
\hline MTC & mixed-treatment comparison \\
\hline MUA & manipulation under anaesthesia \\
\hline MWM & mobilisation with movement \\
\hline NICE & National Institute for Health and Clinical Excellence \\
\hline NSAID & non-steroidal anti-inflammatory drug \\
\hline NTIS & National Technical Information Service \\
\hline OLS & ordinary least squares \\
\hline PCS & physical component summary \\
\hline PSWD & pulsed short-wave diathermy \\
\hline QALY & quality-adjusted life-year \\
\hline QoL & quality of life \\
\hline RCT & randomised controlled trial \\
\hline RMSE & root-mean-square error \\
\hline $\mathrm{SD}$ & standard deviation \\
\hline SDQ & Shoulder Disability Questionnaire \\
\hline SE & standard error \\
\hline SF-6D & Short Form questionnaire-6 Dimensions \\
\hline SF-36 & Short Form questionnaire-36 items \\
\hline
\end{tabular}




$\begin{array}{ll}\text { SMD } & \text { standardised mean difference } \\ \text { SPADI } & \text { Shoulder Pain and Disability Index } \\ \text { SRQ } & \text { Shoulder Rating Questionnaire } \\ \text { SWD } & \text { short-wave diathermy } \\ \text { TENS } & \text { transcutaneous electrical nerve stimulation } \\ \text { UCLA } & \text { University of California Los Angeles } \\ \text { VAS } & \text { visual analogue scale }\end{array}$

All abbreviations that have been used in this report are listed here unless the abbreviation is well known (e.g. NHS), or it has been used only once, or it is a non-standard abbreviation used only in figures/tables/appendices, in which case the abbreviation is defined in the figure legend or in the notes at the end of the table. 


\section{Executive summary}

\section{Background}

Frozen shoulder is a condition in which movement of the shoulder becomes restricted. The condition can vary in severity from mild pain and/or less significant restriction of movement to severe pain and/or severely restricted movement. Key characteristics are gradual onset of shoulder stiffness, severe pain, especially at night, and restriction in active and passive range of movement of the shoulder. Frozen shoulder can be described as either primary (idiopathic), if the aetiology is unknown, or secondary, when it can be attributed to another cause. It is commonly a self-limiting condition, of approximately $1-3$ years' duration, although incomplete resolution can occur.

\section{Objectives}

To (1) evaluate the clinical effectiveness of strategies currently used in the NHS for the management of primary frozen shoulder and identify the most appropriate intervention by stage of condition; (2) collate patients' views and experiences of the interventions by way of a systematic review; (3) assess the cost-effectiveness of the different interventions in order to inform the development of a decision model; (4) develop a decision-analytic model to estimate the cost-effectiveness of alternative treatment options for frozen shoulder; (5) make recommendations for clinical practice; and (6) identify any gaps in the evidence, undertake value of information analysis to assess the potential value of future research on interventions for frozen shoulder and make specific recommendations for further research.

\section{Methods}

\section{Clinical effectiveness}

A systematic review was undertaken. Nineteen databases, including MEDLINE, EMBASE and the Cochrane Central Register of Controlled Trials (CENTRAL), were searched up to March 2010 for published and unpublished studies and without language restrictions. The websites of relevant organisations were scanned. The reference lists of systematic reviews were also checked to identify studies. Updated searches of MEDLINE and EMBASE were undertaken in January 2011.

Randomised controlled trials (RCTs) evaluating physical therapies, arthrographic distension, steroid injection, sodium hyaluronate injection, manipulation under anaesthesia (MUA), capsular release or watchful waiting, alone or in combination, were eligible for inclusion. Patients with primary frozen shoulder (with or without diabetes) were included. Relevant comparators were any of the treatments, no treatment or placebo. Quasi-experimental studies were included in the absence of randomised trials. For MUA and capsular release, case series of at least 50 participants with primary frozen shoulder were also eligible in the absence of controlled study designs. The main outcomes of interest were pain, range of movement, function and disability, quality of life, time to recovery, return to work and recreation, and adverse events.

Two researchers independently screened studies for relevance based on the inclusion criteria. One reviewer extracted data and assessed study quality; this was checked by a second reviewer. 
Final value data were extracted where available and if not change from baseline was extracted. Disagreements were resolved by consensus and, if necessary, a third researcher was consulted.

A narrative and tabular summary of key study characteristics, quality assessment and results was undertaken. Outcomes were reported as the mean difference (MD) and 95\% confidence interval (CI), except for pain, for which the standardised mean difference (SMD) was calculated. Length of follow-up was classified as short term ( 4 weeks to 3 months), medium term ( $>3$ months and $\leq 6$ months) and long term ( $>6$ months). Where appropriate, individual study results were combined in a pair-wise meta-analysis grouped by type of intervention and comparator. A mixed-treatment comparison (MTC) evaluating the effectiveness of the interventions compared with placebo was undertaken as an exploratory analysis. The MTC was performed for the outcome of pain up to 3 months only because this was the only outcome and time point for which a network was available. Comparisons were described as statistically significant (at the 5\% level) when the credibility interval (CrI) did not cross 0 for the SMD.

\section{Patients' views}

MEDLINE, the Cumulative Index to Nursing and Allied Health Literature (CINAHL) and PsycINFO were searched in June 2010 to identify qualitative studies of patients' views of treatments, with searches being restricted to English-language papers published from 1980 onwards. The review processes followed those of the effectiveness review.

\section{Economic analysis}

A systematic review of full economic evaluations meeting the intervention and population inclusion criteria of the clinical review was performed. The intention was to develop a decisionanalytical model; however, because of the paucity of evidence for key components of the modelling (including the structure) the development of a model was not possible. To provide some useful information for the decision-maker, we undertook a number of investigatory exercises. First, we elicited details on resource use from clinicians for the interventions of interest and produced cost estimates based on national sources. Second, we mapped two important clinical outcomes, pain [using visual analogue scale (VAS) $0-100 \mathrm{~mm}$ ] and quality of life [using the Short Form questionnaire-36 items (SF-36) mental and physical component score], to the European Quality of Life-5 Dimensions (EQ-5D). This allowed us to estimate quality-adjusted life-years (QALYs) for some of the interventions included in the clinical effectiveness review. Finally, having these two components allowed us to present a tentative cost-effectiveness analysis for some of the included interventions.

\section{Results}

\section{Clinical effectiveness}

The searches yielded 8883 citations. Thirty-two relevant studies were identified, one of which was a cost-utility analysis conducted alongside a separately published study of effectiveness.

Data from studies with a low risk of bias were sparse, in particular for the more invasive treatments (MUA, distension and capsular release). Twenty-eight RCTs, one quasi-experimental study (for 'supervised neglect') and two case series (for capsular release) were included. A total of 18 studies did not report an adequate method of randomisation (therefore these studies described as RCTs may have been quasi-experimental studies); 24 did not report an adequate method of allocation concealment; and 13 did not have blinded outcome assessment. For most of the studies it was unclear whether they were adequately powered to detect a statistically significant difference between groups. Across most trials there did not appear to be systematic methods for recording of adverse events. 
For most comparisons there were insufficient studies with a similar intervention and comparator to allow quantitative pooling. Primarily a narrative synthesis was undertaken. Because of the lack of data on stage of frozen shoulder and outcomes of patients with and without diabetes, it was not possible to undertake planned subgroup analyses.

\section{Conservative interventions}

A single quasi-experimental study, with a high risk of bias, reported significant short-, mediumand long-term benefit for function and disability with supervised neglect compared with physical therapy.

A further 11 studies investigated a physical therapy. The majority of these were various forms of physiotherapy. The comparators were an alternative physiotherapy modality or a control group. Most of the studies had a high risk of bias. Only single studies were available for specific modalities. The majority of studies comparing two active interventions reported no significant difference in outcome between therapies. For studies that did report a benefit with an intervention, this tended to be evident for some outcomes only within the study. This may reflect the effect of the intervention or may be related to poor study quality. There was evidence from one study of a statistically significant short-term benefit with short-wave diathermy (SWD) plus stretching compared with home exercise only. There was evidence from the same study for a benefit with SWD compared with a heat pack for range of movement; however, in the same study there was no evidence of benefit for function and disability. There was also evidence from a single study that laser therapy had benefit over placebo laser in the short and medium term for pain and function and disability. Both studies had some risk of bias. The content, intensity and quality of the background home exercise that all groups received was also unclear. A further study, also at some risk of bias, found marginally significant improvement for function and disability and range of movement with high-grade mobilisation techniques compared with low-grade mobilisation techniques; however, there was no evidence of benefit for pain or quality of life in this study.

Three RCTs compared acupuncture with another treatment and they all had a high risk of bias. It was possible to calculate the MD and 95\% CI for only one of the studies, which compared electroacupuncture and inferential electrotherapy. There was no significant difference between the two groups in pain or function and disability at short-, medium- and long-term follow-up.

Six studies evaluated steroid injection. The majority of the available data were from two multiarm studies that were of satisfactory quality, although one had some risk of bias. Both studies evaluated a single intra-articular steroid injection in patients with frozen shoulder of $<6$ months' duration. The comparators were home exercise alone, physiotherapy alone (both with placebo injection) and steroid injection followed by physiotherapy. For pain there was a short-term statistically significant benefit with steroid injection compared with placebo (SMD -1.15, 95\% CI -1.62 to -0.67 ; two RCTs).There was no difference compared with physiotherapy (SMD -0.22 , $95 \%$ CI -0.65 to 0.20 ; two RCTs). When steroid injection was provided in conjunction with physiotherapy, there was an added benefit for pain over physical therapy alone (SMD - $0.98,95 \%$ CI -1.43 to -0.52 ; two RCTs). There was also benefit with the combined intervention over steroid injection alone (SMD $-0.75,95 \%$ CI -1.20 to -0.29 ; two RCTs), although there was substantial heterogeneity. The results for function and disability and range of movement were broadly consistent with the results for pain. Based on a single study, there was no statistically significant benefit for quality of life with a steroid injection alone compared with placebo or physiotherapy alone. However, there was a benefit for quality of life when physiotherapy was added to steroid injection compared with placebo and physiotherapy alone. There was no evidence of benefit for the combined intervention over steroid injection alone. 


\section{Invasive interventions}

The data from high-quality RCTs of invasive interventions were even more limited than for conservative interventions. In addition, there was considerable variability between the studies in how the procedures were delivered, making comparability between studies difficult.

Four RCTs assessed MUA. A single, satisfactory-quality study compared MUA with home exercise alone. There was no significant difference between groups in pain, function and disability, range of movement or working ability at short-, medium- or long-term follow-up. A study with some risk of bias compared MUA with arthrographic distension. There was greater improvement in pain and function and disability at 6 months with arthrographic distension than with MUA in participants with adhesive-stage primary frozen shoulder. The remaining two studies had a high risk of bias.

Three RCTs investigated distension with steroid injection, two of which used arthrographic distension. A single satisfactory study reported a significant improvement in one of two function and disability measures with arthrographic distension including steroid compared with home exercise (plus placebo arthrography). There was no evidence of benefit for range of movement or pain. A second study, with some risk of bias, compared arthrographic distension including steroid with steroid alone. There was benefit with distension for a single range of movement measure at 6 weeks. There was no evidence of benefit for other outcomes.

Two case series of more than 50 participants were identified that investigated capsular release. The best-quality case series had an average length of follow-up of 10 months (range 3 to 29 months). There was a significant improvement in function and disability, working ability and a measure of range of movement amongst participants in this study.

Three RCTS, all at high risk of bias, investigated sodium hyaluronate. The best-quality study reported a benefit with two injections of sodium hyaluronate compared with home exercise. However, there was no consistent evidence across outcomes of a benefit compared with 10 daily sessions of physical therapy or a single steroid injection.

\section{Mixed-treatment comparison}

Nine interventions formed part of a connected network with placebo. Steroid combined with physiotherapy was the only treatment that showed a statistically and clinically significant beneficial treatment effect compared with placebo (SMD -1.58, 95\% CrI -2.96 to -0.42). Overall, there was no clear difference in the treatment effects of any of the interventions, that is, the CrIs overlapped. This analysis was based on only a subset of the evidence, which may explain why the findings are only partly supportive of the main analysis. Given the possible heterogeneity of placebos and study populations, and the inclusion of poor-quality studies, there is some uncertainty regarding the results of the MTC.

\section{Patients' views}

No studies were identified from the searches that explored patients' views or experiences of treatments for frozen shoulder.

\section{Cost-effectiveness}

The review identified one economic evaluation, conducted in the Netherlands. High-grade mobilisation was compared with low-grade mobilisation, from a societal perspective. The evaluation failed to deal with parameter uncertainty and dismissed differences in QALYs based on statistical inference. The evaluation was of reasonable quality, with a risk of bias, but failed to provide an answer to the broader question being posed. 
Based on resource-use estimates obtained from clinical experts, average costs were estimated for the interventions included in the review. The costs for an unguided steroid injection varied

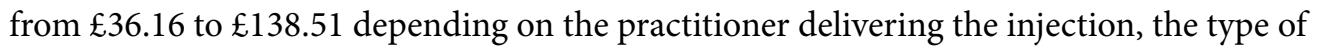
steroid used and where the practitioner is based (i.e. the setting). These costs suggest that a physiotherapist delivering treatment in a community setting is the cheapest option and a rheumatologist delivering treatment in a hospital setting is the most expensive. A guided steroid injection (considered less popular in the NHS than unguided) produced estimated costs ranging from $£ 299.68$ to $£ 475.56$. These costs were mainly influenced by who delivered the injection. Physiotherapy was estimated to cost between $£ 98.75$ and $£ 126.75$ dependent on setting. The addition of a steroid injection to physiotherapy presents a plethora of scenarios dependent on practitioner, steroid choice and setting; these costs range between $£ 121.43$ and $£ 607.31$.

Manipulation under anaesthesia was estimated to cost $£ 1446$ and capsular release $£ 2204$, both of which included rehabilitation physiotherapy. Arthrographic distension was estimated to cost approximately $£ 114.84$, depending on the choice of steroid injection. A range of other resource use and costing scenarios is presented.

A mapping analysis explored the relationship between pain measured with a pain VAS and the SF-36 mental and physical components [mental component summary (MCS) and physical component summary (PCS)] and the EQ-5D. Ordinary least squares (OLS) regression was used as the main statistical technique. The fit of all of the models was poor. However, the data suggest that all models (whether they are based on pain VAS or SF-36 PCS and MCS scores) estimate more accurate predictions for less severe health states, but overpredict the value of more severe EQ-5D states. Using the OLS model we mapped the reported mean pain VAS at baseline and at 3 months of follow-up onto the EQ-5D. The same was carried out for SF-36 PCS and MCS. The findings of the mapping suggest that there is a positive relationship between outcomes: a decreasing pain VAS score (less pain) is accompanied by an increasing (better) EQ-5D score; an increasing EQ-5D score (i.e. better health status) appears to be generally accompanied by increasing SF-36 scores (i.e. higher scores $=$ a better quality of life). The estimates were then used to predict EQ-5D scores at baseline and 3 months for a subset of the trials included in the clinical effectiveness review. Subsequently this allowed the tentative estimation of QALYs and calculation of cost-effectiveness ratios. The results suggested that steroid alone may be more cost-effective than steroid plus physiotherapy or physiotherapy alone.

\section{Conclusions}

\section{Implications for health care}

- There may be short-term benefit from adding a single intra-articular steroid injection to home exercise for patients with primary frozen shoulder of $<6$ months' duration. In the same population there may also be benefit from adding physiotherapy (including mobilisation in 8-10 sessions over 4 weeks) to a single steroid injection.

- Based on a single study, and for some outcomes only, there may be benefit from adding SWD to passive mobilisation and home exercise. Based on a single study, and for some outcomes only, high-grade mobilisation may be more effective than low-grade mobilisation in a population of patients who have already had physiotherapy and/or steroid injection.

Given the paucity of economic evidence it is not possible to make any conclusions regarding the most cost-effective interventions. 


\section{Recommendations for research}

There are large gaps in the evidence for the effectiveness and cost-effectiveness of all of the interventions investigated. Taking into account the views of health-care professionals in a recent survey, and the interventions that are most commonly used in the NHS, we suggest that the following should be given priority in future high-quality RCTs assessing effectiveness and cost-effectiveness:

- A 'standard care' package of high-quality conservative management. This should be fully specified in any future trial and we suggest that it should involve a structured protocol of high-quality education, advice, home exercise and monitoring.

- Steroid injection, in particular an investigation of the effectiveness and cost-effectiveness of multiple injections and whether there is any added benefit from providing physiotherapy after steroid injection, over and above steroid injection plus standard care.

- Physical therapies, specifically physiotherapy interventions, which have a component that involves mobilisation or exercises. Research is required to establish whether there is any benefit from having physiotherapy alone, over and above a standard package of care

- Intensive interventions, specifically arthrographic distension, MUA and arthroscopic capsular release.

- Important subgroups in any future RCTs are phase of frozen shoulder and patients with diabetes. These trials should collect data on resource use and use a utility measure to allow assessment of cost-effectiveness. A systematic approach to recording adverse events will also be important.

The large number of treatment options for frozen shoulder and the limited evidence for their effectiveness and cost-effectiveness makes prioritisation of these difficult and they are not listed in order of importance. We suggest that an appropriate starting point would be a multi-arm trial that compares the effectiveness and cost-effectiveness of interventions of differing intensity and costs: high-quality conservative management, steroid injection (possibly in conjunction with arthrographic distension) and surgical management (MUA and capsular release).

\section{Funding}

Funding for this study was provided by the Health Technology Assessment programme of the National Institute for Health Research. 


\section{Chapter 1}

\section{Background}

\section{The decision problem}

The commissioning brief requested an evidence synthesis comparing the clinical effectiveness and cost-effectiveness of different treatments for frozen shoulder to determine the most appropriate management strategy. The aims of the project were to:

1. evaluate, by way of a systematic review, the clinical effectiveness (including adverse effects) of strategies currently used in the NHS for the management of frozen shoulder and identify the most appropriate intervention by stage of condition, specifically physical therapies, steroid and other shoulder injections, manipulation under anaesthesia (MUA), arthrographic distension, capsular release, watchful waiting and combinations of these interventions

2. collate, by way of a systematic review, patients' views and experiences of the interventions being assessed in the review

3. identify, by way of a systematic review, the cost-effectiveness of the different interventions in order to inform the development of a decision model

4. develop a decision-analytic model to estimate the cost-effectiveness of alternative treatment options for frozen shoulder

5. make recommendations for clinical practice

6. identify any gaps in the evidence, undertake value of information analysis to assess the potential value of future research on interventions for frozen shoulder and make specific recommendations for further research.

\section{Frozen shoulder}

Frozen shoulder is a painful condition in which movement of the shoulder becomes severely restricted. The condition can vary in severity from mild to severe pain and/or from some to severe restriction in movement. It was first described in 1875 by the French pathologist Duplay, who named it péri-arthrite scapula-humérale. The most well-known definition and name for the condition was provided in 1934 by an American surgeon EA Codman. He defined it as coming on slowly 'with pain usually felt near the insertion of the deltoid; inability to sleep on the affected side; painful and incomplete elevation and external rotation; restriction of both spasmodic and mildly adherent type; atrophy of the spinati; little local tenderness; [and] X-rays negative except for bone atrophy' and named it 'frozen shoulder.' However, there is an acknowledged absence of a specific definition of the condition ${ }^{2,3}$ and of a diagnostic label, ${ }^{3}$ with additional names for frozen shoulder including adhesive capsulitis, painful stiff shoulder, retractile capsulitis, Checkrein shoulder, monoarticular arthritis and steroid-sensitive arthritis. ${ }^{4}$ Throughout this report the term 'frozen shoulder' will be used.

Frozen shoulder is typically characterised as having three overlapping phases: ${ }^{5}$

1. phase 1 , in which there is progressive stiffening and loss of motion in the shoulder with increasing pain on movement, which may be worse at night (months 2-9), usually referred to as the painful phase 
2. phase 2 , in which there is a gradual decrease in pain but stiffness remains and there is considerable restriction in the range of movement (months 4-12), usually referred to as the stiffening or 'freezing' phase

3. phase 3 , in which there is an improvement in range of movement (months 12-42), usually referred to as the resolution phase.

There are different views about the underlying fundamental process: inflammation, scarring and scarring produced in reaction to inflammation. ${ }^{6}$ Bunker describes a pathology of fibrous contracture of the rotator interval and coracohumeral ligament of the shoulder joint. ${ }^{7}$ In addition to the capsular contracture, there is often a reduced joint volume: 3-4 ml compared with the normal volume of $10-15 \mathrm{ml} .{ }^{8}$ The formation of new blood vessels in the synovial membrane, which is most marked in the rotator interval area, is a feature of the early stage of the condition. In the stiff phase this declines and thick white scar tissue can be seen and palpated within the capsule at arthroscopic and open surgery. Histology also shows thickening and contracture of the capsule. A significant increase in fibroblasts, which lay down scar tissue and myofibroblasts, which contract scar tissue, has been identified. ${ }^{8}$ In addition, pathological studies have identified the presence of inflammatory cells (mast cells, $\mathrm{T}$ cells, B cells and macrophages), suggesting a process of inflammation leading to scarring. ${ }^{9}$

The cumulative incidence of frozen shoulder is estimated at 2.4 per 1000 population per year based on a Dutch general practice sample..$^{10} \mathrm{~A}$ large UK-based primary care study found that frozen shoulder affected $8.2 \%$ of men and $10.1 \%$ of women of working age. ${ }^{11}$ In contrast, frozen shoulder was estimated to affect only $0.75 \%$ of the UK population based on a specialist shoulder surgeon's hospital care experience. ${ }^{8}$ This discrepancy in estimated prevalence may be explained by the fact that only the most resistant cases of frozen shoulder are seen in a hospital setting. ${ }^{6}$ Frozen shoulder most commonly occurs in people in their mid-50s and is thought to be slightly more common in women than in men.

Frozen shoulder can be described as either primary (idiopathic), whereby the aetiology is unknown, or secondary, when it can be attributed to another cause. Secondary frozen shoulder has been defined as that associated with diabetes (although some classify this as primary frozen shoulder), trauma, cardiovascular disease and hemiparesis. The incidence of frozen shoulder is reported to be $10-36 \%$ amongst people with diabetes, who tend not to respond as well to treatment as those without diabetes. ${ }^{5}$ The proportion of frozen shoulder attributed to trauma varies $(9-33 \%)$ and the trauma is often not particularly severe. There is also a discrepancy between the extent of trauma and severity of subsequent frozen shoulder. ${ }^{4}$ These uncertainties mean that in practice it can be difficult to differentiate between primary and secondary frozen shoulder.

Although for most people frozen shoulder is a self-limiting condition of approximately $1-3$ years' duration, it can be extremely painful and debilitating; people with the condition may struggle with basic daily activities and be worn down by sleep disturbance as a result of the pain. ${ }^{12}$ As well as interfering with domestic and social activities, it can affect the ability to work. There may not be a complete resolution for all patients and there is variation across case series in the proportion of patients who do not regain full shoulder motion, ${ }^{5}$ possibly a reflection of variation in how outcome was assessed. Based on the largest series of patients with a mean follow-up of 4.4 years from onset of symptoms, $59 \%$ had normal or near normal shoulders, $35 \%$ had mild to moderate symptoms with pain being the most common complaint and $6 \%$ had severe symptoms at follow-up. ${ }^{13}$ Recurrence is unusual although it is estimated that the other shoulder becomes affected in $6-17 \%$ of patients within 5 years. 


\section{Diagnosis and management}

Diagnosis, in both primary and secondary settings, is based on clinical examination and medical history. A key alerting feature is restriction of shoulder movement in all directions - passive and active range of movement. ${ }^{14}$ Blood tests, radiography and ultrasound are usually normal and not routinely required unless history or physical examination suggests the need to rule out other pathologies, for example if rheumatoid arthritis or osteoarthritis is suspected. Frozen shoulder is commonly managed in the primary care setting. In a UK study of patterns of referral and diagnosis of shoulder conditions it was estimated that $22 \%$ of patients were referred to secondary care, up to 3 years following initial presentation, although most referrals occurred within 3 months. ${ }^{15}$ There is little evidence available on referral patterns in relation to frozen shoulder specifically. ${ }^{12}$

There are a number of management options, both surgical and non-surgical, but there is no consensus about management. For the purpose of the report we have classified the interventions as conservative and invasive (Table 1).

The aims of treatment, depending on stage of condition, are pain relief, increasing arm movement, reducing the duration of symptoms and return to normal activities for the patient. Treatment options include:

- Watchful waiting or 'supervised neglect', which involves explaining the condition to the patient, and education and advice about mobilisation within pain limits and use of pain relief.

- Oral medications such as non-steroidal anti-inflammatory drugs (NSAIDs) and oral steroids. Although the use of oral steroids is described in the literature they are not a commonly used intervention in the UK.

- Gentle exercise supervised by a physiotherapist or as part of a home exercise programme.

- Physical therapies to help regain range of movement and prevent further stiffness. These encompass the wide range of techniques used by physiotherapists as well as acupuncture, and chiropractic and osteopathic techniques. Several different regimes have been described in the literature including supervised exercises, mobilisation, physiotherapy and use of electrotherapeutic interventions such as laser therapy and ultrasound. Mobilisation is therapist-applied passive movement of joints or other structures performed in such a way that they are always within the control of the patient. Electrotherapies include transcutaneous nerve stimulation (TENS), interferential therapy, short-wave diathermy (SWD) and pulsed short-wave diathermy (PSWD), and ultrasound. TENS and interferential therapy consist of electric pulses or currents and have an analgesic effect. SWD and PSWD use radio frequency energy to generate heat in tissues, which has an analgesic effect and reduces muscle spasm and joint stiffness. Ultrasound, mechanical vibration at very high speed, also generates heat

TABLE 1 Classification of interventions for frozen shoulder

\begin{tabular}{ll}
\hline Conservative treatment & Steroid injections \\
& Physical therapy \\
& Acupuncture \\
Invasive treatment & Sodium hyaluronate \\
& MUA \\
& Distension \\
& Capsular release \\
\hline
\end{tabular}


and has the same therapeutic effect as SWD and PSWD. ${ }^{6}$ Low-level laser therapy is nonthermal and is believed to reduce pain and inflammation, although the exact mechanism of its effect is unknown. ${ }^{16}$ Acupuncture is a form of ancient Chinese medicine in which fine needles are inserted into the skin at certain points on the body. Chiropractic uses a range of manual therapies, with an emphasis on manipulation of the spine, whereas osteopathy uses gentle stretching, massage and manipulation of muscles and joints. ${ }^{17}$

- Intra-articular corticosteroid injections to reduce inflammation and provide pain relief. A range of different doses and number of injections is described in the literature. This intervention is usually delivered in the primary care setting but also in the secondary care setting, depending on how services are organised in a particular region.

- Arthrographic distension (also called hydrodilatation), which involves controlled dilatation of the joint capsule with sterile saline or other solution such as local anaesthetic or steroid, guided by radiological imaging (arthrography). The procedure is performed under local anaesthetic.

- MUA, in which the shoulder is freed by rotation while the patient is under short general anaesthesia. This can be undertaken as a day procedure.

- Arthroscopic capsular release, a surgical procedure conducted under general or regional anaesthesia during which the contracted tissue is released. Open capsular release is another surgical option, usually recommended in those resistant to arthroscopic intervention. Both can be undertaken as a day procedure.

These interventions can be used individually or in combination, depending on the disease stage. There is currently not a consensus about the overall management of the condition and the sequence in which treatments should be offered to patients. A recent survey of $303 \mathrm{UK}$ health-care professionals [general practitioner (GPs), including those with a special interest, physiotherapists, advanced scope physiotherapists and orthopaedic surgeons] found that the professional groups had different views on the most appropriate treatment pathway for frozen shoulder ${ }^{18}$ However, there appears to be a fairly consistent view that the treatments used should depend on the phase of the disease and/or that a step-up approach should be adopted in terms of the degree of treatment invasiveness. ${ }^{5,12,18,19}$ There is a suggestion that aggressive mobilisation should be avoided in the early, severely painful phase..$^{12,14,19}$ Surgical intervention is generally, although not exclusively, used when the condition is resistant to the other interventions, ${ }^{19}$ although there is no consensus as to what time point or level of pain should indicate surgical intervention. ${ }^{18}$

The most commonly used or recommended interventions for the painful phase, in the recent UK survey of health-care professionals, were conservative treatment (watchful waiting, education, oral pain relief) and physical therapy (mainly physiotherapy and mobilisation), each recommended in one-third of responses, and intra-articular steroid injection, recommended in $18 \%$ of responses. For patients in the resolution phase, surgery was the most preferred option (mainly MUA and arthroscopic capsular release), recommended in almost half of the responses, followed by conservative treatment (12\%) and physical therapy (19\%).

\section{Previous systematic reviews}

Several systematic reviews had previously been undertaken on interventions for frozen shoulder, some of which focused on shoulder pain in general and included a range of conditions. Those interventions evaluated were oral steroids, ${ }^{20}$ corticosteroid injections, ${ }^{21-23}$ physiotherapy, ${ }^{24-26}$ acupuncture, ${ }^{27}$ arthrographic distension ${ }^{28}$ and multiple interventions ${ }^{29}$ (Table 2). None of the 
TABLE 2 Previous systematic reviews

\begin{tabular}{lll}
\hline Study & Intervention & End date for literature search \\
\hline Alvado $2001^{26}$ & Physical therapy & 1999 \\
Arroll 2005 & Corticosteroids & 2004 \\
Buchbinder 2006 & Oral steroids & 2005 \\
Buchbinder 2008 & Arthrographic distension & 2006 \\
Buchbinder 200321 & Corticosteroids & 2002 \\
Cleland $2002^{25}$ & Physical therapy & 2000 \\
Green $1998^{29}$ & Multiple intervention & 1995 \\
Green $2003^{24}$ & Physiotherapy & 2002 \\
Green $2005^{27}$ & Acupuncture & 2003 \\
Shah 200722 & Corticosteroid injections & 2006 \\
\hline
\end{tabular}

literature searches was recent and the reviews required updating (Table 2). In addition, some of the reviews did not report results by type of shoulder complaint. A recent review of systematic reviews on frozen shoulder noted the tendency to focus on a single intervention and that updated reviews were required. ${ }^{30}$

It is apparent from previous reviews that there is variation in how frozen shoulder is defined across studies. A review of 21 randomised controlled trials (RCTs) of interventions for frozen shoulder could not derive a consistent description of the condition from the trials investigating this patient group. ${ }^{2}$ Although the RCTs required that participants had restricted shoulder movement, there was inconsistency across trials in the number of degrees of restriction, the type of restriction (active or passive) and the direction of the restriction (abduction or external rotation). ${ }^{2}$ This highlights the difficulty of applying a strict definition for frozen shoulder within the context of a systematic review.

\section{Focus of the synthesis}

We undertook a systematic review of a range of NHS-relevant interventions for the treatment of primary frozen shoulder with the aim of informing a decision-analytic model. Previous reviews have tended to assess single treatments. The main focus was on comparing the main treatment options for frozen shoulder and identifying the most appropriate treatment by stage of frozen shoulder. Although variations within treatments were included in the synthesis, the main interest was in questions such as whether there is additional benefit from providing physiotherapy following steroid injection. Given the range of interventions being considered and the possibility that all treatment options would not have been compared in head-to-head in trials we planned to undertake a mixed-treatment comparison (MTC) in addition to pair-wise meta-analysis. MTC is an extension of traditional meta-analysis in which trials comparing the same intervention and the same comparator are pooled to estimate an overall treatment effect. A MTC overcomes the limitations of standard meta-analysis when there are no or few head-to-head comparisons or when the decision problem requires the comparison of several interventions. ${ }^{31,32}$ A ranking of interventions, based on the probability that each treatment is best,,$^{33}$ can be produced. This is of particular value when several treatment options are under consideration. 



\section{Chapter 2}

\section{Methods}

\section{Overview}

There were four planned components for the assessment: a systematic review of the clinical effectiveness of the interventions, a systematic review of patients' views and experiences of the treatments, a systematic review of studies assessing the cost-effectiveness of the treatments and an assessment of the cost-effectiveness of the alternative treatment options for primary frozen shoulder. At various stages of the assessment the review team consulted with an advisory group including clinicians, an economist, researchers undertaking other research on frozen shoulder, service users and a specialist in dissemination.

\section{Review of clinical effectiveness and cost-effectiveness}

The systematic review of studies of clinical effectiveness and cost-effectiveness was undertaken following the general principles outlined in Centre for Reviews and Dissemination (CRD) guidance. $^{34}$

\section{Literature searches}

The literature searches sought to identify papers on the use of physical therapies, steroid and other shoulder injections, MUA, arthrographic distension, capsular release, watchful waiting and combinations of these interventions for people with a primary diagnosis of frozen shoulder. A range of study types [systematic reviews, RCTs, economic evaluations, quasi-experimental studies (i.e. with a control group), case series of at least 50 participants] were eligible for inclusion and so the use of a study type filter in the search strategy was not considered appropriate.

The following databases were searched: MEDLINE, MEDLINE In-Process \& Other NonIndexed Citations, Cumulative Index to Nursing and Allied Health (CINAHL), EMBASE, Science Citation Index, BIOSIS Previews, Physiotherapy Evidence Database (PEDro), Cochrane Database of Systematic Reviews (CDSR), Database of Abstracts of Reviews of Effects (DARE), Health Technology Assessment (HTA) database, Cochrane Central Register of Controlled Trials (CENTRAL), PASCAL, Manual, Alternative and Natural Therapy (MANTIS), Latin American and Caribbean Health Sciences Literature (LILACS) and NHS Economic Evaluation Database (NHS EED). In the study protocol the Office of Health Economics Health Economic Evaluations Database (OHE HEED) was listed as one of the databases to be searched but access to this database was not available at the time that the searches were conducted. The searches were not restricted by language. The databases were searched from inception up to March 2010 (22 March for MEDLINE). Update searches were undertaken of MEDLINE and EMBASE on 5 January 2011.

In addition, information on studies in progress, unpublished research or research reported in the grey literature was sought by searching a range of relevant databases including Conference Proceedings Citation Index: Science, Health Management Information Consortium (HMIC), 
ClinicalTrials.gov and National Technical Information Service (NTIS). Furthermore, the reference lists of relevant systematic reviews were checked to identify further studies.

The websites of some relevant organisations were also scanned: the National Institute of Arthritis and Musculoskeletal and Skin Diseases (NIAMS) (www.niams.nih.gov/default.asp), the British Elbow \& Shoulder Society (BESS) (www.bess.org.uk/), the National Physiotherapy Research Network (www.csp.org.uk/director/members/research/networkingandsupport/ nationalphysiotherapyresearchnetwork.cfm) and the Primary Care Rheumatology Society (www. pcrsociety.org/).

The search strategy was developed using the MEDLINE database as the primary database (Table 3). In terms of the PICOS formula (patient, intervention, comparator, outcome, study design), the search strategy focused on population and intervention. The strategy was constructed so that lines 1-18 contain the various terms for frozen shoulder with these being combined at line 19. In the remainder of the strategy potential interventions for this condition are grouped together and then subsequently combined with line 19 . For example, lines 28-30 seek to retrieve records for arthroscopy, arthroscopic release or capsular release, all combined in line 31 and subsequently further combined with line 19 so that line 32 potentially identifies records containing terms for both frozen shoulder and arthroscopy. A similar approach has been used throughout the strategy with each of the potential interventions.

One of the particular challenges in constructing this search strategy was that there is no $\mathrm{MeSH}$ directly corresponding to 'frozen shoulder' or 'adhesive capsulitis.' The nearest MeSH available, 'bursitis', refers to knee joints and elbow joints as well as the shoulder joint so it is inevitable that redundant records will be identified by the search. The inclusion of free-text terms in the strategy is therefore essential.

During the development of the search strategy a small number of records were identified in which neither the title of the paper nor the abstract contained reference to a potential intervention. As the article titles appeared to indicate that the papers could be potentially useful, lines 1-6 at the beginning of the MEDLINE search strategy focus simply on identifying and retrieving records that have 'frozen shoulder', etc. in the title of the record. This assumes that when the term appears in the title there is a high likelihood that it could be relevant even if no intervention is specifically mentioned.

The strategies from all of the databases are given in full in Appendix 1.

\section{Inclusion and exclusion criteria}

Studies were included in the review if they met the criteria below, which are also summarised in Table 4.

\section{Population}

Participants with idiopathic (primary) frozen shoulder were included. We took a pragmatic approach and included studies based on the authors' definition of frozen shoulder to ensure that we identified all of the relevant evidence. Ideally, only patients with loss of active and passive external rotation of the involved shoulder with normal radiographic findings would be included. This would allow for exclusion of patients with arthritis of the shoulder, which can present as a similar clinical picture. However, such information is not always available in reports of trials. 


\section{TABLE 3 MEDLINE search strategy}

OvidSP interface; Ovid MEDLINE(R), 1950 to March Week 22010

3158 records identified, update search (5 January 2011) identified 98 records

1. (frozen adj6 shoulder\$).ti. (272)

2. (stiff\$adj3 shoulder\$).ti. (93)

3. (adhesive adj (capsulitis or capsulitides)).ti. (165)

4. ((bursitis or bursitides) adj6 shoulder\$).ti. (27)

5. ((capsulitis or capsulitides) adj6 shoulder\$).ti. (89)

6. 1 or 2 or 3 or 4 or $5(552)$

7. (frozen adj6 shoulder\$).ab. (466)

8. (stiff $\$ a d j 3$ shoulder\$).ab. (307)

9. exp bursitis/ (3095)

10. (adhesive adj (capsulitis or capsulitides)).ab. (298)

11. ((bursitis or bursitides) adj6 shoulder $\$$ ).ab. (45)

12. ((capsulitis or capsulitides) adj6 shoulder\$).ab. (161)

13. ((periarthritis or peri-arthritis or periarthritides or peri-arthritides or peri-capsulitis or pericapsulitis) adj6 shoulder\$).ti,ab. (175)

14. shoulder pain/ (1799)

15. (shoulder\$adj3 (pain or pains or painful or complain\$)).ti,ab. (3905)

16. Shoulder Impingement Syndrome/ (929)

17. (shoulder\$adj6 impinge\$).ti,ab. (496)

18. subacromial impingement syndrome.ti,ab. (110)

19.7 or 8 or 9 or 10 or 11 or 12 or 13 or 14 or 15 or 16 or 17 or $18(8926)$

20. Arthrography/ (3671)

21. (arthrograph\$adj6 (distension\$or distention\$)).ti,ab. (36)

22. (arthrogram\$adj6 (distension\$or distention\$)).ti,ab. (1)

23. (glenohumeral adj6 (distension\$or distention\$)).ti,ab. (9)

24. Dilatation/ (7623)

25. (dilatation or hydrodilat\$).ti,ab. (34,251)

26. or/20-25 (43,572)

27. 19 and 26 (213)

28. Arthroscopy/ $(12,449)$

29. (arthroscop\$adj6 (releas\$or decompress\$or capsulotom\$)).ti,ab. (568)

30. ((capsular adj2 releas\$) or interventional microadhesiolysis or capsulotomy).ti,ab. (1953)

31. or/28-30 (14,377)

32. 19 and 31 (778)

33. Injections, Intra-Articular/ (4409)

34. 33 and 19 (259)

35. injections/ $(29,104)$

36. 35 and 19 (67)

37. ((bursa $\$ o r$ intrabursa $\$$ or intra bursa $\$ o r$ periartic $\$$ or peri artic\$or intraartic $\$ o r$ intra artic\$) adj3 inject\$).ti,ab. (3026)

38. 37 and 19 (132)

39. ((subacromial or acromioclavicular or glenohumeral) adj3 inject\$).ti,ab. (144)

40. ((extra articular or extraarticular or shoulder\$) adj3 inject\$).ti,ab. (153)

41.34 or 36 or 38 or 39 or 40 (572)

42. exp Physical Therapy Modalities/ $(95,938)$

43. (physiotherapy or physiotherapies or physical therap\$or manual therap\$).ti,ab. $(16,547)$

44. (passive adj (motion or movement)).ti,ab. (1400)

45. CPM.ti,ab. (3594) 


\section{TABLE 3 MEDLINE search strategy (continued)}

46. muscle stretching exercises/ (333)

47. (stretching or stretches).ti,ab. $(15,092)$

48. (mobilisation or mobilization).ti,ab. $(32,663)$

49. (exercise\$adj2 (program\$or strength\$or intervention\$or training or prescription\$or prescrib\$)).ti,ab. $(15,121)$

50. (exercise\$adj2 (therap\$or therapeutic)).ti,ab. (2787)

51. ((home or supervis\$) adj2 exercis\$).ti,ab. (1536)

52. ((pendular or pendulum) adj exercis\$).ti,ab. (13)

53. ((isokinetic or resist\$) adj2 exercise \$).ti,ab. (2412)

54. or/42-53 (165,811)

55. 19 and 54 (1251)

56. exp Musculoskeletal Manipulations/ (9623)

57. chiropractic\$.ti,ab. (2520)

58. osteopath\$.ti,ab. (3141)

59. (manipulat\$adj3 (anesthesia or anaesthesia or anesthetic\$or anaesthetic\$)).ti,ab. (317)

60. MUA.ti,ab. (752)

61.56 or 57 or 58 or 59 or $60(15,100)$

62. 19 and 61 (223)

63. (TENS or ALTENS).ti,ab. (4717)

64. ((electric\$adj2 stimulat\$) or (transcutaneous adj2 stimulat\$) or (transdermal adj2 electrostimulat\$) or (cutaneous adj2 electrostimulat\$) or electroanalgesia or electro analgesia).ti,ab. $(43,717)$

65. (muscle adj2 stimulat\$).ti,ab. (4333)

66. (neuromodulation or neuro modulation or neurostimulation or neuro stimulation).ti,ab. (2177)

67. interferential.ti,ab. (204)

68. or/63-67 (53,102)

69. 19 and 68 (79)

70. biofeedback.ti,ab. (3972)

71. Biofeedback, Psychology/ (5327)

72. or/70-71 (6440)

73. 19 and $72(18)$

74. cryotherapy/ (2779)

75. ice/ (2686)

76. diathermy/ (2482)

77. hyperthermia, induced/ (9561)

78. hot temperature/ $(82,248)$

79. ((cold or ice or heat or hot) adj (pack\$or therap\$or treat\$)).ti,ab. $(10,182)$

80. (thermograph\$or thermotherap\$or thermo therap\$or hypertherm\$or hyper therm\$or diatherm\$or cryotherap\$or cryo therap\$).ti,ab. $(33,180)$

81. or/74-80 (123,890)

82. 19 and 81 (103)

83. exp Laser Therapy/ $(41,566)$

84. ultrasonic therapy/ (6801)

85. ultrasound.ti,ab. (105,682)

86. Ultrasonography, Interventional/ (9172)

87. (electrotherapeutic adj (intervention\$or treat\$)).ti,ab. (7)

88. or/83-87 (153,673)

89. 19 and 88 (375)

90. magnetic field therapy/(279)

91. pulsed electromagnetic field therapy.ti,ab. (22)

92. ((electromagnetic\$or magnetic\$) adj3 field\$).ti,ab. $(17,098)$ 
TABLE 3 MEDLINE search strategy (continued)

93. (biomagnetic $\$$ or bio magnetic\$or pulsed signal).ti,ab. (345)

94. PEMF.ti,ab. (264)

95. or/90-94 $(17,449)$

96. 19 and 95 (16)

97. nerve block/ $(10,624)$

98. neuromuscular blockade/ (1083)

99. (nerve adj2 block\$).ti,ab. (5976)

100. or/97-99 $(14,316)$

101. 19 and 100 (129)

102. exp Acupuncture Therapy/ $(12,086)$

103. acupuncture\$.ti,ab. (9788)

104. (electroacupuncture\$or electro acupuncture\$).ti,ab. (1968)

105. (osteopuncture\$or osteo puncture\$).ti,ab. (1)

106. (perioste\$adj3 (stimulat\$or therap\$or needling)).ti,ab. (134)

107. or/102-106 (13,946)

108. and 107 (110)

109. massage/ (3834)

110. (massag\$or acupressure or shiatsu or shiatzu or zhi ya or chih ya).ti,ab. (5864)

111. 09 or 110 (7657)

112. 19 and 111 (61)

113. (rehabilitat\$adj2 (program\$or protocol\$)).ti,ab. (8627)

114. 19 and 113 (125)

115. ((watch\$adj2 wait\$) or (conservative adj2 treat\$)).ti,ab. $(20,170)$

116. 19 and 115 (243)

117. (management adj2 (decision\$or option\$or choice\$)).ti,ab. (6499)

118. 19 and 117 (6)

119. 114 or 116 or $118(361)$

120. 6 or 27 or 32 or 41 or 55 or 62 or 69 or 73 or 82 or 89 or 96 or 101 or 108 or 112 or 119 (3236)

121. limit 120 to yr="1966 -Current" (3158)

\section{TABLE 4 Inclusion criteria}

\begin{tabular}{ll}
\hline $\begin{array}{l}\text { Population } \\
\text { Intervention }\end{array}$ & $\begin{array}{l}\text { Participants with idiopathic (primary) frozen shoulder (adhesive capsulitis) as defined by the authors, with or without diabetes } \\
\text { Physical therapies, arthrographic distension, steroid injection, sodium hyaluronate injection, MUA, capsular release, watchful } \\
\text { waiting }\end{array}$ \\
$\begin{array}{l}\text { Comparator } \\
\text { Outcomes }\end{array}$ & $\begin{array}{l}\text { Any of the above, no treatment or placebo } \\
\text { Study design }\end{array}$ \\
$\begin{array}{l}\text { RCTs; in the absence of randomised trials, quasi-experimental studies (i.e. with a control group). If controlled trials were not } \\
\text { available for MUA or capsular release, case series of at least } 50 \text { participants }\end{array}$ \\
$\begin{array}{l}\text { Studies of } \\
\text { economic } \\
\text { evaluations }\end{array}$
\end{tabular}

ROM, range of movement. 
Studies of general shoulder conditions were included only if outcome data were reported separately for participants with primary frozen shoulder, and studies of mixed populations of primary and secondary frozen shoulder were included only when at least $90 \%$ of the participants had primary frozen shoulder or data were reported separately for the two groups. Frozen shoulder in people with diabetes is defined as primary in some classifications and as secondary in others. Patients with diabetes were included as primary frozen shoulder.

\section{Intervention}

The following interventions, or combinations of these, were eligible for inclusion:

- physical therapies (including physiotherapy, acupuncture, chiropractic and osteopathy interventions); physiotherapy encompasses a wide range of techniques including mobilisation, biofeedback, ultrasound and laser therapy and all therapies falling under the physiotherapy umbrella were included

- distension

- steroid and other shoulder injections such as sodium hyaluronate

- MUA

- capsular release (arthroscopic and open)

- the approach of 'watchful waiting' (as defined by the authors but including education and advice about mobilisation within pain limits, home exercise and use of pain relief).

There are a number of other treatments that have been researched for frozen shoulder that are not commonly used on the NHS, such as radiotherapy, collagenase injection, salmon calcitonin and antibodies to tumour necrosis factor- $\alpha$. These interventions were excluded. Sodium hyaluronate is not licensed for use in frozen shoulder but a recent survey reported its use by a few respondents and therefore it was included in the review to establish the evidence base.

\section{Comparator}

Studies using any of the above treatments as a comparator (including studies comparing different regimens of the same intervention), no treatment or placebo were included. The two exceptions to this were acupuncture and sodium hyaluronate. Dose-ranging studies of sodium hyaluronate were excluded. Studies of acupuncture were included only when the comparator was one of the other interventions of interest in the review. Therefore, studies comparing more than one type of acupuncture or comparing acupuncture to an alternative therapy such as moxibustion were excluded.

\section{Outcomes}

The outcomes of interest were pain (e.g. at rest, on movement, at night); range of movement (e.g. internal and external rotation, elevation); function and disability; quality of life; time to recovery, return to work and recreation; and adverse events.

\section{Study design}

Only RCTs were eligible for inclusion where this level of evidence was available on an intervention/management strategy. In the absence of randomised trials, quasi-experimental studies (i.e. with a control group) were included. If controlled trials were not available for MUA or capsular release, case series of at least 50 participants were included. It is unclear what size a good-quality case series should be and therefore this was chosen as an arbitrary cut-off; it was considered an achievable size of case series for this field while maximising the possibility of a representative sample of patients.

The intention was to include and update systematic reviews if (1) they fulfilled all the relevant inclusion criteria, (2) they had no significant sources of error and bias and (3) they were reported 
in detail and the raw data are available from the report or authors to allow an update of the synthesis (if searches are more than 12 months out of date). Otherwise they would be screened as an additional source of studies. Previous systematic reviews focused on individual interventions and had variable inclusion criteria and therefore updating of individual reviews was not appropriate. The reference lists of reviews were screened for additional studies.

\section{Full economic evaluations}

Full economic evaluations that met the population and intervention inclusion criteria were eligible for inclusion. A full economic evaluation was defined as any study in which a comparison of two or more relevant alternatives was undertaken with costs and outcomes examined separately for each alternative. This included cost-effectiveness analyses (including cost-consequence analysis), in which health outcomes are expressed in natural units; cost-utility analysis, in which benefits are measured in utility units or utility-weighted life-years; and cost-benefit analyses, in which benefits are measured in monetary form using approaches such as 'willingness to pay' or 'human capital approach'.

\section{Screening and study selection}

Two researchers independently screened all titles and abstracts identified from the searches to identify potentially relevant studies. Full manuscripts of potentially relevant studies were ordered and two researchers independently assessed the relevance of each study using the criteria above. Disagreements were resolved by consensus and, if necessary, a third researcher was consulted.

\section{Data extraction}

Descriptive data extracted included study design, number randomised, loss to follow-up, country, setting, inclusion criteria, population characteristics, description of the intervention including duration and intensity, concomitant treatments and outcome measures used. These data were extracted by one researcher using a pre-piloted standardised data extraction form in Evidence for Policy and Practice Information (EPPI)-Reviewer 3 (EPPI-Centre, Institute of Education, University of London, UK). Number of participants randomised, number included in each analysis, mean and standard deviation (SD) were extracted into a pre-piloted Microsoft Excel 2007 spreadsheet (Microsoft Corporation, Redmond, WA, USA). Descriptive and outcome data were checked by a second reviewer and discrepancies were resolved through discussion and, if necessary, a third opinion was sought.

For continuous outcomes the post-intervention (final value) mean and SD for each group were extracted, where available, as first preference. Otherwise, change scores (the difference between baseline and follow-up) and SD for each group were extracted, and the between-group difference in change and SD where data for the individual groups were not available. Data available only in graph format were not extracted; authors were contacted for the actual data. Where only median and ranges were reported, these were extracted. Unadjusted data were extracted to allow for covariate analyses in the MTC. Where unadjusted data were not available, adjusted data were extracted and the type of adjustment recorded (two studies ${ }^{35,36}$ ). Standard data imputation methods were used, where necessary, to calculate SDs. ${ }^{37}$ If the SD or standard error (SE) was not reported, the variance was calculated from the $95 \%$ confidence interval (CI) or $p$-value (three studies $^{38-40}$ ). Where this information was not available, the SD was imputed based on the average SD across all interventions for that outcome (two studies ${ }^{35,41}$ ). As a final means of imputing SD, a simulation model written in the $\mathrm{R}$ programme was used. The model repeatedly sampled from the possible range of values of the outcome measure being used (e.g. 0-9 in the case of a Likert 
0-9 scale used to measure pain) to identify sequences whose mean equalled the mean reported in the trial of interest. Where the two means matched, the model calculated a SD; finally, the mean of these SDs was calculated and used in our analysis (two studies ${ }^{42,43}$ ). The $\mathrm{R}$ code is presented in Appendix 3.

Where the number of participants in an analysis was unclear, and the information was not available from the authors, the number randomised minus the number of dropouts was used.

\section{Assessment of risk of bias}

Quality assessment was also undertaken by one researcher and checked by a second with discrepancies resolved by consensus or recourse to a third researcher if necessary. Studies were assessed using the checklist in Appendix 4. The criteria for assessing randomised and nonrandomised trials were based on recent CRD guidance, ${ }^{34}$ the criteria for case series are based on those used in a recent systematic review including case series. ${ }^{44}$ The quality of economic evaluations was assessed using a modified version of the Drummond checklist. ${ }^{45}$

\section{Synthesis}

The synthesis had two main components: (1) a narrative synthesis, including pair-wise comparisons and (2) a MTC. The analysis was based on the protocol and an a priori analysis plan. The primary outcomes of interest were patient-assessed pain intensity, function and disability, quality of life and range of movement. Given that the symptoms of frozen shoulder change over time (with pain being the strongest characteristic of the early stages but not later), it was not appropriate to use a single primary outcome.

\section{Narrative synthesis and pair-wise comparisons}

A narrative and tabular summary of key study characteristics, quality assessment and results was undertaken. Studies were grouped by the main intervention of interest in the study and then by comparator. Where appropriate, based on clinical and statistical heterogeneity and the necessary data being available, individual study results were combined in a pair-wise meta-analysis based on type of intervention and comparator using RevMan 5 (The Cochrane Collaboration, The Nordic Cochrane Centre, Copenhagen, Denmark). ${ }^{46} \mathrm{~A}$ random-effects model was used except when there were fewer than three studies when a fixed-effect model was used. Heterogeneity was assessed using the chi-squared test and the $I^{2}$ statistic. A chi-squared test with $p$-values $<0.1$, and $I^{2}$ values $>50 \%$ were taken to indicate that there was substantial statistical heterogeneity. ${ }^{34,47}$ When a quantitative synthesis was not appropriate the results of studies were discussed in a narrative. Studies reporting median rather than mean values were discussed in the narrative synthesis only.

\section{Outcomes}

Pain was measured using several different outcome scales; therefore, the standardised mean difference (SMD) was calculated. Final value and change scores were not combined together as SMDs, as the difference in SD reflects differences in measurement. Scales were amended, where necessary, so that an increase in score equated to an increase in pain according to the Cochrane Handbook. ${ }^{48}$ The SMD measure used was Hedges' $g$ as this is considered appropriate for studies with small sample sizes. ${ }^{49}$

The interpretation of the SMD is not as intuitive as the interpretation of the original measures of treatment effect. To address this issue, the pooled SMD was back transformed to an original 
measure by multiplying it with a 'typical' SD of one of the pain measures. A visual analogue scale (VAS) 0-100 mm was chosen, as this measure of pain, compared with others used by studies included in the review, had the largest volume of literature regarding minimum clinically important differences (MCIDs) in pain. This included one study performed in a shoulder-specific population. ${ }^{50}$ Transformation from SMD to VAS $0-100 \mathrm{~mm}$ was made using a representative pooled SD at baseline from one of the included trials. ${ }^{51}$ It should be noted that this was only appropriate for pooled rather than individual study SMDs. ${ }^{52}$ The reduction in pain recorded as a SMD can be interpreted on a VAS $0-100 \mathrm{~mm}$ using Table 5. No data could be located on the MCID in pain for individuals with frozen shoulder; however, it is suggested that a difference of $14 \mathrm{~mm}$ on a 100-mm VAS scale is the MCID for individuals with rotator cuff disease, a shoulder disorder also characterised by pain and restricted range of movement. ${ }^{50}$ Given that the betweengroup MCID is thought to approximate $40 \%$ of that within individuals, ${ }^{6,53}$ the MCID between groups for rotator cuff disorder can be estimated to be approximately $5.6 \mathrm{~mm}$. This value is therefore used as a proxy for the between-group MCID for frozen shoulder.

A wide range of function and disability scales were reported in the studies. These outcomes were not converted to a SMD as they were not considered similar enough. This was because there were differences between some scales in the aspects of function and disability assessed and in the weighting given to similar components. In addition, the correlation between some of the included scales was at best moderate. ${ }^{54-56}$

Because of the large number of different range of movement measures used, following discussion with the advisory group these were prioritised for the analyses. The passive range of movement outcomes of interest were passive external rotation, passive internal rotation and passive abduction; and the active range of movement outcomes were active external rotation, active internal rotation and active abduction. When passive and active range of movement was not reported separately, unspecified external rotation, internal rotation and abduction were used in the synthesis. When active internal rotation was not reported but hand behind back was, this was used as a proxy measure, although there is some evidence that the correlation between the two is low to moderate. ${ }^{57}$

\section{Interventions}

As stated above, studies were grouped by type of intervention in the narrative synthesis and pair-wise comparisons. In addition, subgroups were created within interventions. These subgroups were specified a priori to identify clinically similar studies for pooling in the pair-wise comparisons and MTC to guide the exploration of similarities and differences within classes of interventions in the narrative synthesis. These subgroups were as follows:

- Active physical therapy and physical therapy without mobilisation. Active physical therapy (or physical therapy with mobilisation) was defined as an intervention in which at least part of the intervention involved the patient's body being wholly or partly in motion, such as exercise, mobilisation and stretching (with or without passive techniques such as heat treatment). Interventions in which there was no such active mobilisation or stretching and

TABLE 5 Standardised mean differences and equivalent reductions on the VAS $0-100 \mathrm{~mm}$ scale

\begin{tabular}{llllll}
\hline \multicolumn{7}{c}{ Reduction in pain score } & & & \\
\hline SMD & -0.3 & -0.5 & -1.0 & -1.5 & -2.0 \\
VAS 0-100 mm & -5.6 & -9.15 & -18.3 & -27.45 & -36.6 \\
\hline
\end{tabular}


the participant was the passive recipient of a therapy such as laser therapy, TENS, ultrasound, heat treatment or ice packs alone were classified as physical therapy without mobilisation.

- Steroid injections were grouped by the number of injections - three or fewer injections and more than three injections. This was based on advice from the advisory group that patients in the UK are unlikely to receive more than three injections for frozen shoulder; therefore, this was the cut-off used.

\section{Length of follow-up}

Follow-up of $\leq 4$ weeks was not included in the analysis as it was not considered to be informative. Where studies did not report the same length of follow-up, outcomes were pooled grouped by short-, medium- and long-term follow-up. For short-term follow-up the data point from each study at 3 months' follow-up or the closest data point before 3 months' follow-up was used. For the outcome of pain, where pair-wise comparisons were possible, the same short-term follow-up data used for the MTC at 3 months were pooled. This was to enable comparison of results from the pair-wise comparisons and the MTC. For medium-term follow-up the data point at 6 months or the closest data point before 6 months was used. For long-term follow-up the data point at 12 months or the closest data point before 12 months was used. Data were also presented (when reported) at multiple times within a follow-up period (e.g. 6 weeks and 3 months). When only a narrative synthesis was possible, data were discussed using the same categories.

\section{Unit of analysis error}

Some of the included studies had a unit of analysis error, that is, patients were randomised to the intervention but outcome was reported by shoulder when a participant had two frozen shoulders. In these instances the number of patients was used as the denominator in the analysis as only a few patients had more than one shoulder involved; therefore, the difference between number of shoulders and number of participants was small. ${ }^{58}$

\section{Multi-arm trials}

Where pooling of multi-arm trials required the use of a control group more than once in the same meta-analysis, the number of participants in the control group was divided approximately evenly among the comparisons, with the means and SDs left unchanged. Where different arms were assessing minor variations of a class of intervention these were combined before pooling. ${ }^{37}$

\section{Subgroup analyses}

Subgroup analyses were planned to explore the influence of the following study characteristics in the MTC and pair-wise comparisons:

- inclusion of patients with diabetes

- stage of frozen shoulder

- study quality (with studies stratified by concealment of allocation and by blinding of outcome assessors).

Because of the small number of studies suitable for pooling it was only possible to explore these factors in the narrative.

\section{Mixed-treatment comparison}

A MTC was undertaken. A MTC, also referred to as network meta-analysis, goes beyond the scope of the standard pair-wise meta-analysis in which direct within-trial results are pooled in a weighted manner, and provides a mechanism for bringing together both direct and indirect evidence. The approach allows data networks that include A versus B trials, B versus $C$ trials and A versus $C$ trials to be used to make inferences about the relative efficacy of all treatments. ${ }^{33}$ This allows estimation of comparisons not previously considered in a clinical trial to be made. As the 
summary treatment effect from each trial is used to inform the MTC, the randomisation of the trial is maintained within the analysis.

As with standard meta-analysis, to facilitate this type of analysis a number of conditions need to hold:

- Homogeneity assumption - that trials are sufficiently homogeneous to be quantitatively combined.

- Consistency assumption - that had treatment $\mathrm{C}$ been included in a trial comparing interventions $\mathrm{A}$ and $\mathrm{B}$, then the treatment effect $\mathrm{dAC}$ would be equivalent to that obtained from a trial of interventions $A$ and $C .{ }^{33}$ Assuming consistency, the treatment effect dAC is the sum of the treatment effects $\mathrm{AAB}$ and $\mathrm{dBC}$.

- Methodological similarity - that trial methods are similar as relative effects from trials may be associated with the quality of the study, particularly when subjective outcome measures are used. ${ }^{59}$

The MTC analysis was conducted in WinBUGS version 1.4.3 (MRC Biostatistics Unit, Cambridge, UK), ${ }^{60}$ which is a Bayesian analysis software that utilises Markov chain Monte Carlo (MCMC) methods. A Bayesian framework involves a formal combination of a prior probability distribution (which reflects our belief about the possible values of the pooled effect) and a likelihood function (which informs the distribution of the pooled effect based on the observed data) to obtain a posterior probability distribution of the pooled effect. As the outcome for this analysis was the treatment effect difference, a normal likelihood distribution was used for the treatment effect data. A random-effects model was specified as it is likely that, although the treatment effects may be drawn from a common distribution, because of clinical and methodological variations, the true effect size is unlikely to be exactly the same.

Mixed-treatment comparison methods can be applied only to a connected network of RCTs. Because of the diversity of outcomes reported in the clinical trials the number of network options was limited. We were unable to standardise function and disability measures and given the variety reported no network was available. Range of movement outcomes were also not reported in a systematic manner in trials, leaving no network available. As previously stated it was possible to standardise the different pain outcome measures using the SMD; therefore, pain was the only outcome available to undertake a MTC.

Some studies reported more than one type of pain outcome; therefore, for the MTC one pain measure was selected from each study using a hierarchy based on the clinical significance of the type of pain. This was established following discussions with the advisory group, including individuals with frozen shoulder, and after exploring the literature. When more than one pain outcome was reported in a study a single measure was chosen for the MTC analysis in the following order: overall pain, pain at night, pain on activity, any other type of pain (excluding pain at rest) and pain at rest.

Only RCTs reporting final values and SDs (or when data were available to calculate final values or to impute SDs) were eligible for inclusion in the MTC. The model fit was assessed using the residual deviance. A value close to the number of data points in the data set reflects a 'good fit'. The MCMC simulation requires initial values to be specified for all parameters that are modelled as distributions. It is anticipated that following a sufficient burn-in period the chain will approach a stationary distribution. Convergence to a stable estimate should take place regardless of initial values. This was assessed using diagnostic tools provided within the WinBUGS package, including graphical time series trace of starting values for the selected parameters, the BrooksGelman-Rubin statistic and posterior distributions. The first 10,000 iterations were used as 
'burn-in' and discarded; subsequently, a further 100,000 iterations were performed. Uncertainty was presented using the upper and lower limits of $95 \%$ credible intervals (CrIs), which describe the bounds within which it is believed there is a $95 \%$ chance that the true value lies.

The selection of the prior distributions is extremely important, particularly when there is limited effectiveness data. In a situation in which we have no information and we wish to include non-informative priors, it is important to check that the selections are truly non-informative. Prior distributions are given to those parameters being estimated by the model. The combining of the prior and the data gives us the posterior distributions from which we sample. The prior for the between-study SD was set to be a uniform distribution with a range 0 to 2 . This covered the range of treatment effects within a particular comparison. However, when data are sparse, non-informative priors have an unintentionally large influence on the precision of the treatment effect, which can lead to different statistical inferences.$^{61}$ For this reason we undertook a number of sensitivity analyses to ensure that our prior was non-informative and allowed the model to reach convergence. For each network, a sensitivity analysis was performed by changing the range of the uniform prior distribution on the SD from 0-2 to 0-0.8, 0-5, 0-10 and, as a final check for stability for network 1, 0-15. An additional sensitivity analysis was also performed by repeating each analysis using a burn-in of 30,000 iterations.

\section{Systematic review of patients' views of interventions for frozen shoulder}

Given the range of possible treatment options for frozen shoulder, patient preference is an important factor in the treatments received by people with frozen shoulder as well as the sequence in which they try treatments when more than one type of treatment is necessary. We therefore undertook a systematic review of studies of patients' views of treatments for frozen shoulder. Three databases (CINAHL, MEDLINE and PsycINFO) were searched on 3 June 2010 to identify qualitative studies, with searches being restricted to English-language papers published from 1980 onwards. The search strategies used combined a set of terms for frozen shoulder/ adhesive capsulitis with a qualitative search filter. ${ }^{62}$ The search strategies used are given in full in Appendix 2.

Studies investigating patients' views or experiences about the treatments included in the main review were eligible for inclusion. Only English-language qualitative studies were included; expert opinion, letters containing no data on patient views, editorials and discussion papers were excluded.

The processes for study selection, data extraction and quality assessment followed those of the main review. Studies were selected independently by two researchers and disagreements were resolved through discussion.

The intention was to extract data on study aim, participant characteristics, methods of collecting data on patient views and experiences, method of analysis, results in the form of a summary of key themes arising from the analysis and authors' conclusions. Study quality was to be assessed using a tool developed by Hawker et al. ${ }^{63}$ and a narrative synthesis undertaken.

\section{Assessment of cost-effectiveness}

The intention was to develop a decision-analytic model, following National Institute for Health and Clinical Excellence (NICE) guidance on methods for technology appraisal, ${ }^{64}$ to estimate the cost-effectiveness of the different treatments for frozen shoulder. The specific objectives outlined 
in the proposal for the cost-effectiveness analysis were to (1) assess the cost-effectiveness of the named interventions for frozen shoulder to inform clinical practice and (2) identify the key uncertainties relating to the cost-effectiveness analysis and to use these to inform future research priorities. We reviewed the literature to:

- identify any existing modelling in a similar population

- identify treatments for this condition

- identify sequences or order of treatments

- explore whether treatments (or sequences of treatment) were influenced by phase of condition

- identify any potential sources of data to populate the model

- identify studies most relevant to the UK decision-maker.

The economic literature for this clinical area was found to be scant. The development of a full economic model was not possible. We present a full discussion around the issues of modelling treatments for frozen shoulder in Chapter 3 (see Decision model), but, in brief, a lack of clinical evidence and consensus regarding treatment options made the structuring and populating of a model unachievable.

In an attempt to present some information that will be useful to the decision-maker we undertook a number of supplementary investigative exercises. First, we used the advisory group to elicit details of resource use associated with the interventions for which evidence has been identified in the systematic review. In conjunction with the advisory group, cost estimates for these procedures were produced. Second, as our initial aim was to express health benefits in terms of quality-adjusted life-years (QALYs), we investigated the available evidence to identify utility outcomes. A search of the published literature was undertaken to find any published studies that had measured and reported health utility as an outcome. Following the NICE reference case $^{64}$ the European Quality of Life-5 Dimensions (EQ-5D) should be used to elicit individuals' preference for health states. The EQ-5D measures health on five attributes: mobility, self-care, usual activity, pain/discomfort and anxiety/depression. These data are then combined with the UK social tariff, which is based on 10 -year time trade-off data, ${ }^{65}$ and used to weight the time spent in a given health state. Third, we explored the possibility of mapping outcomes presented in the clinical trials from the systematic review to the EQ-5D using established methods. The aim of the mapping was to generate changes in EQ-5D and QALYs for the interventions assessed in the clinical review. We then present some basic exploratory calculations to identify interventions that might be cost-effective and worthy of further investigation, although we acknowledge that these calculations are extremely uncertain. 



\section{Chapter 3}

\section{Results}

\section{Assessment of clinical effectiveness}

\section{Study selection}

The searches identified 8883 records, 8341 from the original searches undertaken in March 2010, 521 from the update searches undertaken in MEDLINE and EMBASE in January 2011 and 21 from subsequent reference checking (Figure 1). On the basis of reviewing title and abstracts 8161 papers were excluded; 722 papers were ordered for a more detailed evaluation. Additionally, an author sent in one paper. Of the 723 papers, 67 were published in languages other than English. These included Chinese (22 papers), Russian (19 papers), Italian (6 papers), Japanese (6 papers), Dutch (5 papers), French (2 papers), Croatian (1 paper), German (1 paper), Hebrew (1 paper), Korean (1 paper), Norwegian (1 paper), Slovak (1 paper) and Turkish (1 paper).

After a detailed evaluation, 691 papers were excluded from the review, including four for which there was no translator available, and five that could not be obtained through the British Library (see Appendix 5 for a list of excluded studies). The main reasons for excluding studies from the review were that they were not a clinical study (146 studies) or they did not meet the study design criteria (193 studies) or the population criteria (244 studies). Of the studies excluded on the basis

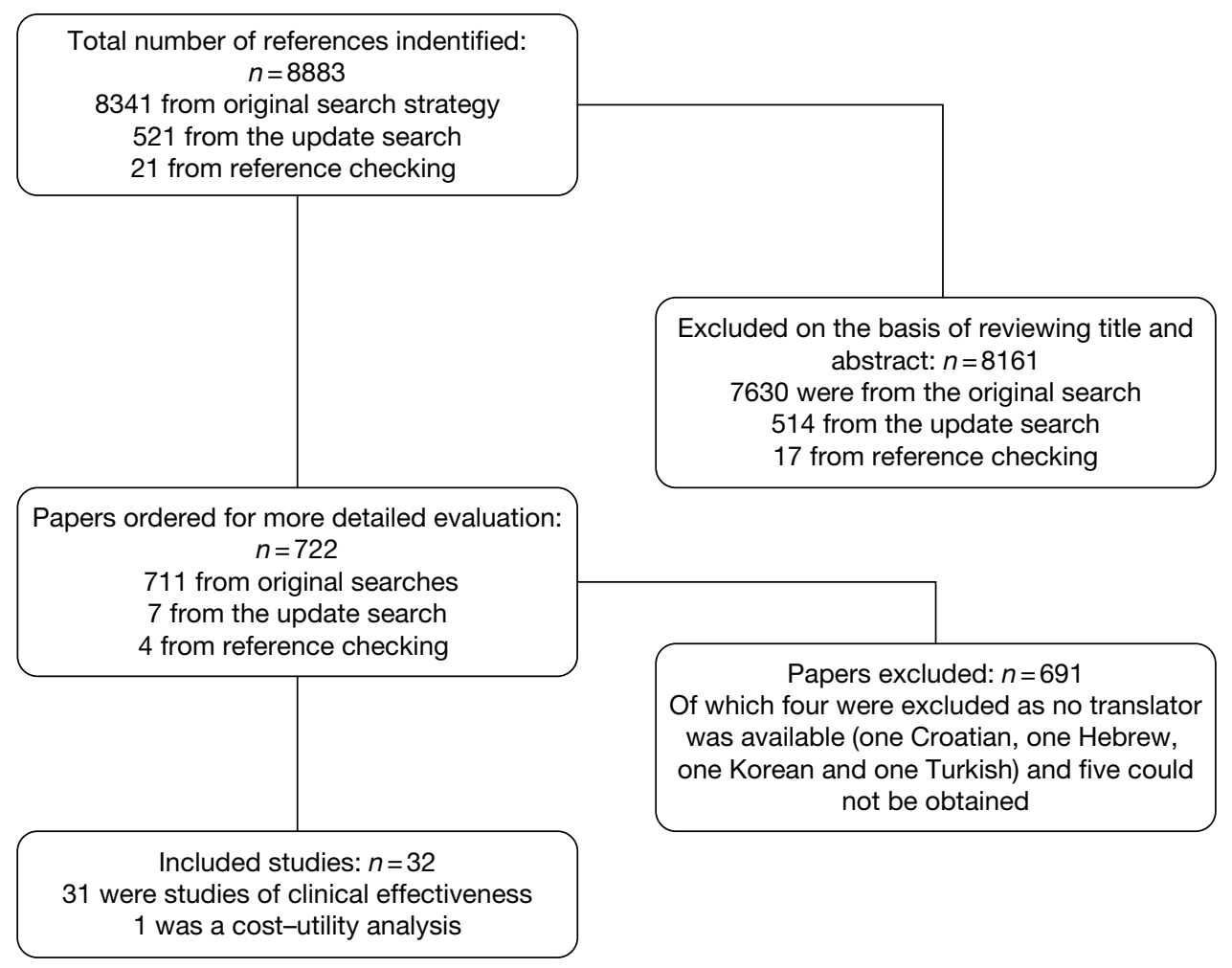

FIGURE 1 Flow chart of study selection. 
of population, in 42 studies it was unclear if the patients had primary frozen shoulder, whereas in 17 studies $<90 \%$ of patients in each study had primary frozen shoulder.

There were 32 studies included in the review, one of which was a cost-utility analysis conducted alongside a separately published study of effectiveness. Of the 32 studies, 28 were published in English, 2 in Chinese, 1 in Japanese and 1 in Norwegian.

Studies were grouped by the main treatment intervention of interest: six studies were of steroid injection (with and without physical therapy), three of sodium hyaluronate injection, twelve of physical therapy, three of acupuncture, four of MUA, three of distension and two of capsular release. One study, by Calis et al. ${ }^{66}$ fell into more than one category and was reported in all relevant sections (steroid injection, sodium hyaluronate injection and physical therapy). In one of the physical therapy studies there was a 'supervised neglect' comparator. The included studies were from a wide range of countries (Turkey, Canada, UK, USA, Italy, Japan, Hong Kong, China, Taiwan, the Netherlands, Singapore, Thailand, Greece, Pakistan, Finland, Australia, Denmark and Norway).

Although a quantitative synthesis was planned this was largely not possible, as few studies could be pooled. The overall quality of the studies was poor and there were few studies of the same comparators. Within the classes of intervention there was considerable variability between studies in various aspects of the intervention such as dose (e.g. of steroid or sodium hyaluronate injection), and duration and intensity of treatment, especially in terms of physical therapy and home exercise programmes. The physical therapy interventions encompassed a range of combinations of 'active' and 'passive' components. There was variability in the outcomes reported, the tools used to measure individual outcomes, time of follow-up and type of data reported. As a result there were few situations in which it was appropriate to pool and undertake the planned subgroup analyses. It was therefore necessary to primarily use narrative synthesis to present the findings of this review. The main analysis was the narrative synthesis with pair-wise comparisons where appropriate. This is presented first with studies grouped by intervention. This is followed by the MTC. This was a secondary, exploratory analysis because of the small number of studies that connected in the network.

\section{Steroid injection}

\section{Study characteristics}

Six RCTs investigated steroid injections, with or without the addition of physiotherapy, in the treatment of primary frozen shoulder. A summary of the study characteristics is reported in Table 6, with further details available in Appendix 6. All were full papers of studies conducted in the UK, ${ }^{41,67}$ Turkey, $^{6,68}$ Canada $^{35}$ and the USA ${ }^{42}$ between 1981 and 2008. There was one twoarmed trial, ${ }^{68}$ one three-armed trial ${ }^{67}$ and four four-armed trials ${ }^{35,41,42,66}$

The steroids administered were methylprednisolone acetate and triamcinolone hexacetonide. Two studies used a single injection of $20 \mathrm{mg}$ triamcinolone hexacetonide, ${ }^{41,67}$ one of which also administered lidocaine,${ }^{67}$ and three studies used a single injection of $40 \mathrm{mg}$ methylprednisolone acetate or triamcinolone hexacetonide. ${ }^{35,66,68}$ The remaining study administered three $40-\mathrm{mg}$ injections of methylprednisolone acetate plus lidocaine, one each week for 3 weeks. ${ }^{42}$ In one of the studies the injection was guided using fluoroscopy. ${ }^{35}$ Five of the studies reported administering an intra-articular injection, ${ }^{35,41,42,66,68}$ one of which compared an anterior and a lateral approach, ${ }^{42}$ and in one study it was unclear. ${ }^{67}$

The comparators were steroid injection plus physiotherapy, ${ }^{35,41,67}$ placebo injection, ${ }^{35,41,42,68}$ physiotherapy with ${ }^{35,41}$ and without ${ }^{66,67}$ placebo injection and home exercise alone. ${ }^{66}$ 
TABLE 6 Studies of steroid injections

\begin{tabular}{|c|c|c|c|}
\hline $\begin{array}{l}\text { Study details } \\
\text { and study } \\
\text { design }\end{array}$ & $\begin{array}{l}\text { Participants randomised } \\
\text { (total } n \text { and by group) }\end{array}$ & Trial treatments & Condition-related inclusion criteria \\
\hline $\begin{array}{l}\text { Bal 2008 } \\
\text { Two-arm RCT }\end{array}$ & $\begin{array}{l}n=80 \text { (82 shoulders) } \\
\text { Steroid injection: } 40 \text { (42) } \\
\text { Placebo injection: } 40(40)\end{array}$ & $\begin{array}{l}\text { Steroid: methylprednisolone acetate, } 40 \mathrm{mg} \text {, } \\
1 \mathrm{ml} \text {, one injection } \\
\text { Placebo: saline, } 0.9 \% \text { sodium chloride, } 1 \mathrm{ml} \text {, } \\
\text { one injection }\end{array}$ & $\begin{array}{l}\text { Presence of shoulder pain with limitation of } \\
\text { both active and passive movements of the } \\
\text { glenohumeral joint of } \geq 25 \% \text { in at least two } \\
\text { directions. Symptom duration between } 6 \text { weeks } \\
\text { and } 6 \text { months }\end{array}$ \\
\hline $\begin{array}{l}\text { Calis } 2006^{66} \\
\text { Four-arm RCT }\end{array}$ & $\begin{array}{l}n=90 \text { (95 shoulders) } \\
\text { Sodium hyaluronate } \\
\text { injection: } 24 \text { (27) } \\
\text { Steroid injection: } 25 \text { (26) } \\
\text { PT: } 21 \text { (22) } \\
\text { No intervention (home } \\
\text { exercise only): } 20(20)\end{array}$ & $\begin{array}{l}\text { Sodium hyaluronate: } 30 \mathrm{mg} \text {, one injection } \\
\text { once weekly for } 2 \text { weeks. } \\
\text { Steroid: triamcinolone acetonide, } 40 \mathrm{mg} \text {, one } \\
\text { injection } \\
\text { PT: } 10 \text { daily sessions at least } 45 \text { minutes }\end{array}$ & $\begin{array}{l}\text { History of pain for at least } 1 \text { month; limited active } \\
\text { and passive shoulder movement; decreased } \\
\text { passive ROM of } 20 \% \text { or more, in at least three } \\
\text { movements, according to the American Medical } \\
\text { Association guide for the evaluation of permanent } \\
\text { impairment }\end{array}$ \\
\hline $\begin{array}{l}\text { Carette } 2003^{35} \\
\text { Four-arm RCT }\end{array}$ & $\begin{array}{l}n=93 \\
\text { Steroid injection: } 23 \\
\text { Placebo injection: } 23 \\
\text { Steroid + PT: } 26 \\
\text { PT + placebo: } 23\end{array}$ & $\begin{array}{l}\text { Steroid: triamcinolone hexacetonide, } 40 \mathrm{mg} \text {, } \\
2 \mathrm{ml} \text {, one injection } \\
\text { Placebo: saline, } 2 \mathrm{ml} \text {, one injection } \\
\text { PT: } 12 \times 1 \text {-hour sessions, } 3 \text { times per week } \\
\text { for } 4 \text { weeks }\end{array}$ & $\begin{array}{l}\text { Adhesive capsulitis defined as the presence of } \\
\text { shoulder pain with limitation of both active and } \\
\text { passive movements of the glenohumeral joint } \\
\text { of } \geq 25 \% \text { in at least two directions compared } \\
\text { with contralateral shoulder or normal values. } \\
\text { Symptomatic for }<1 \text { year }\end{array}$ \\
\hline $\begin{array}{l}\text { Dacre } 198967 \\
\text { Three-arm } \\
\text { RCT }\end{array}$ & $\begin{array}{l}n=66 \text { (data reported for } \\
62 \text { ) } \\
\text { Steroid injection: } 22 \\
\text { Steroid + PT: } 20 \\
\text { PT: } 20\end{array}$ & $\begin{array}{l}\text { Steroid: triamcinolone, } 20 \mathrm{mg}, 1 \mathrm{ml} \text { with } 2 \% \\
\text { lidocaine, one injection } \\
\text { PT: 4-6 weeks' duration }\end{array}$ & $\begin{array}{l}\text { Painful stiff shoulder for at least } 4 \text { weeks; inability } \\
\text { to use arm with restriction of movement and } \\
\text { loss of full function; pain at night causing sleep } \\
\text { disturbance and inability to lie on affected side }\end{array}$ \\
\hline $\begin{array}{l}\text { Rizk } 1991^{42} \\
\text { Four-arm RCT }\end{array}$ & $\begin{array}{l}n=48 \\
\text { Steroid, (IA) + PT: } 16 \\
\text { Steroid, (IB) + PT: } 16 \\
\text { Placebo injection (IA) + PT: } 8 \\
\text { Placebo injection (IB) + PT: } 8\end{array}$ & $\begin{array}{l}\text { Steroid: methylprednisolone acetate, } 40 \mathrm{mg} \text {, } \\
1 \mathrm{ml} \text { with } 2 \mathrm{ml} \text { of } 1 \% \text { lidocaine. Once weekly } \\
\text { for } 3 \text { weeks IA (using anterior approach) or } \\
\text { IB (using lateral approach) } \\
\text { PT: } 11 \text { weeks } \\
\text { Placebo: } 1 \% \text { lidocaine once-weekly for } \\
3 \text { weeks IA or IB }\end{array}$ & $\begin{array}{l}\text { Total ROM }<50 \% \text { of normal range (i.e. }<320^{\circ} \text { ); } \\
\text { shoulder pain for }<6 \text { months, nocturnal } \\
\text { accentuation of pain, no effusion in the } \\
\text { glenohumeral joint }\end{array}$ \\
\hline $\begin{array}{l}\text { Ryans } 2005^{41} \\
\text { Four-arm RCT }\end{array}$ & $\begin{array}{l}n=80 \\
\text { Steroid injection: } 20 \\
\text { Steroid + PT: } 20 \\
\text { PT + placebo injection: } 20 \\
\text { Placebo injection: } 20\end{array}$ & $\begin{array}{l}\text { Steroid: triamcinolone, } 20 \mathrm{mg}, 1 \mathrm{ml} \text { and } \\
\text { normal saline, } 2 \mathrm{ml} ; 1.5 \mathrm{ml} \text { injected by an } \\
\text { anterior approach and } 1.5 \mathrm{ml} \text { by a lateral } \\
\text { approach } \\
\text { Placebo: saline ( } 3 \mathrm{ml}) \text { administered as per } \\
\text { steroid } \\
\text { PT: eight sessions over } 4 \text { weeks }\end{array}$ & $\begin{array}{l}\text { Painful shoulder in the } 5 \text { th cervical (C5) } \\
\text { dermatome distribution of between } 4 \text { weeks' } \\
\text { and } 6 \text { months' duration. Restriction of active } \\
\text { and passive ROM in both external rotation and } \\
\text { glenohumeral abduction of }>25 \% \text { compared with } \\
\text { other shoulder }\end{array}$ \\
\hline
\end{tabular}

IA, intra-articular; IB, intrabursal; PT, physiotherapy; ROM, range of movement.

Five of the six trials evaluated a physical therapy regimen as part of the intervention. ${ }^{35,41,42,66,67}$ These varied between studies but all consisted of physiotherapy modalities. They included combinations of exercises, mobilisation techniques, TENS, ultrasound, ice, heat packs and interferential therapy. All of the studies appeared to include some form of mobilisation as part of the physiotherapy, although details were scant in some studies. The duration of physiotherapy ranged from 4 to 11 weeks, although, with the exception of one study, duration was $<6$ weeks. One study tailored the physiotherapy depending on whether participants had acute or chronic-like symptoms. ${ }^{35}$ 
Five studies reported that participants in all groups were asked to undertake home exercises, although the level of detail reported varied between studies. Bal et al. ${ }^{68}$ had a substantial home exercise programme: participants were asked to undertake shoulder stretching and stabilising exercises five times per day over 12 weeks, using a heat pack before exercise and a cold pack after. Carette et al. ${ }^{35}$ asked participants to carry out active and auto-assisted exercises twice daily over 12 weeks. In the study by Calis et al. ${ }^{66}$ stretching and Codman's exercises were performed. Details of home exercise were not reported in the other two studies. ${ }^{41,42}$ The study by Dacre et al. ${ }^{67}$ did not have a home exercise component.

There was also some variability between studies in concomitant treatments. Four studies reported that paracetamol of varying doses was available to participants: $1500 \mathrm{mg}$ per day when needed in one study, ${ }^{68}$ a supply (dose unspecified) in one study, ${ }^{35}$ one to two tablets every 4-6 hours (a maximum of eight per day) in another study ${ }^{41}$ and paracetamol when needed in the remaining study ${ }^{66}$ Another study stated that all participants were advised to continue NSAIDs. ${ }^{42}$ One study did not report whether participants received concomitant treatment. ${ }^{67}$

The inclusion criteria varied between the six studies, although four of the studies used similar criteria for the extent of restriction of movement. ${ }^{35,41,66,68}$ Three of the studies included participants with frozen shoulder of $<6$ months' duration. ${ }^{35,41,42}$ Where reported, frozen shoulder was diagnosed through presence of pain and range of movement, clinical diagnosis, presence of symptoms, laboratory tests and radiography. The method of diagnosis was unclear in two studies. ${ }^{42,68}$ Three studies included a small number of participants with diabetes: $6 \%$ in the study by Carette et al. ${ }^{35} 5 \%$ in the study by Dacre et al. ${ }^{67}$ and $6 \%$ in the study by Ryans et al. ${ }^{41}$ None of the studies reported results separately for patients with diabetes. The participants' stage of frozen shoulder at baseline was not reported in any of the studies. Where reported, the mean duration of frozen shoulder amongst the included participants ranged from $13^{42}$ to $21^{35}$ weeks. None of the studies reported whether participants had received any previous treatment for frozen shoulder. The mean age of participants ranged from 54 to 57 years and the proportion of women ranged from $42 \%$ to $63 \%$.

\section{Quality assessment}

Quality varied between the included studies. Four studies did not report the method of randomisation; ${ }^{42,66-68}$ therefore, it was unclear whether these were truly randomised studies as stated by the authors. In addition, in two of these studies it was unclear whether the intervention groups were comparable at baseline. ${ }^{67,68}$ These four studies therefore have a potentially high risk of bias even though they met other criteria such as blinding of outcome assessment. Only one of these studies was adequately powered for at least one outcome. ${ }^{67} \mathrm{Bal}$ et al. ${ }^{68}$ also reported substantial loss to follow-up (20\%, all in the placebo group). Dacre et al. ${ }^{67}$ and Rizk et al. ${ }^{42}$ reported considerably less loss to follow-up ( $6 \%$ and $8 \%$ respectively), whereas Calis et al. ${ }^{66}$ reported that there were no dropouts.

The remaining two studies were considered of satisfactory quality although one had some risk of bias. ${ }^{41}$ Both reported an adequate method of randomisation and Carette et al..$^{35}$ also reported an adequate method of allocation concealment; it was unclear whether Ryans et al. ${ }^{41}$ used an adequate method because, although sealed envelopes were used, it was not stated whether these were opaque or sequentially numbered. Both studies met most of the remaining criteria including reported blinding of outcome assessment. Ryans et al. ${ }^{41}$ reported that participants were blinded to type of injection. Carette et al. ${ }^{35}$ attempted to blind the injection; however, the method used (covering the syringe with foil) may not have been reliable. Blinding of the physiotherapy treatment was not possible. Carette et al..$^{35}$ also reported an intention-to-treat analysis. The main limitation of this study was that it may not have been sufficiently powered; $17 \%$ were lost to follow-up and there appears to be an imbalance between treatment groups, with greater loss to 
follow-up in the steroid group. Ryans et al. ${ }^{41}$ did not report an intention-to-treat analysis and there was also a high proportion lost to follow-up (27\%), with a higher number of dropouts in the steroid only and placebo groups. As with the Carette study, the Ryans study may not have been sufficiently powered. Full details of study quality are reported in Appendix 8.

\section{Pain}

All six studies assessed pain but Calis et al..$^{66}$ and Dacre et al.$^{67}$ reported data in graphical form only (Table 7). Consequently, a SMD could not be calculated and these two studies could not be included in a meta-analysis. Calis et al. ${ }^{66}$ reported that there was significantly greater improvement in pain in the steroid group than in the placebo group $(p=0.02)$. Dacre et al. ${ }^{67}$ evaluated steroid, steroid and physiotherapy, and physiotherapy alone. This study reported that all three groups showed a significant reduction in pain after 6 weeks $(p<0.001)$ with mean measures improving by $49-66 \%$, and further improvements at 6 months. Improvements were reported to be similar in all three treatment groups.

Of the four remaining studies, three reported final mean values and SDs were reported or could be imputed. Both Carette et al..$^{35}$ and Rizk et al.$^{42}$ reported pain overall and Ryans et al..$^{41}$ reported daytime pain at rest (Table 7). Each of the three studies reported pain using a different scale.

The study by Bal et al. ${ }^{68}$ reported mean change and SDs at 12 weeks for pain at night measured on the VAS 0-100 mm. Although a SMD could be calculated for this study, it was inappropriate to pool this SMD with those SMDs calculated from final value means, as the difference in SD reflects not differences in measurement scale, but differences in the reliability of the measurements. ${ }^{48}$

Table 8 provides the SMDs and $95 \%$ CIs. The outcome data for individual groups in the included trials are available in Appendix 7.

\section{Steroid versus placebo}

Three studies reported pain at short-term follow-up. Two studies reported final value mean pain for short-term follow-up: Carette et al. ${ }^{35}$ reported data at 6 weeks and 3 months and Ryans et $a l .^{41}$ reported data at 6 weeks (Table 8). As stated in the methods section, to allow comparison of the results of the meta-analysis with the MTC for pain, time points closest to 3 months were pooled, that is, 3-month data for Carette et al. ${ }^{35}$ were pooled with 6-week data for Ryans et al. ${ }^{41}$ There was a significant decrease in pain with steroid injection compared with placebo: pooled SMD $-1.15,95 \%$ CI -1.62 to -0.67 (Figure 2). When back transformed to a 0-100 mm VAS scale,

TABLE 7 Pain outcomes in studies evaluating steroid injections

\begin{tabular}{|c|c|c|c|}
\hline Study & $\begin{array}{l}\text { Pain outcome } \\
\text { assessed }^{a}\end{array}$ & Scale used & Data reported and time of follow-up \\
\hline Bal $2008^{68}$ & Pain at night & VAS $0-100 \mathrm{~mm}$ & Mean change at 2 and 12 weeks (baseline median and IQR reported only) \\
\hline Calis $2006^{66}$ & Pain severity & VAS $^{b}$ & $\begin{array}{l}\text { Data reported in graphs only; } p \text {-value for between-group difference reported at } \\
3 \text { months }\end{array}$ \\
\hline $\begin{array}{l}\text { Carette } \\
2003^{35}\end{array}$ & Pain overall & $\begin{array}{l}\text { SPADI 5-item pain } \\
\text { subscale }\end{array}$ & Change from baseline at 6 weeks and 3, 6 and 12 months \\
\hline Dacre $1989^{67}$ & Pain at night & VAS $0-10 \mathrm{~cm}$ & Data presented in graphs only \\
\hline Rizk $1991^{42}$ & Pain overall & Likert (0-5) & Final mean values at 4,11 and 24 weeks. SD not reported \\
\hline Ryans $2005^{41}$ & Daytime pain at rest & VAS $0-100 \mathrm{~mm}$ & Change from baseline at 6 and 16 weeks \\
\hline
\end{tabular}

IQR, interquartile range; SPADI, Shoulder Pain and Disability Index.

a Lower scores indicated better outcome.

b Unit of measurement (cm or $\mathrm{mm}$ ) not specified. 
this equates to a mean difference (MD) of $21 \mathrm{~mm}$ (95\% CI -29.7 to $-12.3 \mathrm{~mm}$ ). No data could be located on the MCID in pain for individuals with frozen shoulder. However, it is suggested that a difference of $14 \mathrm{~mm}$ on a 100-mm VAS scale is the MCID for individuals with rotator cuff disease, a shoulder disorder also characterised by pain and restricted range of movement. ${ }^{50}$ Given that the between-group MCID is thought to approximate $40 \%$ of that within individuals, ${ }^{6,53}$ the MCID between groups for rotator cuff disorder can be estimated to be approximately $5.6 \mathrm{~mm}$. Using this as a proxy for between-group MCID for frozen shoulder, this suggests that there is a clinically significant decrease in pain with steroid compared with placebo. There was no statistical heterogeneity between the studies $\left(I^{2}=0 \%\right)$. When 6-week data for Carette et al. ${ }^{35}$ and Ryans et al. ${ }^{41}$ were pooled the results were similar (pooled SMD $-1.30,95 \% \mathrm{CI}-1.78$ to -0.81 ; MD $23.8 \mathrm{~mm}, 95 \%$ CI 32.6 to $14.8 \mathrm{~mm}$ ).

Bal et al.$^{68}$ reported mean change in pain at 12 weeks. There was no significant difference in pain with steroid compared with placebo (Table 8).

\section{Steroid versus physiotherapy combined with placebo}

Two studies reported data at short-term follow-up: Carette et al. ${ }^{35}$ reported data at 6 weeks and 3 months and Ryans et al. ${ }^{41}$ reported data at 6 weeks (Table 8). In both studies physiotherapy was combined with placebo. When 3-month data from Carette et al. ${ }^{35}$ were pooled with 6-week data from Ryans et al ${ }^{41}$ there was no significant difference in pain between steroid and physiotherapy (Figure 3). When back transformed to a $0-100 \mathrm{~mm}$ VAS scale, this equates to a MD of $-4 \mathrm{~mm}$ ( $95 \% \mathrm{CI}-11.9$ to $3.7 \mathrm{~mm}$ ), which suggests that there may be no clinically significant difference between treatments. There was no statistical heterogeneity between studies $\left(I^{2}=0 \%\right)$. When 6-week data for Carette et al. ${ }^{35}$ and Ryans et $a l .{ }^{41}$ were pooled there was a marginally significant decrease in pain with steroid compared with physiotherapy combined with placebo (pooled SMD $-0.51,95 \%$ CI -0.94 to -0.07 ). However, when back transformed to a $0-100 \mathrm{~mm}$ VAS scale, this equates to a MD of $-9.3 \mathrm{~mm}$ ( $95 \%-17.2$ to $-1.2 \mathrm{~mm}$ ), which, as with the pooled 3-month and 6-week data, suggests that there may be no clinically significant difference between treatments.

\section{Steroid combined with physiotherapy versus placebo}

Two studies reported pain at short-term follow-up: Carette et al. ${ }^{35}$ reported data at 6 weeks and 3 months and Ryans et al. ${ }^{41}$ reported data at 6 weeks (Table 8). When 3-month data from Carette et al. ${ }^{35}$ were pooled with 6 -week data from Ryans et al. ${ }^{41}$ there was a statistically significant decrease in pain with steroid combined with physiotherapy compared with placebo: pooled SMD $-1.88,95 \%$ CI -2.43 to -1.33 (Figure 4). When back transformed to a $0-100 \mathrm{~mm}$ VAS scale, this equates to a MD of $-34.40 \mathrm{~mm}$ ( $95 \% \mathrm{CI}-44.47$ to $-24.34 \mathrm{~mm})$. This suggests that there is a clinically significant decrease in pain with steroid combined with physiotherapy compared with placebo. There was no statistical heterogeneity between studies $\left(I^{2}=3 \%\right)$. When 6 -week data for Carette et al..$^{35}$ and Ryans et al. ${ }^{41}$ were pooled the results were similar (pooled SMD - 2.31, 95\% CI -2.89 to -1.72 ; $\mathrm{MD}-42.3 \mathrm{~mm}, 95 \% \mathrm{CI}-52.9$ to $-31.5 \mathrm{~mm}$ ).

\section{Steroid combined with physiotherapy versus physical therapy combined with placebo}

Three studies reported pain for short-term follow-up. In all studies physiotherapy was combined with a placebo injection. Carette et al. ${ }^{35}$ reported pain at 6 weeks and 3 months, Rizk et al. ${ }^{42}$ reported pain at 11 weeks and Ryans et al. ${ }^{41}$ reported pain at 6 weeks (Table 8 ).

When 3-month data from Carette et al.$^{35}$ were pooled with 11-week data from Rizk et al..$^{42}$ and 6-week data from Ryans et al. ${ }^{41}$ there was a significant decrease in pain with steroid combined with physiotherapy compared with physiotherapy: pooled SMD $-0.57,95 \% \mathrm{CI}-0.94$ to -0.20 (Figure 5). However, there was evidence of substantial statistical heterogeneity $\left(I^{2}=79 \%\right)$. When 6-week data for Carette et al. ${ }^{35}$ were pooled with 11-week data from Rizk et al. ${ }^{42}$ and 6-week data 


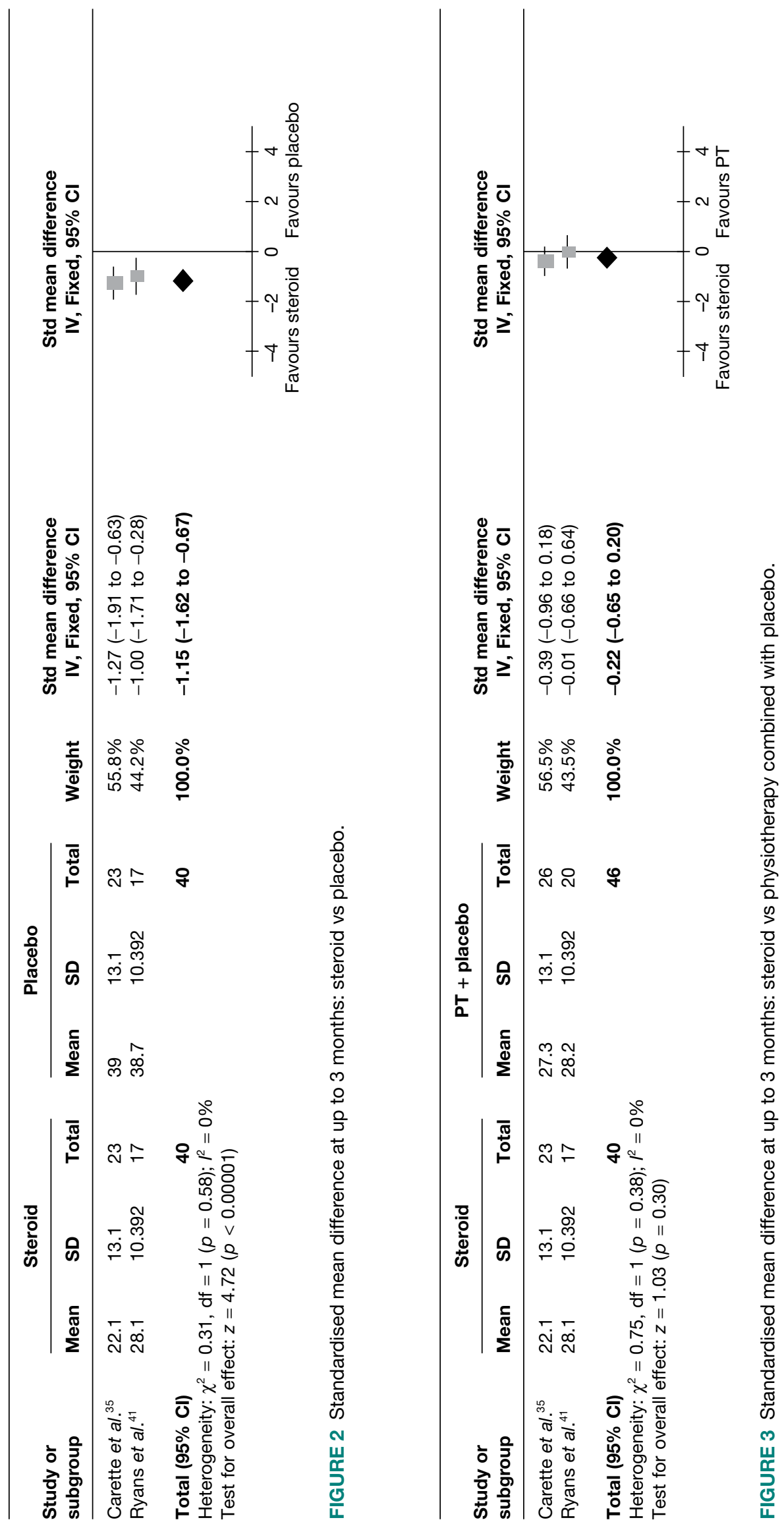




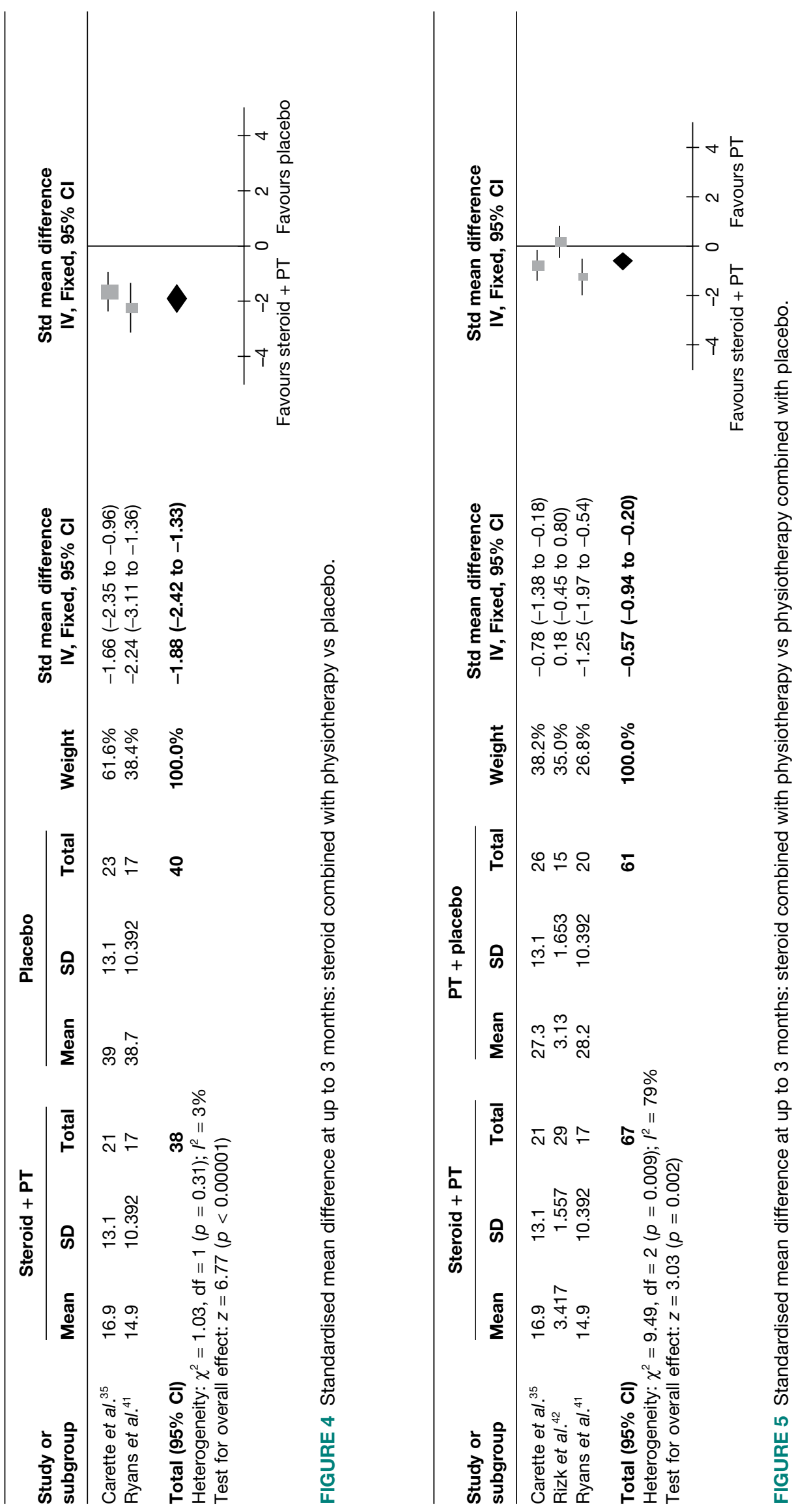


from Ryans et al., ${ }^{41}$ the results were similar (pooled SMD $-0.86,95 \%$ CI -1.25 to $-0.47, I^{2}=89 \%$ ). Potential sources of heterogeneity may be the type of steroid used, different lengths of follow-up and proportion of patients with diabetes. Furthermore, the studies by Carette et al. ${ }^{35}$ and Ryans et $a l^{41}$ were assessed to have a potentially lower risk of bias than the study by Rizk $e t$ al. ${ }^{42}$

Given the differences in study quality, a sensitivity analysis was performed by excluding Rizk et al. ${ }^{42}$ from the meta-analyses (Figure 6). Pooling 3-month data from Carette et al. ${ }^{35}$ and 6-week data from Ryans et al. ${ }^{41}$ resulted in a significant decrease in pain with steroid combined with physiotherapy compared with physiotherapy: pooled SMD $-0.98,95 \% \mathrm{CI}-1.43$ to -0.52 . Furthermore, there was no longer evidence of statistical heterogeneity $\left(I^{2}=0 \%\right)$. When back transformed to a $0-100 \mathrm{~mm}$ VAS, the pooled SMD equates to a $\mathrm{MD}$ of $-17.93 \mathrm{~mm}(95 \% \mathrm{CI}-26.2$ to $-9.5 \mathrm{~mm}$ ), which suggests that there is also a clinically significant decrease in pain with steroid combined with physiotherapy compared with physiotherapy. When 6-week data for Carette et al. ${ }^{35}$ and Ryans et al. ${ }^{41}$ were pooled the results were similar (pooled SMD $-1.51,95 \% \mathrm{CI}-2.00$ to -1.02 ; MD $27.6 \mathrm{~mm}, 95 \%$ CI 36.6 to $18.7 \mathrm{~mm}$ ).

The study by Rizk et al. ${ }^{42}$ also reported pain for medium-term follow-up. At 24 weeks there was no significant difference between steroid combined with physiotherapy and physiotherapy (see Table 8).

\section{Steroid combined with physiotherapy versus steroid}

Carette et al. ${ }^{35}$ reported data at 6 weeks and 3 months and Ryans et al.$^{41}$ reported data at 6 weeks (Table 8). When 3-month data from Carette et al..$^{35}$ were pooled with 6-week data from Ryans et al. ${ }^{41}$ there was a significant difference in pain with steroid combined with physiotherapy compared with steroid alone: pooled SMD $-0.75,95 \%$ CI -1.20 to -0.29 (Figure 7 ). When back transformed to a $0-100 \mathrm{~mm}$ VAS, this equates to a $\mathrm{MD}$ of $-13.7 \mathrm{~mm}$ ( $95 \% \mathrm{CI}-21.96$ to $-5.3 \mathrm{~mm}$ ), which suggests that there may be no clinically significant difference between treatments. However, there was evidence of substantial statistical heterogeneity $\left(I^{2}=70 \%\right)$; therefore, this result should be interpreted with caution. When 6-week data for Carette et al..$^{35}$ and Ryans et al. ${ }^{41}$ were pooled, there was a significant decrease in pain with steroid combined with physiotherapy compared with steroid alone (pooled SMD -1.00, 95\% CI - 1.47 to -0.53 ). When back transformed to a $0-100 \mathrm{~mm}$ VAS, this equates to a $\mathrm{MD}$ of $-18 \mathrm{~mm}$ ( $95 \%$ CI 26.9 to $9.7 \mathrm{~mm}$ ). This suggests that there may be a clinically significant reduction in pain with steroid combined with physiotherapy compared with steroid alone. Furthermore, there was no evidence of statistical heterogeneity $\left(I^{2}=0 \%\right)$.

\section{Function and disability}

Four of the six studies assessed function and disability; however, only the Shoulder Pain and Disability Index (SPADI) total score and SPADI 8-item disability subscales were common to more than one study and follow-up times varied (Table 9). Results were presented as change from baseline data for all but one of the studies. ${ }^{66}$ Table 10 provides the between-group difference in means and 95\% CIs. The outcome data for the individual groups in the included trials are available in Appendix 7.

\section{Steroid versus placebo}

Two studies reported SPADI total score and SPADI 8-item subscale score for steroid versus placebo injection (Table 10). . $^{35,68}$

Carette et al.$^{35}$ reported SPADI total score at 6 weeks and found a significant effect in favour of the steroid injection group (MD $-17.8,95 \% \mathrm{CI}-31.96$ to -3.64$)$. 

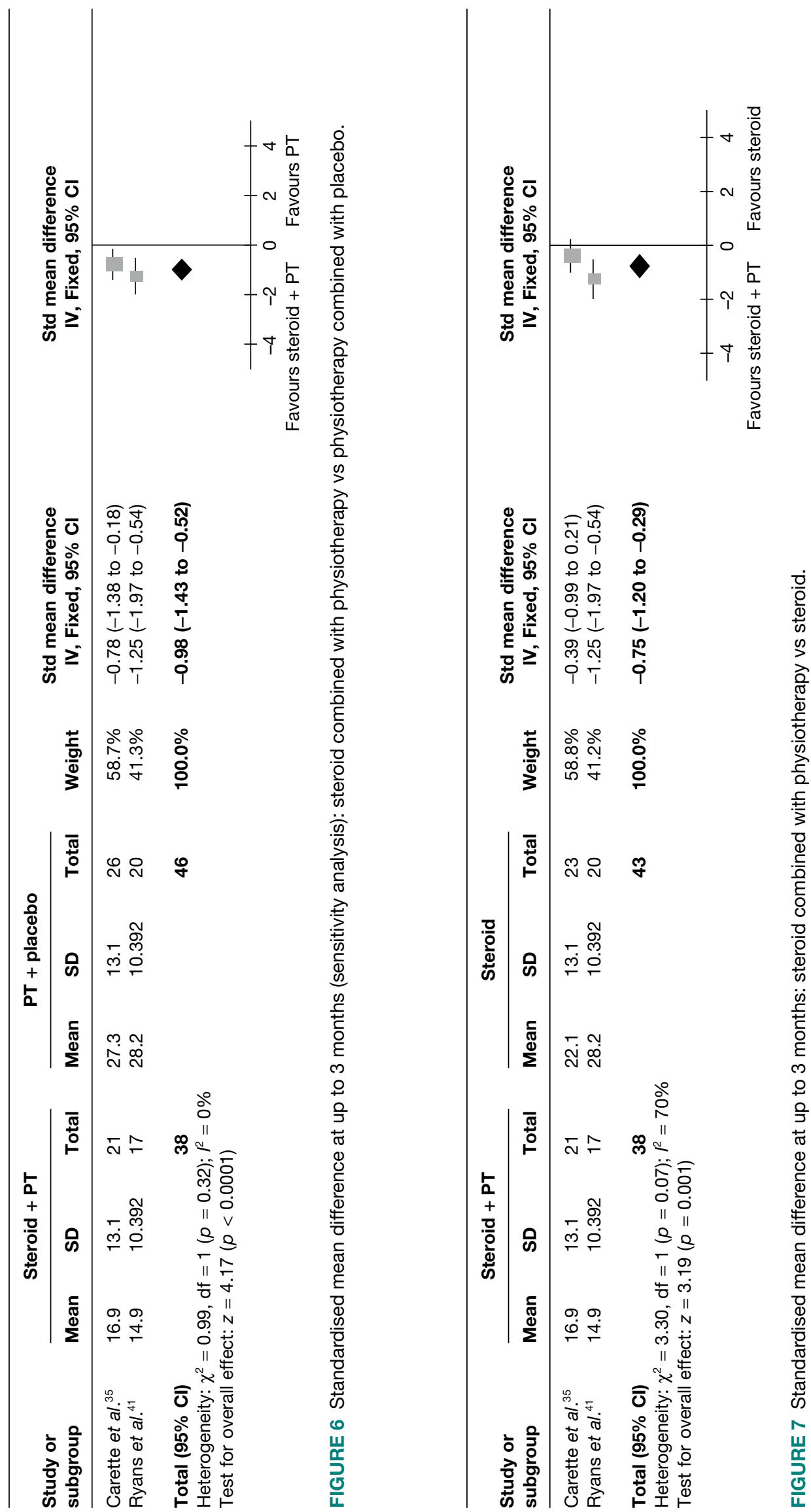
TABLE 8 Pain results from studies of steroid injections (SMD and 95\% Cl)

\begin{tabular}{|c|c|c|c|}
\hline Study & Short term ( $\leq 3$ months) & $\begin{array}{l}\text { Medium term (>3 and } \\
\leq 6 \text { months) }\end{array}$ & Long term ( $>6$ and $\leq 12$ months \\
\hline \multicolumn{4}{|c|}{ Steroid vs placebo } \\
\hline Bal $2008^{68}$ & $-0.05(-0.55 \text { to } 0.45)^{\mathrm{a}}$ & & \\
\hline \multirow[t]{2}{*}{ Carette $2003^{35}$} & $-1.55(2.22 \text { to }-0.89)^{b}$ & & \\
\hline & $-1.27(-1.91 \text { to }-0.63)^{\mathrm{c}, \mathrm{d}}$ & & \\
\hline Ryans $2005^{41}$ & $-1.00(-1.71 \text { to }-0.28)^{b, d}$ & & \\
\hline \multicolumn{4}{|c|}{ Steroid vs physiotherapy + placebo } \\
\hline \multirow[t]{2}{*}{ Carette $2003^{35}$} & $-0.94(-1.53 \text { to }-0.35)^{b}$ & & \\
\hline & $-0.39(-0.96 \text { to } 0.18)^{c}$ & & \\
\hline Ryans $2005^{41}$ & $-0.01(-0.66 \text { to } 0.64)^{b}$ & & \\
\hline \multicolumn{4}{|c|}{ Steroid + physiotherapy vs placebo } \\
\hline \multirow[t]{2}{*}{ Carette $2003^{35}$} & $-2.36(-3.15 \text { to }-1.58)^{\mathrm{b}}$ & & \\
\hline & $-1.66(-2.35 \text { to }-0.96)^{\mathrm{c}, \mathrm{d}}$ & & \\
\hline Ryans $2005^{41}$ & $-2.24(-3.11 \text { to }-1.36)^{b, d}$ & & \\
\hline \multicolumn{4}{|c|}{ Steroid + physiotherapy vs physiotherapy + placebo } \\
\hline \multirow[t]{2}{*}{ Carette $2003^{35}$} & $-1.75(-2.43 \text { to }-1.07)^{b}$ & & \\
\hline & $-0.78(-1.38 \text { to }-0.18)^{\mathrm{c}, \mathrm{d}}$ & & \\
\hline Rizk $1991^{42}$ & $0.18(-0.45 \text { to } 0.80)^{\mathrm{e}}$ & $0.06(-0.56 \text { to } 0.68)^{\dagger}$ & \\
\hline Ryans $2005^{41}$ & $-1.25(-1.97 \text { to }-0.54)^{\mathrm{b}, \mathrm{d}}$ & & \\
\hline \multicolumn{4}{|c|}{ Steroid + physiotherapy vs steroid } \\
\hline \multirow[t]{2}{*}{ Carette $2003^{35}$} & $-0.81(-1.43,-0.19)^{b}$ & & \\
\hline & $-0.39(-0.99 \text { to } 0.21)^{c}$ & & \\
\hline Ryans $2005^{41}$ & $-1.25(-1.97 \text { to }-0.54)^{b, d}$ & & \\
\hline
\end{tabular}

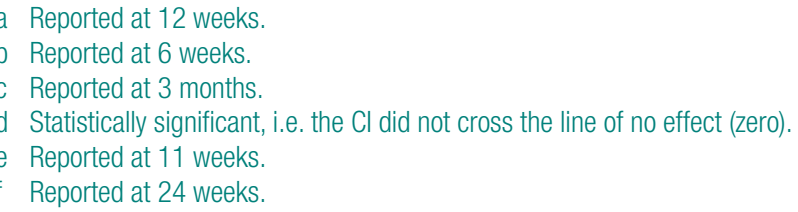

Both studies reported 3-month follow-up and were pooled at this time point, although it should be noted that the study by Bal et al. ${ }^{68}$ was at high risk of bias. When the two studies were pooled (Figure 8$)$ statistical heterogeneity was high $\left(I^{2}=80 \%\right)$; therefore, the treatment effect should be treated with considerable caution. The study by Carette $\mathrm{et} \mathrm{al.} .^{35}$ reported a significantly greater decrease in SPADI total score in the steroid group than in the placebo and home exercise group (MD -16.10, 95\% CI -30.26 to -1.94 ), whereas in the Bal et al. study ${ }^{68}$ the treatment effect was in favour of placebo and home exercise, although the CIs crossed the line of no effect. Only Carette et al. ${ }^{35}$ reported SPADI total score for medium- and long-term follow-up; there was no significant difference between groups for these later follow-up times (Table 10).

Carette et al. ${ }^{35}$ reported SPADI 8-item disability subscale score at 6 weeks and found a significant effect in favour of the steroid injection group (MD -13.80, 95\% CI -27.53 to -0.07 ).

As with SPADI total score, the same two studies reported SPADI 8-item disability subscale score and were available for meta-analysis at 3 months. ${ }^{35,68}$ Again, the pooled treatment effect should be treated with considerable caution because of high heterogeneity (Figure 9). Carette et al. ${ }^{35}$ 
TABLE 9 Function and disability outcomes in studies evaluating steroid injections

\begin{tabular}{|c|c|c|}
\hline Study & Function and disability outcomes assessed & Data reported and time of follow-up \\
\hline \multirow[t]{3}{*}{ Bal $2008^{68}$} & $\begin{array}{l}\text { UCLA Shoulder score (35-point scale: }<28 \text { poor, 29-33 good, 34-35 } \\
\text { excellent as defined by the authors) }\end{array}$ & \multirow[t]{3}{*}{ Change from baseline at 12 weeks } \\
\hline & SPADI total score $(0-100)^{\mathrm{a}}$ & \\
\hline & SPADI 8-item disability subscale $(0-100)^{a}$ & \\
\hline Calis $2006^{66}$ & Constant score & $\begin{array}{l}\text { Final value data at } 15 \text { days and } 3 \text { months (3-month data } \\
\text { used in analysis) }\end{array}$ \\
\hline \multirow{2}{*}{$\begin{array}{l}\text { Carette } \\
2003^{35}\end{array}$} & SPADI total score $(0-100)^{\mathrm{a}}$ & \multirow{2}{*}{$\begin{array}{l}\text { Change from baseline at } 6 \text { weeks and 3, } 6 \text { and } \\
12 \text { months }^{b}\end{array}$} \\
\hline & SPADI 8-item disability subscale $(0-100)^{a}$ & \\
\hline \multirow[t]{2}{*}{$\begin{array}{l}\text { Ryans } \\
2005^{41}\end{array}$} & $\begin{array}{l}\text { Shoulder Disability Questionnaire (SDQ) (0-22) }{ }^{\mathrm{a}, \mathrm{c}} \text { (score } \geq 5 \text { was } \\
\text { defined by authors as significant disability) }\end{array}$ & \multirow[t]{2}{*}{ Change from baseline at 6 and 16 weeks } \\
\hline & Self-assessed global function (100-mm VAS, 0-100)a & \\
\hline
\end{tabular}

UCLA, University of California Los Angeles, CA, USA.

a Higher scores indicated worse outcomes.

b All analyses adjusted for gender.

c Also known as Crofts score.

reported a significantly greater decrease in SPADI 8-item subscale score in the steroid group than with placebo and home exercise (MD -14.30,95\% CI -28.03 to -0.57), whereas in the Bal et al. study ${ }^{68}$ the treatment effect was in favour of placebo and home exercise, although the CIs crossed the line of no effect.

Only Carette et al..$^{35}$ reported SPADI 8 -item subscale score for medium- and long-term follow-up; there was no significant difference between groups for this outcome (Table 10).

Ryans et al. ${ }^{41}$ reported results of the Shoulder Disability Questionnaire (SDQ) and global function at 6 and 16 weeks. There was no significant difference between the steroid group and the placebo group at either time point for either outcome (Table 10). Bal et al. ${ }^{68}$ reported University of California Los Angeles (UCLA) Shoulder score with steroid injection versus placebo and both groups were similar at the 3-month follow-up [steroid group median 32.5, interquartile range (IQR) 6.2; placebo group median 31.5, IQR 7.7; no $p$-value reported].

\section{Steroid versus home exercise}

Calis et al. ${ }^{66}$ reported Constant score for short-term follow-up; steroid injection was associated with a significantly better outcome than home exercise alone (MD 9.10, 95\% CI 2.98 to 15.22).

\section{Steroid versus physiotherapy}

Calis et al ${ }^{66}$ compared steroid with physiotherapy (without placebo injection) using the Constant score. At 3 months there was no significant difference between the two treatment groups (MD $-5.80,95 \%$ CI -11.80 to 0.20$)$.

\section{Steroid versus physiotherapy combined with placebo}

Two studies compared steroid with physiotherapy plus placebo injection. ${ }^{35,41}$ One study reported SPADI total score ${ }^{35}$ and the other the SDQ and a global function score. ${ }^{41}$

There was a significantly greater improvement in SPADI total score in the steroid group than in the physiotherapy group at 6 weeks in the study by Carette et al. ${ }^{35}$ (MD $-14.50,95 \%$ CI -28.25 to -0.75), but not at 3 months or for medium- and long-term follow-up or in the SPADI 8-item disability subscale score between treatment groups at any follow-up time in the same study 


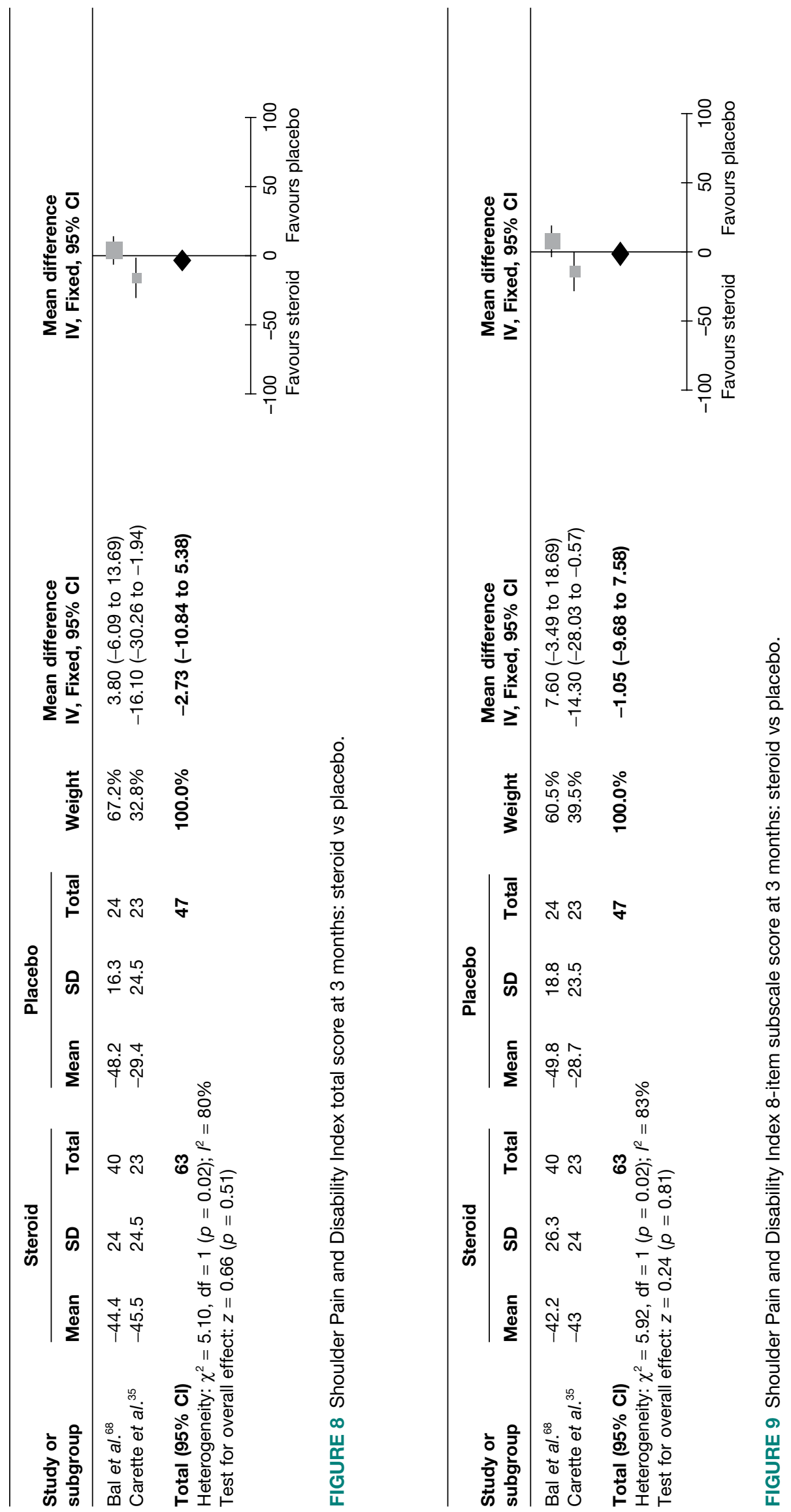


(Table 10). ${ }^{35}$ There was also no significant difference between treatment groups in either SDQ or global function score at 6 or 16 weeks in the study by Ryans et al. ${ }^{41}$

\section{Steroid combined with physiotherapy versus placebo}

Two studies investigated steroid combined with physiotherapy versus placebo. ${ }^{35,41}$ One study reported SPADI total score ${ }^{35}$ and the other the SDQ and a global function score. ${ }^{41}$

There was a significant improvement in total SPADI score with steroid in combination with physiotherapy compared with placebo in the study by Carette et al. ${ }^{35}$ at 6 weeks (MD -27.60 , $95 \% \mathrm{CI}-42.03$ to -13.17 ) and 3 months (MD $-21.00,95 \% \mathrm{CI}-35.43$ to -6.57 ), although not at medium- (MD -14.10, 95\% CI -28.53 to 0.33 ) or long-term follow-up (MD -1.10, 95\% CI -15.53 to 13.33) (Table 10). ${ }^{35}$ There was also a significant improvement in SPADI 8-item disability subscale score with steroid in combination with physiotherapy compared with placebo in the same study at 6 weeks (MD $-23.90,95 \% \mathrm{CI}-37.89$ to -9.91$)$ and 3 months (MD $-20.00,95 \%$ CI -33.99 to -6.01 ) but not at medium- and long-term follow-up.

There was a significant improvement in both SDQ (MD -4.70, 95\% CI -7.85 to -1.55) and global function score (MD $-21.00,95 \% \mathrm{CI}-34.54$ to -7.46$)$ with steroid in combination with physiotherapy compared with placebo at 6 weeks in the study by Ryans et al. ${ }^{41}$ At 16 weeks there was no significant difference between groups in this study (Table 10).

\section{Steroid combined with physiotherapy versus physiotherapy combined with placebo}

Two studies investigated the effect of steroid combined with physiotherapy versus physiotherapy (plus placebo injection) on function and disability using SPADI total score ${ }^{35}$ and the SDQ and a global function score. ${ }^{41}$

There was a significant improvement in total SPADI score with steroid in combination with physiotherapy compared with physiotherapy in the study by Carette et al..$^{35}$ at 6 weeks (MD $-24.30,95 \% \mathrm{CI}-38.33$ to -10.27 ), although not at 3 months (MD $-12.50,95 \% \mathrm{CI}-26.53$ to 1.53) or for medium- and long-term follow-up (Table 10). ${ }^{35}$ Similarly, there was a significant improvement in SPADI 8-item disability subscale score with steroid in combination with physiotherapy compared with physiotherapy in the same study at 6 weeks (MD $-21.60,95 \% \mathrm{CI}$ -35.34 to -7.86 ) but not at any of the other follow-up points. ${ }^{35}$

Ryans et al. ${ }^{41}$ also showed a significant improvement in SDQ score with steroid combined with physiotherapy compared with physiotherapy at 6 weeks (MD $-4.30,95 \% \mathrm{CI}-7.76$ to -0.84 ) but not at 16 weeks, and there was no significant difference in global function score at either follow-up time.

\section{Steroid combined with physiotherapy versus steroid}

Two studies compared steroid injection followed by physiotherapy with steroid alone. One study reported a SPADI total score and SPADI 8-item disability subscale score; ${ }^{35}$ another study reported SDQ and global function score. ${ }^{41}$

There was no significant difference between treatment groups in SPADI total or SPADI 8-item disability subscale score at short-, medium- or long-term follow-up in the study by Carette $e t$ al. ${ }^{35}$ or between treatment groups in either SDQ or global function for short- or medium-term follow-up in the study by Ryans et al. (Table 10). ${ }^{41}$ 
TABLE 10 Function and disability results of studies of steroid injections (MD and 95\% Cl)

\begin{tabular}{|c|c|c|c|}
\hline Study & Short term ( $\leq 3$ months) & Medium term ( $>3$ and $\leq 6$ months) & Long term ( $>6$ and $\leq 12$ months) \\
\hline \multicolumn{4}{|c|}{ Steroid vs placebo } \\
\hline \multicolumn{4}{|c|}{ SPADI total score } \\
\hline Bal $2008^{68}$ & $3.80(-6.09 \text { to } 13.69)^{\mathrm{a}}$ & & \\
\hline Carette $2003^{35}$ & $\begin{array}{l}-17.8(-31.96 \text { to }-3.64)^{\mathrm{b}, \mathrm{c}} \\
-16.10(-30.26 \text { to }-1.94)^{\mathrm{a}, \mathrm{b}}\end{array}$ & $-12.90(-27.06 \text { to } 1.26)^{d}$ & $-2.90(-17.06 \text { to } 11.26)^{\mathrm{e}}$ \\
\hline \multicolumn{4}{|c|}{ SPADI 8-item subscale } \\
\hline Bal $2008^{68}$ & $7.60(-3.49 \text { to } 18.69)^{\mathrm{a}}$ & & \\
\hline Carette $2003^{35}$ & $\begin{array}{l}-13.80(-27.53 \text { to }-0.07)^{\mathrm{b}, \mathrm{c}} \\
-14.30(-28.03 \text { to }-0.57)^{\mathrm{a}, \mathrm{b}}\end{array}$ & $-7.10(-20.83 \text { to } 6.63)^{d}$ & $0.80(-12.93 \text { to } 14.53)^{\mathrm{e}}$ \\
\hline \multicolumn{4}{|l|}{$S D Q$} \\
\hline Ryans $2005^{41}$ & $-3.00(-6.44 \text { to } 0.44)^{c}$ & $-1.20(-5.63 \text { to } 3.23)^{\dagger}$ & \\
\hline \multicolumn{4}{|l|}{ Global function } \\
\hline Ryans $2005^{41}$ & $-11.60(-27.11 \text { to } 3.91)^{c}$ & $5.10(-15.92 \text { to } 26.12)^{\dagger}$ & \\
\hline \multicolumn{4}{|c|}{ UCLA Shoulder score } \\
\hline Bal $2008^{68}$ & $\begin{array}{l}\text { Steroid: median } 32.5(\operatorname{IQR} 6.2)^{\mathrm{a}} \\
\text { Placebo: median } 31.5(\mathrm{IQR} 7.7)^{\mathrm{a}} \\
\text { No } p \text {-value }\end{array}$ & & \\
\hline \multicolumn{4}{|c|}{ Steroid vs home exercise } \\
\hline \multicolumn{4}{|l|}{ Constant score } \\
\hline Calis $2006^{66}$ & $9.10(2.98 \text { to } 15.22)^{a, b}$ & & \\
\hline \multicolumn{4}{|c|}{ Steroid vs physiotherapy } \\
\hline \multicolumn{4}{|l|}{ Constant score } \\
\hline Calis $2006^{66}$ & $-5.80(11.80 \text { to } 0.20)^{\mathrm{a}}$ & & \\
\hline \multicolumn{4}{|c|}{ Steroid vs physiotherapy + placebo } \\
\hline \multicolumn{4}{|c|}{ SPADI total score } \\
\hline Carette $2003^{35}$ & $\begin{array}{l}-14.50(-28.25 \text { to }-0.75)^{c} \\
-7.60(-21.35 \text { to } 6.15)^{a}\end{array}$ & $-8.20(-21.95 \text { to } 5.55)^{d}$ & $-4.60(-18.35 \text { to } 9.13)^{\mathrm{e}}$ \\
\hline \multicolumn{4}{|c|}{ SPADI 8-item subscale } \\
\hline Carette $2003^{35}$ & $\begin{array}{l}-11.50(-24.97 \text { to } 1.97)^{\mathrm{c}} \\
-5.30(-18.77 \text { to } 8.17)^{\mathrm{a}}\end{array}$ & $-5.30(-18.77 \text { to } 8.17)^{d}$ & $-2.60(-16.07 \text { to } 10.87)^{e}$ \\
\hline \multicolumn{4}{|l|}{$S D Q$} \\
\hline \multicolumn{4}{|l|}{ Global function } \\
\hline Ryans $2005^{41}$ & $-2.30(-18.68 \text { to } 14.08)^{c}$ & $7.10(-13.43 \text { to } 27.63)^{\dagger}$ & \\
\hline \multicolumn{4}{|c|}{ Steroid + physiotherapy vs placebo } \\
\hline \multicolumn{4}{|c|}{ SPADI total score } \\
\hline Carette $2003^{35}$ & $\begin{array}{l}-27.60(-42.03 \text { to }-13.17)^{\mathrm{b}, \mathrm{c}} \\
-21.00(-35.43 \text { to }-6.57)^{\mathrm{a}, \mathrm{b}}\end{array}$ & $-14.10(-28.53 \text { to } 0.33)^{d}$ & $-1.10(-15.53 \text { to } 13.33)^{\mathrm{e}}$ \\
\hline
\end{tabular}


TABLE 10 Function and disability results of studies of steroid injections (MD and 95\% Cl) (continued)

\begin{tabular}{|c|c|c|c|}
\hline Study & Short term ( $\leq 3$ months) & Medium term ( $>3$ and $\leq 6$ months) & Long term ( $>6$ and $\leq 12$ months) \\
\hline \multicolumn{4}{|c|}{ SPADI 8-item subscale } \\
\hline Carette $2003^{35}$ & $\begin{array}{l}-23.90(-37.89 \text { to }-9.91)^{\mathrm{b}, \mathrm{c}} \\
-20.00(-33.99 \text { to }-6.01)^{\mathrm{a}, \mathrm{b}}\end{array}$ & $-11.60(-25.59 \text { to } 2.39)^{d}$ & $0.30(-13.69 \text { to } 14.29)^{\mathrm{e}}$ \\
\hline \multicolumn{4}{|l|}{$S D Q$} \\
\hline Ryans $2005^{41}$ & $-4.70(-7.85 \text { to }-1.55)^{b, c}$ & $-1.00(-5.12 \text { to } 3.12)^{\dagger}$ & \\
\hline \multicolumn{4}{|l|}{ Global function } \\
\hline Ryans $2005^{41}$ & $-21.00(-34.54 \text { to }-7.46)^{\mathrm{b}, c}$ & $1.50(-17.96 \text { to } 20.96)^{\dagger}$ & \\
\hline \multicolumn{4}{|c|}{ Steroid + physiotherapy vs physiotherapy + placebo } \\
\hline \multicolumn{4}{|c|}{ SPADI total score } \\
\hline Carette $2003^{35}$ & $\begin{array}{l}-24.30(-38.33 \text { to }-10.27)^{\mathrm{b}, \mathrm{c}} \\
-12.50(-26.53 \text { to } 1.53)^{\mathrm{a}}\end{array}$ & $-9.40(-23.43 \text { to } 4.63)^{d}$ & $-2.80(-16.83 \text { to } 11.23)^{e}$ \\
\hline \multicolumn{4}{|c|}{ SPADI 8-item subscale } \\
\hline Carette $2003^{35}$ & $\begin{array}{l}-21.60(-35.34 \text { to }-7.86)^{\mathrm{b}, \mathrm{c}} \\
-11.00(-24.74 \text { to } 2.74)^{\mathrm{a}}\end{array}$ & $-9.80(-23.54 \text { to } 3.94)^{d}$ & $-3.10(-16.84 \text { to } 10.64)^{e}$ \\
\hline \multicolumn{4}{|l|}{$S D Q$} \\
\hline Ryans $2005^{41}$ & $-4.30(-7.76 \text { to }-0.84)^{b, c}$ & $-2.00(-5.96 \text { to } 1.96)^{\dagger}$ & \\
\hline \multicolumn{4}{|l|}{ Global function } \\
\hline Ryans $2005^{41}$ & $-11.70(-26.22 \text { to } 2.82)^{c}$ & $3.50(-15.42 \text { to } 22.42)^{t}$ & \\
\hline \multicolumn{4}{|c|}{ Steroid + physiotherapy vs steroid } \\
\hline \multicolumn{4}{|c|}{ SPADI total score } \\
\hline Carette $2003^{35}$ & $\begin{array}{l}9.80(-4.63 \text { to } 24.23)^{\mathrm{c}} \\
4.9(-9.53 \text { to } 19.33)^{\mathrm{a}}\end{array}$ & $1.20(-13.23 \text { to } 15.63)^{d}$ & $-1.8(-16.23 \text { to } 12.63)^{\mathrm{e}}$ \\
\hline \multicolumn{4}{|c|}{ SPADI 8-item subscale } \\
\hline Carette $2003^{35}$ & $\begin{array}{l}10.10(-4.04 \text { to } 24.24)^{\mathrm{c}} \\
5.70(-8.44 \text { to } 19.84)^{\mathrm{a}}\end{array}$ & $4.50(-9.64 \text { to } 18.64)^{d}$ & $0.50(-13.64 \text { to } 14.64)^{e}$ \\
\hline \multicolumn{4}{|l|}{$S D Q$} \\
\hline Ryans $2005^{41}$ & $1.70(-2.37 \text { to } 5.77)^{b}$ & $-0.20(-4.43 \text { to } 4.03)^{\dagger}$ & \\
\hline \multicolumn{4}{|l|}{ Global function } \\
\hline Ryans $2005^{41}$ & $9.40(-4.93 \text { to } 23.73)^{c}$ & $3.60(-15.49 \text { to } 22.69)^{\dagger}$ & \\
\hline
\end{tabular}

\footnotetext{
a Reported at 3 months or 12 weeks.

b Statistically significant, i.e. the $\mathrm{Cl}$ did not cross the line of no effect (zero).

c Reported at 6 weeks.

d Reported at 6 months.

e Reported at 12 months.

f Reported at 16 weeks.
} 


\section{Range of movement}

The range of movement measurements of interest in the review were external rotation, internal rotation and abduction (both passive and active); if internal rotation was not available but hand behind back was reported, this was used as a proxy measure. Five studies reported at least one of these measures (Table 11). Change from baseline data was available from three of these studies ${ }^{35,41,68}$ and final value data was available from one study. ${ }^{66} \mathrm{~A}$ fifth study, by Rizk $e t$ al. ${ }^{42}$ reported only means with no measures of variance or $p$-values with which to impute SDs. Table 12 provides the between-group differences in means and 95\% CIs. The outcome data for the individual groups in the included trials are available in Appendix 7.

\section{Steroid versus placebo}

One study by Bal et al. ${ }^{68}$ reported passive internal rotation at 12 weeks. There was a significantly greater improvement in the placebo group compared with steroid injection (MD $-28.50^{\circ}, 95 \% \mathrm{CI}$ $-41.56^{\circ}$ to $\left.-15.44^{\circ}\right)$. This result contradicts the original analysis in the paper, which reported that there was no statistically significant difference between the two groups, using the Mann-Whitney $U$-test.

Three studies reported passive external rotation at short-term follow-up, two at 6 weeks ${ }^{35,41}$ and two at 3 months. ${ }^{35,68}$ Improvement in passive external rotation was significantly greater with steroid injection than with placebo injection at 6 weeks (pooled MD $9.48^{\circ}, 95 \%$ CI $2.76^{\circ}$ to $16.19^{\circ}$; two RCTs) (Figure 10). There was no significant heterogeneity between these studies $\left(I^{2}=0 \%\right)$

Meta-analysis of the two studies that reported passive external rotation at 3 months showed no significant difference between the steroid and the placebo groups (Figure 11) ${ }^{35,68}$ This pooled result was associated with substantial heterogeneity $\left(I^{2}=80 \%\right)$ and should therefore be treated with caution. The study by Carette $\mathrm{et} \mathrm{al.} .^{35}$ showed a significant improvement in passive external rotation in the steroid group compared with placebo whereas the study by Bal et al ${ }^{68}$ showed no significant difference between groups (Table 11). There were several possible sources of heterogeneity, in particular differences in the risk of bias.

Two RCTs reported passive external rotation for medium-term follow-up: 16 weeks ${ }^{41}$ and 6 months. ${ }^{35,41}$ There was no significant difference between steroid and placebo groups in passive external rotation ( $\mathrm{MD} 4.67^{\circ}, 95 \% \mathrm{CI}-2.21^{\circ}$ to $11.55^{\circ}$ ) (Figure 12). There was some evidence of

TABLE 11 Range of movement (ROM) outcomes in studies evaluating steroid injections

\begin{tabular}{|c|c|c|}
\hline Study & ROM outcomes assessed ${ }^{a}$ & Data reported and time of follow-up \\
\hline Bal $2008^{68}$ & $\begin{array}{l}\text { Passive internal rotation }\left({ }^{\circ}\right) \text {, passive external rotation }\left({ }^{\circ}\right) \text {, passive } \\
\text { abduction }\left({ }^{\circ}\right)\end{array}$ & Change from baseline at 12 weeks \\
\hline $\begin{array}{l}\text { Calis } \\
2006^{66}\end{array}$ & Passive abduction $\left({ }^{\circ}\right)$, passive external rotation $\left(^{\circ}\right)$ & $\begin{array}{l}\text { Final value data at } 15 \text { days and } 3 \text { months (3-month data used } \\
\text { in analysis) }\end{array}$ \\
\hline $\begin{array}{l}\text { Carette } \\
2003^{35}\end{array}$ & $\begin{array}{l}\text { Passive external rotation }\left({ }^{\circ}\right) \text {, passive abduction }\left({ }^{\circ}\right) \text {, hand behind } \\
\text { back }(\mathrm{cm})\end{array}$ & Change from baseline at 6 weeks and 3,6 and 12 months ${ }^{b}$ \\
\hline Rizk $1991^{42}$ & $\begin{array}{l}\text { Passive internal rotation }\left({ }^{\circ}\right) \text {, passive external rotation }\left(^{\circ}\right) \text { and } \\
\text { passive abduction }\left({ }^{\circ}\right)\end{array}$ & $\begin{array}{l}\text { Mean only (no measure of variance) for weeks } 1-11,15 \text { weeks } \\
\text { and } 6 \text { months ( } 6 \text { weeks, } 11 \text { weeks and } 6 \text { months used in } \\
\text { analysis) }{ }^{c}\end{array}$ \\
\hline $\begin{array}{l}\text { Ryans } \\
2005^{41}\end{array}$ & Passive external rotation $\left({ }^{\circ}\right)$ & Change from baseline at 6 and 16 weeks \\
\hline
\end{tabular}

a Higher ROM indicates better outcome (appears to be measured in degrees but not specifically stated).

b All analyses adjusted for gender.

c Means only reported, SDs calculated if possible. 

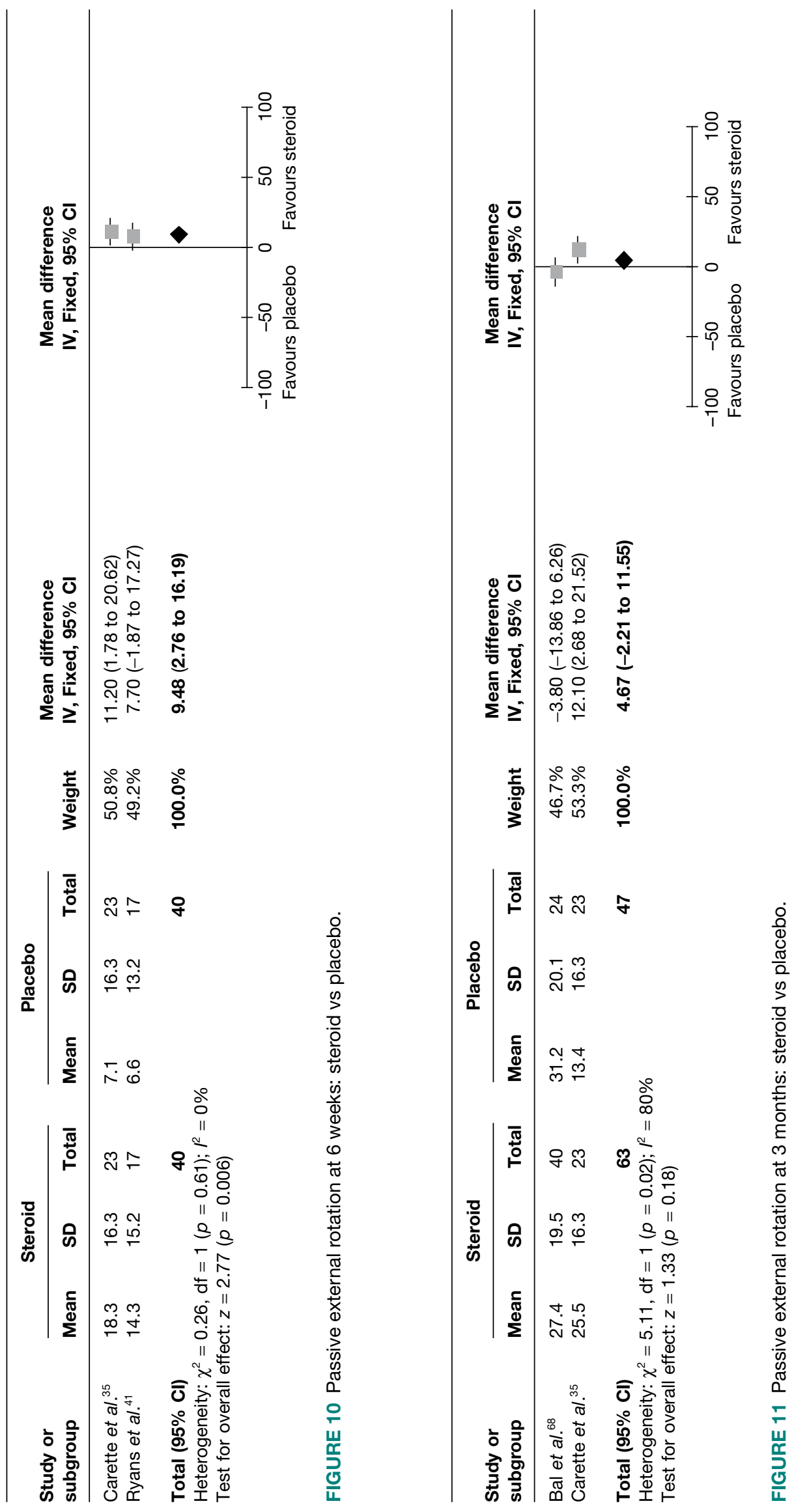
moderate statistical heterogeneity $\left(I^{2}=43 \%\right)$. Both studies appeared clinically similar although the follow-up time points differed. Carette et al. ${ }^{35}$ was the only study to examine passive external rotation at long-term follow-up and found no significant difference between steroid and placebo groups (Table 12). ${ }^{35}$

Carette $e$ t $a^{35}$ reported passive abduction and hand behind back at 6 weeks and 3, 6 and 12 months. There was no significant difference between groups at any of the time points for either outcome (Table 11).

\section{Steroid versus home exercise}

Calis et al. ${ }^{66}$ reported passive abduction and passive external rotation at 3 months. There was a significantly greater improvement in the steroid group compared with home exercise alone in both passive abduction ( $\mathrm{MD} 16.80^{\circ}, 95 \% \mathrm{CI} 6.60^{\circ}$ to $27.00^{\circ}$ ) and passive external rotation (MD $8.00^{\circ}, 95 \%$ CI $2.48^{\circ}$ to $13.52^{\circ}$ ).

\section{Steroid versus physiotherapy}

Calis et al. ${ }^{66}$ reported passive external rotation and passive abduction at 3 months. There was significantly greater passive external rotation at 3 months with physiotherapy than with steroid $\left(\mathrm{MD}-10.80^{\circ}, 95 \% \mathrm{CI}-16.94^{\circ}\right.$ to $\left.-4.66^{\circ}\right)$. There was no significant difference between treatment groups for passive abduction (Table 12).

\section{Steroid versus physiotherapy combined with placebo}

Three studies reported passive external rotation at time points up to 3 months. ${ }^{35,41,66}$ Two of the studies involved physiotherapy sessions over 12 weeks and in both physiotherapy groups a placebo injection was also given. ${ }^{35,41}$ Both studies reported data at 6 weeks and one ${ }^{35}$ reported data at 3 months; 6-week data were pooled. There was no significant difference in passive external rotation between groups at 6 weeks (Figure 13). There was substantial statistical heterogeneity between the studies $\left(I^{2}=64 \%\right)$.

Carette et al. ${ }^{35}$ reported passive external rotation, and passive abduction at 3 months. There was no significant difference between treatment groups for both outcomes (Table 12).

Medium-term follow-up data were available for the studies by Carette et al. ${ }^{35}$ and Ryans et al. ${ }^{41}$ Carette et al. ${ }^{35}$ reported passive external rotation at 6 months and Ryans et al.$^{41}$ at 16 weeks. There was no significant difference between groups (Figure 14). There was no significant difference between groups in passive external rotation at 12 months in the Carette et al. study. ${ }^{35}$ Similarly, the same study showed no significant difference between treatment groups in passive abduction or hand behind back at medium- or long-term follow-up (Table 12).

\section{Steroid combined with physiotherapy versus placebo}

Two studies reported passive external rotation at time points up to 3 months. ${ }^{35,41}$ Both studies reported data at 6 weeks and one ${ }^{35}$ reported data at 3 months. Data at 6 weeks were pooled. The meta-analysis (Figure 15) indicated that passive external rotation was significantly greater with steroid injection plus physiotherapy than with placebo injection at 6 weeks (pooled MD 16.99 ${ }^{\circ}$, $95 \%$ CI $10.01^{\circ}$ to $23.97^{\circ}$ ).

Passive external rotation was significantly greater with steroid injection plus physiotherapy than with placebo injection at 3 months (MD $17.60^{\circ}, 95 \%$ CI $7.90^{\circ}$ to $27.30^{\circ}$ ) in the study by Carette et al. $^{35}$

Medium-term follow-up data were also available up to 6 months: Carette et al. ${ }^{35}$ reported passive external rotation at 6 months and Ryans $e t$ al. ${ }^{41}$ at 16 weeks. The pooled estimate showed that 


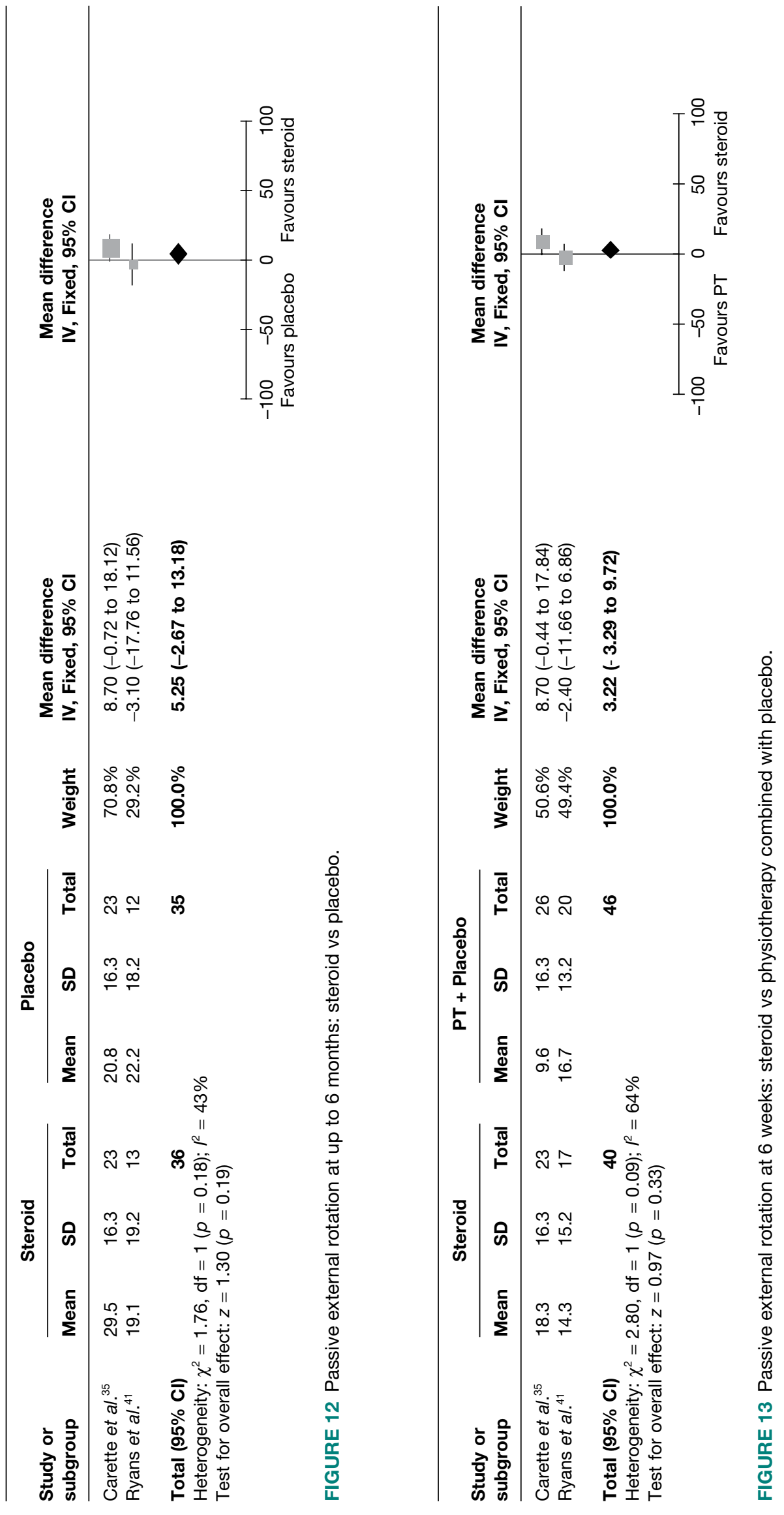




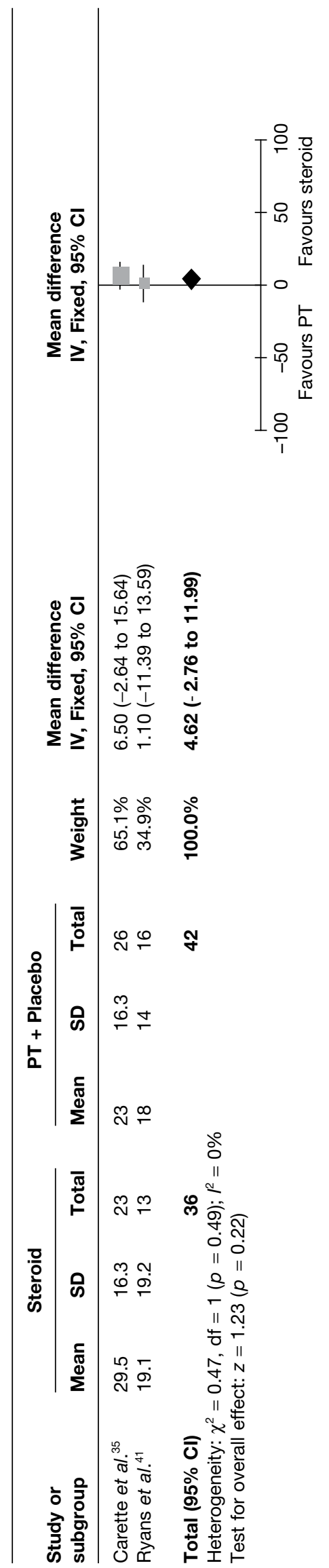

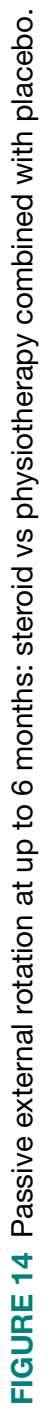

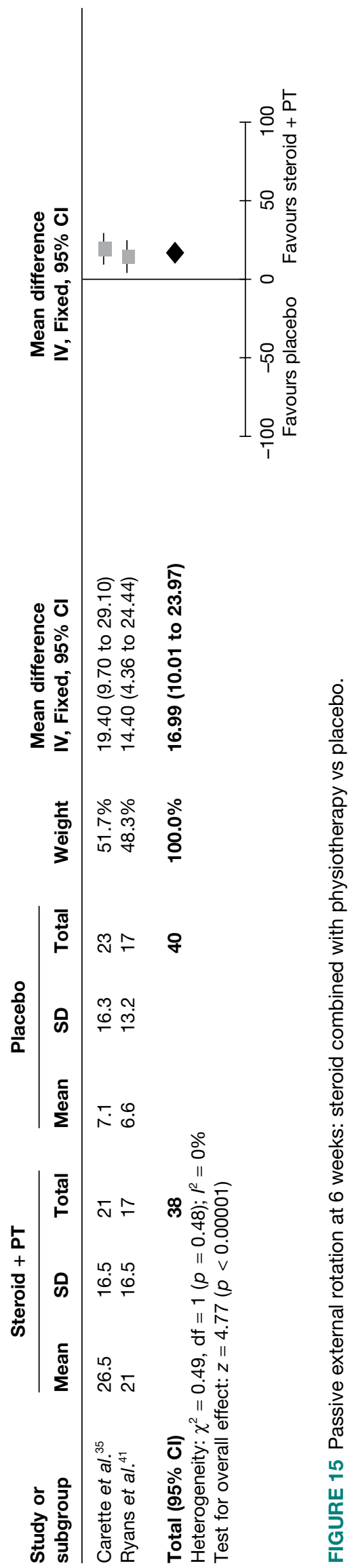


passive external rotation was significantly greater with steroid injection plus physiotherapy than with placebo injection at medium-term follow-up (pooled MD $8.14^{\circ}, 95 \%$ CI $0.18^{\circ}$ to $16.09^{\circ}$ ); however, there was substantial statistical heterogeneity $\left(I^{2}=70 \%\right)$ (Figure 16). There were a number of possible sources of heterogeneity including the dose of steroid used, different length of follow-up and the proportion of participants with diabetes. There was no significant difference between groups at 12 months in the single study reporting long-term follow-up (Table 12). ${ }^{35}$

Carette et al.$^{35}$ reported passive abduction at 6 weeks and 3, 6 and 12 months. Improvement of passive abduction was significantly greater in the steroid plus physiotherapy group than with placebo injection at 6 weeks (MD $15.30^{\circ}, 95 \%$ CI $7.14^{\circ}$ to $23.46^{\circ}$ ), 3 months (MD $16.30^{\circ}, 95 \%$ CI $8.14^{\circ}$ to $24.46^{\circ}$ ) and 6 months (MD $13.00^{\circ}, 95 \%$ CI $4.84^{\circ}$ to $21.16^{\circ}$ ). There was no significant difference between treatment groups at 12 months (Table 12).

In the same study there was a significantly greater improvement in hand behind back for the steroid with physiotherapy group than with placebo injection at 6 weeks $(\mathrm{MD}-9.00 \mathrm{~cm}, 95 \% \mathrm{CI}$ $-15.80 \mathrm{~cm}$ to $-2.20 \mathrm{~cm}$ ) and 3 months (MD $-7.10 \mathrm{~cm}, 95 \% \mathrm{CI}-13.90 \mathrm{~cm}$ to $-0.30 \mathrm{~cm}$ ), but not at any of the other time points (Table 12).

\section{Steroid combined with physiotherapy versus physiotherapy combined with placebo}

Three studies reported passive external rotation at time points up to 3 months. ${ }^{35,41,42}$ All studies reported data at 6 weeks ${ }^{35,41,42}$ and two also reported data at 3 months. ${ }^{35,42}$ Because of SDs not being available for one study, ${ }^{42}$ data were pooled from two of the studies for 6-week follow-up. ${ }^{35,41}$ The increase in passive external rotation was significantly greater with steroid injection plus physiotherapy than with physiotherapy (plus placebo injection) at 6 weeks (pooled MD 10.80 $95 \% \mathrm{CI} 4.02^{\circ}$ to $17.58^{\circ}$ ) (Figure 17 ). There was moderate to high heterogeneity between the studies $\left(I^{2}=70 \%\right)$; the results of this analysis should therefore be considered with caution.

The study by Carette et al. ${ }^{35}$ also showed that the increase in passive external rotation was significantly greater with steroid injection plus physiotherapy than with physiotherapy at 3 months (MD $13.00^{\circ}, 95 \%$ CI $3.56^{\circ}$ to $22.44^{\circ}$ ).

Medium-term data were also available from Carette et al. ${ }^{35}$ and Ryans et al. ${ }^{41}$ The pooled estimate (Figure 18) showed that passive external rotation was significantly greater with steroid injection plus physiotherapy than with physiotherapy at up to 6 months (pooled MD $7.36^{\circ}, 95 \% \mathrm{CI} 0.04^{\circ}$ to $\left.14.68^{\circ}\right)$. The study by Carette et al..$^{35}$ showed no significant difference between groups at 12 months (Table 12).

Carette et al..$^{35}$ reported passive abduction at 6 weeks and 3, 6 and 12 months. Improvement of passive abduction was significantly greater in the steroid plus physiotherapy group than with physiotherapy at 6 weeks (MD $11.30^{\circ}, 95 \%$ CI $3.40^{\circ}$ to $19.20^{\circ}$ ), 3 months (MD $10.40^{\circ}$, $95 \%$ CI $2.50^{\circ}$ to $18.30^{\circ}$ ) and 6 months (MD $7.90^{\circ}, 95 \% \mathrm{CI}-0.00^{\circ}$ to $15.80^{\circ}$ ). There was no significant difference between treatment groups at 12 months (Table 12).

\section{Steroid versus steroid and physiotherapy}

Two studies reported passive external rotation for short-term follow-up; ${ }^{35,41}$ both studies reported data at 6 weeks and one ${ }^{35}$ reported data at 3 months; the 6-week data were pooled. Passive external rotation was significantly greater with steroid injection plus physiotherapy than with steroid alone at 6 weeks (pooled $\mathrm{MD}-7.52^{\circ}, 95 \% \mathrm{CI}-14.70^{\circ}$ to $-0.34^{\circ}$ ). This analysis was not associated with statistical heterogeneity $\left(I^{2}=0 \%\right.$ ) (Figure 19). The study by Carette et al. ${ }^{35}$ showed no significant difference between treatment groups at 3 months (Table 12). 


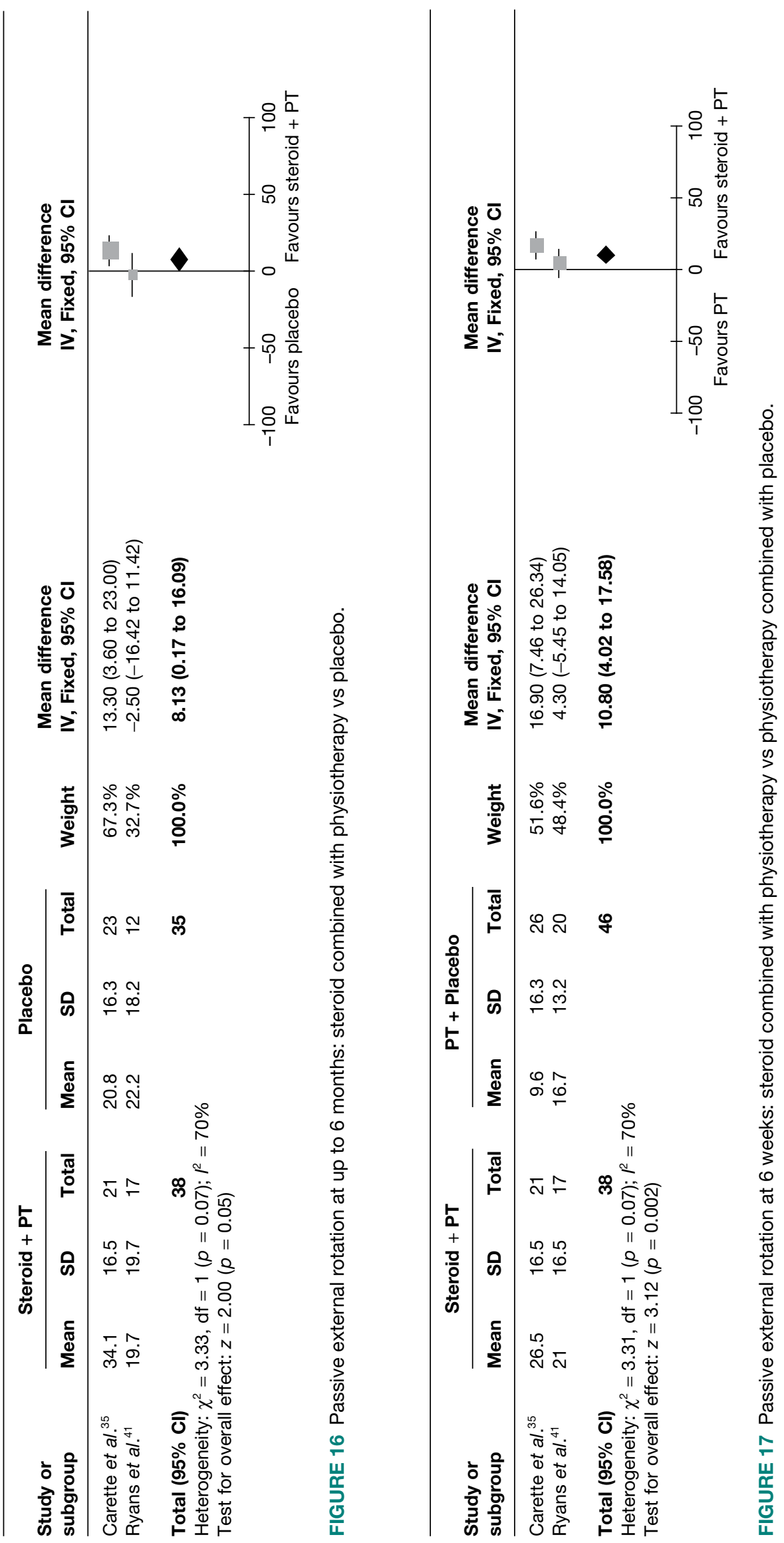




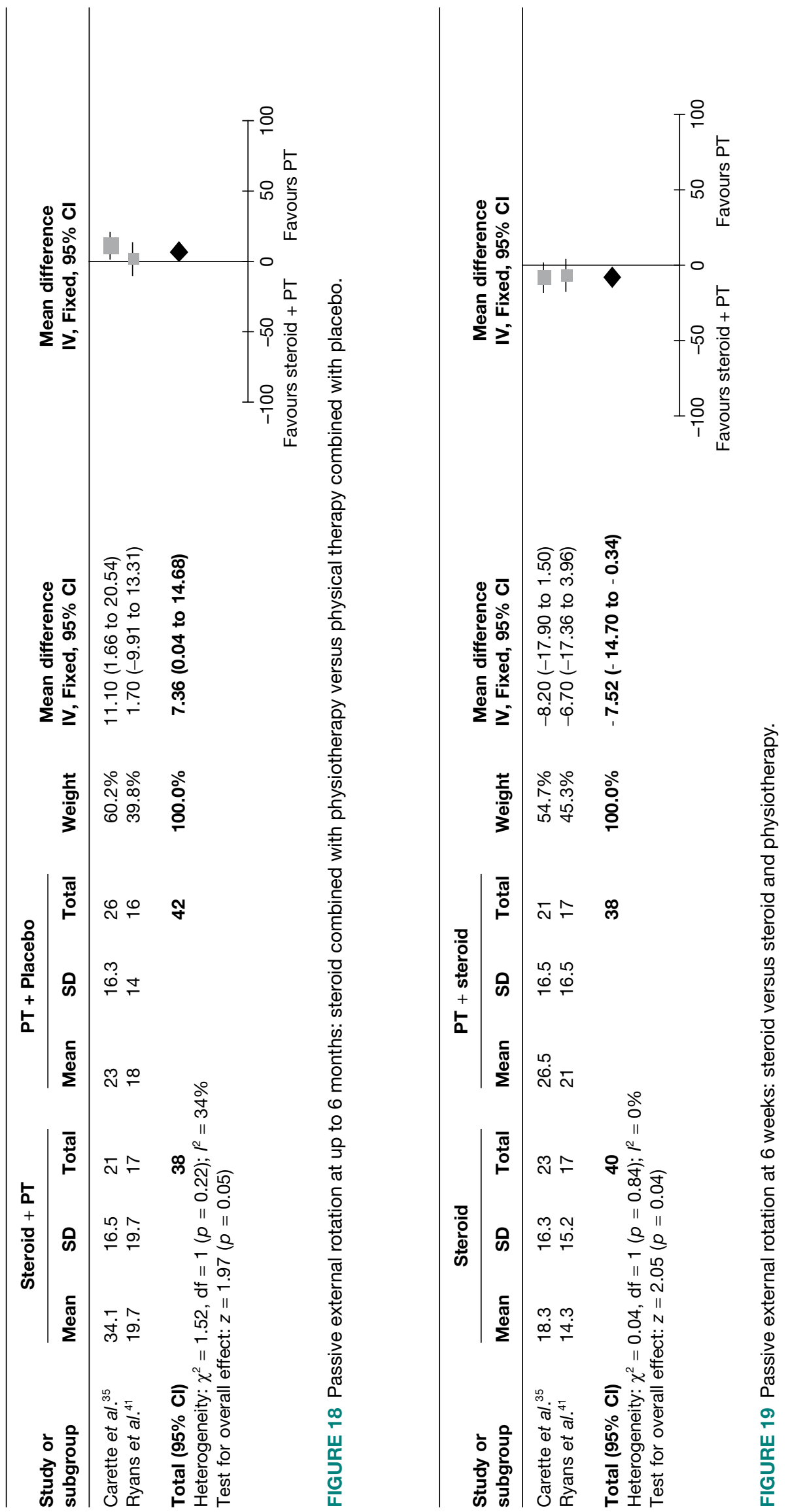




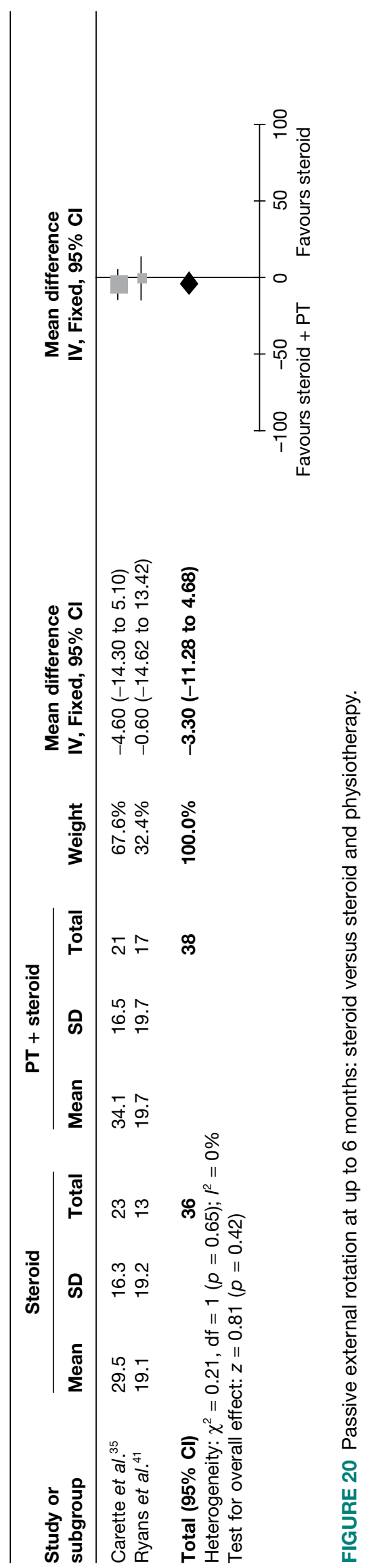


TABLE 12 Range of movement results of studies of steroid injections (MD and 95\% Cl)

\begin{tabular}{|c|c|c|c|}
\hline Study & $\begin{array}{l}\text { Short term } \\
\text { ( } \leq 3 \text { months) }\end{array}$ & $\begin{array}{l}\text { Medium term } \\
\text { ( }>3 \text { and } \leq 6 \text { months) }\end{array}$ & $\begin{array}{l}\text { Long term } \\
\text { ( }>6 \text { and } \leq 12 \text { months) }\end{array}$ \\
\hline \multicolumn{4}{|c|}{ Steroid vs placebo } \\
\hline \multicolumn{4}{|c|}{ Passive internal rotation $\left({ }^{\circ}\right)$} \\
\hline Bal $2008^{68}$ & $-28.50(-41.56 \text { to }-15.44)^{a, b}$ & & \\
\hline \multicolumn{4}{|c|}{ Passive external rotation $\left({ }^{\circ}\right)$} \\
\hline Bal $2008^{68}$ & $-3.80(-13.86 \text { to } 6.26)^{\mathrm{a}}$ & & \\
\hline \multirow[t]{2}{*}{ Carette $2003^{35}$} & 11.20 (1.78 to 20.62) $)^{\mathrm{b}, \mathrm{c}}$ & $8.70(-0.72 \text { to } 18.12)^{d}$ & $0.40(-9.02 \text { to } 9.82)^{\mathrm{e}}$ \\
\hline & $12.10(2.68 \text { to } 21.52)^{\mathrm{a}, \mathrm{b}}$ & & \\
\hline Ryans $2005^{41}$ & $7.70(-1.87 \text { to } 17.27)^{c}$ & $-3.10(-17.76 \text { to } 11.56)^{\dagger}$ & \\
\hline \multicolumn{4}{|c|}{ Passive abduction $\left({ }^{\circ}\right)$} \\
\hline Bal $2008^{68}$ & $3.60(-10.90 \text { to } 18.10)^{\mathrm{a}}$ & & \\
\hline \multirow[t]{2}{*}{ Carette $2003^{35}$} & $7.20(-0.83 \text { to } 15.23)^{c}$ & $7.80(-0.23 \text { to } 15.83)^{d}$ & $-1.20(-9.23 \text { to } 6.83)^{\mathrm{e}}$ \\
\hline & $12.30(4.27 \text { to } 20.33)^{\mathrm{a}}$ & & \\
\hline \multicolumn{4}{|c|}{ Hand behind back (cm) } \\
\hline \multirow[t]{2}{*}{ Carette $2003^{35}$} & $-0.50(-7.15 \text { to } 6.15)^{\mathrm{c}}$ & $-0.50(-7.15 \text { to } 6.15)^{d}$ & $2.80(-3.85 \text { to } 9.4)^{\mathrm{e}}$ \\
\hline & $-1.10(-7.75 \text { to } 5.55)^{\mathrm{a}}$ & & \\
\hline \multicolumn{4}{|c|}{ Steroid vs home exercise } \\
\hline \multicolumn{4}{|c|}{ Passive external rotation ( ${ }^{\circ}$ ) } \\
\hline Calis $2006^{66}$ & $8.00(2.48 \text { to } 13.52)^{a, b}$ & & \\
\hline \multicolumn{4}{|c|}{ Passive abduction ( ${ }^{\circ}$ ) } \\
\hline Calis $2006^{66}$ & $16.80(6.60 \text { to } 27.00)^{\mathrm{a}, \mathrm{b}}$ & & \\
\hline \multicolumn{4}{|c|}{ Steroid vs physiotherapy } \\
\hline \multicolumn{4}{|c|}{ Passive external rotation $\left({ }^{\circ}\right)$} \\
\hline Calis $2006^{66}$ & $-10.80(-16.94 \text { to }-4.66)^{a, b}$ & & \\
\hline \multicolumn{4}{|c|}{ Passive abduction (') } \\
\hline Calis $2006^{66}$ & $-8.10(-19.07 \text { to } 2.87)^{\mathrm{a}}$ & & \\
\hline \multicolumn{4}{|c|}{ Steroid vs physiotherapy + placebo } \\
\hline \multicolumn{4}{|c|}{ Passive external rotation ( ${ }^{\circ}$ ) } \\
\hline \multirow[t]{2}{*}{ Carette $2003^{35}$} & $8.70(-0.44 \text { to } 17.84)^{c}$ & $6.50(-2.64 \text { to } 15.64)^{d}$ & $-0.80(-9.94 \text { to } 8.34)^{\mathrm{e}}$ \\
\hline & $7.50(-1.64 \text { to } 16.64)^{\mathrm{a}}$ & & \\
\hline Ryans $2005^{41}$ & $-2.40(-11.66 \text { to } 6.86)^{c}$ & $1.10(-11.39 \text { to } 13.59)^{t}$ & \\
\hline \multicolumn{4}{|c|}{ Passive abduction $\left({ }^{\circ}\right)$} \\
\hline \multirow[t]{2}{*}{ Carette $2003^{35}$} & $3.20(-4.57 \text { to } 10.97)^{c}$ & $2.70(-5.07 \text { to } 10.47)^{d}$ & $-1.90(-9.67 \text { to } 5.87)^{\mathrm{e}}$ \\
\hline & $6.40(-1.37 \text { to } 14.17)^{\mathrm{a}}$ & & \\
\hline \multicolumn{4}{|c|}{ Hand behind back (cm) } \\
\hline \multirow[t]{2}{*}{ Carette $2003^{35}$} & $-0.80(-7.30 \text { to } 5.70)^{c}$ & $0.70(-5.80 \text { to } 7.20)^{d}$ & $2.90(-3.60 \text { to } 9.40)^{e}$ \\
\hline & $0.10(-6.40 \text { to } 6.60)^{\mathrm{a}}$ & & \\
\hline
\end{tabular}


TABLE 12 Range of movement results of studies of steroid injections (MD and 95\% Cl) (continued)

\begin{tabular}{|c|c|c|c|}
\hline Study & $\begin{array}{l}\text { Short term } \\
\text { ( } \leq 3 \text { months) }\end{array}$ & $\begin{array}{l}\text { Medium term } \\
\text { ( }>3 \text { and } \leq 6 \text { months) }\end{array}$ & $\begin{array}{l}\text { Long term } \\
\text { ( }>6 \text { and } \leq 12 \text { months) }\end{array}$ \\
\hline \multicolumn{4}{|c|}{ Steroid + physiotherapy vs placebo } \\
\hline \multicolumn{4}{|c|}{ Passive external rotation $\left({ }^{\circ}\right.$ ) } \\
\hline \multirow[t]{2}{*}{ Carette $2003^{35}$} & $19.40(9.70 \text { to } 29.10)^{b, c}$ & $13.30(3.60 \text { to } 23.00)^{\mathrm{b}, \mathrm{d}}$ & $7.60(-2.10 \text { to } 17.30)^{\mathrm{e}}$ \\
\hline & $17.60(7.90 \text { to } 27.30)^{a, b}$ & & \\
\hline Ryans $2005^{41}$ & $-2.50(-16.42 \text { to } 11.42)^{c}$ & $-2.50(-16.42 \text { to } 11.42)^{\dagger}$ & \\
\hline \multicolumn{4}{|c|}{ Passive abduction $\left({ }^{\circ}\right)$} \\
\hline \multirow[t]{2}{*}{ Carette $2003^{35}$} & $15.30(7.14 \text { to } 23.46)^{b, c}$ & $13.00(4.84 \text { to } 21.16)^{b, d}$ & $5.70(-2.46,13.86)^{\mathrm{e}}$ \\
\hline & $16.30(8.14,24.46)^{a, b}$ & & \\
\hline \multicolumn{4}{|c|}{ Hand behind back (cm) } \\
\hline \multirow[t]{2}{*}{ Carette $2003^{35}$} & $-9.00(-15.80 \text { to }-2.20)^{\mathrm{b}, \mathrm{c}}$ & $-5.90(-12.70 \text { to } 0.90)^{d}$ & $-1.50(-8.30 \text { to } 5.30)^{\mathrm{e}}$ \\
\hline & $-7.10(-13.90 \text { to }-0.30)^{\mathrm{a}, \mathrm{b}}$ & & \\
\hline \multicolumn{4}{|c|}{ Steroid + physiotherapy vs physiotherapy + placebo } \\
\hline \multicolumn{4}{|c|}{ Passive external rotation $\left({ }^{\circ}\right)$} \\
\hline \multirow[t]{2}{*}{ Carette $2003^{35}$} & $16.90(7.46 \text { to } 26.34)^{\mathrm{b}, \mathrm{c}}$ & $11.10(1.66 \text { to } 20.54)^{\mathrm{b}, \mathrm{d}}$ & $6.40(-3.04 \text { to } 15.84)^{e}$ \\
\hline & $13.00(3.56 \text { to } 22.44)^{\mathrm{a}, \mathrm{b}}$ & & \\
\hline Ryans $2005^{41}$ & $4.30(-5.45 \text { to } 14.05)^{c}$ & $1.70(-9.91 \text { to } 13.31)^{\dagger}$ & \\
\hline \multicolumn{4}{|c|}{ Passive abduction $\left({ }^{\circ}\right)$} \\
\hline \multirow[t]{2}{*}{ Carette $2003^{35}$} & $11.30(3.40 \text { to } 19.20)^{b, c}$ & $7.90(-0.00 \text { to } 15.80)^{d}$ & $5.00(-2.90 \text { to } 12.90)^{e}$ \\
\hline & $10.40(2.50 \text { to } 18.30)^{a, b}$ & & \\
\hline \multicolumn{4}{|c|}{ Hand behind back (cm) } \\
\hline \multirow[t]{2}{*}{ Carette $2003^{35}$} & $-9.30(-15.96 \text { to }-2.64)^{\mathrm{b}, \mathrm{c}}$ & $-4.70(-11.36 \text { to } 1.96)^{d}$ & $-1.40(-8.06 \text { to } 5.26)^{\mathrm{e}}$ \\
\hline & $-5.90(-12.56 \text { to } 0.76)^{a, b}$ & & \\
\hline \multicolumn{4}{|c|}{ Steroid + physiotherapy vs steroid } \\
\hline \multicolumn{4}{|c|}{ Passive external rotation $\left({ }^{\circ}\right)$} \\
\hline \multirow[t]{2}{*}{ Carette $2003^{35}$} & $-8.20(-17.90 \text { to } 1.50)^{c}$ & $-4.60(-14.30 \text { to } 5.1)^{d}$ & $-7.20(-16.90 \text { to } 2.50)^{\mathrm{e}}$ \\
\hline & $-5.50(-15.20 \text { to } 4.20)^{\mathrm{a}}$ & & \\
\hline Ryans $2005^{41}$ & $-6.70(-17.36 \text { to } 3.96)^{b, c}$ & $-0.60(-14.62 \text { to } 13.42)^{f}$ & \\
\hline \multicolumn{4}{|c|}{ Passive abduction ( $\left.{ }^{\circ}\right)$} \\
\hline \multirow[t]{2}{*}{ Carette $2003^{35}$} & $-8.10(-16.26 \text { to } 0.06)^{c}$ & $-5.20(-13.36 \text { to } 2.96)^{d}$ & $-6.90(-15.06 \text { to } 1.26)^{\mathrm{e}}$ \\
\hline & $-4.00(-12.16 \text { to } 4.16)^{\mathrm{a}}$ & & \\
\hline \multicolumn{4}{|c|}{ Hand behind back (cm) } \\
\hline \multirow[t]{2}{*}{ Carette $2003^{35}$} & $8.50(1.70 \text { to } 15.30)^{\mathrm{b}, \mathrm{c}}$ & $5.40(-1.40 \text { to } 12.20)^{d}$ & $4.30(-2.50 \text { to } 11.10)^{e}$ \\
\hline & $6.00(-0.80 \text { to } 12.80)^{\mathrm{a}}$ & & \\
\hline
\end{tabular}

\footnotetext{
a Reported at 3 months or 12 weeks.

b Statistically significant, i.e. the $\mathrm{Cl}$ did not cross the line of no effect (zero).

c Reported at 6 weeks.

d Reported at 6 months.

e Reported at 12 months.

f Reported at 16 weeks.
} 
Data were also available for medium-term follow-up for the same two studies. The pooled analysis showed no significant difference between groups and the analysis was not associated with statistical heterogeneity $\left(I^{2}=0 \%\right)$ (Figure 20). The study by Carette et al. ${ }^{35}$ showed no significant difference between treatment groups at long-term follow-up (Table 12).

Carette et al..$^{35}$ reported passive abduction at 6 weeks and 3, 6 and 12 months. At 6 weeks improvement of passive abduction was significantly greater in the steroid plus physiotherapy group than with steroid alone (MD $-8.10,95 \% \mathrm{CI}-16.26$ to 0.06 ); there were no significant differences between treatment groups at later follow-up times (Table 12).

Similarly, for hand behind back, there was a significantly better improvement in the steroid with physiotherapy group than with steroid alone at 6 weeks (MD 8.50, 95\% CI 1.70 to 15.30) but not at later follow-up (Table 12).

\section{Quality of life}

Only Carette et al. ${ }^{35}$ reported quality of life data following treatment (Table 13). ${ }^{35}$ Table 14 provides between-group differences in means and associated 95\% CIs. Further outcome data can be found in Appendix 7.

There was a statistically significant improvement in the physical component of the SF-36 with steroid plus physiotherapy compared with placebo at 6 months (MD 7.10, 95\% CI 0.86 to 13.34). There was no statistically significant difference between these two groups at any of the other time points evaluated.

There was a statistically significant improvement with steroid plus physiotherapy compared with physiotherapy (plus placebo injection) in the physical component at 6 weeks (MD 5.30, 95\% CI 0.03 to 10.57) and in the mental component at 6 months (MD 8.20,95\% CI 1.98 to 14.42) and 12 months (MD 6.90, 95\% CI 0.68 to 13.12). There was no statistically significant difference between these two treatments groups at any of the other time points evaluated.

For the comparisons steroid compared with placebo, steroid compared with physiotherapy (plus placebo injection) and steroid compared with physiotherapy plus steroid, the study by Carette et al. ${ }^{35}$ showed no statistically significant difference between treatment groups at any of the time points evaluated.

TABLE 13 Quality of life outcomes in studies that evaluated steroid injections

\begin{tabular}{lll}
\hline Study & QoL outcomes assessed & $\begin{array}{l}\text { Data reported and time point before and closest to } \mathbf{3} \text { and } \\
\mathbf{6} \text { months }\end{array}$ \\
\hline Carette & SF-36 physical component & Change from baseline at 6 weeks and 3,6 and 12 months \\
$2003^{35}$ & SF-36 mental component & \\
Ryans & SF-36 scores: physical function, role limitation physical, role & Baseline data only reported \\
$2005^{41}$ & limitation mental, social functioning, mental health, vitality, bodily & \\
& pain, health perception, change in health &
\end{tabular}

SF-36, Short Form questionnaire-36 items.

a All analyses adjusted for gender. 
TABLE 14 Quality of life results of studies of steroid injections (MD and 95\% Cl)

\begin{tabular}{|c|c|c|c|}
\hline Study & Short term ( $\leq 3$ months) & Medium term ( $>3$ and $\leq 6$ months) & Long term ( $>6$ and $\leq 12$ months) \\
\hline \multicolumn{4}{|c|}{ Steroid vs placebo } \\
\hline \multicolumn{4}{|c|}{ SF-36 physical component } \\
\hline \multirow[t]{2}{*}{ Carette $2003^{35}$} & $1.90(-3.36 \text { to } 7.16)^{\mathrm{a}}$ & $3.30(-1.96 \text { to } 8.56)^{c}$ & $1.00(-4.26 \text { to } 6.26)^{d}$ \\
\hline & $5.10(-0.16 \text { to } 10.36)^{b}$ & & \\
\hline \multicolumn{4}{|c|}{ SF-36 mental component } \\
\hline \multirow[t]{2}{*}{ Carette $2003^{35}$} & $-1.10(-7.23 \text { to } 5.03)^{\mathrm{a}}$ & $1.70(-5.34 \text { to } 8.74)^{c}$ & $0.30(-5.83 \text { to } 6.43)^{d}$ \\
\hline & $1.00(-5.13 \text { to } 7.13)^{b}$ & & \\
\hline \multicolumn{4}{|c|}{ Steroid vs physiotherapy } \\
\hline \multicolumn{4}{|c|}{ SF-36 physical component } \\
\hline \multirow[t]{2}{*}{ Carette $2003^{35}$} & $3.30(-1.97 \text { to } 8.57)^{\mathrm{a}}$ & $1.00(-4.27 \text { to } 6.27)^{c}$ & $1.70(-3.57 \text { to } 6.97)^{d}$ \\
\hline & $3.70(-1.57 \text { to } 8.97)^{\mathrm{b}}$ & & \\
\hline \multicolumn{4}{|c|}{ SF-36 mental component } \\
\hline \multirow[t]{2}{*}{ Carette $2003^{35}$} & $-0.50(-6.61 \text { to } 5.61)^{\mathrm{a}}$ & $2.80(-3.31 \text { to } 8.91)^{c}$ & $1.10(-5.01 \text { to } 7.21)^{d}$ \\
\hline & $-1.60(-7.71 \text { to } 4.51)^{\mathrm{b}}$ & & \\
\hline \multicolumn{4}{|c|}{ Steroid combined with physiotherapy vs placebo } \\
\hline \multicolumn{4}{|c|}{ SF-36 physical component } \\
\hline \multirow[t]{2}{*}{ Carette $2003^{35}$} & $3.90(-1.36 \text { to } 9.16)^{a}$ & $2.00(-3.26 \text { to } 7.26)^{c}$ & $1.40(-3.86 \text { to } 6.66)^{d}$ \\
\hline & $3.60(-1.66 \text { to } 8.86)^{b}$ & & \\
\hline \multicolumn{4}{|c|}{ SF-36 mental component } \\
\hline \multirow[t]{2}{*}{ Carette $2003^{35}$} & $3.10(-3.14 \text { to } 9.34)^{\mathrm{a}}$ & $7.10(0.86 \text { to } 13.34)^{c, e}$ & $6.10(-0.14 \text { to } 12.34)^{d}$ \\
\hline & $5.40(-0.84 \text { to } 11.64)^{b}$ & & \\
\hline \multicolumn{4}{|c|}{ Steroid combined with physiotherapy vs physiotherapy } \\
\hline \multicolumn{4}{|c|}{ SF-36 physical component } \\
\hline \multirow[t]{2}{*}{ Carette $2003^{35}$} & $5.30(0.03 \text { to } 10.57)^{\mathrm{a}, \mathrm{e}}$ & $-0.30(-5.57 \text { to } 4.97)^{c}$ & $2.10(-3.17 \text { to } 7.37)^{d}$ \\
\hline & $2.20(-3.07 \text { to } 7.47)^{b}$ & & \\
\hline \multicolumn{4}{|c|}{ SF-36 mental component } \\
\hline \multirow[t]{2}{*}{ Carette $2003^{35}$} & $3.70(-2.52 \text { to } 9.92)^{\mathrm{a}}$ & $8.20(1.98 \text { to } 14.42)^{c, e}$ & $6.90(0.68 \text { to } 13.12)^{\mathrm{d}, e}$ \\
\hline & $2.80(-3.42 \text { to } 9.02)^{b}$ & & \\
\hline \multicolumn{4}{|c|}{ Steroid combined with physiotherapy vs steroid } \\
\hline \multicolumn{4}{|c|}{ SF-36 physical component } \\
\hline \multirow[t]{2}{*}{ Carette $2003^{35}$} & $-2.00(-7.26 \text { to } 3.26)^{\mathrm{a}}$ & $1.30(-3.96 \text { to } 6.56)^{c}$ & $-0.40(-5.66 \text { to } 4.86)^{d}$ \\
\hline & $1.50(-3.76 \text { to } 6.76)^{b}$ & & \\
\hline \multicolumn{4}{|c|}{ SF-36 mental component } \\
\hline \multirow[t]{2}{*}{ Carette $2003^{35}$} & $-4.20(-10.44 \text { to } 2.04)^{\mathrm{a}}$ & $-5.40(-11.64 \text { to } 0.84)^{c}$ & $-5.80(-12.04 \text { to } 0.44)^{d}$ \\
\hline & $-4.40(-10.64 \text { to } 1.84)^{b}$ & & \\
\hline
\end{tabular}

\footnotetext{
a Reported at 6 weeks.

b Reported at 3 months or 12 weeks.

c Reported at 6 months.

d Reported at 12 months.

e Statistically significant, i.e. the $\mathrm{Cl}$ did not cross the line of no effect (zero).
} 
TABLE 15 Adverse events in studies evaluating steroid injections

\begin{tabular}{ll}
\hline Study & Adverse events reported \\
\hline Bal $2008^{68}$ & No side effects were noted during the drug or exercise therapy sessions \\
Calis $2006^{66}$ & Not reported \\
Carette $2003^{35}$ & Not reported \\
Dacre $1989^{67}$ & No patients had adverse reactions \\
Rizk $1991^{42}$ & No withdrawals due to adverse effects \\
Ryans $2005^{41}$ & Not reported \\
\hline
\end{tabular}

\section{Adverse events}

Three of the studies that investigated steroids reported adverse events. ${ }^{42,67,68}$ There was a limited amount of information reported regarding adverse events in the included studies (Table 15). None of the studies reported any details regarding how adverse event data were collected or recorded; therefore, the data should be treated with caution. Two reported that no adverse effects were reported in any of the treatment groups $s^{67,68}$ and one reported that there were no withdrawals due to adverse effects. ${ }^{42}$

\section{Summary}

The six included steroid injection studies were diverse in terms of the intervention: two used a single steroid injection of $20 \mathrm{mg}$, three a single injection of $40 \mathrm{mg}$ and one used three injections of $40 \mathrm{mg}$ administered over 3 weeks. Five of the studies reported using an intra-articular injection. There was also some variation between studies in the physiotherapy provided, although most of the studies used a programme of $<6$ weeks' duration that included mobilisation and home exercise for all groups. Five of the studies were multi-armed trials. Steroid injection was compared with placebo in three studies, home exercise in one study and physiotherapy alone in four studies (with or without placebo). The effect of steroid injection followed by physiotherapy was also assessed. This combined intervention was compared with placebo, steroid alone and physiotherapy (with placebo) alone.

Information about previous treatments that participants had received and stage of frozen shoulder was limited. Outcomes were not reported by stage of frozen shoulder or by whether or not participants had diabetes. As a result, it was not possible to explore in the synthesis the effect of these variables on outcome. It was unclear whether four of the studies were truly randomised and therefore these studies had a potentially high risk of bias. ${ }^{42,66-68}$ The fifth study was of adequate quality, ${ }^{35}$ whereas the sixth was of reasonable quality but may have a risk of bias. ${ }^{41}$

For most of the comparisons, data were available from only one or two studies, which may have been underpowered to detect an effect. There were sufficient data to pool the two best-quality studies for some of the outcomes, although in some instances statistical heterogeneity was high. In addition, although both studies used a single intra-articular injection, one evaluated a guided injection of $40 \mathrm{mg}$ of steroid ${ }^{35}$ and the other used a $20-\mathrm{mg}$ dose and the injection appeared to be unguided. ${ }^{41}$ For most outcomes and comparisons it was necessary to undertake a narrative synthesis.

\section{Steroid alone}

There was evidence of short-term benefit with steroid injection compared with placebo for pain, function and disability, and range of movement but not quality of life. The evidence on function and disability was mixed and this may reflect differences in study quality and/or clinical differences. There was also evidence of short-term benefit with steroid injection compared with 
home exercise alone for function and disability and two measures of range of movement from one study with a potentially high risk of bias. There was no consistent evidence across outcomes for a benefit with steroid injection compared with physical therapy.

\section{Steroid followed by physiotherapy}

There was consistent evidence across all outcomes (pain, function and disability, range of movement and quality of life) of a short-term benefit with steroid injection followed by physiotherapy compared with placebo or physiotherapy; however, this was based on a small number of studies. There was also evidence of better quality of life with the combined treatment in the medium term. When steroid injection plus physiotherapy was compared with steroid alone there was a short-term beneficial reduction in pain and a better range of movement at the 6-week follow-up with the combined intervention but not at 3 months or later and there was no benefit for any other outcomes.

In conclusion, a small number of diverse studies were identified, four of which had a potentially high risk of bias. Based on the best available evidence, there was a benefit, mainly short term, with a single steroid. There was an added benefit with providing physiotherapy in addition to steroid injection compared with home exercise alone and physiotherapy alone for several outcomes. There was also benefit with the combined intervention compared with steroid alone for pain and for range of movement at 6 weeks but not the other outcomes. There was insufficient evidence to conclude with reasonable certainty in what clinical situations steroid injection, with or without physiotherapy, is most likely to be effective for primary frozen shoulder.

\section{Sodium hyaluronate}

\section{Study characteristics}

Three RCTs investigated sodium hyaluronate in the treatment of primary frozen shoulder. A summary of the study characteristics is reported in Table 16. All were full papers of studies conducted in Turkey, ${ }^{66}$ Italy $^{69}$ and Japan ${ }^{70}$ between 1996 and 2006. There were two two-armed trials $^{69,70}$ and one four-armed trial. ${ }^{66}$

Calis et al.$^{66}$ used a $30-\mathrm{mg}$ injection once weekly for 2 weeks, Takagishi et al. ${ }^{70}$ used $2 \mathrm{mg}$ injected once weekly for 5 weeks and Rovetta et al. ${ }^{69}$ used $20 \mathrm{mg}$ of sodium hyaluronate coadministered with $20 \mathrm{mg}$ of steroid (triamcinolone acetonide) injected at 15-day intervals for the first month then monthly for 6 months and physical therapy (mobilisation or exercises) for 4-12 weeks. The comparators were steroid injection, ${ }^{66,70}$ steroid injection and physiotherapy, ${ }^{69}$ physiotherapy ${ }^{66}$ and no intervention except for home exercise. ${ }^{66}$

Two of the three studies included physiotherapy. ${ }^{66,69}$ Both studies differed in the duration and content of the regimen, but each contained some form of active exercise or mobilisation. Calis et al.$^{66}$ had a regimen of 10 daily sessions consisting of a heat pack applied for 20 minutes, ultrasonic therapy for 5 minutes $\left(1.5 \mathrm{~W} / \mathrm{cm}^{2}\right.$ intensity), TENS for 20 minutes at the patient's level of tolerance, and stretching exercises. In comparison, Rovetta et al. ${ }^{69}$ had a 4 - to 12 -week regimen of the most appropriate therapy for the individual (passive mobilisation, active exercises and facilitation exercises), which was chosen by a physiotherapist. Only Calis et al. ${ }^{66}$ reported that participants had a home exercise programme, which consisted of stretching and Codman exercises, but did not provide any further details.

Two studies reported that patients received concomitant treatments. ${ }^{69,70}$ Rovetta et al. ${ }^{69}$ allowed paracetamol (dose unspecified) to be taken if necessary, whereas Takagishi et al. ${ }^{70}$ stated that participants who were using topical NSAIDs prior to commencing the study were allowed to continue to do so. Calis et al. ${ }^{66}$ allowed paracetamol to be taken if necessary. 
TABLE 16 Studies of sodium hyaluronate with or without physical therapy

\begin{tabular}{|c|c|c|c|}
\hline $\begin{array}{l}\text { Study details and } \\
\text { study design }\end{array}$ & $\begin{array}{l}\text { Participants randomised } \\
\text { (total } n \text { and by group) }\end{array}$ & Trial treatments & Condition-related inclusion criteria \\
\hline $\begin{array}{l}\text { Calis 2006 } \\
\text { Four-arm RCT }\end{array}$ & $\begin{array}{l}n=90 \text { (95 shoulders) } \\
\text { Sodium hyaluronate injection: } \\
24 \text { (27) } \\
\text { Steroid injection: } 25 \text { (26) } \\
\text { PT: } 21 \text { (22) } \\
\text { No intervention (home exercise } \\
\text { only): } 20 \text { (20) }\end{array}$ & $\begin{array}{l}\text { Sodium hyaluronate: } 30 \mathrm{mg} \text {, one } \\
\text { injection once weekly for } 2 \text { weeks } \\
\text { Steroid: triamcinolone acetonide, } \\
40 \mathrm{mg} \text {, one injection } \\
\text { PT: } 10 \text { daily sessions at least } \\
45 \text { minutes }\end{array}$ & $\begin{array}{l}\text { History of pain for at least } 1 \text { month; limited } \\
\text { active and passive shoulder movement; } \\
\text { decreased passive ROM of } \geq 20 \% \text {, in at least } \\
\text { three movements, according to the American } \\
\text { Medical Association guide for the evaluation of } \\
\text { permanent impairment }\end{array}$ \\
\hline $\begin{array}{l}\text { Rovetta } 1998^{69} \\
\text { Two-arm RCT }\end{array}$ & $\begin{array}{l}n=30 \\
\text { Sodium hyaluronate + steroid } \\
\text { injection + PT: } 16 \\
\text { Steroid injections + PT: } 14\end{array}$ & $\begin{array}{l}\text { Sodium hyaluronate: } 20 \mathrm{mg} \text { at } 15- \\
\text { day intervals in the first month, then } \\
\text { monthly for } 6 \text { months } \\
\text { Steroid: triamcinolone acetonide at } \\
\text { 15-day intervals in the first month, } \\
\text { then monthly for } 6 \text { months } \\
\text { PT: for 4-12 weeks }\end{array}$ & $\begin{array}{l}\text { Clinical history of spontaneous shoulder } \\
\text { pain; glenohumeral abduction and forward } \\
\text { flexion }<90^{\circ} \text {, external rotation }<20^{\circ} \text {; clinical } \\
\text { absence of signs of rotator cuff interruption; } \\
\text { cervical examination excluding dysfunction in } \\
\text { this area; plain radiographs in standard views; } \\
\text { sonographic examination showing shrinking } \\
\text { of the joint capsule with increased capsular } \\
\text { echogenicity }\end{array}$ \\
\hline $\begin{array}{l}\text { Takagishi } 1996^{70} \\
\text { Two-arm RCT }\end{array}$ & $\begin{array}{l}n=20 \\
\text { Sodium hyaluronate: } 10 \\
\text { Steroid injection: } 10\end{array}$ & $\begin{array}{l}\text { Sodium hyaluronate: } 2 \mathrm{mg} \text {, one } \\
\text { injection once weekly for } 5 \text { weeks } \\
\text { Steroid: dexamethasone, } 2 \mathrm{mg} \text {, one } \\
\text { injection once weekly for } 5 \text { weeks }\end{array}$ & $\begin{array}{l}\text { Patients with frozen shoulder who have }<120^{\circ} \\
\text { of shoulder joint flexion and those who suffer } \\
\text { from pain on exercise of }>4 \text { on the VAS and } \\
\text { suffer from pain during night-time and daytime }\end{array}$ \\
\hline
\end{tabular}

PT, physiotherapy; ROM range of movement.

The inclusion criteria varied between the three studies. Rovetta et al.$^{69}$ reported that none of the participants had diabetes, whereas Calis et al. ${ }^{66}$ and Takagishi et al.$^{69}$ did not report whether or not any of the participants had diabetes. Frozen shoulder was diagnosed through physical examination, laboratory tests, subacromial impingement test, clinical history, radiography or ultrasound when described. The method of diagnosis was unclear in one study. ${ }^{70}$ The participants' stage of frozen shoulder at baseline was not reported in any of the studies and it was unclear whether participants had received any previous treatments. The mean age of participants ranged from 48 years to 65.8 years. The proportion of women ranged from $63 \%$ to $75 \%$.

\section{Quality assessment}

None of the studies reported the method of randomisation or allocation concealment, and only one stated that blinded outcome assessment was performed; therefore, all three studies have a potentially high risk of bias. The study by Calis et al. ${ }^{66}$ was of higher quality and fulfilled five criteria: the number of participants randomised, treatment groups that were comparable at baseline, blinded outcome assessors, use of intention-to-treat analysis and no unexpected imbalances in dropouts. Rovetta et al.$^{69}$ fulfilled two criteria, stating the number of participants randomised and also having treatment groups that were comparable at baseline, whereas Takagishi et al..$^{70}$ fulfilled only one criterion, which was stating the number of patients randomised. Full details of study quality are reported in Appendix 8.

Pain

All three studies evaluating sodium hyaluronate assessed pain; however, the study by Calis et al. ${ }^{66}$ reported pain in graphical form and therefore could not be included in the analysis. Calis et al. ${ }^{66}$ did, however, report that there was significant improvement in pain severity at 3 months within each of the treatment groups, including the no intervention group $(p<0.001)$. Rovetta et al. ${ }^{69}$ and Takagishi et al..$^{70}$ both used a VAS but assessed different types of pain at different time points (Table 17); therefore, it was inappropriate to pool the two studies. Table 18 provides the SMDs 
TABLE 17 Pain outcomes in studies evaluating sodium hyaluronate

\begin{tabular}{llll}
\hline Study & Pain outcome assessed & Scale used & Data reported and time of follow-up \\
\hline Calis $2006^{66}$ & Pain severity & VAS & Data reported graphically only \\
Rovetta $1998^{69}$ & Pain on passive joint motion & VAS $0-10 \mathrm{~cm}$ & Final value at 6 months \\
Takagishi $1996^{70}$ & Pain on activity & VAS $0-10$ & Final value at 5 weeks \\
\hline
\end{tabular}

TABLE 18 Pain results of studies of sodium hyaluronate (SMD and 95\% Cl)

\begin{tabular}{|c|c|c|c|}
\hline Study & Short term ( $\leq 3$ months) & Medium term ( $>3$ and $\leq 6$ months) & Long term ( $>6$ and $\leq 12$ months) \\
\hline \multicolumn{4}{|c|}{ Sodium hyaluronate combined with steroid and physiotherapy vs steroid combined with physiotherapy } \\
\hline Rovetta $1998^{69}$ & & $-0.78(-1.50 \text { to }-0.06)^{a, b}$ & \\
\hline \multicolumn{4}{|c|}{ Sodium hyaluronate vs steroid } \\
\hline Takagishi $1996^{70}$ & $-0.21(-1.09 \text { to } 0.67)^{c}$ & & \\
\hline
\end{tabular}

and $95 \%$ CIs. The outcome data for the individual groups in the included trials are available in Appendix 7.

\section{Sodium hyaluronate versus steroid}

Takagishi et al. ${ }^{70}$ found no significant difference in pain at short-term follow-up with sodium hyaluronate compared with steroid (see Table 18).

\section{Sodium hyaluronate combined with steroid and physiotherapy versus steroid combined with physiotherapy}

Rovetta et al. ${ }^{69}$ found a significant decrease in pain at 6 months with sodium hyaluronate combined with steroid and physiotherapy compared with steroid combined with physiotherapy (SMD $-0.78,95 \% \mathrm{CI}-1.50$ to -0.06$)$.

\section{Function and disability}

Calis et al. ${ }^{66}$ and Takagishi et al. ${ }^{70}$ both assessed function and disability. Calis et al. assessed function and disability using the Constant score (Table 19), whereas Takagishi et al. ${ }^{70}$ used a Japanese-specific activities of daily living questionnaire. However, Takagishi et al. reported baseline values and a $p$-value for the within-group change from baseline only and therefore could not be included in the analysis. Table 20 provides the MD and 95\% CIs. The outcome data for the individual groups in the included trial are available in Appendix 7.

At 3 months Calis et al. ${ }^{66}$ found a significantly greater improvement in the Constant score with sodium hyaluronate compared with no treatment (MD 8.90, 95\% CI 2.62 to 15.18), but no significant difference in Constant score between sodium hyaluronate and physiotherapy (MD $-6.00,95 \% \mathrm{CI}-12.16$ to 0.16$)$ or between sodium hyaluronate and steroid injection $(\mathrm{MD}-0.20$, $95 \%$ CI -5.86 to 5.46$)$.

\section{Range of movement}

The range of movement measurements of interest were external rotation, internal rotation and abduction (both passive and active); if internal rotation was not available but hand behind back was reported, this was used as a proxy measure of active internal rotation. All three studies 
TABLE 19 Function and disability outcomes in studies evaluating sodium hyaluronate

\begin{tabular}{lll}
\hline Study & Function and disability outcomes assessed & Data reported and time of follow-up \\
\hline Calis $2006^{66}$ & Constant score & Final values at 3 months \\
Takagishi $1996^{70}$ & $\begin{array}{l}\text { Activities of daily living questionnaire (Japanese } \\
\text { Specific) }\end{array}$ & $\begin{array}{l}\text { Baseline data and } p \text {-value for within-group change from } \\
\text { baseline only reported }\end{array}$ \\
\hline
\end{tabular}

TABLE 20 Function and disability results of sodium hyaluronate (MD and 95\% Cl)

\begin{tabular}{|c|c|c|c|}
\hline Study & Short term ( $\leq 3$ months) & Medium term ( $>3$ and $\leq 6$ months) & Long term ( $>6$ and $\leq 12$ months) \\
\hline \multicolumn{4}{|c|}{ Sodium hyaluronate vs no treatment } \\
\hline \multicolumn{4}{|c|}{ Constant score } \\
\hline Calis $2006^{66}$ & $8.90(2.62 \text { to } 15.18)^{a, b}$ & & \\
\hline \multicolumn{4}{|c|}{ Sodium hyaluronate vs physiotherapy } \\
\hline \multicolumn{4}{|c|}{ Constant score } \\
\hline Calis $2006^{66}$ & $-6.00(-12.16 \text { to } 0.16)^{\mathrm{a}}$ & & \\
\hline \multicolumn{4}{|c|}{ Sodium hyaluronate vs steroid } \\
\hline \multicolumn{4}{|c|}{ Constant score } \\
\hline Calis $2006^{66}$ & $-0.20(-5.86 \text { to } 5.46)^{\mathrm{a}}$ & & \\
\hline
\end{tabular}

that evaluated sodium hyaluronate reported at least one of these measurements (Table 21). Table 22 provides the between-group difference in means and 95\% CIs. The outcome data for the individual groups in the included trials are available in Appendix 7.

\section{Sodium hyaluronate versus no treatment}

Calis et al. ${ }^{66}$ reported passive external rotation and passive abduction at 3 months. There was a significantly greater improvement in the sodium hyaluronate group than in the no treatment group in both passive external rotation (MD $8.30^{\circ}, 95 \%$ CI $2.52^{\circ}$ to $14.08^{\circ}$ ) and passive abduction $\left(\mathrm{MD} 12.40^{\circ}, 95 \% \mathrm{CI} 0.14^{\circ}\right.$ to $\left.24.66^{\circ}\right)$.

\section{Sodium hyaluronate versus physiotherapy}

Calis et al. ${ }^{66}$ reported passive external rotation and passive abduction at 3 months. There was significantly greater improvement in the physiotherapy group than in the sodium hyaluronate group in both passive external rotation $\left(\mathrm{MD}-10.50^{\circ}, 95 \% \mathrm{CI}-16.87^{\circ}\right.$ to $-4.13^{\circ}$ ) and passive abduction $\left(\mathrm{MD}-12.50^{\circ}, 95 \% \mathrm{CI}-23.98^{\circ}\right.$ to $\left.-1.02^{\circ}\right)$.

\section{Sodium hyaluronate versus steroid}

Calis et al. ${ }^{66}$ reported passive external rotation and passive abduction at 3 months. There was no significant difference in passive external rotation or passive abduction between groups (see Table 22). Takagishi et al. ${ }^{70}$ reported external rotation at 5 weeks. There was no significant difference between groups (see Table 22). 
TABLE 21 Range of movement (ROM) outcomes in studies evaluating sodium hyaluronate injections

\begin{tabular}{lll}
\hline Study & ROM outcomes assessed $\left(^{\circ}\right)$ & Data reported at time points up to 3 and 6 months \\
\hline Calis $2006^{66}$ & Passive external rotation, passive abduction & Final values at 15 days and 3 months \\
Rovetta $1998^{69}$ & $\begin{array}{l}\text { Internal rotation, external rotation, } \\
\text { abduction }\end{array}$ & Final values at 6 months \\
Takagishi $1996^{70}$ & External rotation & Final values at 5 weeks \\
\hline
\end{tabular}

TABLE 22 Range of movement results of studies of sodium hyaluronate injections (MD and 95\% Cl)

\begin{tabular}{|c|c|c|c|}
\hline Study & Short term ( $\leq 3$ months) & Medium term ( $>3$ and $\leq 6$ months) & Long term ( $>6$ and $\leq 12$ months) \\
\hline \multicolumn{4}{|c|}{ Sodium hyaluronate vs no treatment } \\
\hline \multicolumn{4}{|c|}{ Passive external rotation $\left({ }^{\circ}\right)$} \\
\hline Calis $2006^{66}$ & $8.30(2.52 \text { to } 14.08)^{\mathrm{a}, \mathrm{b}}$ & & \\
\hline \multicolumn{4}{|c|}{ Passive abduction ( $\left.{ }^{\circ}\right)$} \\
\hline Calis $2006^{66}$ & $12.40(0.14 \text { to } 24.66)^{a, b}$ & & \\
\hline \multicolumn{4}{|c|}{ Sodium hyaluronate vs physiotherapy } \\
\hline \multicolumn{4}{|c|}{ Passive external rotation $\left({ }^{\circ}\right.$ ) } \\
\hline Calis $2006^{66}$ & $-10.50(-16.87 \text { to }-4.13)^{\mathrm{a}, \mathrm{b}}$ & & \\
\hline \multicolumn{4}{|c|}{ Passive abduction ( $\left.{ }^{\circ}\right)$} \\
\hline Calis $2006^{66}$ & $-12.50(-23.98 \text { to }-1.02)^{\mathrm{a}, \mathrm{b}}$ & & \\
\hline \multicolumn{4}{|c|}{ Sodium hyaluronate vs steroid } \\
\hline \multicolumn{4}{|c|}{ Passive external rotation $\left({ }^{\circ}\right)$} \\
\hline Calis $2006^{66}$ & $0.30(-5.92 \text { to } 6.52)^{\mathrm{a}}$ & & \\
\hline \multicolumn{4}{|c|}{ Passive abduction $\left(^{\circ}\right)$} \\
\hline Calis $2006^{66}$ & $-4.40(-15.78 \text { to } 6.98)^{\mathrm{a}}$ & & \\
\hline \multicolumn{4}{|l|}{ External rotation $\left({ }^{\circ}\right)$} \\
\hline Takagischi $1996^{70}$ & $-2.00(-21.25 \text { to } 17.28)^{c}$ & & \\
\hline \multicolumn{4}{|c|}{ Sodium hyaluronate + steroid + physiotherapy vs steroid + physiotherapy } \\
\hline \multicolumn{4}{|l|}{ Internal rotation $\left({ }^{\circ}\right)$} \\
\hline Rovetta $1998^{69}$ & & $23.70(13.44 \text { to } 33.96)^{b, d}$ & \\
\hline \multicolumn{4}{|l|}{ External rotation $\left({ }^{\circ}\right)$} \\
\hline Rovetta $1998^{69}$ & & $-0.70(-15.11 \text { to } 13.71)^{d}$ & \\
\hline \multicolumn{4}{|l|}{ Abduction ( $\left.{ }^{\circ}\right)$} \\
\hline Rovetta $1998^{69}$ & & $3.20(-18.70 \text { to } 25.10)^{d}$ & \\
\hline
\end{tabular}

\footnotetext{
a Reported at 3 months.

b Statistically significant i.e. the $\mathrm{Cl}$ did not cross the line of no effect (zero).

c Reported at 5 weeks.

d Reported at 6 months.
} 


\section{Sodium hyaluronate with steroid and physiotherapy versus steroid with physiotherapy}

One study, by Rovetta et al. ${ }^{69}$ reported internal rotation, external rotation and abduction at 6 months only. There was a significantly greater improvement in internal rotation with sodium hyaluronate combined with steroid and physiotherapy than with steroid combined with physiotherapy (MD $23.70^{\circ}, 95 \%$ CI $13.44^{\circ}$ to $33.96^{\circ}$ ). There was no significant difference in mean external rotation or mean abduction between groups (see Table 22).

\section{Quality of life}

None of the three studies that evaluated sodium hyaluronate assessed quality of life.

\section{Adverse events}

None of the three studies evaluating sodium hyaluronate reported whether or not participants experienced any adverse events.

\section{Summary}

The three included studies investigating sodium hyaluronate used variable doses and in one study sodium hyaluronate was combined with steroid injection and physiotherapy. Because of variations in outcomes reported and comparators used it was not possible to pool data in a metaanalysis. For most outcome measures data were available from a single study only. The studies did not report participants' stage of frozen shoulder or previous treatments received.

All of the studies had a potentially high risk of bias; none reported the method of randomisation and therefore it was unclear whether they were truly randomised. Because it was unclear whether all studies had enough participants to detect a statistically significant treatment effect, it should be kept in mind that where studies did not report a statistically significant benefit (i.e. the CI crossed the line of no difference) this should not be interpreted as evidence of no difference between the groups.

One study reported a medium-term benefit in pain outcome when sodium hyaluronate was added to steroid and physiotherapy. The same study also reported a significant benefit at medium-term follow-up for internal rotation but not for external rotation or abduction. Another study reported a benefit for function and disability with sodium hyaluronate compared with home exercise but not with physiotherapy or steroid. The same study reported significantly better short-term passive external rotation and abduction with sodium hyaluronate than with home exercise and physiotherapy but not steroid injection. A second study reported no evidence of a benefit for external rotation with sodium hyaluronate compared with steroid.

In conclusion, a small number of diverse studies of sodium hyaluronate were identified, all of which may have had a high risk of bias. There was insufficient evidence to make conclusions with any certainty about the effectiveness of sodium hyaluronate for primary frozen shoulder and in what situations it is likely to be effective.

\section{Physical therapy}

\section{Study characteristics}

Eleven RCTs and one controlled trial investigated various types of physical therapy in the treatment of primary frozen shoulder. A summary of the study characteristics is reported in Table 23, with further details available in Appendix 6. Eleven were full papers and one ${ }^{71}$ was available in abstract form only. One was a Chinese-language paper. ${ }^{72}$ The studies were published in the Netherlands, ${ }^{40,73}$ Turkey, ${ }^{51,66,74}$ Hong Kong, ${ }^{75}$ Singapore, ${ }^{76}$ Thailand, ${ }^{77}$ Greece, ${ }^{16}$ Taiwan,${ }^{78}$ $\mathrm{China}^{72}$ and the $\mathrm{UK}^{71}$ between 1994 and 2009. There were nine two-armed trials, ${ }^{16,40,51,72-74,76-78}$ two three-armed trials ${ }^{71,75}$ and one four-armed trial. ${ }^{66}$ 
TABLE 23 Studies including physical therapies

\begin{tabular}{|c|c|c|c|}
\hline $\begin{array}{l}\text { Study details } \\
\text { and study design }\end{array}$ & $\begin{array}{l}\text { Participants randomised } \\
\text { (total } n \text { and by group) }\end{array}$ & Trial treatments & Condition-related inclusion criteria \\
\hline $\begin{array}{l}\text { Calis 2006 } \\
\text { Four-arm RCT }\end{array}$ & $\begin{array}{l}n=90 \text { (95 shoulders) } \\
\text { Sodium hyaluronate } \\
\text { injection: } 24 \text { (27) } \\
\text { Steroid injection: } 25 \text { (26); } \\
\text { PT: } 21 \text { (22); } \\
\text { No intervention (home } \\
\text { exercise only): } 20 \text { (20) }\end{array}$ & $\begin{array}{l}\text { Sodium hyaluronate: } 30 \mathrm{mg} \text {, one injection once } \\
\text { weekly for } 2 \text { weeks } \\
\text { Steroid: triamcinolone acetonide, } 40 \mathrm{mg} \text {, one } \\
\text { injection } \\
\text { PT: } 10 \text { daily sessions: heat pack applied for } \\
20 \text { minutes; ultrasonic therapy for } 5 \text { minutes } \\
\text { (1.5W/cm² intensity); TENS for } 20 \text { minutes; and } \\
\text { stretching exercises }\end{array}$ & $\begin{array}{l}\text { History of pain for at least } 1 \text { month; limited } \\
\text { active and passive shoulder movement; } \\
\text { decreased passive ROM of } \geq 20 \% \text {, in at } \\
\text { least three movements, according to the } \\
\text { American Medical Association guide for } \\
\text { the evaluation of permanent impairment }\end{array}$ \\
\hline $\begin{array}{l}\text { Diercks } 2004^{73} \\
\text { Two-arm } \\
\text { controlled trial }\end{array}$ & $\begin{array}{l}n=77 \\
\text { PT: } 32 \\
\text { Supervised neglect: } 45\end{array}$ & $\begin{array}{l}\text { PT: active PT; duration and frequency not reported } \\
\text { Supervised neglect: education and home exercise } \\
\text { within painless range }\end{array}$ & $\begin{array}{l}\text { Idiopathic frozen shoulder using Lundberg } \\
\text { criteria: }>50 \% \text { motion restriction of the } \\
\text { glenohumeral joint in all directions for at } \\
\text { least } 3 \text { months }\end{array}$ \\
\hline $\begin{array}{l}\text { Dogru } 2008^{51} \\
\text { Two-arm RCT }\end{array}$ & $\begin{array}{l}n=50 \\
\text { Ultrasound + PT: } 25 \\
\text { Sham + PT: } 25\end{array}$ & $\begin{array}{l}\text { Ultrasound + PT: active PT; } 10 \times 50 \text {-minute } \\
\text { sessions over } 2 \text { weeks of superficial heat (heat } \\
\text { packs) ( } 20 \text { minutes), ultrasound ( } 10 \text { minutes) and } \\
\text { exercise programme ( } 20 \text { minutes) } \\
\text { Sham ultrasound + PT: same as above except that } \\
\text { the ultrasound machine was not switched to 'on' }\end{array}$ & $\begin{array}{l}\text { Shoulder pain of at least } 3 \text { months' } \\
\text { duration; at least } 25 \% \text { loss of shoulder } \\
\text { motion in all planes; pain on motion of at } \\
\text { least } 40 \mathrm{~mm} \text { on VAS }\end{array}$ \\
\hline $\begin{array}{l}\text { Dundar } 2009^{74} \\
\text { Two-arm RCT }\end{array}$ & $\begin{array}{l}n=57 \\
\text { Continuous passive motion: } \\
29 \\
\text { PT: } 28\end{array}$ & $\begin{array}{l}\text { Continuous passive motion: } 20 \times 1 \text {-hour sessions } \\
\text { over } 4 \text { weeks using an external motorised device } \\
\text { Conventional PT: active PT, } 20 \times 1 \text {-hour sessions } \\
\text { over } 4 \text { weeks }\end{array}$ & $\begin{array}{l}\text { Frozen shoulder patients with gradually } \\
\text { increasing shoulder pain and stiffness } \\
\text { (painful phase and stiff phase) }\end{array}$ \\
\hline $\begin{array}{l}\text { Leung } 2008^{75} \\
\text { Three-arm RCT }\end{array}$ & $\begin{array}{l}n=30 \\
\text { SWD + stretching: } 10 \\
\text { Heat pack + stretching: } 10 \\
\text { No intervention: } 10\end{array}$ & $\begin{array}{l}\text { SWD + stretching: three } 20 \text {-minute sessions } \\
\text { per week for } 4 \text { weeks, machine frequency of } \\
27.12 \mathrm{MHz} \text {, followed by stretching exercises } \\
\text { Heat pack + stretching: superficial heat }\left(63^{\circ} \mathrm{C}\right) \\
\text { with electrical heat pack; stretching exercises as } \\
\text { above } \\
\text { No intervention: home exercise only }\end{array}$ & $\begin{array}{l}\text { Idiopathic frozen shoulder with shoulder } \\
\text { pain and limited shoulder movement for at } \\
\text { least } 8 \text { weeks }\end{array}$ \\
\hline $\begin{array}{l}\text { Maricar 199976 } \\
\text { Two-arm RCT }\end{array}$ & $\begin{array}{l}n=54 \\
\text { PT + exercise: } 16^{\mathrm{a}} \\
\text { Exercise only: } 16^{\mathrm{a}}\end{array}$ & $\begin{array}{l}\text { PT + exercise: active PT, eight weekly sessions } \\
\text { and from week } 5 \text { a 15-minute exercise circuit of } \\
\text { nine exercises } \\
\text { Exercise only: } 15 \text {-minute exercise circuit of nine } \\
\text { exercises }\end{array}$ & $\begin{array}{l}\text { Major complaint of limitation in shoulder } \\
\text { range of motion with secondary complaint } \\
\text { of pain; able to place arms behind head } \\
\text { and back reaching vertebral column; } \\
\text { 90-degree abduction (late stage } 2 \text { to } \\
\text { stage 3) }\end{array}$ \\
\hline $\begin{array}{l}\text { Pajareya } 2004^{77} \\
\text { Two-arm RCT }\end{array}$ & $\begin{array}{l}n=122 \\
\text { PT: } 61 \\
\text { No intervention: } 61\end{array}$ & $\begin{array}{l}\text { PT: SWD ( } 20 \text { minutes) + active PT three times a } \\
\text { week for } 3 \text { weeks } \\
\text { No intervention: information only }\end{array}$ & $\begin{array}{l}\text { Shoulder pain and limitation of a passive } \\
\text { range of shoulder motion in all directions } \\
\text { that interfered with activities of daily living }\end{array}$ \\
\hline $\begin{array}{l}\text { Stergioulas } \\
2008^{16} \\
\text { Two-arm RCT }\end{array}$ & $\begin{array}{l}n=74 \\
\text { Laser therapy: } 37 \\
\text { Placebo laser: } 37\end{array}$ & $\begin{array}{l}\text { Laser therapy: } 12 \text { sessions over } 8 \text { weeks: two per } \\
\text { week in the first } 4 \text { weeks, then one per week } \\
\text { Placebo laser: regimen as above }\end{array}$ & $\begin{array}{l}\text { Painful and limited passive glenohumeral } \\
\text { mobility; more restricted lateral rotation } \\
(<8 \%) \text { relative to abduction and medial } \\
\text { rotation }\end{array}$ \\
\hline $\begin{array}{l}\text { Vermeulen } 2006^{40} \\
\text { Two-arm RCT }\end{array}$ & $\begin{array}{l}n=100 \\
\text { HGMT: } 49 \\
\text { LGMT: } 51\end{array}$ & $\begin{array}{l}\text { HGMT: } 30 \text {-minute session twice weekly for a } \\
\text { maximum of } 12 \text { weeks consisting of } 5 \text {-minute } \\
\text { assessment of ROM in order to apply the } \\
\text { mobilisation technique into the stiffness zone. } \\
\text { Mobilisation techniques were applied with } \\
\text { intensities according to Maitland grades III and IV } \\
\text { LGMT: } 30 \text {-minute session twice weekly for a } \\
\text { maximum of } 12 \text { weeks consisting of } 5 \text {-minute } \\
\text { assessment of ROM in order to apply the } \\
\text { mobilisation technique within the pain-free } \\
\text { zone. Mobilisation techniques were performed } \\
\text { according to Maitland grades I and II }\end{array}$ & $\begin{array}{l}\geq 50 \% \text { loss of passive movement of } \\
\text { shoulder joint relative to non-affected } \\
\text { side, in one or more of three movement } \\
\text { directions (abduction in frontal plane, } \\
\text { forward flexion or external rotation); } \\
\text { duration of complaints } \geq 3 \text { months }\end{array}$ \\
\hline
\end{tabular}


TABLE 23 Studies including physical therapies (continued)

\begin{tabular}{|c|c|c|c|}
\hline $\begin{array}{l}\text { Study details } \\
\text { and study design }\end{array}$ & $\begin{array}{l}\text { Participants randomised } \\
\text { (total } n \text { and by group) }\end{array}$ & Trial treatments & Condition-related inclusion criteria \\
\hline $\begin{array}{l}\text { Wies } 2003^{71} \\
\text { Three-arm RCT }\end{array}$ & $\begin{array}{l}n=30 \\
\text { Osteopathy: } 10 \\
\text { PT: } 10 \\
\text { Control: } 10\end{array}$ & $\begin{array}{l}\text { Osteopathy: the Niel-Asher technique, consisting } \\
\text { of a progression of deep tissue manipulation, for } \\
9 \text { weeks } \\
\text { PT: manual therapy and therapeutic exercise for } \\
9 \text { weeks } \\
\text { Control: breathing exercises + massage + ROM } \\
\text { exercises for } 9 \text { weeks }\end{array}$ & Patients with primary frozen shoulder \\
\hline $\begin{array}{l}\text { Yang } 2007^{78} \\
\text { Two-arm RCT }\end{array}$ & $\begin{array}{l}n=30 \text { (28 started) } \\
\text { ERM + MRM: } 14 \\
\text { MWM + MRM: } 14\end{array}$ & $\begin{array}{l}\text { ERM + MRM: } 30 \text { minutes twice per week for } \\
3 \text { weeks each } \\
\text { MWM + MRM: } 30 \text { minutes twice per week for } \\
3 \text { weeks each }\end{array}$ & $\begin{array}{l}\text { Painful stiff shoulder for at least } 3 \text { months; } \\
\text { ROM losses of at least } 25 \% \text { compared } \\
\text { with the non-involved shoulder in at least } \\
\text { two of glenohumeral flexion, abduction or } \\
\text { medial or lateral rotation }\end{array}$ \\
\hline $\begin{array}{l}\text { Yan } 2005^{72} \\
\text { Two-arm RCT }\end{array}$ & $\begin{array}{l}n=54 \\
\text { Dumb-bell gymnastics: } 26 \\
\text { Barehanded exercises: } 28\end{array}$ & $\begin{array}{l}\text { Dumb-bell gymnastics: } 5-10 \text { minutes, two to } \\
\text { three times a day for } 3 \text { months using dumb-bells } \\
\text { weighing } 2-5 \mathrm{~kg} \\
\text { Barehanded exercises: performed for } 3 \text { months }\end{array}$ & $\begin{array}{l}\text { 'Standard shoulder periarthritis diagnostic } \\
\text { criteria' }\end{array}$ \\
\hline
\end{tabular}

PT, physiotherapy; ROM, range of movement.

a Number analysed in each group and number randomised not reported by group.

A variety of physical therapy regimens were used, most of which were physiotherapy modalities. These included various combinations of exercises (up to and beyond the pain threshold), stretching, manipulation of the glenohumeral joint, ultrasound, superficial heat, SWD, laser therapy, Codman's exercises, wall-climbing exercises, continuous passive motion, manual therapy, dumb-bell gymnastics and massage. For the purposes of the synthesis, the interventions were grouped based on whether or not at least one component involved mobilisation (of any type) under the supervision of a therapist. Therapies that involved some form of mobilisation (with or without other physical therapies) are described as active therapies. Studies using interventions such as laser therapy or TENS without any supervised mobilisation or exercise were classified as therapy without mobilisation.

Seven compared at least two forms of physical therapy. $40,71,72,74-76,78$ One of the studies had what was described as a 'supervised neglect' intervention. ${ }^{73}$ This study by Diercks and Stevens was the only study identified that explicitly used a supervised neglect intervention; therefore, this study was included although it was not a RCT. ${ }^{73}$ Undertaken in the Netherlands, a physiotherapy intervention of unspecified duration was used that involved a standardised protocol, carried out by a therapist, of active exercises up to and beyond the pain threshold. The supervised neglect comparator involved providing patients with an explanation of the natural course of the disease and instructions not to exercise in excess of their pain threshold, to undertake pendulum exercises and active exercises within the painless range and to resume all activities that were tolerated. In the Calis et al. study, physiotherapy was compared with sodium hyaluronate and steroid injection ${ }^{66}$ and in the Pajareya et al. study, physiotherapy was compared with no intervention. ${ }^{77}$

A single study did not have an arm with an active mobilisation component. This study by Stergioulas ${ }^{16}$ compared laser therapy with placebo laser (with home exercise). The remaining studies compared multiple active physical therapies. One compared ultrasound and physiotherapy with sham ultrasound and physiotherapy; ${ }^{51}$ one compared 1-hour sessions of continuous passive motion with physiotherapy $;^{74}$ one compared osteopathy with physiotherapy and with breathing exercises, massage and range of movement exercises; ${ }^{71}$ one compared 
end-range mobilisation (ERM) and mid-range mobilisation (MRM) with mobilisation with movement (MWM) and MRM $;^{78}$ one compared passive joint mobilisations and exercises with exercises only; ${ }^{76}$ one compared SWD plus stretching with heat pack plus stretching ${ }^{75}$ one compared dumb-bell gymnastics with barehanded exercises ${ }^{72}$ and one compared high-grade mobilisation techniques (HGMT) with low-grade mobilisation techniques (LGMT) (both followed by Codman pendular exercises). ${ }^{40}$ All of the physiotherapy arms included an active mobilisation component. The intervention duration ranged from 2 weeks to 3 months.

Six studies reported that home exercise was used as part of the intervention, although details were limited in some studies. Where specified the home exercise routines included daily Codman and stretching exercises, ${ }^{66}$ Codman, stretching and active range of movement exercises, ${ }^{51}$ daily passive range of movement and pendulum exercises, ${ }^{74}$ pendulum and pain-free exercises ${ }^{16}$ and daily stretches. ${ }^{75}$ Home exercise was discouraged in one study ${ }^{78}$ Analgesic use also differed between studies where reported. NSAIDs or other analgesics were prescribed when necessary in one study, ${ }^{73}$ paracetamol was taken when necessary in another ${ }^{66}$ paracetamol was allowed to a maximum of $1000 \mathrm{mg}$ daily in another, ${ }^{51} 400 \mathrm{mg}$ of ibuprofen was given daily for 3 weeks in one study ${ }^{77}$ and prescribed and non-prescribed pain medication was allowed in one study. ${ }^{40}$ No NSAIDs or other analgesics were allowed in one RCT, with a week washout period before the study commenced. ${ }^{74}$

The inclusion criteria varied between the studies. All included patients with some limitation of movement, although the extent of this varied where reported. Primary frozen shoulder was diagnosed through range of movement, biochemical analyses, radiography and clinical diagnosis where reported. Four studies did not report how frozen shoulder was diagnosed. ${ }^{40,71,77,78}$ Three included participants with diabetes: $37 \%$ in the study by Dogru et al. ${ }^{51} 16 \%$ in the study by Vermeulen et al. ${ }^{40}$ and $33 \%$ in the study by Pajareya et al. ${ }^{77}$ Separate data were not presented for this subgroup. The mean duration of frozen shoulder at baseline ranged from 3 months to 6.8 years. The stage of frozen shoulder included was reported by few studies: stage 1 or 2 in the study by Dundar et al. ${ }^{74}$ 'stiff stage' in the studies by Yan ${ }^{72}$ and Leung and Cheing ${ }^{75}$ and late stage 2 or 3 in the study by Maricar and Chok. ${ }^{76}$ Outcome was not reported separately by stage of frozen shoulder. Only one study, Vermeulen et al. ${ }^{40}$ reported on whether participants had received any previous treatment for frozen shoulder. In this study $81 \%$ of participants had previously received physical therapy, $61 \%$ steroid injections and $6 \%$ surgery. The proportion of female participants ranged from $20 \%$ to $86 \%$ and the mean age of participants ranged from 50 to 62.5 years.

\section{Quality assessment}

The quality of the studies was generally poor. Only one was of satisfactory quality, reporting the number randomised and both an appropriate method of randomisation and adequate allocation concealment. ${ }^{78}$ This study by Yang et al. also met most of the remaining criteria including comparability at baseline, blinding of outcome assessors and intention-to-treat analysis; however, the study may not be adequately powered to detect significant differences between groups and there was a large number of dropouts (23\%). The main limitation of this study was the study design for the purposes of our review; only limited data were suitable for inclusion in our analysis as different sequences of the three same mobilisations were compared at end of treatment (see Appendix 6). ${ }^{78}$

Leung and Cheing, ${ }^{75}$ Stergioulas ${ }^{16}$ and Vermeulen et al.$^{40}$ reported an appropriate method of randomisation; Leung and Cheing ${ }^{75}$ and Vermeulen et al..$^{40}$ did not report whether an adequate method of allocation concealment was used; and Stergioulas ${ }^{16}$ reported that allocation was performed using opaque envelopes but it was unclear whether the envelopes were numbered sequentially. Although these three studies fulfilled other criteria, such as comparability at baseline 
and blinding of outcome assessors, there was still a possible risk of bias. Leung and Cheing ${ }^{75}$ reported that there was no loss to follow-up and loss to follow-up was $15 \%$ in the Stergioulas $\operatorname{study}^{16}$ (the majority in the laser group) and $4 \%$ in the Vermeulen study $y^{40}$ (with equal numbers from each group). Vermeulen et al..$^{40}$ also used intention-to-treat analysis and was adequately powered for one outcome.

Calis et al. ${ }^{66}$ Dogru et al. ${ }^{51}$ Dundar et al..${ }^{74}$ Pajareya et al. ${ }^{77}$ and Yan ${ }^{72}$ reported the number of participants randomised. It was unclear whether the method of assignment was truly random in any of these studies and allocation concealment was not reported; therefore, they potentially have high risk of bias. These studies also fulfilled few of the remaining criteria. Dogru et al. ${ }^{51}$ and Calis et al ${ }^{66}$ reported blinding of outcome assessment. Dropouts were relatively high in the Pajareya study ${ }^{77}$ (12\%, the majority in the control group) and the reporting of satisfaction results was also unclear. There were no or very few dropouts in the Calis et al. ${ }^{66}$ Dogru et al. ${ }^{51}$ Yan $^{72}$ and Dundar et al. ${ }^{74}$ studies. Calis et al..$^{66}$ also used intention-to-treat analysis.

The number of participants randomised was inconsistently reported in the study by Maricar and Chok. ${ }^{76}$ Neither Maricar and $\mathrm{Chok}^{76}$ or Wies et al. ${ }^{71}$ reported the method of randomisation or allocation concealment, so were at high risk of bias. Few of the remaining criteria were met. Dropouts were high in the study by Maricar and Chok $(41 \%)^{76}$ and not reported by Wies et al. ${ }^{71}$

Allocation to groups was not randomised in the study by Diercks and Stevens ${ }^{73}$ and met none of the quality criteria (including blinding of outcome assessment) except comparability at baseline (there were no significant differences in age, sex or duration of disease). This study was quasiexperimental with a successive cohort as the physical therapy group. This study was therefore at a high risk of bias. The paper reported that there was no loss to follow-up.

Pain

Six studies assessed pain (Table 24). The study by Pajareya et al. ${ }^{77}$ reported analgesic use at 3 weeks only and was therefore excluded from the synthesis. Calis et al. ${ }^{66}$ reported data in graphical form only; consequently, the SMD could not be calculated and this study could not be included in a meta-analysis. Calis et al ${ }^{66} \mathrm{did}$, however, report that there was significant improvement in pain severity at 3 months within both the physical therapy group and the no intervention group $(p<0.001)$.

The remaining four studies each evaluated different physical therapies using different comparators; therefore, it was not appropriate to pool the studies in a meta-analysis. ${ }^{16,40,51,74}$

TABLE 24 Pain outcomes in studies evaluating physical therapies

\begin{tabular}{|c|c|c|c|}
\hline Study & $\begin{array}{l}\text { Pain outcome } \\
\text { assessed }\end{array}$ & Scale used & Data reported and time of follow-up \\
\hline Calis $2006^{66}$ & Pain severity & VAS & Data reported graphically only \\
\hline Dogru $2008^{51}$ & Pain overall & $\begin{array}{l}\text { SPADI 5-item pain } \\
\text { subscale }\end{array}$ & Final mean values at 2 weeks and 3 months \\
\hline Dundar $2009^{74}$ & Pain overall & $\begin{array}{l}\text { SPADI 5-item pain } \\
\text { subscale }\end{array}$ & Final mean values at 4 weeks and 12 weeks \\
\hline Pajareya $2004^{77}$ & Analgesic use & No. of tablets & Median at 3 weeks \\
\hline $\begin{array}{l}\text { Stergioulas } \\
2008^{16}\end{array}$ & Pain overall & VAS 0-100mm & Final mean values at 4,8 and 12 weeks \\
\hline $\begin{array}{l}\text { Vermeulen } \\
2006^{40}\end{array}$ & Pain at night & VAS 0-100 mm & $\begin{array}{l}\text { Mean change at 3, } 6 \text { and } 12 \text { months (baseline median and IQR } \\
\text { reported only) }\end{array}$ \\
\hline
\end{tabular}


Table 25 provides the SMDs and 95\% CIs. The outcome data for individual groups in the included trials are available in Appendix 7.

\section{Physical therapy versus physical therapy}

There was no significant difference in pain between continuous passive motion and conventional physiotherapy at short-term follow-up (12 weeks) (Table 25). ${ }^{74}$ There was no significant benefit from adding ultrasound to a 20-minute supervised exercise programme of Codman's exercises, wall-climbing and joint stretching for patient's tolerance at short-term follow-up ( 3 months) (Table 25)..$^{5}$ There was no significant difference in pain between HGMT and LGMT at any time point (Table 25). ${ }^{40}$

\section{Physical therapy without mobilisation versus control}

There was a significant reduction in pain with laser compared with placebo laser at 8 weeks (SMD $-1.71,95 \%$ CI -2.29 to -1.12 ) and 16 weeks (SMD $-1.89,95 \%-2.49$ to -1.29$)$ (Table 25). ${ }^{16}$

\section{Function and disability}

Ten of the twelve studies that assessed physical therapies reported function and disability outcomes (Table 26). SPADI score ${ }^{16,51,71,74,77}$ and Constant score ${ }^{66,73,74}$ were common to several studies; however, the data could not be pooled in meta-analysis as the comparisons varied substantially. Short- and medium-term data were available from these studies. Final value data were available from six studies. ${ }^{16,51,66,73-75}$ Pajareya et al. ${ }^{77}$ included only baseline data, so this study was not included in the analysis. Change from baseline data was available from the study by Vermeulen et al. ${ }^{40}$ Yang et al. ${ }^{78}$ reported mean percentage of change only. Table 27 provides between-group differences and 95\% CIs. Because of differences between interventions and comparators it was not appropriate to pool these studies. Outcome data for individual studies are available in Appendix 7.

\section{Physical therapy versus control}

Diercks and Stevens ${ }^{73}$ reported a statistically significant improvement in Constant score with supervised neglect compared with physiotherapy at 3 (MD $-16.37,95 \% \mathrm{CI}-21.46$ to -11.28 ), 6 (MD -15.40 95\% CI -20.50 to -10.30 ), 12 (MD -17.74, 95\% CI -22.75 to -12.73 ), 18 (MD $-16.13,95 \% \mathrm{CI}-22.20$ to -10.06 ) and 24 (MD 9.22, 95\% CI -15.69 to -2.75 ) months' follow-up. It is worth noting that there were several factors that could have biased this result. The

TABLE 25 Pain results of studies of physical therapy (SMD and 95\% Cl)

\begin{tabular}{|c|c|c|c|}
\hline Study & Short term ( $\leq 3$ months) & $\begin{array}{l}\text { Medium term (>3 and } \\
\leq 6 \text { months) }\end{array}$ & Long term ( $>6$ and $\leq 12$ months) \\
\hline \multicolumn{4}{|c|}{ Physical therapy vs physical therapy } \\
\hline Dundar $2009^{74}$ & $-0.31(-0.83 \text { to } 0.21)^{\mathrm{a}}$ & & \\
\hline Dogru $2008^{51}$ & $0.28(-0.28 \text { to } 0.85)^{b}$ & & \\
\hline Vermeulen $2006^{40}$ & $-0.15(-0.55 \text { to } 0.24)^{b}$ & $-0.27(-0.67 \text { to } 0.12)^{c}$ & $-0.33(-0.73 \text { to } 0.06)^{d}$ \\
\hline \multicolumn{4}{|c|}{ Physical therapy without mobilisation vs control } \\
\hline Stergioulas $2008^{16}$ & $-1.71(-2.29 \text { to }-1.12)^{e, f}$ & $-1.89(-2.49 \text { to }-1.29)^{f, 9}$ & \\
\hline $\begin{array}{ll}\text { a } & \text { Reported at } 12 \mathrm{w} \\
\text { b } & \text { Reported at } 3 \text { mo } \\
\text { c } & \text { Reported at } 6 \text { mo } \\
\text { d } & \text { Reported at } 12 \mathrm{~m} \\
\text { e } & \text { Reported at } 8 \text { we } \\
\text { f } & \text { Statistically signif } \\
\text { g } & \text { Reported at } 16 \text { w }\end{array}$ & the $\mathrm{Cl}$ did not cross the line & (zero). & \\
\hline
\end{tabular}


TABLE 26 Function and disability outcomes of studies evaluating physical therapy

\begin{tabular}{|c|c|c|}
\hline Study & Outcomes assessed & Data reported and time of follow-up \\
\hline Calis $2006^{66}$ & Constant score & Final value data at 15 days and 3 months \\
\hline Diercks $2004^{73}$ & $\begin{array}{l}\text { Constant score ( } \geq 80 \text { points indicates a normal functioning } \\
\text { shoulder) }\end{array}$ & $\begin{array}{l}\text { Final value data at } 3,6,9,12,15,18,21 \text { and } 24 \text { months } \\
\text { reported }\end{array}$ \\
\hline \multirow[t]{2}{*}{ Dogru $2008^{51}$} & SPADI total score $(0-100)^{\mathrm{a}}$ & \multirow[t]{2}{*}{ Final value data at 2 weeks and 3 months $s^{b, c}$} \\
\hline & SPADI 8-item disability subscale $(0-100)^{a}$ & \\
\hline \multirow[t]{2}{*}{ Dundar $2009^{74}$} & SPADI total score $(0-100)^{\mathrm{a}}$ & \multirow[t]{2}{*}{ Final value data at 4 weeks and 12 weeks } \\
\hline & Constant score & \\
\hline Leung $2008^{75}$ & ASES assessment (score out of 100 ) & Final value data reported at 2, 4 and 8 weeks \\
\hline \multirow[t]{2}{*}{ Pajareya $2004^{77}$} & SPADI total score $(0-100)^{a}$ & 3-week data only (not included) \\
\hline & Global rating of pain and disability & Baseline data only \\
\hline Stergioulas $2008^{16}$ & $\begin{array}{l}\text { SPADI total score (0-100), Croft score (out of 22), DASH } \\
\text { (\%) and HAQ (two or three items scored 0-3) }\end{array}$ & Final value data reported at 4,8 and 16 weeks \\
\hline \multirow[t]{4}{*}{ Vermeulen $2006^{40}$} & Shoulder rating questionnaire (Dutch-language version) & Change from baseline at 3, 6 and 12 months \\
\hline & $(17-100)$ & \multirow{3}{*}{$\begin{array}{l}\text { Percentage of participants reporting 'much worse or no } \\
\text { change' and 'better or much better' at } 3,6 \text { and } 12 \text { months }\end{array}$} \\
\hline & SDQ (Dutch-language version) $(0-100)^{\mathrm{a}}$ & \\
\hline & 5-point Likert scale ( $1=$ much worse; $5=$ much better) & \\
\hline Wies $2003^{71}$ & SPADI $(0-100)^{\mathrm{a}}$ & Change from baseline at 9 weeks \\
\hline Yang $2007^{78}$ & FLEX-SF (1-50) & Mean percentage of change from baseline at 6 weeks \\
\hline
\end{tabular}

DASH, Disabilities of the Arm, Shoulder and Hand; FLEX-SF, Flexilevel Scale of Shoulder Function; HAQ, Health Assessment Questionnaire.

a Higher scores indicated worse outcomes.

TABLE 27 Function and disability results of studies of physical therapy (MD and $95 \% \mathrm{Cl}$ )

\begin{tabular}{|c|c|c|c|}
\hline Study & Short term ( $\leq 3$ months) & Medium term ( $>3$ and $\leq 6$ months) & Long term ( $>6$ and $\leq 12$ months) \\
\hline \multicolumn{4}{|c|}{ Physical therapy vs control } \\
\hline \multicolumn{4}{|l|}{ Constant score } \\
\hline Calis $2006^{66}$ & $14.90(8.32 \text { to } 21.48)^{a, b}$ & & \\
\hline \multirow[t]{3}{*}{ Diercks $2004^{73}$} & $-16.37(-21.46 \text { to }-11.28)^{a, b}$ & $-15.40(-20.50 \text { to }-10.30)^{b, c}$ & $-17.74(-22.75 \text { to }-12.73)^{\mathrm{b}, \mathrm{d}}$ \\
\hline & & & $-16.13(-22.20 \text { to }-10.06)^{b, e}$ \\
\hline & & & $-9.22(-15.69 \text { to }-2.75)^{b, f}$ \\
\hline \multirow[t]{2}{*}{ Leung $2008^{75}$} & $\begin{array}{l}\text { SWD vs home exercise: } 17.50 \text { (1.76 } \\
\text { to } 33.24)^{b, g}\end{array}$ & & \\
\hline & $\begin{array}{l}\text { HP vs home exercise: } 4.00(-10.38 \\
\text { to } 18.38)^{g}\end{array}$ & & \\
\hline \multicolumn{4}{|c|}{ Physical therapy vs physical therapy } \\
\hline \multicolumn{4}{|c|}{ SPADI total score } \\
\hline Dogru $2008^{51}$ & $4.50(-6.36 \text { to } 15.36)^{\mathrm{a}}$ & & \\
\hline Dundar $2009^{74}$ & $0.17(-0.73 \text { to } 1.07)^{\mathrm{a}}$ & & \\
\hline \multirow[t]{3}{*}{ Wies $2003^{71}$} & $\begin{array}{l}\text { PT vs osteopathy: }-19.90(-40.11 \text { to } \\
0.31)^{\mathrm{h}}\end{array}$ & & \\
\hline & $\begin{array}{l}\text { PT vs control: }-4.00(-22.47 \text { to } \\
14.47)^{\mathrm{h}}\end{array}$ & & \\
\hline & $\begin{array}{l}\text { Osteopathy vs control: } 15.90(-2.04 \\
\text { to } 33.84)^{\mathrm{h}}\end{array}$ & & \\
\hline \multicolumn{4}{|c|}{ SPADI 8-item subscale } \\
\hline Dogru $2008^{51}$ & $3.10(-8.44 \text { to } 14.64)^{\mathrm{a}}$ & & \\
\hline \multicolumn{4}{|l|}{ Constant score } \\
\hline Dundar $2009^{74}$ & $-3.37(-8.28 \text { to } 1.54)^{\mathrm{a}}$ & & \\
\hline
\end{tabular}


TABLE 27 Function and disability results of studies of physical therapy (MD and 95\% Cl) (continued)

\begin{tabular}{|c|c|c|c|}
\hline Study & Short term ( $\leq 3$ months) & Medium term ( $>3$ and $\leq 6$ months) & Long term ( $>6$ and $\leq 12$ months) \\
\hline \multicolumn{4}{|l|}{ FLEX-SF } \\
\hline \multirow[t]{2}{*}{ Yang $2007^{78}$} & ERM + MRM: 19.9\% (SD 8.1\%)i & & \\
\hline & MWM + MRM: 17.25\% (SD 12.2\%)' & & \\
\hline \multicolumn{4}{|l|}{ ASES } \\
\hline Leung $2008^{75}$ & HP vs SWD: $13.50(-2.16 \text { to } 29.16)^{9}$ & & \\
\hline \multicolumn{4}{|c|}{ Shoulder rating questionnaire } \\
\hline Vermeulen $2006^{40}$ & $2.40(-4.00 \text { to } 8.80)^{\mathrm{a}}$ & $4.50(-2.39 \text { to } 11.39)^{c}$ & $6.60(-0.63 \text { to } 13.83)^{d}$ \\
\hline \multicolumn{4}{|l|}{$S D Q$} \\
\hline Vermeulen $2006^{40}$ & $-5.20(-14.95 \text { to } 4.55)^{\mathrm{a}}$ & $-5.70(-17.42 \text { to } 6.02)^{c}$ & $-11.20(-22.60 \text { to } 0.20)^{d}$ \\
\hline \multicolumn{4}{|c|}{ Physical therapy without mobilisation vs control } \\
\hline \multicolumn{4}{|l|}{ SPADI } \\
\hline Stergioulas $2008^{16}$ & $-14.11(-19.50 \text { to }-8.72)^{b, g}$ & $-13.83(-18.88 \text { to }-8.78)^{b, j}$ & \\
\hline \multicolumn{4}{|l|}{ Croft score } \\
\hline Stergioulas $2008^{16}$ & $-4.34(-6.34 \text { to }-2.34)^{b, g}$ & $-7.13(-9.18 \text { to }-5.08)^{\mathrm{bj} j}$ & \\
\hline \multicolumn{4}{|l|}{ DASH score } \\
\hline Stergioulas $2008^{16}$ & $-9.24(-14.35 \text { to }-4.13)^{\mathrm{b}, \mathrm{g}}$ & $-10.51(-15.37 \text { to }-5.65)^{b, j}$ & \\
\hline \multicolumn{4}{|l|}{$H A Q$ score } \\
\hline Stergioulas $2008^{16}$ & $-0.75(-1.09 \text { to }-0.41)^{\mathrm{b}, \mathrm{g}}$ & $-0.31(-0.64 \text { to } 0.02)^{\mathrm{j}}$ & \\
\hline
\end{tabular}

FLEX-SF, Flexilevel Scale of Shoulder Function; HP, heat pack; PT, physiotherapy.

a Reported at 3 months or 12 weeks.

b Statistically significant, i.e. the $\mathrm{Cl}$ did not cross the line of no effect (zero).

c Reported at 6 months or 24 weeks.

d Reported at 12 months.

e Reported at 18 months.

f Reported at 24 months.

g Reported at 8 weeks.

h Reported at 9 weeks.

i Reported at 6 weeks.

j Reported at 16 weeks.

physiotherapy group were prescribed exercises up to and beyond the pain threshold whereas the supervised neglect group were advised to exercise within the painless range. Also, as a successive cohort was recruited after 2 years (the physiotherapy group), treatment protocols may have changed during the time that elapsed (although the authors did report that a standardised treatment protocol was used). Although the baseline characteristics reported were similar between groups it is possible that there may have been unmeasured differences between groups.

Leung and Cheing ${ }^{75}$ found significant improvement in American Shoulder and Elbow Surgeons (ASES) score with SWD plus stretching compared with home exercise (MD 17.50, 95\% CI 1.76 to 33.24). There was no significant difference between heat pack plus stretching and home exercise (Table 27).

Calis et al. ${ }^{66}$ reported that Constant score was significantly improved with physiotherapy plus home exercise compared with home exercise alone (MD 14.90, 95\% CI 8.32 to 21.48). 


\section{Physical therapy versus physical therapy}

The study by Dogru et al. ${ }^{51}$ which compared ultrasound and physiotherapy with sham ultrasound and physiotherapy, found no significant difference between treatment groups in SPADI total score or the SPADI 8-item disability subscale at 3 months (Table 27).

The intention in the study by Yang et al. ${ }^{78}$ was to compare two different sequences of three types of mobilisation (each type of mobilisation was delivered over a 3-week period). The two groups received the three types of mobilisation but in a different order over 12 weeks (see Appendix 6). Because at the end of the 12 weeks both groups had received the same treatments, only 6-week data were extracted for ERM and MRM versus MWM and MRM. The change in FLEX-SF score was similar at 6 weeks for ERM and MRM (19.9\%, SD 8.1\%) and MWM and MRM $(17.25 \%$, SD $12.2 \%)$

The study by Dundar et al., ${ }^{74}$ which compared continuous passive motion with physiotherapy, found no significant difference between treatment groups in SPADI total score or the Constant score (Table 27).

Leung and Cheing ${ }^{75}$ found no significant difference between SWD and heat pack groups in terms of ASES score (Table 27).

In the study by Vermeulen et al..$^{40}$ there was no significant difference between HGMT and LGMT in the shoulder rating questionnaire score (Table 27). There was also no significant improvement in SDQ score with HGMT compared with LGMT at any time point (Table 27); however, it should be noted that the $95 \%$ CI marginally crossed the line of no effect at 12 months (MD -11.20, $95 \%$ CI -22.60 to 0.20 ). There was no significant difference in the proportions of patients who reported '(much) worse or no change' at 3 months (13\% vs 12\%), 6 months (13\% vs 10\%) or 12 months ( $9 \%$ vs $18 \%$ ) and 'better or much better' at 3 months ( $87 \%$ vs $88 \%) 6$ months ( $87 \%$ vs $90 \%$ ) or 12 months (91\% vs $82 \%)$.

There was no significant difference in SPADI score with physiotherapy compared with osteopathy in the study by Wies et al. $7^{71}$ however, it should be noted that the $95 \%$ CI marginally crossed the line of no effect (MD -19.90, 95\% CI -40.11 to 0.31 ). There was no significant difference between either the physiotherapy or osteopathy group and the control group (breathing exercises, range of movement exercises and massage) (Table 27).

\section{Physical therapy without mobilisation versus control}

Stergioulas ${ }^{16}$ reported several measures of function and disability. At 8 weeks laser treatment was associated with significantly improved SPADI score (MD -14.11, 95\% CI -19.50 to -8.72), Croft score (MD -4.34, 95\% CI -6.34 to -2.34) Disabilities of the Arm, Shoulder and Hand (DASH) score (MD -9.24, 95\% CI -14.35 to -4.13) and Health Assessment Questionnaire (HAQ) score (MD -0.75, 95\% CI -1.09 to -0.41) compared with placebo laser. At 16 weeks laser treatment was associated with significantly improved SPADI score (MD -13.83, 95\% CI -18.88 to -8.78), Croft score (MD $-7.13,95 \% \mathrm{CI}-9.18$ to -5.08 ) and DASH score (MD -10.51, 95\% CI - 15.37 to -5.65 ). There was no significant difference in HAQ score between groups at 16 weeks (Table 27).

\section{Range of movement}

Eleven of the included studies that investigated physical therapy reported a range of movement measure of interest (Table 28). All of the studies except two reported short- or medium-term follow-up. One presented data at 24 months ${ }^{73}$ and one at 12 months. ${ }^{40}$ Six studies reported final value data and one $\mathrm{e}^{77}$ presented data that were not included in the analysis as follow-up was 1 $<4$ weeks. One study reported change from baseline data. ${ }^{40}$ Maricar and $\mathrm{Chok}^{76}$ reported only 
TABLE 28 Range of movement outcomes in studies evaluating physical therapy

\begin{tabular}{|c|c|c|}
\hline Study & Outcomes assessed & Data reported and time of follow-up \\
\hline Calis $2006^{66}$ & Passive abduction, passive external rotation $\left({ }^{\circ}\right)$ & Final value data at 15 days and 3 months \\
\hline Diercks $2004^{73}$ & $\begin{array}{l}\text { External rotation, internal rotation (ROM part of Constant score, } \\
\text { number of points out of } 10 \text { correspond to extent of movement) }\end{array}$ & Final value data at 24 months $\mathrm{s}^{\mathrm{a}}$ \\
\hline Dogru $2008^{51}$ & External rotation, internal rotation, passive abduction $\left({ }^{\circ}\right)$ & Final value data at 2 weeks and 3 months ${ }^{b}$ \\
\hline Dundar $2009^{74}$ & $\begin{array}{l}\text { Passive external rotation, passive internal rotation, passive } \\
\text { abduction }\left({ }^{\circ}\right)\end{array}$ & Final value data at 4 weeks and 12 weeks \\
\hline Leung $2008^{75}$ & $\begin{array}{l}\text { External rotation (arm by side) }\left(^{\circ}\right) \text {, external rotation (arm at } 90 \\
\left({ }^{\circ}\right) \text { ), hand behind back }(\mathrm{cm})\end{array}$ & Final value data at 2, 4 and 8 weeks \\
\hline Maricar $1999^{76}$ & External rotation $\left({ }^{\circ}\right)$, internal rotation $\left({ }^{\circ}\right)$, hand behind back $(\mathrm{cm})$ & $\begin{array}{l}\text { Graphs and } p \text {-values only reported at 3, 5, } 7 \text { and } \\
8 \text { weeks }\end{array}$ \\
\hline Pajareya $2004^{77}$ & External rotation, internal rotation & 3-week data only (not included in analysis) \\
\hline Stergioulas $2008^{16}$ & Active abduction, external rotation (in neutral abduction) $\left(^{\circ}\right)$ & Final value data at 4,8 and 16 weeks \\
\hline Vermeulen $2006^{40}$ & $\begin{array}{l}\text { Active abduction, active external rotation, passive abduction, } \\
\text { passive external rotation }\left(^{\circ}\right)\end{array}$ & Change from baseline at 3, 6 and 12 months \\
\hline Wies $2003^{71}$ & Active abduction $\left({ }^{\circ}\right)$ & Change from baseline at 9 weeks \\
\hline Yang $2007^{78}$ & $\begin{array}{l}\text { Humeral lateral rotation (external rotation), humeral medial } \\
\text { rotation (internal rotation) }\left({ }^{\circ}\right)\end{array}$ & Mean percentage of change from baseline at 6 weeks \\
\hline
\end{tabular}

graphs and $p$-values, presented narratively. Because of the differences between the interventions and comparators meta-analyses were not performed. Table 29 provides between-group differences and 95\% CIs. Outcome data for individual studies are available in Appendix 7.

\section{Physical therapy versus control}

In the study by Calis et al. ${ }^{66}$ there was a significant improvement in passive abduction (MD $24.90^{\circ}, 95 \% \mathrm{CI} 14.59^{\circ}$ to $35.21^{\circ}$ ) and passive external rotation (MD $18.80^{\circ}, 95 \% \mathrm{CI} 13.11^{\circ}$ to $24.49^{\circ}$ ) in the physiotherapy group compared with home exercise alone.

In the study by Leung and Cheing ${ }^{75}$ there was a significant improvement in external rotation with arm by side (MD $21.00^{\circ}, 95 \%$ CI $4.95^{\circ}$ to $37.05^{\circ}$ ) with SWD plus stretching compared with home exercise. Hand behind back was also significantly greater in the SWD plus stretching group compared with the home exercise group (MD $-7.00 \mathrm{~cm}, 95 \% \mathrm{CI}-13.14 \mathrm{~cm}$ to $-0.86 \mathrm{~cm}$ ). There was no significant difference between either SWD or heat pack (both with stretching exercises) and home exercise in external rotation with arm at $90^{\circ}$, and no significant difference between heat pack and home exercise in external rotation with arm by side or hand behind back (Table 29).

The study by Diercks and Stevens ${ }^{73}$ reported that at 24 months the physiotherapy group had a median of 8 points for the Constant score for external rotation, compared with 10 points in the supervised neglect group, although no measure of variance was provided. This corresponds to a greater range of motion in the supervised neglect group in this plane. For internal rotation, both groups reported a median of 8 points, corresponding to the position dorsum of the 12th dorsal vertebra.

\section{Physical therapy versus physical therapy}

Dogru et al. ${ }^{51}$ found no significant difference between ultrasound and sham ultrasound in external rotation, internal rotation and abduction (Table 29). 
There was also no significant difference in the study by Dundar et al. ${ }^{74}$ between continuous passive motion and physical therapy in passive external rotation, passive internal rotation and passive abduction (Table 29).

The study by Wies et al..$^{71}$ showed a significantly greater improvement in active abduction in the osteopathy group (MD $45.20^{\circ}, 95 \%$ CI $16.87^{\circ}$ to $73.53^{\circ}$ ) and physiotherapy groups (MD $38.80^{\circ}$, $95 \%$ CI $5.76^{\circ}$ to $71.84^{\circ}$ ) compared with control (breathing exercises, massage and exercise). There was no significant difference between the physiotherapy and osteopathy groups (Table 29).

Yang et $a l . .^{78}$ reported that the mean percentage of change in external rotation was similar between ERM plus MRM (mean 36.4\%, SD 24.3\%) and MWM plus MRM (mean 34.2\%, SD 13.3\%).

However, there was a greater change in internal rotation with MWM + MRM (45.6\%, SD 38.5\%) compared with ERM and MRM (20.5\%, SD 24.4\%) (Table 29).

Leung and Cheing ${ }^{75}$ found that there was a significant improvement in external rotation both with arm by side (MD $29.50^{\circ}, 95 \%$ CI $14.28^{\circ}$ to $44.72^{\circ}$ ) and with arm at $90^{\circ}$ abduction (MD $30.10^{\circ}, 95 \%$ CI $13.51^{\circ}$ to $46.69^{\circ}$ ) with SWD compared with heat pack. There was also a significant improvement in hand behind back with SWD compared with heat pack $(\mathrm{MD}-12.30 \mathrm{~cm}, 95 \% \mathrm{CI}$ $-18.79 \mathrm{~cm}$ to $-5.81 \mathrm{~cm}$ ) (Table 29).

Maricar and $\mathrm{Chok}^{76}$ compared passive joint mobilisations and exercises with exercises only and found no significant difference in external rotation or internal rotation between treatment groups at weeks five, seven or eight.

The study by Vermeulen et al. ${ }^{40}$ showed a significant improvement in active abduction at 12 months with HGMT compared with LGMT (MD $12.60^{\circ}$, $95 \%$ CI $0.06^{\circ}$ to $25.14^{\circ}$ ). There was also a significant improvement with HGMT in passive abduction at 3 months (MD $13.10^{\circ}, 95 \%$ CI $1.62^{\circ}$ to $24.58^{\circ}$ ) and 12 months (MD $12.50^{\circ}, 95 \%$ CI $1.03^{\circ}$ to $23.97^{\circ}$ ), and in passive external rotation at 12 months (MD $6.50^{\circ}, 95 \% \mathrm{CI} 0.27^{\circ}$ to $12.73^{\circ}$ ). There was no significant difference between groups in active external rotation at any of the time points, or in passive external rotation, active abduction or passive abduction at the remaining time points (Table 29).

\section{Physical therapy without mobilisation versus control}

There was no significant difference in external rotation or active abduction at short- or mediumterm follow-up between laser and placebo laser (Table 29). ${ }^{16}$

\section{Quality of life}

Two studies, one by Dogru et al..$^{51}$ and the other by Vermeulen et al. ${ }^{40}$ reported quality of life using the physical and mental components of the SF-36 (Table 30). Data at 3 months' follow-up were available (Table 31). As the interventions varied substantially these studies were not pooled. Outcome data for the individual groups are available in Appendix 7.

\section{Physical therapy versus physical therapy}

There was no significant difference between ultrasound and sham ultrasound groups in the SF-36 physical or mental components at 3 months' follow-up in the study by Dogru et al. (Table 31). ${ }^{51}$

There was no significant difference between HGMT and LGMT groups in the SF-36 physical or mental components at any time point in the study by Vermeulen et al. (Table 31). ${ }^{40}$

\section{Other}

Two studies reported other outcomes of interest (Table 32). Pajareya et al. ${ }^{77}$ reported satisfaction with treatment and percentage of patients reporting treatment success. Yan ${ }^{72}$ also reported the 
TABLE 29 Range of movement results of studies of physical therapy (MD and 95\% Cl)

\begin{tabular}{|c|c|c|c|}
\hline Study & Short term ( $\leq 3$ months) & Medium term ( $>3$ and $\leq 6$ months) & Long term ( $>6$ and $\leq 12$ months) \\
\hline \multicolumn{4}{|c|}{ Physical therapy vs control } \\
\hline \multicolumn{4}{|l|}{ External rotation } \\
\hline \multirow[t]{2}{*}{ Diercks $2004^{73}$} & & & PT: median = 8 points $^{\mathrm{a}}$ \\
\hline & & & Supervised neglect: median $=10$ \\
\hline \multirow[t]{4}{*}{ Leung $2008^{75}$} & $\begin{array}{l}\text { SWD vs home exercise, arm by side: } \\
21.00^{\circ}\left(4.95^{\circ} \text { to } 37.05^{\circ}\right)^{\mathrm{b}, \mathrm{c}}\end{array}$ & & \\
\hline & $\begin{array}{l}\text { SWD vs home exercise, arm at } 90^{\circ} \text { : } \\
11.60^{\circ}\left(-6.58^{\circ} \text { to } 29.78^{\circ}\right)^{\mathrm{b}}\end{array}$ & & \\
\hline & $\begin{array}{l}\text { HP vs home exercise, arm by side: } \\
-8.50^{\circ}\left(-28.19^{\circ} \text { to } 11.19^{\circ}\right)^{\mathrm{b}}\end{array}$ & & \\
\hline & $\begin{array}{l}\text { HP vs home exercise, arm at } 90^{\circ} \text { : } \\
-18.50^{\circ}\left(-41.15^{\circ} \text { to } 4.15^{\circ}\right)^{\mathrm{b}}\end{array}$ & & \\
\hline \multicolumn{4}{|l|}{ Internal rotation } \\
\hline \multirow[t]{2}{*}{ Diercks $2004^{73}$} & & & PT: median $=8$ points $^{\mathrm{a}}$ \\
\hline & & & Supervised neglect: median $=8$ \\
\hline \multicolumn{4}{|c|}{ Hand behind back } \\
\hline \multirow[t]{2}{*}{ Leung $2008^{75}$} & $\begin{array}{l}\text { SWD }+ \text { stretching vs home exercise: } \\
-7.00 \mathrm{~cm}(-13.14 \mathrm{~cm} \text { to }-0.86 \mathrm{~cm})^{\mathrm{b}, \mathrm{c}}\end{array}$ & & \\
\hline & $\begin{array}{l}\mathrm{HP}+\text { stretching vs home exercise: } \\
5.30 \mathrm{~cm}(-0.93 \mathrm{~cm} \text { to } 11.53 \mathrm{~cm})^{b}\end{array}$ & & \\
\hline
\end{tabular}

Passive external rotation

Calis $2006^{66} \quad 18.80^{\circ}\left(13.11^{\circ} \text { to } 24.49^{\circ}\right)^{c, d}$

Passive abduction

Calis $2006^{66} \quad 24.90^{\circ}\left(14.59^{\circ} \text { to } 35.21^{\circ}\right)^{c, d}$

\section{Physical therapy vs physical therapy}

External rotation

\begin{tabular}{|c|c|}
\hline Dogru $2008^{51}$ & $-9.70^{\circ}\left(-19.51^{\circ} \text { to } 0.11^{\circ}\right)^{d}$ \\
\hline Dundar $2009^{74}$ & $0.76^{\circ}\left(-7.40^{\circ} \text { to } 8.92^{\circ}\right)^{d}$ \\
\hline \multirow[t]{2}{*}{ Yang $2007^{78}$} & ERM + MRM: 36.4\% (SD 24.3\%) \\
\hline & MWM + MRM: $34.2 \%($ SD 13.3\%) \\
\hline \multirow[t]{2}{*}{ Leung $2008^{75}$} & $\begin{array}{l}\text { HP vs SWD, arm by side: } 29.50^{\circ} \\
\left(14.28^{\circ} \text { to } 44.72^{\circ}\right)^{b, c}\end{array}$ \\
\hline & $\begin{array}{l}\text { HP vs SWD, } 90 \text { degree abduction: } \\
30.10^{\circ}\left(13.51^{\circ} \text { to } 46.69^{\circ}\right)^{b, c}\end{array}$ \\
\hline
\end{tabular}

Internal rotation

$\begin{array}{ll}\text { Dogru 200851 } & -3.50^{\circ}\left(-11.67^{\circ} \text { to } 4.67^{\circ}\right)^{\mathrm{d}} \\ \text { Yang 200778 } & \text { ERM + MRM: 20.5\% (SD 24.4\%) } \\ & \text { MWM + MRM: 45.6\% (SD 38.5\%) }\end{array}$

Passive internal rotation

Dundar $2009^{74} \quad 0.92^{\circ}\left(-8.34^{\circ} \text { to } 10.18^{\circ}\right)^{d}$

Passive abduction

$\begin{array}{ll}\text { Dogru 2008 } & -0.20^{\circ}\left(-16.06^{\circ} \text { to } 15.66^{\circ}\right)^{\mathrm{d}} \\ \text { Dundar 2009 } & -4.42^{\circ}\left(-11.83^{\circ} \text { to } 2.99^{\circ}\right)^{\mathrm{d}} \\ {\text { Vermeulen } 2004^{40}} & 13.10^{\circ}\left(1.62^{\circ} \text { to } 24.58^{\circ}\right)^{\mathrm{c}, \mathrm{d}}\end{array}$


TABLE 29 Range of movement results of studies of physical therapy (MD and 95\% Cl) (continued)

\begin{tabular}{|c|c|c|c|}
\hline Study & Short term ( $\leq 3$ months) & Medium term ( $>3$ and $\leq 6$ months) & Long term ( $>6$ and $\leq 12$ months) \\
\hline \multicolumn{4}{|l|}{ Active abduction } \\
\hline Vermeulen, $2004^{40}$ & $10.00^{\circ}\left(-2.16^{\circ} \text { to } 22.16^{\circ}\right)^{d}$ & $8.90^{\circ}\left(-4.67^{\circ} \text { to } 22.47^{\circ}\right)^{f}$ & $12.60^{\circ}\left(0.06^{\circ} \text { to } 25.14^{\circ}\right)^{\mathrm{c}, g}$ \\
\hline \multirow[t]{3}{*}{ Wies $2003^{71}$} & $\begin{array}{l}\text { Physical therapy vs osteopathy: }-6.40^{\circ} \\
\left(-32.77^{\circ} \text { to } 19.97^{\circ}\right)^{\mathrm{h}}\end{array}$ & & \\
\hline & $\begin{array}{l}\text { Physical therapy vs control: } 38.80^{\circ} \\
\left(5.76^{\circ} \text { to } 71.84^{\circ}\right)^{c, h}\end{array}$ & & \\
\hline & $\begin{array}{l}\text { Osteopathy vs control: } 45.20^{\circ}\left(16.87^{\circ}\right. \\
\left.\text { to } 73.53^{\circ}\right)^{c, h}\end{array}$ & & \\
\hline \multicolumn{4}{|c|}{ Active external rotation } \\
\hline Vermeulen $2004^{40}$ & $2.30^{\circ}\left(-2.81^{\circ} \text { to } 7.41^{\circ}\right)^{d}$ & $2.70^{\circ}\left(-2.36^{\circ} \text { to } 7.76^{\circ}\right)^{\dagger}$ & $4.90^{\circ}\left(-0.67^{\circ} \text { to } 10.47^{\circ}\right)^{\mathrm{g}}$ \\
\hline \multicolumn{4}{|c|}{ Passive external rotation } \\
\hline Vermeulen $2004^{40}$ & $1.40^{\circ}\left(-3.48^{\circ} \text { to } 6.28^{\circ}\right)^{d}$ & $4.10^{\circ}\left(-0.97^{\circ} \text { to } 9.17^{\circ}\right)^{\dagger}$ & $6.50^{\circ}\left(0.27^{\circ} \text { to } 12.73^{\circ}\right)^{\mathrm{c}, 9}$ \\
\hline \multicolumn{4}{|l|}{ Hand behind back } \\
\hline Leung $2008^{75}$ & $\begin{array}{l}\text { Heat pack vs SWD: }-12.30 \mathrm{~cm} \\
(-18.79 \mathrm{~cm} \text { to }-5.81 \mathrm{~cm})^{\mathrm{b}, \mathrm{c}}\end{array}$ & & \\
\hline \multicolumn{4}{|c|}{ Physical therapy without mobilisation vs control } \\
\hline \multicolumn{4}{|l|}{ External rotation } \\
\hline Stergioulas $2008^{16}$ & $2.05^{\circ}\left(-2.75^{\circ} \text { to } 6.85^{\circ}\right)^{b}$ & $4.19^{\circ}\left(-0.74^{\circ} \text { to } 9.12^{\circ}\right)^{i}$ & \\
\hline \multicolumn{4}{|l|}{ Active abduction } \\
\hline Stergioulas $2008^{16}$ & $5.47^{\circ}\left(-0.40^{\circ} \text { to } 11.34^{\circ}\right)^{b}$ & $5.20^{\circ}\left(-1.60^{\circ} \text { to } 12.00^{\circ}\right)^{i}$ & \\
\hline \multicolumn{4}{|c|}{$\begin{array}{ll}\text { a } & \text { Reported at } 24 \text { months. } \\
\text { b } & \text { Reported at } 8 \text { weeks. } \\
\text { c } & \text { Statistically significant, i.e. the } \mathrm{Cl} \text { did } \\
\text { d } & \text { Reported at } 3 \text { months or } 12 \text { weeks. } \\
\text { e } & \text { Reported at } 6 \text { weeks. } \\
\text { f } & \text { Reported at } 6 \text { months. } \\
\text { g } & \text { Reported at } 12 \text { months. } \\
\text { h } & \text { Reported at } 9 \text { weeks. } \\
\text { i } & \text { Reported at } 16 \text { weeks. }\end{array}$} \\
\hline Study & Outcomes assessed & Data reported and time of follow-up & \\
\hline \multirow[t]{2}{*}{ Dogru $2008^{51}$} & SF-36 physical component & \multirow{2}{*}{\multicolumn{2}{|c|}{ Final value data at 2 weeks and 3 months ${ }^{\mathrm{a}}$ (3-month data included in analysis) }} \\
\hline & SF-36 mental component & & \\
\hline \multirow{2}{*}{$\begin{array}{l}\text { Vermeulen } \\
2004^{40}\end{array}$} & SF-36 physical component & \multirow{2}{*}{\multicolumn{2}{|c|}{ Change from baseline at 3, 6 and 12 months }} \\
\hline & SF-36 mental component & & \\
\hline
\end{tabular}

a Unclear whether 3 months from commencement of treatment or following treatment. 
TABLE 31 Quality of life results of studies of physical therapy (MD and 95\% Cl)

\begin{tabular}{|c|c|c|c|c|}
\hline Study & Short term ( $\leq 3$ months) & \multicolumn{2}{|c|}{ Medium term (>3 and $\leq 6$ months) } & Long term ( $>6$ and $\leq 12$ months) \\
\hline \multicolumn{5}{|c|}{ Physical therapy vs physical therapy } \\
\hline \multicolumn{5}{|c|}{ SF-36 physical component } \\
\hline Dogru $2008^{51}$ & $-0.40(-5.22 \text { to } 4.42)^{\mathrm{a}}$ & \multirow{2}{*}{\multicolumn{2}{|c|}{$2.10(-5.08 \text { to } 9.28)^{b}$}} & \\
\hline Vermeulen $2004^{40}$ & $0.60(-6.03 \text { to } 7.23)^{\mathrm{a}}$ & & & $0.40(-7.78 \text { to } 8.58)^{c}$ \\
\hline \multicolumn{5}{|c|}{ SF-36 mental component } \\
\hline Dogru $2008^{51}$ & $1.00(-5.19 \text { to } 7.19)^{\mathrm{a}}$ & \multirow{2}{*}{\multicolumn{2}{|c|}{$0.30(-7.95 \text { to } 8.55)^{b}$}} & \\
\hline Vermeulen $2004^{40}$ & $4.10(-3.91 \text { to } 12.11)^{\mathrm{a}}$ & & & $-2.50(-8.36 \text { to } 3.36)^{c}$ \\
\hline \multicolumn{5}{|c|}{$\begin{array}{l}\text { a Reported at } 3 \text { months. } \\
\text { b Reported at } 6 \text { months. } \\
\text { c Reported at } 12 \text { months. }\end{array}$} \\
\hline Study & Outcomes assessed & & Data reported an & time of follow-up \\
\hline \multirow[t]{2}{*}{ Pajareya $2004^{77}$} & \multicolumn{2}{|c|}{$\begin{array}{l}\text { Satisfaction (number of patients reporting that they were } \\
\text { 'very satisfied', 'moderately satisfied', 'unsatisfied' or 'very } \\
\text { unsatisfied') }\end{array}$} & \multicolumn{2}{|l|}{ Data at 3 weeks } \\
\hline & \multicolumn{2}{|c|}{ Treatment success (\% success) } & \multicolumn{2}{|c|}{ Treatment success at 3, 6, 12 and 24 weeks } \\
\hline Yan $2005^{72}$ & \multicolumn{2}{|l|}{ Rate of improvement (\%) } & \multicolumn{2}{|c|}{$\begin{array}{l}\text { Rate of improvement (excellent, good, average and bad) at } \\
3 \text { months }\end{array}$} \\
\hline
\end{tabular}

percentage of patients describing their rate of improvement as bad, average, good and excellent at 3 months. These data are summarised in Table 33 and further information for the individual studies is available in Appendix 7.

\section{Physical therapy versus control}

In the study by Pajareya et al. ${ }^{77}$ similar proportions of each group reported that they had successful treatment at 6 weeks ( $61.4 \%$ vs $60.8 \%)$. At 12 weeks, $76.8 \%$ in the physiotherapy compared with $60.8 \%$ in the control group reported successful treatment, and at 24 weeks the proportions were $80.4 \%$ in the physiotherapy group and $82.4 \%$ in the control group.

In the same study the number of patients 'very satisfied' at 3 weeks was five in the physical therapy group versus one in the control group; the numbers 'moderately satisfied, 'unsatisfied' and 'very unsatisfied' were 7 versus 1, 24 versus 13 and 23 versus 45 in the physical therapy group versus the control group respectively. ${ }^{77}$

\section{Physical therapy versus physical therapy}

$\mathrm{Yan}^{72}$ compared dumb-bell exercises with barehanded exercises and found that a greater number of patients performing dumb-bell exercises than those performing barehanded exercises reported an excellent rate of improvement at 3 months ( 24 vs 0 ).

\section{Adverse events}

Two studies reported adverse events. ${ }^{74,77}$ In the study by Pajareya et al..$^{77}$ patients in the physiotherapy group were asked whether they had pain that persisted for $>2$ hours after treatment or more disability the next morning. There were 10 episodes of pain in the physiotherapy group (in four patients) that persisted for $>2$ hours after treatment. Patients 
TABLE 33 Other outcome results from studies of physical therapy

\begin{tabular}{|c|c|c|c|}
\hline Study & Short term ( $\leq 3$ months) & Medium term ( $>3$ and $\leq 6$ months) & Long term ( $>6$ and $\leq 12$ months) \\
\hline \multicolumn{4}{|c|}{ Physical therapy vs control } \\
\hline \multicolumn{4}{|c|}{ Treatment success } \\
\hline \multirow[t]{3}{*}{ Pajareya $2004^{77}$} & $\%$ of patients & & $\%$ of patients ${ }^{c}$ \\
\hline & $\begin{array}{l}\text { Physical therapy: } 61.4 \%,{ }^{\text {a }} \text { control: } \\
60.8 \%\end{array}$ & & $\begin{array}{l}\text { Physical therapy: } 80.4 \% \text {, control: } \\
82.4 \%\end{array}$ \\
\hline & $\begin{array}{l}\text { Physical therapy: } 76.8 \%,{ }^{b} \text { control: } \\
60.8 \%\end{array}$ & & \\
\hline \multicolumn{4}{|l|}{ Satisfaction } \\
\hline \multirow[t]{5}{*}{ Pajareya $2004^{77}$} & No. of patients ${ }^{d}$ & & \\
\hline & $\begin{array}{l}\text { 'very satisfied': physical therapy } 5 \text {, } \\
\text { control } 1\end{array}$ & & \\
\hline & $\begin{array}{l}\text { 'moderately satisfied': physical therapy } \\
7, \text { control } 1\end{array}$ & & \\
\hline & $\begin{array}{l}\text { 'unsatisfied': physical therapy } 24, \\
\text { control } 13\end{array}$ & & \\
\hline & $\begin{array}{l}\text { 'very unsatisfied': physical therapy } 23 \text {, } \\
\text { control } 45\end{array}$ & & \\
\hline \multicolumn{4}{|c|}{ Physical therapy vs physical therapy } \\
\hline \multicolumn{4}{|c|}{ Rate of improvement } \\
\hline \multirow[t]{5}{*}{ Yan $2005^{72}$} & No. of patients ${ }^{b}$ & & \\
\hline & $\begin{array}{l}\text { Bad: dumb-bell exercises } 0 \text {, } \\
\text { barehanded } 7\end{array}$ & & \\
\hline & $\begin{array}{l}\text { Average: dumb-bell exercises } 0 \text {, } \\
\text { barehanded } 16\end{array}$ & & \\
\hline & $\begin{array}{l}\text { Good: dumb-bell exercises } 2 \text {, } \\
\text { barehanded } 5\end{array}$ & & \\
\hline & $\begin{array}{l}\text { Excellent: dumb-bell exercises } 24 \text {, } \\
\text { barehanded } 0\end{array}$ & & \\
\hline
\end{tabular}

a Reported at 6 weeks.

Reported at 3 months or 12 weeks.

c Reported at 6 months or 24 weeks.

d Reported at 3 weeks.

were also asked whether the trial drugs and/or treatment programme upset them in any way and were examined for signs of echymosis or burn during range of movement evaluation. Side effects of NSAIDs were reported but it was unclear which treatment groups these applied to (see Appendix 6). Dundar et al..$^{74}$ stated that no side effects were observed during the study. No further information regarding how adverse events were measured or assessed was reported. The study by Stergioulas ${ }^{16}$ reported that there were no complications. Adverse events did not appear to have been assessed in the remaining nine studies.

\section{Summary}

Twelve studies were included that investigated various types of physical therapy, without use of steroid injection. They were very diverse in the physical therapies evaluated, although most evaluated physiotherapy modalities. The comparators were another physical therapy or control. With the exception of one study, ${ }^{16}$ at least one component of the intervention involved active mobilisation or exercise. Half the studies reported that participants were advised to undertake home exercise. 
One study of satisfactory quality was identified and three studies were of reasonable quality but had some risk of bias. The other studies had a potentially high risk of bias. Because of the considerable variability of the interventions investigated in the studies and different outcomes measures used it was not appropriate to pool any studies in a meta-analysis. The studies did not report outcome by stage of frozen shoulder or the presence of diabetes and information was very limited regarding previous treatments received.

\section{Physical therapy versus control}

There was evidence from one study (with some risk of bias) that there was a significant shortterm benefit for function and disability with SWD in conjunction with stretching compared with home exercise and for some but not all measures of range of movement. A further study with some risk of bias reported significant short- and medium-term benefit with laser therapy (plus home exercise) compared with home exercise alone for pain and function and disability but not range of movement. Another study with a potentially high risk of bias reported significant short-term benefit with physiotherapy (plus home exercise) compared with home exercise alone for function and disability and two ranges of movement. One study reported significant short-, medium- and long-term benefit for function and disability with supervised neglect compared with physiotherapy; however, this study was at high risk of bias.

\section{Comparing physical therapies}

The majority of studies comparing two physical therapies reported no significant difference in outcome between therapies; however, with the exception of three studies, ${ }^{40,75,78}$ these all had a potentially high risk of bias. A single study with some risk of bias found a significant benefit of SWD compared with a heat pack (both with stretching) for three ranges of movement; however, in this same study there was no significant difference in benefit for function and disability. Another study with some risk of bias found a significant improvement in one measure of function and disability and in three ranges of movement with HGMT compared with LGMT, but no benefit for pain. A single satisfactory study found no benefit from providing ERM followed by MRM compared with MWM followed by MRM. Based on single studies with a high risk of bias there was no significant difference between continuous passive motion and conventional physical therapy, between mobilisation with and without ultrasound or between joint mobilisation and exercise. A single study with a high risk of bias reported that daily exercises using dumb-bells were significantly more effective than exercises without dumb-bells. One study available only as an abstract and at high risk of bias reported a significant benefit of physiotherapy and osteopathy compared with breathing and range of movement exercises combined with massage in one measure of range of movement. There was no significant difference between physiotherapy and osteopathy techniques.

In conclusion, the studies identified were diverse and mainly of poor quality. With the exception of physiotherapy including SWD, only single studies were identified of any one physical approach. Based on the best available evidence there may be benefit from SWD and stretching and from HGMT in patients who have already had physiotherapy or a steroid injection. There was insufficient evidence to make conclusions with any certainty about the best mode of physical therapy for primary frozen shoulder.

\section{Acupuncture}

Study characteristics

Three RCTs investigated acupuncture in the treatment of primary frozen shoulder. A summary of study characteristics is reported in Table 34. All were full papers of studies conducted in Hong Kong, ${ }^{79} \mathrm{China}^{80}$ and Taiwan ${ }^{81}$ between 2006 and 2008. There was one two-armed trial ${ }^{80}$ and two three-armed trials. ${ }^{79,81}$ Sample sizes ranged from 74 to 360. 
TABLE 34 Studies of acupuncture with or without physical therapy

\begin{tabular}{|c|c|c|c|}
\hline $\begin{array}{l}\text { Study details } \\
\text { and study } \\
\text { design }\end{array}$ & $\begin{array}{l}\text { Participants } \\
\text { randomised (total } n \\
\text { and by group) }\end{array}$ & Trial treatments & $\begin{array}{l}\text { Condition-related } \\
\text { inclusion criteria }\end{array}$ \\
\hline $\begin{array}{l}\text { Cheing } 2008^{79} \\
\text { Three-arm RCT }\end{array}$ & $\begin{array}{l}n=74 \\
\text { Electroacupuncture: } 25 \\
\text { Interferential } \\
\text { electrotherapy: } 24 \\
\text { Control: } 25\end{array}$ & $\begin{array}{l}\text { Electroacupuncture: } 10 \text { sessions, two to three times per week over } \\
\text { a 4-week period. Three points needled (one trigger point, LI15 and } \\
\text { ST38) with a needle of } 0.30 \times 40 \mathrm{~mm} \text { diameter } \\
\text { Interferential electrotherapy: } 10 \times 20 \text {-minute sessions over } 4 \text { weeks } \\
\text { at } 80-120 \mathrm{~Hz} \text {. Four suction-type electrodes were placed around the } \\
\text { shoulder region in a coplanar arrangement } \\
\text { Control: no treatment for } 4 \text { weeks }\end{array}$ & $\begin{array}{l}\text { Localised pain over one } \\
\text { shoulder, night pain and } \\
\text { restricted active and } \\
\text { passive shoulder motion }\end{array}$ \\
\hline $\begin{array}{l}\text { Fang } 2006^{80} \\
\text { Two-arm RCT }\end{array}$ & $\begin{array}{l}n=360 \\
\text { Electroacupuncture: } 174 \\
\text { TENS: } 186\end{array}$ & $\begin{array}{l}\text { Electroacupuncture: } 10 \times 40 \text {-minute sessions every other day. Four } \\
\text { acupuncture points [Waiguan (SJ5) and Hegu (LI4) and, alternating } \\
\text { each session, Jianliao (SJ14) and Jian qian (Ex-UE) or Jianyu (LI15) } \\
\text { and Naoshu (SI10)]; } 0.30 \times 40 \mathrm{~mm} \text { needles inserted to depth of } \\
20-25 \mathrm{~mm} \\
\text { TENS: } 10 \times 40 \text {-minute sessions every other day. Electrodes placed } \\
\text { at four acupuncture points [Waiguan (SJ5) and Hegu (LI4) and, } \\
\text { alternating each session, Jianliao (SJ14) and Jian qian (Ex-UE) } \\
\text { or Jianyu (LI15) and Naoshu (SI10)]. High frequency (100 Hz) for } \\
10 \text { minutes and low frequency ( } 2 \mathrm{~Hz}) \text { for } 30 \text { minutes. Intensity of } \\
\text { current } 10 \pm 2 \mathrm{~mA}\end{array}$ & $\begin{array}{l}\text { Used 'recognised criteria' } \\
\text { (no further details provided }\end{array}$ \\
\hline $\begin{array}{l}\text { Ma } 2006{ }^{81} \\
\text { Three-arm RCT }\end{array}$ & $\begin{array}{l}n=75 \\
\text { Acupuncture: } 30 \\
\text { Acupuncture +PT: } 15 \\
\text { PT: } 30\end{array}$ & $\begin{array}{l}\text { Acupuncture: 15-minute session twice a week for } 4 \text { weeks. Each } \\
\text { session consisted of therapeutic principles in promoting flow of qi } \\
\text { and blood, driving out the wind and cold, removing dampness and } \\
\text { activating meridians; therapeutic methods on three yang meridians } \\
\text { of the hand; and prescriptions with jianijiao, jianyu (LI15), fengchi } \\
\text { (GB20), hegu (LI4) and yanglingquan (GB34) } \\
\text { PT. five sessions }\end{array}$ & $\begin{array}{l}\text { Shoulder pain for at least } \\
3 \text { months, could not lift } \\
\text { arms more than } 135^{\circ}\end{array}$ \\
\hline
\end{tabular}

PT, physiotherapy.

One study used acupuncture ${ }^{81}$ and two studies used electroacupuncture, ${ }^{79,80}$ The number of acupuncture points needled ranged from three to five. Although needle point prescriptions varied between studies, two studies both included Hegu (LI4) and Jianyu (LI15) needle points. ${ }^{80,81}$ In the acupuncture study, acupuncture was administered in a course of eight sessions. In both studies of electroacupuncture, the intervention was administered in a course of 10 sessions, with acupuncture points needled to a depth of $15-25 \mathrm{~mm}$ and electricity administered when 'De qi' sensation was felt. Further details of the electrical stimulation used were reported in one study only $^{79}$ and consisted of 2-100-Hz electrical stimulation at a pulse of 100-400 microseconds for 20 minutes. The comparators were interferential electrotherapy, ${ }^{79}$ waiting list control, ${ }^{79} \mathrm{TENS},{ }^{80}$ physiotherapy $^{81}$ and acupuncture with physiotherapy. ${ }^{81}$

One study included a physiotherapy regimen. ${ }^{81}$ This consisted of a heat pack for 15 minutes, joint mobilisation for 5-10 minutes and active shoulder exercises for 5-10 minutes, five times per week for 4 weeks. One study reported concomitant treatment of a home exercise programme of mobilisation exercises five times a day over 6 months. ${ }^{79}$ Two studies did not report whether participants received any concomitant treatment. ${ }^{80,81}$

The inclusion criteria varied between the three studies. It was unclear in all studies whether they included participants with diabetes. Frozen shoulder was diagnosed by an orthopaedic surgeon, through clinical examination and through clinical history. The participants' stage of frozen shoulder was not reported in two studies. ${ }^{79,81}$ In the other study participants were reported 
to have either stage 1 (described by the authors as pre-adhesive) frozen shoulder, that is, they had shoulder pain that increased at night with range of motion normal or only slightly affected, or stage 2 (described by the authors as adhesive) frozen shoulder, that is, a reduction in pain but severely affected range of motion. ${ }^{80}$ None of the studies reported whether participants had received previous treatment for frozen shoulder. Where reported, the mean age of participants ranged from 51.5 to 56.4 years and the proportion of women from $52 \%$ to $55 \%$.

\section{Quality assessment}

None of the studies reported the method of randomisation or allocation concealment; therefore, these three studies are potentially at high risk of bias. The study by Cheing et al. ${ }^{79}$ was of the highest quality and fulfilled five criteria: the number randomised, the comparability of treatment groups at baseline, double blinded, blinding of outcome assessors to treatment allocation and no unexpected imbalances in dropouts between groups. This study, however, did not use intention-to-treat analysis. Fang et al. ${ }^{80}$ fulfilled two criteria: the number of participants randomised and the blinding of participants. Ma et al..$^{81}$ also fulfilled two criteria: the number of participants randomised and the comparability of treatment groups at baseline for important prognostic factors.

The studies by Fang et al. ${ }^{80}$ and Ma et al. ${ }^{81}$ reported outcome measures at $\leq 4$ weeks from baseline and are therefore excluded from the subsequent synthesis. Furthermore, the waiting list control arm for the study by Cheing et al..$^{79}$ reported outcomes at 4 weeks from baseline only. The following synthesis is therefore based on the study by Cheing et al. ${ }^{79}$ and compares electroacupuncture with interferential therapy only. This study reported on the outcomes of pain and of function and disability only.

Pain

Cheing et al. ${ }^{79}$ reported results of 'pain at the moment' at approximately 2, 4 and 7 months using a $0-10 \mathrm{~cm}$ VAS. There was no significant difference in pain between the electroacupuncture and interferential therapy groups at 2 months (SMD 0.35, 95\% CI -0.23 to 0.93), 4 months (SMD $0.21,95 \% \mathrm{CI}-0.37$ to 0.78 ) or 7 months (SMD $0.21,95 \% \mathrm{CI}-0.37$ to 0.78 ).

\section{Function and disability}

Cheing et al. ${ }^{79}$ reported results of the Constant score at 2, 4 and 7 months. There was no significant difference in Constant score between the electroacupuncture and interferential therapy groups at 2 months (MD $-2.80,95 \% \mathrm{CI}-5.88$ to 0.28 ), 4 months (MD 3.10, 95\% CI -1.53 to 7.73 ) or 7 months (MD $-1.70,95 \% \mathrm{CI}-4.76$ to 1.36 ).

\section{Adverse events}

None of the three studies that evaluated acupuncture or electroacupuncture reported on whether or not participants experienced any adverse events.

\section{Summary}

Three studies compared acupuncture with another treatment, although only one provided data beyond 4 weeks' follow-up. This study had a potentially high risk of bias and it was unclear whether it had enough participants to detect a difference between groups. Based on a single study, there was no statistically significant difference between electroacupuncture and inferential electrotherapy in pain or function and disability at short-, medium- or long-term follow-up. There was insufficient evidence to make conclusions with any certainty about the effectiveness of acupuncture for primary frozen shoulder and in what situations it is likely to be effective. 


\section{Manipulation under anaesthesia}

\section{Study characteristics}

Four RCTs investigated MUA in the treatment of primary frozen shoulder. A summary of study characteristics is available in Table 35 and full details are provided in Appendix 6. All were full papers of studies conducted in Pakistan, ${ }^{82}$ the $\mathrm{UK}^{38,83}$ and Finland ${ }^{39}$ between 2007 and 2009. All were two-arm trials and sample sizes ranged from $36^{38}$ to $125 .{ }^{39}$

The MUA procedure was described in three of the $\mathrm{RCTs}^{38,39,83}$ and was performed by a physician ${ }^{39}$ or, in the two UK studies, by an orthopaedic surgeon. ${ }^{38,83}$ In two studies participants received a steroid injection (triamcinolone) in conjunction with MUA, $30 \mathrm{mg}^{38}$ in one of the UK studies and $80 \mathrm{mg}^{82}$ in the study from Pakistan.

A single study, by Amir-us-Saqlain et al. ${ }^{82}$ reported use of a physiotherapy programme following MUA. The other three studies advised participants in the intervention and control groups to undertake home exercises. Kivimaki et al..$^{39}$ appeared to have the most intensive home exercise programme, although details were scanty in all the studies; participants in this study received physiotherapy advice in two sessions and written instructions for a daily training programme including pendulum and stretching exercises. Quraishi et al. ${ }^{38}$ reported a home exercise programme of pendulum exercises and wall-climbing movements; specific details were not provided by Jacobs et al. ${ }^{83}$

TABLE 35 Studies of MUA

\begin{tabular}{|c|c|c|c|}
\hline $\begin{array}{l}\text { Study details } \\
\text { and study } \\
\text { design }\end{array}$ & $\begin{array}{l}\text { Participants randomised } \\
\text { (total } n \text { and by group) }\end{array}$ & Trial treatments & Condition-related inclusion criteria \\
\hline $\begin{array}{l}\text { Amir-us- } \\
\text { Saqlain } \\
2007^{82} \\
\text { Two-arm RCT }\end{array}$ & $\begin{array}{l}n=43 \\
\text { MUA + steroid } \\
\text { injection + manipulated } \\
\text { extremity + PT: } 23 \\
\text { MUA + steroid injection + PT: } \\
20\end{array}$ & $\begin{array}{l}\text { MUA: no further details } \\
\text { Steroid: } 80 \mathrm{mg} \text { Kenacort (triamcinolone) with } \\
2 \% \text { xylocaine } \\
\text { Manipulated extremity: kept in } 160^{\circ} \text { of } \\
\text { abduction with } 90^{\circ} \text { of external rotation for } \\
24 \text { hours } \\
\text { PT: daily } 30 \text {-minute sessions for } 3 \text { weeks }\end{array}$ & $\begin{array}{l}\text { History of pain and stiffness of the shoulder } \\
\text { joint with no identifiable cause, restriction of } \\
\text { glenohumeral motion }<50 \% \text { of abduction and } \\
<50 \% \text { of external rotation compared with } \\
\text { the contralateral shoulder joint and normal } \\
\text { radiography. All symptoms had to be of } \\
3 \text { weeks' duration }\end{array}$ \\
\hline $\begin{array}{l}\text { Jacobs } \\
2009^{83} \\
\text { Two-arm RCT }\end{array}$ & $\begin{array}{l}n=53 \\
\text { MUA: } 28 \\
\text { Steroid + distension: } 25\end{array}$ & $\begin{array}{l}\text { MUA: manipulation of arm into full adduction } \\
\text { and forward flexion, full external rotation, full } \\
\text { internal rotation and full abduction } \\
\text { Steroid + distension: three steroid and } \\
\text { distension treatments at 6-week intervals: } \\
\text { triamcinolone, } 40 \mathrm{mg}, 1 \mathrm{ml} ; 2 \% \text { lidocaine, } 5 \mathrm{ml} \text {; } \\
0.25 \% \text { bupivacaine, } 10 \mathrm{ml} \text {; air, } 5 \mathrm{ml}\end{array}$ & Patients with primary frozen shoulder \\
\hline $\begin{array}{l}\text { Kivimaki } \\
2007^{39} \\
\text { Two-arm RCT }\end{array}$ & $\begin{array}{l}n=125 \\
\text { MUA: } 65 \\
\text { Home exercise: } 60\end{array}$ & $\begin{array}{l}\text { MUA: manipulation of arm into flexion and } \\
\text { abduction and rotation into internal and external } \\
\text { rotation } \\
\text { Home exercise: PT advice in two sessions } \\
\text { and written instructions for a daily training } \\
\text { programme }\end{array}$ & $\begin{array}{l}\text { Gradually increasing shoulder pain and } \\
\text { stiffness, shoulder mobility of } \leq 140^{\circ} \text { in } \\
\text { elevation and } 30^{\circ} \text { in external rotation }\end{array}$ \\
\hline $\begin{array}{l}\text { Quraishi } \\
2007^{38} \\
\text { Two -arm RCT }\end{array}$ & $\begin{array}{l}n=36 \\
\text { MUA+steroid: } 17(18 \\
\text { shoulders) } \\
\text { Arthrographic distension: } \\
19(20)\end{array}$ & $\begin{array}{l}\text { MUA: a specific protocol was followed to ensure } \\
\text { safe breakage of adhesions by using a short } \\
\text { lever arm } \\
\text { Steroid: triamcinolone acetonide, } 30 \mathrm{mg} \text { and } \\
2 \% \text { lidocaine, } 2 \mathrm{ml} \\
\text { Distension: normal saline (10-55 ml) injected to } \\
\text { progressively distend capsule rupture }\end{array}$ & $\begin{array}{l}\text { Stage } 2 \text { adhesive capsulitis, global loss of } \\
\text { active and passive shoulder movement, } \\
\text { restriction of rotation }<50 \% \text { of normal, and } \\
\text { normal anteroposterior and axillary lateral } \\
\text { radiography of glenohumeral joint }\end{array}$ \\
\hline
\end{tabular}


Each of the studies had a different comparator. In the study by Amir-us-Saqlain $e t$ al. ${ }^{82}$ from Pakistan, the intervention being assessed was the effect of keeping the manipulated extremity in abduction and external rotation for 24 hours following MUA. Both groups received MUA, including a steroid injection, followed by physical therapy and the experimental group also had the extremity kept in a fixed position following MUA. In the two UK studies, Jacobs et al. ${ }^{83}$ compared MUA with distension in combination with steroid injection; and Quraishi et al..$^{38}$ compared MUA, including a steroid injection, with arthrographic distension. Kivimaki et al. ${ }^{39}$ compared MUA (plus home exercise) with home exercise alone, which involved physiotherapy advice in two sessions and written instructions for home exercise.

The inclusion criteria varied between studies in terms of extent of restriction of movement. ${ }^{38,39,82}$ Jacobs et al. ${ }^{83}$ did not specify the symptomatic inclusion criteria other than that patients with primary frozen shoulder were included. Quraishi et al. ${ }^{38}$ included only patients with stage 2 frozen shoulder. The stage of frozen shoulder was described as painful and stiff or in the 'freezing' phase in two RCTs, ${ }^{39,83}$ and the majority of participants reported pain and stiffness in one RCT (the remainder had either pain or stiffness). ${ }^{82}$ At baseline the duration of frozen shoulder ranged from a mean or median of 10 weeks to 40 weeks. Two studies reported on whether participants had received previous treatment for frozen shoulder. Quraishi et al. ${ }^{38}$ reported that $44 \%$ had previously received physical therapy, $61 \%$ a steroid injection and $31 \%$ physical therapy combined with steroid injections. Kivimaki et al. ${ }^{39}$ alluded to participants having received previous treatment by reporting that in the 3 months prior to randomisation there was no difference between treatment groups in terms of physical therapy, massage or chiropractic manipulations received. The mean or median age of the participants ranged from 53 to 57 years and the proportion of women ranged from $58 \%$ to $68 \%$. It was unclear whether participants with diabetes were included in one study, ${ }^{82}$ one study reported that there were no participants with diabetes ${ }^{83}$ and in the remaining two studies the proportions with diabetes were $14 \%{ }^{39}$ and $17 \% .^{38}$

\section{Quality assessment}

The included RCTs generally varied in quality. All of the studies reported the number of participants randomised and comparability at baseline, although one reported only comparability for range of movement. ${ }^{82}$ The Amir-us-Saqlain et al. ${ }^{82}$ and Jacobs et al. ${ }^{83}$ studies did not report the method of randomisation and so it was unclear whether these studies were truly randomised, and they did not report allocation concealment or whether the outcome assessors were blinded, although this would not have been possible in the study by Amir-us-Saqlain et al ${ }^{82}$ These studies therefore had a potentially high risk of bias. Additionally, Jacobs et al. ${ }^{83}$ was not adequately powered, loss to follow-up was high (19\%) and it was unclear whether imbalances in dropouts were adjusted for. Although the authors stated that intention-to-treat analysis was performed, not all patients were included in the analysis. It was unclear whether the study by Amir-us-Saqlain $e t$ $a l .{ }^{82}$ was adequately powered and loss to follow-up was high (23\%).

Both Kivimaki et al..$^{39}$ and Quraishi et al.$^{38}$ reported appropriate randomisation methods; however, only Kivimaki et al.$^{39}$ reported allocation concealment and blinding of outcome assessors and was therefore classified as of adequate quality. Loss to follow-up was high in this study (34\% at 6 months) and was greater in the MUA group than in the control group; the analysis did not appear to be intention to treat. The study did not appear to be adequately powered at the 6- and 12-month follow-ups. Although Quraishi et al. ${ }^{38}$ reported that the investigator was blinded, it was not clear whether outcome assessment was blinded or whether there was allocation concealment and therefore this study may be at risk of bias. Loss to follow-up was relatively low in this study $(8 \%)$ although it was unclear whether the study was adequately powered. Full details of study quality are reported in Appendix 8. 


\section{Manipulation under anaesthesia versus home exercise}

Kivimaki et al. ${ }^{39}$ compared MUA plus home exercise with home exercise alone. MDs and 95\% CIs were reported by the authors for pain, function and disability, passive abduction, passive internal rotation, external rotation and working ability at the follow-up points of 6 weeks and 3, 6 and 12 months from baseline (Table 36). The outcome data for the individual groups are available in Appendix 7.

Pain

There was no significant difference in pain intensity between MUA and home exercise at 6 weeks or 3,6 or 12 months (Table 37 ).

\section{Function and disability}

There was no significant difference in Constant score between treatment groups at 6 weeks or 3, 6 or 12 months (Table 37).

\section{Range of movement}

There was no significant difference in passive abduction, passive internal rotation or external rotation between treatment groups at 6 weeks or 3, 6 or 12 months (Table 37).

\section{Working ability}

There was no significant difference in the change in working ability between treatment groups at 6 weeks or 3,6 or 12 months (Table 37).

\section{Adverse events}

The authors reported that there were no major complications during manipulation.

\section{Manipulation under anaesthesia versus distension}

Two studies compared MUA with distension; ${ }^{38,83}$ however, both MUA and distension differed between studies and so the data for pain and function and disability that were available could not be considered for pooling.

One RCT by Jacobs et al. ${ }^{83}$ compared MUA with steroid injection in combination with distension treatment. Only limited data were available for pain, function and disability and quality of life, which were presented as mean regression coefficients up to 16 weeks (and 24 months for quality of life) (Table 38). The other RCT by Quraishi et al..$^{38}$ compared MUA plus steroid injection with arthrographic distension. This study reported means and ranges for pain and function and disability at 2 and 6 months. The percentage of patients who were satisfied with treatment was also reported (Table 38). The outcome data for the individual groups are available in Appendix 7.

TABLE 36 Outcomes assessed in the study of MUA versus home exercise ${ }^{39}$

\begin{tabular}{|c|c|}
\hline Outcomes assessed & Data reported and time of follow-up \\
\hline Pain intensity: Likert scale $(0-10)^{\mathrm{a}}$ & MD at 6 weeks and 3,6 and 12 months (converted to SMD) \\
\hline $\begin{array}{l}\text { Function and disability: modified version of SDQ (14 activities of daily } \\
\text { living in previous } 24 \text { hours, scored out of } 28)^{\mathrm{a}}\end{array}$ & MD at 6 weeks and 3,6 and 12 months \\
\hline $\begin{array}{l}\text { ROM: passive abduction }\left({ }^{\circ} \text { ), passive internal rotation }(\mathrm{cm}) \text {, external }\right. \\
\text { rotation }\left({ }^{\circ}\right) \text { (and flexion, not included in analysis) }\end{array}$ & MD at 6 weeks and 3,6 and 12 months \\
\hline Working ability (0-10) & MD at 6 weeks and 3,6 and 12 months \\
\hline Adverse events & Narrative summary \\
\hline
\end{tabular}

ROM, range of movement.

a Higher score indicates worse outcome.

b SMD imputed from $95 \%$ Cls. 
TABLE 37 Results of the study of MUA versus home exercise (MD and $95 \% \mathrm{Cl})^{39}$

\begin{tabular}{|c|c|c|c|}
\hline & Short term ( $\leq 3$ months) & $\begin{array}{l}\text { Medium term (>3 and } \\
\leq 6 \text { months) }\end{array}$ & Long term ( $>6$ and $\leq 12$ months) \\
\hline \multicolumn{4}{|c|}{ Pain intensity (SMD and 95\% CI) } \\
\hline & $0.2(-0.64 \text { to } 1.02)^{\mathrm{a}}$ & $-0.35(-0.78 \text { to } 0.09)^{c}$ & $-0.28(-0.73 \text { to } 0.16)^{d}$ \\
\hline & $0.07(-0.32 \text { to } 0.46)^{b}$ & & \\
\hline \multicolumn{4}{|l|}{ Function and disability } \\
\hline & $4(-3.8 \text { to } 11.8)^{\mathrm{a}}$ & $-1.7(-5.3 \text { to } 1.9)^{c}$ & $0(-3.2 \text { to } 3.2)^{d}$ \\
\hline & $0.3(-2.69 \text { to } 2.75)^{b}$ & & \\
\hline \multicolumn{4}{|l|}{ ROM } \\
\hline \multirow[t]{2}{*}{ Passive abduction $\left({ }^{\circ}\right)$} & $10(-3.2 \text { to } 23.2)^{\mathrm{a}}$ & $9(-4 \text { to } 22)^{c}$ & $7(-5 \text { to } 19)^{d}$ \\
\hline & $9(-6 \text { to } 24)^{b}$ & & \\
\hline \multirow[t]{2}{*}{ Passive internal rotation (cm) } & $4(-9 \text { to } 9)^{a}$ & $-2(-7.4 \text { to } 3.4)^{c}$ & $-1(-4.1 \text { to } 6.1)^{\mathrm{d}}$ \\
\hline & $-3(-7.4 \text { to } 2.4)^{b}$ & & \\
\hline \multirow[t]{2}{*}{ External rotation $\left({ }^{\circ}\right)$} & $5(-2 \text { to } 12)^{\mathrm{a}}$ & $6(-2 \text { to } 14)^{c}$ & $4(-4.1 \text { to } 12.2)^{d}$ \\
\hline & $6(-3 \text { to } 15)^{b}$ & & \\
\hline \multicolumn{4}{|l|}{ Working ability } \\
\hline & $0.4(-4.2 \text { to } 1.8)^{\mathrm{a}}$ & $0.5(-0.6 \text { to } 1.6)^{c}$ & $0.1(-0.8 \text { to } 1.0)^{\mathrm{d}}$ \\
\hline & $0(-0.8 \text { to } 0.8)^{b}$ & & \\
\hline
\end{tabular}

ROM, range of movement.

a Reported at 6 weeks.

b Reported at 3 months.

c Reported at 6 months.

d Reported at 12 months.

\section{Pain}

The study by Jacobs et al. ${ }^{83}$ showed no significant difference in pain intensity between treatment groups up to 16 weeks. The mean regression coefficient (SE) was -2.77 (0.33) for MUA and -2.75 (0.42) for distension combined with steroid injection (95\% CI -1.11 to 1.15$)$.

In the study by Quraishi et al. ${ }^{38}$ at 2 months' follow-up the mean VAS score was 4.7 (range 0 to 8.5) in the MUA plus steroid arm and 2.4 (range 0 to 8 ) in the arthrographic distension arm ( $p$-value not reported). At 6 months there was a significantly greater improvement in VAS in the arthrographic distension group than in the MUA group (SMD 1.52, 95\% CI 0.75 to 2.30 ) (Table 39).

\section{Function and disability}

The study by Jacobs et al..$^{83}$ found no significant difference between treatment groups in the Constant score up to 16 weeks: mean regression coefficient (SE) 3.13 (0.24) for MUA and 3.23 (0.42) for distension plus steroid injection ( $95 \% \mathrm{CI}-1.09$ to 0.81 ).

In the study by Quraishi et al..$^{38}$ at 2 months' follow-up the mean Constant score was 58.5 (range 24 to 90 ) in the MUA plus steroid arm and 57.4 (range 17 to 80 ) in the arthrographic distension arm. At 6 months the Constant score was significantly improved in the arthrographic distension group compared with the MUA group (-6.40, 95\% CI -6.56 to -6.24) (Table 39). 
TABLE 38 Outcomes assessed in the studies of MUA vs distension

\begin{tabular}{|c|c|c|}
\hline Study & Outcomes assessed & Data reported and time of follow-up \\
\hline Jacobs $2009^{83}$ & Pain (not specified): VAS (1-100) ${ }^{a}$ & Mean regression coefficient (mean of 2, 6, 12 and 16 weeks) \\
\hline Quraishi $2007^{38}$ & Pain outcome overall: VAS $(0-10)^{a}$ & Mean and range at 2 and 6 months (no SDs reported) $)^{b}$ \\
\hline Jacobs $2009^{83}$ & Function and disability: Constant score & Mean regression coefficient (mean of 2, 6, 12 and 16 weeks) \\
\hline Quraishi $2007^{38}$ & Function and disability: Constant score & Mean and range at 2 and 6 months (no SDs reported) $)^{b}$ \\
\hline Quraishi $2007^{38}$ & $\begin{array}{l}\text { ROM: external rotation, internal rotation, abduction (and } \\
\text { forward elevation, not included in analysis) }\left(^{\circ}\right)\end{array}$ & $\begin{array}{l}\text { Between-group } p \text {-values at } 6 \text { months (mean values available } \\
\text { only on graph) }\end{array}$ \\
\hline Jacobs $2009^{83}$ & Quality of life: SF-36, all components & $\begin{array}{l}\text { Scores at } 24 \text { months presented in graphs and summarised } \\
\text { narratively in text }\end{array}$ \\
\hline Quraishi $2007^{38}$ & Satisfaction & $\%$ satisfied at 6 months \\
\hline
\end{tabular}

ROM, range of movement.

a Higher score indicates worse outcome.

b A between-group p-value was reported for 6 months' follow-up and this was used to estimate the SD at 6 months; there was no $p$-value for 2 months' follow-up.

TABLE 39 Results of the studies of MUA vs arthrographic distension (SMD and 95\% Cl)

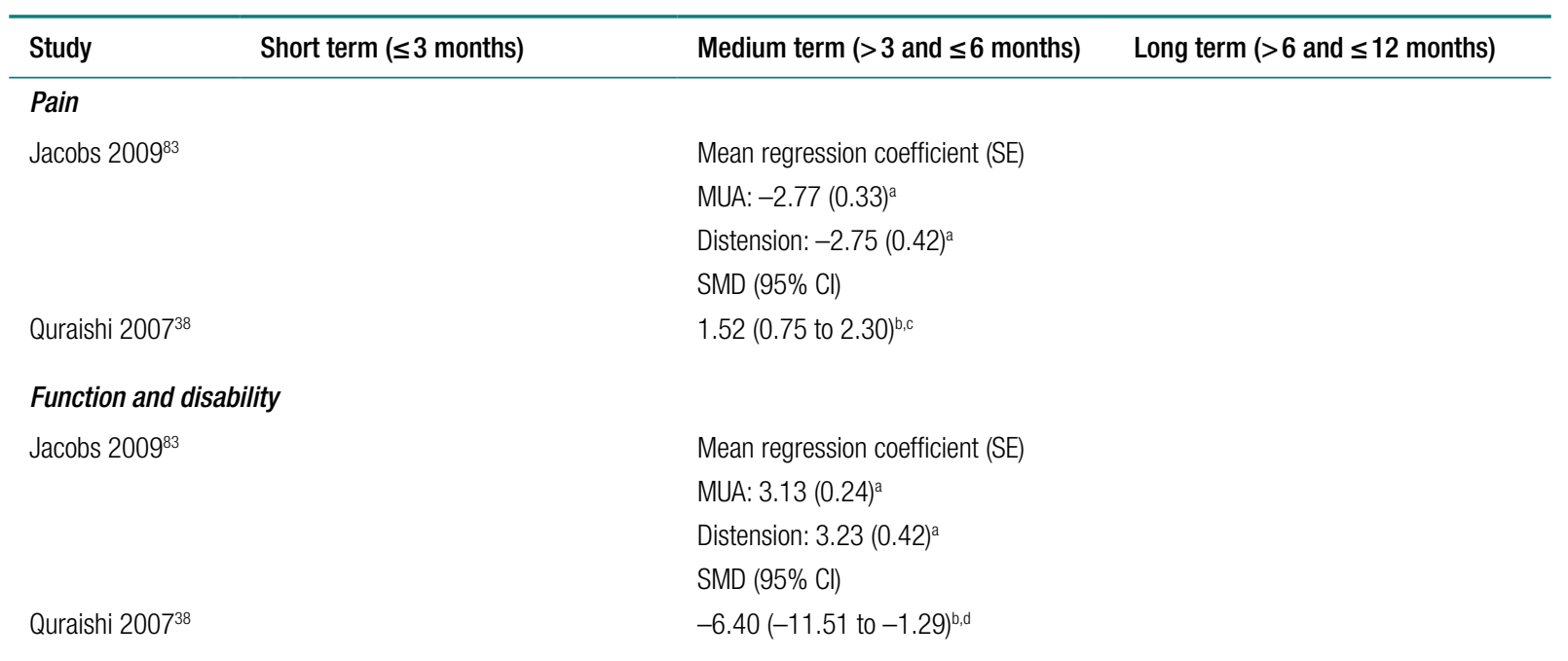

a Reported at 16 weeks.

b Reported at 6 months.

c Statistically significant, i.e. the $\mathrm{Cl}$ did not cross the line of no effect (zero).

\section{Range of movement}

Quraishi et al ${ }^{38}$ reported no statistically significant difference between groups in external rotation $(p=0.13)$, internal rotation $(p=0.48)$ and abduction at 6 months' follow-up $(p=0.62)$ (data available only in a graph).

\section{Quality of life}

Jacobs et al. ${ }^{83}$ reported that all components of the SF-36 improved for all patients during the course of treatment, with the greatest improvements shown in the physical role and bodily pain components. There was no statistically significant difference with respect to change in SF-36 scores between the treatment groups (data available only in graph format, no $p$-values reported). 


\section{Satisfaction}

Quraishi et al. ${ }^{38}$ reported that at final follow-up $81 \%$ of MUA patients were satisfied or very satisfied compared with $94 \%$ of patients who received arthrographic distension. All of the patients with diabetes were satisfied with their outcome (three in both the MUA and arthrographic distension groups).

\section{Adverse events}

The study by Jacobs et al ${ }^{83}$ reported that no systemic or local complications were noted in either treatment group. Quraishi et al. ${ }^{38}$ did not report any information on adverse events.

\section{Manipulation under anaesthesia followed by manipulated extremity kept in fixed position}

Amir-us-Saqlain et al. ${ }^{82}$ assessed the effect of keeping the manipulated extremity in abduction and external rotation for 24 hours following MUA compared with MUA not using the fixed position. Both groups also received a steroid injection and physical therapy. Only measures of range of movement were assessed 12 weeks post treatment (Table 40). Between-group differences in means and 95\% CIs are presented in Table 41. Outcome data for the individual groups are presented in Appendix 7.

\section{Range of movement}

Passive abduction (MD $22.49^{\circ}, 95 \%$ CI $13.14^{\circ}$ to $31.84^{\circ}$ ), passive internal rotation (MD $1.00 \mathrm{~cm}$, $95 \%$ CI $0.71 \mathrm{~cm}$ to $1.29 \mathrm{~cm}$ ), active abduction (MD $28.99^{\circ}, 95 \%$ CI $17.07^{\circ}$ to $40.91^{\circ}$ ) and active internal rotation (MD $0.91 \mathrm{~cm}, 95 \%$ CI $0.49 \mathrm{~cm}$ to $1.33 \mathrm{~cm}$ ) were significantly greater at 12 weeks post treatment in the MUA combined with steroid injection plus manipulated extremity and physiotherapy group than in the MUA combined with steroid injection plus physiotherapy group. There was no significant difference in passive or active external rotation between treatment groups.

TABLE 40 Outcomes assessed in the study of MUA followed by fixed extremity ${ }^{82}$

\begin{tabular}{ll}
\hline Outcomes assessed & Data reported and time of follow-up \\
\hline ROM: passive abduction, passive internal rotation, passive external rotation, active abduction, & ROM 12 weeks post treatment \\
active external rotation, active internal rotation (and active and passive forward flexion, not \\
included in analysis)
\end{tabular}

ROM, range of movement.

a All measured in degrees except internal rotation, which was measured by spinal level.

TABLE 41 Results of the study of MUA followed by fixed extremity (MD and $95 \% \mathrm{CI})^{82}$

\begin{tabular}{llll}
\hline & Short term ( $\leq 3$ months $)$ & $\begin{array}{l}\text { Medium term (>3 and } \\
\leq 6 \text { months })\end{array}$ & $\begin{array}{l}\text { Long term }(>6 \text { and } \\
\leq 12 \text { months })\end{array}$ \\
\hline ROM & & \\
Passive abduction $\left({ }^{\circ}\right)$ & $22.49(13.14 \text { to } 31.84)^{\mathrm{a}}$ & \\
Passive internal rotation $(\mathrm{cm})^{\mathrm{b}}$ & $1.00(0.71 \text { to } 1.29)^{\mathrm{a}}$ & \\
Passive external rotation $\left(^{\circ}\right)$ & $4.84(-2.26$ to 11.94$)$ & \\
Active abduction $\left({ }^{\circ}\right)$ & $28.99(17.07 \text { to } 40.91)^{\mathrm{a}}$ & \\
Active internal rotation $(\mathrm{cm})$ & $0.91(0.49 \text { to } 1.33)^{\mathrm{a}}$ & \\
Active external rotation $\left(^{\circ}\right)$ & $6.62(-3.32$ to 16.56$)$ & \\
\hline
\end{tabular}

ROM, range of movement.

a Statistically significant, $p<0.0001$.

b The paper states that this was measured by spinal level. There is a lack of clarity in the paper, but it appears to be measured in cm. 


\section{Summary}

The four included studies of MUA were diverse in terms of the intervention and the comparator. Some studies used a steroid injection (triamcinolone) in conjunction with MUA. The studies were not considered clinically similar enough to be pooled in a meta-analysis. Across all of the studies information about previous treatments that participants had received and stage of frozen shoulder was limited. It was not possible to explore variation between those with and without diabetes.

Because it was unclear whether all studies had enough participants to detect a statistically significant treatment effect, it should be kept in mind that where studies did not report a significant benefit (i.e. the CI crossed the line of no difference) this should not be interpreted as evidence of no difference between the groups. A single study of adequate quality reported no statistically significant difference between MUA (and home exercise) and home exercise alone in pain, function and disability, range of movement or working ability at 6 weeks and 3, 6 and 12 months. ${ }^{39}$ Follow-up at 6 months and beyond in this study should be treated with some caution because of high loss to follow-up, which was somewhat higher in the MUA group, and also the lack of intention-to-treat analysis. Two studies comparing MUA with distension had mixed findings. One, with a potentially high risk of bias, found no significant difference in pain or function and disability between MUA and distension (which included steroid) up to 16 weeks post treatment. ${ }^{83}$ The second, which had some risk of bias, found a significantly greater improvement in pain and function and disability at 6 months with arthrographic distension than with MUA in conjunction with steroid..$^{38}$ The fourth study, with a potentially high risk of bias, reported that keeping the manipulated extremity in abduction and external rotation for 24 hours following MUA was superior to MUA without this additional process for passive and active abduction and internal rotation at 12 weeks. ${ }^{82}$

In conclusion, there was very little evidence available for MUA and most of the studies identified had limitations. The single adequate study found no evidence of benefit of MUA over home exercise alone. Generalisability is somewhat unclear because of the limited information about previous interventions that participants had received and stage of frozen shoulder.

\section{Distension}

\section{Study characteristics}

Three RCTs investigated distension with steroid injection in the treatment of primary frozen shoulder. A summary of study characteristics is reported in Table 42, with further details available in Appendix 6. All were full papers of studies conducted in Australia, ${ }^{43}$ Denmark $^{84}$ and Norway ${ }^{36}$ and published between 1998 and 2008. All were two-armed trials.

The two most recent studies by Buchbinder et al. ${ }^{43}$ and Tvetia et al. ${ }^{36}$ used arthrographic distension. One injected $40 \mathrm{mg}$ of steroid (in $1 \mathrm{ml}$ ) and up to $82 \mathrm{ml}$ of saline on a single occasion ${ }^{43}$ and the other injected $20 \mathrm{mg}$ of steroid $(2 \mathrm{ml}), 4 \mathrm{ml}$ of local anaesthetic and $10 \mathrm{ml}$ of saline on three occasions at 2-week intervals, ${ }^{36}$ along with contrast medium in the arthrographic distension groups. Both of these studies stated that the procedure continued until rupture occurred.

Buchbinder et al. ${ }^{43}$ had the additional end points of patient termination of the procedure and a maximum of $90 \mathrm{ml}$ having been injected. The older study by Gam et al.$^{84}$ stated that the injection was confirmed by ultrasound and participants were injected with $20 \mathrm{mg}$ of steroid with $19 \mathrm{ml}$ of lidocaine once per week for up to 6 weeks. Details were not provided in this study as to whether distension occurred or how this was established. The two studies of arthrographic distension used different comparators: Buchbinder et al.$^{43}$ used a placebo comparator of arthrogram only, whereas Tveita et al. ${ }^{36}$ used steroid injection (with local anaesthetic). 
TABLE 42 Studies including distension with steroid injection

\begin{tabular}{|c|c|c|c|}
\hline $\begin{array}{l}\text { Study details } \\
\text { and study } \\
\text { design }\end{array}$ & $\begin{array}{l}\text { Participants } \\
\text { randomised (total } n \\
\text { and by group) }\end{array}$ & Trial treatments & Condition-related inclusion criteria \\
\hline $\begin{array}{l}\text { Buchbinder } \\
2004^{43} \\
\text { Two-arm RCT }\end{array}$ & $\begin{array}{l}n=46 \\
\text { Arthrographic } \\
\text { distension + steroid: } 25 \\
\text { Placebo: } 21\end{array}$ & $\begin{array}{l}\text { Arthrogram: the needle was positioned, connected to } \\
\text { the connector tap and tube and } 0.5-1 \mathrm{ml} \text { of contrast } \\
\text { injected and a radiographic image taken. Steroid: } \\
\text { methylprednisolone acetate, } 40 \mathrm{mg}, 1 \mathrm{ml} \text { and up to } \\
82 \mathrm{ml} \text { of normal saline was then injected (total volume } \\
30-90 \mathrm{ml} \text { ) } \\
\text { Distension: the end point of the procedure was filling } \\
\text { of the subscapular bursa, capsular rupture, injection of } \\
\text { the total volume of liquid or the participant requesting } \\
\text { termination of the procedure } \\
\text { Placebo: arthrogram only }\end{array}$ & $\begin{array}{l}\text { Pain and stiffness in predominantly one } \\
\text { shoulder for } \geq 3 \text { months, restriction of } \\
\text { passive ROM of }>30^{\circ} \text { in two or more } \\
\text { planes of movements, measured to } \\
\text { onset of pain }\end{array}$ \\
\hline $\begin{array}{l}\text { Gam } 1998^{84} \\
\text { Two-arm RCT }\end{array}$ & $\begin{array}{l}n=22 \\
\text { Distension + steroid: } 13 \\
\text { Steroid: } 9\end{array}$ & $\begin{array}{l}\text { Distension + steroid: intra-articular injection, confirmed } \\
\text { by ultrasound } \\
\text { Steroid: triamcinolone hexacetonide, } 20 \mathrm{mg} \text {, once per } \\
\text { week for a maximum of } 6 \text { weeks or until no symptoms } \\
\text { Distension: an additional } 19 \mathrm{ml} \text { of lidocaine } 0.5 \%\end{array}$ & $\begin{array}{l}\text { Frozen shoulder of more than } 6 \text { weeks' } \\
\text { duration, nocturnal accentuation } \\
\text { of pain, passive range of shoulder } \\
\text { external rotation }<50 \% \text { of opposite } \\
\text { shoulder }\end{array}$ \\
\hline $\begin{array}{l}\text { Tveita } 2008^{36} \\
\text { Two-arm RCT }\end{array}$ & $\begin{array}{l}n=76 \\
\text { Arthrographic } \\
\text { distension + steroid: } 37 \\
\text { Steroid: } 39\end{array}$ & $\begin{array}{l}\text { Arthrogram: Kaye-Schneider technique was used } \\
\text { Steroid: triamcinolone acetonide, } 20 \mathrm{mg}, 2 \mathrm{ml} \text { and } \\
3-4 \mathrm{ml} \text { of bupivacaine hydrochloride }(5 \mathrm{mg} / \mathrm{ml}) \text {. Three } \\
\text { injections at 2-week intervals } \\
\text { Distension: an additional } 10 \mathrm{ml} \text { of saline. Three } \\
\text { injections at 2-week intervals were given. The capsule } \\
\text { would usually rupture in the wall of the subscapular } \\
\text { recess or sometimes in the wall of the bicipital or } \\
\text { axillary recesses, which was recorded as a loss of } \\
\text { resistance, and contrast leakage was identified by } \\
\text { fluoroscopy. If rupture had not occurred, more contrast } \\
\text { and local anaesthetic was injected until rupture }\end{array}$ & $\begin{array}{l}\text { Limitation of passive movement in } \\
\text { the glenohumeral joint compared with } \\
\text { the unaffected side, }>30^{\circ} \text { for at least } \\
\text { two of forward flexion, abduction or } \\
\text { external rotation; pain in predominantly } \\
\text { one shoulder lasting }>3 \text { months and } \\
<2 \text { years }\end{array}$ \\
\hline
\end{tabular}

Home exercise of pendular exercises and scapular setting were given in the study by Buchbinder et al..$^{43}$ and no manual treatment (e.g. physiotherapy, massage) or other medical interventions were allowed. In contrast, in the Tvetia et al. study ${ }^{36}$ patients were allowed to continue with their current physiotherapy programme; no patients were prescribed new physiotherapy programmes during the study. The study of non-arthrographic distension did not report any information regarding home exercise. ${ }^{84}$

There was some variability in concomitant treatments. Buchbinder et al..$^{43}$ allowed paracetamol and codeine preparations but NSAIDs were not allowed, Tveita et al. ${ }^{36}$ allowed pain medication organised by the patients' primary care physicians and Gam et al. ${ }^{84}$ reported that analgesics were permitted.

The inclusion criteria varied between studies; however, the two most recent studies of arthrographic distension required similar extent of restriction in shoulder movement. ${ }^{36,43}$ The duration of frozen shoulder at baseline ranged from a median or mean of 114 days (approximately 4 months) to 7 months, although the stage at baseline was not reported by any of the studies. Diagnosis was by measuring passive range of movement to onset of pain, ${ }^{43}$ clinical examination, blood samples and radiography or ultrasound, ${ }^{84}$ and clinical history and radiography. ${ }^{36}$ 
A substantial proportion of patients (28\%) had diabetes in the Buchbinder study ${ }^{43}$ the remaining two studies had no patients with diabetes. Buchbinder et al..$^{43}$ also included a small proportion (2.2\%) with secondary frozen shoulder. Buchbinder et al. ${ }^{43}$ and Tveita et al..$^{36}$ also reported that patients had undergone treatment for frozen shoulder before the study: in the Buchbinder study $28 \%$ of patients had received corticosteroid injections whereas in the Tveita study $17 \%$ had undergone physiotherapy. The ages of patients ranged from a mean or median of 47 years to 57.3 years and the proportion of women was either $59 \%$ or $80 \%$.

\section{Quality assessment}

Buchbinder et $a l .{ }^{43}$ was considered of satisfactory quality as the risk of bias was minimised through computerised randomisation and allocation concealment by a biostatistician who kept the assignment scheme. An intention-to-treat analysis was also performed. Patients were blinded to treatment and outcome assessors were also blinded. The treatment groups were also comparable at baseline. The main limitation of this study is that it was not sufficiently powered after dropouts (9\%). The intention-to-treat analysis used in the study was adequately powered based on the authors' power calculation (the sample size to detect a difference in SPADI scores).

Both Gam et al ${ }^{84}$ and Tveita et al. ${ }^{36}$ reported a reliable method of randomisation; however, it was not clear whether an adequate method of allocation concealment had been used. Tveita $e t$ al. ${ }^{36}$ reported that patients were not informed of their actual assignment until the first injection was to be given, and no information regarding allocation concealment was reported by Gam et al ${ }^{84}$ Therefore, these studies are potentially at risk of bias. Gam et al..$^{84}$ reported that outcome assessors were blinded and that the groups were comparable at baseline. In the study by Tveita et al. ${ }^{36}$ there appeared to be a higher proportion with previous shoulder problems and who were on sick leave in the steroid injection group. Tveita et al. ${ }^{36}$ also reported using statistical adjustment to control for baseline differences for the function and disability SPADI outcome but not for range of movement. Tveita et al..$^{36}$ used intention-to-treat analysis and both studies reported the same rate of loss to follow-up (9\%), with similar numbers in each arm.

\section{Pain}

Two studies, one by Buchbinder et al. ${ }^{43}$ and one by Gam et al. ${ }^{84}$ assessed pain. Buchbinder et al. ${ }^{43}$ reported change from baseline data at 3,6 and 12 weeks using a 10-point Likert scale (Table 43). However, the study by Gam et al.$^{84}$ reported data for VAS pain scores at rest and VAS pain scores on function in graphical form only. This study was described narratively.

There was no significant difference in pain between arthrographic distension with steroid and placebo (arthrogram) at 6 weeks (SMD $-0.40,95 \%$ CI -0.99 to 0.19 ) or 12 weeks (SMD -0.15 , $95 \%$ CI -0.73 to 0.44 ) in the study by Buchbinder et al. ${ }^{43}$

In the study by Gam et al..$^{84}$ there was no significant difference between the steroid and distension groups for VAS pain score on function $(p=0.1)$ or VAS pain score at rest $(p=0.1)$ (time points unclear). The authors reported that there was a tendency toward less pain in the distension group. Additionally, the authors reported that analgesic consumption was significantly lower at week 11 in the distension group than in the steroid group $(p=0.008)$. These results were presented in graphical form only.

\section{Function and disability}

Two of the three included studies assessed function and disability. ${ }^{36,43}$ Both reported SPADI total score and one ${ }^{43}$ reported a problem elicitation technique score, a patient preference disability measure in which patients identify their own problems related to the disease that they would like to see improve as a result of treatment. Change from baseline data were available from Buchbinder et al. ${ }^{43}$ and final value data were available from Tveita et al. ${ }^{36}$ (Table 44). These 
TABLE 43 Pain outcomes in studies evaluating distension

\begin{tabular}{lll}
\hline Study & Outcomes assessed & Data reported and time of follow-up \\
\hline $\begin{array}{l}\text { Buchbinder } \\
2004^{43}\end{array}$ & Likert scale, pain overall $(0-10)^{\mathrm{a}}$ & $\begin{array}{l}\text { Change from baseline at } 3,6 \text { and } 12 \text { weeks (6 and } 12 \text { weeks included in } \\
\text { analysis) }\end{array}$ \\
Gam 1998 & $\begin{array}{l}\text { Data presented graphically at } 3,6 \text { and } 12 \text { weeks, } p \text {-values only } \\
\text { VAS pain score at rest }(0-10)^{\mathrm{a}}\end{array}$ & \\
& VAS pain score on function $(0-10)^{\mathrm{a}}$ & \\
& Analgesic consumption & \\
\hline
\end{tabular}

a Higher scores indicate worse outcomes.

TABLE 44 Function and disability outcomes in studies evaluating distension

\begin{tabular}{lll}
\hline Study & Outcomes assessed & Data reported and time of follow-up \\
\hline $\begin{array}{l}\text { Buchbinder } \\
2004^{43}\end{array}$ & SPADI total score $(0-100)^{\mathrm{a}}$ & $\begin{array}{l}\text { Change from baseline at } 3,6 \text { and } 12 \text { weeks (6 and } 12 \text { weeks included in } \\
\text { analysis) }\end{array}$ \\
Tveita 2008 & Problem elicitation technique score & Final value at 6 weeks after last injection \\
\hline
\end{tabular}

a Higher scores indicate worse outcomes.

b Data appeared to be adjusted for baseline differences in groups.

were not pooled as the comparator arms were different between studies: Buchbinder et al. ${ }^{43}$ compared arthrographic distension with placebo whereas Tveita et al. ${ }^{36}$ used steroid injection as a comparator. Table 45 provides the between-group MDs and 95\% CIs. The outcome data for individual groups are available in Appendix 7.

\section{Distension versus placebo}

There was no significant difference between arthrographic distension and placebo in SPADI total score at 6 or 12 weeks in the study by Buchbinder et al..$^{43}$ (Table 45). In contrast, there was a significant improvement in the problem elicitation technique score with distension compared with placebo at 6 weeks (MD -46.00, 95\% CI -80.99 to -11.01) and 12 weeks (MD 54.40, 95\% CI 12.57 to 96.23$)$ in the same study.

\section{Distension versus steroid}

There was no significant difference in SPADI total scores between distension and steroid at 6 weeks in the Tveita et al. study ${ }^{36}$ (Table 45).

\section{Range of movement}

The range of movements of interest in this review that were reported by the studies of distension were passive and active abduction, passive and unspecified external rotation, passive internal rotation and hand behind back. Change from baseline data were available from Buchbinder et al..$^{43}$ and final value data were available from Tveita et al. ${ }^{36}$ (Table 46). It was considered inappropriate to pool the two studies as one had a placebo comparator and one a steroid injection comparator. The study by Gam et al. ${ }^{84}$ provided limited data for extraction and these were summarised narratively. Table 47 summarises the between-group MDs and 95\% CIs. The outcome data for individual groups are available in Appendix 7.

\section{Distension versus placebo}

There was no significant difference between groups in the outcomes active abduction, external rotation or hand behind back at 6 or 12 weeks' follow-up in the study by Buchbinder et al..$^{43}$ (Table 47). 
TABLE 45 Function and disability results of studies of distension (MD and $95 \% \mathrm{Cl}$ )

\begin{tabular}{|c|c|c|c|}
\hline Study & Short term ( $\leq 3$ months) & Medium term ( $>3$ and $\leq 6$ months) & Long term ( $>6$ and $\leq 12$ months) \\
\hline \multicolumn{4}{|c|}{ Distension vs placebo } \\
\hline \multicolumn{4}{|l|}{ SPADI total score } \\
\hline \multirow[t]{2}{*}{ Buchbinder $2004^{43}$} & $5.00(-7.85 \text { to } 17.85)^{\mathrm{a}}$ & & \\
\hline & $4.40(-10.08 \text { to } 18.88)^{b}$ & & \\
\hline \multicolumn{4}{|c|}{ Problem elicitation technique score } \\
\hline \multirow[t]{2}{*}{ Buchbinder, $2004^{43}$} & $46.00(-80.99 \text { to }-11.01)^{\mathrm{a}, \mathrm{c}}$ & & \\
\hline & $54.40(12.57 \text { to } 96.23)^{\mathrm{b}, \mathrm{c}}$ & & \\
\hline \multicolumn{4}{|c|}{ Distension vs steroid } \\
\hline \multicolumn{4}{|l|}{ SPADI total score } \\
\hline Tveita $2008^{36}$ & $-6.00(-14.12 \text { to } 2.12)^{d}$ & & \\
\hline \multicolumn{4}{|c|}{$\begin{array}{l}\text { a Reported at } 6 \text { weeks. } \\
\text { b Reported at } 12 \text { weeks. } \\
\text { c Statistically significant, i.e. the } \mathrm{Cl} \text { did not cross the line of no effect (zero). } \\
\text { d Reported at } 6 \text { weeks after last injection ( } 12 \text { weeks from baseline). }\end{array}$} \\
\hline Study & Outcomes assessed & Data reported and time of follo & I-up \\
\hline Buchbinder $2004^{43}$ & $\begin{array}{l}\text { Active abduction, external rotation in neutral, } \\
\text { hand behind back }\left({ }^{\circ}\right)\end{array}$ & Change from baseline at 3, 6 and & 12 weeks \\
\hline Gam $1998^{84}$ & Passive abduction, passive external rotation $\left({ }^{\circ}\right)$ & $\begin{array}{l}\text { Data presented graphically (numb } \\
\text { at } 3,6 \text { and } 12 \text { weeks, } p \text {-values } 0\end{array}$ & r achieving four levels of improvement) \\
\hline Tveita $2008^{36}$ & $\begin{array}{l}\text { Passive abduction, passive external rotation, } \\
\text { passive internal rotation, active abduction, } \\
\text { active external rotation, active internal } \\
\text { rotation }\left(^{\circ}\right)\end{array}$ & Final value at 6 weeks after last $i$ & ection \\
\hline
\end{tabular}

\section{Distension versus steroid}

The study by Tveita et al. ${ }^{36}$ found that there was a significant improvement in passive abduction with steroid compared with distension at 6 weeks after the last injection (MD $-34.00^{\circ}, 95 \% \mathrm{CI}$ $-40.78^{\circ}$ to $-27.22^{\circ}$ ). There was no significant difference between groups for active abduction, external or internal rotation, or passive internal or external rotation (Table 47).

The study by Gam et al. ${ }^{84}$ reported that there was a significant improvement with distension compared with steroid in external rotation $(p=0.0007)$ but no statistically significant difference in abduction (time point unclear).

\section{Adverse events}

All of the studies investigating distension reported adverse events. These were reported in detail in the study by Buchbinder et al..$^{43}$ and to a lesser extent by Gam et al. ${ }^{84}$ and Tveita. ${ }^{36}$ The adverse events reported by all studies are summarised in Table 48.

Buchbinder et $a l .{ }^{43}$ reported that open-ended questions were used to assess the extent of adverse events. More patients in the arthrographic distension group than in the placebo group reported pain associated with the procedure (16\% vs 5\%) and pain lasting up to 48 hours (12\% vs $5 \%$ ). None of the adverse events was reported as serious and a number of additional adverse events were reported (Table 48). 
TABLE 47 Range of movement results of studies of distension (MD and 95\% Cl)

\begin{tabular}{|c|c|c|c|}
\hline Study & Short term ( $\leq 3$ months) & Medium term ( $>3$ and $\leq 6$ months) & Long term ( $>6$ and $\leq 12$ months) \\
\hline \multicolumn{4}{|c|}{ Distension vs placebo } \\
\hline \multicolumn{4}{|l|}{ Active abduction $\left(^{\circ}\right)$} \\
\hline \multirow[t]{2}{*}{ Buchbinder $2004^{43}$} & $8.90(-8.55 \text { to } 26.35)^{\mathrm{a}}$ & & \\
\hline & $4.50(-15.16 \text { to } 24.16)^{b}$ & & \\
\hline \multicolumn{4}{|l|}{ External rotation $\left(^{\circ}\right)$} \\
\hline \multirow[t]{2}{*}{ Buchbinder $2004^{43}$} & $-6.50(-24.62 \text { to } 11.62)^{a}$ & & \\
\hline & $2.10(-14.50 \text { to } 18.70)^{b}$ & & \\
\hline \multicolumn{4}{|l|}{ Hand behind back $\left(^{\circ}\right)$} \\
\hline \multirow[t]{2}{*}{ Buchbinder $2004^{43}$} & $1.90(-0.76 \text { to } 4.56)^{\mathrm{a}}$ & & \\
\hline & $0.80(-1.95 \text { to } 3.55)^{b}$ & & \\
\hline \multicolumn{4}{|c|}{ Distension vs steroid } \\
\hline \multicolumn{4}{|l|}{ Active abduction $\left({ }^{\circ}\right)$} \\
\hline Tveita $2008^{36}$ & $3.00(-13.00 \text { to } 19.00)^{c}$ & & \\
\hline \multicolumn{4}{|c|}{ Active external rotation $\left(^{\circ}\right)$} \\
\hline Tveita $2008^{36}$ & $2.00(-6.33 \text { to } 10.33)^{c}$ & & \\
\hline \multicolumn{4}{|c|}{ Active internal rotation $\left(^{\circ}\right)$} \\
\hline Tveita $2008^{36}$ & $2.00(-5.88 \text { to } 9.88)^{c}$ & & \\
\hline \multicolumn{4}{|l|}{ Passive abduction $\left({ }^{\circ}\right)$} \\
\hline Tveita $2008^{36}$ & $-34.00(-40.78 \text { to }-27.22)^{\mathrm{c,d}}$ & & \\
\hline \multicolumn{4}{|c|}{ Passive external rotation $\left({ }^{\circ}\right)$} \\
\hline Tveita $2008^{36}$ & $-2.00(-9.42 \text { to } 5.42)^{c}$ & & \\
\hline \multicolumn{4}{|c|}{ Passive internal rotation $\left(^{\circ}\right)$} \\
\hline Tveita $2008^{36}$ & $-3.00(-9.13 \text { to } 3.13)^{c}$ & & \\
\hline
\end{tabular}

a Reported at 6 weeks.

b Reported at 12 weeks.

c Reported at 6 weeks after last injection (time point unclear).

d Statistically significant, i.e. the $\mathrm{Cl}$ did not cross the line of no effect (zero).

TABLE 48 Adverse events in studies investigating distension

\begin{tabular}{|c|c|}
\hline Study & Adverse events reported \\
\hline \multirow{6}{*}{$\begin{array}{l}\text { Buchbinder } \\
2004^{43}\end{array}$} & Total reported adverse events: arthrographic distension ( $n=25): 9(36 \%)$, placebo $(n=21): 1(5 \%)$ \\
\hline & Pain associated with procedure: arthrographic distension $(n=25): 4$ (16\%), placebo $(n=21): 1(5 \%)$ \\
\hline & Increased pain for up to 48 hours after procedure: arthrographic distension $(n=25): 3(12 \%)$, placebo $(n=21): 1(5 \%)$ \\
\hline & Claustrophobia at time of procedure: arthrographic distension $(n=25): 1(4 \%)$, placebo $(n=21): 1(5 \%)$ \\
\hline & Unsettled, anxious and hot: arthrographic distension ( $n=25): 0(0 \%)$, placebo $(n=21): 1(5 \%)$ \\
\hline & Shoulder noisy (i.e. fluid noises): arthrographic distension ( $n=25): 1$ (4\%), placebo $(n=21): 0(0 \%)$ \\
\hline Gam $1998^{84}$ & Two cases of unacceptable pain after injection (one in each treatment group) \\
\hline \multirow[t]{5}{*}{ Tveita $2008^{36}$} & Injections reported as very painful: distension: 5 , steroid: 6 \\
\hline & Other possible side effects reported: distension: 14, steroid: 20 \\
\hline & Complaints of flushing or disturbances in heat regulation: distension: 9 , steroid: 13 \\
\hline & Minor loss of sensation and motor control loss in affected arm: distension: 2, steroid: 2 \\
\hline & Glenohumeral joint infection: treatment group unspecified: 1 \\
\hline
\end{tabular}


Gam et al.$^{84}$ reported that the number and type of side effects were recorded. The only adverse events reported in this study were two cases of unacceptable pain after injection (one in each treatment group); these patients dropped out of the study.

In the study by Tveita et al. ${ }^{36}$ the patients recorded pain intensity related to the injection procedures. Six patients in the steroid group and five in the distension group felt that the injections were very painful. Flushing and disturbances in heat regulation were also common in both groups and a number of other non-serious adverse events were reported (Table 48).

\section{Summary}

Three studies were included that investigated the effects of distension with steroid injection. Two of these studies investigated arthrographic distension ${ }^{36,43}$ and the other investigated non-arthrographic distension..$^{84}$ The comparators evaluated were steroid alone ${ }^{36,84}$ and placebo (arthrogram only). ${ }^{43}$ Only one study was judged to be of satisfactory quality, ${ }^{43}$ whereas the remainder were potentially at risk of bias. Because of the variability in the interventions and comparators it was not considered appropriate to pool any of the studies. Stage of frozen shoulder was not reported in any of these studies; however, previous treatments were described in two of the studies. ${ }^{36,43}$

\section{Distension compared with placebo}

One study of satisfactory quality compared distension with placebo and reported benefit with distension in the short term in one of two measures of function and disability. There was no difference in three measures of range of movement or in pain at function at short-term follow-up. None of the adverse events reported was considered serious.

\section{Distension compared with steroid}

Two studies that were potentially at risk of bias compared distension with steroid. One study that compared arthrographic distension with steroid reported no difference between groups in one measure of function and disability, but reported a benefit in one range of movement in the short term. Some patients in both groups considered the injections as being very painful and one case of glenohumeral joint infection (septic arthritis) was reported. The study that compared non-arthrographic distension with steroid reported no difference between groups in pain at function or at rest, but a significant benefit with distension in one measure of range of movement but not another at short-term follow-up. One patient from each group dropped out as a result of unacceptable pain.

In conclusion, few studies of distension were identified and only single studies of different comparisons were available. Based on one study of satisfactory quality there is a little evidence of potential benefit with distension compared with placebo. There is insufficient evidence to draw conclusions about the efficacy of distension (arthrographic or non-arthrographic) for frozen shoulder.

\section{Capsular release}

Two case series of more than 50 participants were identified that investigated capsular release in the treatment of primary frozen shoulder. A summary of the study characteristics is reported in Table 49, with further details available in Appendix 7. One study was published in Norway in 2007 by Austgulen et al. ${ }^{85}$ the other in Taiwan in 2002 by Chen et al. ${ }^{86}$

The capsular release procedures varied between the two studies. Only a summary of the procedures are provided here; fuller descriptions are available in the original papers. In the study by Chen et al., ${ }^{86}$ the authors stated that the intervention involved distension and debridement, release and manipulation. The degree of joint movement was confirmed arthroscopically 
TABLE 49 Studies including capsular release

\begin{tabular}{|c|c|c|c|}
\hline $\begin{array}{l}\text { Study details } \\
\text { and study design }\end{array}$ & Participants & Treatment & Condition-related inclusion criteria \\
\hline Austgulen $2007^{85}$ & \multirow{2}{*}{$\begin{array}{l}n=66(70 \\
\text { shoulders })\end{array}$} & Arthroscopic capsular and ligament release + PT & \multirow{2}{*}{$\begin{array}{l}\text { Patients with primary frozen shoulder. } \\
\text { Diagnosis of frozen shoulder was } \\
\text { confirmed during anaesthesia: limited } \\
\text { outward rotation of }<20^{\circ} \text { and }<45^{\circ} \\
\text { in abduction with a fixed scapula }\end{array}$} \\
\hline Case series & & $\begin{array}{l}\text { Surgery was performed under general anaesthesia in beach } \\
\text { chair position. Shoulder arthroscopy was performed using normal } \\
\text { technique. Access to the shoulder joint was from behind and } \\
\text { surgical instruments entered from in front in the rotator interval. } \\
\text { The rotator interval was cleaned out and the frontal capsule and } \\
\text { glenohumeral ligament and coracohumeral ligament were split } \\
\text { from biceps tendon to } 60^{\prime} \text { 'clock. Both capsule and ligaments } \\
\text { were split with an Acufex Upbiter Scissor punch. The subacromial } \\
\text { space was inspected and adherences were loosened. The space } \\
\text { and displacement were evaluated. Where spaces were narrow } \\
\text { subacromial decompression was performed until the shoulder could } \\
\text { move at maximal outward rotation and to } 180^{\circ} \text { in abduction. All } \\
\text { patients received aggressive rehabilitation with a physiotherapist } \\
\text { from the first day after surgery }\end{array}$ & \\
\hline Chen $2002^{86}$ & $n=183$ & Arthroscopic brisement (distension, debride, release) followed by & \multirow{2}{*}{$\begin{array}{l}\text { Basic criteria for definition of } \\
\text { idiopathic frozen shoulder }\end{array}$} \\
\hline Case series & cases & $\begin{array}{l}\text { gentle manipulation + PT (further details provided), then the arm } \\
\text { was kept in abduction-external rotation position for } 2 \text { days during } \\
\text { which the patient was confined to bed. Passive and active exercise } \\
\text { of the shoulder was then allowed, with a rehabilitation programme } \\
\text { at the hospital rehabilitation facility }\end{array}$ & \\
\hline
\end{tabular}

PT, physiotherapy.

(after distension by water and the syringe rebound), a synovectomy was performed using an arthroscopic shaver or vaporisation, capsular scar tissue was described by the authors as being debrided and then gentle manipulation was performed. This involved arm elevation in the scapular plane (which was usually associated with audible popping of the contracted capsule) and external rotation followed by internal rotation at varying degrees of abduction. This was done with gradual pressure and stopped if unyielding resistance was met. The authors stated that repetition of these steps led to tearing of the capsular structures. The arm was kept in abductionexternal rotation for 2 days following the procedure.

The Austgulen et al. study ${ }^{85}$ reported that shoulder arthroscopy was performed using normal technique, and the frontal capsule, glenohumeral ligament and coracohumeral ligament were split from the bicep tendon to 6 o'clock. The authors stated that the subacromial space was inspected and adherences loosened. Where the spaces were narrow subacromial decompression was performed until the shoulder could move at maximal outward rotation and at $180^{\circ}$ in abduction.

Both studies included a programme of physiotherapy following the procedure. Austgulen et al ${ }^{85}$ specifically stated that the patients received aggressive rehabilitation from the first day after surgery (no further details reported). This study also reported that participants were asked to perform home exercises (including stretches) every day. In addition, concomitant NSAIDs were given as needed and postoperative oxycodone was given occasionally. ${ }^{85}$ Chen et al. ${ }^{86}$ did not state whether a home exercise routine was undertaken or whether concomitant medication was given.

The inclusion criteria differed between the studies, with Chen et al. ${ }^{86}$ reporting limited criteria (i.e. basic criteria for diagnosis of idiopathic frozen shoulder). Additionally, Austgulen et al. ${ }^{85}$ required that participants had already tried physiotherapy but without a satisfactory result. The nature and intensity of the previous physiotherapy was not reported. The diagnostic criteria 
also varied between studies. In the Austgulen study ${ }^{85}$ diagnosis was based around limited range of movement and confirmed under anaesthesia. Chen et al. ${ }^{86}$ used clinical history, physical examination, radiography and arthrography. Neither study reported the stage of frozen shoulder; the average duration of frozen shoulder at baseline was 13 months (range 3 to 60 months) in the Austgulen study ${ }^{85}$ and 8 months (range not reported) in the Chen study ${ }^{86}$ (it was unclear whether this was mean or median). A total of $17 \%$ of the patients in the Austgulen study had diabetes ${ }^{85}$ but this information was not reported in the study by Chen et al..$^{86}$ In both studies participants had received previous treatment for frozen shoulder. Austgulen et al ${ }^{85}$ reported that participants had received physical therapy, whereas Chen et al ${ }^{86}$ reported that none of the participants had responded to conservative treatment of at least 12 weeks' duration. The average age (mean or median not specified) of participants was 53 years in the Austgulen study, ${ }^{85}$ whereas Chen et al. ${ }^{86}$ reported that ages ranged from 32 years to 79 years. The percentage of women was similar in both studies: $67 \%{ }^{85}$ and $75 \% .{ }^{86}$

\section{Quality assessment}

Both studies were case series, which are inherently at high risk of bias because of the lack of a control group. The Bradford Hill criteria (a group of minimal conditions necessary to provide adequate evidence of a causal relationship between an incidence and a consequence) state that there must be a temporal relationship between the incidence of the factor (in this case surgery) and consequence (recovery). For a condition such as frozen shoulder, which for most people will resolve over a 1- to 3-year period, this relationship cannot be ascertained without a control group. ${ }^{87}$ Austgulen et al.$^{85}$ was considered better quality as this study reported inclusion criteria, the population appeared representative of the frozen shoulder population, SDs were reported and patients were recruited prospectively, although the extent of this was unclear. The number of dropouts was unclear. Chen et al ${ }^{86}$ met only one criterion (reporting of inclusion criteria); the remainder were unclear or not met. There were no dropouts in this study. Further details of quality assessment are available in Appendix 8.

\section{Outcomes available}

Data were sparse for all outcomes assessed (Table 50). The effect on short-term pain was described narratively in Chen $e t$ al. ${ }^{86}$ Function and disability and relevant measures of range of movement (external rotation, internal rotation and abduction; it was not specified whether these were active or passive) were reported at baseline and follow-up and as average gain in range of movement by Chen et al. (no further details available). ${ }^{85,86}$ Additionally, Austgulen et al. ${ }^{85}$ reported data for ability to work, physical activity, sleep at night and satisfaction. Both studies reported adverse events. Numerical results are summarised in Table 51. The complete outcome data for these studies are available in Appendix 7.

Pain

Only one study reported any data regarding pain. Chen et al. ${ }^{86}$ reported that two-thirds of patients complained of pain exacerbation after arthroscopic brisement; $5 \%$ had pain duration of longer than a few weeks. Half of patients had pain relief within 1 month whether in motion or not. After 3 months all except eight shoulders were pain free in any direction of shoulder movement (data were not reported).

\section{Function and disability}

Austgulen et al. ${ }^{85}$ reported a significant improvement in the Oxford Shoulder score from a baseline mean of 41.0 (SD 7.5) to 18.4 (SD 7.3) postoperatively $(p<0.001$ ), a scale for which the best possible outcome is 12 and worst possible outcome is 60 (Table 51).

Similarly, Chen et al. ${ }^{86}$ reported that an improvement in the mean modified ASES score from a baseline score of 41 (SD 13) to 87 (SD 11) was significant $(p<0.005)$ at follow-up (Table 51). 
TABLE 50 Outcomes assessed in studies evaluating capsular release

\begin{tabular}{|c|c|c|}
\hline Study & Outcomes assessed & Data reported and time of follow-up \\
\hline $\begin{array}{l}\text { Chen } \\
2002^{86}\end{array}$ & Pain (reduction of pain): pain component of ASES (modified version) ${ }^{\mathrm{a}}$ & $\begin{array}{l}\text { Pain described narratively at 'a few weeks' and } 1 \text { and } \\
3 \text { months (3-month data used in analysis) }\end{array}$ \\
\hline \multirow[t]{2}{*}{$\begin{array}{l}\text { Austgulen } \\
2007^{85}\end{array}$} & $\begin{array}{l}\text { Function and disability: Oxford Shoulder score (12, best outcome; } 60 \text {, } \\
\text { worst) }\end{array}$ & $\begin{array}{l}\text { Mean and SD postoperatively (average follow-up } \\
10 \text { months, range } 3 \text { to } 29 \text { months) }\end{array}$ \\
\hline & $\begin{array}{l}\text { Function and disability: telephone questionnaire assessing ability to } \\
\text { work, physical activity, sleep at night (all 0, most complaints; } 10 \text {, least } \\
\text { complaints) and patient satisfaction (0, worst; 10, best) }\end{array}$ & $\begin{array}{l}\text { Mean and SD postoperatively (average follow-up } \\
10 \text { months, range } 3 \text { to } 29 \text { months) }\end{array}$ \\
\hline $\begin{array}{l}\text { Chen } \\
2002^{86}\end{array}$ & Function and disability: modified version of ASES $(0-100)^{\mathrm{a}}$ & $\begin{array}{l}\text { Final value mean and } S D^{b} \text { at time of follow-up } \\
\text { (average follow-up } 23 \text { months, range } 6 \text { months to } \\
5 \text { years) }\end{array}$ \\
\hline $\begin{array}{l}\text { Austgulen } \\
2007^{85}\end{array}$ & $\begin{array}{l}\text { ROM: external rotation, abduction (both degrees) (and flexion, not included } \\
\text { in analysis) }\end{array}$ & $\begin{array}{l}\text { Mean and SD postoperatively (average follow-up } \\
10 \text { months, range } 3 \text { to } 29 \text { months) }\end{array}$ \\
\hline $\begin{array}{l}\text { Chen } \\
2002^{86}\end{array}$ & $\begin{array}{l}\text { ROM: external rotation, internal rotation (both degrees) (and elevation, not } \\
\text { included in analysis) }\end{array}$ & Average gain at latest follow-up (not specified) \\
\hline
\end{tabular}

ROM, range of movement.

a Higher score indicates improved outcome.

b Measure of variance unclear but assumed to be a SD.

Austgulen et al. ${ }^{85}$ reported significant improvements from baseline to follow-up $(p<0.001)$ on a 10-point scale in mean working ability (baseline 2.4, SD 2.6; follow-up 7.4, SD 2.5), mean physical activity (baseline 2.3, SD 2.5; follow-up 7.4, SD 2.4) and sleep at night (baseline 1.7, SD 2.5; follow-up 7.2, SD 2.6). This study also reported high satisfaction postoperatively (8.6, SD 1.6 on a 10-point scale) (Table 51).

\section{Range of movement}

Austgulen et al. ${ }^{85}$ reported that there was a significant improvement in mean external rotation from $3^{\circ}\left(\mathrm{SD} 5^{\circ}\right)$ to $39^{\circ}\left(\mathrm{SD} 23^{\circ}\right)(p<0.001)$ and in mean abduction from $34^{\circ}\left(\mathrm{SD} 8^{\circ}\right)$ to $154^{\circ}$ $\left(\mathrm{SD} 37^{\circ}\right)(p<0.001)$ postoperatively.

Chen et al. ${ }^{86}$ reported a similar average gain in external rotation of $35^{\circ}$. The gain in internal rotation was $30^{\circ}$.

\section{Adverse events}

Austgulen et al. ${ }^{85}$ reported that two patients had frozen shoulder again and had repeat surgery. No deep infections, nerve damage or other complications were reported. Chen et al. ${ }^{86}$ found that only one patient experienced complications (superficial wound infection). No further information regarding how adverse events had been assessed was reported in either study.

\section{Summary}

Two studies investigated capsular release. Both were case series and therefore inherently at high risk of bias. One of the studies stated that debrisement was a component of the capsular release procedure and both reported that physiotherapy was undertaken post procedure.

One case series reported that the majority of patients were pain free at movement at short-term follow-up. Statistical significance was not reported. There was evidence of benefit for function and disability from both studies. Each study reported a significant improvement in one function and disability scale. Additionally, one study reported significant benefits in working ability, physical activity, sleep at night and satisfaction with capsular release. One study reported 
TABLE 51 Results of the studies of capsular release

\begin{tabular}{|c|c|c|c|}
\hline Study & Short term ( $\leq 3$ months) & Medium term ( $>3$ and $\leq 6$ months) & Long term ( $>6$ and $\leq 12$ months) \\
\hline \multicolumn{4}{|c|}{ Function and disability } \\
\hline \multicolumn{4}{|c|}{ Oxford Shoulder score } \\
\hline \multirow[t]{2}{*}{ Austgulen $2007^{85}$} & & & Baseline: mean 41.0 (SD 7.5) \\
\hline & & & $\begin{array}{l}\text { Postoperatively: mean } 18.4 \text { (SD 7.3) } \\
(p<0.001)\end{array}$ \\
\hline \multicolumn{4}{|l|}{ Modified ASES } \\
\hline \multirow[t]{2}{*}{ Chen $2002^{86}$} & Baseline: mean 41 (SD 13) & & \\
\hline & $\begin{array}{l}\text { Follow-up: mean } 87(S D 11)^{a} \\
(p<0.005)\end{array}$ & & \\
\hline \multicolumn{4}{|c|}{ Range of movement } \\
\hline \multicolumn{4}{|l|}{ Internal rotation $\left({ }^{\circ}\right)$} \\
\hline Chen $2002^{86}$ & Average gain: 35 & & \\
\hline \multicolumn{4}{|l|}{ External rotation $\left({ }^{\circ}\right)$} \\
\hline \multirow[t]{2}{*}{ Austgulen $2007^{85}$} & & & Baseline: mean 3 (SD 5) \\
\hline & & & $\begin{array}{l}\text { Postoperatively: mean } 39 \text { (SD 23) } \\
(p<0.001)\end{array}$ \\
\hline Chen $2002^{86}$ & Average gain: 30 & & \\
\hline \multicolumn{4}{|l|}{ Abduction ( ${ }^{\circ}$ ) } \\
\hline \multirow[t]{2}{*}{ Austgulen $2007^{85}$} & & & Baseline: mean 34 (SD 8) \\
\hline & & & $\begin{array}{l}\text { Postoperatively: mean } 154 \text { (SD 37) } \\
(p<0.001)\end{array}$ \\
\hline \multicolumn{4}{|l|}{ Other } \\
\hline \multicolumn{4}{|l|}{ Working ability } \\
\hline \multirow[t]{2}{*}{ Austgulen $2007^{85}$} & & & Baseline: mean 2.4 (SD 2.6) \\
\hline & & & $\begin{array}{l}\text { Postoperatively: mean } 7.4 \text { (SD 2.5) } \\
(p<0.001)\end{array}$ \\
\hline \multicolumn{4}{|l|}{ Physical activity } \\
\hline \multirow[t]{2}{*}{ Austgulen $2007^{85}$} & & & Baseline: mean 2.3 (SD 2.5) \\
\hline & & & $\begin{array}{l}\text { Postoperatively: mean } 7.4 \text { (SD 2.4) } \\
(p<0.001)\end{array}$ \\
\hline \multicolumn{4}{|l|}{ Sleep at night } \\
\hline \multirow[t]{2}{*}{ Austgulen $2007^{85}$} & & & Baseline: mean 1.7 (SD 2.5) \\
\hline & & & $\begin{array}{l}\text { Postoperatively: mean } 7.2 \text { (SD 2.6) } \\
(p<0.001)\end{array}$ \\
\hline \multicolumn{4}{|l|}{ Satisfaction } \\
\hline Austgulen $2007^{85}$ & & & Mean (SD) $8.6(1.6)$ \\
\hline
\end{tabular}

a Measure of variance unclear, but assumed to be SD.

evidence of significant benefit in two measures of range of movement with capsular release; the other study reported an improvement in two measures of range of movement, but did not report whether or not these were significant. 
In conclusion, although the evidence available suggested potential benefit from capsular release, these studies were at high risk of bias and cannot be used to draw conclusions regarding the efficacy of this treatment for frozen shoulder.

\section{Mixed-treatment comparison results}

A MTC was performed for pain only. As previously stated in the report, this was because pain was the only outcome for which a network was available.

Analyses were planned for pain recorded at $\leq 3$ months (excluding pain recorded at $\leq 4$ weeks from the start of treatment), $>3$ months and $\leq 6$ months, and $>6$ months and $\leq 12$ months. However, data were only available for $\leq 3$ months.

Four networks were evaluated:

- Network 1: studies of any intervention (i.e. conservative and invasive) and any quality.

- Network 2: studies of any intervention that were at least of satisfactory quality (i.e. method of randomisation was adequate and outcome assessment was blinded). The overall quality of the available trials for the MTC was poor, with most not reporting on the a priori quality criterion of allocation concealment. So that a network of trials could be formed, method of randomisation rather than allocation concealment was used as a quality criterion in the MTC.

- Network 3: studies of non-invasive treatments of any quality, excluding sodium hyaluronate (because it is rarely used in the NHS). ${ }^{18}$

- Network 4: studies of non-invasive treatments that were at least of satisfactory quality (i.e. method of randomisation was adequate and outcome assessment was blinded), excluding sodium hyaluronate.

\section{Interpretation of standardised mean differences}

Reduction in pain recorded as a SMD can be transformed back into the units of one or more of the pain measurement instruments. The decision was made to back transform to a VAS $0-100 \mathrm{~mm}$ as this measure had more substantial information than other pain measures. This included one study performed in a shoulder-specific population. ${ }^{50}$ Transformation from SMD to VAS was made using a representative pooled SD at baseline from one of the included trials. ${ }^{51}$ No data could be located on the MCID in pain for individuals with frozen shoulder. However, it is suggested that a difference of $14 \mathrm{~mm}$ on a 100-mm VAS scale is the MCID for individuals with rotator cuff disease, a shoulder disorder also characterised by pain and restricted range of movement. ${ }^{50}$ Given that the between-group MCID is thought to approximate $40 \%$ of that within individuals ${ }^{6,53}$ the MCID between groups for rotator cuff disorder can be estimated to be approximately $5.6 \mathrm{~mm}$. This value is therefore used as a proxy for the between-group MCID for frozen shoulder. ${ }^{6}$ The reduction in pain recorded as a SMD can be interpreted on a VAS $0-100 \mathrm{~mm}$ scale using Table 52.

From the 31 included trials, the total number of trials potentially available for analysis was nine. The reasons for trials being excluded from the analysis are presented in Table 53.

The nine trials and the 10 interventions they evaluated are shown in Table 54. The placebo used varied between studies and included saline injections, lidocaine injection, placebo laser, sham distension and sham ultrasound. The four studies of physical therapy consisted of physiotherapy with an active mobilisation component. Physical therapies without an active component consisted of laser therapy and interferential therapy. Four studies included steroid injections; 
TABLE 52 Standardised mean differences and equivalent reductions on a VAS 0-100 mm scale

\begin{tabular}{lllccc}
\hline \multicolumn{7}{l}{ Reduction in pain score } \\
\hline SMD & -0.3 & -0.5 & -1.0 & -1.5 & -2.0 \\
VAS 0-100 mm & -5.6 & -9.15 & -18.3 & -27.45 & -36.6 \\
\hline
\end{tabular}

TABLE 53 Reasons for trials being excluded from the MTC

\begin{tabular}{ll}
\hline Reason for exclusion from the MTC & No. of studies excluded \\
\hline Trial did not report pain as an outcome & 9 \\
Trial did not report pain data & 6 \\
Trial reported pain at $\leq 4$ weeks & 2 \\
Trial reported pain at $>3$ months only & 1 \\
Trial reported mean change and baseline medians only & 2 \\
SDs could not be imputed & 1 \\
Trial compared the same class of intervention with each other & 1 \\
\hline
\end{tabular}

\begin{tabular}{l}
\hline \\
\end{tabular}

these were triamcinolone hexacetonide (40 mg once, $20 \mathrm{mg}$ once), methylprednisolone (40 mg once for 3 weeks) and dexamethasone ( $2 \mathrm{mg}$ once weekly for 5 weeks). Steroid injections were either given alone (three studies ${ }^{35,41,70}$ ) or combined with physiotherapy (three studies ${ }^{35,41,42}$ ). Seven of the nine studies included in the review had a home exercise component. ${ }^{16,35,39,41-43,51}$ 
In these nine trials, a variety of pain outcomes and pain scales were reported (Table 55). If a trial reported more than one pain outcome or used more than one scale, only the pain outcome/scale prioritised for use in the analysis (see Chapter 2, Mixed-treatment comparison) is reported.

To check that the non-informative priors used in the analysis were truly non-informative a sensitivity analysis was performed for each network by changing the range of the uniform prior distribution on the SD from $0-2$ to $0-0.8,0-5,0-10$ and, as a final check for stability for network $1,0-15$. An additional sensitivity analysis was also performed by repeating each analysis using a burn-in of 30,000 iterations.

All four networks were evaluated; however, the lack of evidence available to inform networks 2, 3 and 4 led to a lack of stability in the models, particularly a lack of stability in between-study variance. For completeness these analyses have been presented in Appendix 10 although no conclusions can be drawn from the results presented. Only the results for the network of any intervention and of any quality (network 1 ) are presented here. The slightly higher number of trials included in this network allows the model to achieve stability.

\section{Network 1: studies of any intervention and of any quality}

There were nine trials with data that could be used in the analysis of pain at or close to 3 months. These nine trials formed a connected network of nine interventions compared with placebo. The network is presented in Figure 21.

Each line represents one comparison. For example, there were two studies available for the comparison of steroid with placebo. Where there is no line there were no studies available, for example physical therapy without mobilisation versus arthrographic distension and steroid.

The models were run for 100,000 iterations and were not sensitive to the length of burn-in. Stable estimates of treatment effect, CrIs and between-study heterogeneity were produced by the models using a uniform prior of $(0,5),(0,10)$ and $(0,15)$. Convergence, determined by graphical traces and the Brooks-Gelman-Rubin statistic, was achieved. The results of the uniform prior $(0,5)$ are presented here. Results for uniform priors $(0,10)$ and $(0,15)$ are available in Appendix 10.

TABLE 55 Type of pain reported, pain scale used and quality of studies available for MTC analysis

\begin{tabular}{|c|c|c|c|c|}
\hline Study & Type of pain reported & Type of pain scale used & Time point used & At least satisfactory qualitya \\
\hline Buchbinder $2004^{43}$ & Pain overall & Likert scale 0-9 & 12 weeks & $\checkmark$ \\
\hline Carette $2003^{35}$ & Pain overall & SPADI 5-item pain subscale & 3 months & $\checkmark$ \\
\hline Cheing $2008^{79}$ & Pain at the moment & VAS $0-10 \mathrm{~cm}$ & Approx. 2 months & \\
\hline Dogru $2008^{51}$ & Pain overall & SPADI 5-item pain subscale & 3 months & \\
\hline Kivimaki $2007^{39}$ & Pain intensity & Likert scale 0-10 & 3 months & $\checkmark$ \\
\hline Rizk $1991^{42}$ & Pain overall & Likert scale 0-5 & 11 weeks & \\
\hline Ryans $2005^{41}$ & Daytime pain at rest & VAS 0-100 mm & 6 weeks & $\checkmark$ \\
\hline Stergioulas $2008^{16}$ & Pain overall & VAS 0-100 mm & 8 weeks & $\checkmark$ \\
\hline Takagishi $1996^{70}$ & Pain on activity & VAS 0-10 & 5 weeks & \\
\hline
\end{tabular}

a Method of randomisation was adequate and outcome assessment was blinded. 


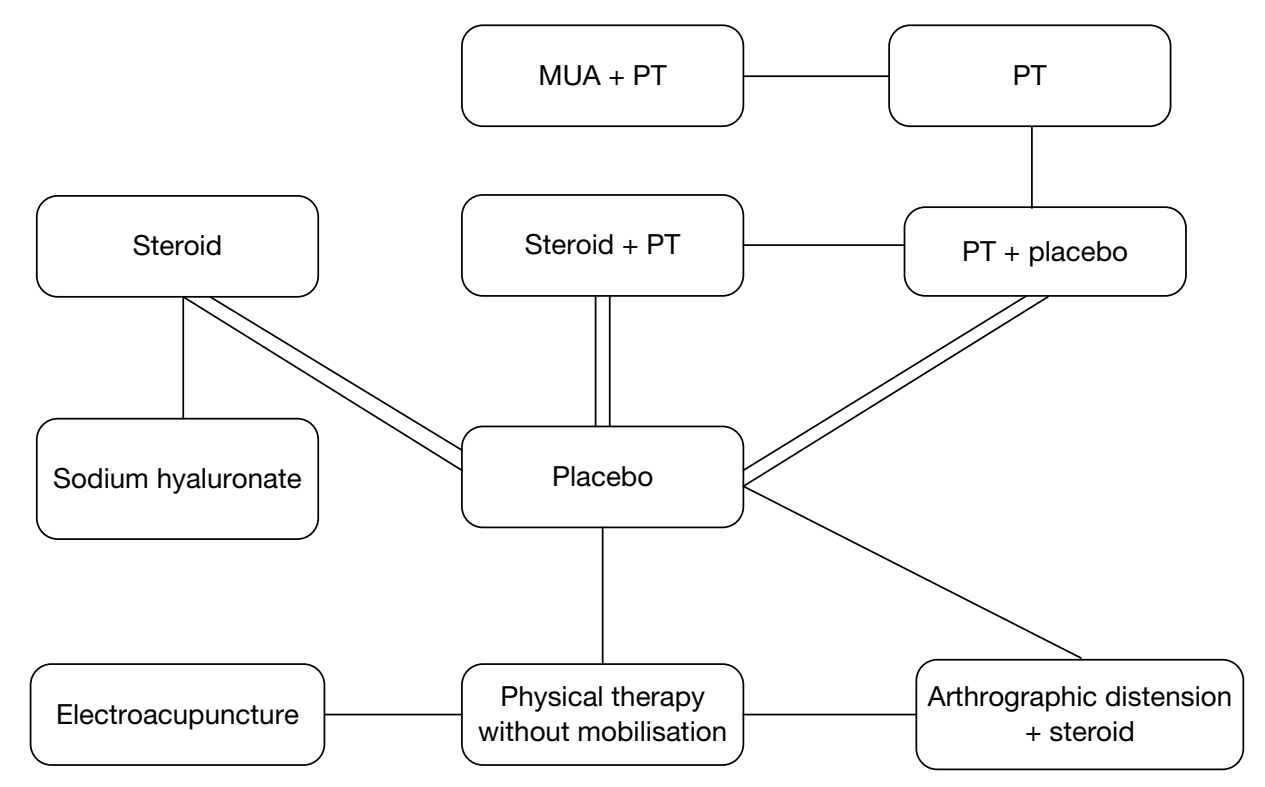

FIGURE 21 Network diagram of studies of any intervention and any quality. PT, physical therapy.

The model was a good fit as the residual deviance was close to the number of data points. Table 56 presents the treatment effects compared with placebo in order of mean effectiveness. Steroid combined with physiotherapy showed a beneficial treatment effect compared with placebo with 95\% CrIs that did not cross the line of no effect. The 95\% CrI of the treatment effect of physical therapy without mobilisation and physiotherapy combined with placebo marginally crossed the line of no effect. The remaining interventions (electroacupuncture, steroid injection, physiotherapy, MUA plus physiotherapy, sodium hyaluronate and arthrographic distension) all crossed the line of no effect. There was no clear difference between the interventions in treatment effects, that is, the CrIs overlapped. These results are also displayed graphically in Figure 22.

Steroid combined with physiotherapy showed a clinically significant as well as statistically significant reduction in pain, with a $95 \% \mathrm{CrI}$ that corresponded to a clinically significant reduction in pain only: $-28.9 \mathrm{~mm}(95 \% \mathrm{CrI}-54.1$ to $-7.7 \mathrm{~mm})$. Although the $\mathrm{MD}$ was clinically significant for most of the other comparisons, the $95 \%$ CrIs contained values that were clinically insignificant. For example, steroid injection had a MD of $-21.0 \mathrm{~mm}$ ( $95 \% \mathrm{CrI}-45.0$ to $3.5 \mathrm{~mm}$ ).

Opportunities for comparison with standard meta-analysis are limited as most of the studies were not pooled in a quantitative synthesis. It was possible to compare with the standard metaanalyses for steroid plus physiotherapy versus placebo and steroid versus placebo. The results of the MTC are consistent with the standard meta-analysis of steroid combined with physical therapy versus placebo presented earlier in this report. This standard meta-analysis contained the same two studies included in the MTC. The results of the meta-analysis also showed that steroid combined with physiotherapy resulted in both a statistically and a clinically significant reduction in pain compared with placebo (pooled SMD $-0.98,95 \% \mathrm{CI}-1.43$ to -0.52 ; back transformed MD $17.93 \mathrm{~mm}, 95 \%$ CI -26.2 to $-9.5 \mathrm{~mm}$ ).

\section{Assumptions}

In a MTC there are three key assumptions: homogeneity, consistency and methodological similarity. 
TABLE 56 Mean reduction in pain compared with placebo with Crls in order of mean treatment effectiveness for pain at 3 months, including studies of any quality

\begin{tabular}{lcll}
\hline Prior: uniform $(0,5)$ & & & \\
\hline Comparator: placebo & & & \\
\hline Treatment & Node number & SMD $(95 \%$ Crl $)$ & VAS 0-100 mm, MD (95\% Crl) \\
\hline $\begin{array}{l}\text { Physical therapy without } \\
\text { mobilisation }\end{array}$ & 4 & $-1.71(-3.46$ to 0.04$)$ & $-31.3(-63.3$ to 0.7$)$ \\
Steroid + physiotherapy & 6 & $-1.58(-2.96 \text { to }-0.42)^{\mathrm{a}}$ & $-28.9(-54.1$ to -7.7$)$ \\
Electroacupuncture & 7 & $-1.36(-3.84$ to 1.14$)$ & $-24.9(-70.3$ to 20.9) \\
Physiotherapy + placebo & 2 & $-1.22(-2.42$ to 0.07$)$ & $-22.3(-44.3$ to 1.2$)$ \\
Steroid & 5 & $-1.15(-2.46$ to 0.19$)$ & $-21.0(-45.0$ to 3.5$)$ \\
Physiotherapy & 3 & $-0.95(-3.09$ to 1.25$)$ & $-17.4(-56.5$ to 22.9$)$ \\
MUA + physiotherapy & 8 & $-0.88(-3.60$ to 1.90$)$ & $-16.1(-65.9$ to 34.8$)$ \\
Sodium hyaluronate & 10 & $-0.21(-1.90$ to 1.47$)$ & $-3.8(-34.8$ to 26.9$)$ \\
Arthrographic distension + steroid & 9 & $-0.14(-1.89$ to 1.62$)$ & $-2.6(-34.6$ to 29.6$)$ \\
\hline
\end{tabular}

Data points: 13; residual deviance: 12.66; $\tau^{2}$ (between-study variance): 0.69 (95\% $\mathrm{Crl} 0.0003$ to 4.58).

a Did not cross line of no effect.

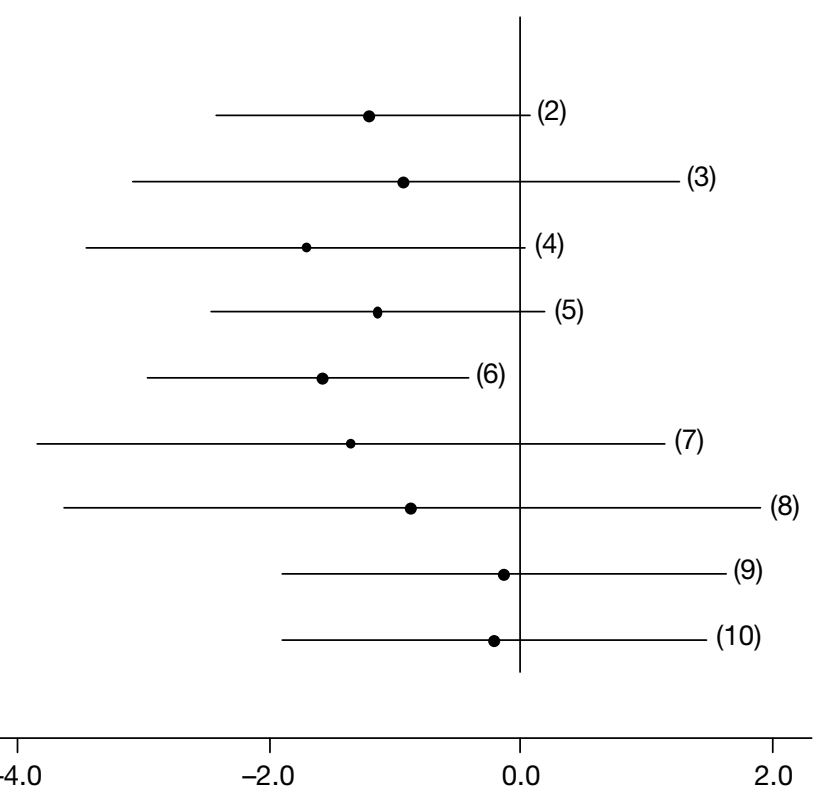

FIGURE 22 Caterpillar plot of the SMD estimates and their 95\% Crls compared with placebo. The treatments associated with each number can be identified from Table 56.

\section{Homogeneity}

The assumption of homogeneity is that trials are sufficiently homogeneous to be quantitatively combined. In the analysis presented, placebo included sham distension, saline injections and placebo laser. Although we believed that it was appropriate to pool different types of placebo, it could be argued that these are not similar enough because of the differing degree of invasiveness. Furthermore, there were differences in the populations of the included studies. 
For all studies of conservative treatments, it was not reported whether participants had received any previous treatment for frozen shoulder. However, for both studies of invasive treatments (MUA combined with physical therapy, and arthrographic distension with steroid) it was reported that at least some participants had undergone previous treatments for frozen shoulder including corticosteroid injections or some form of physical therapy. This would suggest that the populations of the invasive studies had a longer duration and possibly more treatment-resistant forms of frozen shoulder than those in the conservative treatment studies.

\section{Consistency}

The assumption of consistency is that had treatment $\mathrm{C}$ been included in a trial comparing interventions $\mathrm{A}$ and $\mathrm{B}$ then the treatment effect $\mathrm{dAC}$ would be equivalent to that obtained from a trial of interventions A and C. ${ }^{33}$ Assuming consistency, the treatment effect dAC is the sum of the treatment effects $\mathrm{dAB}$ and $\mathrm{dBC}$. However, this assumption requires that the treatments involved belong to closed loops in the network of evidence, such as that seen in a connected polygonal structure.$^{88}$ Given that the structure of the network presented here was of a radiating star, with only one loop of evidence, it was not feasible to assess consistency.

\section{Methodological similarity}

Of the nine studies included in the analysis, five were of at least satisfactory quality (method of randomisation was adequate and outcome assessment was blinded) $)^{16,35,39,41,43}$ and four were not. ${ }^{42,51,70,79}$ To assess the effect of quality on the estimates, a network of studies that were at least of satisfactory quality was evaluated (network 2). However, this model was unstable and, although presented in Appendix 10, the reliability of the data included in the analysis presented is uncertain and does not allow a robust assessment of the impact of quality.

\section{Summary}

Nine interventions formed part of a connected network with placebo and the evidence was informed by all nine trials. Twenty-two studies had to be excluded from the MTC for a range of reasons; therefore, the network was a small subset of the available evidence. Steroid combined with physiotherapy showed a beneficial treatment effect compared with placebo with 95\% CrIs that did not cross the line of no effect. Furthermore, this treatment, when back transformed to a VAS 0-100 mm scale, showed a clinically significant reduction in pain with $95 \%$ CrIs that excluded clinically insignificant values. The remaining treatments had 95\% CrIs that crossed the line of no effect and which contained clinically insignificant values. Overall, there was no clear difference in the treatment effects of any of the interventions, that is, the CrIs overlapped. Although the model was a good fit and gave stable estimates, given the arguable heterogeneity of the placebos and of study populations, and the inclusion of poor-quality studies, the reliability of the results of the MTC is uncertain and they should be interpreted with caution.

\section{Patients' views of interventions for frozen shoulder}

Searches of three databases (CINAHL, MEDLINE and PsycINFO) identified 1067 potentially relevant references (Figure 23). Nine full papers were ordered on the basis of screening titles and abstracts. Of these, two papers could not be obtained within the time frame required; however, it is unlikely that these would have met the inclusion criteria. One paper appeared to focus on the clinical effectiveness of steroid injections ${ }^{89}$ and the remaining paper appeared to examine the quality of life of patients with frozen shoulder but did not appear to address treatments. ${ }^{90}$ None of the remaining six papers met the inclusion criteria; therefore, no data were available on patients' views regarding interventions for frozen shoulder. 


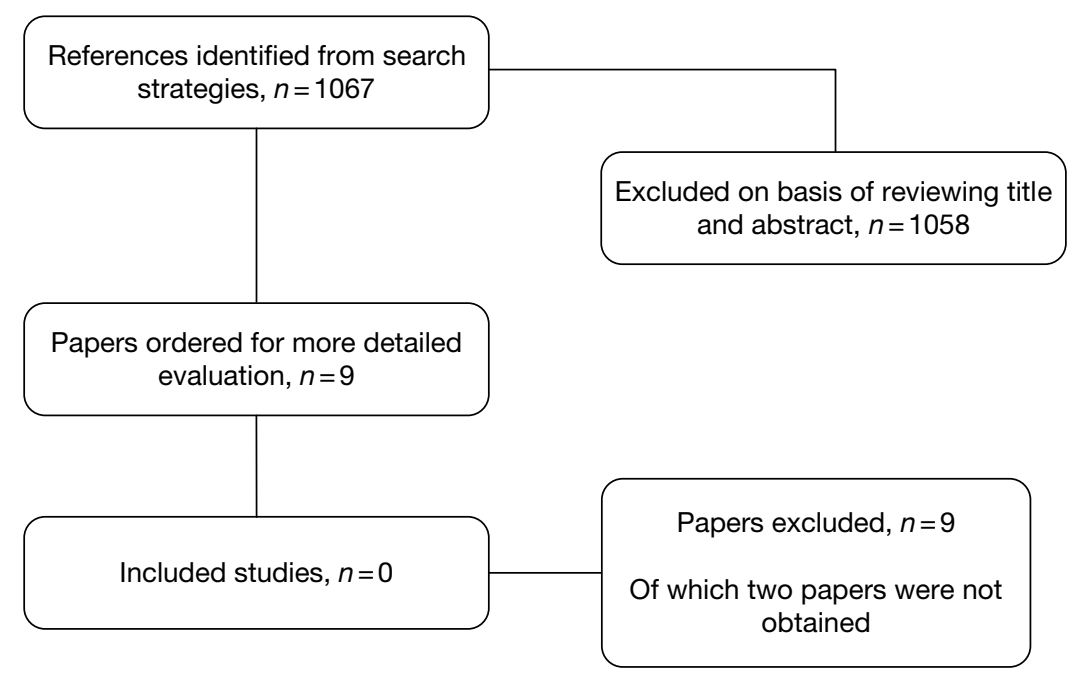

FIGURE 23 Study selection.

\section{Economic analyses}

\section{Previous economic evaluations}

We identified one full economic evaluation, conducted in the Netherlands, that met our inclusion criteria as outlined in Chapter 2 (see Inclusion and exclusion criteria). ${ }^{91}$ The included economic evaluation, a cost-utility analysis, was conducted as part of a clinical study ${ }^{40}$ comparing HGMT with LGMT in a Dutch frozen shoulder population. The clinical study, reported in a separate paper, also met the inclusion criteria for the effectiveness review. ${ }^{40}$ In summary, the clinical study randomised 100 patients to either HGMT or LGMT. Patients were treated twice weekly for 30 minutes during a period of 12 weeks and were encouraged to attend all treatment sessions. From 6 weeks onward, treatment could be reduced in frequency or stopped if a normal range of movement was noticed by the therapist. The study assessed a number of clinical outcomes including active and passive range of movement, shoulder disability (measured by a shoulder rating questionnaire and the SDQ), pain and quality of life (measured by the SF-36). For a full assessment of the study details and quality see Physical therapy. The study was considered to be of reasonable quality, with some risk of bias.

The aim of the economic evaluation component of the study was to compare the two techniques in terms of costs and QALYs from a societal perspective. In addition, the authors also estimated the impact of frozen shoulder on costs and health. Costs were assessed over the 12-month follow-up period and presented as undiscounted annual costs in euros for the price year 2004. All resource-use information was collected from the patients using quarterly cost questionnaires. Wherever possible Dutch standard prices, which were designed to reflect social costs and standardise economic evaluations, were used. Where standard prices were not available, charges were used. Costs included treatment sessions, alternative medicine sessions, hospitalisations (MUA, acromioplasty), home nursing care, medication, travel costs and non-health-care costs (labour and domestic help). All appropriate costs appear to have been included for a societal perspective.

The measure of benefit used in the analysis was QALYS; these were based on the Short Form questionnaire-6 Dimensions (SF-6D) utility index values, which were estimated using SF-36 data collected alongside the study. The SF-6D provides a means for using the SF-36 data to estimate a preference-based single index measure for health using general population values, thereby allowing the authors to obtain QALYs. 
Differences in outcome measures (SF-6D and costs) were tested using double-sided nonparametric bootstrapping. The average estimated QALYs were 0.695 for HGMT and 0.702 for LGMT. The difference of 0.007 in favour of LGMT was reported to be not statistically significant $(p=0.71,95 \% \mathrm{CI}-0.32$ to 0.049$)$. The total reported average annual societal costs were $€ 8809$ for HGMT and $€ 6911$ for LGMT (a cost difference of $€ 1898$ in favour of LGMT). The difference in costs was also reported to be not statistically significant $(p=0.37,95 \% \mathrm{CI}-€ 2551$ to $€ 5711$ ). The authors concluded that the economic analysis does not allow for evidence-based recommendation regarding the preferred treatment.

A full assessment of the quality of this economic evaluation based on the Drummond checklist and the study data extraction/summary are presented in Appendices 11 and 12 respectively. There were a number of limitations to the study including the lack of an incremental analysis, which would not have resulted in an incremental cost-effectiveness ratio (ICER) but would have informed us that LGMT dominates HGMT (i.e. has higher effectiveness and lower costs). The authors dismiss the difference in QALYs and costs based on statistical inference, which is inappropriate. Further probabilistic sensitivity analysis would have allowed parameter uncertainty to be fully characterised and the expected value of further research to be considered. Overall, despite the limitations the analysis was of reasonable quality and the results, although uncertain, provide us with an indication that LGMT may be a more cost-effective option than HGMT.

Although this one economic evaluation provides us with some information regarding the cost-effectiveness of these two physiotherapy techniques, it does not fully address the question posed. The study was conducted in the Netherlands, was limited to two active physiotherapy techniques, failed to appropriately deal with uncertainty and presented utilities using SF-6D, which cannot easily be compared with the preferred EQ-5D measure because of differences in the descriptive systems and values applied to health states. EQ-5D has become the instrument of choice for many agencies including NICE. ${ }^{64}$ The use of one instrument allows more comparability across projects undertaken by these agencies. The small amount of evidence that we are able to glean from this analysis is useful but, given the lack of economic evaluations for other relevant interventions, to further develop and inform decision-making in the UK it was felt that it was essential for us to undertake some additional work.

\section{Decision model}

Based on the preliminary examination of the clinical effectiveness data it was anticipated that any modelling undertaken was likely to be in the form of a simple decision tree. As a tool for modelling decision trees provide an effective method for structuring a problem and combining data from various sources. Possible treatment pathways are laid out in a linear manner and appropriate costs and effects are assigned. The final outcome of interest, in this instance QALYs, is attached to the end of the tree. The proportion of participants achieving particular end points is calculated to give a measure of effect. Costs can be attached to both the end points and the treatments/events within the tree. Total costs for each intervention are calculated by summing the costs associated with each pathway. At each point at which a choice is available there is a chance node; a probability conditional on the previous event is attached to each chance node. This node determines the proportion of individuals who progress down each unique pathway in the tree. A simple example is shown in Figure 24.

We reviewed the literature to identify existing models in a similar population and sequences or order of treatments, to explore whether treatments (or sequences of treatments) were influenced by phase of condition and to identify any potential source data (e.g. unit costs, resources or utilities) to help populate the model. 


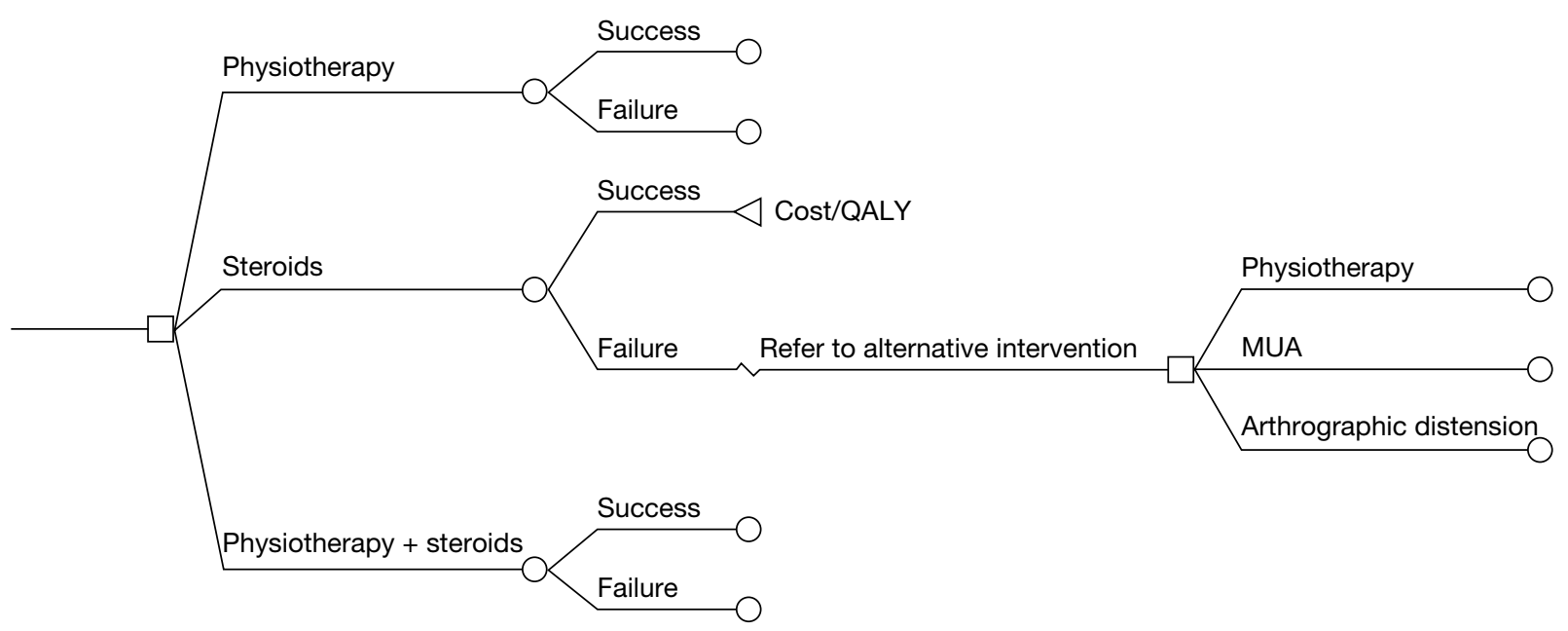

FIGURE 24 Example decision tree.

As outlined earlier, we identified one economic evaluation as part of a clinical study; we did not identify any modelling studies in this area. Further, very few of the trials in the review of effectiveness consider phase of frozen shoulder or previous treatment history of participants. Because of the limited data available, it was not possible in the clinical synthesis to investigate the impact of phase of frozen shoulder on treatment effectiveness. The only potential 'proxy' for phase of condition was duration of frozen shoulder at baseline. The uncertainty associated with such an assumption would need to be carefully considered when drawing conclusions as there is a considerable individual variability in the duration of each phase: it is suggested that the painful freezing stage may last from 2 to 9 months, the adhesive phase from 4 to 12 months and the resolution phase from 12 to 42 months. It was felt that the poor reporting in many of the clinical trials made it difficult to support the assumption that mean duration of frozen shoulder at baseline could be used as a proxy for stage of condition.

Supplementary examination of the available literature failed to provide any clear indication as to potential treatment pathways or treatment sequences. The recent survey of UK health-care professionals suggested that there is a fairly constant view that treatments should depend on the phase of the condition and/or that a step-up approach should be adopted in terms of the degree of treatment invasiveness. ${ }^{18}$ With this in mind the clinical advisory group were presented with two alternative modelling scenarios that were thought to be potentially feasible. These were discussed in depth at a group meeting. Option one involved comparing all possible treatments for each phase of the condition (painful, adhesive and resolution) as mutually exclusive options, ignoring any treatment sequencing and phase of condition. This was considered unrepresentative of clinical practice where treatments would often be given simultaneously or in sequence. The results of this type of model would be uninformative and misleading. Option one was rejected as a viable modelling scenario. Option two involved sequencing treatments dependent on their invasiveness. This would potentially allow treatments such as physiotherapy followed by steroid injections followed by MUA to be evaluated. In essence, all treatments, in all possible sequence order, would be considered. This modelling option required an assumption that the clinical effectiveness of each treatment, as obtained from the included clinical trials, would remain the same regardless of where it was placed in a sequence of treatments or what treatments had been received before it. Again, it was the view of the advisory group that this was not a realistic assumption and in fact the effectiveness of treatments is likely to be linked to the position of the treatment in the sequence. The general consensus within the advisory group surrounding this issue was that the assumptions that would be required were, from a clinical perspective, inappropriate. 
Given the lack of relevant data from the trials included in the review, the consensus from the advisory group that the required assumptions were clinically inappropriate and the confirmatory position regarding the lack of consensus on recommended treatment pathways in recently published articles, ${ }^{18}$ it was decided that it was not feasible or useful in this instance to construct a decision-analytic model. In fact, it was perceived that any attempt to undertake modelling would provide hugely uncertain results, which would not allow any conclusions to be made.

Given the lack of UK data on physiotherapy from the identified economic evaluation ${ }^{91}$ and the lack of economic evaluations of the other relevant interventions we opted to elicit from our clinicians on the advisory group details of the resource use associated with the modalities identified in the review for a frozen shoulder population. The available evidence was then used to obtain utility valuations, which we present as exploratory cost-effectiveness analyses. Our intention, had a de novo model been developed, was to undertake the analysis from an NHS perspective. This perspective has been maintained for the investigative costing exercise. Resources estimated have been valued using national average unit costs (pounds sterling) at 2009-10 prices where possible. Resource use, unit costs and total cost are presented separately, where possible, for each individual treatment. The estimation of resource use gives us only part of the evidence requirements for an economic analysis. The presentation of cost data alone should allow some conjecture about the economic implications of alternative treatments, but does not provide the information required to inform a decision on the cost-effectiveness of these alternatives. To help inform such decisions, the cost estimates obtained from this exercise will be combined with our derived health-related quality of life (HRQoL) estimates (see Mapping from the pain VAS and SF-36 onto the EQ-5D) and presented as ICERs. An ICER is calculated as the difference in the expected cost of two interventions, divided by the difference in the expected QALYs produced by the two interventions. To inform which therapies are of greatest clinical as well as economic value, cost-effectiveness results are usually compared against an acceptable threshold of cost-effectiveness to determine whether a health-care intervention is cost-effective, and a good use of resources, or one that represents poor value for money. The cost-effectiveness threshold for interventions in the English and Welsh NHS is suggested by NICE to be $£ 30,000$ per additional QALY; therefore, we have used this threshold. ${ }^{92}$

The following sections will outline:

1. resource-use estimation and costing

2. mapping from clinical outcomes to EQ-5D

3. estimation of QALYs and ICERs.

\section{Resource-use estimation}

\section{Steroid injections}

Six RCTs investigated steroid injections in the treatment of primary frozen shoulder, either alone or in combination with physical therapy ${ }^{35,41,42,66-68}$ Steroid injections can be delivered by a number of different practitioners using different approaches. We have broken these down into a guided injection delivered by an orthopaedic surgeon, a rheumatologist or a radiologist in a hospital setting; unguided injection delivered by a physiotherapist or a GP in a non-hospital setting; and unguided injection delivered by a physiotherapist, an orthopaedic surgeon or a rheumatologist in a hospital setting. Given the nature of the condition and the individual nature of treatments, it was not possible to be precise about the number of injections that an average individual may receive. It was also suggested by our advisory group that the number of patients receiving guided injection within the NHS was likely to be low. 


\section{Unguided injections}

The clinical experts suggested that it would be rare for individuals to receive more than three injections and on average they would receive two injections spread over various durations of time. Table 57 provides the unit costs of the resources that are used when delivering a steroid injection outside of a hospital setting. We have not included the cost of consumables as these costs are likely to be negligible. The six trials evaluating injections identified in the review of effectiveness assess the use of dexamethasone alone or in conjunction with lidocaine, or triamcinolone alone or in conjunction with lidocaine. We have elected to present costs for both types of steroid although both of the UK trials identified in the review evaluated triamcinolone. ${ }^{41,67}$

Alternative scenarios for the delivery of unguided steroid injections were considered and full details of these are presented in Appendix 13. The base case considered three sessions, an initial session of 20 minutes' duration when an assessment would be undertaken plus two injection sessions of 10-20 minutes' duration. Using an average of 15 minutes for the two injection sessions, and assuming a combined injection of triamcinolone and lidocaine, the total cost if delivered by a physiotherapist is $£ 36.18$. The total cost if delivered by a GP is $£ 108.68$. The choice of steroid injection for the base case was based on the UK trials identified as part of the effectiveness review. ${ }^{41,67}$ The use of a premixed ready-to-inject vial of dexamethasone plus lidocaine would increase the total cost to $£ 39.06$ if delivered by a physiotherapist and $£ 111.56$ if delivered by a GP.

Unguided injections are also delivered within a hospital setting. We further estimated that the costs associated with unguided injections in the hospital setting would be $£ 27.17$ if delivered in a hospital physiotherapy unit, $£ 88.01$ if delivered in an orthopaedic setting and $£ 138.51$ if delivered in a rheumatology setting.

\section{Guided injections}

Guided injections were assumed to be delivered in a hospital setting by key personnel including an orthopaedic surgeon, a rheumatologist or a radiologist. We estimated that the same number of sessions would be required, using the same type of steroid. The variation in the cost of the guided injections is driven by who delivers the injection. For the base case we have again used the cost of a combined injection of triamcinolone and lidocaine, but present the cost of the premixed dexamethasone plus lidocaine to allow a range to be expressed. We have also assumed that on average two injections will be given over three visits. Alternative scenarios showing varying resource-use levels (suggested alternative numbers of sessions/injections) are presented in Appendix 13. Unit costs for guided injections are presented in Table 58.

TABLE 57 Unit costs for unguided steroid injections

\begin{tabular}{lll}
\hline Unguided injection & Unit cost & Source \\
\hline GP & $£ 35$ per consultation & Curtis $2009^{93}$ \\
Physiotherapist & $£ 39$ per hour & Curtis $2009^{93}$ \\
Dexamethasone $40 \mathrm{mg} /$ lidocaine $10 \mathrm{mg}$ & $£ 3.28$ per vial & BNF \\
Dexamethasone $40 \mathrm{mg}$ & $£ 1.00$ per vial & BNF $^{94}$ \\
Triamcinolone $20 \mathrm{mg}$ & $£ 1.49$ per vial & BNF $^{94}$ \\
Lidocaine $5 \mathrm{ml}$ & $35 p$ & BNF $^{94}$ \\
\hline
\end{tabular}

BNF, British National Formulary.

Note: Unit costs ${ }^{93}$ for key health-care personnel reflect average values. Data were not available by staff grade level, e.g. physiotherapist grade 6 or above. 
TABLE 58 Unit costs for guided steroid injections

\begin{tabular}{lll}
\hline Guided injection & Unit cost & Source \\
\hline Orthopaedic outpatient visit, first & $£ 124$ & Department of Health $^{95}$ \\
Orthopaedic outpatient visit, follow-up & $£ 86$ & Department of Health $^{95}$ \\
Rheumatology outpatient visit, first & $£ 211$ & Department of Health $^{95}$ \\
Rheumatology outpatient visit, follow-up & $£ 129$ & Department of Health $^{95}$ \\
Dexamethasone $40 \mathrm{mg} /$ /idocaine $10 \mathrm{mg}$ & $£ 3.28$ per vial & BNF $^{94}$ \\
Dexamethasone $40 \mathrm{mg}$ & $£ 1.00$ per vial & BNF $^{94}$ \\
Physiotherapist outpatient attendance, first & $£ 38$ per hour & Department of Health $^{95}$ \\
Physiotherapist outpatient attendance, follow-up & $£ 29$ per hour & Department of Health $^{95}$ \\
Triamcinolone $20 \mathrm{mg}$ & $£ 1.49$ per vial & BNF $^{94}$ \\
Lidocaine $5 \mathrm{ml}$ & $35 p$ & BNF $^{94}$ \\
\hline
\end{tabular}

BNF, British National Formulary.

Note: Unit costs ${ }^{95}$ for key health-care personnel reflect average values. Data were not available by staff grade level.

The total cost of injections delivered within the orthopaedic setting ranges from $£ 299.68$ to $£ 302.56$ depending on the steroid injected. Injections delivered in the rheumatology setting have a total cost ranging from $£ 472.68$ to $£ 475.56$. These alternative delivery settings have been presented as it is believed that these are all viable options depending on available NHS services within a local area.

\section{Steroid injection plus physiotherapy}

The resource use associated with the combined treatment of steroid injection plus physiotherapy is again related to the setting in which the treatments are given and the method of steroid delivery. A number of scenarios are presented (see Table 59 for relevant unit costs):

- Scenario one: six physiotherapy sessions with an active mobilisation component and two unguided steroid injections all delivered by a physiotherapist. It has been assumed that no additional time was required and the total cost is equal to the total cost of active physiotherapy plus the steroid injections. The total cost ranges from $£ 130.43$ to $£ 133.31$ if delivered by a community physiotherapist, or from $£ 121.43$ to $£ 126.81$ if delivered by a hospital-based physiotherapist, depending on choice of steroid.

- Scenario two: six physiotherapy sessions with an active mobilisation component delivered by a community-based physiotherapist, plus two unguided steroid injections delivered by a GP over three visits. The total cost of this scenario ranges from $£ 235.75$ to $£ 238.75$, depending on choice of steroid.

- Scenario three: six physiotherapy sessions with a mobilisation component delivered by a hospital-based physiotherapist, plus two unguided steroid injections delivered by a GP over three visits. The total cost of this scenario ranges from $£ 226.75$ to $£ 238$, depending on choice of steroid.

- Scenario four: six physiotherapy sessions with a mobilisation component delivered by a community-based physiotherapist, plus two guided steroid injections delivered within the orthopaedic setting. The total cost ranges from $£ 420.23$ to $£ 423.31$, depending on choice of steroid.

- Scenario five: six physiotherapy sessions with a mobilisation component delivered by a community-based physiotherapist, plus two guided steroid injections delivered within the rheumatology setting. The total cost ranges from $£ 605.43$ to $£ 607.31$, depending on choice of steroid. 
- Scenario six: six physiotherapy sessions with an active mobilisation component, plus two guided steroid injections delivered by a hospital-based physiotherapist. The total cost ranges from $£ 160.43$ to 165.81 , depending on choice of steroid.

Alternative scenarios could be estimated by varying the number of injections, delivery method and setting. However, the base-case scenarios presented reflect what we believe are current options in the NHS.

\section{Sodium hyaluronate injection}

Three trials evaluating sodium hyaluronate were identified in the effectiveness review. ${ }^{66,69,70}$ In a recent UK-based survey of treatment recommendations by health-care professionals involved in managing frozen shoulder, sodium hyaluronate injection was recommended by $<1 \%$ for treating the early 'painful' phase or 'resolution' phase of frozen shoulder. ${ }^{18}$ It was further suggested by the clinical advisory group that one injection would typically be delivered over two sessions in a hospital setting. The injection was likely to be delivered by a consultant. However, the costs were not available for sodium hyaluronate for provision under the NHS. It was felt that this intervention was likely to be delivered in a hospital setting, but we were unable to ascertain any costs associated to its delivery that would be borne by the NHS.

\section{Physical therapy}

The broader range of physical therapies are not available through the NHS and therefore have not been costed. Two categories of physiotherapy were costed, physiotherapy with mobilisation (active) and physiotherapy without mobilisation. It was felt by the advisory group that physiotherapy without mobilisation would not be delivered on its own in an NHS setting, but may be used to complement another therapy. Therefore, for the purpose of resource-use estimation, physiotherapy without mobilisation has been considered only as an add-on delivered alongside other therapies.

\section{Active physiotherapy}

It was estimated by the advisory group that physiotherapy with a mobilisation component would on average comprise six sessions each of 30 minutes' duration, followed by a final review session of 15 minutes' duration. Full details of all estimates of resource use and any plausible ranges are presented in Appendix 13.

As can be seen from Table 59, the cost of physiotherapy is dependent on the setting in which the treatment is delivered. We have used all variations in hourly cost to calculate a plausible range of total cost for the delivery of active physical therapy. Within a community setting, active physical therapy is estimated to cost a total of $£ 126.75$. Within a hospital setting the same treatment is estimated to have an average total cost of $£ 98.75$.

\section{Physiotherapy without mobilisation}

The review of effectiveness identified one trial that was classified as 'physiotherapy without mobilisation. ${ }^{16}$ The trial evaluated laser versus placebo laser (plus home exercise). Given that

TABLE 59 Unit costs for active physiotherapy

\begin{tabular}{lll}
\hline Staff delivering treatment & Unit cost & Source \\
\hline Community physiotherapist & $£ 39$ per hour & Curtis 200993 \\
Physiotherapist outpatient attendance, first & $£ 38$ per hour & Department of Health \\
Physiotherapist outpatient attendance, follow-up & $£ 29$ per hour & Department of Health \\
\hline
\end{tabular}

Note: Unit costs ${ }^{93,95}$ for key health-care personnel reflect average values. Data were not available by staff grade level. 
it is unlikely that laser therapy would be delivered without some form of mobilisation (active physiotherapy) within the NHS, we have assumed that laser therapy would be provided in conjunction with an active mobilisation, adding an additional 15 minutes to a 20 - to 30-minute session. The additional cost reflects what we believe is current practice, that is, the delivery of both active mobilisation and other passive modalities such as heat treatment in the same treatment session. The additional cost would be either $£ 49.38$ or $£ 63.38$ depending on the setting.

\section{Acupuncture}

Three trials evaluating acupuncture were identified in the effectiveness review. ${ }^{79-81}$

It was felt by the clinical advisory group that acupuncture, like other physiotherapies, would in clinical practice rarely be given in isolation of other treatments. We have therefore assumed that acupuncture would be delivered in physiotherapy sessions, in either a hospital or a community setting, over the same duration as active physiotherapy, that is, six sessions of 20-30 minutes' duration at a total cost ranging from $£ 117.75$ to $£ 126.75$.

\section{Manipulation under anaesthesia}

The review identified four RCTs investigating MUA in the treatment of primary frozen shoulder. ${ }^{38,39,82,83}$ The resource implications of MUA were difficult to firmly establish. The actual cost of MUA has been estimated at $£ 424$ (range $£ 191-500$ ) based on the HD24C code, which was obtained from the NHS Reference Costs. ${ }^{95}$ In addition, there is a need for extensive rehabilitation physiotherapy, which was estimated on average to consist of two 30-minute sessions per week for 4 months, plus a final review, which would take place with the orthopaedic surgeon. Therefore, in total, the estimated cost of MUA is $£ 1446$. If steroid injections are also used this cost would increase accordingly.

\section{Arthrographic distension}

Three trials evaluating arthrographic distension with steroid injection were identitied in the effectiveness review. ${ }^{36,43,84}$ Arthrographic distension is assumed to be delivered in the hospital setting by a radiologist or an orthopaedic surgeon and involves the use of radiological imaging (e.g. fluoroscopy) to ensure that the injection is accurately placed. The procedure was assumed to require around 15 minutes based on the clinical literature. Because there are no UK trials in the clinical review it is unclear how many visits for injections might be needed. We have therefore conservatively estimated the cost as involving one hospital outpatient visit with injection. The estimated cost of this procedure has been derived from the NHS Reference Costs ${ }^{95}$ at $£ 113$ (range

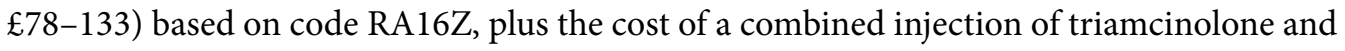

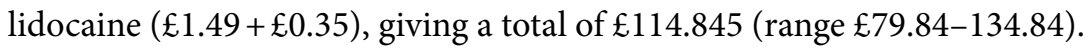

\section{Capsular release}

Two studies evaluating capsular release were identified in the effectiveness review. ${ }^{85,86}$ Capsular release is performed in the hospital setting and delivered by a surgical team, which is likely to comprise anaesthetist, orthopaedic surgeon, anaesthetic nurse, scrub nurse and assistant and recovery nurse. The procedure is likely to take $30-45$ minutes, followed by a further 15 minutes for recovery from anaesthetic. The estimated cost of this procedure has been derived from the NHS Reference Costs ${ }^{95}$ at $£ 1182$ (range $£ 787-1489$ ) based on code HB62C. Like MUA, capsular release requires a follow-up of intensive physiotherapy. We have estimated that the physiotherapy following capsular release would be the same as that following MUA. On average this would consist of two 30-minute sessions per week for 4 months, plus a final review that would take place with the orthopaedic surgeon. Therefore, in total, the estimated cost of capsular release is $£ 2204$ (range $£ 1809-2511$ ). 


\section{Summary}

Average costs were estimated for the interventions included in the review. These ranged from $£ 36.18$ for an unguided steroid injection to $£ 2204$ for capsular release. Where possible, these estimated average costs will be used to inform tentative cost-effectiveness ratios, which will be presented later in the report. They will be combined with HRQoL data estimated by means of a mapping exercise, which is presented in the following section. The aim is to estimate HRQoL by mapping from three clinical outcomes to EQ-5D using an available data set. The main focus of the mapping is to explore the nature and direction of the relationship between different clinical and HRQoL outcome measures. We will then use the EQ-5D estimates to obtain HRQoL outcomes for a subset of clinical trials identified in the review that have presented data on the three relevant outcomes.

\section{Mapping from the pain VAS and SF-36 onto the EQ-5D}

The clinical trials identified in the effectiveness review report outcomes using a variety of measures including pain (overall, at rest, on movement, at night), range of movement (e.g. internal and external rotation, elevation), function and disability, quality of life, time to recovery and return to work and recreation, and adverse events. Of the selection of measures used in the trials, none was designed to generate health state utility values, which are required to calculate QALYs, which can then be used to assess cost-effectiveness. Outcomes from treatments affect two basic components - the quantity and quality of life - both of which are captured in the QALY. There are a variety of standardised 'off-the-shelf' preference-based measures available to derive estimates of HRQoL, such as the EQ-5D, SF-6D, Health Utilities Index (HUI) and 15-dimension utility measure (15D). ${ }^{65,96,97,98}$ These HRQoL values can subsequently be incorporated in economic models for performing cost-effectiveness analyses. They are the preferred benefit measure when undertaking any cost-effectiveness analysis.

An additional search of MEDLINE was conducted to identify health quality of life outcomes that could be used to inform any modelling. The lack of use of generic preference-based measures in existing frozen shoulder clinical studies is a potential current barrier to populating economic models with the best evidence on effectiveness and to thereby allow broader comparisons across different diseases.

The included economic evaluation presented HRQoL in the form of the SF-6D, but it was felt that given the general preference for EQ-5D and the possibility of bias in the clinical study further investigation was warranted. The EQ-5D is the recommended instrument of choice for use in cost-effectiveness analyses by NICE and is the most commonly used instrument.

In the context of this clinical review, it is not clear why HRQoL has not been included in the battery of outcome measures in the clinical trials in frozen shoulder populations to date. Possible reasons why such instruments have not been administered may be because it is not practical or they are too time-consuming for patients to complete in trials in addition to all of the other forms/questionnaires that are used - often across multiple time points - or even because studies were not initially designed to focus on economic questions. It may be that the general perception is that these generic instruments are not sensitive enough.

In a situation such as this, in which such instruments have not been used in clinical studies, a pragmatic alternative is to apply a mapping function to convert non-preference-based health data into one of the generic preference-based measures. Mapping essentially provides a statistical equation that allows the estimation of health utilities and the subsequent calculation of QALYs in clinical studies that do not use any preference-based HRQoL instruments. In the published literature, the EQ-5D was recently reported as being the most popular target measure used for mapping. ${ }^{96}$ 
On the other side of the mapping equation, the most widely used generic measures/instruments that have been mapped include the SF-36 and SF-12 health surveys, which offer the option of presenting scores across eight dimensions or two summary measures derived from the eight dimensions. These two summary measures are the physical component and the mental component. ${ }^{99,100}$ Also, a number of shoulder-specific questionnaires (scores) have been shown to be significantly correlated with the EQ-5D in the literature, in particular the SDQ. ${ }^{55}$ Of particular importance in the current clinical effectiveness review were patient-assessed pain intensity, function and disability, quality of life and range of movement. Pain was the most common outcome measure in the included clinical studies and, for instance, in the MTC analysis, pain was analysed based on the following preference hierarchy: overall pain, pain at night, pain on activity, any other type of pain (excluding pain at rest) and pain at rest. Furthermore, and within the context of the current clinical effectiveness review, three of the studies reported the SF-36 as an outcome measure. As mentioned earlier in the clinical sections of the report, two studies meeting the full inclusion criteria reported the mean physical and mental component summary scores. ${ }^{31,47}$ One additional study, which did not meet the analysis criteria, reported the means for the eight dimensions of the SF-36. ${ }^{81}$ The outcomes were reported over the time horizon of 4 weeks and as a result the study was excluded from further analysis. Therefore, in line with the decision made as part of the clinical review we elected not to map from this evidence base, but rather to utilise the potentially more reliable component summary scores that had been reported in the other two trials..$^{35,51}$

In a recent review of mapping studies, simple additive models that employed a utility index score as the dependent variable and the main effects of either total or instrument dimension scores as independent variables performed almost as well as more complex models comprising many variables. ${ }^{99}$ With this in mind, and as the main focus of this analysis was to explore the nature and direction of the relationship between different outcome measures, we chose to follow a simple approach and use mean pain VAS score (as it was a main outcome in the clinical review) and mean SF-36 physical and mental component summary scores (as it is one of the most widely used generic quality-of-life measures in mapping studies). These were important outcomes in the clinical effectiveness review.

To undertake an exploratory mapping analysis of this nature we required a data set in which the two measures being mapped had been administered specifically to a frozen shoulder population. We were unable to obtain a data set of this nature for a frozen shoulder population; however, we obtained a UK data set involving patients with rotator cuff disease from the SAPPHIRE RCT. ${ }^{101}$ Both measures chosen for the exploratory mapping analyses were already calculated and readily available in the SAPPHIRE data set. ${ }^{102}$

The selection of outcomes on which to map was a pragmatic decision based on the data available in the SAPPHIRE data set. The relationship is estimated using a range of techniques and statistical specifications. Furthermore, we compare the mapping approaches tested in terms of their predictive performance in the SAPPHIRE trial population. Within the context of the current review, we conducted an analysis to explore whether or not relationships between the pain VAS and SF-36 and the EQ-5D exist and the nature of those relationships. In doing so, we considered that we might be able to demonstrate the need for the collection of utility EQ-5D data in future clinical trials and offer a potential alternative approach for frozen shoulder populations for whom there is currently a lack of HRQoL data.

The SAPPHIRE trial compared the efficacy of corticsosteroid $(40 \mathrm{mg} / \mathrm{ml}$ triamcinolone acetonide) versus local anaesthetic (1\% lidocaine) injection in subjects presenting to GPs with (acute) shoulder pain (in one or both shoulders) persisting no longer than 12 months, and with diagnosis of rotator cuff tendonitis. ${ }^{102}$ The trial evaluated the impact of giving GPs 
TABLE 60 Patient baseline characteristics and mean reported scores

\begin{tabular}{|c|c|c|c|c|c|c|c|c|}
\hline \multirow[b]{2}{*}{ Study } & \multirow{2}{*}{$\begin{array}{l}\text { Treatment } \\
\text { arms }\end{array}$} & \multirow{2}{*}{$\begin{array}{l}\text { Duration of shoulder } \\
\text { condition }^{\mathrm{a}}\end{array}$} & \multicolumn{2}{|c|}{ Patient characteristics } & \multirow{2}{*}{$\begin{array}{l}\text { EQ-5D } \\
\text { (mean) }\end{array}$} & \multirow{2}{*}{$\begin{array}{l}\text { SF-36 PCS } \\
\text { (mean) }\end{array}$} & \multirow{2}{*}{$\begin{array}{l}\text { SF-36 MCS } \\
\text { (mean) }\end{array}$} & \multirow{2}{*}{$\begin{array}{l}\text { Pain VAS } \\
\text { (mean) } \\
(0-100 \mathrm{~mm})\end{array}$} \\
\hline & & & Age (years) & $\%$ female & & & & \\
\hline $\begin{array}{l}\text { Dogru } \\
\text { (Turkey) }{ }^{51}\end{array}$ & 2 & $\begin{array}{l}\text { Shoulder pain of at least } \\
3 \text { months' duration }\end{array}$ & $\begin{array}{l}53.9-56.8 \\
\text { (55.4 total) }\end{array}$ & 57 (total) & - & $36.6-38.9$ & $42-43.5$ & $78-80.8$ \\
\hline $\begin{array}{l}\text { Stergioulas } \\
\text { (Greece) }^{16}\end{array}$ & 2 & $\begin{array}{l}\text { Duration of frozen } \\
\text { shoulder at baseline of } \\
26.5-27.1 \text { weeks }\end{array}$ & $55.5-56.8$ & 37 & - & - & - & $67.03-70.9$ \\
\hline $\begin{array}{l}\text { Carette } \\
\text { (Canada) }^{35}\end{array}$ & 4 & $\begin{array}{l}\text { Adhesive capsulitis of } \\
<1 \text { year's duration, } \\
\text { mean duration of current } \\
\text { episode of shoulder pain } \\
20.3-22.1 \text { weeks }\end{array}$ & $54.2-56.5$ & $\begin{array}{l}46.2- \\
66.7\end{array}$ & - & $35.2-37.6$ & $43.1-49.8$ & - \\
\hline $\begin{array}{l}\text { SAPPHIRE } \\
\text { trial } \\
(\mathrm{UK})^{102,104}\end{array}$ & 2 & $\begin{array}{l}\text { Shoulder pain persisting } \\
\text { for } \leq 12 \text { months, median } \\
\text { duration of shoulder pain } \\
6.5-8 \text { weeks }\end{array}$ & $55-59$ & $51-55$ & $\begin{array}{l}0.453- \\
0.512\end{array}$ & $\begin{array}{l}35.96- \\
38.89\end{array}$ & $\begin{array}{l}44.64- \\
46.54\end{array}$ & $68.43^{b}$ \\
\hline
\end{tabular}

a Based on (study-specific) condition-related inclusion criteria.

b Not reported in the trial publications and thus estimated from the SAPPHIRE data set for the current analyses.

additional training in diagnosing rotator cuff pathologies and in performing shoulder injections, or no training. Trial participants were followed up at 1 and 3 months, with a final follow-up at 6-12 months post randomisation. The outcome measures were the SF-36, EQ-5D, British $\mathrm{SDQ}^{103}$ and VAS to assess the extent of shoulder pain (at night, in daytime at rest, in daytime on movement).

The population characteristics for the two studies included in the clinical review that reported mean overall pain VAS scores, ${ }^{16,51}$ using a 0 - to 100 - $\mathrm{mm}$ scale, and the two studies included in the clinical review that reported mean SF-36 physical and mental component summary scores ${ }^{35,51}$ are reported in Table 60. The population characteristics of the participants in the SAPPHIRE trial ${ }^{102,104}$ are also shown in Table 60.

The three studies included in the clinical effectiveness review reporting mean pain VAS scores and/or mean SF-36 physical component summary (PCS) and mental component summary (MCS) scores and the SAPPHIRE trial include populations with broadly similar proportions of men and women and of a similar age and duration of shoulder condition or pain and with similar mean pain VAS and SF-36 PCS and MCS scores.

The mapping presented here is an exploratory exercise, based on a patient population with very similar baseline characteristics as the frozen shoulder population. We examine the relationship between the EQ-5D and pain as measured by a VAS 0-100 mm and the EQ-5D and SF-36 (specifically the SF-36 PCS and MCS) health surveys.

\section{Methods}

The following sections present the methods used in estimating HRQoL data to inform our tentative analysis and potentially inform other economic evaluations.

\section{Instruments}

Pain visual analogue scale Pain was measured on a scale from 0 to $100 \mathrm{~mm}$ in both the studies included in the clinical review (see Table 60) and the SAPPHIRE data set. Values anchored on 
the VAS at 0 generally represent no pain whereas values anchored at 100 generally represent the worst pain possible. Both studies have data available as mean values. When more than one type of pain was measured in any single study, data were selected for the mapping exercise based on the pain hierarchy as reported earlier in the clinical sections of this report.

SF-36 The SF-36 questionnaire covers different aspects of HRQoL and the items can be pooled in eight different subscales: physical functioning, role - physical, body pain, general health, vitality, social functioning, role - emotional, and mental health. ${ }^{107}$ Patient scores are rated on a 0 (worst)-100 (best) scale for each subscale. These eight subscales can be pooled in two different clusters: the PCS and the MCS. The calculation of the SF-36 summary scores (PCS and MCS) is based on a published algorithm which uses a standardised scoring system with mean $=50$ and $\mathrm{SD}=10$. The two component summary scores are used for the current regression analyses.

EQ-5D The EQ-5D is a five-item questionnaire encompassing the domains of mobility, self-care, usual activities, pain/discomfort and anxiety/depression. Respondents are asked to indicate whether they have no problems, some problems or major problems in each domain, with responses scored as 1,2 and 3 respectively. To produce a combined utility health score, in which 1 is full health and 0 is equivalent to being dead, individuals' answers about the extent of their problems in each of the five health domains are then weighted. The weights, based on a large UK study, are used to take into consideration population preferences for each health domain. ${ }^{65}$ The resulting descriptive system defines 243 health states. The worst-valued health state is -0.594 (worse than death), whereas the best-valued health state is 1.0 (perfect health).

\section{Data set}

We used the SAPPHIRE trial data set and measurements at 3 months for the primary (base-case) analysis. The number of participants with complete outcomes data at 3 months was (1) 141 for the EQ-5D and pain VAS and (2) 133 for the EQ-5D and SF-36 PCS and MCS. The time point of 3 months was chosen as it represented a clinically relevant time point in the frozen shoulder trials included in this review. The data sets for (1) and (2) were then each divided into two by taking a random sample of $60 \%$ of the data and separating that data from the remaining $40 \%$ to provide an estimation data set and a validation data set respectively. In the case of (1), the EQ-5D and pain VAS, 85 measurements were available for the estimation data set and 56 measurements for validation. For (2), the EQ-5D and SF-36 PCS and MCS, 80 measurements were available for the estimation data set and 53 measurements for validation.

It should be noted that the collection of new data to validate a model's predictions is the preferred approach. ${ }^{108}$ However, in many situations this is neither practical nor possible and an alternative approach is to split the data in hand into two parts. The first part is used to estimate the model coefficients (estimation sample) and the second part is used to assess the prediction accurary of the model (validation sample). Thus, data splitting provides a data set to measure the 'in-use' (or within-sample) prediction accuracy of the model and simulates the complete or partial replication of the data set, in this case the SAPPHIRE trial data set.

\section{Statistical analysis}

Econometric estimation methods tested Ordinary least squares (OLS) regression was used as the main statistical technique in the analysis. The target outcome (dependent variable) was the overall EQ-5D index score, and the explanatory (independent) variables were (1) the pain VAS and (2) the SF-36 PCS and MCS scores. Based on the theoretical limitations of OLS modelling approaches reported in the empirical econometric literature, ${ }^{100,109,110}$ the current exploratory investigation estimates the EQ-5D index scores also using TOBIT (an ecometric model named after its developer James Tobin, ${ }^{111}$ which allows for upper censoring of the dependent variable, 
EQ-5D, at 1.0; a caveat is that it produces biased estimates in the presence of heteroscedasticity or non-normality) and CLAD (censored least absolute deviations, which produces consistent estimates in the presence of heteroscedasticity or non-normality, whereas also allowing for censoring at either the upper or the lower bound).

Regression analyses All regressions were conducted in Stata version 11 (StataCorp LP, College Station, TX, USA) and specifically included the estimation of the following models and included independent variables. The general regression models for (1) the EQ-5D and pain VAS and (2) the EQ-5D and SF-36 PCS and MCS were defined as:

$$
\mathrm{EQ}-5 \mathrm{D}_{\mathrm{i}}=\alpha+\beta_{1} \times{\text { pain } V A S_{\mathrm{i}}+\varepsilon_{\mathrm{i}}}
$$

[Equation 1]

where EQ-5D represents the EQ-5D health state index value, $\mathrm{i}=1,2, \ldots, n$ represents individual respondents, painVAS represents the pain score, $\alpha$ is the intercept and $\varepsilon$ is the error term, and

$$
\mathrm{EQ}-5 \mathrm{D}_{\mathrm{i}}=\alpha+\beta_{1} \times P C S_{\mathrm{i}}+\beta_{2} \times M C S_{\mathrm{i}}+\varepsilon_{\mathrm{i}}
$$

[Equation 2]

where EQ-5D represents the EQ-5D health state index value, $\mathrm{i}=1,2, \ldots, n$ represents individual respondents, PCS represents the SF-36 PCS score, MCS represents the SF-36 MCS score, $a$ is the intercept and $\varepsilon$ is the error term. Variations of the first model were explored by including squared terms. Variations of the second model were explored by including squared and interaction terms.

We conducted three sets of analyses using alternative approaches relating to the choice of estimation and validation samples from the main SAPPHIRE data set:

- Regression models using main effects with and without squared terms and interaction term (using individual-level data at 3 months):

- for (1), EQ-5D and pain VAS, total $n=141$, estimation data set $=85(60 \%)$, validation data set $=56(40 \%)$

- for (2), EQ-5D and SF-36 PCS and MCS, total $n=133$, estimation data set $=80(60 \%)$, validation data set $=53(40 \%)$.

- Regression models using main effects with and without squared terms and interaction term (using individual-level data at 1, 3 and 12 months):

- for (1), EQ-5D and pain VAS, total $n=491$, estimation data set $=295$ (60\%), validation data set $=196$

- for (2), EQ-5D and SF-36 PCS and MCS, total $n=467$, estimation data set $=280(60 \%)$, validation data set $=187$.

Data for all time points (1, 3 and 12 months) were pooled to increase the sample size and the statistical precision of the estimates with correction for the clustering of several responses per patient by using the option 'cluster' in the regress command in Stata version $11 .{ }^{112}$

- Prediction models using the main effects with and without squared terms and interaction term (using models estimated from 3-month data and used to predict EQ-5D scores at 12 months):

- for (1), EQ-5D and pain VAS, validation data set $=171$

- for (2), EQ-5D and SF-36 PCS and MCS, validation data set $=163$.

Assessment of models' performance Model fit (explanatory performance) and predictive/ mapping performance were based on assessments of the adjusted $R^{2}$, mean error (ME), mean absolute error (MAE) and root-mean-square error (RMSE), as well as the percentage of individual predictions within $0.10,0.05$ and 0.01 of their actual EQ-5D utility values (arbitrary 
cut-off values). Descriptive statistics and plots of actual versus predicted EQ-5D scores were also generated to examine the pattern of errors across EQ-5D scale, for example to assess whether they were evenly distributed across poor/better health states.

\section{Results from the mapping exercise \\ Pattern of EQ-5D, pain visual analogue scale and SF-36 PCS and MCS scores}

EQ-5D and pain visual analogue scale The mean EQ-5D scores (ordered according to health state severity) in the SAPPHIRE population vary over a wide range (e.g. from negative utility values to 'perfect health' as indicated by utility values of 1.0; Table 61 and Figure 25). In contrast, the SAPPHIRE trial mean pain VAS scores vary over a narrower range (e.g. highest mean VAS score $=60$, when a maximum pain VAS of 100 is possible). An increasing EQ-5D score (i.e. better health status) appears to be generally accompanied by decreasing pain VAS scores (i.e. lower scores $=$ less pain).

EQ-5D and SF-36 PCS and MCS Figure 26 and Table 62 show that mean EQ-5D scores (ordered according to health state severity) in the SAPPHIRE population vary over a wide range. In contrast, the SAPPHIRE trial mean SF-36 PCS and MCS scores vary over a relatively narrow range. An increasing EQ-5D score (i.e. better health status) appears to be generally accompanied by increasing SF-36 PCS and MCS scores (i.e. higher scores = a better quality of life).

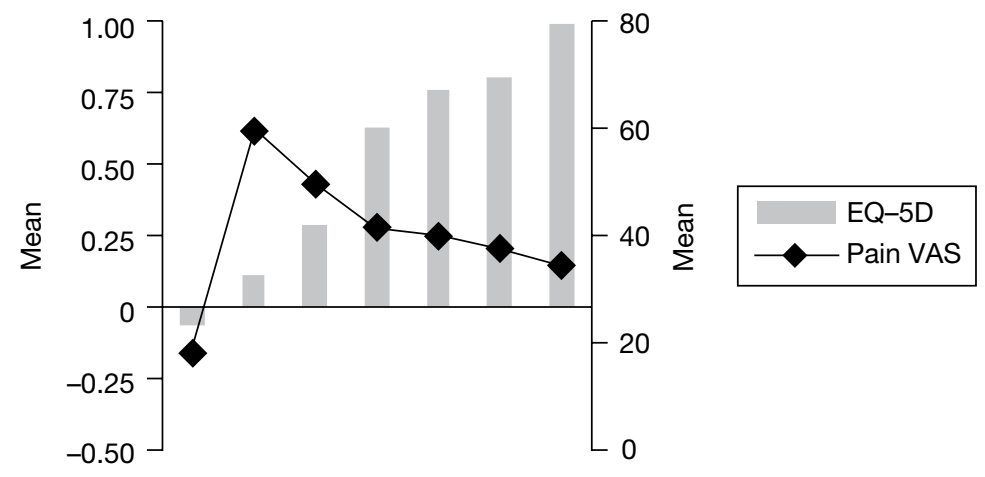

FIGURE 25 Pattern of EQ-5D scores and pain VAS scores within the SAPPHIRE data set (for complete responses) at 3 months' follow-up (base case).

TABLE 61 Pattern of EQ-5D scores and pain VAS scores within the SAPPHIRE data set (with complete responses at 3 months)

\begin{tabular}{lrll}
\hline EQ-5D grouping & \multicolumn{1}{c}{$\boldsymbol{n}$} & EQ-5D (mean) & Pain VAS (mean) \\
\hline$<0$ & 8 & -0.05 & 18.13 \\
$0-0.249$ & 15 & 0.08 & 60 \\
$0.25-0.499$ & 1 & 0.26 & 50 \\
$0.5-0.699$ & 52 & 0.63 & 41.87 \\
$0.7-0.799$ & 24 & 0.75 & 40.17 \\
$0.8-0.899$ & 26 & 0.83 & 38.88 \\
$0.9-1.0^{a}$ & 15 & 1.0 & 34.67 \\
Full index & 15 & 1.0 & 34.67 \\
Overall & 141 & 0.63 & 37.63 \\
\hline
\end{tabular}

a All values were equal to 1.0 , i.e. full index. 
TABLE 62 Pattern of EQ-5D scores and SF-36 PCS and MCS scores within the SAPPHIRE data set (with complete responses at 3 months)

\begin{tabular}{lccll}
\hline EQ-5D grouping & $\boldsymbol{n}$ & EQ-5D (mean) & SF-36 PCS (mean) & SF-36 MCS (mean) \\
\hline$<0$ & 10 & -0.07 & 35.96 & 45.70 \\
$0-0.249$ & 18 & 0.11 & 39.18 & 47.35 \\
$0.25-0.499$ & 1 & 0.26 & 36.64 & 41.14 \\
$0.5-0.699$ & 44 & 0.63 & 39.68 & 42.01 \\
$0.7-0.799$ & 21 & 0.75 & 44.07 & 50.23 \\
$0.8-0.899$ & 28 & 0.82 & 42.91 & 53.2 \\
$0.9-1.0^{a}$ & 0 & NA & NA & A \\
Full index & 11 & 1.0 & 40.68 & 48.95 \\
Overall & 133 & 0.59 & 40.79 & 47.21 \\
\hline
\end{tabular}

NA, not applicable (no values fell within this grouping).

a All values were equal to 1.0, i.e. full index.
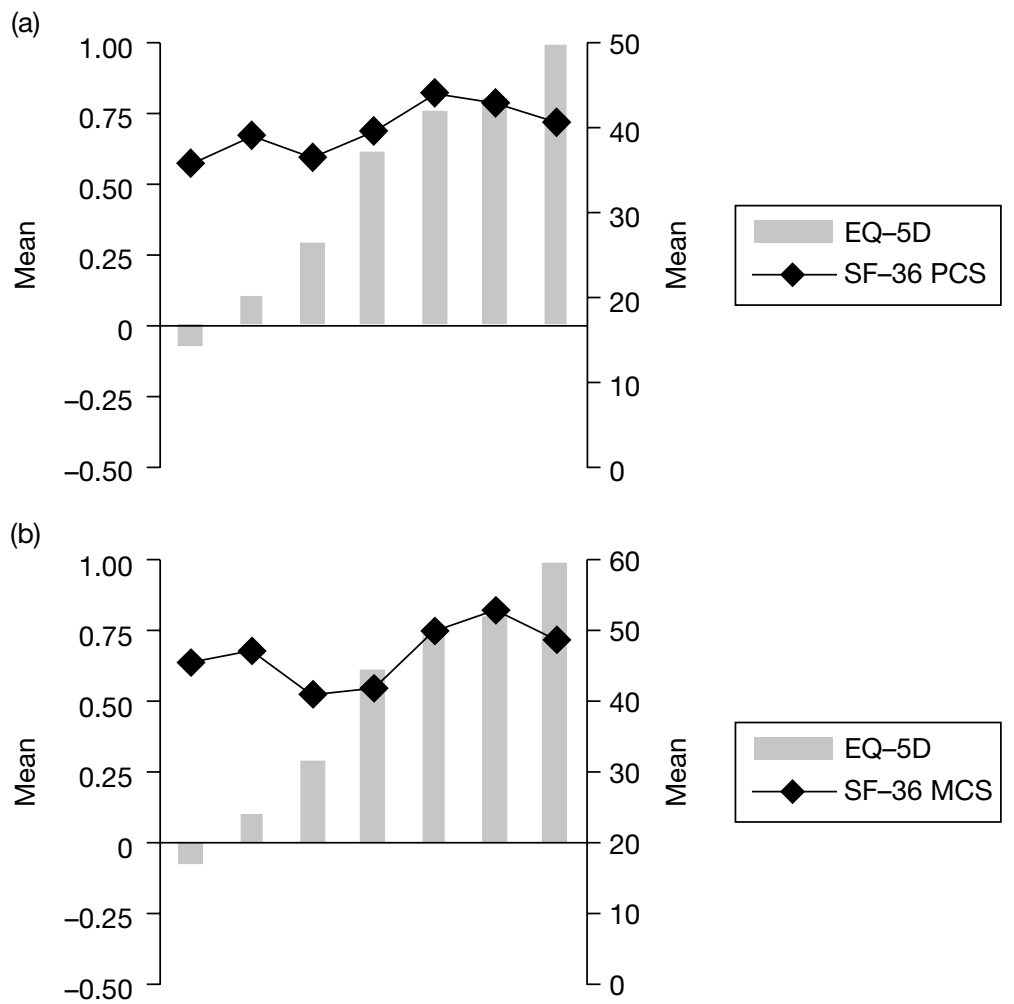

FIGURE 26 Pattern of EQ-5D scores and SF-36 PCS and MCS scores within the SAPPHIRE data set (for complete responses) at 3 months' follow-up (base case).

\section{Regression analyses}

EQ-5D and pain visual analogue scale Table 63 shows the results of the regression of the pain VAS scores onto the EQ-5D using the 3-month data set (base case). The explanatory power and fit of both OLS models was poor, with an adjusted $R^{2}$ ranging from -0.0014 to 0.0081 . The TOBIT and CLAD models including main effects and squared terms were still relatively poor although they produced the smallest MAEs ( 0.2158 and 0.1877 respectively) compared with the two OLS models. However, all models predicted only between $16 \%$ and $23 \%$ of scores to within 0.05 of actual EQ-5D utility values. 


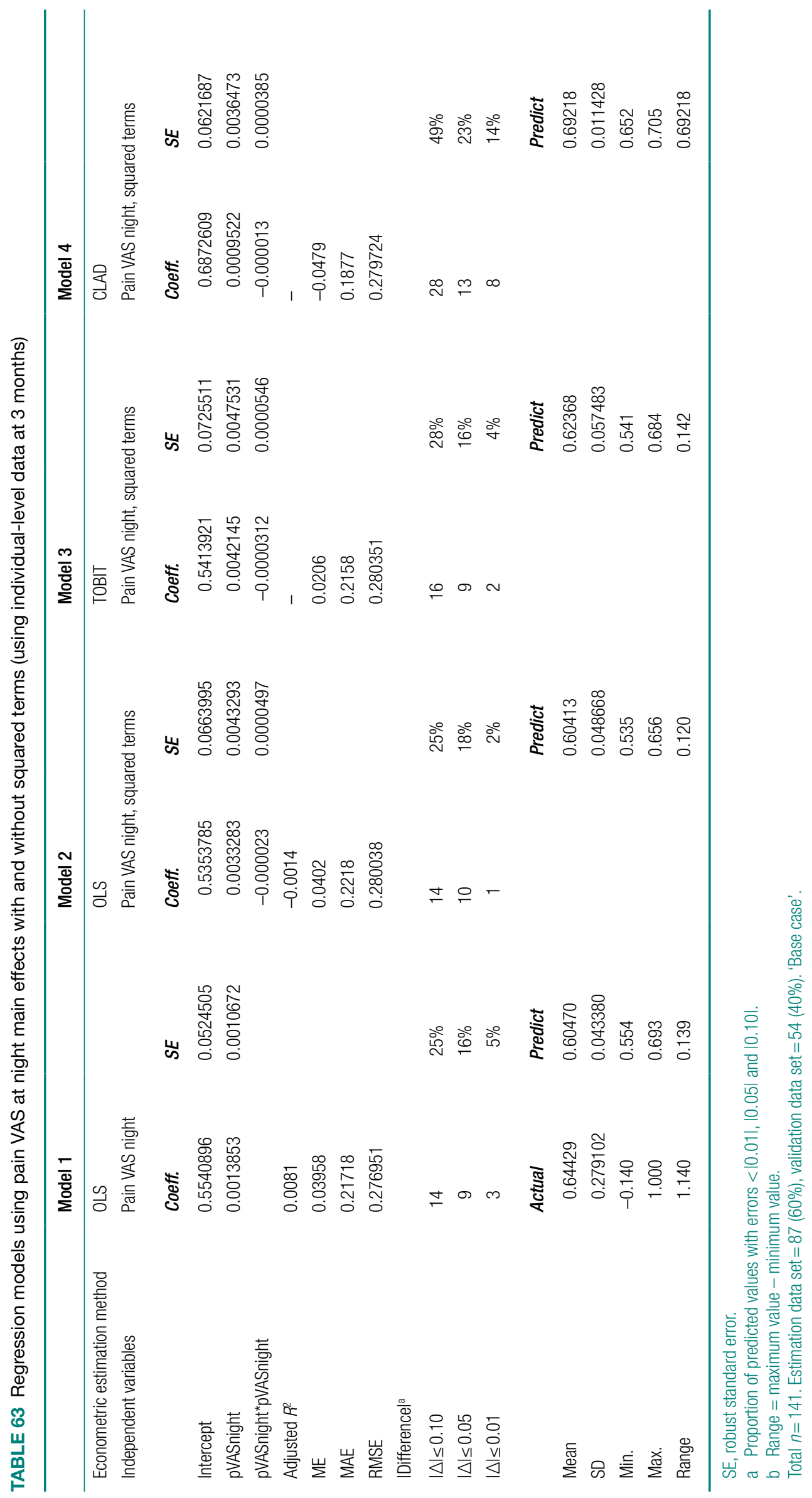


Appendix 14 shows the results of the regression of the pain VAS scores onto the the EQ-5D using individual-level data at 1,3 and 12 months. Compared with using data from 3 months only, the explanatory power and fit improves but only slightly. The two OLS models generated an adjusted $R^{2}$ of 0.1009 . Errors were generally similar across all models with RMSEs ranging from 0.26403 to 0.26689 . Models $1-4$ were able to predict between $20 \%$ and $26 \%$ of the scores to within 0.05 of actual EQ-5D utility values. The predictive performances of all models were generally similar.

Appendix 14 also shows the results of the regression of the pain VAS scores onto the EQ-5D using models estimated from 3-month data and used to predict EQ-5D scores at 12 months. Predicting EQ-5D scores at 12 months based on the mapping functions derived from the 3-month data set did not perform as well as the analyses using data from all time points. Models 1-4 were able to predict between $11 \%$ and $21 \%$ of the scores to within 0.05 of actual EQ-5D utility values.

EQ-5D and SF-36 PCS and MCS Table 64 shows the results of the regression of the SF-36 PCS and MCS scores onto the EQ-5D using the 3-month data set (base case). The explanatory power and fit of the three OLS models was poor, with an adjusted $R^{2}$ ranging from 0.0147 to 0.0830 . The simplest OLS model including only main effects produced the smallest MAEs (0.21135) compared with the other models. However, models $1-5$ were only able to predict between $13 \%$ and $25 \%$ of the scores to within 0.05 of actual EQ-5D utility values.

Appendix 14 shows the results of the regression of the SF-36 PCS and MCS scores onto the EQ-5D using individual-level data at 1, 3 and 12 months. Compared with using data only from 3 months, the explanatory power and fit of all models improves. The three OLS models generated an adjusted $R^{2}$ ranging from 0.3840 to 0.4284 . Errors are smaller, with the smallest MAE and RMSE for model 5 (CLAD) of 0.1814 and model 1 (OLS) of 0.1889 respectively. Models $1-5$ were able to predict between $70 \%$ and $78 \%$ of the scores to within 0.05 of actual EQ-5D utility values but only $41-55 \%$ to within 0.01 of actual EQ-5D utility values. The predictive performances of all models are generally similar.

Appendix 14 also shows the results of the regression of the SF-36 PCS and MCS scores onto the EQ-5D using models estimated from 3-month data and used to predict EQ-5D scores at 12 months. Predicting EQ-5D scores at 12 months based on the mapping functions derived from the 3-month data set was somewhat less accurate than the analyses using data from all time points. Models $1-5$ were able to predict between $31 \%$ and $58 \%$ of the scores to within 0.05 of actual EQ-5D utility values.

Finally, the data suggest that all models (whether they are based on pain VAS or SF-36 PCS and MCS scores) predict better for less severe health states, but overpredict the value of more severe EQ-5D states.

\section{Potential implications for application to frozen shoulder populations}

The population used in mapping studies should cover the range of clinical and demographic characteristics of the population on which the mapping function is ultimately to be applied. The two populations should be sufficiently homogeneous that relationships between outcomes in one population can be used to infer relationships about outcomes in the other population. The current exploratory analyses were undertaken on a UK data set of patients with rotator cuff disease and not on the specific population of interest in the current review, that is, frozen shoulder patients. However, we considered the patients in the SAPPHIRE trial data set to exhibit similar enough characteristics to patients with frozen shoulder (see Table 60) to be potentially useful for drawing inferences for this population from the mapping analyses. Our findings indicated that, of the models estimated, the simple OLS model including only the main effects of (1) pain VAS mapped onto the EQ-5D and (2) SF-36 PCS and MCS mapped onto the EQ-5D 


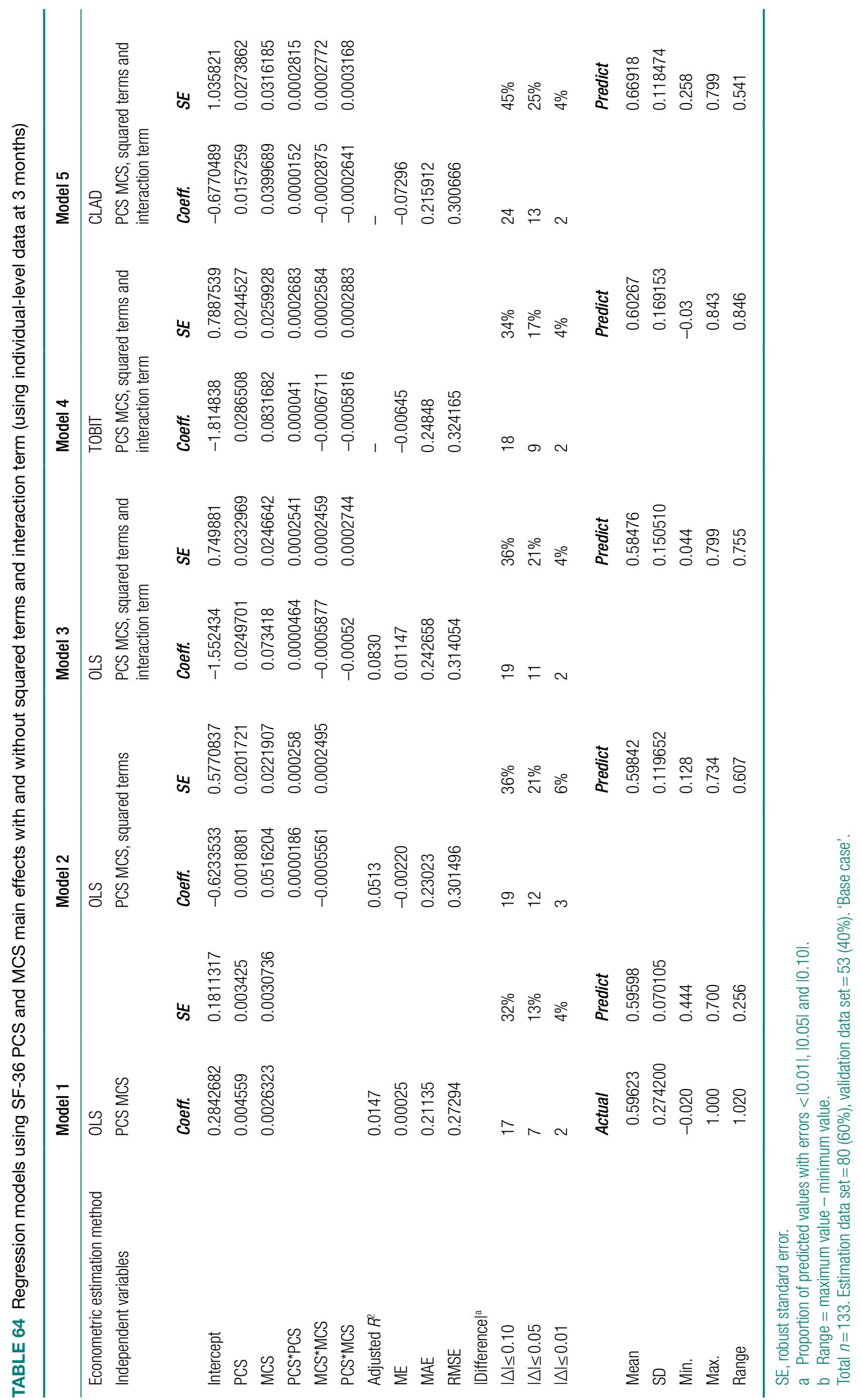


performed just as well as some of the more complex models/approaches. If we apply this mapping function to the two studies in the review that did report data for (1) mean pain VAS ${ }^{16,51}$ and (2) mean SF-36 PCS and MCS scores ${ }^{35,51}$ then hypothetically it becomes possible to derive utilities based on the EQ-5D, which in principal can then be used in performing assessments of the costeffectiveness (cost-utility) of two or more treatments in patients with frozen shoulder.

Table 65 illustrates the results of this process using data from the two frozen shoulder trials in the clinical effectiveness review that reported mean pain VAS scores ${ }^{16,51}$ (see Appendix 7 for full details and data extraction tables for these studies). Using the OLS1 model function/equations we mapped the reported mean pain VAS at baseline and after 3 months of follow-up onto the EQ-5D. The corresponding EQ-5D scores can then be predicted at baseline and after 3 months of follow-up.

Table 66 illustrates the same using data from the two frozen shoulder trials in the clinical effectiveness review that reported the SF-36 PCS and MCS ${ }^{35,51}$ (see Appendix 7 for full details and data extraction tables for these studies).

TABLE 65 Cost-effectiveness results from mapping onto EQ-5D (pain VAS scale 0-100 mm)

\begin{tabular}{|c|c|c|c|c|c|c|c|c|}
\hline \multirow[b]{2}{*}{ Study } & \multirow[b]{2}{*}{ Intervention } & \multicolumn{2}{|c|}{$\begin{array}{l}\text { EQ-5D estimated } \\
\text { from mapping }\end{array}$} & \multirow{2}{*}{$\begin{array}{l}\text { Mean QALYs } \\
\text { over } 3 \text { months }\end{array}$} & \multirow{2}{*}{$\begin{array}{l}\text { Estimated cost } \\
\text { per patient }\end{array}$} & \multirow{2}{*}{$\begin{array}{l}\text { QALY } \\
\text { difference }\end{array}$} & \multirow{2}{*}{$\begin{array}{l}\text { Cost } \\
\text { difference }\end{array}$} & \multirow[b]{2}{*}{ ICER } \\
\hline & & Baseline & 3 months & & & & & \\
\hline \multirow[t]{2}{*}{$\begin{array}{l}\text { Dogru } \\
(\text { Turkey) })^{51}\end{array}$} & $\begin{array}{l}\text { Ultrasound + } \\
\text { physiotherapy }\end{array}$ & 0.518 & 0.695 & 0.15163 & $£ 148.13$ & -0.00163 & 0 & \\
\hline & $\begin{array}{l}\text { Sham ultrasound + } \\
\text { physiotherapy }\end{array}$ & 0.527 & 0.699 & 0.15325 & $£ 148.13$ & & & $\begin{array}{l}\text { Benefit } \\
\text { favours }\end{array}$ \\
\hline \multirow[t]{2}{*}{$\begin{array}{l}\text { Stergioulas } \\
\text { (Greece) }^{16}\end{array}$} & $\begin{array}{l}\text { Physical therapy } \\
\text { (laser) }\end{array}$ & 0.55 & 0.687 & 0.15463 & $£ 49.38$ & 0.00350 & 0 & $\begin{array}{l}\text { Benefit } \\
\text { favours }\end{array}$ \\
\hline & $\begin{array}{l}\text { Control (placebo } \\
\text { laser) }\end{array}$ & 0.562 & 0.647 & 0.15113 & $£ 49.38$ & & & \\
\hline
\end{tabular}

Note: Studies reporting pain VAS data $(0-100 \mathrm{~mm})$ as medians rather than means were excluded from the theoretical mapping analysis. Cheapest resource-use scenarios as presented in Resource-use estimation have been used to obtain costs.

TABLE 66 Cost-effectiveness results from mapping onto EQ-5D (SF-36 PCS and MCS)

\begin{tabular}{|c|c|c|c|c|c|c|c|c|}
\hline \multirow[b]{2}{*}{ Study } & \multirow[b]{2}{*}{ Intervention } & \multicolumn{2}{|c|}{$\begin{array}{l}\text { EQ-5D estimated from } \\
\text { mapping }\end{array}$} & \multirow{2}{*}{$\begin{array}{l}\text { Mean QALYs } \\
\text { over } 3 \text { months }\end{array}$} & \multirow{2}{*}{$\begin{array}{l}\text { Estimated cost } \\
\text { per patient }\end{array}$} & \multirow{2}{*}{$\begin{array}{l}\text { QALY } \\
\text { difference }\end{array}$} & \multirow{2}{*}{$\begin{array}{l}\text { Cost } \\
\text { difference }\end{array}$} & \multirow[b]{2}{*}{ ICER } \\
\hline & & Baseline & 3 months & & & & & \\
\hline \multirow[t]{4}{*}{$\begin{array}{l}\text { Carette } \\
\text { (Canada) }^{35}\end{array}$} & $\begin{array}{l}\text { Steroid injection + } \\
\text { physiotherapy }\end{array}$ & 0.51 & 0.673 & 0.14788 & $£ 121.43$ & & Dominated & \\
\hline & $\begin{array}{l}\text { Physiotherapy+ } \\
\text { placebo injection }\end{array}$ & 0.587 & 0.702 & 0.16113 & $£ 117.75$ & & Dominated & \\
\hline & Steroid injection & 0.583 & 0.739 & 0.16525 & $£ 36.18$ & 0.01275 & $£ 3.68$ & $£ 288.63$ \\
\hline & Placebo injection & 0.571 & 0.649 & 0.15250 & $£ 32.50$ & & & \\
\hline \multirow[t]{2}{*}{$\begin{array}{l}\text { Dogru } \\
\text { (Turkey) }{ }^{51}\end{array}$} & $\begin{array}{l}\text { Ultrasound + } \\
\text { physiotherapy }\end{array}$ & 0.564 & 0.647 & 0.15138 & $£ 148.13$ & 0.00537 & 0 & $\begin{array}{l}\text { Benefit } \\
\text { favours }\end{array}$ \\
\hline & $\begin{array}{l}\text { Sham ultrasound + } \\
\text { physiotherapy }\end{array}$ & 0.522 & 0.646 & 0.14600 & $£ 148.13$ & & & \\
\hline
\end{tabular}

Note: Cheapest resource use scenarios as presented in Resource-use estimation have been used to obtain costs. 
Based on these EQ-5D estimates QALYs have been approximated by applying the area under the curve (AUC) method, which is implemented by summing the areas of the geometrical shapes obtained by linearly interpolating between utility scores over the study period. Using the UK estimated costs obtained from our resource-use assessment we have presented tentative costeffectiveness results, which may allow some inference about the cost-effectiveness of some of these interventions.

These calculations are based on the assumption that no further downstream costs will be incurred by the patient. Also, because of the methods we are using we have only been able to compare interventions within trials, not across trials. Any future evaluation should consider all relevant interventions within the same analysis.

Tables 65 and 66 show the baseline and 3-month EQ-5D scores that have been obtained using the estimates provided by the mapping and the QALYs obtained based on these estimates. The costs presented in these tables represent the cheapest UK scenarios presented in Resource-use estimation. In some instances, because of interventions requiring the same resource and therefore costing the same, no ICERs have been presented. For the study evaluating interventions that incur different costs, ${ }^{35}$ the incremental results suggest that physiotherapy (with placebo injection) and steroid injection with physiotherapy were both dominated (less effective and more costly) by steroid injection alone. Steroid injection has an ICER of $£ 288.63$, which is well below the widely used threshold of $£ 30,000$.

These results are tentative. Their purpose is to try to help inform further research, not to directly inform decision-making. Actual QALY gains, and incremental cost per QALY estimates, can differ based on how the utilities were derived: directly from preference-based measures or predicted on the basis of mapping. ${ }^{113}$ This is neatly demonstrated by the results of the Dogru study, which, depending on whether mapping from the VAS or SF-36, suggest QALY gains in favour of alternative interventions. ${ }^{51}$ Furthermore, the choice of direct measure used in costutility analyses matters (e.g. EQ-5D, SF-6D, HUI, 15D) and may, in an economic evaluation, lead to different treatment options being considered more or less cost-effective relative to one another. ${ }^{114}$ These are all issues outside the scope of this research.

\section{Summary}

There were no studies identified in the clinical review that provided utilities data needed for generating cost per QALY estimates in assessments of the cost-effectiveness of alternative treatment options for frozen shoulder.

- The exploratory analysis presents an alternative approach to deriving utility estimates by mapping from outcomes measured in a trial onto a preference-based measure of QoL.

- For mapping to be of any value, a degree of overlap is required between the descriptive systems of the measures being used. The results of the exploratory analysis suggest that a statistical association between the EQ-5D and pain VAS and the EQ-5D and the SF-36 PCS and MCS measures potentially exists, specifically that a decreasing pain VAS score (less pain) is accompanied by an increasing (better) EQ-5D score and similarly that increasing (better) SF-36 PCS and MCS scores are accompanied by an increasing (better) EQ-5D score.

- There is insufficient evidence available to make conclusions about the effectiveness of treatments for frozen shoulder in terms of QoL outcomes based on patient preference measures. 


\section{Chapter 4}

\section{Discussion}

The aims of the project were to undertake a systematic review of the clinical effectiveness and

cost-effectiveness of treatments used for the management of idiopathic frozen shoulder, by stage of condition, in order to make recommendations for clinical practice and identify gaps in the evidence. The project also aimed to develop a decision-analytic model to estimate the costeffectiveness of the alternative treatment options and undertake value of information analysis to assess the potential value of future research on interventions for frozen shoulder.

Both conservative and more invasive treatment options were included in the review. Conservative treatments are generally considered to be education about the condition with advice for selfmanagement, physiotherapy and corticosteroid injection, either alone or alongside physiotherapy. Physiotherapy and steroid injection are delivered in a range of NHS settings including primary care, delivered mainly by physiotherapists and GPs, secondary tier specialist musculoskeletal clinics and a hospital setting. More invasive treatments that were included in the review were distension, MUA and capsular release, which are delivered in a hospital setting. Sodium hyaluronate does not appear to be commonly used in the NHS but there appeared to be a growing literature on its use and it was therefore included. This was classified with the more invasive treatments, mainly because it would be delivered in a hospital setting.

\section{Principal findings}

\section{Clinical effectiveness}

Data from studies with a low risk of bias were sparse, in particular for the more invasive treatments. For most comparisons the necessary data were not available or it was inappropriate to statistically pool studies because of their variability. Primarily a narrative synthesis was undertaken. For the majority of studies it was unclear whether they had sufficient power to detect a statistically significant difference between groups; therefore, where there is no evidence of an effect it cannot be assumed that there is no effect. Information on outcome by stage of condition was not available. Based on the duration of frozen shoulder and the inclusion criteria, the trials included mixed populations with idiopathic frozen shoulder, probably mainly people in the first painful phase and the second stiffening stage. In addition, only five of the studies were undertaken in a UK NHS setting. RCTs were available for all of the interventions except capsular release, for which case series only were available, and watchful waiting or 'supervised neglect', for which a quasi-experimental study was available. However, some of the RCTs may in fact have been quasi-experimental. Across most trials there did not appear to be systematic methods for recording of adverse events.

\section{Conservative interventions}

Although several of the studies had a home exercise only control group, the information provided was often limited in terms of the extent of educational advice provided. The single study of 'supervised neglect', perhaps more appropriately called watchful waiting, was the only study to formally assess a broader intervention of self-management. However, as with the reporting of many complex interventions, there was insufficient information for replication in a clinical setting. ${ }^{73}$ The finding from this study from the Netherlands, that supervised neglect was associated with a better outcome than physiotherapy (of unspecified duration), should be treated 
with considerable caution because of limitations in the study design. The lack of high-quality research on watchful waiting was surprising given that in a recent survey of UK professionals it was a commonly used treatment in the painful phase of frozen shoulder. ${ }^{18}$

A further 11 studies evaluated physical therapy, mainly physiotherapy with active mobilisation. The comparators were a control or another form of physical therapy. Because of the diversity of the interventions and comparators only single studies were available for individual therapies and most of the studies had a high risk of bias. Based on a single study there was evidence of a statistically significant short-term benefit of SWD plus stretching (12 sessions over 4 weeks) compared with home exercise only and compared with a heat pack plus stretching for some outcomes. ${ }^{75}$ There was also evidence from a single study that laser therapy (12 sessions over 8 weeks) had benefit over home exercise alone in the short and medium term. ${ }^{16}$ Both studies had some risk of bias and the content, intensity and quality of the home exercise comparator was unclear. A further study, also at some risk of bias and in a population in which the majority had already received physiotherapy and/or a steroid injection, found marginally significant improvements for function and disability and improvement in range of movement with HGMT compared with LGMT. ${ }^{40}$ None of these studies was undertaken in a UK setting.

Only studies comparing acupuncture with another of the treatments of interest were included, not studies comparing different methods of acupuncture. Only three studies were identified, none of which was from the UK, and all had a potentially high risk of bias. ${ }^{79-81}$ There was insufficient evidence to make conclusions about the effectiveness of acupuncture for primary frozen shoulder.

Six studies evaluated steroid injections, although the majority of the available data were from two multi-arm studies that were of satisfactory quality, one with some risk of bias. ${ }^{35,41}$ Both studies evaluated a single intra-articular steroid injection in patients with frozen shoulder of $<6$ months' duration. The comparators were home exercise alone, physiotherapy alone (both with placebo injection) and steroid injection followed by physiotherapy. One used a guided injection and the other appeared to use an unguided approach. The physiotherapy was of 4 weeks' duration in both studies, with one including twelve sessions and the other eight. The actual content of the sessions varied between the studies, although both used mobilisation techniques. All groups in both studies were also given a home exercise programme.

Given that steroid injection is administered to reduce inflammation and provide pain relief, pain outcome is of particular interest for this intervention. For pain there was a short-term statistically significant benefit (up to 3 months) with steroid injection compared with home exercise alone but not compared with 4 weeks of physiotherapy. When steroid injection was provided in conjunction with physiotherapy, there was an added benefit for pain over physical therapy alone and over steroid injection alone. This was broadly consistent with the evidence for function and disability and range of movement. Only one of these studies used a QoL outcome measure (SF36). There was no statistically significant benefit for QoL with a steroid injection alone compared with home exercise or physiotherapy alone. However, there was a benefit for QoL when physiotherapy was added to steroid injection over either home exercise or physiotherapy alone but not steroid injection alone. Overall, the evidence suggests that there is some evidence of a short-term benefit from adding steroid injection to physiotherapy alone; however, there remains some uncertainty as to whether this adds any substantial clinical benefit over steroid alone (with home exercise).

\section{Invasive interventions}

The data from high-quality RCTs of invasive interventions were even more limited than for conservative interventions and only two were undertaken in a UK setting. MUA, distension and capsular release are most commonly used when conservative treatments have failed, although 
for most of these studies it was unclear what treatments participants had already received, if any. In addition, there was considerable variability between the studies in how the procedures were delivered, making comparability between studies difficult.

There was insufficient evidence on all three interventions to make any robust recommendations regarding their use. Based on a single, satisfactory quality study, there was no evidence, across several outcomes, that MUA was better than a conservative intervention involving provision of advice in two sessions from a physical therapist plus written instructions for a daily training programme. ${ }^{39} \mathrm{~A}$ study from the UK, with some risk of bias, reported greater improvement across outcomes with arthrographic distension than with MUA in participants with second stage (stiffening or 'freezing' stage) primary frozen shoulder. ${ }^{38}$

There was no consistent evidence that arthrographic distension was associated with a better outcome than home exercise only or steroid injection. A single satisfactory study comparing arthrographic distension including steroid with placebo arthrography (with home exercise) reported a significant improvement with distension in function and disability measured using a patient-defined scale, but not in another function measure, range of movement or pain. ${ }^{43} \mathrm{~A}$ second study with some risk of bias reported a benefit with arthrographic distension including steroid compared with steroid alone but only for a single range of movement measure at 6 weeks and not for other range of movement outcomes or a measure of function and disability. ${ }^{36}$

Only case series were available for capsular release. The lack of a control group presents particular problems in assessing the effectiveness of an intervention in a condition such as frozen shoulder in which the condition normally resolves within a 3-year period. Based on the best-quality case series, undertaken in Norway with the procedure performed by a single surgeon, there was a significant improvement in several outcomes based on an average length of follow-up of 10 months (range 3 to 29 months). ${ }^{85}$ All patients had tried physiotherapy previously without a satisfactory result.

Based on three studies of sodium hyaluronate, all at high risk of bias, there was insufficient evidence to recommend use of this treatment in preference to steroid or physical therapy. The best-quality study reported a benefit with two injections of sodium hyaluronate compared with home exercise but there was no consistent evidence across outcomes of a benefit over 10 daily sessions of physical therapy or single steroid injection. ${ }^{66}$

\section{Mixed-treatment comparison}

A MTC was undertaken for the outcome of pain with the aim of bringing together both direct and indirect evidence in a connected network, thereby allowing inferences about the relative efficacy of all treatments to be made. Nine interventions formed part of a connected network with placebo and the evidence was informed by nine trials. A total of 22 studies had to be excluded from the MTC for a range of reasons; therefore, the network was a small subset of the available evidence. Of the nine interventions compared with placebo (plus home exercise) in the MTC, only steroid combined with physiotherapy and steroid alone were compared with placebo in the direct evidence synthesis (i.e. standard meta-analysis). The results from the MTC were consistent with the direct evidence synthesis of steroid combined with physiotherapy versus placebo. In both analyses the combined intervention showed a beneficial and clinically significant effect. In contrast, the results of the MTC were inconsistent with the direct evidence synthesis of steroid compared with placebo. In the MTC the $95 \%$ CrIs crossed the line of no effect. In the direct evidence synthesis, steroid showed both a statistically and a clinically significant beneficial effect compared with placebo. The remaining interventions included in the MTC (physiotherapy, physiotherapy combined with placebo, physiotherapy without mobilisation, electroacupuncture, sodium hyaluronate, MUA combined with physiotherapy, and arthrographic distension with steroid) had $95 \%$ CrIs that crossed the line of no effect. 


\section{Gaps in the clinical effectiveness evidence}

There were several gaps in the evidence regarding conservative treatment options for primary frozen shoulder. Watchful waiting is the most conservative treatment option. However, it remains unclear what constitutes watchful waiting. It would seem inappropriate to do absolutely nothing and this is unlikely to happen in clinical practice. The intervention of supervised neglect evaluated by Diercks and Stevens ${ }^{73}$ included provision of education about the condition. This included an explanation of the natural course of the disease and advice not to exercise in excess of the pain threshold and to carry out pendulum exercises and active exercises within this painless range, with resumption of all activities that were tolerated. In addition, NSAIDs and analgesics were prescribed where necessary. Details were not provided about how the information was delivered and any methods used to maximise compliance with the home exercise programme and the extent of any monitoring, for example in the form of primary care review appointments, were unclear. In our view, education about the condition and guidance for self-management including home exercises are appropriate elements of a standard care package. Methods of maximising compliance with any advice may also be important. Several of the studies used a home exercise control group but it was often unclear what this consisted of and the extent and quality of education provided. Therefore, it was unclear in most studies whether the control group were receiving a high-quality standard care package or whether they were simply provided with a leaflet of exercises. In our view, the former is the most appropriate comparator to use in trials to establish any additional benefit from any more intensive interventions such as a package of physiotherapy treatment or steroid injection. The benefit of a physiotherapy intervention over a structured high-quality conservative intervention of education and home exercise using methods to maximise compliance has not been adequately addressed. Physiotherapy is the most commonly used NHS physical therapy and is therefore of particular interest.

It also remains unclear, based on the research evidence available, what constitutes an optimal physiotherapy intervention in terms of the essential components, number and length of sessions and overall duration and the type of physiotherapy. It also remains unclear at what stage and severity of frozen shoulder such an intervention would be of greatest benefit and whether any effect is similar in people with and without diabetes.

In clinical practice, patients can receive a second or third steroid injection when there has been no benefit from the first injection or when symptoms deteriorate again. There was no evidence available on the effectiveness of a second or third injection provided in a way that reflects NHS clinical practice. One US study evaluated the effect of once-weekly injections for 3 weeks rather than on the basis of the patients' response to the first injection. ${ }^{42}$

The role of the more invasive interventions in the treatment pathway for frozen shoulder remains unclear and further research is required to establish their effectiveness. There was considerable overlap in the duration of symptoms between the participants in the trials of invasive and conservative treatments. This suggests that the populations may have been similar across the more conservative and invasive interventions. In most studies it was unclear whether participants had received conservative treatments first that had not been effective, whether they were presenting for treatment having tried self-management or whether their preference was for an intervention that involved a shorter duration of treatment.

\section{Comparison with other effectiveness research}

We are aware of three systematic reviews that have been published since our assessment started: one of corticosteroid injections compared with physiotherapy for frozen shoulder; ${ }^{115}$ one of sodium hyaluronate injection for chronic painful shoulder; ${ }^{116}$ and one of conservative and surgical interventions for frozen shoulder. ${ }^{117} \mathrm{~A}$ guideline based on a systematic review has also recently been published focusing on diagnosis, assessment and physiotherapy management of 
frozen shoulder. ${ }^{6}$ Our conclusions regarding steroid injections are similar to those of Blanchard and colleagues ${ }^{115}$ who concluded that corticosteroid injections have a greater effect in the short term than physiotherapy but that the results of the review should be interpreted with caution because of the limited data available and variability between the studies. The recent guideline also comes to similar conclusions to the Blanchard review and our review regarding steroids compared with physiotherapy. ${ }^{6}$

The review of conservative and surgical interventions, undertaken by Favejee et al., ${ }^{117}$ highlighted similar limitations in the literature to our review. They identified the lack of an unambiguous definition for frozen shoulder and the lack of information on phase of condition as well as a need for further research. However, they made somewhat stronger conclusions about the effectiveness of some interventions than our review has done or those of Blanchard et al. ${ }^{115}$ and Hanchard et $a l .{ }^{6}$ have done. They concluded that there was strong evidence for the short-term effectiveness of steroid injections and laser therapy and moderate evidence for the effectiveness of mobilisation techniques in the short and long term as well as evidence for the effectiveness of arthrographic distension alone and in addition to active physiotherapy in the short term. The Favejee et al. ${ }^{117}$ review used a broad definition of frozen shoulder that did not appear to be restricted to primary frozen shoulder. For example, the conclusion about strong evidence for the effectiveness of laser therapy is based on three trials only, one of which was of a primary frozen shoulder population. The other two trials were excluded from our review. The review of sodium hyaluronate is not directly comparable with our review as a range of chronic shoulder conditions were included, as well as dose-ranging studies. The review concluded that sodium hyaluronate injection was modestly more effective than steroid injection for function and there was an improvement in pain and function compared with placebo. ${ }^{116}$

\section{Patient views}

We were unable to identify any papers that explored patients' views or experiences of treatments for frozen shoulder. As treatment for frozen shoulder can vary substantially and is often guided by patient preference, this was considered an important area for investigation; however, the lack of available evidence did not allow us to explore this further. Although no papers exploring patients' views were found, there were limitations to this review because of time constraints. Our searches were restricted to searches of three databases for English-language studies and unpublished studies may have been missed. Nonetheless, there does appear to be a clear need for research in this area.

\section{Cost-effectiveness review}

A single Dutch cost-effectiveness study met the inclusion criteria for the systematic review. ${ }^{91}$ This was conducted as part of a trial that was included in the clinical effectiveness review. ${ }^{40}$ The trial reported statistically significant improvement in range of movement with HGMT compared with LGMT in a population in which the majority of patients had already received physiotherapy and/or steroid injection. There was no significant difference in QoL, although the CIs were wide. There was also no significant difference in pain or function and disability, although for one of the function and disability scales the CIs just crossed the line of no effect. The economic analysis was of reasonable quality with some risk of bias and was conducted outside of the UK (the Netherlands), which may limit generalisability. It showed that LGMT may be a cost-effective option compared with HGMT.

Our searches failed to identify any modelling studies conducted in the relevant population or similar populations/conditions. We did identify a limited number of economic evaluations conducted alongside clinical trials in patients with unilateral shoulder pain, chronic shoulder complaints and acute and subacute shoulder complaints. ${ }^{91,118-121}$ We considered these populations to be similar enough to allow some comparisons to be made, although these populations were 
not the focus of our research and we did not undertake a systematic review in these patient populations. The economic evaluations did not meet our inclusion criteria but we anticipated that they would provide some useful information to inform our research. One of these studies, James et al., ${ }^{119}$ was conducted in the UK and allowed some comparison of the cost estimates obtained in the clinical trial and the estimates obtained from our advisory group for the frozen shoulder population. Because of the limited evidence available to us through the review, alternative methods of obtaining evidence were pursued. The clinical advisors on the project were utilised and primary data from a clinical trial in a similar population identified.

\section{Cost estimates}

The estimated resource use provided by our clinical advisory group allowed the calculation of average treatment costs from the perspective of the UK NHS for the interventions identified in the systematic review. The estimated average costs for therapies involving administration of steroid injections ranged from $£ 36.18$ to $£ 138.51$ for unguided injections and from $£ 299.68$ to $£ 475.56$ for guided injections. Variations in costs were attributable to the type of healthcare professionals involved and the delivery setting. The costs of therapies involving active physiotherapy alongside steroids were estimated to vary over a wide range, from $£ 121.43$ to $£ 607.31$, and reflected practice variations in regard to number of physiotherapy sessions, delivery setting and the steroid used.

Physiotherapy alone, involving some form of mobilisation as a component, was estimated to range from $£ 98.75$ to $£ 126.75$. Acupuncture was estimated to cost from $£ 117.75$ to $£ 126.75$. Difference in costs was mainly due to community versus hospital-based setting.

The estimated average costs associated with surgical interventions ranged from $£ 1213$ to $£ 1522$ for MUA, from $£ 79.84$ to $£ 134.84$ for arthrographic distension and from $£ 1809$ to $£ 2511$ for capsular release. The difference in costs was driven by the difference in the hospital resources utilised in performing MUA arthrographic distension and capsular release. This is reflected in the different NHS reference cost codes under which these interventions are reported by hospitals and subsequently costed.

\section{Comparison with costs of other research}

The validation of these estimates is difficult given the lack of evidence available. James $e t$ al. ${ }^{119}$ conducted a cost-consequence analysis comparing local corticosteroid injection and physiotherapy for the treatment of new episodes of unilateral shoulder pain in UK primary care. The analysis collected resource-use data from patients enrolled in the trial from entry until 6 months post randomisation. The study reports an average cost of $£ 114.60$ for those patients receiving the physiotherapy intervention. This is very similar to our estimate of an average cost of $£ 112.75$ for physiotherapy (average based on community/hospital setting). They also report an average cost of $£ 71.28$ for the steroid group, which compares favourably with our average estimate of $£ 73.87$ for an unguided injection (average based on alternative delivery setting and choice of steroid). Although these costs appear to be close, the uncertainty remains on how closely either set of costings would reflect clinical practice. Patients throughout the James et al. study had access to additional treatments that were included in the resource-use figures. So the average cost for the physiotherapy group also included costs for resources such as radiography, non-trial injections, etc. In addition, costs were presented for 2003 and we have not inflated them to match our 2009 price year. This may suggest that our cost estimates are a little on the high side given the difference in price years and the fact that our costs consider only resources for one specific treatment and not the additional resources that a patient may consume by choosing to access alternative treatments or specialists alongside the primary prescribed treatment. 
As with the single included economic evaluation for frozen shoulder, the majority of economic evaluations identified were conducted in the Netherlands. Although some provided useful data for the comparability of utility estimates they did not provide us with a clear picture of the resource utilisation of the alternative treatments that we have considered in this review. Van den Hout et al. ${ }^{91}$ which met the inclusion criteria, presented societal costs of $€ 8809$ for HGMT and $€ 6911$ for LGMT. Enough data were presented to allow costs from the perspective of the health-care provider to be estimated and these range from €2552 for HGMT to €2293 for LGMT. Because of differences in health-care systems the cost analysis used charges, which are relevant in the Netherlands, rather than costs, which are relevant for the NHS setting. So even if we convert euros into British pounds a like-for-like comparison would be difficult. In our view, the estimates obtained from our clinical advisory group represent the most reliable resource estimates and, although we acknowledge that there is some uncertainty surrounding the estimates because of the lack of evidence available, it was not possible to investigate this further. The need for a clearer picture of the resources used as patients progress through their treatment pathway is evident and is lacking in the current evidence base.

\section{Quality of life and utility data}

To fully evaluate HRQOL it is necessary to consider all of the ways that the condition being treated and the intervention being delivered impact on patient health outcomes. This means measuring the effect of the condition and the treatment on both duration and quality of life. The use of QALYs allows both of these concerns to be captured and reflected in one outcome. The QALY integrates mortality and morbidity to convey health status in terms of equivalent well years of life. A key component of the QALY is the utility values, which can be measured using a variety of methods. The measurement of utilities is an area of ongoing debate and research, which is beyond the scope of this discussion.

The clinical trials identified in the effectiveness review reported a variety of outcomes, mainly pain, range of movement and function and disability, with some measuring QoL, time to recovery and return to work and recreation, and adverse events. There seems little agreement across the trials regarding the tools with which these outcomes should be measured or which is the primary outcome. This may be related to the changing, progressive nature of the condition. In the early painful stages those outcomes that capture pain are likely to be more important; however, as the condition progresses the function and disability outcomes may become more important. When faced with a plethora of outcomes such as this it raises the question of why HRQoL, which may capture all of these outcomes, has not been routinely measured alongside those outcomes more traditionally considered clinical outcomes. We found only one study reporting HRQoL (utility outcomes) in the frozen shoulder population ${ }^{91}$ and only scant data on HRQoL in patient populations considered similar.

It is unclear why these data have not been collected in the clinical trials identified in the effectiveness review. Possible reasons may be because it was not practical or was too timeconsuming for patients to complete in addition to other measures or possibly because the studies were not designed to focus on economic questions and therefore utilities were not thought to be important. These reasons are only conjecture; further research is required to establish why HRQoL does not appear to be considered a relevant outcome in these types of patients. However, this lack of utility data hinders any decision analysis being undertaken. When outcome measures used in trials are limited to condition-specific outcomes the ability to evaluate the costeffectiveness of one treatment against another treatment in a different population is lost.

Because of the potential for bias in the one study presenting HRQoL data and the use of the less popular SF-6D tool, ${ }^{91}$ we undertook a mapping exercise. Our aim was to try and establish 
a link between the outcomes considered in the clinical trials and HRQoL. The EQ-5D is the most popular instrument of choice for the measurement of utilities and is recommended in the NICE reference case. ${ }^{64}$ The decision was made to use this instrument in a mapping exercise. The mapping investigated the relationship between QoL, as measured by two components of the SF-36, and HRQoL, as measured by the EQ-5D, and pain, as measured by a VAS $0-100 \mathrm{~mm}$, and EQ-5D. The SF-36 was used as this is a widely used and accepted tool for measuring QoL and was used in two of the included studies in the effectiveness review: one compared ultrasound and placebo $;^{51}$ the other compared steroid injection, steroid injection plus physiotherapy, physiotherapy alone and placebo. ${ }^{35}$ A VAS $0-100 \mathrm{~mm}$ was used as it was readily available in the data set used for mapping and was a scale used in the clinical trials. The mapping was based on data from the SAPPHIRE trial. ${ }^{40}$ The complete data set was made available by the authors of the trial. The findings of the mapping suggest that there is a positive relationship between outcomes: a decreasing pain VAS score (less pain) is accompanied by an increasing (better) EQ-5D score; an increasing EQ-5D score (i.e. better health status) appears to be generally accompanied by increasing SF-36 scores (i.e. higher scores $=$ a better quality of life). Two trials included in the review of effectiveness, Dogru et al.${ }^{51}$ and Stergioulas, ${ }^{16}$ used pain VAS $(0-100 \mathrm{~mm})$. Using the prediction equation obtained from mapping pain VAS and the results of the two trials we were able to estimated incremental QALY improvements, which ranged from -0.00163 to 0.00350 . Two trials included in the review, Carette et al. ${ }^{35}$ and Dogru et al. ${ }^{51}$ used SF-36 PCS and MCS as an outcome measure. Using the prediction equation obtained from the mapping and the results of the two trials we were able to estimate incremental QALY improvements, which ranged from 0.00537 to 0.01275 .

Although the mapping was conducted on data from a similar population, primary collection of utility data would be required to validate that the positive relationship remains in the frozen shoulder population.

Those economic evaluations in similar populations that have reported EQ-5D data ${ }^{18,120,121}$ have demonstrated improvements from baseline ranging from 0.06 to 0.16 , which are comparable to those in the SAPPHIRE trial. These trials included a variety of interventions including an education and activation programme, manipulative therapy, which comprised specific manipulation and mobilisation techniques, and graded exercise therapy delivered by a physiotherapist. All were compared with usual care, which was variable in its content and the depth of its description. The populations in these trials had a higher baseline EQ-5D value (range 0.51 to 0.587 for pain VAS; $0.518-0.562$ for SF-36 PCS/MCS) than the population from the SAPPHIRE trial (range 0.453 to 0.512 ). ${ }^{102}$ This may suggest that the population of the SAPPHIRE trial, each of whom had a confirmed diagnosis of rotator cuff tendonitis, differs from populations with other shoulder complaints. It is likely that this population may be comparable to a population of frozen shoulder patients in the painful phase of the condition; however, we have no evidence to support this other than the comparability of the SF-36 scores across the SAPPHIRE population and the frozen shoulder populations in Carette et al..$^{35}$ and Dogru et al. ${ }^{51}$ These data suggest that the populations have very similar QoL scores at baseline. Further investigation of these issues is warranted but is outside the scope of this research.

None of those economic evaluations presenting EQ-5D data presented QALYs. It is not clear why QALYs were not derived. One study provided justification, which was that the minimal changes in clinical effectiveness of the EQ-5D outcome provided no additional information when analysed in a cost-effectiveness analysis. ${ }^{113}$ It is not clear that this is valid justification for not deriving QALY outcomes. 


\section{Strengths and limitations of the review}

To our knowledge this is the first systematic review that has included all NHS-relevant interventions in a single synthesis. A total of 19 electronic databases were searched including potential sources of unpublished data. Unlike several previous reviews there were no language restrictions. Full papers of 67 studies in 12 languages other than English were screened for eligibility, of which four were subsequently included in the review. Risk of bias was assessed and taken into consideration in the synthesis. Whenever possible, the treatment effect for individual studies was reported with a 95\% CI, even when quantitative synthesis was not undertaken.

The key limitation of this technology assessment was the lack of data available. This was despite comprehensive searches without language restrictions across a range of sources of studies. There was insufficient evidence to make robust conclusions about the effectiveness of any of the interventions. Because of the lack of evidence on stage of frozen shoulder in the included trials, it was not possible to undertake the planned synthesis exploring the influence of stage of frozen shoulder or the presence of diabetes on the effectiveness of the various interventions. Even where there were several trials of a single intervention, there was such diversity in the intervention and comparators used and such poor reporting of outcome data that there were few instances in which the planned quantitative synthesis was possible or appropriate. Given that most of the included studies had a small number of participants and may have been underpowered, this was unfortunate.

Unlike some other reviews that have looked at shoulder complaints across the board as a single condition, we attempted to reduce variability due to the nature of the shoulder condition by including only patients with primary frozen shoulder. However, despite this, it is likely that the population included is diverse because of variability in how the included studies defined frozen shoulder. Together with the lack of information on impact of stage, this means that even for those interventions for which there is some evidence of effectiveness, there is some uncertainty as to what primary frozen shoulder populations this applies to.

Because the studies were often poorly reported it was difficult in some instances to be certain that the population included those with primary frozen shoulder and not those with secondary frozen shoulder. As a result we may have excluded studies that did in fact include a primary frozen shoulder population. Comparing the results of our review with those of other recent reviews it is unlikely that the inclusion of such studies would have altered our conclusions.

The variability in the outcome measures used was a further limitation of the data available. Pain, and function and disability were the most commonly used end points in clinical trials for frozen shoulder and there was considerably variability in the measures used to assess these three outcomes. Studies included in the review measured pain used the verbal numeric rating scales or VAS scales (including the SPADI 5-item subscale), which have been shown to be highly correlated with one another. ${ }^{122}$ The decision to standardise results of these studies using the SMD enabling pooling of studies was therefore appropriate. In direct contrast the outcome of function and disability was measured by a number of different shoulder-specific scales, which have been shown to have low to moderate correlation with one another. This indicates that different shoulder-specific function and disability scales may not be measuring the same thing. ${ }^{54,55,123,124} \mathrm{On}$ the basis of this we did not use a standardised score for function and disability outcomes, which meant that a quantitative pooling of these data, for the most part, was not possible.

To aid interpretation, pooled SMDs for the outcome of pain were back transformed to a 0-100 mm VAS. As no between-group, or, for that matter, within-group, MCID on the $0-100 \mathrm{~mm}$ 
VAS was available for frozen shoulder, a proxy was derived from the within-group MCID of a rotator cuff disease population. Although this proxy represents the best available betweengroup MCID for frozen shoulder at this time and has been used elsewhere, ${ }^{6}$ any implied clinical significance should be seen as tentative rather than definitive. In addition, such an approach was appropriate only for pooled estimates.

Several studies that were included in the review were not included in the synthesis because the period of follow-up was $\leq 4$ weeks. Such short follow-up periods are unlikely to capture meaningful changes in outcome measures.

\section{Mixed-treatment comparison}

A key factor to consider in evaluating the strengths and limitations of the MTC undertaken is whether the assumption that the studies are sufficiently homogeneous to be quantitatively combined has been met. Arguable sources of heterogeneity in the MTC were the fact that the placebos were not identical between studies, given the differing nature of the interventions; and that there was differential reporting of the use of previous treatments for frozen shoulder associated with the invasiveness of treatment, which may indicate that populations between trials varied in the treatment resistance of frozen shoulder. Furthermore, as in the main synthesis, there was a paucity of high-quality studies. However, the model was a good fit and gave stable estimates and it is interesting that there was no clear difference between the interventions in effectiveness and no single intervention was substantially better than the others. Given the possible heterogeneity of placebos and of study populations, and the inclusion of poor-quality studies, there is some uncertainty regarding the results of the MTC, as there is with the main synthesis.

\section{Economic evidence}

The lack of available data made the development of a decision-analytic model impossible. We found little evidence on treatment related to stage of condition, treatment pathways, the impact on quality of life and associated resource use and no information on utilities. Without making a number of questionable assumptions, modelling was not possible. Following clinical advice it was decided that insufficient evidence was available to inform the structure of a model or to guide and justify our assumptions. Although it was not possible to build a decision model, the assessment has estimated the costs for the various treatments included in the review and investigated the link between QoL and utilities where possible. We have not been able to quantify the uncertainty in our cost estimates and the use of a small group of clinical experts to derive the estimates is a limitation. Despite this limitation we believe that the estimates obtained may provide useful information as input to (future) cost-effectiveness assessments of treatments in frozen shoulder populations.

The findings of the exploratory mapping analysis suggest that there is likely to be a relationship between the SF-36 and the EQ-5D, and VAS and the EQ-5D in frozen shoulder populations. Caution is needed, however, as further data on QoL outcomes using both non-preference-based and preference-based measures are now needed. This will allow full validation of the nature and direction of the relationship between the instruments and investigation of whether utilities might be a useful outcome measure in frozen shoulder populations. It is clear from the results obtained that the relationship between the two clinical outcomes and EQ-5D is not the same. This is demonstrated in the Dogru et al. study ${ }^{51}$ by the different direction of QALY benefit, which favours the intervention over the control when VAS is mapped to EQ-5D and the control over the intervention when SF-36 is mapped. The choice of clinical outcome to be included in the mapping is obviously very important and how this choice is made warrants further research. It is also unclear whether the use of more complex statistical methods would alter the predictive performance of the SF-36 PCS and MCS mapping onto the EQ-5D utilities, compared with the models tested in the exploratory analysis, for example whether including all eight dimensions 
of the SF-36 profile in mapping onto the EQ-5D changes the nature of the relationship. These methodological investigations are beyond the scope of this report.

Mapping is a substitute for primary measurement of utilities and is subject to a number of potential limitations. In this instance the main limitations are likely to be that it was not performed directly using individual-level patient data drawn from frozen shoulder trials but used a UK data set of patients with rotator cuff disease (the SAPPHIRE trial); and the small study sample ( $n=141$ for pain VAS, and $n=133$ for SF-36 PCS/MCS in the base case presented). It would be possible to further validate the apparent relationship between the SF-36/VAS and the EQ-5D through the use of an external data set (i.e. an alternative trial). However, to fully measure the apparent relationship between these two outcomes the collection of primary data will be required, which will have greater validity.

\section{Improving the evidence on the effectiveness and cost-effectiveness}

This assessment highlighted considerable gaps in the evidence for the effectiveness and costeffectiveness of all of the interventions investigated for frozen shoulder. Given the range of treatment options a multi-arm trial would be the most appropriate way forward. This raises the question of which interventions should be given priority for future research on treatments for frozen shoulder and where resources would best be spent in undertaking future research. Given the lack of an economic model we were not in a position to characterise the decision uncertainty and present a value of information analysis to support recommendations about where future resources for research should focus.

Given that clinically there is a fairly consistent view that a stepped-up approach should be used in terms of invasiveness of treatment, most patients are likely to receive only the conservative treatments. It could be argued, therefore, that this is the most important area on which to focus the research effort. However, given the lack of consensus across professional groups regarding the most appropriate care pathway and the apparent overlap in patients receiving treatments of varying invasiveness, it may be inappropriate to put research effort into conservative treatments at the expense of the invasive treatments. The costing exercise we undertook found that the invasive treatments may be substantially more expensive than the conservative options. It would therefore be important to establish whether these interventions produce benefits for the patient and are cost-effective choices.

In a recent survey of 303 health-care professionals involved in the management of frozen shoulder, $88 \%$ of respondents, including GPs, physiotherapists and orthopaedic surgeons, considered that there was a need for a RCT comparing treatments, particularly in the early painful phase. ${ }^{18}$ Several interventions were supported for future research: watchful waiting and education (classified as conservative), physical therapy, injections and surgery (encompassing MUA and capsular release). Overall preferences for interventions requiring further research differed between the professional groups. The interventions that received least support were arthrographic distension, sodium hyaluronate injection, oral painkillers and oral steroids. There was support for research on physiotherapy and mobilisation techniques but much less so for individual therapies such as acupuncture, ultrasound, heat, laser therapy and electroacupuncture. A recently published guideline from the Chartered Society for Physiotherapy has also suggested that there may not be much to be gained from evaluating specific modalities as stand-alone treatments. ${ }^{6}$

Any future trials should give more serious attention to the control group used. Home exercise (with or without a placebo) was the most commonly reported control in the included studies. 
However, in general the content of this control intervention was poorly reported. There is a large gap in the evidence about the effectiveness of a high-quality conservative intervention of education, home exercise and self-management of pain with support and monitoring. Any future trial should pay careful attention to the control condition to clearly establish what additional benefit other interventions provide. In addition, the maximum length of follow-up in most studies was 3 months. Future trials should therefore have follow-up periods that are of sufficient length to allow determination of whether interventions are effective in the medium and long term.

There is a view clinically that stage of frozen shoulder is an important aspect to consider when making treatment choices. This is not reflected in how most trials have been undertaken to date, although it is unclear why not. Obviously it makes recruitment to trials easier if the population is not restricted in this way. Stratification within trials may be a more pragmatic approach. Another possible factor explaining the lack of consideration for stage is the wide range of countries in which the trials have been undertaken. It is clear, however, that any future research should not neglect consideration of stage. Because of the lack of evidence on treatment effects in patients with diabetes, this will be an important subgroup. This reflects the recent survey that highlighted those with diabetes as an important subgroup. ${ }^{18}$

Populations included in the review were diverse because of considerable variation in how frozen shoulder was defined in the individual studies. This is unsurprising given that there is a noted absence of a standard definition and classification for frozen shoulder. ${ }^{2,3}$ The variations in study populations together with the lack of information on the stage of frozen shoulder mean that, even for those interventions for which there is some evidence of effectiveness, there is some uncertainty as to which frozen shoulder populations these apply to. Although potential classifications for primary and secondary frozen shoulder have been put forward, ${ }^{3}$ a consensus on the definition of frozen shoulder is sorely needed to enable the generalisability of further research to be assessed.

Given that studies of clinimetric properties (i.e. validity, reproducibility, responsiveness and ease of scoring) of various shoulder-specific disability scores have conflicting findings, it is difficult to suggest the most appropriate score to use. ${ }^{125,126}$ Further research is therefore needed to determine the clinimetric properties of shoulder-specific function and disability scores. This should conclude with a consensus on the most appropriate tools to assess shoulder function, both in research and in clinical practice. For assessing the clinical meaningfulness of betweengroup differences in future trials, consideration should be given to the recommendations of the Initiative on Methods, Measurement and Pain Assessment in Clinical Trials (IMPACT). ${ }^{127}$ This group suggests that the clinical meaningfulness of group differences in trials, in terms of pain, should be established using a multifactorial evaluation of factors such as statistically significant treatment effects, responder analysis, effect size compared with that of other established treatments, safety and tolerability, and results for other end points.

Based on the available evidence we were unable to structure a simple decision tree, but, given the complex nature of the treatment pathways, future decision-analytic modelling undertaken in this area will need to take into account the nature of any sequential decision-making processes and treatments rather than just a single therapy. This will require tangible evidence of current treatment patterns and resource use in frozen shoulder populations. There may be benefit in undertaking an elicitation exercise with a range of health-care professionals to prioritise the interventions to be compared and underpin the design of any future trial.

There is a current lack of studies providing data on HRQoL specific to frozen shoulder populations. This information is required to enable assessments of cost-utility to be undertaken. 
The inclusion of preference-based QoL measures alongside clinical trials in frozen shoulder populations is a necessity. Whether the EQ-5D as an instrument is considered to be the most appropriate for use in frozen shoulder populations has yet to be determined and further research is required to address this and other questions on the use of HRQoL measures in clinical studies. Mapping is always a second-best solution to using a preference-based (generic or conditionspecific) measure in the first place, but it is often the approach followed for pragmatic reasons and so this remains an important area of research. Primary data collection in frozen shoulder populations is now needed. 



\section{Chapter 5}

\section{Conclusions}

\section{Implications for service provision}

- There may be short-term benefit for patients with frozen shoulder of $<6$ months' duration of adding a single intra-articular steroid injection to home exercise. There is also shortterm benefit for the same population of adding physiotherapy to a single intra-articular steroid injection. This is based on two studies that varied in steroid dose and in whether the injection was guided, making it difficult to determine how the steroid intervention should be delivered. Although neither study explicitly provided information on stage of frozen shoulder, only patients with frozen shoulder of $<6$ months' duration were included, suggesting that these patients were likely to be at the initial painful stage. Based on two studies of physiotherapy that included mobilisation in 8-12 sessions over a 4-week period, it is unclear what is the most effective physiotherapy to provide with injection in terms of content and duration.

- Based on a single study, and for some outcomes only, there may be benefit from adding SWD to passive mobilisation and home exercise.

- Based on a single study, HGMT may be more effective for some outcomes, but not others, than LGMT in a population that has already received physiotherapy and/or a steroid injection. In the study, high-grade mobilisation consisted of twice-weekly, 30-minute sessions for a maximum of 12 weeks during which Maitland grade III and IV mobilisation was applied into the stiffness zone. Low-grade mobilisation was delivered according to the same schedule but consisted of Maitland grade I and II mobilisation applied within the pain-free zone. LGMT may be more cost-effective than HGMT.

- Given the paucity of economic evidence no conclusions can be made about the costeffectiveness of the different interventions.

\section{Suggested research priorities}

There are large gaps in the evidence for the effectiveness and cost-effectiveness of all of the interventions investigated. Taking into account the gaps in the evidence, the views of health-care professionals in a recent survey ${ }^{18}$ and the interventions that are most commonly used in the NHS, we suggest that the following should be given priority in future high-quality RCTs assessing effectiveness and cost-effectiveness:

- A 'standard care' package of high-quality conservative management. This should be fully specified in any future trial and we suggest that it should involve a structured protocol of high-quality education, advice, home exercise and monitoring/support to encourage completion of the home exercises.

- Steroid injection, in particular an investigation of the effectiveness and cost-effectiveness of multiple injections and whether there is any added benefit from providing physiotherapy after steroid injection over and above that obtained with steroid injection plus high-quality conservative management. 
- Physical therapies, specifically physiotherapy interventions that have a component which involves mobilisation or exercises. Research is required to establish whether there is any benefit from having physical therapy alone over and above that of high-quality conservative management.

- Intensive interventions, specifically arthrographic distension, MUA and arthroscopic capsular release. Although there is also a gap in the evidence regarding the effectiveness of distension, in the recent survey of health-care professionals this intervention did not receive as much support as a topic for future research as the other intensive interventions.

The large number of treatment options for frozen shoulder and the limited evidence for their effectiveness and cost-effectiveness makes prioritisation of these difficult and they are not listed in order of importance. We suggest that an appropriate starting point would be a multi-arm trial that compares the effectiveness and cost-effectiveness of interventions of differing intensity and costs: high-quality conservative management, steroid injection (possibly in conjunction with arthrographic distension) and surgical management (MUA and capsular release).

Important subgroups in any future RCTs are phase of frozen shoulder and patients with diabetes. These trials should collect data on resource use and use a utility measure to allow assessment of cost-effectiveness. A systematic approach to recording adverse events will also be important. 


\section{Acknowledgements}

$\mathrm{M}$ any thanks to all members of the advisory group for the many helpful contributions.

Many thanks to Jonathan Minton for the help provided with study selection.

\section{Contribution of authors}

Emma Maund contributed to writing the protocol, study selection, data extraction, quality assessment, data analysis and report writing.

Dawn Craig contributed to writing the protocol, undertaking the economic analysis and report writing.

Sara Suekarran contributed to writing the protocol, study selection, data extraction, quality assessment, data analysis and report writing.

Aileen Rae Nielson was involved in the economic analysis and report writing.

Kath Wright developed the search strategies, undertook the searches and wrote the literature search methods section.

Stephen Brealey, Laura Dennis, Lorna Goodchild, Nigel Hanchard, Amar Rangan, Gerry Richardson and James Robertson provided clinical and/or methodological input and advice throughout the project and commented on the protocol and drafts of the report.

Catriona McDaid was responsible for writing the protocol, contributed to study selection, data extraction, quality assessment, data analysis and report writing and had overall responsibility for co-ordinating the project. 



\section{References}

1. Codman E. Rupture of the supraspinatus tendon and other lesions in or about the subacromial bursa. Malabar, FL: Krieger; 1965.

2. Schellingerhout JM, Verhagen AP, Thomas S, Koes BW. Lack of uniformity in diagnostic labeling of shoulder pain: time for a different approach. Man Therap 2008;13:478-83.

3. Zuckerman J, Rokito A. Frozen shoulder: a consensus definition. J Shoulder Elbow Surg 2010;20:322-5.

4. de Jong BA. The painful stiff shoulder. PhD thesis. Amsterdam: University of Amsterdam; 1991.

5. Dias R, Cutts S, Massoud S. Frozen shoulder. BMJ 2005;331:1453-6.

6. Hanchard N, Goodchild L, Thompson J, O'Brien T, Davison D, Richardson C, et al. Evidencebased clinical guidelines for the diagnosis, assessment and physiotherapy management of contracted (frozen) shoulder. London: Chartered Society of Physiotherapy; 2011. URL: www. csp.org.uk/skipp. Accessed 11 March 2011.

7. Bunker TD. Frozen shoulder: unravelling the enigma. Ann R Coll Surg Engl 1997;79:210-13.

8. Bunker TD. Time for a new name for frozen shoulder - contracture of the shoulder. Shoulder Elbow 2009;1:4-9.

9. Hand GCR, Athanasou NA, Matthews T, Carr AJ. The pathology of frozen shoulder. J Bone Joint Surg Br 2007;89:928-32.

10. van der Windt DA, Koes BW, de Jong BA, Bouter LM. Shoulder disorders in general practice: incidence, patient characteristics, and management. Ann Rheum Dis 1995;54:959-64.

11. Walker-Bone K, Palmer KT, Reading I, Coggon D, Cooper C. Prevalence and impact of musculoskeletal disorders of the upper limb in the general population. Arthritis Care Res 2004;51:642-51.

12. Dawson J, Shepperd S, Carr A. An overview of factors relevant to undertaking research and reviews on the effectiveness of treatment for frozen shoulder. Shoulder Elbow 2010;2:232-7.

13. Hand C, Clipsham K, Rees JL, Carr AJ. Long-term outcome of frozen shoulder. J Shoulder Elbow Surg 2008;17:231-6.

14. New Zealand Guidelines Group. The diagnosis and management of soft tissue shoulder injuries and related disorders. Wellington: New Zealand Guidelines Group; 2004. URL: www.nzgg. org.nz. Accessed 9 March 2009.

15. Linsell L, Dawson J, Zondervan K, Rose P, Randall T, Fitzpatrick R, et al. Prevalence and incidence of adults consulting for shoulder conditions in UK primary care; patterns of diagnosis and referral. Rheumatology 2006;45:215-21.

16. Stergioulas A. Low-power laser treatment in patients with frozen shoulder: preliminary results. Photomed Laser Surg 2008;26:99-105.

17. NHS Choices. Health $A-Z$ - conditions and treatments. URL: www.nhs.uk/Conditions/Pages/ hub.aspx. Accessed 11 March 2011.

18. Dennis L, Brealey S, Rangan A, Rookmoneea M, Watson J. Managing idiopathic frozen shoulder: a survey of health professionals' current practice and research priorities. Shoulder Elbow 2010;2:294-300. 
19. Manske RC, Prohaska D. Clinical commentary and literature review: diagnosis, conservative and surgical management of adhesive capsulitis. Shoulder Elbow 2010;2:238-54.

20. Buchbinder R, Green S, Youd JM, Johnston Renea V. Oral steroids for adhesive capsulitis. Cochrane Database Syst Rev 2006; Issue 4, Art. No. CD006189.

21. Buchbinder R, Green S, Youd JM. Corticosteroid injections for shoulder pain. Cochrane Database Syst Rev 2003; Issue 1, Art. No. CD004016.

22. Shah N, Lewis M. Shoulder adhesive capsulitis: systematic review of randomised trials using multiple corticosteroid injections. Br J Gen Pract 2007;57:662-7.

23. Arroll B, Goodyear-Smith F. Corticosteroid injections for painful shoulder: a meta-analysis. Br J Gen Pract 2005;55:224-8.

24. Green S, Buchbinder R, Hetrick SE. Physiotherapy interventions for shoulder pain. Cochrane Database Syst Rev 2003; Issue 2, Art. No. CD004258.

25. Cleland J, Durall CJ. Physical therapy for adhesive capsulitis: systematic review. Physiotherapy 2002;88:450-7.

26. Alvado A, Pelissier J, Benaim C, Petiot S, Herisson C. [Physical therapy of frozen shoulder: literature review.] Ann Readapt Med Phys 2001;44:59-71.

27. Green S, Buchbinder R, Hetrick SE. Acupuncture for shoulder pain. Cochrane Database Syst Rev 2005; Issue 2, Art. No. CD005319.

28. Buchbinder R, Green S, Youd JM, Johnston Renea V, Cumpston M. Arthrographic distension for adhesive capsulitis (frozen shoulder). Cochrane Database Syst Rev 2008; Issue 1, Art. No. CD007005.

29. Green S, Buchbinder R, Glazier R, Forbes A. Systematic review of randomised controlled trials of interventions for painful shoulder: selection criteria, outcome assessment, and efficacy. BMJ 1998;316:354-60.

30. Rookmoneea M, Dennis L, Brealey S, Rangan A, White B, McDaid C, et al. The effectiveness of interventions in the management of patients with primary frozen shoulder. J Bone Joint Surg Br 2010;92B:1267-72.

31. Lu G, Ades AE. Combination of direct and indirect evidence in mixed treatment comparisons. Stat Med 2004;23:3105-24.

32. Caldwell DM, Ades AE, Higgins JP. Simultaneous comparison of multiple treatments: combining direct and indirect evidence. BMJ 2005;331:879-900.

33. Sutton A, Ades A, Cooper N, Abrams K. Use of indirect and mixed treatment comparisons for technology assessments. Pharmacoeconomics 2008;26:753-67.

34. Centre for Reviews and Dissemination. Systematic reviews: CRD's guidance for undertaking reviews in health care. York: University of York; 2009.

35. Carette S, Moffet H, Tardif J, Bessette L, Morin F, Fremont P, et al. Intraarticular corticosteroids, supervised physiotherapy, or a combination of the two in the treatment of adhesive capsulitis of the shoulder: a placebo-controlled trial. Arthritis Rheum 2003;48:829-38.

36. Tveita EK, Tariq R, Sesseng S, Juel NG, Bautz-Holter E. Hydrodilatation, corticosteroids and adhesive capsulitis: a randomized controlled trial. BMC Musculoskelet Disord 2008;9:53.

37. Higgins J, Deeks JJ. Chapter 7: selecting studies and collecting data. In Higgins J, Green S, editors. Cochrane handbook for systematic reviews of interventions. Version 5.0.1 (updated 
September 2008). The Cochrane Collaboration; 2008. URL: www.cochrane-handbook.org. Accessed 9 March 2009.

38. Quraishi NA, Johnston P, Bayer J, Crowe M, Chakrabarti AJ. Thawing the frozen shoulder. A randomised trial comparing manipulation under anaesthesia with hydrodilatation. J Bone Joint Surg Br 2007;89:1197-200.

39. Kivimaki J, Pohjolainen T, Malmivaara A, Kannisto M, Guillaume J, Seitsalo S, et al. Manipulation under anesthesia with home exercises versus home exercises alone in the treatment of frozen shoulder: a randomized, controlled trial with 125 patients. J Shoulder Elbow Surg 2007;16:722-6.

40. Vermeulen HM, Rozing PM, Obermann WR, le Cessie S, Vliet Vlieland TPM. Comparison of high-grade and low-grade mobilization techniques in the management of adhesive capsulitis of the shoulder: randomized controlled trial. Phys Ther 2006;86:355-68.

41. Ryans I, Montgomery A, Galway R, Kernohan WG, McKane R. A randomized controlled trial of intra-articular triamcinolone and/or physiotherapy in shoulder capsulitis. Rheumatology 2005;44:529-35.

42. Rizk TE, Pinals RS, Talaiver AS. Corticosteroid injections in adhesive capsulitis: investigation of their value and site. Arch Phys Med Rehabil 1991;72:20-2.

43. Buchbinder R, Green S, Forbes A, Hall S, Lawler G. Arthrographic joint distension with saline and steroid improves function and reduces pain in patients with painful stiff shoulder: results of a randomised, double blind, placebo controlled trial. Ann Rheum Dis 2004;63:302-9.

44. Rodgers M, McKenna C, Palmer S, Chambers D, van Hout S, Golder S, et al. Curative catheter ablation in atrial fibrillation and typical atrial flutter: systematic review and economic evaluation. Health Technol Assess 2008;12(34).

45. Drummond MF. Methods for the economic evaluation of healthcare programmes. 3rd edn. Oxford: Oxford University Press; 2005.

46. Review Manager (RevMan) [computer program.] Version 5. Copenhagen: The Nordic Cochrane Centre, The Cochrane Collaboration; 2008.

47. Higgins JP, Thompson SG, Deeks JJ, Altman DG. Measuring inconsistency in meta-analysis. BMJ 2003;327:557-60.

48. Deeks JJ, Higgins JPT, Altman DG. Chapter 9: analysing data and undertaking metaanalyses. In Higgins JPT, Green S, editors. Cochrane handbook for systematic reviews of interventions. Version 5.0.1 (updated September 2008): The Cochrane Collaboration; 2008. URL: www.cochrane-handbook.org. Accessed 9 March 2009.

49. Borenstein M, Hedges LV, Higgins JPT, Rothstein HR. Introduction to meta-analysis. Oxford: John Wiley; 2009.

50. Tashiian RZ, Deloacha J, Porucznik CA, Powell AP. Minimal clinically important differences (MCID) and patient acceptable symptomatic state (PASS) for visual analog scales (VAS) measuring pain in patients treated for rotator cuff disease. J Shoulder Elbow Surg 2009;18:927-32.

51. Dogru H, Basaran S, Sarpel T. Effectiveness of therapeutic ultrasound in adhesive capsulitis. Joint Bone Spine 2008;75:445-50.

52. Scholten R, de Beurs E, Bouter L. Is the standardised mean difference a suitable measure of treatment effect? In: The best evidence for health care: the role of The Cochrane Collaboration Università S. Tommaso d’Aquino, Rome, Italy; 1999. p. 9. 
53. Finch E, Brooks D, Stratford PW, Mayo NE. Physical rehabilitation outcome measures: a guide to enhanced clinical decision making. 2nd edn. Toronto: Lippincott, Williams and Wilkins; 2002.

54. Placzek J, Lukens S, Badalanmenti S, Roubal P, Freeman D, Walleman K, et al. Shoulder outcome measures: a comparison of 6 functional tests. Am J Sports Med 2004;32:1270-7.

55. Paul A, Lewis M, Shadforth M, Croft P, van der Windt D, Hay E. A comparison of four shoulder-specific questionnaires in primary care. Ann Rheum Dis 2004;63:1293-9.

56. Romeo A, Bach B, O’Halloran K. Scoring systems for shoulder conditions. Am J Sports Med 1996;24:472-6.

57. Ginn K, Cohen M, Herbert R. Does hand-behind-back range of motion accurately reflect shoulder internal rotation? J Shoulder Elbow Surg 2006;15:311-4.

58. Higgins JPT, Deeks JJ, Altman DG. Chapter 16: Special topics in statistics. In Higgins JPT, Green S, editors. Cochrane handbook for systematic reviews of interventions. Version 5.0.1 (updated September 2008). The Cochrane Collaboration, 2008. URL: www.cochranehandbook.org. Accessed 9 March 2009.

59. Song F, Harvey I, Lilford R. Adjusted indirect comparison may be less biased than direct comparison for evaluating new pharmaceutical interventions. J Clin Epidemiol 2008;61:455-63.

60. Lunn DJ, Thomas A, Best N, Spiegelhalter D. WinBUGS - a Bayesian modelling framework: concepts, structure, and extensibility. Stat Comput 2000;10:325-37.

61. Lambert PC, Sutton AJ, Burton PR, Abrams KR, Jones DR. How vague is vague? A simulation study of the impact of the use of vague prior distributions in MCMC using WinBUGS. Stat Med 2005;24:2401-28.

62. Flemming K, Briggs M. Electronic searching to locate qualitative research: evaluation of three strategies. J Adv Nurs 2007;57:95-100.

63. Hawker S, Payne S, Kerr C, Hardey M, Powell J. Appraising the evidence: reviewing disparate data systematically. Qual Health Res 2002;12:1284-99.

64. National Institute for Health and Clinical Excellence. Guide to the methods of technology appraisal. London: National Institute for Health and Clinical Excellence; 2008.

65. Dolan P. Modeling valuations for EuroQol health states. Med Care 1997;35:1095-108.

66. Calis M, Demir H, Ulker S, Kirnap M, Duygulu F, Calis HT. Is intraarticular sodium hyaluronate injection an alternative treatment in patients with adhesive capsulitis? Rheumatol Int 2006;26:536-40.

67. Dacre JE, Beeney N, Scott DL. Injections and physiotherapy for the painful stiff shoulder. Ann Rheum Dis 1989;48:322-5.

68. Bal A, Eksioglu E, Gulec B, Aydog E, Gurcay E, Cakci A. Effectiveness of corticosteroid injection in adhesive capsulitis. Clin Rehabil 2008;22:503-12.

69. Rovetta G, Monteforte P. Intraarticular injection of sodium hyaluronate plus steroid versus steroid in adhesive capsulitis of the shoulder. Int J Tissue React 1998;20:125-30.

70. Takagishi K, Saito A, Segawa K, Takahira N, Itomitu S. Evaluation of intra-articular injection in patients with so-called Gojyukata: comparison hyaluronate and steroid. Jpn J Med Pharm Sci 1996;35:377-81.

71. Wies J. A pilot randomised placebo controlled trial of osteopathic and physiotherapy treatment for frozen shoulder. J Osteopath Med 2003;6:40. 
72. Yan F. [Comparison of dumbbell gymnastics and bare-handed exercise in ameliorating the symptoms of shoulder periarthritis.] Chin J Clin Rehab 2005;9:187-9.

73. Diercks RL, Stevens M. Gentle thawing of the frozen shoulder: a prospective study of supervised neglect versus intensive physical therapy in seventy-seven patients with frozen shoulder syndrome followed up for two years. J Shoulder Elbow Surg 2004;13:499-502.

74. Dundar U, Toktas H, Cakir T, Evcik D, Kavuncu V. Continuous passive motion provides good pain control in patients with adhesive capsulitis. Int J Rehabil Res 2009;32:193-8.

75. Leung MSF, Cheing GLY. Effects of deep and superficial heating in the management of frozen shoulder. J Rehabil Med 2008;40:145-50.

76. Maricar NN, Chok B. A comparison of the effect of manual therapy with exercise therapy and exercise therapy alone for stiff shoulders. Physiother Singapore 1999;2:99-104.

77. Pajareya K, Chadchavalpanichaya N, Painmanakit S, Kaidwan C, Puttaruksa P, Wongsaranuchit $\mathrm{Y}$. Effectiveness of physical therapy for patients with adhesive capsulitis: a randomized controlled trial. J Med Assoc Thai 2004;87:473-80.

78. Yang J-1, Chang C-W, Chen S-Y, Wang S-F, Lin J-J. Mobilization techniques in subjects with frozen shoulder syndrome: randomized multiple-treatment trial. Phys Ther 2007;87:1307-15.

79. Cheing GLY, So EML, Chao CYL. Effectiveness of electroacupuncture and interferential eloctrotherapy in the management of frozen shoulder. J Rehabil Med 2008;40:166-70.

80. Fang J-Q, Zhang Y, Xuan L-H, Liu K-Z, Chen L. [Observation on clinical therapeutic effect of transcutaneous point electric stimulation on periarthritis of shoulder at different stages.] Zhongguo Zhenjiu 2006;26:11-14.

81. Ma T, Kao MJ, Lin IH, Chiu YL, Chien C, Ho TJ, et al. A study on the clinical effects of physical therapy and acupuncture to treat spontaneous frozen shoulder. Am J Chin Med 2006;34:759-75.

82. Amir-us-Saqlain H, Zubairi A, Taufiq I. Functional outcome of frozen shoulder after manipulation under anaesthesia. J Pak Med Assoc 2007;57:181-5.

83. Jacobs LG, Smith MG, Khan SA, Smith K, Joshi M. Manipulation or intra-articular steroids in the management of adhesive capsulitis of the shoulder? A prospective randomized trial. $J$ Shoulder Elbow Surg 2009;18:348-53.

84. Gam AN, Schydlowsky P, Rossel I, Remvig L, Jensen EM. Treatment of 'frozen shoulder' with distension and glucorticoid compared with glucorticoid alone. A randomised controlled trial. Scand J Rheumatol 1998;27:425-30.

85. Austgulen OK, Oyen J, Hegna J, Solheim E. [Arthroscopic capsular release in treatment of primary frozen shoulder.] Tidsskr Nor Laegeforen 2007;127:1356-8.

86. Chen S-K, Chien S-H, Fu Y-C, Huang P-J, Chou P-H. Idiopathic frozen shoulder treated by arthroscopic brisement. Kaohsiung J Med Sci 2002;18:289-94.

87. Höfler M. The Bradford Hill considerations on causality: a counterfactual perspective. Emerg Themes Epidemiol 2005;2:11.

88. Salanti G, Higgins J, Ades A, Ioannidis J. Evaluation of networks of randomized trials. Stat Methods Med Res 2008;17:279-301.

89. Ucuncu F, Capkin E, Karkucak M, Ozden G, Cakirbay H, Tosun M, et al. A comparison of the effectiveness of lanmark-guided injections and ultrasonography-guided injections for shoulder pain. Clin J Pain 2009;25:786-9. 
90. Weber MD. Factors that predict change in health-related qualityof life of patients with adhesive capsulitis. Jackson, MS: University of Mississippi Medical Centre; 2001.

91. van den Hout WB, Vermeulen HM, Rozing PM, Vliet Vlieland TPM. Impact of adhesive capsulitis and economic evaluation of high-grade and low-grade mobilisation techniques. Aust J Physiother 2005;51:141-9.

92. National Institute for Health and Clinical Excellence. Guide to the methods of technology appraisal. London: NICE; 2004. URL: www.nice.org.uk/aboutnice/howwework/devnicetech/ technologyappraisalprocessguides/guide_to_the_methods_of_technology_appraisal_ reference_n0515.jsp. Accessed 9 March 2009.

93. Curtis L. Unit costs of health and social care 2009. Canterbury: Personal Social Services Research Unit, University of Kent; 2010.

94. British Medical Association and Royal Pharmaceutical Society of Great Britain. British national formulary. No. 60. London: BMA and RPS; 2010.

95. Department of Health. NHS reference costs 2008-2009; 2010. URL: www.dh.gov.uk/prod_ consum_dh/groups/dh_digitalassets/@dh/@en/@ps/documents/digitalasset/dh_118329.pdf. Accessed 2 February 2011.

96. Brazier J, Roberts J, Deverill M. The estimation of a preference-based measure of health from the SF-36. J Health Econ 2002;21:271-92.

97. Feeny D, Furlong W, Torrance G, Goldsmith C, Zhu Z, DePaw S, et al. Multi-attribute and single-attribute utility functions for the Health Utilities Index Mark 3 system. Med Care 2002;40:113-28.

98. Sintonen $\mathrm{H}$. The 15D instrument of health-related quality of life: properties and applications. Ann Med 2001;33:328-36.

99. Brazier JE, Yang Y, Tsuchiya A, Rowen DL. A review of studies mapping (or cross walking) non-preference based measures of health to generic preference-based measures. Eur J Health Econ 2010;11:215-25.

100. Rowen D, Brazier J, Roberts J. Mapping SF-36 onto the EQ-5D index: how reliable is the relationship? Health Qual Life Outcomes 2009;7:27.

101. Watson J, Helliwell P, Morton V, Adebajo A, Dickson J, Russell I, et al. Shoulder acute pain in primary healthcare: is retraining effective for GP principals? SAPPHIRE - a randomized controlled trial. Rheumatology 2008;47:1795-802.

102. Watson J, Helliwell P, Morton V, Adebajo A, Dickson J, Russell I, et al. Shoulder acute pain in primary healthcare: is retraining effective for GP principals? SAPPHIRE-a randomized controlled trial. Rheumatology 2008;48:558-63.

103. Croft P, Pope D, Zonca M, O'Neill T, Silman A. Measurement of shoulder related disability: results of a validation study. Ann Rheum Dis 1994;53:525-8.

104. McKenna C, Bojke L, Manca A, Adebajo A, Dickson J, Helliwell P, et al. Shoulder acute pain in primary health care: is retraining GPs effective? The SAPPHIRE randomized trial: a costeffectiveness analysis. Rheumatology 2009;48:558-63.

105. Hurvitz AP, Hodapp KL, Jadgchew J, Solomon DJ, Stolldorf HS, Provencher MT. Central serous chorioretinopathy resulting in altered vision and color perception after glenohumeral corticosteroid injection. Orthopedics 2009; 32:pii. URL: http://orthosupersite.com/view. asprID=A1926. Accessed 22 March 2010.

106. Lee KS. Musculoskeletal ultrasound: how to evaluate for Morton's neuroma. AJR Am J Roentgenol 2009;193:W172. 
107. Ware J, Sherbourne C. The MOS 36-item short-form health survey (SF-36): conceptual framework and item selection. Med Care 1992;30:473-81.

108. Snee RD. Validation of regression models. Methods and examples. Technometrics 1977;19:415-28.

109. Chay K, Powell J. Semiparametric censored regression models. J Econ Persp 2001;15:29-42.

110. Sullivan PWG. Mapping the EQ-5D from the SF-12: US general population preferences in a nationally representative sample. Med Decis Making 2006;26:401-9.

111. Tobin J. Estimation of relationships for limited dependent variables. Econometrica 1958;26:22-36.

112. Crott R, Briggs A. Mapping the QLQ-C30 quality of life cancer questionnaire to EQ-5D patient preferences Eur J Health Econ 2010;1 1:427-34.

113. Barton G, Sach T, Jenkinson C, Avery A, Doherty M, Muir K. Do estimates of cost-utility based on the EQ-5D differ from those based on the mapping of utility scores? Health Qual Life Outcomes 2008;6:51.

114. Sach T, Barton G, Jenkinson C, Doherty M, Avery A, Muir K. Comparing cost-utility estimates: does the choice of EQ-5D or SF-6D matter? Med Care 2009;47:889-94.

115. Blanchard V, Barr S, Cerisola FL. The effectiveness of corticosteroid injections compared with physiotherapeutic interventions for adhesive capsulitis: a systematic review. Physiotherapy 2010;96:95-107.

116. Saito S, Furuya T, Kotake S. Therapeutic effects of hyaluronate injections in patients with chronic painful shoulder: a meta-analysis of randomized controlled trials. Arthritis Care Res 2010;62:1009-18.

117. Favejee MM, Huisstede BM, Koes BW. Frozen shoulder: the effectiveness of conservative and surgical interventions - systematic review. Br J Sports Med 2010;45:19-56.

118. de Bruijn C, Goossens M, de Bie R, Ament A, Geraets J, Dinant GJ. Cost-effectiveness of an education and activation program for patients with acute and subacute shoulder complaints compared to usual care. Int J Technol Assess Health Care 2007;23:80-8.

119. James M, Stokes EA, Thomas E, Dziedzic K, Hay EM. A cost consequences analysis of local corticosteroid injection and physiotherapy for the treatment of new episodes of unilateral shoulder pain in primary care. Rheumatology 2005;44:1447-51.

120. Geraets JJXR, Goossens MEJB, de Bruijn CPC, de Groot IJM, Koke AJS, Pelt RAGB, et al. Cost-effectiveness of a graded exercise therapy program for patients with chronic shoulder complaints. Int J Technol Assess Health Care 2006;22:76-83.

121. Bergman GJ, Winter JC, van Tulder MW, Meyboom-De Jong B, Postema K, van der Heijden GJ. Manipulative therapy in addition to usual medical care accelerates recovery of shoulder complaints at higher costs: economic outcomes of a randomized trial. BMC Musculoskelet Disord 2010;11:200.

122. Holdgate A, Asha S, Craig J, Thompson J. Comparison of a verbal numeric rating scale with the visual analogue scale for the measurement of acute pain. Emerg Med (Fremantle) 2003;15:441-6.

123. Beaton D, Richards R. Assessing the reliability and responsiveness of 5 shoulder questionnaires. J Shoulder Elbow Surg 1998;7:565-72.

124. Kirkley A, Griffin S, Dainty K. Scoring systems for the functional assessment of the shoulder. Arthroscopy 2003;19:1109-20. 
125. Bot S, Terwee C, van der Windt D, Bouter L, Dekker J, de Vet H. Clinimetric evaluation of shoulder disability questionnaires: a systematic review of the literature. Ann Rheum Dis 2004;63:335-41.

126. Staples MP, Forbes A, Green S, Buchbinder R. Shoulder-specific disability measures showed acceptable construct validity and responsiveness. J Clin Epidemiol 2010;63:163-70.

127. Dworkin RH, Turk DC, McDermott MP, Peirce-Sandner S, Burke LB, Cowan P, et al. Interpreting the clinical importance of group differences in chronic pain clinical trials: IMMPACT recommendations. Pain 2009;146:238-44.

128. NCT00160784. Arthroscopy and manipulation vs a home therapy program in the treatment of adhesive capsulitis. URL: http://ClinicalTrials.gov/show/NCT00160784. Accessed 6 April 2010.

129. NCT00840229. Capsular and intra-articular corticosteroid injection for adhesive capsulitis (frozen shoulder): a randomised, double blind, placebo controlled trial. URL: http:// ClinicalTrials.gov/show/NCT00840229. Accessed 6 April 2010.

130. NCT00679887. Chronic shoulder pain treated by pressures with the thumbs on the trigger points. URL: http://ClinicalTrials.gov/show/NCT00679887. Accessed 6 April 2010.

131. NCT00261196. Collagenase in the treatment of adhesive capsulitis (frozen shoulder). URL: http://ClinicalTrials.gov/show/NCT00261196. Accessed 6 April 2010.

132. NCT00873158. Dynamic splinting for patients with adhesive capsulitis. URL: http:// ClinicalTrials.gov/show/NCT00873158. Accessed 6 April 2010.

133. NCT01087229. Effectiveness of an oxygen-nitrous oxide mixture during physical therapy for frozen shoulder. URL: http://ClinicalTrials.gov/show/NCT01087229. Accessed 6 April 2010.

134. NCT00884065. Effectiveness of diacutaneous fibrolysis in painful shoulder. URL: http:// ClinicalTrials.gov/show/NCT00884065. Accessed 6 April 2010.

135. NCT00415441. Effectiveness of physiotherapy for chronic shoulder pain. URL: http:// ClinicalTrials.gov/show/NCT00415441. Accessed 6 April 2010.

136. NCT00742846. Effects of intra-articular versus subacromial steroid injections on clinical outcomes in adhesive capsulitis. URL: http://ClinicalTrials.gov/show/NCT00742846. Accessed 6 April 2010.

137. NCT00694538. Efficacy of interferential laser therapy in shoulder pain. URL: http:// ClinicalTrials.gov/show/NCT00694538. Accessed 6 April 2010.

138. NCT00680472. HKT-500 in adult patients with shoulder pain. URL: http://ClinicalTrials. gov/show/NCT00680472. Accessed 6 April 2010.

139. NCT00377624. HUPS: Hyalgan Use in Painful Shoulder. URL: http://ClinicalTrials.gov/ show/NCT00377624. Accessed 6 April 2010.

140. NCT00211718. Intra-articular injection of botulinum toxin type A for shoulder pain. URL: http://ClinicalTrials.gov/show/NCT00211718. Accessed 6 April 2010.

141. NCT00929305. Low level laser light therapy and chronic neck and shoulder pain. URL: http://ClinicalTrials.gov/show/NCT00929305. Accessed 6 April 2010.

142. NCT00172601. Mobilization techniques in patients with frozen shoulder: a randomized multiple-treatment trial. URL: http://ClinicalTrials.gov/show/NCT00172601. Accessed 6 April 2010.

143. NCT00587626. Proposal to evaluate the efficacy of the InterX 5000 in the treatment of chronic neck and shoulder pain. URL: http://ClinicalTrials.gov/show/NCT00587626. Accessed 6 April 2010. 
144. NCT00163124. A randomized controlled trial of best approach to care compared to diversified chiropractic adjustive technique. URL: http://ClinicalTrials.gov/show/ NCT00163124. Accessed 6 April 2010.

145. NCT00992927. Should the joint capsule of the painful stiff shoulder be ruptured during intra-articular hydraulic distension? URL: http://ClinicalTrials.gov/show/NCT00992927. Accessed 6 April 2010.

146. NCT00875862. Shoulder adhesive capsulitis and ambulatory continuous interscalene nerve blocks. URL: http://ClinicalTrials.gov/show/NCT00875862. Accessed 6 April 2010.

147. NCT01029600. Surgery or capsular distention with steroid in the treatment of primary frozen shoulder? URL: http://ClinicalTrials.gov/show/NCT01029600. Accessed 6 April 2010.

148. Anonymous. Steroid injections vs PT for stiff shoulder. Joint Lett 1999;5:119.

149. Anonymous. UK physio challenges Dutch findings. Van der Heijden GJM et al. No effect of bipolar interferential electrotherapy and pulsed ultrasound for soft tissue shoulder disorders: a randomised controlled trial. Ann Rheum Dis 1999;58;530-40. Physiother Frontline 2000;6:22.

150. Anonymous. Pearls. Phys Sportsmed 2001;29:21.

151. Anonymous. Statistical reanalysis of four recent acupuncture trials. Acupunct Med 2004;22:226.

152. Anonymous. Acupuncture for shoulder pain ( $n=130)$. Acupunct Med 2005;23:41.

153. Anonymous. Manipulative therapies. Focus Altern Complement Ther 2005;10:237.

154. Anonymous. Application of fascial manipulation technique in chronic shoulder pain: anatomical basis and clinical implications. SportEX Dynamics 2009;22:4.

155. Ahmad I, Askar Z, Durrani Z, Idrees M, Ayaz M, Hakim A, et al. Intraarticular injection of methylprednisolone for idiopathic frozen shoulder. J Med Sci 2009;17:16-18.

156. Ahn K, Lee Y-J, Kim E-H, Yang S-M, Lim T-K, Kim Y-S, et al. Interventional microadhesiolysis: a new nonsurgical release technique for adhesive capsulitis of the shoulder. BMC Musculoskelet Disord 2008;9:12.

157. Ainsworth R, Dziedzic K, Hiller L, Daniels J, Bruton A, Broadfield J. A prospective double blind placebo-controlled randomized trial of ultrasound in the physiotherapy treatment of shoulder pain. Rheumatology 2007;46:815-20.

158. Alegre Marcet C. [Trimethylacetate of prednisolone for intra-articular injection in therapy of several disorders of the locomotor apparatus.] Rev Clin Esp 1959;74:32-5.

159. Alexander LD, Gilman DRD, Brown DR, Brown JL, Houghton PE. Exposure to low amounts of ultrasound energy does not improve soft tissue shoulder pathology: a systematic review. Phys Ther 2010;90:14-25.

160. Allano G, Katz D, Fournier-Boursier A. [Physiotherapy of interscalenic blocks in painful or stiff shoulders.] Kinesither Sci 2005;452:21-4.

161. Altman RD, Moskowitz R, Jacobs S, Daley M, Udell J, Levin R, et al. A double-blind, randomized trial of intra-articular injection of sodium hyaluronate (hyalgan $(\mathrm{R})$ ) for the treatment of chronic shoulder pain. Arthritis Rheum 2005;52:S461.

162. Andersen NH, Sojbjerg JO, Johannsen HV, Sneppen O. Frozen shoulder: arthroscopy and manipulation under general anesthesia and early passive motion. J Shoulder Elbow Surg 1998;7:218-22. 
163. Andersen NH, Johannsen HV, Sneppen O, Sojbjerg JO. [Frozen shoulder. Arthroscopy and manipulation in general anesthesia, followed by early passive mobilization.] Ugeskr Laeger 1996;158:147-50.

164. Andren L, Lundberg BJ. Treatment of rigid shoulders by joint distension during arthrography. Acta Orthop Scand 1965;36:45-53.

165. Ankermann KJ, Gobisch F. [The frozen shoulder: results of a 10-year study.] Z Arztl Fortbild (Jena) 1986;80:975-80.

166. Aoki T. [Clinical study of tiaprofenic acid on frozen shoulder.] Jpn Pharmacol Therapeut 1982;10:605-39.

167. Aoki T, Yamamoto M, Kawaji W, Yamamoto R, Sugawara S, Ogawa R. [Clinical evaluation of $\mathrm{CN}-100$ on lumbago, cervicobrachial syndrome and periarthritis scapulohumeralis. Multicenter double-blind study in comparison with indomethacin.] Jpn Pharmacol Therapeut 1991;19:197-222.

168. Arias M, Alcala T. Frozen shoulder: a new approach to its treatment with local anaesthesia. Eur J Phys Rehabil Med 1992;2:11-14.

169. Arslan S, Celiker R. Comparison of the efficacy of local corticosteroid injection and physical therapy for the treatment of adhesive capsulitis. Rheumatol Int 2001;21:20-3.

170. Atra E, Sarmento P, Pizzanelli M. [Comparative study between glucametacin and indomethacin in the treatment of acute periarthritis of the shoulder.] Arq Bras Med 1986;60:423-30.

171. Avetisova EG, Ovchinnikova IP, Yankovskaya AN. Physio therapeutic treatment of patients with scapulo humeral peri arthritis. Kazanskii Meditsinskii Zhurnal 1980;61:22-3.

172. Awad T, Losada M, Losada A. [Treatment of scapulo-humeral periarthritis with alphachymotrypsin and rehabilitation.] Rev Med Chil 1967;95:372-6.

173. Azevedo DC, de Carvalho SC, Leal EWPS, Damasceno SP, Ferreira ML. [Influence of the range of motion (ROM) limitation on the shoulder flexibility improvement after a six-week training.] Rev Bras Med Esporte 2008;14:119-21.

174. Badalamente M, Ang W, Selby RM, Safran M. Enzymatic capsulotomy for adhesive capsulitis. American Academy of Orthopaedic Surgeons Annual Meeting, Chicago, IL, 22-26 March 2006. URL: www3.aaos.org/education/anmeet/anmt2006/podium/ppr06_22.cfm. Accessed

175. Badalamente MA, inventor, Advance Biofactures Corporation, assignee. Methods for treating adhesive capsulitis. United States patent NIH-M01RR01710, PC A04/MF A01; 2009.

176. Bancheri C, Di Loreto C, Occhi P. [Evaluation of the effectiveness of peri- and intraarterial somatostatin 14 in gonarthrosis and scapulo-humeral periarthritis.] G Ital Ricerche Clin Terapeutiche 1993;14:93-7.

177. Baslund B, Thomsen BS, Jensen EM. Humero-scapular periarthrosis. Ugeskr Laeger 1991;153:170-3.

178. Batra YK, Chari P, Negi ON. Comparison of acupuncture and placebo in treatment of chronic shoulder pain. Am J Acupunct 1985;13:69-71.

179. Battisti E, Bianciardi L, Albanese A, Piazza E, Rigato M, Galassi G, et al. [The new magnetic therapy TAMMEF in the treatment of simple shoulder pain.] Clin Ter 2007;158:397-401.

180. Battisti E, Albanese A, Bianciardi L, Fiaschi AI, Rigato M, Vittoria A, et al. TAMMEF therapy in the treatment of shoulder periarthritis: efficacy and safety. Environmentalist 2009;29:190-5. 
181. Baumann F. Ventral capsular denervation: an operative treatment of periarthropathia humero-scapularis. Arch Orthop Trauma Surg 1981;98:13-17.

182. Baumgartner H, Wagenhauser FJ. [Results of mobilization under narcosis.] Orthopade 1981;10:238-41.

183. Baums MH, Spahn G, Nozaki M, Steckel H, Schultz W, Klinger HM. Functional outcome and general health status in patients after arthroscopic release in adhesive capsulitis. Knee Surg Sports Traumatol Arthrosc 2007;15:638-44.

184. Beaufils P, Prevot N, Boyer T, Allard M, Dorfmann H, Frank A, et al. [Gleno-humeral arthroscopic arthrolysis for shoulder stiffness. Apropos of 26 cases.] Rev Chir Orthop Reparatrice Appar Mot 1996;82:608-14.

185. Beaufils P, Prevot N, Boyer T, Allard M, Dorfmann H, Frank A, et al. Arthroscopic release of the glenohumeral joint in shoulder stiffness: a review of 26 cases. Arthroscopy 1999;15:49-55.

186. Beckerman H, Bouter LM, van der Heijden GJ, de Bie RA, Koes BW. Efficacy of physiotherapy for musculoskeletal disorders: what can we learn from research? $\mathrm{Br} J \mathrm{Gen}$ Pract 1993;43:73-7.

187. Bell S, Coghlan J, Richardson M. Hydrodilatation in the management of shoulder capsulitis. Australas Radiol 2003;47:247-51.

188. Bellmann H, Zacharias J, Hasert V, Kretschmar KH, Weidenbach H. [Use of hylase 'Dessau' in periarthritis humero-scapularis (Duplay syndrome)]. Zentralbl Chir 1969;94:1288-304.

189. Bennett WF. Addressing glenohumeral stiffness while treating the painful and stiff shoulder arthroscopically. Arthroscopy 2000;16:142-50.

190. Beres I, Staba R. [Treatment of periarthritis humeroscapularis by acupuncture.] Acta Chir Orthop Traumatol Cech 1979;46:436-40.

191. Berger MR, Froimson AI. Frozen shoulder. Nurse Pract 1980;5:28-9,32.

192. Berghs BM, Sole-Molins X, Bunker TD. Arthroscopic release of adhesive capsulitis. J Shoulder Elbow Surg 2004;13:180-5.

193. Berglezov MA, Vial'ko VV. [Complex treatment of brachio-scapular periarthrisis using laser therapy.] Klin Khir 1986:31-2.

194. Bergman GJD, Winters JC, van der Heijden GJMG, Postema K, Meyboom-de Jong B. Groningen Manipulation Study. The effect of manipulation of the structures of the shoulder girdle as additional treatment for symptom relief and for prevention of chronicity or recurrence of shoulder symptoms. Design of a randomized controlled trial within a comprehensive prognostic cohort study. J Manipulative Physiol Ther 2002;25:543-9.

195. Bergman GJD, Winters JC, Groenier KH, Pool JJM, Meyboom-de Jong B, Postema K, et al. Manipulative therapy in addition to usual medical care for patients with shoulder dysfunction and pain: a randomized, controlled trial. Ann Intern Med 2004;141:432-9.

196. Bergman G, Winters J, Groenier K, Pool J, Meyboom-de Jong B, Postema K, et al. [Additional manual therapy in patients with shoulder complaints: a randomized effect study.] Huisarts Wet 2005;48:438-43.

197. Bergman GJ, Winters JC, Groenier KH, Meyboom-de Jong B, Postema K, van der Heijden GJ. Manipulative therapy in addition to usual care for patients with shoulder complaints: results of physical examination outcomes in a randomized controlled trial. J Manipulative Physiol Ther 2010;33:96-101. 
198. Bertoft ES. Painful shoulder disorders from a physiotherapeutic view: a review of literature. Crit Rev Phys Rehab Med 1999;11:229-77.

199. Bettermann AA. [Periarthritis humeroscapularis: alternative pain treatment with magnet plasters.] Ther Ggw 1982;121:487-92.

200. Bicer A, Ozisik S, Aksik SC, Erdogan C. [Comparison of local corticosteroid injection and conventional physical therapy in management of the painful shoulder.] Turkiye Klinikleri $J$ Med Sci 2005;25:506-12.

201. Bierner SM. Manipulation in the treatment of frozen shoulder. Orthopedics 1989;12:356.

202. Bilgici A, Kuru O, Alayli G, Ulusoy H. [Static stretching therapy with traction in adhesive capsulitis of the shoulder.] J Rheumatol Med Rehabil 2002;13:102-7.

203. Bingol U, Altan L, Yurtkuran M. Low-power laser treatment for shoulder pain. Photomed Laser Surg 2005;23:459-64.

204. Biswas AK, Sur BN, Gupta CR. Treatment of periarthritis shoulder. J Indian Med Assoc 1979;72:276-7.

205. Blaine T, Moskowitz R, Udell J, Skyhar M, Levin R, Friedlander J, et al. Treatment of persistent shoulder pain with sodium hyaluronate: a randomized, controlled trial. A multicenter study. J Bone Joint Surg Am 2008;90:970-9.

206. Blanchard TK, Bearcroft PW, Maibaum A, Hazelman BL, Sharma S, Dixon AK. Magnetic resonance imaging or arthrography for shoulder problems: a randomised study. Eur J Radiol 1999;30:5-10.

207. Blauth W. [Diagnosis and therapy of frozen shoulder.] Hefte Unfallheilkd 1989:68-81.

208. Booi GA, Vanhorn JR, Vanrens TJG. Results and comparison of a Neer acromioplasty versus manipulation under anesthetics of the painful shoulder. Acta Orthop Scand 1986;57:480.

209. Bosch Olives V, Llurba Llurba J, Peinado Vistuer A. [Electrophysiotherapy in the treatment of a frozen shoulder.] Rev Esp Reum Enferm Osteoartic 1967;12:149-56.

210. Boyer T, Dorfmann H, Berquet R. [Frozen shoulder.] Rhumatologie 1994;46:111-16.

211. Boylan M. Massage and exercise reduces pain and improves function in frozen shoulder. J Aus Tradit Med Soc 2005;11:15.

212. Boylan M. Soft tissue massage improves range of motion, function and pain in shoulder pain. J Aus Tradit Med Soc 2005;11:177-8.

213. Boyles RE, Flynn TW, Whitman JM. Manipulation following regional interscalene anesthetic block for shoulder adhesive capsulitis: a case series. Man Therap 2005;10:164-71.

214. Bratanova KA. [Medical exercises in the treatment of periarthritis of the shoulder.] Med Sestra 1978;37:41-2.

215. Brigo B, Campaccu R, Schinina V. [Reflex therapy in functional rehabilitation: treatment of scapulohumeral periarthritis by needle puncture.] Riabilitazione 1981;14:25-32.

216. Brox JI. Regional musculoskeletal conditions: shoulder pain. Best Pract Res Clin Rheumatol 2003; 17:33-56.

217. Buchbinder R, Green S, Hall S, Lawler G, Forbes A. Efficacy of hydrodilatation for frozen shoulder (FS): results of a randomised double-blind placebo-controlled trial. Aust NZ J Med 2000;30:526.

218. Buchbinder R, Green S, Youd JM. Corticosteroid injections for shoulder pain. Cochrane Database Syst Rev 2003; Issue 1, Art. No. CD004016. 
219. Buchbinder R, Hoving JL, Green S, Hall S, Forbes A, Nash P. Short course prednisolone for adhesive capsulitis (frozen shoulder or stiff painful shoulder): a randomised, double blind, placebo controlled trial. Ann Rheum Dis 2004;63:1460-9.

220. Buchbinder R, Green S. Effect of arthrographic shoulder joint distension with saline and corticosteroid for adhesive capsulitis. Br J Sports Med 2004;38:384-5.

221. Buchbinder R, Youd JM, Green S, Stein A, Bennell K, Harris A, et al. Physiotherapy (manual therapy and directed exercise) following arthrographic distension of the glenohumeral joint for adhesive capsulitis: a randomized double-blind placebo-controlled trial. Arthritis Rheum 2006;54(Suppl. 9):813.

222. Buchbinder R, Youd JM, Green S, Stein A, Bennell K, Forbes A, et al. Physiotherapy following hydrodilatation for adhesive capsulitis: a randomised placebo-controlled double blind trial. Intern Med J 2006;36:A67.

223. Buchbinder R, Green S, Youd JM, Johnston RV. Oral steroids for adhesive capsulitis. Cochrane Database Syst Rev 2006; Issue 4, Art. No. CD006189.

224. Buchbinder R, Youd JM, Green S, Stein A, Forbes A, Harris A, et al. Efficacy and costeffectiveness of physiotherapy following glenohumeral joint distension for adhesive capsulitis: a randomized trial. Arthritis Rheum 2007;57:1027-37.

225. Buchbinder R, Green S, Youd JM, Johnston RV, Cumpston M. Arthrographic distension for adhesive capsulitis (frozen shoulder). Cochrane Database Syst Rev 2008; Issue 1, Art. No. CD007005.

226. Bulgen DY, Binder AI, Hazleman BL, Dutton J, Roberts S. Frozen shoulder: prospective clinical study with an evaluation of three treatment regimens. Ann Rheum Dis 1984;43:353-60.

227. Bumin G, Can F. Effects of iontophoresis and phonophoresis methods on pain in cases with shoulder periarthritis. Pain Clinic 2001;13:159-62.

228. Bunker TD. Frozen shoulder. Curr Orthop 1998;12:193-201.

229. Calabro JJ. Indomethacin in acute painful shoulder bursitis and/or tendinitis. Semin Arthritis Rheum 1982;12(Suppl. 1):130-1.

230. Caldwell KD, Nuys SR, Brown CL. Evaluation of mobilization in the treatment of frozen shoulder. Arthritis Rheum 1986;29(Suppl. 4):S147.

231. Callinan N, McPherson S, Cleaveland S, Voss DG, Rainville D, Tokar N. Effectiveness of hydroplasty and therapeutic exercise for treatment of frozen shoulder. J Hand Ther 2003;16:219-24.

232. Camarinos J, Marinko L. Effectiveness of manual physical therapy for painful shoulder conditions: a systematic review. J Man Manip Ther 2009;17:206-15.

233. Caniggia M, Carnevale I, Passarello F. [Analgesic and antiflogistic properties of intra- and periarticular thymopentin.] Riforma Med 1989;104:417-19.

234. Capone M, Stancati MT, Tolla V, Chiatti R, Muscolo V, Pasquale M. [Observations on the administration of sodium edetate in calcified scapulohumeral periarthritis. Ionophoresis and mesotherapy: comparison of two techniques.] Ortop Traumatol Oggi 1994;14:163-8.

235. Carette S, Moffet H, Tardif J, Bessette L, Fremont P, Blanchette C, et al. Intraarticular (IA) corticosteroids, physiotherapy or the combination of both in patients with adhesive capsulitis of the shoulder: a placebo controlled trial. J Rheumatol 2002;29:13. 
236. Carey TS. Adding single-point acupuncture to physiotherapy for painful shoulder improved function and reduced pain. ACP J Club 2008;149:JC4-12.

237. Carter B. Clients' experiences of frozen shoulder and its treatment with Bowen technique. Complement Ther Nurs Midwifery 2002;8:204-10.

238. Casanova G. [Crenotherapy and bone and joint surgery.] Presse Therm Clim 1988;125:95-7.

239. Castellarin G, Ricci M, Vedovi E, Vecchini E, Sembenini P, Marangon A, et al. Manipulation and arthroscopy under general anesthesia and early rehabilitative treatment for frozen shoulders. Arch Phys Med Rehabil 2004;85:1236-40.

240. Castelli PG, Schneider L, Sartori G, Camisassa R, Grandi G. [Release arthroscopy for posttraumatic frozen shoulder: a review of 19 cases.] Minerva Ortop Traumatol 2006;57:399-404.

241. Champion G. Frozen shoulder. Ann Rheum Dis 2005;64:795-6.

242. Chang WH, Im SH, Ryu JA, Lee SC, Kim JS. The effects of scapulothoracic bursa injections in patients with scapular pain: a pilot study. Arch Phys Med Rehabil 2009;90:279-84.

243. Chavero Carrasco V, Salguero Molpeceres O, Lopez Ruano P, Alvarez Montero S. [Treatment of shoulder pain; non steroidal anti-inflammatory drug (NSAID) or intraarticular injection?]. Rev Med Fam Comun 2002;12:341-3.

244. Chavez-Lopez MA, Navarro-Soltero LA, Rosas-Cabral A, Gallaga A, Huerta-Yanez G. Methylprednisolone versus triamcinolone in painful shoulder using ultrasound-guided injection. Mod Rheumatol 2009;19:147-50.

245. Checchia GA, Pezzoli R, Gorini L, Amadori L, Foresti A, Gazzi A. [Electrotherapy in the treatment of shoulder pain.] Riabilitazione 1991;24:121-7.

246. Chen SK, Lin SY, Liao JS. Primary and secondary frozen shoulder. J Surg Ass Rep China 1985;18:121-8.

247. Chen SK, Lin SY, Liao JS. [An orthopaedic study of frozen shoulder.] Gaoxiong Yi Xue Ke Xue Za Zhi 1988;4:1-9.

248. Chen Y, Zheng K. New exploration of needling manipulations at point Tianzong. J Tradit Chin Med 2002;22:38-41.

249. Chen ZS, Yang RK. [Effects of alkalinizing administration of suprascapular nerve block on the periarthritis of shoulder-induced pain and the function of joint movement.] Chin J Clin Rehab 2005;9:8-9.

250. Chen L. [Comparison of therapeutic effects between normal acupuncture and shallow needling with short-needle on periarthritis of shoulder.] Zhongguo Zhenjiu 2006;26:647-8.

251. Chen S-J, Li H, Zhang J-W. [Control study of therapeutic effects of three phase acupuncture method and routine acupuncture method on periarthritis of shoulder.] Zhongguo Zhenjiu 2006;26:421-3.

252. Chen S-K, Chou P-H, Lue Y-J, Lu Y-M. Treatment for frozen shoulder combined with calcific tendinitis of the supraspinatus. Kaohsiung J Med Sci 2008;24:78-84.

253. Chen JF, Ginn KA, Herbert RD. Passive mobilisation of shoulder region joints plus advice and exercise does not reduce pain and disability more than advice and exercise alone: a randomised trial. Aust J Physiother 2009;55:17-23.

254. Cheng P, Shi WM, He J, Lang HT, Xi CL, Wu MJ. [Efficacy and safety of infrared rays and Qingpeng paste in treating periarthritis of shoulder.] J Clin Rehab Tissue Eng Res 2008;12:1353-6. 
255. Cherkashin VV, Burtseva NI. [Exercise therapy in the complex treatment of scapulohumeral periarthritis.] Vopr Kurortol Fizioter Lech Fiz Kult 1969;34:236-9.

256. Cho S-H, Shin B-C, Kim I-H. Effects of myofascial-meridian stimulation therapy (MMST) on shoulder pain. Int J Neurosci 2005;115:1175-81.

257. Ciapetti A, Salaffi F, Garofalo J, Stancati A, Filippucci E, Grassi W. Painful shoulder: shortterm efficacy of a randomized controlled trial of local steroid injection versus physiotherapy. Ann Rheum Dis 2006;65(Suppl. 2):238.

258. Cinar M, Akpinar S, Derincek A, Circi E, Uysal M. Comparison of arthroscopic capsular release in diabetic and idiopathic frozen shoulder patients. Arch Orthop Trauma Surg 2010;130:401-6.

259. Cloke DJ, Watson H, Purdy S, Steen IN, Williams JR. A pilot randomized, controlled trial of treatment for painful arc of the shoulder. J Shoulder Elbow Surg 2008;17(Suppl. 1):17-21.

260. Cohen NP, Levine WN, Marra G, Pollock RG, Flatow EL, Brown AR, et al. Indwelling interscalene catheter anesthesia in the surgical management of stiff shoulder: a report of 100 consecutive cases. J Shoulder Elbow Surg 2000;9:268-74.

261. Compernolle P, Vansteenland H, Rosselle N. [Adhesive capsulitis of the shoulder.] Acta Belg Med Phys 1987;10:71-81.

262. Connolly J, Regen E, Evans OB. The management of the painful, stiff shoulder. Clin Orthop Relat Res 1972;84:97-103.

263. Connolly JF. Exercises to loosen the shoulder joint. J Musculoskeletal Med 1998;15:57.

264. Coombes WN. Distension-manipulation for the treatment of adhesive capsulitis (frozen shoulder syndrome). Clin Orthop Relat Res 1984:309-10.

265. Corazza G, Basaglia N. [Rehabilitation of patients with periarthritis humeroscapularis.] Riabilitazione 1982;15:185-91.

266. Corbeil V, Dussault RG, Leduc BE, Fleury J. [Adhesive capsulitis of the shoulder: a comparative study of arthrography with intra-articular corticotherapy and with or without capsular distension.] Can Assoc Radiol J 1992;43:127-30.

267. Cossu M, Conti B, Celestini M. [EDTA ionophoresis in calcified periarthritis of the shoulder. New aspects.] Riabilitazione 1993;26:19-23.

268. Coudane H, Sommelet J, Mole D, Schmitt D. [Arthroscopy of the shoulder in scapulohumeral periarthritis.] Rev Chir Orthop Reparatrice Appar Mot 1988;74:289.

269. Crawshaw DP, Helliwell P, Hensor EM, Hay EM, Aldous SJ, Conaghan PG. Is there a window of opportunity for physiotherapy after steroid injections for shoulder pain? Results from a large randomised trial. Rheumatology 2009;48:I96.

270. Dacre JE, Beeney N, Scott DL. Physiotherapy or steroids for the painful stiff shoulder? $B r J$ Rheumatol 1987;26(Suppl. 2):1-13.

271. Dahan TH, Fortin L, Pelletier M, Petit M, Vadeboncoeur R, Suissa S. Double blind randomized clinical trial examining the efficacy of bupivacaine suprascapular nerve blocks in frozen shoulder. J Rheumatol 2000;27:1464-9.

272. Dal Conte G, Rivoltini P, Combi F. [Pulsed magnetic field therapy for calcific periarthritis of the shoulder joint.] Riabilitazione 1990;23:27-33.

273. Danneskiold-Samsoe B. [Manipulation of frozen shoulder?]. Ugeskr Laeger 1996;158:145. 
274. de Jong BA, Dahmen R, Hogeweg JA, Marti RK. Intra-articular triamcinolone acetonide injection in patients with capsulitis of the shoulder: a comparative study of two dose regimens. Clin Rehabil 1998;12:211-15.

275. de la Serna AR, Moreno MA, Pereira AA. Preliminary study to evaluate the efficacy and tolerance of joint lavage in shoulder pain vs intra-articular injection of hyaluronic acid. Osteoarthr Cartil 2004;12:S143-4.

276. de Macedo JM, Goldstein RC, Marinho MASDO, Pena LW, Machado JKS. [Frozen shoulder: results of the treatment with suprascapular nerve block.] Rev Bras Ortop 2000;35:131-6.

277. de Seze S, Debeyre J, Denis A. [Surgical treatment of certain forms of periarthritis of the shoulder.] Rev Rhum Mal Osteoartic 1950;17:172-5.

278. Debeyre J. [Surgical treatment of scapulohumeral periarthritis and of painful shoulder.] Minerva Ortop 1971;22:371-3.

279. Degen IL. [Magnetotherapy of humeroscapular periarthritis.] Ortop Travmatol Protez 1974;35:66-8.

280. Denicolai F, Dettoni A, Piolatto G, Sibelli P. [Assessment of the comparative efficacy of orgotein and betamethasone in scapulohumeral periarthritis and tendinopathies.] Minerva Ortop 1986;37:79-86.

281. Desproges-Gotteron R. [Treatment of recalcitrant periarthritis of the shoulder.] Rev Med Limoges 1980;11:1-2.

282. Devitt M. Acupuncture and frozen shoulder. Treatment plus exercise better than exercise alone. Acupunct Today 2002;3:1,5.

283. Deyle GD, Bang MD. Examination and treatment of the shoulder: a literature-based approach. Orthop Phys Ther Clin N Am 1999;8:83-115.

284. DiMarcantonio T. Multiple collagenase injections effective, safe for treating 'frozen shoulder'. Orthop Today 2006;26:62-3.

285. Diwan DB, Murrell GAC. An evaluation of the effects of the extent of capsular release and of postoperative therapy on the temporal outcomes of adhesive capsulitis. Arthroscopy 2005;21:1105-13.

286. Dodenhoff RM, Levy O, Wilson A, Copeland SA. Manipulation under anesthesia for primary frozen shoulder: effect on early recovery and return to activity. J Shoulder Elbow Surg 2000;9:23-6.

287. Dogru H, Basaran S, Sarpel T. [Effectiveness of therapeutic ultrasound in adhesive capsulitis.] Rev Rhum Ed Fr 2008;75:625-31.

288. Dorian R, Miletic M. [Local cryotherapeutical or heat applications in patients with painful shoulder stiffness.] Med Welt 1985;36:157-8.

289. D’Orta S, Germani M, Parente F, Bigazzi G, Pietrogrande V. [Double-blind study on acupuncture treatment of frozen shoulder.] Ortop Traumatol Oggi 1985;5:37-44.

290. Drakos MC, Green DM, Dodson CC, Allen AA, Warren RF. Shoulder dislocation after mobilization procedures for adhesive capsulitis. Orthopedics 2008;31:pii.

291. Duke O, Zecler E, Grahame R. Anti-inflammatory drugs in periarthritis of the shoulder: a double-blind, between-patient, study of naproxen versus indomethacin. Rheumatol Rehabil 1981;20:54-9.

292. Duschatko DM. Certified pilates and gyrotonics trainer. J Bodyw Mov Ther 2000;4:13-19. 
293. Echternach JL. Audioanalgesia as an adjunct to mobilization of the chronic frozen shoulder. Phys Ther 1966;46:839-46.

294. Ekelund AL, Rydell N. Combination treatment for adhesive capsulitis of the shoulder. Clin Orthop Relat Res 1992:105-9.

295. Elleuch MH, Baklouti S, Gdoura H, Keskes H, Sellami S. [The rehabilitation in the frozen shoulder syndrome.] Rhumatologie 1994;46:77-80.

296. Elleuch MH, Yahia A, Ghroubi S, Kharrat O, Mezghanni M, Ayedi K. [The contribution of capsular distension to the treatment of primary adhesive capsulitis of the shoulder: a comparative study versus rehabilitation.] Ann Readapt Med Phys 2008;51:722-8.

297. Erlendsson F. [Frozen shoulder.] Ugeskr Laeger 1996;158:2139-40.

298. Ernst E, Dong H. Encouraging long-term results for acupuncture for neck and shoulder pain. Focus Altern Complement Ther 2004;9:297-8.

299. Ernst E. [Physical therapy for shoulder pains?]. MMW Fortschr Med 2009;151:28.

300. Escalante Triay FJ. [Combined use of cobamamide and thiocolchicoside in treatment of pain syndromes accompanied by muscle contracture.] Invest Med Int 1981;8:272-8.

301. Esposito S, Ragozzino A, Russo R, Minelli S, Tuccillo M. [Arthrography in the diagnosis and treatment of idiopathic adhesive capsulitis.] Radiol Med 1993;85:583-7.

302. Eulert J, Apoil A, Dautry P. [Pathogenesis and surgical treatment of 'periarthritis humeroscapularis.'] Z Orthop Ihre Grenzgeb 1981;119:25-30.

303. Famaey JP, Ginsberg F. Treatment of periarthritis of the shoulder: a comparison of ibuprofen and diclofenac. J Int Med Res 1984;12:238-43.

304. Famaey JP, Broux G, Cleppe D. Ionization with Voltaren. A multi-centre trial. J Belge Med Phys Rehabil 1982;5:55-60.

305. Fan Z-Y, Huang Z-B, Lai S-H, Zhong W, Guo R-S. [Clinical experience on the manipulative treatment of cervical frozen shoulder.] Zhongguo Gushang 2008;21:796.

306. Fareed DO, Gallivan WR Jr. Office management of frozen shoulder syndrome. Treatment with hydraulic distension under local anesthesia. Clin Orthop Relat Res 1989:177-83.

307. Farrell CM, Sperling JW, Cofield RH. Manipulation for frozen shoulder: long-term results. J Shoulder Elbow Surg 2005;14:480-4.

308. Feng Zg. Two-hundred and ten cases of shoulder periarthritis treated by needling Lingxia and Sanjian. J Tradit Chin Med 2003;23:201-2.

309. Filshie J. Acupuncture improves short- and long-term pain and disability in patients with shoulder pain compared with a non-penetrating sham treatment. Focus Altern Complement Ther 2005;10:124-5.

310. Flannery O, Mullett $\mathrm{H}$, Colville J. Adhesive shoulder capsulitis: does the timing of manipulation influence outcome? Acta Orthop Belg 2007;73:21-5.

311. Foster NE, Vas J. Trial suggests that adding single-point acupuncture to a package of physiotherapy is better for shoulder pain patients than adding mock TENS to the same physiotherapy package of care. Focus Altern Complement Ther 2008;13:276-8.

312. Fuhr AW, Menke JM. Status of activator methods chiropractic technique, theory, and practice. J Manipulative Physiol Ther 2005;28:e1-e20.

313. Fujiwara M, Tamura K, Odera K, Tada K, Takaya Y, Ikeda N. Injection therapy of high molecular sodium hyaluronate (ARTZ) for patients with periarthritis scapulohumeralis: 
evaluation of combined therapy with non-steroidal anti-inflammatory drugs. Jpn J Med Pharm Sci 1993;30:965-70.

314. Fukuhara F. [Clinical evaluation of the OTC product K98-01 (KINKAN) on the shoulder stiffness, backache, confusion, sprain.] Jpn Pharmacol Therapeut 1999;27:255-62.

315. Fura M, Lenartowski E, Soroka L. [Methods and results of treatment of primary painful shoulder.] Chir Narzadow Ruchu Ortop Pol 1981;46:65-8.

316. Gabrhelik T, Adamus M, Michalek P, Pieran M. [Invasive therapy for shoulder pain.] Bolest 2009;12:101-6.

317. Gado K, Emery P. Intra-articular guanethidine injection for resistant shoulder pain: a preliminary double blind study of a novel approach. Ann Rheum Dis 1996;55:199-201.

318. Galarraga B. Adhesive capsulitis of the shoulder: a review article. CPD Rheumatology 2002;3:54-8.

319. Galgano RC. Manipulative therapy for patients with shoulder symptoms. Ann Intern Med 2005;142:226.

320. Garrido FV, Muñoz F, Monasterioguren XV, Ortiz AM, Orejón CO, Hernández PS, et al. [Effectiveness of hyperthermia at $434 \mathrm{MHz}$ after manipulation under anesthesia in the treatment of frozen shoulder.] Fisoterapia 2009;31:203-12.

321. Gaspar PD, Willis FB. Adhesive capsulitis and dynamic splinting: a controlled, cohort study. BMC Musculoskelet Disord 2009;10:111.

322. Gavant ML, Rizk TE, Gold RE, Flick PA. Distention arthrography in the treatment of adhesive capsulitis of the shoulder. J Vasc Interv Radiol 1994;5:305-8.

323. Geraets JJXR, Goossens MEJB, de Bruijn CPC, Koke AJA, de Bie RA, Pelt RAGB, et al. A behavioural treatment for chronic shoulder complaints: concepts, development, and study design. Aust J Physiother 2004;50:33-8.

324. Geraets JJXR, Goossens MEJB, de Groot IJM, de Bruijn CPC, de Bie RA, Dinant G-J, et al. Effectiveness of a graded exercise therapy program for patients with chronic shoulder complaints. Aust J Physiother 2005;51:87-94.

325. Gerber C, Espinosa N, Perren TG. Arthroscopic treatment of shoulder stiffness. Clin Orthop Relat Res 2001:119-28.

326. Gilula LA, Schoenecker PL, Murphy WA. Shoulder arthrography as a treatment modality. AJR Am J Roentgenol 1978;131:1047-8.

327. Ginn KA, Herbert R, Khouw W, Lee R. A randomised controlled clinical trial of the effectiveness of a physiotherapy treatment for shoulder pain. Proceedings of the 12th International Congress of the World Confederation for Physical Therapy, Washington, DC, 25-30 June 1995.

328. Ginn KA, Herbert RD, Khouw W, Lee R. A randomized, controlled clinical trial of a treatment for shoulder pain. Phys Ther 1997;77:802-9.

329. Ginn K. Corticosteroid injections are more effective than joint mobilisations and exercise for managing painful stiff shoulders. Aust J Physiother 1999;45:320.

330. Ginn KA, Cohen ML. Exercise therapy for shoulder pain aimed at restoring neuromuscular control: a comparative clinical trial. J Rehabil Med 2001;37:115-22.

331. Ginn KA, Cohen ML. Conservative treatment for shoulder pain: prognostic indicators of outcome. Arch Phys Med Rehabil 2004;85:1231-5. 
332. Ginn KA, Cohen ML. Exercise therapy for shoulder pain aimed at restoring neuromuscular control: a randomized comparative clinical trial. J Rehabil Med 2005;37:115-22.

333. Ginn K. Do passive mobiliztions [sic] applied to shoulder region joints improve the treatment outcome in patients with shoulder pain? N Z J Physiother 2009;37:148.

334. Ginsberg F, Famaey JP. Double-blind, randomized crossover study of the percutaneous efficacy and tolerability of a topical indomethacin spray versus placebo in the treatment of tendinitis. J Int Med Res 1991;19:131-6.

335. Gobezie R, Pacheco IH, Petit CJ, Millett PJ. Dislocation and instability after arthroscopic capsular release for refractory frozen shoulder. Am J Orthop 2007;36:672-4.

336. Goh GJ, Over KE, Daroszewska A, Whitehouse GH, Bucknall RC. The value of arthrography in steroid injection of the shoulder joint. Br J Rheumatol 1997;36:709-10.

337. Gotte S, Spreitzer FO, Stratz T. [Percutaneous treatment of periarthritis humeroscapularis with a new formulation of etofenamate.] Z Allgemeinmed 1986;62:1198-201.

338. Gotter G. Comparative evaluation of tenoxicam and piroxicam in the treatment of humeroscapular periarthritis. Eur J Rheumatol Inflamm 1987;9:95-7.

339. Graber MA. Treating shoulder pain: a randomized trial. J Fam Pract 1997;45:103-4.

340. Grabovoi AF, Grishko AI, Rodichkin VA, Rudenko AT, Gozhenko IS. [Blockade of the suprascapular nerve in the complex treatment of humero-scapular periarthritis.] Vestn Khir Im I I Grek 1986;136:65-6.

341. Grammont P, Lelaurin G, Lemaire JP. [Surgical treatment of chronic periarthritis of the shoulder joint.] Acta Orthop Belg 1982;48:762-74.

342. Green S, Buchbinder R, Hetrick S. Physiotherapy interventions for shoulder pain. Cochrane Database Syst Rev 2003; Issue 2, Art. No. CD004258.

343. Green S. Physiotherapy and injection better than injection alone or physiotherapy alone for improving range of motion in adhesive capsulitis. Aust J Physiother 2003;49:145.

344. Green S, Buchbinder R, Hetrick S. Acupuncture for shoulder pain. Cochrane Database Syst Rev 2005; Issue 2, Art. No. CD005319.

345. Green S, Buchbinder R, Glazier R, Forbes A. Interventions for shoulder pain. Cochrane Database Syst Rev 2006; Issue 4, Art. No. CD001156.

346. Green S, Alexander M. Australian Physiotherapy Association: shoulder pain position statement [with systematic review.] URL: www.physiotherapy.asn.au/. Accessed 19 April 2010.

347. Grete J. [Physical therapy in impaired mobility and pain in the shoulder.] Therapiewoche 1981;31:6318-25.

348. Griggs SM, Ahn A, Green A. Idiopathic adhesive capsulitis. A prospective functional outcome study of nonoperative treatment. J Bone Joint Surg Am 2000;82-A:1398-407.

349. Grossi E, Monza GC, Pollavini S, Bona L. NSAID ionisation in the management of softtissue rheumatism: role played by the drug, electrical stimulation and suggestion. Clin Exp Rheumatol 1986;4:265-7.

350. Grubbs N. Frozen shoulder syndrome: a review of literature. J Orthop Sports Phys Ther 1993;18:479-87.

351. Gruehn F. [On humeroscapular periarthritis.] Hippokrates 1965;36:66-71. 
352. Gu J. The effect of audio-frequency electrotherapy and carbon dioxide laser treated 96 cases of periarthritis of shoulder. Acta Acad Med Hubei 1992;13:88-9.

353. Gudushauri ON, Goguadze DM. [Ambulatory treatment of humero-scapular periarthritis, humeral epicondylitis and radial styloiditis by local injections of hydrocortisone.] Ortop Travmatol Protez 1975:24-6.

354. Guerra de Hoyos JA, Andres Martin Mdel C, Bassas y Baena de Leon E, Vigara Lopez M, Molina Lopez T, Verdugo Morilla FA, et al. Randomised trial of long term effect of acupuncture for shoulder pain. Pain 2004;112:289-98.

355. Guler-Uysal F, Kozanoglu E. Comparison of the early response to two methods of rehabilitation in adhesive capsulitis. Swiss Med Wkly 2004;134:353-8.

356. Guo XY, Li YF. [Etiological factor of periarthritis of shoulder and advantages of integrated traditional Chinese medicine and western medicine in treatment.] Chin J Clin Rehab 2006;10:150-2.

357. Guo C-Q, Zhang F-M, Sha Y, Ma G-H, Liu Y-X, Liu Y-G, et al. [Randomized controlled trials of acupuncture at Tiaokou (ST 38) for treatment of periarthritis of shoulder.] Zhongguo Zhenjiu 2006;26:544-6.

358. Guo C-Q, Rui N, Liu Y-G, Liu Y-X, Sha Y, Ma G-H. [Multi-central randomized controlled observation on clinical therapeutic effect of the new Bian-stone therapy on scapulohumeral periarthritis.] Zhongguo Zhenjiu 2007;27:633-7.

359. Gusarova SA. [Massage in humeroscapular periarthosis.] Med Sestra 1989;48:36-8.

360. Gwilym SE, Mullett H, Levy O. The one-stop diagnostic clinic in shoulder surgery: costs and benefits. Clin Manag 2007;15:37-41.

361. Habib GS. Systemic effects of intra-articular corticosteroids. Clin Rheumatol 2009;28:749-56.

362. Haines JF, Hargadon EJ. Manipulation as the primary treatment of the frozen shoulder. $J R$ Coll Surg Edinb 1982;27:271-5.

363. Hall FM. Adhesive capsulitis. Radiology 1988;169:579.

364. Hall FM. Frozen shoulder. Radiology 2005;235:713-14.

365. Hamdan TA, Al-Essa KA. Manipulation under anaesthesia for the treatment of frozen shoulder. Int Orthop 2003;27:107-9.

366. Hamer J, Kirk JA. Physiotherapy and the frozen shoulder: a comparative trial of ice and ultrasonic therapy. N Z Med J 1976;83:191-2.

367. Han EJ. The comparison of the effectiveness in auricular acupuncture between acupuncture points and tender points for frozen shoulder. Proceedings of the 12th International Pain Clinic: World Society of Pain Clinicians, Turin, Italy, 2006. pp. 205-8.

368. Hando BR. Glenohumeral joint translational manipulation under anesthesia for the treatment of adhesive capsulitis: a case series study and findings from immediate postmanipulation arthroscopic visualization. 2010 Combined Sections Meeting (CSM), San Diego, California, February 17-20, 2010. J Orthop Sports Phys Ther 2010;40(Suppl. 1):A32.

369. Hannafin JA, Strickland SM. Frozen shoulder. Curr Opin Orthop 2000;11:271-5.

370. Harryman DT. Shoulders: frozen and stiff. Instr Course Lect 1993;42:247-57.

371. Harryman DT, Matsen FA, Sidles JA. Arthroscopic management of refractory shoulder stiffness. Arthroscopy 1997;13:133-47. 
372. Hart BF. Frozen shoulder treated with acupuncture and nutrition. Am J Acupunct 1976;4:365-7.

373. Hauzeur JP. [Conservative treatment of the painful shoulder. Review of the literature.] Rev Med Brux 2004;25:A411-5.

374. Hay EM, Thomas E, Paterson SM, Dziedzic K, Croft PR. A pragmatic randomised controlled trial of local corticosteroid injection and physiotherapy for the treatment of new episodes of unilateral shoulder pain in primary care. Arthritis Rheum 2001;44(Suppl. 9):72.

375. Hay EM, Thomas E, Paterson SM, Dziedzic K, Croft PR. A pragmatic randomised controlled trial of local corticosteroid injection and physiotherapy for the treatment of new episodes of unilateral shoulder pain in primary care. Ann Rheum Dis 2003;62:394-9.

376. He J, Chen X-L, Li Z-B, Zhang W, Fu W-Y, Gong J-D. Research of the clinical results of scapulohumeral periarthritis treated with comprehensive therapy. J Shanghai Med Univ 2000;27:141-6.

377. Heber A, Eulert J. [Conservative treatment of periarthritis humeroscapularis.] Therapiewoche 1983;33:580-4.

378. Helbig B, Wagner P, Dohler R. Mobilization of frozen shoulder under general anaesthesia. Acta Orthop Belg 1983;49:267-74.

379. Heller B, Tarricone R. Oxaprozin versus diclofenac in NSAID-refractory periarthritis pain of the shoulder. Curr Med Res Opin 2004;20:1279-90.

380. Hempel V. [Suprascapular nerve blockade in treatment of periarthritis humeroscapularis.] Therapiewoche 1983;33:584-6.

381. Herold HZ. [Frozen shoulder.] Harefuah 1982;103:26-7.

382. Heuleu JN, Courtillon A, Nahon E. Physiotherapy for a painful shoulder. Gaz Med Fr 1979;86:4087, 4091-2.

383. Hieber F. [Periarthritis stiffness of the shoulder and its therapy.] Hippokrates 1967;38:888-94.

384. Ho C-YC, Sole G, Munn J. The effectiveness of manual therapy in the management of musculoskeletal disorders of the shoulder: a systematic review. Man Therap 2009;14:463-74.

385. Hoenle R. [Radiotherapy for relief of pain in recalcitrant periarthritis humeroscapularis.] Therapiewoche 1983;33:5269-75.

386. Hollingworth GR, Ellis RM, Hattersley TS. Comparison of injection techniques for shoulder pain: results of a double blind, randomised study. BMJ 1983;287:1339-41.

387. Hollis R, Lahav A, West HS Jr. Manipulation of the shoulder using Codman's paradox. Orthopedics 2006;29:971-3.

388. Hong CZ, Lin JC, Bender LF. Magnetic necklace: its therapeutic effectiveness on neck and shoulder pain application of a static magnetic field as a physical therapy modality. Arch Phys Med Rehabil 1982;63:462-6.

389. Hormusjee DN, Mehta SD. Our experience in manipulative therapy for periarthritis of shoulder. Indian J Surg 1980;42:297-300.

390. Hossain S, Jacobs LGH, Hashmi R. The long-term effectiveness of steroid injections in primary acromioclavicular joint arthritis: a five-year prospective study. J Shoulder Elbow Surg 2008;17:535-8. 
391. Hosseini H, Agneskirchner JD, Lobenhoffer P. [Arthroscopic capsular release in the management of refractory adhesive capsulitis. Technique and results.] Unfallchirurg 2006;109:212-18.

392. Hsu SY, Chan KM. Arthroscopic distension in the management of frozen shoulder. Int Orthop 1991;15:79-83.

393. Hu XD. [63 cases of periarthritis of shoulder joint treated by scalp-acupuncture and bodyneedling.] Shanghai J Acupunct Moxibustion 1993;12:30.

394. Hu Y. Clinical application of the point Xuanzhong. J Tradit Chin Med 2003;23:275-7.

395. Hu J, Yan J-T. [Evaluation of clinical reports in treating periarthritis of shoulder by manipulation with the theory of evidence based medicine.] Zhong Xi Yi Jie He Xue Bao 2004;2:185-8.

396. Hu J. Acupuncture treatment of shoulder pain. J Tradit Chin Med 2006;26:78-9.

397. Huang WEI. Analysis on therapeutic effects of 46 cases of periarthritis of shoulder treated by needling jiansanzhen with magnetic pole needle and massage. World J Acup-Moxi 1996;6:31-4.

398. Hulstyn MJ, Weiss AP. Adhesive capsulitis of the shoulder. Orthop Rev 1993;22:425-33.

399. Hummel-Berry KH. Assessment of shoulder function and functional impact of clinic physical therapy versus home exercise for patients with shoulder stiffness: a randomized controlled trial. $\mathrm{PhD}$ thesis. Washington, DC: University of Washington; 2001.

400. Ibrahim T, Rahbi H, Beiri A, Jeyapalan K, Taylor GJS. Adhesive capsulitis of the shoulder: the rate of manipulation following distension arthrogram. Rheumatol Int 2006;27:7-9.

401. Ide J, Takagi K. Early and long-term results of arthroscopic treatment for shoulder stiffness. J Shoulder Elbow Surg 2004;13:174-9.

402. Imai R, Kusaka Y, Sakakida K. [Clinical studies on Voltaren suppository for stiff shoulder.] Jpn Pharmacol Therapeut 1983;11:373-86.

403. Indeck W. Office management of frozen shoulder syndrome. Clin Orthop Relat Res 1990:309-10.

404. Ingram-Rice B. Occupational therapy perspective. J Bodyw Mov Ther 2000;4:20-6.

405. Itel R. [Exercise therapy and physical therapy measures in the treatment of shoulder and arm pain.] Ther Umsch 1988;45:812-19.

406. Itzkowitch D, Ginsberg F, Leon M, Bernard V, Appelboom T. Peri-articular injection of tenoxicam for painful shoulders: a double-blind, placebo controlled trial. Clin Rheumatol 1996;15:604-9.

407. Ivanov VA, Iakovleva EA. [Auricular reflexotherapy of humeroscapular periarthritis.] Ortop Travmatol Protez 1986:44-5.

408. Jacchia GE, Cosco Mazzuca R. [Mobilization under anesthesia in periarthritis of the shoulder.] Arch Putti Chir Organi Mov 1968;23:231-41.

409. Jacobs LG, Barton MA, Wallace WA, Ferrousis J, Dunn NA, Bossingham DH. Intraarticular distension and steroids in the management of capsulitis of the shoulder. $B M J$ 1991;302:1498-501.

410. Jacobs L, Barton M, Wallace W, Ferrousis J, Dunn NBD. Intra-articular distension and steroids in the management of capsulitis of the shoulder. J Orthop Med 1992;14:40-4. 
411. Jacobs LGH, Smith MG, Khan SA. Comparision of manipulation under anaesthetic and distension injections in the management of frozen shoulder. J Bone Joint Surg Br 2005;87-B(Suppl. 2):162-3.

412. Jayson MI. Frozen shoulder: adhesive capsulitis. BMJ 1981;283:1005-6.

413. Jensen I, Nygren A, Gamberale F, Goldie I, Westerholm P, Jonsson E. The role of the psychologist in multidisciplinary treatments for chronic neck and shoulder pain: a controlled cost-effectiveness study. Scand J Rehabil Med 1995;27:19-26.

414. Ji XP. Shoulder pain. J Tradit Chin Med 1988;8:310-12.

415. Jia H, Li Q. Treatment of periomarthritis with scalp acupuncture therapy: a report of 210 cases. J Tradit Chin Med 1993;13:199-201.

416. Jia C-S, Li X-F, Ma X-S, Shi J, Liu E-J. [Comparison of analgesic effects between subcutaneous penetration needling and straight needling of otopoints in the treatment of scapulohumeral periarthritis.] Zhen Ci Yan Jiu 2008;33:339-42.

417. Jiang YK. Frozen shoulder effectively treated by single needle penetration acupuncture. Am J Acupunct 1982;10:189.

418. Jiang D, Liu W. Thick needle treatment for periomitis. J Tradit Chin Med 1991;11:112-14.

419. Jin D, Li Z. Acupuncture and the elevation manipulation of massage for treatment of frozen shoulder. J Tradit Chin Med 2003;23:212-13.

420. Johnson AJ, Godges JJ, Zimmerman GJ, Ounanian LL. The effect of anterior versus posterior glide joint mobilization on external rotation range of motion in patients with shoulder adhesive capsulitis. J Orthop Sports Phys Ther 2007;37:88-99.

421. Jones DS, Chattopadhyay C. A randomised trial to compare suprascapular nerve block with intra-articular injections for the treatment of frozen shoulder in primary care. $\mathrm{Br} \mathrm{J}$ Rheumatol 1997;36(Suppl. 2):25.

422. Jones DS, Chattopadhyay C. Suprascapular nerve block for the treatment of frozen shoulder in primary care: a randomized trial. Br J Gen Pract 1999;49:39-41.

423. Joshi GP, McCarroll SM. Intra-articular morphine for the management of frozen shoulder. Anaesthesia 1992;47:627.

424. Judet H, Judet J. [Surgery in the treatment of refractory forms of stiff and painful shoulder.] Rev Chir Orthop Reparatrice Appar Mot 1985;71(Suppl. 2):75-7.

425. Jurgel J, Rannama L, Gapeyeva H, Ereline J, Kolts I, Paasuke M. Shoulder function in patients with frozen shoulder before and after 4-week rehabilitation. Medicina (Kaunas) 2005;41:30-8.

426. Kalke SJ, Perera SD, Dasgupta B. Ultrasound in the evaluation of shoulder pain. Arthritis Rheum 1999;42(Suppl. 9):150.

427. Kanai S, Taniguchi N, Kawamoto M, Endo H, Higashino H. Effect of static magnetic field on pain associated with frozen shoulder. Pain Clinic 2004;16:173-9.

428. Kanai S, Taniguchi N. Effect of polarity exchangeable permanent magnet on frozen shoulder pain. Pain Clinic 2006;18:37-45.

429. Kaptelin AF, Larina LE. [Complex conservative treatment of brachio-scapular periarthritis.] Ortop Travmatol Protez 1976:60-4.

430. Karatas GK, Meray J. Suprascapular nerve block for pain relief in adhesive capsulitis: comparison of 2 different techniques. Arch Phys Med Rehabil 2002;83:593-7. 
431. Karkan Z, Mikes K, Vurm F, Maresova B. [Our experiences with the therapy of the stiffening shoulder.] Rehabilitacia 1985;18:31-8.

432. Katz JN, Solomon DH, Schaffer JL, Horsky J, Burdick E, Bates DW. Outcomes of care and resource utilization among patients with knee or shoulder disorders treated by general internists, rheumatologists, or orthopedic surgeons. Am J Med 2000;108:28-35.

433. Kay NR. The clinical diagnosis and management of frozen shoulders. Practitioner 1981;225:164-7.

434. Kay NRM, Eyres KS. The role of manipulation for the frozen shoulder. J Orthop Rheumatol 1990;3:203-12.

435. Keilholz L, Seegenschmiedt MH, Kutzki D, Sauer R. [Periarthritis humeroscapularis (PHS). Indications, technique and outcome of radiotherapy.] Strahlenther Onkol 1995;171:379-84.

436. Kent H. Physical medicine and rehabilitation of the painful shoulder. J Okla State Med Assoc 1961;54:433-7.

437. Kent JM. Help for the stiff, sore shoulder. Patient Care 1985;19:55-8, 61, 64-5.

438. Keyl W. [Mobilization of the stiff shoulder under anesthesia - indication, technique and results.] Z Orthop Ihre Grenzgeb 1982;120:574-5.

439. Khan AA, Mowla A, Shakoor MA, Rahman MR. Arthrographic distension of the shoulder joint in the management of frozen shoulder. Mymensingh Med J 2005;14:67-70.

440. Khan JA, Devkota P, Acharya BM, Pradhan NMS, Shreshtha SK, Singh M, et al. Manipulation under local anesthesia in idiopathic frozen shoulder - a new effective and simple technique. Nepal Med Coll J 2009;11:247-53.

441. Khitrov NA. [Periarthritis of the shoulder joint: variants of the course and treatment with artrofoon.] Ter Arkh 2007;79:40-6.

442. Kirillova RA. Diagnosis and treatment of humeroscapular periarthritis. Vrach Delo 1986:15-17.

443. Kivimaki J, Seitsalo S. Treatment of adhesive capsulitis (frozen shoulder) with manipulation under anaesthesia. Eur J Phys Rehabil Med 1996;6:59-61.

444. Kivimaki J, Pohjolainen T. Manipulation under anesthesia for frozen shoulder with and without steroid injection. Arch Phys Med Rehabil 2001;82:1188-90.

445. Klinger H-M, Otte S, Baums MH, Haerer T. Early arthroscopic release in refractory shoulder stiffness. Arch Orthop Trauma Surg 2002;122:200-3.

446. Knebl JA, Shores JH, Gamber RG, Gray WT, Herron KM. Improving functional ability in the elderly via the Spencer technique, an osteopathic manipulative treatment: a randomized, controlled trial. J Am Osteopath Assoc 2002;102:387-96.

447. Kneer W, Eulert J. [Operative treatment of periarthritis humeroscapularis.] Therapiewoche 1983;33:588-94.

448. Kneer W, Kuhnau S, Bias P, Haag RF. [Dimethylsulfoxide (DMSO) gel for the treatment of acute tendopathy. A multicentric, placebo-controlled randomized study.] Fortschr Med 1994;112:142-6.

449. Knusel O. [Die transkutane elektrische nervenstimulation beim weichteilrheumatismus: eine kontrollierte untersucherblinde studie an 60 patienten mit levator-scapulae-syndrom.] Z Phys Med Bal Med Klim 1984;13:337-9. 
450. Koel G. Comment on: A prospective double-blind placebo-controlled randomized trial of ultrasound in the physiotherapy treatment of shoulder pain. Rheumatology 2008;47:229-30.

451. Kong JC, Lee MS, Shin BC. Randomized clinical trials on acupuncture in Korean literature: a systematic review. Evid Based Complement Alternat Med 2009;6:41-8.

452. Kostadinov D, Michailov S, Dimitrov M. [Treatment of patients with periarthritis of the shoulder joint using the stimulator 'Arfai'.] Kurortol Fizioter 1980;17:16-20.

453. Koubaa S, Ben Salah FZ, Lebib S, Miri I, Dziri C, Ben Hammouda M, et al. [Capsular distension and physical therapy in treatment of adhesive capsulitis.] Tunis Med 2006;84:621-5.

454. Kovacs E. Comparative examinations with pelsonin and indometacin by double blind method in patients suffering from locomotor diseases. Ther Hung 1982;30:180-3.

455. Kucukdeveci AA, Sahin M, Tuncer S, Gok H. Effectiveness of electrical stimulation in shoulder pain due to intrinsic mechanical shoulder problems. Ann Rheum Dis 2005;64(Suppl. 3):387.

456. Kuijpers T, van Tulder MW, van der Heijden GJ, Bouter LM, van der Windt DA. Costs of shoulder pain in primary care consulters: a prospective cohort study in the Netherlands. BMC Musculoskelet Disord 2006;7:83.

457. Kulenkampff HA, Reichelt A. [Studies on conservative and operative treatment of the supraspinatus syndrome.] Aktuel Rheumatol 1989;14:70-6.

458. Kuptniratsaikul S, Kuptniratsaikul V, Tejapongvorachai T, Itiravivong P. A capsular dilatation facilitated shoulder manipulation for treating patients with frozen shoulder. J Med Assoc Thai 2002;85(Suppl. 1):163-9.

459. Kurtais Gursel Y, Ulus Y, Bilgic A, Dincer G, van der Heijden GJMG. Adding ultrasound in the management of soft tissue disorders of the shoulder: a randomized placebo-controlled trial. Phys Ther 2004;84:336-43.

460. Laidley S. Shiatsu massage for frozen shoulder. Positive Health 2004;101:52-4.

461. Lanfranchi R, Volpi A. [Results of the clinical trial of intra-articular thiocolchicoside in scapulo-humeral periarthritis.] Minerva Med 1968;59:4678-81.

462. Laroche M, Ighilahriz O, Moulinier L, Constantin A, Cantagrel A, Mazieres B. Adhesive capsulitis of the shoulder: an open study of 40 cases treated by joint distention during arthrography followed by an intraarticular corticosteroid injection and immediate physical therapy. Rev Rhum Engl Ed 1998;65:313-19.

463. Laskowski I, Schuckmann W. [Contribution to operative treatment of periarthritis humeroscapularis.] Beitr Orthop Traumatol 1985;32:337-41.

464. LaStayo P, Jaffe R. Assessment and management of shoulder stiffness: a biomechanical approach. J Hand Ther 1994;7:122-30.

465. Latham BA. Injections and physiotherapy for the painful stiff shoulder. Ann Rheum Dis 1989;48:703.

466. Laznicky J. [Experiences with spa treatment in humeroscapular periarthropathy in spa Teplice.] Rehabilitacia 1989;22:207-13.

467. Lech O, Subbrack G, Valenzuela Neto C. Capsulite adesiva ('ombro congelado'): abordagem multidisciplinar. Rev Bras Ortop 1993;28:617-24. 
468. Leclaire R, Bourgouin J. Electromagnetic treatment of shoulder periarthritis: a randomized controlled trial of the efficiency and tolerance of magnetotherapy. Arch Phys Med Rehabil 1991;72:284-7.

469. Lee M, Haq AM, Wright V, Longton EB. Periarthritis of the shoulder: a controlled trial of physiotherapy. Physiotherapy 1973;59:312-15.

470. Lee PN, Lee M, Haq AM, Longton EB, Wright V. Periarthritis of the shoulder: trial of treatments investigated by multivariate analysis. Ann Rheum Dis 1974;33:116-19.

471. Lee WK, Maguire MG. Injection of the shoulder. Med J Aust 1986;144:669.

472. Lee H, Hong K, Kim Y, Yim Y, Ahn T, Kang W. A clinical trial of acupuncture treatment for frozen shoulder. J Korean Acupunct Mox Soc 2006;23:165-77.

473. Lee H-J, Lim K-B, Kim D-Y, Lee K-T. Randomized controlled trial for efficacy of intraarticular injection for adhesive capsulitis: ultrasonography-guided versus blind technique. Arch Phys Med Rehabil 2009;90:1997-2002.

474. Lee MS, Choi TY, Kim JI, Choi SM. Using Guasha to treat musculoskeletal pain: a systematic review of controlled clinical trials. Chin Med [serial online] 2010;5. URL: www.ncbi.nlm.nih. gov/pmc/articles/PMC2827462/. Accessed 6 January 2010.

475. Lehmann JF, Erickson DJ, Martin GM, Krusen FH. Comparison of ultrasonic and microwave diathermy in the physical treatment of periarthritis of the shoulder; study of the effects of ultrasonic and microwave diathermy when employed in conjunction with massage and exercise. Arch Phys Med Rehabil 1954;35:627-34.

476. Levenets VN, Iurchenko AP, Gertsen GI. [Humeroscapular periarthrosis and its treatment.] Ortop Travmatol Protez 1982:24-7.

477. Li ZM. 235 cases of frozen shoulder treated by manipulation and massage. J Tradit Chin Med 1984;4:213-15.

478. Li H-M. Treatment of 84 cases of shoulder periarthritis by tuina plus acupuncture. J Acu Tuina Sci 2003;1:47-9.

479. Liang HC, Lien IN. Comparative study in the management of frozen shoulder. Taiwan Yi Xue Hui Za Zhi 1973;72:243-7.

480. Liao B-N, Zhang S, Zhang L-M. [Clinical observation on 'Jianying point'-injection combined with massage for treatment of periarthritis of shoulder.] Zhongguo Zhenjiu 2007;27:261-3.

481. Liaw SC. The effect and timing of physiotherapy on change in range of motion and function in frozen shoulder. Physiother Singapore 2000;3:82-6.

482. Lidstrom A. [The 'frozen' shoulder. Pathologic anatomy and treatment with mobilization.] Nord Med 1963;69:125-9.

483. Liebolt FL. Frozen shoulder. Passive exercises for treatment. N Y State J Med 1970;70:2085.

484. Liem D, Meier F, Thorwesten L, Marquardt B, Steinbeck J, Poetzl W. The influence of arthroscopic subscapularis tendon and capsule release on internal rotation strength in treatment of frozen shoulder. Am J Sports Med 2008;36:921-6.

485. Liu X. [Periarthritis of shoulder therapy by combination of drug hammer and exercise in 83 cases.] Chin J Clin Rehab 2003;7:1055.

486. Liu HP, Wang C, Xu SF. [Comparison of different treatments for relieving pain and improving joint movement disorder in patients with frozen shoulder.] Chin J Clin Rehab 2004;8:3946-7. 
487. Lin JG, Chen CT, Lu TW, Lin YS, Chen HL, Chen YS. Quantitative evaluation of the motion of frozen shoulders treated with acupuncture by puncturing from Tiaokou (St. 38) towards Chengshan (U.B. 57). Biomed Eng 2005;17:31-7.

488. Lin H-C, Li J-S, Lo S-F, Shih Y-F, Lo C-Y, Chen S-Y. Isokinetic characteristics of shoulder rotators in patients with adhesive capsulitis. J Rehabil Med 2009;41:563-8.

489. Livinya BY. Osteoreflexotherapy of humerocapsular periarthritis. Latvijas Psr Zinatnu Akademijas Vestis 1989:121-3.

490. Long J, Peer G, Cwik J. The triscapular block. A new application of therapy of periscapular pain and glenohumeral restriction. Reg Anesth 1987;12:70-5.

491. Lorbach O, Anagnostakos K, Scherf C, Seil R, Kohn D, Pape D. Nonoperative management of adhesive capsulitis of the shoulder: oral cortisone application versus intra-articular cortisone injections. J Shoulder Elbow Surg 2010;19:172-9.

492. Loyd JA, Loyd HM. Adhesive capsulitis of the shoulder: arthrographic diagnosis and treatment. South Med J 1983;76:879-83.

493. Lu S. Scalp acupuncture therapy and its clinical application. J Tradit Chin Med 1991;11:272-80.

494. Lu J, Sun J-H, Fu Z-H, Yuan J-H, Li J, Ji A-Q. [Transient therapeutic effect and safety of superficial needling therapy for treatment of periarthritis of shoulder.] Zhongguo Zhenjiu 2008;28:414-16.

495. Ludwig J, Hedtmann DA. [Orthopedic treatment of shoulder pain.] Z Orthop Ihre Grenzgeb 1998;136:OA10-OA13.

496. Lundberg BJ. Arthrography and manipulation in rigidity of shoulder joint. Acta Orthop Scand 1965;36:35-44.

497. Luo F, Shen Y, Liu YQ, Wang EZ, Wang BG. [Intraarticular injection of sodium hyaluronate plus nerve and pain block with betamethasone in treatment of periarthritis of shoulder.] Chin J Clin Rehab 2005;9:266-7.

498. Luziatelli S, Colagrosso B, Alaimo A. [Clinical data on intraarticular administration of orgotein; comparison with a corticosteroid.] G Med Mil 1984;134:490-3.

499. Ma SH, Sun Y, Yan XM. [Effect of suprascapular nerve block combined with triole nerve block for scapulohumeral periarthritis.] Chin J Clin Rehab 2004;8:4698-9.

500. Maiotti M, Massoni C, Basile A, Cedrone P. Role of arthroscopic arthrolysis in stiff shoulder. Eur J Sports Traumat Rel Res 2001;23:94-9.

501. Maitland GD. Treatment of the glenohumeral joint by passive movement. Physiotherapy 1983;69:3-7.

502. Mao YR, Huang DF, Ding JX, Xu GQ, Xu YL, Zhao M. [Analysis of extracorporeal shock wave therapy in immediate treatment of musculoskeletal disorders.] Chin J Clin Rehab 2003;7:3216-17.

503. Marcus A. A combined treatment approach to shoulder pain using orthopaedic medicine and acupuncture technique. J Orthop Med 1994;16:75-9.

504. Mardjuadi A, Dequeker J. Double-blind trial comparing bufexamac infiltrations with triamcinolone acetonide infiltrations in patients with periarthritis of the shoulder. Curr Med Res Opin 1978;5:401-5. 
505. Marx RG, Malizia RW, Kenter K, Wickiewicz TL, Hannafin JA. Intra-articular corticosteroid injection for the treatment of idiopathic adhesive capsulitis of the shoulder. HSS J 2007;3:202-7.

506. Massoud SN, Pearse EO, Levy O, Copeland SA. Operative management of the frozen shoulder in patients with diabetes. J Shoulder Elbow Surg 2002;11:609-13.

507. Mattara L, Trotta F, Biasi D, Cervetti R. Evaluation of the efficacy and tolerability of a new locally acting preparation of flurbiprofen in scapulohumeral periarthritis. Eur J Rheumatol Inflamm 1994;14:15-20.

508. Mavrikakis ME, Sfikakis PP, Kontoyannis SA, Antoniades LG, Kontoyannis DA, Moulopoulou DS. Clinical and laboratory parameters in adult diabetics with and without calcific shoulder periarthritis. Calcif Tissue Int 1991;49:288-91.

509. Mayerhofer H. [Enhancement of effect of a high dosage salicylate ointment by iontophoresis.] Z Allgemeinmed 1981;57:634-6.

510. McClatchie L, Laprade J, Martin S, Jaglal SB, Richardson D, Agur A. Mobilizations of the asymptomatic cervical spine can reduce signs of shoulder dysfunction in adults. Man Therap 2009;14:369-74.

511. McHardy A, Hoskins W, Pollard H, Onley R, Windsham R. Chiropractic treatment of upper extremity conditions: a systematic review. J Manipulative Physiol Ther 2008;31:146-59.

512. McKeever DC. Thawing the frozen shoulder. Clin Orthop 1958;11:168-76.

513. McQuay HJ, Moore RA, Eccleston C, Morley S, Williams AC. Systematic review of outpatient services for chronic pain control. Health Technol Assess 1997;1.

514. Meijer EM, Sluiter JK, Heyma A, Sadiraj K, Frings-Dresen MH. Cost-effectiveness of multidisciplinary treatment in sick-listed patients with upper extremity musculoskeletal disorders: a randomized, controlled trial with one-year follow-up. Int Arch Occup Environ Health 2006;79:654-64.

515. Melzer C, Wallny T, Wirth CJ, Hoffmann S. Frozen shoulder - treatment and results. Arch Orthop Trauma Surg 1995;114:87-91.

516. Mencke M, Wieden TE, Hoppe M, Porschke W, Hoffmann O, Herget HF. Acupuncture of shoulder pain and low back pain. Two prospective double-blind studies. Akupunktur 1988;16:204-15.

517. Menkes CJ. [Scapulo-humeral periarthritis: efficacy, safety and therapeutic benefit of etodolac (600 mg daily) versus piroxicam (40/20 mg daily)]. Rhumatologie 1990;42:195-200.

518. Mert M, Atakanli LK, Temizel M. The comparison of treatment response of diabetic and non-diabetic adhesive capsulitis to physical therapy. Diabetes 2009;58(Suppl. 1):2159.

519. Meyer MP, Ter Waarbeek B. [Humeroscapular periarthritis and exercise therapy.] Ned Tijdschr Geneeskd 1952;96:2297-301.

520. Miccoli G, Angiolini G, Vannini A. [Association of a benzofurane derivative and kinesitherapy in the treatment of periarthritis of the shoulder.] Clin Ter 1964;31:310-23.

521. Michlovitz SL, Ann Harris B, Watkins MP. Therapy interventions for improving joint range of motion: a systematic review. J Hand Ther 2004;17:118-31.

522. Miller MD, Rockwood CA, Wirth MA. Thawing the frozen shoulder - the 'patient' patient. South Med J 1991;84:2S55.

523. Miller JS, Stanley I, Moore K. Videotaped exercise instruction: a randomised controlled trial in musculoskeletal physiotherapy. Physiother Theory Pract 2004;20:145-54. 
524. Miller JS, Litva A, Gabbay M. Motivating patients with shoulder and back pain to self-care: can a videotape of exercise support physiotherapy? Physiotherapy 2009;95:29-35.

525. Mior S. Manipulation and mobilization in the treatment of chronic pain. Clin J Pain 2001;17(Suppl. 4):70-6.

526. Miszczyk L, Wozniak G, Walichiewicz P, Spindel J. The effectiveness of radiotherapy for painful humeroscapular periarthritis (PHS). Nowotwory 2005;55:49-53.

527. Mizuno S. [Frozen shoulder.] Nippon Rinsho 1976;34:3552-4.

528. Mohindra Y, Jain S, Sain A. R. compound (Alarsin) in periarthritis shoulder. Clinical trial. Med Surg 1987;27:9-10.

529. Molsberger AF, Schneider T, Gotthardt H, Drabik A. German Randomized Acupuncture Trial for chronic shoulder pain (GRASP). A pragmatic, controlled, patient-blinded, multicentre trial in an outpatient care environment. Pain 2010;151:146-54.

530. Monreal González RJ, Díaz Ramos H, León Santana P, Paredtes González LF. [Capsulitis adhesiva del hombro: tratamiento con distensión hidráulica y anestesia local.] Rev Cuba Ortop Traumatol 2006;20. URL: http://scielo.sld.cu/pdf/art/v20n2/ort0526.pdf. Accessed 26 March 2010.

531. Montemagni P, Carnazza G. [Diagnosis and arthroscopic treatment of frozen shoulder.] Minerva Ortop Traumatol 1989;40:85-90.

532. Moore ME, Berk SN. Acupuncture for chronic shoulder pain. An experimental study with attention to the role of placebo and hypnotic susceptibility. Ann Intern Med 1976;84:381-4.

533. Morgan B, Jones AR, Mulcahy KA, Finlay DB, Collett B. Transcutaneous electric nerve stimulation (TENS) during distension shoulder arthrography: a controlled trial. Pain 1995;64:265-7.

534. Morris CR, Morris AJ. Intravenous pulse corticosteroids for the treatment of frozen shoulder. Arthritis Rheum 1994;37(Suppl. 9):241.

535. Moutounet J, Chevrot A, Godefroy D. [Radioguided puncture-filtration treatment of intractable calcifying periarthritis of the shoulder.] J Radiol 1984;65:569-72.

536. Mueller EE, Mead S, Schulz BF, Vaden MR. A placebo-controlled study of ultrasound treatment for periarthritis. Am J Phys Med 1954;33:31-5.

537. Mund-Hoym WD. [Degree of efficacy of salicylate therapy of periarthropathia humeroscapularis.] Med Welt 1987;38:1500-2.

538. Munting E. Ultrasonic therapy for painful shoulders. Physiotherapy 1978;64:180-1.

539. Murnaghan GF, McIntosh D. Hydrocortisone in painful shoulder. A controlled trial. Lancet 1955:798-800.

540. Musil D, Sadovsky P, Stehlik J, Filip L, Vodicka Z. [Arthroscopic capsular release in frozen shoulder syndrome.] Acta Chir Orthop Traumatol Cech 2009;76:98-103.

541. Nabeta T. [Effect of a tender point acupuncture therapy for shoulder stiffness: a randomized controlled trial.] J Jpn Acupunct Moxibustion Soc 2000;50:328.

542. Nabeta T, Kawakita K. Relief of chronic neck and shoulder pain by manual acupuncture to tender points: a sham-controlled randomized trial. Complement Ther Med 2002;10:217-22.

543. Naglic N, Klopcic-Spevak M. The effect of TENS during kinesiotherapy on chronic shoulder pain. Int J Rehabil Res 2009;32(Suppl. 1):10-11. 
544. Naredo E, Cabero F, Beneyto P, Cruz A, Mondejar B, Uson J, et al. A randomized comparative study of short term response to blind injection versus sonographicguided injection of local corticosteroids in patients with painful shoulder. J Rheumatol 2004;31:308-14.

545. Narouze SN, Govil H, Guirguis M, Mekhail NA. Continuous cervical epidural analgesia for rehabilitation after shoulder surgery: a retrospective evaluation. Pain Physician 2009;12:189-94.

546. Nash P, Hazleman BL. Frozen shoulder. Baillieres Clin Rheumatol 1989;3:551-66.

547. Neviaser TJ. Adhesive capsulitis. In Mcginty JBE, editor. Operative arthroscopy. New York, NY: Raven Press; 1991. pp. 561-6.

548. Ng CY, Amin AK, Narborough S, McMullan L, Cook R, Brenkel IJ. Manipulation under anaesthesia and early physiotherapy facilitate recovery of patients with frozen shoulder syndrome. Scott Med J 2009;54:29-31.

549. Nicholson GG. The effects of passive joint mobilization on pain and hypomobility associated with adhesive capsulitis of the shoulder. J Orthop Sports Phys Ther 1985;6:238-46.

550. Nicholson GP. Arthroscopic capsular release for stiff shoulders: effect of etiology on outcomes. Arthroscopy 2003;19:40-9.

551. Nicolova L. Efficiency of interferential current in scapulo-humeral periarthritis calcarea. J Radiol Electrol Arch Electr Med 1966;47:521.

552. Nikolova L. [Low-frequency treatment with interference current of patients with periarthritis humeroscapularis.] MMW Munch Med Wochenschr 1970;112:472-6.

553. Nobuhara K, Sugiyama D, Ikeda H, Makiura M. Contracture of the shoulder. Clin Orthop Relat Res 1990:105-10.

554. Noel E. Treatment of calcific tendinitis and adhesive capsulitis of the shoulder. Rev Rhum Engl Ed 1997;64:619-28.

555. Noskov SM, Fetelego OI, Krasivina IG, Dolgova LN. [Alflutop in local therapy of shoulder periarthritis.] Ter Arkh 2005;77:57-60.

556. Nouijai A, Mahi M, El J, Ghazi M, Mounach A, Achemlal L, et al. Arthrographic joint distension with saline and steroid followed by continuous passive motion improves function in patients with frozen shoulder: an open study. Ann Rheum Dis 2006;65(Suppl. 2):612.

557. Nykanen M. Pulsed ultrasound treatment of the painful shoulder a randomized, doubleblind, placebo-controlled study. Scand J Rehabil Med 1995;27:105-8.

558. Ogilvie-Harris DJ, Biggs DJ, Fitsialos DP, MacKay M. The resistant frozen shoulder. Manipulation versus arthroscopic release. Clin Orthop Relat Res 1995:238-48.

559. Ogilvie-Harris DJ, Myerthall S. The diabetic frozen shoulder: arthroscopic release. Arthroscopy 1997;13:1-8.

560. Ohshima Y. [Intra-articular injection therapy of high molecular weight sodium hyaluronate (SPH) on periarthritis of the shoulder.] Jpn Pharm Ther 1987;15:341-56.

561. Ohta K, Yano T. [Comparison study between superficial and deep acupuncture on the neck and shoulder stiffness - as index of muscle hardness, surface EMG and deep hemodynamics.] J Jpn Assoc Phys Med Balneol Climatol 2005;68:122-33.

562. Okamura K. [Arthroscopic shoulder surgery.] Hokkaido J Med Sci 1995;70:375-81. 
563. Olejarova M. [Local nonsteroidal antiinflamatory drugs from the point of view of evidencebased medicine.] Bolest 2004;7:93-9.

564. Omari A, Bunker TD. Open surgical release for frozen shoulder: surgical findings and results of the release. J Shoulder Elbow Surg 2001;10:353-7.

565. Orr B. Treating shoulder complaints in general practice. Study's results must be viewed in context of Dutch, not British, physiotherapy practice. BMJ 1997;315:681.

566. Ortolani M, Tarantello G, Cognolatto F, Faggin A. [Manipulation by McMennel's technique in treatment of noncalcified periarthritis of the shoulder.] Riabilitazione 1985;18:155-65.

567. Othman A, Taylor G. Manipulation under anaesthesia for frozen shoulder. Int Orthop 2002;26:268-70.

568. Ou HC. [An observation of four physiotherapies for 249 cases with shoulder periarthritis.] Chin J Phys Therap 1989;12:32-3.

569. Ozaki J, Nakagawa Y, Sakurai G, Tamai S. Recalcitrant chronic adhesive capsulitis of the shoulder. Role of contracture of the coracohumeral ligament and rotator interval in pathogenesis and treatment. J Bone Joint Surg Am 1989;71:1511-15.

570. Ozaki J. Frozen shoulder - operative management and pathogenesis. Surg Shoulder 1995;1085:275-8.

571. Ozaki J. Pathomechanics and operative management of chronic frozen shoulder. Ann Chir Gynaecol 1996;85:156-8.

572. Pages IH, Herrmann H, Retter D. [Magnetic field therapy with Poljus 1-first experimental and clinical results.] $Z$ Physiother 1982;34:205-7.

573. Pap G, Liebau C, Meyer M, Merk H. [Results of mobilization under anesthesia in adhesive capsulitis in relation to stage of the disease.] Z Orthop Ihre Grenzgeb 1998;136:13-17.

574. Parker RD, Froimson AI, Winsberg DD, Arsham NZ. Frozen shoulder. Part II: treatment by manipulation under anesthesia. Orthopedics 1989;12:989-90.

575. Parker RD, Froimson AI, Winsberg DD, Arsham NZ. Frozen shoulder. Part I: chronology, pathogenesis, clinical picture, and treatment. Orthopedics 1989;12:869-73.

576. Parsons JL, Shepard WL, Fosdick WM. DMSO an adjutant to physical therapy in the chronic frozen shoulder. Ann N Y Acad Sci 1967;141:569-71.

577. Patel M, Gutzwiller F, Paccaud F, Marazzi A. A meta-analysis of acupuncture for chronic pain. Int J Epidemiol 1989;18:900-6.

578. Patte D, Goutallier D. ['Reparative' and 'delivering' conceptions in treatment of the periarthritis of the shoulder.] Acta Orthop Belg 1983;49:299-309.

579. Pearsall AW, Osbahr DC, Speer KP. An arthroscopic technique for treating patients with frozen shoulder. Arthroscopy 1999;15:2-11.

580. Peng AT, Behar S, Yue SJ. Long-term therapeutic effects of electro-acupuncture for chronic neck and shoulder pain: a double blind study. Acupunct Electrother Res 1987;12:37-44.

581. Peng J-M, Lu H, Hu X-B. [Observation on therapeutic effect of Shaoshanhuo needling method on periarthritis of shoulder.] Zhongguo Zhenjiu 2006;26:581-2.

582. Petri M, Dobrow R, Neiman R, Whiting-O’Keefe Q, Seaman WE. Randomized, doubleblind, placebo-controlled study of the treatment of the painful shoulder. Arthritis Rheum 1987;30:1040-5. 
583. Petukhov IA. Poly clinical diagnosis and treatment of shoulder scapular periarthritis. Zdravookhr Beloruss 1979:53-5.

584. Philadelphia Panel. Philadelphia Panel evidence-based clinical practice guidelines on selected rehabilitation interventions for shoulder pain. Phys Ther 2001;81:1719-30.

585. Piotte F, Gravel D, Moffet H, Fliszar E, Roy A, Nadeau S, et al. Effects of repeated distension arthrographies combined with a home exercise program among adults with idiopathic adhesive capsulitis of the shoulder. Am J Phys Med Rehabil 2004;83:537-46.

586. Pittler MH. Static magnets for reducing pain. Focus Altern Complement Ther 2008;13:5-10.

587. Placzek JD, Roubal PJ, Freeman DC, Kulig K, Nasser S, Pagett BT. Long-term effectiveness of translational manipulation for adhesive capsulitis. Clin Orthop Relat Res 1998:181-91.

588. Polimeni V, Panuccio A, Furfari P, Crupi D, Barreca G, Forgione C, et al. Preliminary study on the efficacy of various rehabilitation therapies for shoulder pain. Eura Medicophys 2003;39:59-63.

589. Politano B, Jucopill AN, Gherardi GM, Loda M. [Review of 11 cases of surgically treated Duplay's disease.] Minerva Ortop 1982;33:1025-8.

590. Pollock RG, Duralde XA, Flatow EL, Bigliani LU. The use of arthroscopy in the treatment of resistant frozen shoulder. Clin Orthop Relat Res 1994:30-6.

591. Poonam Singh, Singh PD. Manipulation for periarthritis of the shoulder in tribal population of Chotanagpur-Ranchi. Indian Med J 1990;84:247-8.

592. Qiu Q-Z. [Treatment of severe scapulohumeral periarthritis with combined use of suprascapular nerve blockade and manipulation therapy: a report of 49 cases.] Zhong Xi Yi Jie He Хиe Bao 2006;4:82-3.

593. Quigley TB. Checkrein shoulder - a type of frozen shoulder - diagnosis and treatment by manipulation and ACTH or cortisone. N Engl J Med 1954;250:188-92.

594. Quigley TB. Checkrein shoulder. A type of 'frozen' shoulder diagnosis and treatment by manipulation and ACTH or cortisone. Clin Orthop 1982;164:4-9.

595. Quin CE. 'Frozen shoulder'. Evaluation of treatment with hydrocortisone injections and exercises. Ann Phys Med 1965;10:22-5, 29.

596. Quraishi NA, Johnston P, Bayer J, Crowe M, Chakrabarti A. Thawing the frozen shoulder: a randomised controlled trial comparing manipulation under anaesthesia with hydrodilatation. J Bone Joint Surg Br 2006;89B:1197-200.

597. Quraishi NA, Johnston P, Bayer J, Crowe M, Chakrabarti A. Thawing the frozen shoulder - a randomised controlled trial of manipulation under anaesthetic versus hydrodilatation. J Bone Joint Surg Br 2008;90B(Suppl. 2):215.

598. Radaelli E, Veneziani GA, Rossi A. [Manual medicine in treatment of adhesive capsulitis.] Riabilitazione 1984;17:3-12.

599. Rainbow DM, Weston JP, Brantingham JW, Globe G, Lee F. A prospective clinical trial comparing chiropractic manipulation and exercise therapy vs chiropractic mobilization and exercise therapy for treatment of patients suffering from adhesive capsulitis/frozen shoulder. J Am Chiropr Assoc 2008;45:12-28.

600. Ramsey ML, Getz CL, Parsons BO. What's new in shoulder and elbow surgery. J Bone Joint Surg Am 2009;91:1283-93.

601. 600. Refior HJ, Melzer C. [Shoulder stiffness and mobilization under anesthesia.] Hefte Unfallheilkd 1984;170:145-9. 
602. Reichmister JP, Friedman SL. Long-term functional results after manipulation of the frozen shoulder. Md Med J 1999;48:7-11.

603. Ren YM, Shang JB, Wu XM, Chen YF, He XF. [Treatment of periarthritis humeroscapularis with medical ozone injection.] Chin J Interven Imag Therap 2006;3:341-3.

604. Rendeiro DG, Majkowski GR, Lee IE, Gill NW III, Jensen DA, Deyle GS, et al. The effectiveness of translational manipulation under interscalene block for the treatment of adhesive capsulitis of the shoulder: a prospective clinical trial. J Man Manip Ther 2006;14:180.

605. Revel M, Ghanem N. [Adhesive capsulitis of the shoulder.] Rev Prat 1999;49(Suppl. 13):1406-8.

606. Rey B, Gerber NJ. Shoulder pain trials. In Schlapbach P, Gerber NJ, editors. Rheumatology, Vol. 14. Physiotherapy: controlled trials and facts. Basel, Switzerland: S. Karger Ag; 1991. pp. 91-8.

607. Rhind V, Downie WW, Bird HA. Naproxen and indomethacin in periarthritis of the shoulder. Rheumatol Rehabil 1982;21:51-3.

608. Rich FR, Waligora M. Chronic shoulder pain: evaluation and management. Physician Assist 1985;9:66-7, 70-2, 81-2.

609. Richardson AT. Ernest Fletcher Lecture. The painful shoulder. Proc R Soc Med 1975;68:731-6.

610. Rigato M, Battisti E, Fortunato M, Giordano N. Comparison between the analgesic and therapeutic effects of a musically modulated electromagnetic field (TAMMEF) and those of a $100 \mathrm{~Hz}$ electromagnetic field: blind experiment on patients suffering from cervical spondylosis or shoulder periarthritis. J Med Eng Technol 2002;26:253-8.

611. Ritchie LD. A clinical evaluation of flurbiprofen LAT and piroxicam gel: a multicentre study in general practice. Clin Rheumatol 1996;15:243-7.

612. Ritzmann P. ['Frozen shoulder': intraarticular corticosteroids lead to faster pain relief than physiotherapy.] Praxis 1999;88:1369-70.

613. Rizk TE, Christopher RP, Higgins AC. Adhesive capsulitis - a new therapeutic approach. Arch Phys Med Rehabil 1982;63:501.

614. Rizk TE, Christopher RP, Pinals RS, Higgins AC, Frix R. Adhesive capsulitis (frozen shoulder): a new approach to its management. Arch Phys Med Rehabil 1983;64:29-33.

615. Rizk TE, Gavant ML, Pinals RS. Treatment of adhesive capsulitis (frozen shoulder) with arthrographic capsular distension and rupture. Arch Phys Med Rehabil 1994;75:803-7.

616. Roberts HJ. Local Injection of a preparation containing 2 betamethasone esters in the treatment of noninfectious musculoskeletal disorders. J Am Geriatr Soc 1965;13:275-91.

617. Romoli M, van der Windt D, Giovanzana P, Masserano G, Vignali F, Quirico E, et al. International research project to devise a protocol to test the effectiveness of acupuncture on painful shoulder. J Altern Complement Med 2000;6:281-7.

618. Rompe G. [Remedial gymnastic treatment in degenerative affections of the shoulder.] Krankengymnastik 1981;33:727-35.

619. Roques CF, Railhac JJ, Arbus L. [Arthrography and electromyography of shoulder periarthriti.] Rhumatologie 1984;36:5-12. 
620. Roubal PJ, Dobritt D, Placzek JD. Glenohumeral gliding manipulation following interscalene brachial plexus block in patients with adhesive capsulitis. J Orthop Sports Phys Ther 1996;24:66-77.

621. Rowlingson JC, Arasi R. The use of suprascapular nerve blocks in the management of shoulder pain. Reg Anesth 1986;11:156-9.

622. Saadat Niaki A, Siaie M, Sadeghi H, Khatibi H. The use of steroids for the management of chronic shoulder pain by interventional techniques. Arch Iran Med 2005;8:127-30.

623. Saadeh CK, Ramos LG, Malacara JM, Mitchell A, Shelton D, Christian N, et al. Ultrasound guided joint injection: comparison with outcomes in blinded injections. Ann Rheum Dis 2005;64(Suppl. 3):187.

624. Saeidian SR, Hemmati AA, Haghighi MH. Pain relieving effect of short-course, pulse prednisolone in managing frozen shoulder. J Pain Palliat Care Pharmacother 2007;21:27-30.

625. Saggini R, Zoppi M, Vecchiet F, Gatteschi L, Obletter G, Giamberardino MA. Comparison of electromotive drug administration with ketorolac or with placebo in patients with pain from rheumatic disease: a double-masked study. Clin Ther 1996;18:1169-74.

626. Sakeni RA, Al-Nimer MSM. Comparison between intraarticular triamcinolone acetonide and methylprednisolone acetate injections in treatment of frozen shoulder. Saudi Med J 2007;28:707-12.

627. Sanders WE, Hooper WE. Recalcitrant chronic adhesive capsulitis of the shoulder. J Bone Joint Surg Am 1990;72:1269-70.

628. Sandor R. Adhesive capsulitis: optimal treatment of 'frozen shoulder'. Phys Sportsmed 2000;28:23-5, 27, 29.

629. Sandor R, Brone S. Exercising the frozen shoulder. Phys Sportsmed 2000;28:83-4.

630. Santalena G, Favalli E, Ligniere GC. [Local infiltrative therapy of the painful shoulder.] Riv It Biol Med 1998;18:84-7.

631. Sauvain MJ. [Periarthritis of the shoulder.] Ther Umsch 1985;42:695-8.

632. Scendoni P. [Therapy of periarthropathies.] Clin Ter 1987;122:357-66.

633. Scheef SE. [The treatment of humero-scapular periarthritis.] ZFA (Stuttgart) 1979;55:1474.

634. Schieroni MP, Revello MP. [Medical, physical and rehabilitative treatment in a case load of 250 patients affected by scapulo-humeral periarthritis.] Minerva Med 1985;76:941-3.

635. Schomacher J. The effect of anterior versus posterior glide joint mobilization on external rotation range of motion in patients with shoulder adhesive capsulitis. J Orthop Sports Phys Ther 2007;37:413.

636. Schultheis A, Reichwein F, Nebelung W. [Frozen shoulder syndrome.] MMW Fortschr Med 2009;151:36-7.

637. Schultze J, Schlichting G, Galalae R, Koltze H, Kimmig B. [Results of radiation therapy in periarthritis humeroscapularis.] Rontgenpraxis 2004;55:160-4.

638. Schulz J. Frozen shoulder. Med J Aust 1986;144:503.

639. Schwitalle M, Betz U, Eckardt A, Rompe JD, Meurer A, Karbowski A. Rehabilitation success after shoulder manipulation under anaesthesia supported by continuous interscalene block. Eur J Phys Rehabil Med 1998;8:44-7.

640. Scott NA, Guo B, Barton PM, Gerwin RD. Trigger point injections for chronic nonmalignant musculoskeletal pain: a systematic review. Pain Med 2009;10:54-69. 
641. Segmuller HE, Taylor DE, Hogan CS, Saies AD, Hayes MG. Arthroscopic treatment of adhesive capsulitis. J Shoulder Elbow Surg 1995;4:403-8.

642. Sharma RK, Bajekal RA, Bhan S. Frozen shoulder syndrome. A comparison of hydraulic distension and manipulation. Int Orthop 1993;17:275-8.

643. Sharma SPS, Kvale AK, Baerheim AB. Outcome in shoulder capsulitis (frozen shoulder) treated with corticosteroid and corticosteroid with distension: a randomised pilot study. Int Musculoskelet Med 2009;31:75-80.

644. Shchekotov GM. [Humero-scapular periarthritis and its treatment with hydrocortisone.] Vestn Khir Im I I Grek 1977;118:66-8.

645. Shchepina TP. [Physical therapy methods used in the rehabilitation of patients with periarthritis of the shoulder joint.] Kurortol Fizioter 1989;26:33-5.

646. Shehab D, Adham N. Comparative effectiveness of ultrasound and transcutaneous electrical stimulation in treatment of periarticular shoulder pain. Physiother Can 2000;52:208-10, 214.

647. Shiraishi T, Kubo T, Matsunaga T, Sugimoto K, Miyamoto H, Okumura SI, et al. [Clinical study on tizanidine hydrochloride (Ternelin) therapy of tinnitus patients with shoulder stiffness.] Practica Otologica 1991;84:1173-8.

648. Sileghem A, Verstraeten A, Dequeker J. Double-blind, randomized, parallel-group study of the efficacy and safety of proglumetacin and naproxen in periarthritis of the shoulder or elbow. Curr Ther Res Clin Exp 1991;50:93-100.

649. Simpson JK, Budge R. Treatment of frozen shoulder using distension arthrography (hydrodilatation): a case series. Australas Chiropr Osteopathy 2004;12:25-35, 37-8.

650. Singh OP, Goel MK, Goel SC, Singh GK. Periarthritis shoulder-treatment by manipulation and physiotherapy. Indian J Orthop 1980;14:201-3.

651. Singh LN, Singh Ak J, Singh YN, Devi Sh P. Manipulation under anaesthesia for treatment of adhesive capsulitis with early physiotherapy. A pilot study. J Med Soc 2010;24:12-15.

652. Skorogliadov AV, Sakalov DA, Seregin GI, Len'kova NA. [Conduction blocks in the complex treatment of humeroscapular periarthritis.] Ortop Travmatol Protez 1990:10-13.

653. Skorogliadov AV, Gudkov VS, Okhotskaia OV, Sakalov DA. [A method of treatment of humeroscapular periarthritis.] Sov Med 1990:102-5.

654. Skoroglyadov AV, Tektumanidze OV. Scapulohumeral periarthritis. Sov Med 1986:56-60.

655. Slullitel D, Slullitel M, Blasco A, Periotti G, Yucra V, Cagliero G. Capsulitis adhesiva. Rev Argent Artrosc 2000;7:28-32.

656. Smidt N, de Vet HCW, Bouter LM, Dekker J, Arendzen JH, de Bie RA, et al. Effectiveness of exercise therapy: a best-evidence summary of systematic reviews. Aust J Physiother 2005;51:71-85.

657. Snow M, Boutros I, Funk L. Posterior arthroscopic capsular release in frozen shoulder. Arthroscopy 2009;25:19-23.

658. Sokk J, Gapeyeva H, Ereline J, Kolts I, Paasuke M. Shoulder muscle strength and fatigability in patients with frozen shoulder syndrome: the effect of 4-week individualized rehabilitation. Electromyogr Clin Neurophysiol 2007;47:205-13.

659. Spacca G, Cacchio A, Forgacs A, Monteforte P, Rovetta G, Rovati S. Analgesic efficacy of a lecithin-vehiculated diclofenac epolamine gel in shoulder periarthritis and lateral epicondylitis: a placebo-controlled, multicenter, randomized, double-blind clinical trial. Drugs Exp Clin Res 2005;31:147-54. 
660. Speed C, Hazleman B. Shoulder pain. Clin Evid 2004:1613-32.

661. Speed C. Shoulder pain. Clin Evid 2006:1543-60.

662. Spier W. [Conservative therapy of shoulder stiffness.] Hefte Unfallheilkd 1984;170:142-4.

663. Spresser K. Extracorporeal shock wave therapy. J Am Chiropr Assoc 2002;39:46.

664. Srivastava KP, Bhan BL, Bhatia IL. Scapulohumeral periarthritis. A clinical study and evaluation of end results of its treatment. J Indian Med Assoc 1972;59:275-8.

665. Srour F. [Use of cold for the management of acute retractile capsulitis of the shoulder joint.] Kinesither Rev 2008;83:29-33.

666. Stavnichii GV. [Massage in humeroscapular periarthritis.] Feldsher i Akusherka 1985;50:61-3.

667. Stavnichii GV. [Humeroscapular periarthritis. Its treatment by massage.] Med Sestra 1986;45:11-13.

668. Steinbrocker O, Argyros TG. Frozen shoulder: treatment by local injections of depot corticosteroids. Arch Phys Med Rehabil 1974;55:209-13.

669. Stoddard A. Adhesive capsulitis of the shoulder and the sedimentation rate. Br J Phys Med 1955;18:9-10.

670. Stodell MA, Sturrock RD. Frozen shoulder. Lancet 1981;2:527.

671. Stoker DJ, Bruckner FE. Intra-articular injections in capsulitis. BMJ 1991;303:123.

672. Stratz T, Stauber A, Muller W. [Local treatment of periarthropathies with the 5-HT3receptor-antagonist tropisetron.] Z Orthop Ihre Grenzgeb 2002;140:394-8.

673. Strobel G. [Long-term therapeutic effect of different intra-articular injection treatments of the painful shoulder: effect on pain, mobility and work capacity.] Rehabilitation 1996;35:176-8.

674. Strunce JB, Walker MJ, Boyles RE, Young BA. The immediate effects of thoracic spine and rib manipulation on subjects with primary complaints of shoulder pain. J Man Manip Ther 2009;17:230-6.

675. Sun SX. ECIWO acupuncture treatment of 151 cases of scapulohumeral periarthritis. Proceedings of the 3rd International Congress of Eciwo Biology, 1996. pp. 161-3.

676. Sun KO, Chan KC, Lo SL, Fong DY. Acupuncture for frozen shoulder. Hong Kong Med J 2001;7:381-91.

677. Sun G. Clinical experience in application of the acupoint futu. J Tradit Chin Med 2002;22:132-3.

678. Surenkok O, Aytar A, Baltaci G. Acute effects of scapular mobilization in shoulder dysfunction: a double-blind randomized placebo-controlled trial. J Sport Rehabil 2009;18:493-501.

679. Swirski J. [Results of ultrasound treatment of humero-scapular periarthritis.] Reumatologia 1971;9:159-63.

680. Taller AJ. [Treatment of periarthritis humeroscapularis with pirprofen. Double-blind study on 101 outpatients.] Fortschr Med 1985;103:197-9.

681. Talybov FI, Apenysheva NP. [Thermography in patients with scapulohumeral periarthritis.] Vopr Revm 1981:64-6.

682. Talybov Yu F, Badokina GI, Sysoev VF, Agababova ER. [Scintigraphy with the use of 99mTcpyrophosphate in patients with scapulohumeral periarthritis.] Ter Arkh 1981;53:25-8. 
683. Tam G. Effects of LLLT on the periarthritis of the shoulder: a clinical study on different treatments with low level laser therapy, corticosteroid injections or a wait-and-see policy. 15th International Congress of the European Medical Laser Association (EMLA), Helsinki, Finland, 2010. pp. S32-3.

684. Tamai K, Yamato M, Hamada J, Mashitori H, Saotome K. Response of frozen shoulder to intraarticular corticosteroid and hyaluronate: a quantitative assessment with dynamic magnetic resonance imaging. Dokkyo J Med Sci 1999;26:235-41.

685. Tamai K, Mashitori H, Ohno W, Hamada Ji, Sakai H, Saotome K. Synovial response to intraarticular injections of hyaluronate in frozen shoulder: a quantitative assessment with dynamic magnetic resonance imaging. J Orthop Sci 2004;9:230-4.

686. Tan W. The recent status of physical medicine and rehabilitation in China. J Jpn Ass Phys Med Balneol Climatol 1995;59:10-14.

687. Taskaynatan MA, Ozgul A, Yilmaz B, Kalyon TA. [Suprascapular nerve block and steroid injection in nonspecific shoulder pain.] J Rheumatol Med Rehabil 2004;15:9-15.

688. Taskaynatan MA, Yilmaz B, Ozgul A, Yazicioglu K, Kalyon TA. Suprascapular nerve block versus steroid injection for non-specific shoulder pain. Tohoku J Exp Med 2005;205:19-25.

689. Taverna E, Parrini M, Cabitza P. [Lasertherapy vs placebo in the treatment of some bone and joints pathology.] Minerva Ortop Traumatol 1990;41:631-6.

690. Teys P, Bisset L, Vicenzino B. The initial effects of a Mulligan's mobilization with movement technique on range of movement and pressure pain threshold in pain-limited shoulders. Man Therap 2008; 13:37-42.

691. Thakur S. Frozen shoulder. J Indian Med Assoc 1991;89:299-300.

692. Thomas D, Williams RA, Smith DS. The frozen shoulder: a single blind controlled study of manipulation treatment compared with local hydrocortisone. Aust N Z J Med 1981;11:726-7.

693. Thumb N, Kolarz G, Scherak O, Mayrhofer F. The efficacy and safety of fentiazac and diclofenac sodium in peri-arthritis of the shoulder: a multi-centre, double-blind comparison. J Int Med Res 1987;15:327-34.

694. Todorov N. [Treatment of humeroscapular periarthritis by an impulse ultrahigh-frequency field.] Vopr Kurortol Fizioter Lech Fiz Kult 1972;37:410-11.

695. Toker S, Kilincoglu V, Toker A, Gulcan E, Erturer E, Sahin L. Short-term outcome of combined corticosteriod and local anaestetic therapy with home-based exercise programme in painful shoulder conditions. Trop J Pharm Res 2008;7:1123-7.

696. Toplicanec M. [Use of cryotherapy in the treatment of a stiff and painful shoulder.] Reumatizam 1986;33:22-8.

697. Trehan RK, Patel S, Hill AM, Curtis MJ, Connell DA. Is it worthwhile to offer repeat hydrodilatation for frozen shoulder after 6 weeks? Int J Clin Pract 2010;64:356-9.

698. Tripathi RP, Verma AN, Sahu SC, Prasad BK. Treatment of periarthritis of the shoulder with intra-articular human placental extract: (a preliminary report). Clinician 1979;43:370-4.

699. Tsarin VI. [Manual therapy in the treatment of the humeroscapular periarthrosis syndrome.] Med Sestra 1990;49:41-3.

700. Tsukayama H, Yamashita H. Systematic review of clinical trials on acupuncture in the Japanese literature. Clin Acupunct Orient Med 2002;3:105-13.

701. Tsun-Nin L. Injection of single acupuncture locus in treatment of posterior shoulder pain. A preliminary study. Orthop Rev 1977;6:63-6. 
702. Turner-Stokes L. MRI and arthroscopic surgery: a combined breakthrough in management of shoulder pain. Ann Rheum Dis 1996;55:405-6.

703. Tuzlukov AP, Gorbatovskaia NS. [Treatment of periarthritis of the shoulder joint.] Ortop Travmatol Protez 1990:59-60.

704. Ucuncu F, Capkin E, Karkucak M, Ozden G, Cakirbay H, Tosun M, et al. A comparison of the effectiveness of landmark-guided injections and ultrasonography guided injections for shoulder pain. Clin J Pain 2009;25:786-9.

705. Ueno Y, Kuramoto K, Konno N, Koizumi T, Hoshiba T, Nunomura T, et al. [Investigation on result of use after launch of ARTZ and ARTZ dispo: evaluation on the efficacy, safety and utility in the medication for osteoarthritis of the knee and periarthritis of the shoulder.] Jpn Pharmacol Therapeut 1995;23:313-22.

706. Uhlemann C, Callies R, Hippius M. [Practical aspects of repeated iontophoresis per day.] $Z$ Physiother 1991;43:240-4.

707. Uhthoff HK. Open surgical release for frozen shoulder. J Shoulder Elbow Surg 2002;11:200.

708. Uitvlugt G, Detrisac DA, Johnson LL, Austin MD, Johnson C. Arthroscopic observations before and after manipulation of frozen shoulder. Arthroscopy 1993;9:181-5.

709. Ulmer G. [Shoulder mobilization under anesthesia in complete and partial shoulder stiffness.] Helv Chir Acta 1982;48:611-14.

710. Vad VB, Sakalkale D, Warren RF. The role of capsular distention in adhesive capsulitis. Arch Phys Med Rehabil 2003;84:1290-2.

711. Valtonen EJ. Methylprednisolone acetate in the management of periarthritis of the shoulder and external humeral epicondylitis resistant to physical therapy. Ann Med Intern Fenn 1967;56:61-3.

712. Valtonen EJ. Subacromial betamethasone therapy. II. Subacromial betamethasone and methylprednisolone injections in treatment of frozen shoulder and supra spinam tendinitis. A comparative trial. Ann Chir Gynaecol Fenn 1974;188(Suppl.):9-16.

713. Valtonen EJ. Subacromial betamethasone therapy. I. The effect of subacromial injection of betamethasone in cases of painful shoulder resistant to physical therapy. Ann Chir Gynaecol Fenn 1974;188(Suppl.):5-8.

714. van de Weg FB, van der Windt DAWM, Winters JC. [Injection therapy in shoulder disorders, yes or no? A systematic review of the literature.] Huisarts Wet 2004;47:178-83.

715. van den Dolder PA, Roberts DL. A trial into the effectiveness of soft tissue massage in the treatment of shoulder pain. Aust J Physiother 2003;49:183-8.

716. van der Heijden GJ, van der Windt DA, Kleijnen J, Koes BW, Bouter LM. Steroid injections for shoulder disorders: a systematic review of randomized clinical trials. Br J Gen Pract 1996;46:309-16.

717. van der Heijden GJ, Leffers P, Wolters PJ, Verheijden JJ, van Mameren H, Houben JP, et al. No effect of bipolar interferential electrotherapy and pulsed ultrasound for soft tissue shoulder disorders: a randomised controlled trial. Ann Rheum Dis 1999;58:530-40.

718. van der Windt D. Steroid injections or physiotherapy for capsulitis of the shoulder: a randomised clinical in primary care. In Shoulder disorders in primary care. Amsterdam: Vrije Universiteit; 1997. pp. 107-23. 
719. van der Windt DA, Koes BW, Deville W, Boeke AJ, de Jong BA, Bouter LM. Effectiveness of corticosteroid injections versus physiotherapy for treatment of painful stiff shoulder in primary care: randomised trial. BMJ 1998;317:1292-6.

720. van der Windt DA, van der Heijden GJ, van den Berg SG, ter Riet G, de Winter AF, Bouter LM. Ultrasound therapy for musculoskeletal disorders: a systematic review. Pain 1999;81:257-71.

721. Arroll B. Corticosteroid injections were superior to physiotherapy for painful stiff shoulder. Evid Based Med 1999;4:118.

722. van der Windt D, Koes B, Boeke J, Deville W, de Jong B, Bouter L. [Corticosteroid injections or physiotherapy for painful, stiff shoulder? A randomized study in a general practice.]

Huisarts Wet 2000;43:420-5, 429.

723. van der Windt DAWM, Bouter LM. Physiotherapy or corticosteroid injection for shoulder pain? Ann Rheum Dis 2003;62:385-7.

724. van Laack W, Hennes A, Refisch A. [Mobilization of the partially stiff shoulder under anesthesia (ankylosing humeroscapular periarthritis)]. Z Orthop Ihre Grenzgeb 1987;125:669-73.

725. van Royen BJ, Pavlov PW. Treatment of frozen shoulder by distension and manipulation under local anaesthesia. Int Orthop 1996;20:207-10.

726. van Royen B, Pavlov P. Frozen shoulder - manipulation after glenohumeral distension under local anesthesia. Acta Orthop Scand 1996;67:22.

727. Vanni G, Galante M, Franchignoni FP. [Orgotein in treatment of scapulohumeral periarthritis in hemiplegic patients.] Riabilitazione 1985;18:251-6.

728. Vas J, Ortega C, Olmo V, Perez-Fernandez F, Hernandez L, Medina I, et al. Single-point acupuncture and physiotherapy for the treatment of painful shoulder: a multicentre randomized controlled trial. Rheumatology 2008;47:887-93.

729. Vaughn BF, Beam JW, Duschatko DM, Ingram-Rice B. Adhesive capsulitis: the 'frozen shoulder' syndrome. J Bodyw Mov Ther 2000;4:3-4.

730. Venturi R. [Medical treatment of acute periarthritis of the shoulder by drug injection directly into the scapulo-humeral joint and into the subachromial-deltoid bursa. Arthrographic and anatomical research.] Chir Organi Mov 1979;65:157-76.

731. Verkhozina EA. Treatment of traumatic synovitis arthritis and peri arthritis with ultrasound. Zdravookhr Beloruss 1972;18:58-9.

732. Vermeulen HM, Verhoef J, Rozing PM, Obermann WR, Le Cessie S, van Houwelingen JC, et al. A comparison of end-range and mid-range mobilization techniques in the treatment of adhesive capsulitis: a randomized controlled trial. Ann Rheum Dis 2004;63(Suppl. 1):248.

733. Vermeulen E. Physical therapy for shoulder disorders. Ned Tijdschr Fysiotherapie 2007;117:90-1.

734. Verstraete A, Deckers Y, Dequeker J. Treatment of periarthritis: a single-blind evaluation of 2 injectable corticosteroids. Clin Trials J 1985;22:471-6.

735. Vigano R, Guarino A, Nannei AM. [Use of laser therapy associated with a lysine ketoprofen gel in various pathologies of orthopedic importance.] Clin Ter 1986;118:13-17.

736. Volhard J. [The treatment of acute humeroscapuar periarthritis.] Med Welt 1968;3:188. 
737. von Knorre VB, Keitel W. [Comparative study of therapy: ultrasound, cryotherapy and intra-articulare cortisonoids to treat alterations of the shoulder joint due to inflammation.] Z Physiother 1990;42:221-5.

738. Vrettos BC, Neumann L, MacKie A, Damrel D, Wallace WA. One-stage arthroplasty of the ipsilateral shoulder and elbow. J Shoulder Elbow Surg 2005;14:425-8.

739. Wagenhau FJ. Treatment of humeroscapular periarthritis. Dtsch Med Wochenschr 1969;94:1579-81.

740. Walach H, Guthlin C, Konig M. Efficacy of massage therapy in chronic pain: a pragmatic randomized trial. J Altern Complement Med 2003;9:837-46.

741. Waldburger M, Meier JL, Gobelet C. The frozen shoulder: diagnosis and treatment. Prospective study of 50 cases of adhesive capsulitis. Clin Rheumatol 1992;11:364-8.

742. Wallny T, Melzer C, Wagner U, Wirth CJ, Schmitt O. ['Primary' shoulder stiffness: illness duration and therapeutic comparison.] Z Orthop Ihre Grenzgeb 1997;135:222-7.

743. Walsh DM, Howe TE, Johnson MI, Sluka KA. Transcutaneous electrical nerve stimulation for acute pain. Cochrane Database Syst Rev 2009; Issue 2, Art. No. CD006142.

744. Wang W, Yin X, He Y, Wei J, Wang J, Di F. Treatment of periarthritis of the shoulder with acupuncture at the Zhongping (foot) extrapoint in 345 cases. J Tradit Chin Med 1990;10:209-12.

745. Wang J, Wang W, Wang S. Treatment of periarthritis humeroscapularis with acupuncture and acupoint blocking. J Tradit Chin Med 1993;13:262-3.

746. Wang W. 78 cases of periomarthritis treated with acupuncture. J Tradit Chin Med 1995; 15:46-7.

747. Wang LC. [ 50 cases of periarthritis of shoulder joint treated by electro-acupuncture and point injection therapy.] Shanghai J Acupunct Moxibustion 1997;16:20.

748. Wang W, Yu ZL, Xie J, Xu AS, Fang J. [Relief of pain and improvement of dysfunction in shoulder periarthritis following Qi acupoint specific acupuncture versus conventional acupuncture.] Chin J Clin Rehab 2005;9:48-50.

749. Wang SS, Trudelle-Jackson EJ. Comparison of customized versus standard exercises in rehabilitation of shoulder disorders. Clin Rehabil 2006;20:675-85.

750. Wang Z-L, Chen L-F, Zhu W-M. [Observation on the transient analgesic effect of abdominal acupuncture TENS on pain of neck, shoulder, loin and legs.] Zhongguo Zhenjiu 2007;27:657-9.

751. Wang J-P, Huang T-F, Hung S-C, Ma H-L, Wu J-G, Chen T-H. Comparison of idiopathic, post-trauma and post-surgery frozen shoulder after manipulation under anesthesia. Int Orthop 2007;31:333-7.

752. Wang Q-H, Du P-B, Gong H-T. [Effects of adhesion releasing under nerve block and arthrolysis on adhesive scapulohumeral periarthritis: a comparative study.] Chin Med J 2008;88:177-8.

753. Warner JJ, Allen A, Marks PH, Wong P. Arthroscopic release for chronic, refractory adhesive capsulitis of the shoulder. J Bone Joint Surg Am 1996;78:1808-16.

754. Wassef MR. Suprascapular nerve block. A new approach for the management of frozen shoulder. Anaesthesia 1992;47:120-4.

755. Watson L, Dalziel R, Story I. Frozen shoulder: a 12-month clinical outcome trial. J Shoulder Elbow Surg 2000;9:16-22. 
756. Weber M, Prim J, Bugglin R, Michel BA, Gerber H. Long-term follow up of patients with frozen shoulder after mobilization under anesthesia, with special reference to the rotator cuff. Clin Rheumatol 1995;14:686-91.

757. Weber KG. [The 7-point shoulder treatment program.] Krankengymnastik 2001;53:976-9.

758. Weiser HI. [Mobilization under local anesthesia for painful primary frozen shoulder.] Harefuah 1976;90:215-19.

759. Weiser HI. Painful primary frozen shoulder mobilization under local anesthesia. Arch Phys Med Rehabil 1977;58:406-8.

760. Wen L. [Analysis on effect of functional exercises to promote rehabilitation of patients wih periarthritis of shoulder.] Chin Nurs Res 2009;23:1925-6.

761. White AE, Tuite JD. The accuracy and efficacy of shoulder injections in restrictive capsulitis. J Bone Joint Surg Br 1995;77-B(Suppl. 1):13.

762. White AET, Tuite JD. The accuracy and efficacy of shoulder injections in restrictive capsulitis. J Orthop Rheumatol 1996;9:37-40.

763. White A. Chronic shoulder pain. Acupunct Med 2009;27:138.

764. Widiastuti-Samekto M, Sianturi GP. Frozen shoulder syndrome: comparison of oral route corticosteroid and intra-articular corticosteroid injection. Med J Malaysia 2004;59:312-16.

765. Wies JT, Niel Asher S, Latham M, Hazleman B, Speed CA. A pilot randomised placebo controlled trial of osteopathic and physiotherapy treatment for frozen shoulder. $\mathrm{Br} J$ Rheumatol 2003;42(Suppl. 1):147.

766. Wiley AM. Frozen shoulder: surgical applications. Surg Shoulder 1995;1085:269-74.

767. Wiley AM, Vastamaki M, Roth JH. Arthroscopy of the frozen shoulder. In Vastamaki M, Roth JH, editors. Hand and upper extremity; arthroscopy of the upper extremity. New York: Churchill Livingstone; 1997. pp. 135-42.

768. Winters JC, Sobel JS, Arendzen JH, Meyboom-de Jong B. [The effect of corticosteroid injections in shoulder disorders.] Huisarts Wet 1995;38:164-8.

769. Winters JC, Sobel JS, Groenier KH, Arendzen HJ, Meyboom-de Jong B. Comparison of physiotherapy, manipulation, and corticosteroid injection for treating shoulder complaints in general practice: randomised, single blind study. BMJ 1997;314:1320-5.

770. Winters JC, Jorritsma W, Groenier KH, Sobel JS, Meyboom-de Jong B, Arendzen HJ. Treatment of shoulder complaints in general practice: long term results of a randomised, single blind study comparing physiotherapy, manipulation, and corticosteroid injection. $B M J$ 1999;318:1395-6.

771. Winters JC. [The small art of the short term. Corticosteroid injections in the treatment of shoulder complaints.] Pharm Weekbl 2000;135:690-3.

772. Worsdorfer O, Wasmer G. [Operative treatment of shoulder stiffness.] Hefte Unfallheilkd 1984;170:150-8.

773. Xiao JB, She SZ, Song WG, Zhang BG, Zeng ZW. [Effects of super laser on the affected limb of acute and chronic pain 315 cases.] Chin J Clin Rehab 2006;10:116-18.

774. Xie KY, Zhao GF, Lu JM. Treatment of 103 cases of periarthritis of the shoulder by acupoint laser irradiation. J Tradit Chin Med 1988;8:265-6. 
775. Xu X, Fang JQ, Zhang Y, Liu KZ, Han CP. Treatment of early-stage adhesive shoulder periarthritis with transcutaneous electric stimulation on acupoints. J Acu Tuina Sci 2006;4:353-5.

776. Xu B. [Bone setting manipulation of Shangsiyuan chuobanchu for treatment of periarthritis of shouder.] Zhongguo Gushang 2008;21:871.

777. Xu K-S, Huang M-W, Yao L-Y, Chen J, Su J, Zheng J-Q. [Clinically randomized controlled trials of moxibustion with salt in bamboo circle for treatment of periarthritis of shoulder.] Zhongguo Zhenjiu 2009;29:77-80.

778. Yamaguchi K, Sethi N, Bauer GS. Postoperative pain control following arthroscopic release of adhesive capsulitis: a short-term retrospective review study of the use of an intra-articular pain catheter. Arthroscopy 2002;18:359-65.

779. Yamaguchi K, Buchbinder R, Green S, Forbes A, Hall S, Lawler G. Arthrographic distension with saline and steroid reduced pain and disability and improved range of motion in the short term in patients with painful stiff shoulder. J Bone Joint Surg Series A 2004;86:1837.

780. Yamaguchi K, Hollis R. Arthroscopic release of frozen shoulder. Semin Arthroplasty 2008;19:50-3.

781. Yamamoto R, Namiki O, Iwata H, Shinmei M, Mikasa M, Ogawa N. [Randomized comparative study of sodium hyaluronate (SPH) on periarthritis scapulohumeralis.] Jpn J Clin Pharmacol Ther 1988;19:717-33.

782. Yamamoto R, Tabata S, Mikasa M, Takagishi K, Ogawa N. [Dose-range finding study of SLM-10 (sodium hyaluronate injection) in patients with periarthritis scapulohumeralis.] Jpn Pharmacol Therapeut 1993;21(Suppl. 2):265-86.

783. Yamamoto R, Tabata S, Mikasa M, Takagishi K, Ogawa N. [Clinical trial of SLM-10 (sodium hyaluronate injection) compared with ARTZ in patients with periarthritis scapulohumeralis.] Jpn Pharmacol Therapeut 1993;21:285-307.

784. Yamshon LJ. Frozen shoulder: methods for bringing about early mobilization. Calif Med 1958;89:333-4.

785. Yan XM, Li XY, Sun Y. [Quantitative evaluation of the enhancement of therapeutic effect in pain therapy by combining nerve block and linear polarized near-infrared ray irradiation.] Chin J Clin Rehab 2005;9:44-5.

786. Yang J, Lu K. Sixty-four cases of scapulohumeral periarthritis treated by auricular plaster therapy. J Tradit Chin Med 2006;26:179-80.

787. Yao HH, Chen YH. The effects of comprehensive rehabilitation therapy on scapulohumeral periarthritis. Chin J Clin Rehab 2002;6:2494.

788. Yaya Huaman R, Blasco Olcina R, Garcia Fernandez M, Vilches Padilla JJ. [A study of 93 cases of painful shoulder and shoulder-band syndrome treated with hydergine.] Rev Esp Otoneurooftalmol Neurocir 1975;33:131-5.

789. Yegudin-Ash J, Afzal M, Cavaliere LF, Lee D, Zelicof S, Weinstein A. A comparative study of ultrasonography with magnetic resonance imaging in patients with painful shoulder using arthroscopy as a gold standard. Arthritis Rheum 1998;41(Suppl. 9):260.

790. Yigiter K, Kerem M. A comparison of the effects of two different iontophoresis applications on pain in patients with adhesive capsulitis of the shoulder. Pain Clinic 2002;14:49-53.

791. Yuan DW. Bioholographic acupuncture therapy for 44 cases of scapulohumeral periarthritis. Shanghai J Acupunct Moxibustion 1995;14:68. 
792. Zachepa AE. [Surgical treatment of humero-scapular periarthroses.] Ortop Travmatol Protez 1991:37-8.

793. Zancan A, Gialanella B, Luisa A, Della Marta ME, D’Alessandro G, Casale R. [Telethermographic effects and a comparative clinical assessment of the treatment of shoulder periarthritis using ultrasound.] G Ital Med Lav 1993;15:55-8.

794. Zeilig G, Guibert R. Terra Quant device for shoulder pain. Double blind trial. Positive Health 2005. URL: www.positivehealth.com/article-view.php?articleid=1778. Accessed 3 June 2010.

795. Zeng X-L, Lei L-M, Lin Z, Wang Z-T. [Clinical observation on acupuncture at pain point combined with moxibustion at Zusanli (ST 36) for treatment of periarthritis of shoulder.] Zhongguo Zhenjiu 2005;25:326-8.

796. Zhang M. Treatment of peri-omarthritis with acupuncture at Yanglingquan (GB 34). J Tradit Chin Med 1991;11:9-10.

797. Zhang W, Oetliker H. Acupuncture for pain control: a review of controlled clinical trials. In Schlapbach P, Gerber NJ, editors. Rheumatology, Vol. 14. Physiotherapy: controlled trials and facts. Basel, Switzerland: S. Karger Ag; 1991. pp. 171-88.

798. Zhang X, Wang Zd, Ou Ck. [Needle scalpel versus acupuncture for scapulohumeral periarthritis in a staged treatment.] Chin J Clin Rehab 2006;10:11-13.

799. Zhang GX. [Treatment of acute injury of soft tissue around shoulder joint by exercise needling and electroacupuncture as main.] Zhongguo Zhen Jiu 2008;28:485-8.

800. Zhang T-W, Cheng S-D, Wang H-F, Xu H-L. [Shoulder-loosening techniques on severe frozen shoulder.] Zhongguo Gushang 2009;22:718-19.

801. Zhao L, Xia J, Fan J. [The treatment of computer middle frequency on periorthritis of shoulder.] Chin J Clin Rehab 2003;7:335.

802. Zhao H, Zhao T, Liu B-Y, Liu Z-S. [Observation on therapeutic effects of different needling methods at tiaokou (ST 38) on periarthritis of shoulder.] Zhongguo Zhenjiu 2006;26:729-31.

803. Zhou G, Yang Y. [Clinical observations on the treatment of 76 shoulder periarthritis cases by meridional differentiation-based distant opposing acupuncture.] Shanghai J Acupunct Moxibustion 2004;23:15.

804. Zhu BW. [182 cases of periarthritis of shoulder joint treated by acupuncture and moxibustion and massage therapy.] Shanghai J Acupunct Moxibustion 1997;16:23-4.

805. Zhu ZZ, Liu CM, Feng WX. [Analgesic effects of traditional Chinese medicine iontophoresis and rehabilitation training on periarthritis of shoulder: a randomized controlled study.] Chin J Clin Rehab 2004;8:2698-9.

806. Zivkovic M. Comparative analysis of the use of kinesi therapy and other methods of physical medicine in the treatment of scapulo humeral peri-arthritis. 12th International Rheumatological Congress. Prague: JR Geigy SA; 1969.

807. Zuecker W, Knuth C. Treatment of shoulder pain by appropriate methods of therapeutic swimming. Z Physiother 1977;29:407-9.

808. Cyriax J, Russel G. Textbook of orthopaedic medicine. Volume 2, 9th edn. London: Ballière Tindall; 1977.

809. Maitland GD. Treatment of the glenohumeral joint by passive movement. Physiotherapy 1983;69:3-7.

810. Kaltenborn FM. Manual therapy for the extremity joints. Oslo: Olaf Norlis Bokhander; 1976. 
811. Vermeulen HM, Obermann WR, Burger BJ, Kok GJ, Rozing PM, van Den Ende CH. Endrange mobilization techniques in adhesive capsulitis of the shoulder joint: a multiple-subject case report. Phys Ther 2000;80:1204-13.

812. Mulligan BR. Mobilisations with movement. J Manual Manipulative Ther 1993;1:154-6. 


\section{Appendix 1}

\section{Search strategies for effectiveness review}

\section{BIOSIS Previews}

Interface: Web of Science (ISI) on Web of Knowledge

Search date: 30 March 2010

Strategy: as below

Records identified: 800

\begin{tabular}{|c|c|c|}
\hline \multirow[t]{2}{*}{ \# 20} & 800 & \#19 OR \#17 OR \#15 OR \#13 OR \#11 OR \#9 OR \#5 OR \#3 \\
\hline & & Databases=PREVIEWS Timespan=All Years \\
\hline \multirow[t]{2}{*}{ \# 19} & 140 & \#18 AND \#1 \\
\hline & & Databases=PREVIEWS Timespan=All Years \\
\hline \multirow[t]{2}{*}{ \# 18} & 33,082 & 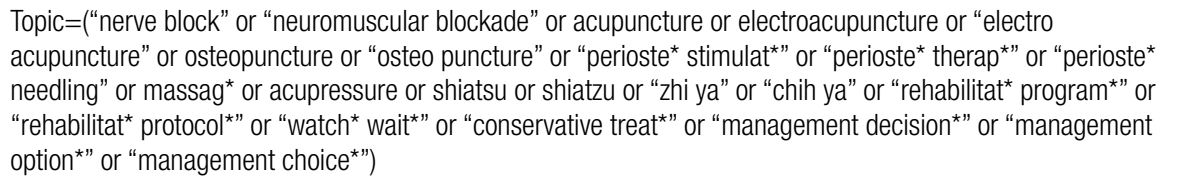 \\
\hline & & Databases=PREVIEWS Timespan=All Years \\
\hline \multirow[t]{2}{*}{ \# 17} & 454 & \#16 AND \#1 \\
\hline & & Databases=PREVIEWS Timespan=1969-2010 \\
\hline \multirow[t]{2}{*}{ \# 16} & $>100,000$ & 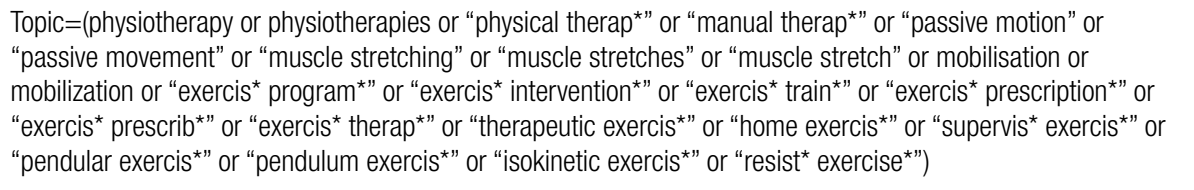 \\
\hline & & Databases=PREVIEWS Timespan=1969-2010 \\
\hline \multirow[t]{2}{*}{ \# 15} & 38 & $\# 14$ AND \#1 \\
\hline & & Databases=PREVIEWS Timespan=1969-2010 \\
\hline \multirow[t]{2}{*}{ \# 14} & $>100,000$ & 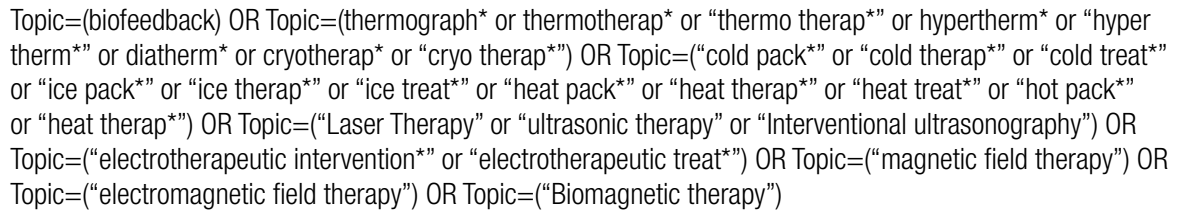 \\
\hline & & Databases=PREVIEWS Timespan=1969-2010 \\
\hline \multirow[t]{2}{*}{ \# 13} & 31 & \#12 AND \#1 \\
\hline & & Databases=PREVIEWS Timespan=1969-2010 \\
\hline \multirow[t]{2}{*}{ \# 12} & 50,394 & $\begin{array}{l}\text { Topic=(TENS or ALTENS) OR Topic=("electric* stimulat*" or "transcutaneous stimulat*”or "transdermal } \\
\text { electrostimulat"or "cutaneous electrostimulat”"or electroanalgesia or "electro analgesia") OR Topic=("muscle } \\
\text { stimulat*”) OR Topic=(neuromodulation or "neuro modulation" or neurostimulation or "neuro stimulation") OR } \\
\text { Topic=(interferential) }\end{array}$ \\
\hline & & Databases=PREVIEWS Timespan=1969-2010 \\
\hline \multirow[t]{2}{*}{ \# 11} & 30 & $\# 10$ AND \#1 \\
\hline & & Databases=PREVIEWS Timespan=1969-2010 \\
\hline \multirow[t]{2}{*}{ \# 10} & 5938 & $\begin{array}{l}\text { Topic }=\left(\text { chiropractic }^{\star}\right) \text { OR Topic }=\left(\text { osteopath }^{\star}\right) \text { OR Topic }=\left(\text { manipulat }{ }^{\star} \text { and }(\text { anesthesia or anaesthesia or anesthetic }\right. \\
\left.\left.\text { or anaesthetic }{ }^{\star}\right)\right) \text { OR Topic }=(\text { MUA) }\end{array}$ \\
\hline & & Databases=PREVIEWS Timespan=1969-2010 \\
\hline
\end{tabular}




\begin{tabular}{|c|c|c|}
\hline \multirow[t]{2}{*}{$\# 9$} & 112 & \#8 OR \#7 \\
\hline & & Databases=PREVIEWS Timespan=1969-2010 \\
\hline \multirow[t]{2}{*}{ \# 8} & 39 & 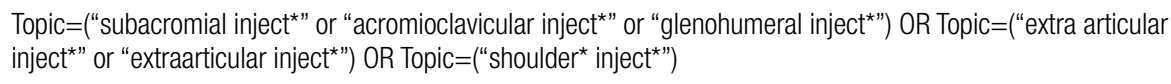 \\
\hline & & Databases=PREVIEWS Timespan=1969-2010 \\
\hline \multirow[t]{2}{*}{ \# 7} & 81 & \#6 AND \#1 \\
\hline & & Databases=PREVIEWS Timespan=1969-2010 \\
\hline \multirow[t]{2}{*}{ \# 6} & 85,801 & 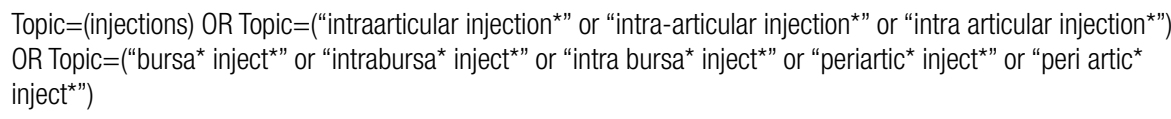 \\
\hline & & Databases=PREVIEWS Timespan=1969-2010 \\
\hline \multirow[t]{2}{*}{ \# 5} & 152 & \#4 AND \#1 \\
\hline & & Databases=PREVIEWS Timespan $=1969-2010$ \\
\hline \multirow[t]{2}{*}{ \# 4} & 5743 & $\begin{array}{l}\text { Topic=(Arthroscopy) OR Topic=("arthroscop* releas*" or "arthroscop* decompress" or "arthroscop* } \\
\text { capsulotom*") OR Topic=("capsular releas"” or "interventional microadhesiolysis" or capsulotomy) }\end{array}$ \\
\hline & & Databases=PREVIEWS Timespan=1969-2010 \\
\hline \multirow[t]{2}{*}{ \# 3} & 11 & \#2 AND \#1 \\
\hline & & Databases=PREVIEWS Timespan=1969-2010 \\
\hline \multirow[t]{2}{*}{ \# 2} & 25,371 & $\begin{array}{l}\text { Topic=("arthrograph* distension*" or "arthograph* distention*”) OR Topic=("arthrogram* distension*" } \\
\text { or "arthogram* distention*”) OR Topic=("glenohumeral distension*" or "glenohumeral distention*”) OR } \\
\text { Topic=(dilatation or hydrodilat*) OR Topic=(arthography) }\end{array}$ \\
\hline & & Databases=PREVIEWS Timespan=1969-2010 \\
\hline \multirow[t]{2}{*}{ \#1 } & 2354 & $\begin{array}{l}\text { Topic=("frozen shoulder*”) OR Topic=("stiff* shoulde*”) OR Topic=("adhesive capsulitis") OR Topic=("shoulder } \\
\text { bursitis") OR Topic=(periarthritis or peri-arthritis or periarthritides or peri-arthritides or peri-capsulitis or } \\
\text { pericapsulitis) OR Topic=("shoulder pain*" or "pain* shoulder" or "shoulder complaint”") OR Topic=("shoulder* } \\
\text { impinge*") OR Topic=("subacromial impingement syndrome") }\end{array}$ \\
\hline & & Databases=PREVIEWS Timespan=1969-2010 \\
\hline
\end{tabular}

\section{Cumulative Index to Nursing and Allied Health Literature (CINAHL)}

Interface: EBSCO

Search date: 26 March 2010

Strategy: as below

Records identified: 1757

\begin{tabular}{|c|c|}
\hline S73 & $S 18$ or $S 22$ or $S 27$ or $S 33$ or $S 40$ or $S 51$ or $S 56$ or $S 62$ or $S 66$ or $S 70$ or $S 72$ \\
\hline S72 & s14 and s71 \\
\hline S71 & 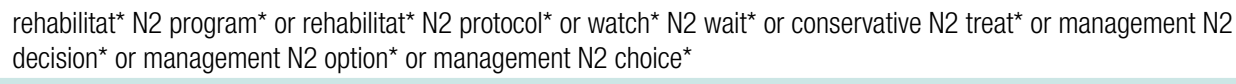 \\
\hline S70 & S14 AND S69 \\
\hline S69 & S67 or S68 \\
\hline S68 & ( massag* or acupressure ) or ( shiatsu or shiatzu ) or zhi ya or chih ya \\
\hline S67 & (MH “Massage”) \\
\hline S66 & S14 and S65 \\
\hline S65 & $\mathrm{S} 63$ or $\mathrm{S} 64$ \\
\hline S64 & electroacupuncture ${ }^{\star}$ or osteopuncture* or perioste* $\mathrm{N} 3$ stimulat* $^{\star}$ or perioste ${ }^{*} \mathrm{~N} 3$ therap* or perioste ${ }^{\star} \mathrm{N} 3$ needling \\
\hline S63 & (MH "Acupuncture+") \\
\hline S62 & S14 AND S61 \\
\hline S61 & $\mathrm{S} 57$ or $\mathrm{S} 58$ or $\mathrm{S} 59$ or $\mathrm{S} 60$ \\
\hline S60 & neuromuscular block ${ }^{*}$ \\
\hline
\end{tabular}




\begin{tabular}{|c|c|}
\hline S59 & (MH “Nerve Block”) \\
\hline S58 & pulsed electromagnetic field therapy or electromagnetic ${ }^{\star} \mathrm{N} 3$ field $^{\star}$ or biomagnetic ${ }^{\star}$ or pulsed signal or PEMF \\
\hline S57 & (MH “Magnetics+”) \\
\hline S56 & S14 AND S55 \\
\hline S55 & $\mathrm{S} 52$ or $\mathrm{S} 53$ or $\mathrm{S} 54$ \\
\hline S54 & electrotherapeutic N2 intervention* or electrotherapeutic N2 treat* or laser N2 therap* or ultrasound N2 therap* \\
\hline S53 & (MH “Ultrasonography+”) \\
\hline S52 & (MH “Lasers+") \\
\hline S51 & S14 AND S50 \\
\hline S50 & $S 43$ or $S 44$ or $S 45$ or $S 46$ or $S 47$ or $S 48$ or $S 49$ \\
\hline S49 & $\begin{array}{l}\text { ( thermograph*or thermotherap* or hypertherm* or diatherm }{ }^{*} \text { or cryotherap* }{ }^{\star} \text { ) or thermo therap* or hyper therm or cryo } \\
\text { therap* or thermo graph* }\end{array}$ \\
\hline S48 & hot N2 pack* or hot N2 therap* or hot N2 treat* \\
\hline S47 & $\begin{array}{l}\text { cold N2 pack* or cold N2 therap* or cold N2 treat }{ }^{*} \text { or ice N2 pack* or ice N2 therap* or ice N2 treat* or heat N2 pack* or heat } \\
\text { N2 therap }{ }^{*} \text { or heat N2 treat* }\end{array}$ \\
\hline S46 & (MH “Hyperthermia, Induced") \\
\hline S45 & (MH “Ice”) \\
\hline S44 & (MH “Diathermy") \\
\hline S43 & (MH “Cryotherapy”) or (MH “Heat-Cold Application”) \\
\hline S42 & S14 AND S41 \\
\hline S41 & (MH “Biofeedback”) \\
\hline S40 & S14 AND S39 \\
\hline S39 & $\begin{array}{l}\text { (TENS or ALTENS ) or electric* N2 stimulat* or transcutaneous N2 stimulat* or transdermal N2 electrostimulat* or cutaneous } \\
\text { N2 electrostimulat* or electroanalgesia or muscle N2 stimulat* or neuromodulation or neurostimulation or interferential }\end{array}$ \\
\hline S38 & S14 and S37 \\
\hline S37 & S34 or S35 or S36 \\
\hline S36 & $\begin{array}{l}\text { chiropractic }^{*} \text { or osteopath* or manipulat* N3 anesthesia or manipulat* N3 anaesthesia or manipulat* N3 anesthetic }{ }^{*} \text { or } \\
\text { manipulat* }^{*} 3 \text { anaesthetic* or MUA }\end{array}$ \\
\hline S35 & (MH “Manual Therapy+") \\
\hline S34 & (MH “Manipulation, Chiropractic") or (MH “Manipulation, Orthopedic”) or (MH “Manipulation, Osteopathic") \\
\hline S33 & S14 and S32 \\
\hline S32 & $\mathrm{S} 28$ or $\mathrm{S} 29$ or $\mathrm{S} 30$ or $\mathrm{S} 31$ \\
\hline S31 & 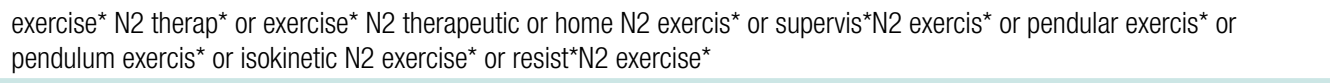 \\
\hline S30 & 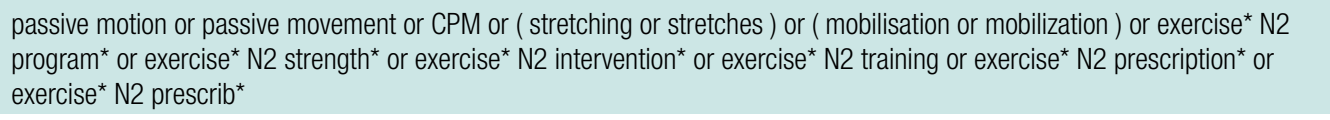 \\
\hline S29 & physiotherapy or physiotherapies or physical therap* or manual therap* \\
\hline S28 & (MH “Physical Therapy+") \\
\hline S27 & S24 or S25 or S26 \\
\hline S26 & $\begin{array}{l}\text { subacromial N3 inject* or acromioclavicular N3 inject* or glenohumeral N3 inject* or extraarticular N3 inject* or shoulder* N3 } \\
\text { inject* }\end{array}$ \\
\hline S25 & 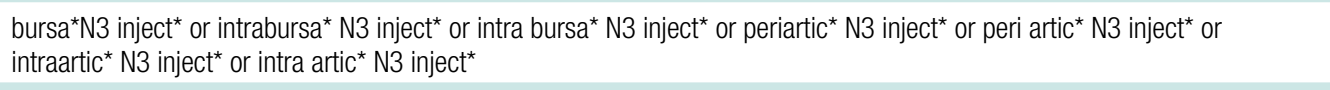 \\
\hline S24 & S14 and S23 \\
\hline S23 & (MH “Injections+") \\
\hline S22 & S14 and S21 \\
\hline S21 & $\mathrm{S} 19$ or S20 \\
\hline S20 & $\begin{array}{l}\text { arthroscop* N6 releas* or arthroscop* N6 decompress* or arthroscop* N6 capsulotom or capsular N2 releas* or } \\
\text { interventional microadhesiolysis or capsulotomy }\end{array}$ \\
\hline S19 & (MH “Arthroscopy”) \\
\hline S18 & S14 and S17 \\
\hline S17 & $\mathrm{S} 15$ or $\mathrm{S} 16$ \\
\hline
\end{tabular}




\begin{tabular}{|c|c|}
\hline S16 & $\begin{array}{l}\text { arthrograph* N6 distension* or arthrograph* N6 distention* or arthrogram* N6 distension* or arthrogram* N6 distention* or } \\
\text { glenohumeral N6 distension* or glenohumeral N6 distention* or ( dilatation or hydrodilat* ) }\end{array}$ \\
\hline S15 & (MH “Arthrography") \\
\hline S14 & $\mathrm{S} 1$ or $\mathrm{S} 2$ or $\mathrm{S} 3$ or $\mathrm{S} 4$ or $\mathrm{S} 5$ or $\mathrm{S} 6$ or $\mathrm{S} 7$ or $\mathrm{S} 8$ or $\mathrm{S} 9$ or $\mathrm{S} 10$ or $\mathrm{S} 11$ or $\mathrm{S} 12$ or $\mathrm{S} 13$ \\
\hline S13 & subacromial impingement syndrome \\
\hline S12 & shoulder* N6 impinge* \\
\hline S11 & (MH “Shoulder Impingement Syndrome") \\
\hline S10 & shoulder* N3 pain or shoulder* N3 pains or shoulder* N3 painful or shoulder N3 complain* \\
\hline S9 & (MH “Shoulder Pain") \\
\hline S8 & $\begin{array}{l}\text { periarthritis N6 shoulder* or peri-arthritis N6 shoulder* or periarthritides N6 shoulder* or peri-arthritides N6 shoulder* or } \\
\text { pericapsulitis N6 shoulder* or peri-capsulitis N6 shoulder* }\end{array}$ \\
\hline S7 & capsulitis N6 shoulder* \\
\hline S6 & bursitides N6 shoulder* \\
\hline S5 & bursitis N6 shoulder* \\
\hline S4 & adhesive N3 capsulitis \\
\hline S3 & (MH “Adhesive Capsulitis") \\
\hline S2 & stiff* N3 shoulder* \\
\hline S1 & frozen N6 shoulder* \\
\hline
\end{tabular}

\section{Cochrane Central Register of Controlled Trials (CENTRAL)}

Interface: Cochrane Library Issue 32010

Search date: 23 March 2010

Strategy: as below

Records identified: 539

\begin{tabular}{|c|c|c|}
\hline ID & Search & Hits \\
\hline$\# 1$ & (frozen NEAR/6 shoulder*):ti,ab,kw & 65 \\
\hline \#2 & 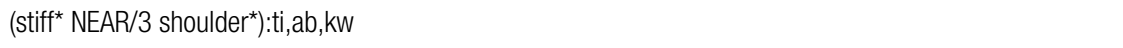 & 63 \\
\hline \#3 & MeSH descriptor Bursitis explode all trees & 127 \\
\hline \#4 & (adhesive NEXT (capsulitis or capsulitides)):ti,ab,kw & 44 \\
\hline \#5 & (bursitis or bursitides) NEAR/6 shoulder*ti,ab,kw & 11 \\
\hline \#6 & (capsulitis or capsulitides) NEAR/6 shoulder* & 39 \\
\hline \#7 & 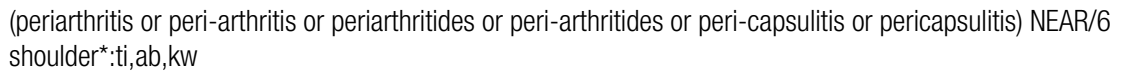 & 77 \\
\hline \#8 & MeSH descriptor Shoulder Pain explode all trees & 240 \\
\hline$\# 9$ & (shoulder* NEAR/3 (pain or pains or painful or complain*)):ti,ab,kw & 682 \\
\hline \#10 & MeSH descriptor Shoulder Impingement Syndrome explode all trees & 84 \\
\hline$\# 11$ & (shoulder* NEAR/6 impinge*):ti,ab,kw & 107 \\
\hline$\# 12$ & "subacromial impingement syndrome":ti,ab,kw & 20 \\
\hline$\# 13$ & (\#1 OR \#2 OR \#3 OR \#4 OR \#5 OR \#6 OR \#7 OR \#8 OR \#9 OR \#10 OR \#11 OR \#12) & 964 \\
\hline$\# 14$ & MeSH descriptor Arthrography, this term only & 148 \\
\hline \#15 & (arthrograph* NEAR/6 (distension* or distention*)):ti,ab,kw & 10 \\
\hline \#16 & $\begin{array}{l}\text { (arthrogram* NEAR/6 (distension* or distention*)):ti,ab,kw or (glenohumeral NEAR/6 (distension* or } \\
\text { distention*)):ti,ab,kw }\end{array}$ & 3 \\
\hline$\# 17$ & MeSH descriptor Dilatation, this term only & 281 \\
\hline \#18 & (dilatation or hydrodilat*):ti,ab,kw & 2541 \\
\hline \#19 & (\#14 OR \#15 OR \#16 OR \#17 OR\#18) & 2695 \\
\hline \#20 & (\#13 AND \#19) & 25 \\
\hline
\end{tabular}




\begin{tabular}{|c|c|c|}
\hline \#21 & MeSH descriptor Arthroscopy explode all trees & 835 \\
\hline \#22 & (arthroscop* NEAR/6 (releas* or decompress* or capsulotom*)):ti,ab,kw & 64 \\
\hline \#23 & (capsular NEAR/2 releas`):ti,ab,kw or “interventional microadhesiolysis":ti,ab,kw or (capsulotomy):ti,ab,kw & 197 \\
\hline \#24 & (\#21 OR \#22 OR \#23) & 1048 \\
\hline \#25 & (\#13 AND \#24) & 51 \\
\hline \#26 & MeSH descriptor Injections, Intra-Articular explode all trees & 628 \\
\hline \#27 & (\#13 AND \#26) & 62 \\
\hline \#28 & MeSH descriptor Injections explode all trees & 15,394 \\
\hline \#29 & (\#13 AND \#28) & 115 \\
\hline \#30 & $\begin{array}{l}\text { (bursa* or intrabursa* or intra bursa* or periartic* or peri artic }{ }^{\star} \text { or intraartic* or intra artic }{ }^{\star} \text { ) NEAR/3 } \\
\text { inject* } t^{\star} \mathrm{ti}, \mathrm{ab}, \mathrm{kw}\end{array}$ & 813 \\
\hline \#31 & (\#13 AND \#30) & 79 \\
\hline \#32 & $\begin{array}{l}\text { (subacromial or acromioclavicular or glenohumeral) NEAR/3 inject*:ti,ab,kw or (extra articular or extraarticular } \\
\text { or shoulder*) NEAR/3 inject*:ti,ab,kw }\end{array}$ & 72 \\
\hline \#33 & (\#27 OR \#29 OR \#31 OR \#32) & 169 \\
\hline \#34 & MeSH descriptor Physical Therapy Modalities explode all trees & 9583 \\
\hline \#35 & $\begin{array}{l}\text { (physiotherapy or physiotherapies or (physical therap*) or (manual therap*)):ti,ab,kw or (passive NEXT (motion } \\
\text { or movement)):ti,ab,kw or (CPM):ti,ab,kw }\end{array}$ & 10,737 \\
\hline \#36 & MeSH descriptor Muscle Stretching Exercises explode all trees & 82 \\
\hline \#37 & 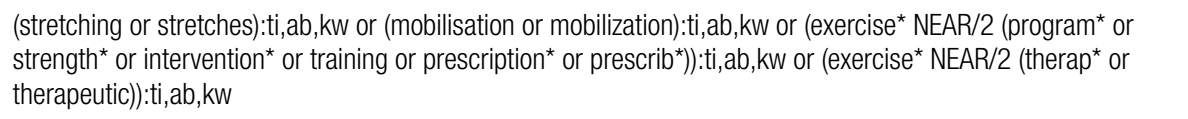 & 10,643 \\
\hline \#38 & 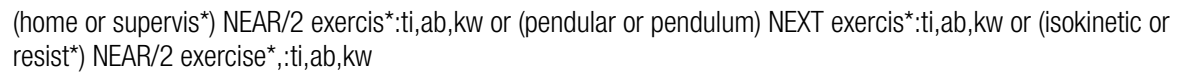 & 1658 \\
\hline \#39 & (\#34 OR \#35 OR \#36 OR \#37 OR \#38) & 23,617 \\
\hline \#40 & (\#13 AND \#39) & 300 \\
\hline \#41 & MeSH descriptor Musculoskeletal Manipulations explode all trees & 4745 \\
\hline \#42 & $\begin{array}{l}\left(\text { chiropractic*}^{\star}\right): t i, a b, k w \text { or (osteopath*):ti,ab,kw or (manipulat* NEAR/3 (anesthesia or anaesthesia or anesthetic } \\
\left.\text { or anaesthetic }{ }^{\star}\right): t i, a b, k w \text { or (MUA):ti,ab,kw }\end{array}$ & 728 \\
\hline \#43 & (\#41 OR \#42) & 5242 \\
\hline \#44 & (\#13 AND \#43) & 119 \\
\hline \#45 & $\begin{array}{l}\text { (TENS or ALTENS):ti,ab,kw or (electric* NEAR/2 stimulat*) or (transcutaneous NEAR/2 stimulat*) or } \\
\text { (transdermal NEAR/2 electrostimulat*) or (cutaneous NEAR/2 electrostimulat*) or electroanalgesia or (electro } \\
\text { analgesia):ti,ab,kw or (muscle NEAR/2 stimulat*):ti,ab,kw or (neuromodulation or (neuro modulation) or } \\
\text { neurostimulation or (neuro stimulation)):ti,ab,kw or (interferential):ti,ab,kw }\end{array}$ & 17,526 \\
\hline \#46 & (\#13 AND \#45) & 76 \\
\hline \#47 & MeSH descriptor Biofeedback (Psychology) explode all trees & 710 \\
\hline \#48 & (biofeedback):ti,ab,kw & 1348 \\
\hline \#49 & (\#47 OR \#48) & 1348 \\
\hline$\# 50$ & (\#13 AND \#49) & 6 \\
\hline \#51 & MeSH descriptor Cryotherapy explode all trees & 866 \\
\hline \#52 & MeSH descriptor Ice explode all trees & 63 \\
\hline$\# 53$ & MeSH descriptor Diathermy explode all trees & 620 \\
\hline \#54 & MeSH descriptor Hyperthermia, Induced explode all trees & 981 \\
\hline \#55 & MeSH descriptor Hot Temperature explode all trees & 1079 \\
\hline \#56 & $\begin{array}{l}\text { (cold or ice or heat or hot) NEXT (pack* or therap* or treat*):ti,ab,kw or (thermograph* or thermotherap* } \\
\text { or (thermo therap*) or hypertherm* or (hyper therm*)):ti,ab,kw or (diatherm* or cryotherap* or (cryo } \\
\text { therap*)):ti,ab,kw }\end{array}$ & 2278 \\
\hline \#57 & (\#51 OR \#52 OR \#53 OR \#54 OR \#55 OR \#56) & 4273 \\
\hline \#58 & (\#13 AND \#57) & 51 \\
\hline$\# 59$ & MeSH descriptor Laser Therapy explode all trees & 2372 \\
\hline \#60 & MeSH descriptor Ultrasonic Therapy explode all trees & 523 \\
\hline
\end{tabular}




\begin{tabular}{|c|c|c|}
\hline \#61 & MeSH descriptor Ultrasonography, Interventional explode all trees & 529 \\
\hline \#62 & (ultrasound):ti,ab,kw or (electrotherapeutic NEAXT (intervention* or treat*)):ti,ab,kw & 5557 \\
\hline \#63 & (\#59 OR \#60 OR \#61 OR \#62) & 8267 \\
\hline \#64 & (\#13 AND \#63) & 58 \\
\hline \#65 & MeSH descriptor Magnetic Field Therapy explode all trees & 369 \\
\hline \#66 & $\begin{array}{l}\left.\text { "pulsed electromagnetic field therapy":ti,ab,kw or (electromagnetic* or magnetic }{ }^{\star}\right) \text { NEAR/3 field*:ti,ab,kw or } \\
\text { (biomagnetic* or (bio magnetic*) or (pulsed signal)):ti,ab,kw or (PEMF):ti,ab,kw }\end{array}$ & 793 \\
\hline$\# 67$ & (\#65 OR \#66) & 1121 \\
\hline \#68 & (\#13 AND \#67) & 12 \\
\hline \#69 & MeSH descriptor Nerve Block explode all trees & 1994 \\
\hline \#70 & MeSH descriptor Neuromuscular Blockade explode all trees & 266 \\
\hline \#71 & (nerve NEAR/2 block*):ti,ab,kw & 2595 \\
\hline \#72 & (\#69 OR \#70 0R \#71) & 2854 \\
\hline \#73 & (\#13 AND \#72) & 49 \\
\hline \#74 & MeSH descriptor Acupuncture Therapy explode all trees & 1830 \\
\hline \#75 & 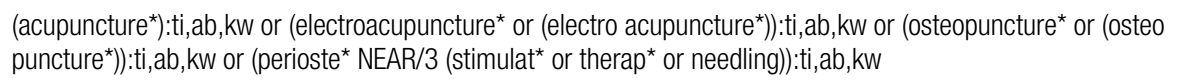 & 4213 \\
\hline \#76 & (\#74 OR \#75) & 4239 \\
\hline \#77 & (\#13 AND \#76) & 58 \\
\hline \#78 & MeSH descriptor Massage explode all trees & 595 \\
\hline \#79 & (massag* or acupressure or shiatsu or shiatzu or (zhi ya) or (chih ya)):ti,ab,kw & 1277 \\
\hline \#80 & (\#78 OR \#79) & 1277 \\
\hline \#81 & (\#13 AND \#80) & 20 \\
\hline \#82 & $\begin{array}{l}\left(\text { rehabilitat* NEAR/2 }\left(\text { program* }^{*} \text { or protocol }{ }^{\star}\right)\right): t i, a b, k w \text { or }\left(\text { watch }^{*} \text { NEAR/2 wait*) or (conservative NEAR/2 }\right. \\
\left.\left.\text { treat*):ti,ab,kw or (management NEAR/2 (decision* or option }{ }^{*} \text { or choice }{ }^{\star}\right)\right): t i, a b, k w\end{array}$ & 2974 \\
\hline \#83 & (\#13 AND \#82) & 34 \\
\hline \#84 & $\begin{array}{l}\text { (\#20 OR \#25 OR \#33 OR \#40 OR \#44 OR \#46 OR \#50 OR \#58 OR \#64 OR \#68 OR \#73 OR \#77 OR \#81 OR } \\
\text { \#83) }\end{array}$ & 588 \\
\hline
\end{tabular}

\section{Clinicaltrials.gov}

Interface: http://clinicaltrials.gov/

Search date: 31 March 2010

Strategy: two separate searches carried out, one for 'frozen shoulder' and a second for 'adhesive capsulitis'

Records identified: 29

\section{Cochrane Database of Systematic Reviews (CDSR)}

Interface: Cochrane Library Issue 32010

Search date: 23 March 2010

Strategy: as CENTRAL strategy above

Records identified: 17

\section{Conference Proceedings Citation Index: Science}

Interface: Web of Science (ISI) via Web of Knowledge

Search date: 30 March 2010

Search strategy: as below 
Records identified: 82

\begin{tabular}{|c|c|c|}
\hline \multirow[t]{2}{*}{ \# 42} & 82 & $\begin{array}{l}\text { \#41 OR \#40 OR \#39 OR \#38 OR \#37 OR \#36 OR \#35 OR \#34 OR \#33 OR \#32 OR \#31 OR \#30 OR \#29 OR \#28 OR \#27 } \\
\text { OR \#26 OR \#25 OR \#24 OR \#23 OR \#22 }\end{array}$ \\
\hline & & Databases $=$ CPCI-S Timespan $=1966-2010$ \\
\hline \multirow[t]{2}{*}{ \# 41} & 1 & \#21 AND \#1 \\
\hline & & Databases $=$ CPCI-S Timespan=1966-2010 \\
\hline \multirow[t]{2}{*}{ \# 40} & 7 & \#20 AND \#1 \\
\hline & & Databases $=$ CPCI-S Timespan=1966-2010 \\
\hline \multirow[t]{2}{*}{ \# 39} & 2 & $\# 19$ AND \#1 \\
\hline & & Databases $=$ CPCI-S Timespan $=1966-2010$ \\
\hline \multirow[t]{2}{*}{ \# 38} & 11 & \#18 AND \#1 \\
\hline & & Databases $=$ CPCI-S Timespan $=1966-2010$ \\
\hline \multirow[t]{2}{*}{ \# 37} & 0 & \#17 AND \#1 \\
\hline & & Databases $=$ CPCI-S Timespan=1966-2010 \\
\hline \multirow[t]{2}{*}{ \# 36} & 0 & $\# 16$ AND \#1 \\
\hline & & Databases $=$ CPCl-S Timespan=1966-2010 \\
\hline \multirow[t]{2}{*}{ \# 35} & 5 & $\# 15$ AND \#1 \\
\hline & & Databases $=$ CPCI-S Timespan=1966-2010 \\
\hline \multirow[t]{2}{*}{ \# 34} & 0 & \#14 AND \#1 \\
\hline & & Databases $=$ CPCI-S Timespan $=1966-2010$ \\
\hline \multirow[t]{2}{*}{ \# 33} & 0 & $\# 13$ AND \#1 \\
\hline & & Databases $=$ CPCI-S Timespan=1966-2010 \\
\hline \multirow[t]{2}{*}{ \# 32} & 0 & $\# 12$ AND \#1 \\
\hline & & Databases $=$ CPCI-S Timespan $=1966-2010$ \\
\hline \multirow[t]{2}{*}{ \# 31} & 2 & $\# 11$ AND \#1 \\
\hline & & Databases $=$ CPCI-S Timespan $=1966-2010$ \\
\hline \multirow[t]{2}{*}{ \# 30} & 0 & $\# 10$ AND \#1 \\
\hline & & Databases=CPCI-S Timespan=1966-2010 \\
\hline \multirow[t]{2}{*}{ \# 29} & 0 & \#9 AND \#1 \\
\hline & & Databases=CPCI-S Timespan=1966-2010 \\
\hline \multirow[t]{2}{*}{ \# 28} & 12 & \#8 AND \#1 \\
\hline & & Databases $=$ CPCI-S Timespan $=1966-2010$ \\
\hline \multirow[t]{2}{*}{ \# 27} & 7 & \#7 AND \#1 \\
\hline & & Databases=CPCI-S Timespan=1966 -2010 \\
\hline \multirow[t]{2}{*}{ \# 26} & 35 & \#6 AND \#1 \\
\hline & & Databases=CPCI-S Timespan=1966-2010 \\
\hline \multirow[t]{2}{*}{ \# 25} & 0 & \#5 AND \#1 \\
\hline & & Databases $=$ CPCI-S Timespan $=1966-2010$ \\
\hline \multirow[t]{2}{*}{ \# 24} & 13 & \#4 AND \#1 \\
\hline & & Databases $=$ CPCI-S Timespan $=1966-2010$ \\
\hline \multirow[t]{2}{*}{ \# 23} & 12 & \#3 AND \#1 \\
\hline & & Databases=CPCI-S Timespan=1966-2010 \\
\hline \multirow[t]{2}{*}{ \# 22} & 1 & \#2 AND \#1 \\
\hline & & Databases $=$ CPCI-S Timespan $=1966-2010$ \\
\hline \multirow[t]{2}{*}{ \# 21} & 2325 & Topic=("management decision”" or "management option" or "management choice ${ }^{\star ” ") ~}$ \\
\hline & & Databases=CPCI-S Timespan=1966 -2010 \\
\hline \multirow[t]{2}{*}{ \# 20} & 1814 & Topic=("rehabilitat* program"” or "rehabilitat* protoco|*" or "watch* wait"” or "conservative treat*") \\
\hline & & Databases=CPCI-S Timespan=1966-2010 \\
\hline \multirow[t]{2}{*}{ \# 19} & 427 & $\begin{array}{l}\text { Topic=("perioste* stimulat*" or "perioste* therap" or "perioste* needling" or massag* or acupressure or shiatsu or shiatzu or } \\
\text { "zhi ya" or "chih ya") }\end{array}$ \\
\hline & & Databases $=$ CPCI-S Timespan=1966-2010 \\
\hline
\end{tabular}




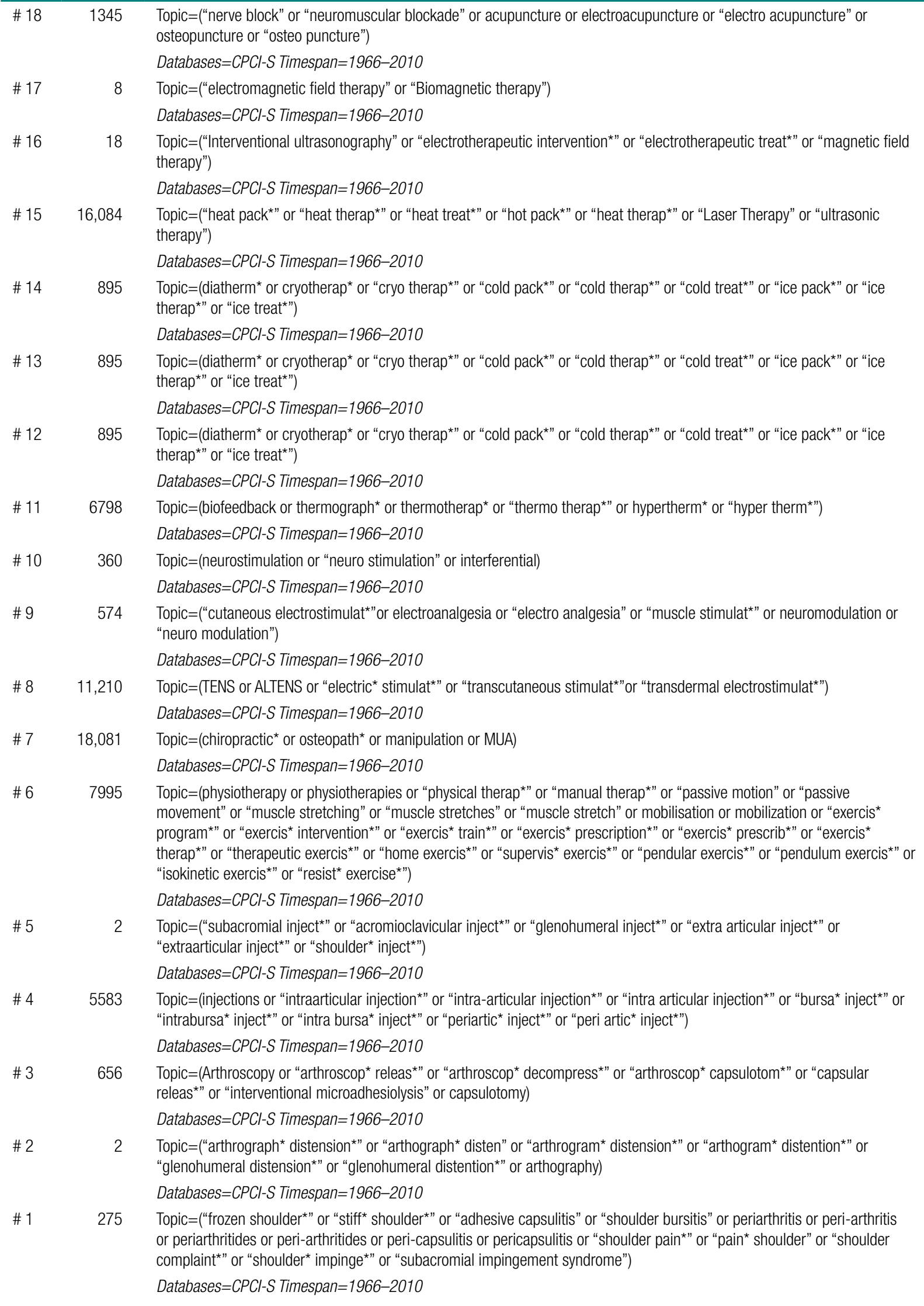




\section{Database of Abstracts of Reviews of Effects (DARE)}

Interface: Cochrane Library Issue 32010

Search date: 23 March 2010

Strategy: as CENTRAL strategy above

Records identified: 17

\section{EMBASE}

Interface: OvidSP

Search date: 22 March 2010

Strategy: as below

Records identified: 3785

An update search carried out on 5 January 2011 identified a further 521 records.

Database: EMBASE <1980 to 2010 Week 11>

1. humeroscapular periarthritis/(766)

2. (frozen adj6 shoulder\$).ti. (215)

3. (stiff\$adj3 shoulder\$).ti. (73)

4. (adhesive adj (capsulitis or capsulitides)).ti. (161)

5. ((bursitis or bursitides) adj6 shoulder\$).ti. (17)

6. ((capsulitis or capsulitides) adj6 shoulder\$).ti. (86)

7. ((periarthritis or peri-arthritis or periarthritides or peri-arthritides or peri-capsulitis or pericapsulitis) adj6 shoulder\$).ti. (70)

8. 1 or 2 or 3 or 4 or 5 or 6 or 7 (1071)

9. (frozen adj6 shoulder\$).ab. (440)

10. (stiff\$adj3 shoulder\$).ab. (313)

11. exp bursitis/(1723)

12. (adhesive adj (capsulitis or capsulitides)).ab. (279)

13. ((bursitis or bursitides) adj6 shoulder\$).ab. (51)

14. ((capsulitis or capsulitides) adj6 shoulder\$).ti,ab. (183)

15. ((periarthritis or peri-arthritis or periarthritides or peri-arthritides or peri-capsulitis or pericapsulitis) adj6 shoulder\$).ab. (98)

16. shoulder pain/(5140)

17. (shoulder $\$$ adj3 (pain or pains or painful or complain\$)).ab. (3349)

18. Shoulder Impingement Syndrome/(680)

19. (shoulder\$adj6 impinge\$).ab. (364)

20. subacromial impingement syndrome.ab. (96)

21. 9 or 10 or 11 or 12 or 13 or 14 or 15 or 16 or 17 or 18 or 19 or 20 (9277)

22. Arthrography/(2891)

23. (arthrograph\$adj6 (distension\$or distention\$)).ti,ab. (32)

24. (arthrogram\$adj6 (distension\$or distention\$)).ti,ab. (1)

25. (glenohumeral adj6 (distension\$or distention\$)).ti,ab. (7)

26. Dilatation/(107)

27. (dilatation or hydrodilat\$).ti,ab. $(28,266)$

28. or/22-27 $(31,214)$

29. 21 and 28 (368)

30. Shoulder arthroscopy/(361) 
31. (arthroscop\$adj6 (releas\$or decompress\$or capsulotom\$)).ti,ab. (535)

32. ((capsular adj2 releas\$) or capsulotomy or interventional microadhesiolysis).ti,ab. (1787)

33. or $/ 30-32(2581)$

34. 21 and $33(280)$

35. injection/(11,806)

36. 35 and $21(61)$

37. ((bursa $\$$ or intrabursa $\$$ or intra bursa $\$$ or periartic $\$$ or peri artic $\$$ or intraartic $\$$ or intra artic $\$$ ) adj3 inject\$).ti,ab. (2681)

38. 37 and $21(141)$

39. ((subacromial or acromioclavicular or glenohumeral) adj3 inject\$).ti,ab. (137)

40. ((extra articular or extraarticular or shoulder\$) adj3 inject\$).ti,ab. (136)

41. 36 or 38 or 39 or $40(400)$

42. exp physiotherapy/(24,873)

43. (physiotherapies or physical therap\$or manual therap\$).ti,ab. (8968)

44. (passive adj (motion or movement)).ti,ab. (1271)

45. CPM.ti,ab. (3193)

46. muscle stretching/or stretching exercise/(3543)

47. (stretching or stretches).ti,ab. $(12,491)$

48. (mobilisation or mobilization).ti,ab. $(28,856)$

49. (exercise\$adj2 (program\$or strength\$or intervention\$or training or prescription\$or prescrib\$)).ti,ab. $(14,622)$

50. (exercise\$adj2 (therap\$or therapeutic)).ti,ab. (2185)

51. ((home or supervis\$) adj2 exercis\$).ti,ab. (1554)

52. ((pendular or pendulum) adj exercis\$).ti,ab. (16)

53. ((isokinetic or resist\$) adj2 exercise\$).ti,ab. (2223)

54. or $/ 42-53(91,822)$

55. 21 and $54(1248)$

56. orthopedic manipulation/(222)

57. chiropractic\$.ti,ab. (1998)

58. osteopath\$.ti,ab. (2213)

59. (manipulat\$adj3 (anesthesia or anaesthesia or anesthetic $\$$ or anaesthetic\$)).ti,ab. (284)

60. MUA.ti,ab. (3446)

61. 56 or 57 or 58 or 59 or 60 (7965)

62. 21 and 61 (109)

63. (TENS or ALTENS).ti,ab. (4441)

64. ((electric $\$$ adj2 stimulat $\$$ ) or (transcutaneous adj2 stimulat\$) or (transdermal adj2 electrostimulat\$) or (cutaneous adj2 electrostimulat\$) or electroanalgesia or electro analgesia).ti,ab. (36,123)

65. (muscle adj2 stimulat\$).ti,ab. (3878)

66. (neuromodulation or neuro modulation or neurostimulation or neuro stimulation).ti,ab. (2484)

67. interferential.ti,ab. (208)

68. or/63-67 (45,043)

69. 21 and $68(108)$

70. biofeedback.ti,ab. (3532)

71. 21 and 70 (7)

72. cryotherapy/(7404)

73. ice/(2468)

74. diathermy/(2178) 
75. hyperthermia/(11,961)

76. thermograph\$.ti,ab. (2186)

77. ((cold or ice or heat or hot) adj (pack\$or therap\$or treat\$)).ti,ab. (7854)

78. (thermotherap\$or thermo therap\$or hypertherm\$or hyper therm\$or diatherm\$or cryotherap\$or cryo therap\$).ti,ab. $(24,875)$

79. or/72-78 $(45,638)$

80. 21 and $79(140)$

81. low level laser Therapy/(4456)

82. ultrasound $/(42,405)$

83. echography/(111,055)

84. (electrotherapeutic adj (intervention\$or treat\$)).ti,ab. (4)

85. or/81-84 $(154,738)$

86. 21 and 85 (717)

87. magnetotherapy/(317)

88. pulsed electromagnetic field therapy.ti,ab. (26)

89. ((electromagnetic\$or magnetic\$) adj3 field\$).ti,ab. $(14,142)$

90. (biomagnetic\$or bio magnetic\$or pulsed signal).ti,ab. (332)

91. PEMF.ti,ab. (223)

92. or/87-91 $(14,579)$

93. 21 and $92(23)$

94. nerve block/(9791)

95. neuromuscular blocking/(6800)

96. nerve block $\$ . t i, a b . ~(4505)$

97. or/94-96 $(17,905)$

98. 21 and 97 (141)

99. exp Acupuncture/(14,373)

100. acupuncture\$.ti,ab. (8902)

101. (electroacupuncture\$or electro acupuncture\$).ti,ab. (1536)

102. (osteopuncture\$or osteo puncture\$).ti,ab. (2)

103. (perioste $\$$ adj 3 (stimulat\$or therap\$or needling)).ti,ab. (114)

104. or/99-103 $(15,019)$

105. 21 and $104(166)$

106. massage/(4230)

107. (massag\$or acupressure or shiatsu or shiatzu or zhi ya or chih ya).ti,ab. (4120)

108. 106 or $107(6434)$

109. 21 and $108(86)$

110. (rehabilitat\$adj2 (program\$or protocol\$)).ti,ab. (8634)

111. 21 and 110 (143)

112. ((watch\$adj2 wait\$) or (conservative\$adj2 treat\$)).ti,ab. $(20,913)$

113. 21 and $112(267)$

114. (management adj2 (decision\$or option\$or choice\$)).ti,ab. (6144)

115. 21 and $114(10)$

116. 111 or 113 or $115(404)$

117. 8 or 29 or 34 or 41 or 55 or 62 or 69 or 71 or 80 or 86 or 93 or 98 or 105 or 109 or $116(3785)$ 


\section{Health Management Information Consortium (HMIC)}

Interface: OvidSP

Search date: 25 March 2010

Strategy: as below

Records identified: 8

Database: HMIC Health Management Information Consortium <January 2010>

1. (frozen adj6 shoulder\$).ti,ab. (0)

2. (stiff\$adj3 shoulder\$).ti,ab. (4)

3. (adhesive adj (capsulitis or capsulitides)).ti,ab. (0)

4. ((bursitis or bursitides) adj6 shoulder $\$$ ).ti,ab. (0)

5. ((capsulitis or capsulitides) adj6 shoulder\$).ti,ab. (0)

6. ((periarthritis or peri-arthritis or periarthritides or peri-arthritides or peri-capsulitis or pericapsulitis) adj6 shoulder\$).ti,ab. (0)

7. (shoulder\$adj3 (pain or pains or painful or complain\$)).ti,ab. (34)

8. (shoulder\$adj6 impinge\$).ti,ab. (0)

9. subacromial impingement syndrome.ti,ab. (0)

10. 1 or 2 or 3 or 4 or 5 or 6 or 7 or 8 or 9 (35)

11. (arthrograph\$adj6 (distension\$or distention\$)).ti,ab. (0)

12. (arthrogram\$adj6 (distension\$or distention\$)).ti,ab. (0)

13. (glenohumeral adj6 (distension\$or distention\$)).ti,ab. (0)

14. (dilatation or hydrodilat\$).ti,ab. (20)

15. (arthroscop\$adj6 (releas\$or decompress\$or capsulotom\$)).ti,ab. (0)

16. ((capsular adj2 releas\$) or interventional microadhesiolysis or capsulotomy).ti,ab. (0)

17. ((bursa\$or intrabursa $\$ o r$ intra bursa $\$$ or periartic\$or peri artic\$or intraartic\$or intra artic\$) adj3 inject\$).ti,ab. (2)

18. ((subacromial or acromioclavicular or glenohumeral) adj3 inject\$).ti,ab. (0)

19. ((extra articular or extraarticular or shoulder\$) adj3 inject\$).ti,ab. (0)

20. (physiotherapy or physiotherapies or physical therap\$or manual therap\$).ti,ab. (732)

21. (passive adj (motion or movement)).ti,ab. (0)

22. CPM.ti,ab. (6)

23. (stretching or stretches).ti,ab. (42)

24. (mobilisation or mobilization).ti,ab. (97)

25. (exercise $\$$ adj2 (program\$or strength\$or intervention\$or training or prescription\$or prescrib\$)).ti,ab. (229)

26. (exercise\$adj2 (therap\$or therapeutic)).ti,ab. (27)

27. ((home or supervis\$) adj2 exercis\$).ti,ab. (40)

28. ((pendular or pendulum) adj exercis\$).ti,ab. (0)

29. ((isokinetic or resist\$) adj2 exercise\$).ti,ab. (11)

30. chiropractic\$.ti,ab. (55)

31. osteopath\$.ti,ab. (80)

32. (manipulat\$adj3 (anesthesia or anaesthesia or anesthetic\$or anaesthetic\$)).ti,ab. (2)

33. MUA.ti,ab. (0)

34. (TENS or ALTENS).ti,ab. (27)

35. ((electric $\$$ adj2 stimulat $\$$ ) or (transcutaneous adj2 stimulat\$) or (transdermal adj2 electrostimulat\$) or (cutaneous adj2 electrostimulat\$) or electroanalgesia or electro analgesia).ti,ab. (9)

36. (muscle adj2 stimulat\$).ti,ab. (5)

37. (neuromodulation or neuro modulation or neurostimulation or neuro stimulation).ti,ab. (1) 
38. interferential.ti,ab. (5)

39. biofeedback.ti,ab. (5)

40. ((cold or ice or heat or hot) adj (pack\$or therap\$or treat\$)).ti,ab. (18)

41. (thermograph\$or thermotherap\$or thermo therap\$or hypertherm\$or hyper therm $\$$ or diatherm\$or cryotherap\$or cryo therap\$).ti,ab. (90)

42. ((laser or ultrasonic) adj2 (therap\$or treat\$)).ti,ab. (25)

43. ultrasound.ti,ab. (260)

44. (electrotherapeutic adj (intervention\$or treat\$)).ti,ab. (0)

45. pulsed electromagnetic field therapy.ti,ab. (0)

46. ((electromagnetic\$or magnetic\$) adj3 field\$).ti,ab. (121)

47. (biomagnetic\$or bio magnetic\$or pulsed signal).ti,ab. (0)

48. PEMF.ti,ab. (0)

49. (nerve adj2 block\$).ti,ab. (2)

50. neuromuscular block\$.ti,ab. (3)

51. acupuncture\$.ti,ab. (118)

52. (electroacupuncture $\$$ or electro acupuncture $\$$ ).ti,ab. (1)

53. (osteopuncture $\$$ or osteo puncture\$).ti,ab. (0)

54. (perioste\$adj3 (stimulat\$or therap\$or needling)).ti,ab. (0)

55. (massag\$or acupressure or shiatsu or shiatzu or zhi ya or chih ya).ti,ab. (67)

56. (rehabilitat\$adj2 (program\$or protocol\$)).ti,ab. (182)

57. ((watch\$adj2 wait\$) or (conservative adj2 treat\$)).ti,ab. (30)

58. (management adj2 (decision\$or option\$or choice\$)).ti,ab. (353)

59. or/11-58 (2475)

60. 10 and $59(8)$

61. limit 60 to $\mathrm{yr}=$ " 1966 -Current" (8)

62. from 61 keep 1-8 (8)

\section{Health Technology Assessment (HTA) database}

Interface: Cochrane Library Issue 32010

Search date: 23 March 2010

Strategy: as CENTRAL strategy above

Records identified: 3

\section{Latin American and Caribbean Health Sciences Literature (LILACS)}

Interface: http://bases.bireme.br/cgi-bin/wxislind.exe/iah/online/?IsisScript=iah/iah. xis\&base $=$ LILACS\&lang $=i \&$ form $=F$

Search date: 26 March 2010

Strategy: shoulder joint [Subject descriptor] or adhesive capsulitis [Words] or bursitis or periarthritis [Words]

Records identified: 298

\section{Manual, Alternative and Natural Therapy (MANTIS)}

Interface: Dialog Classic

Search date: 30 March 2010

Strategy: as below

Records identified: 189 
? s frozen (6W) shoulder?/ti,ab,de

1317 FROZEN/TI,AB,DE

6053 SHOULDER?/TI,AB,DE

S1 172 FROZEN (6W) SHOULDER?/TI,AB,DE

? s stiff? (3W) shoulder?/ti,ab,de

3859 STIFF?/TI,AB,DE

6053 SHOULDER?/TI,AB,DE

S2 33 STIFF? (3W) SHOULDER?/TI,AB,DE

? s adhesive (W) (capsulitis or capsulitides)/ti,ab,de

437 ADHESIVE/TI,AB,DE

149 CAPSULITIS/TI,AB,DE

0 CAPSULITIDES/TI,AB,DE

S3 115 ADHESIVE (W) (CAPSULITIS OR CAPSULITIDES)/TI,AB,DE

? s (bursitis or bursitides) (6W) shoulder?/ti,ab,de

373 BURSITIS/TI,AB,DE

0 BURSITIDES/TI,AB,DE

6053 SHOULDER?/TI,AB,DE

S4 52 (BURSITIS OR BURSITIDES) (6W) SHOULDER?/TI,AB,DE

? s (capsulitis or capsulitides) (6W) shoulder?/ti,ab,de

149 CAPSULITIS/TI,AB,DE

0 CAPSULITIDES/TI,AB,DE

6053 SHOULDER?/TI,AB,DE

S5 41 (CAPSULITIS OR CAPSULITIDES) (6W) SHOULDER?/TI,AB,DE

? s (periarthritis or peri-arthritis or periarthritides or peri-arthritides or peri-capsulitis or pericapsulitis) (6W) shoulder?/ti,ab,de

54 PERIARTHRITIS/TI,AB,DE

0 PERI-ARTHRITIS/TI,AB,DE

0 PERIARTHRITIDES/TI,AB,DE

0 PERI-ARTHRITIDES/TI,AB,DE

0 PERI-CAPSULITIS/TI,AB,DE

3 PERICAPSULITIS/TI,AB,DE

6053 SHOULDER?/TI,AB,DE

S6 29 (PERIARTHRITIS OR PERI-ARTHRITIS OR PERIARTHRITIDES OR

PERI-ARTHRITIDES OR PERI-CAPSULITIS OR PERICAPSULITIS)

(6W) SHOULDER?/TI,AB,DE

? s shoulder? (3W) (pain or pains or painful or complain?)/ti,ab,de

6053 SHOULDER?/TI,AB,DE

$40861 \mathrm{PAIN} / \mathrm{TI}, \mathrm{AB}, \mathrm{DE}$

400 PAINS/TI,AB,DE

3210 PAINFUL/TI,AB,DE

4803 COMPLAIN?/TI,AB,DE

S7 1170 SHOULDER? (3W) (PAIN OR PAINS OR PAINFUL OR

COMPLAIN?)/TI,AB,DE

? s shoulder? (6W) impinge?/ti,ab,de

6053 SHOULDER?/TI,AB,DE 
994 IMPINGE?/TI,AB,DE

S8 192 SHOULDER? (6W) IMPINGE?/TI,AB,DE

? s subacromial (W) impingement $(\mathrm{W})$ syndrome/ti,ab,de

235 SUBACROMIAL/TI,AB,DE

934 IMPINGEMENT/TI,AB,DE

15975 SYNDROME/TI,AB,DE

S9 28 SUBACROMIAL (W) IMPINGEMENT (W) SYNDROME/TI,AB,DE

? s s1:s9

S10 1532 S1:S9

? s arthrograph? (6W) (distension? or distention?)/ti,ab,de

365 ARTHROGRAPH?/TI,AB,DE

204 DISTENSION?/TI,AB,DE

107 DISTENTION?/TI,AB,DE

S11 6 ARTHROGRAPH? (6W) (DISTENSION? OR DISTENTION?)/TI,AB,DE

? s arthrogram? (6W) (distension? or distention?)/ti,ab,de

126 ARTHROGRAM?/TI,AB,DE

204 DISTENSION?/TI,AB,DE

107 DISTENTION?/TI,AB,DE

S12 0 ARTHROGRAM? (6W) (DISTENSION? OR DISTENTION?)/TI,AB,DE

? s glenohumeral (6W) (distension? or distention?)/ti,ab, de

758 GLENOHUMERAL/TI,AB,DE

204 DISTENSION?/TI,AB,DE

107 DISTENTION?/TI,AB,DE

S13 1 GLENOHUMERAL (6W) (DISTENSION? OR DISTENTION?)/TI,AB,DE

? s dilatation or hydrodilat?/ti,ab,de

301 DILATATION

4 HYDRODILAT?/TI,AB,DE

S14 304 DILATATION OR HYDRODILAT?/TI,AB,DE

? s s11:s14

S15 310 S11:S14

? s s 10 and s15

1532 S10

$310 \mathrm{~S} 15$

S16 11 S10 AND S15

? s arthroscop? (6W) (releas? or decompress? or capsulotom?)/ti,ab,de

2147 ARTHROSCOP?/TI,AB,DE

7144 RELEAS?/TI,AB,DE

2479 DECOMPRESS?/TI,AB,DE

52 CAPSULOTOM?/TI,AB,DE

S17 96 ARTHROSCOP? (6W) (RELEAS? OR DECOMPRESS? OR

CAPSULOTOM?)/TI,AB,DE

? s capsular (2W) releas? or interventional (W) microadhesiolysis or capsulotomy/ti,ab,de 


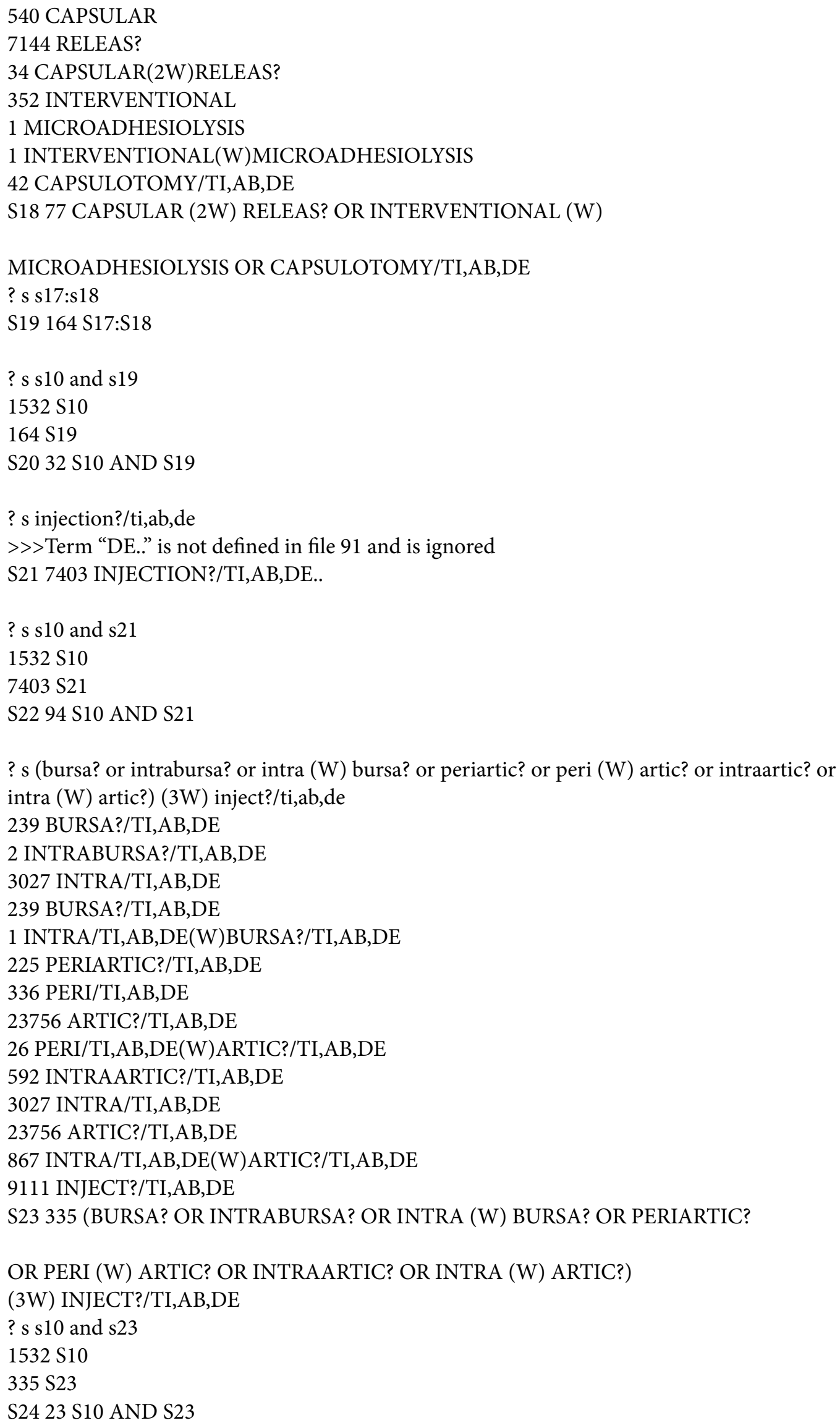


? s (subacromial or acromioclavicular or glenohumeral) (3W) inject?/ti,ab,de

235 SUBACROMIAL/TI,AB,DE

282 ACROMIOCLAVICULAR/TI,AB,DE

758 GLENOHUMERAL/TI,AB,DE

9111 INJECT?/TI,AB,DE

S25 24 (SUBACROMIAL OR ACROMIOCLAVICULAR OR GLENOHUMERAL) (3W)

INJECT?/TI,AB,DE

? s (extra (W) articular or extraarticular or shoulder?) (3W) inject?/ti,ab,de

1137 EXTRA/TI,AB,DE

6439 ARTICULAR/TI,AB,DE

227 EXTRA/TI,AB,DE(W)ARTICULAR/TI,AB,DE

143 EXTRAARTICULAR/TI,AB,DE

6053 SHOULDER?/TI,AB,DE

9111 INJECT?/TI,AB,DE

S26 17 (EXTRA (W) ARTICULAR OR EXTRAARTICULAR OR SHOULDER?) (3W)

INJECT?/TI,AB,DE

? s s 22 or s 24 or s 25 or s 26

$94 \mathrm{~S} 22$

$23 \mathrm{~S} 24$

$24 \mathrm{~S} 25$

17 S26

S27 113 S22 OR S24 OR S25 OR S26

? s physiotherapy or physiotherapies or physical (W) therap? or manual(W) therap?/ti,ab,de 1824 PHYSIOTHERAPY

1 PHYSIOTHERAPIES

27577 PHYSICAL

58031 THERAP?

5273 PHYSICAL(W)THERAP?

3870 MANUAL/TI,AB,DE

58031 THERAP?/TI,AB,DE

662 MANUAL/TI,AB,DE(W)THERAP?/TI,AB,DE

S28 7178 PHYSIOTHERAPY OR PHYSIOTHERAPIES OR PHYSICAL (W) THERAP?

ORy MANUAL(W) THERAP?/TI,AB,DE

? s passive $(\mathrm{W})$ (motion or movement)/ti,ab, de

3275 PASSIVE/TI,AB,DE

$12870 \mathrm{MOTION} / \mathrm{TI}, \mathrm{AB}, \mathrm{DE}$

8522 MOVEMENT/TI,AB,DE

S29 395 PASSIVE (W) (MOTION OR MOVEMENT)/TI,AB,DE

? s CPM/ti,ab,de

S30 117 CPM/TI,AB,DE

? s (stretching or stretches)/ti,ab,de

1453 STRETCHING/TI,AB,DE

200 STRETCHES/TI,AB,DE

S31 1555 (STRETCHING OR STRETCHES)/TI,AB,DE 
? s (mobilisation or mobilization)/ti,ab,de

248 MOBILISATION/TI,AB,DE

1532 MOBILIZATION/TI,AB,DE

S32 1749 (MOBILISATION OR MOBILIZATION)/TI,AB,DE

? s exercise? (2W) (program? or strength? or intervention? or training or prescription? or prescrib?)/ti,ab,de

21844 EXERCISE?/TI,AB,DE

21676 PROGRAM?/TI,AB,DE

13678 STRENGTH?/TI,AB,DE

20015 INTERVENTION?/TI,AB,DE

15534 TRAINING/TI,AB,DE

2502 PRESCRIPTION?/TI,AB,DE

2943 PRESCRIB?/TI,AB,DE

S33 3545 EXERCISE? (2W) (PROGRAM? OR STRENGTH? OR INTERVENTION? OR TRAINING OR PRESCRIPTION? OR PRESCRIB?)/TI,AB,DE

? s exercise? (2W) (therap? or therapeutic)/ti,ab,de

21844 EXERCISE?/TI,AB,DE

58031 THERAP?/TI,AB,DE

15559 THERAPEUTIC/TI,AB,DE

S34 1871 EXERCISE? (2W) (THERAP? OR THERAPEUTIC)/TI,AB,DE

? s (home or supervis?) (2W) exercis?/ti,ab,de

$5568 \mathrm{HOME} / \mathrm{TI}, \mathrm{AB}, \mathrm{DE}$

1564 SUPERVIS?/TI,AB,DE

21993 EXERCIS?/TI,AB,DE

S35 448 (HOME OR SUPERVIS?) (2W) EXERCIS?/TI,AB,DE

? s (pendular or pendulum) (W) exercis?/ti,ab,de

30 PENDULAR/TI,AB,DE

209 PENDULUM/TI,AB,DE

21993 EXERCIS?/TI,AB,DE

S36 5 (PENDULAR OR PENDULUM) (W) EXERCIS?/TI,AB,DE

? s (isokinetic or resist?) (2W) exercise?/ti,ab,de

1921 ISOKINETIC/TI,AB,DE

7695 RESIST?/TI,AB,DE

21844 EXERCISE?/TI,AB,DE

S37 720 (ISOKINETIC OR RESIST?) (2W) EXERCISE?/TI,AB,DE

? s s28:s37

S38 14795 S28:S37

? s s 10 and s 38

$1532 S 10$

14795 S38

S39 313 S10 AND S38

? s chiropractic?/ti,ab,de

S40 18676 CHIROPRACTIC?/TI,AB,DE 
? s osteopath?/ti,ab,de

S41 8326 OSTEOPATH?/TI,AB,DE

? s manipulat? (3W) (anesthesia or anaesthesia or anesthetic? or anaesthetic? or musculoskeletal)/

ti,ab,de

8789 MANIPULAT?/TI,AB,DE

1764 ANESTHESIA/TI,AB,DE

384 ANAESTHESIA/TI,AB,DE

860 ANESTHETIC?/TI,AB,DE

293 ANAESTHETIC?/TI,AB,DE

6860 MUSCULOSKELETAL/TI,AB,DE

S42 237 MANIPULAT? (3W) (ANESTHESIA OR ANAESTHESIA OR ANESTHETIC?

OR ANAESTHETIC? OR MUSCULOSKELETAL)/TI,AB,DE

? s MUA/ti,ab,de

S43 41 MUA/TI,AB,DE

? s s $40: s 43$

S44 26435 S40:S43

? s s 10 and s44

$1532 \mathrm{~S} 10$

$26435 \mathrm{~S} 44$

S45 168 S10 AND S44

? s (TENS or ALTENS)/ti,ab,de

367 TENS/TI,AB,DE

4 ALTENS/TI,AB,DE

S46 369 (TENS OR ALTENS)/TI,AB,DE

? s electric? (2W) stimulat? or transcutaneous $(2 \mathrm{~W})$ stimulat? or transdermal $(2 \mathrm{~W})$

electrostimulat? or cutaneous (2W) electrostimulat? or electroanalgesia or electro(W)analgesia/

ti,ab,de

5115 ELECTRIC?

13078 STIMULAT?

2781 ELECTRIC?(2W)STIMULAT?

716 TRANSCUTANEOUS

13078 STIMULAT?

592 TRANSCUTANEOUS(2W)STIMULAT?

184 TRANSDERMAL

116 ELECTROSTIMULAT?

0 TRANSDERMAL(2W)ELECTROSTIMULAT?

1545 CUTANEOUS

116 ELECTROSTIMULAT?

1 CUTANEOUS(2W)ELECTROSTIMULAT?

7 ELECTROANALGESIA

565 ELECTRO/TI,AB,DE

1688 ANALGESIA/TI,AB,DE

2 ELECTRO/TI,AB,DE(W)ANALGESIA/TI,AB,DE

S47 2821 ELECTRIC? (2W) STIMULAT? OR TRANSCUTANEOUS (2W) STIMULAT?

OR TRANSDERMAL (2W) ELECTROSTIMULAT? OR CUTANEOUS (2W)

ELECTROSTIMULAT? OR ELECTROANALGESIA OR

ELECTRO(W)ANALGESIA/TI,AB,DE 
? s muscle (2W) stimulat?/ti,ab,de

25048 MUSCLE/TI,AB,DE

13078 STIMULAT?/TI,AB,DE

S48 317 MUSCLE (2W) STIMULAT?/TI,AB,DE

? s neuromodulation or neuro (W) modulation or neurostimulation or neuro (W) stimulation/

ti,ab,de

60 NEUROMODULATION

492 NEURO

1698 MODULATION

1 NEURO(W)MODULATION

47 NEUROSTIMULATION

492 NEURO/TI,AB,DE

8205 STIMULATION/TI,AB,DE

0 NEURO/TI,AB,DE(W)STIMULATION/TI,AB,DE

S49 102 NEUROMODULATION OR NEURO (W) MODULATION OR

NEUROSTIMULATION OR NEURO (W) STIMULATION/TI,AB,DE

? s interferential/ti,ab,de

S50 76 INTERFERENTIAL/TI,AB,DE

? s s $46: 550$

S51 3178 S46:S50

? s s 10 and s51

1532 S10

3178 S51

S52 44 S10 AND S51

? s biofeedback/ti,ab,de

S53 1148 BIOFEEDBACK/TI,AB,DE

? s s 10 and s53

1532 S 10

1148 S53

S54 2 S10 AND S53

? s (cold or ice or heat or hot) (W) (pack? or therap? or treat?)/ti,ab,de

$2091 \mathrm{COLD} / \mathrm{TI}, \mathrm{AB}, \mathrm{DE}$

805 ICE/TI,AB,DE

2522 HEAT/TI,AB,DE

$1323 \mathrm{HOT} / \mathrm{TI}, \mathrm{AB}, \mathrm{DE}$

1446 PACK?/TI,AB,DE

58031 THERAP?/TI,AB,DE

79900 TREAT?/TI,AB,DE

S55 306 (COLD OR ICE OR HEAT OR HOT) (W) (PACK? OR THERAP? OR

TREAT?)/TI,AB,DE

? s thermograph? or thermotherap? or thermo $(\mathrm{W})$ therap? or hypertherm? or hyper $(\mathrm{W})$ therm? or diatherm? or cryotherap? or cryo(W) therap?/ti,ab,de

413 THERMOGRAPH?

45 THERMOTHERAP?

32 THERMO 


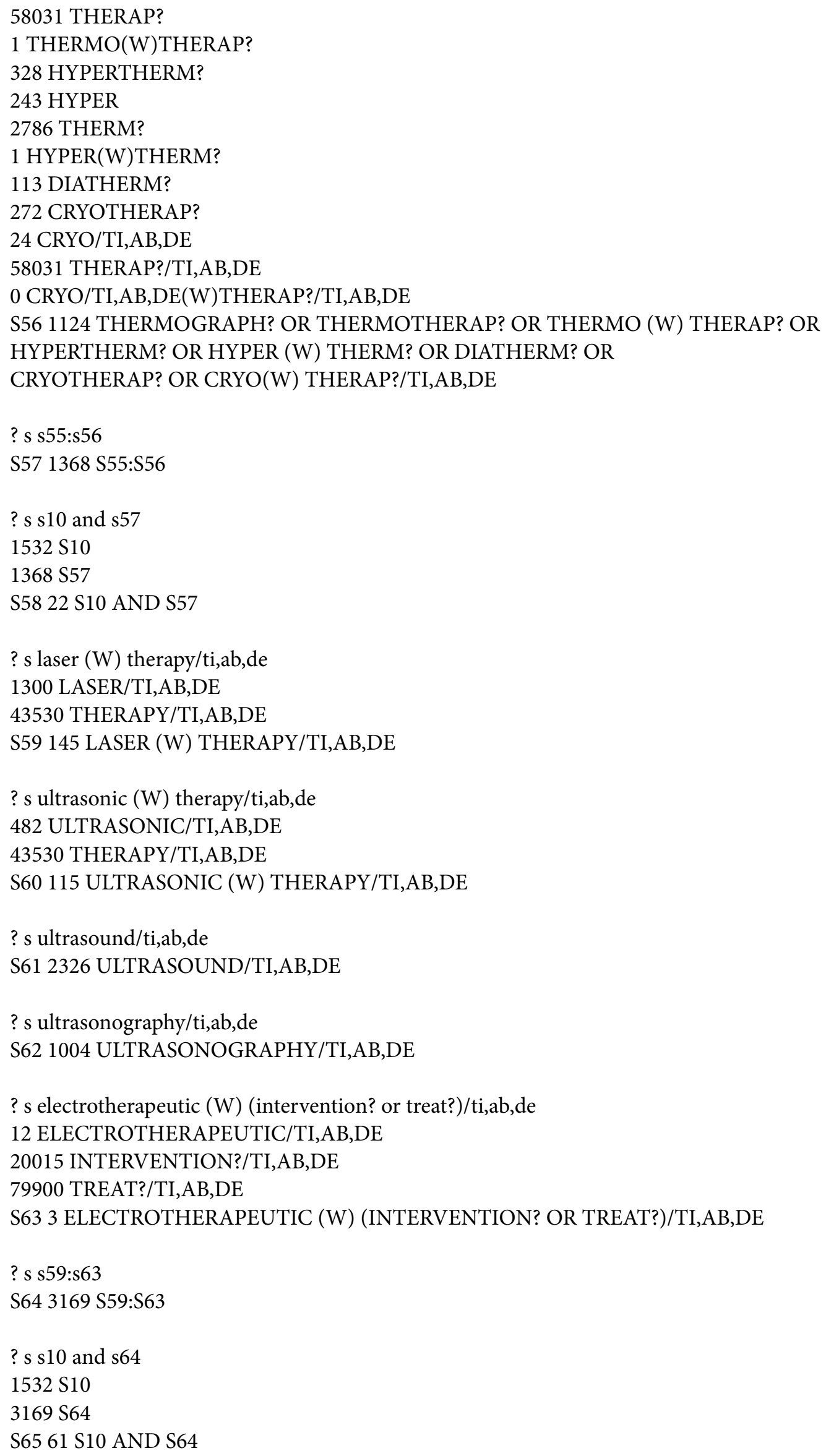


? s pulsed (W) electromagnetic (W) field (W) therapy/ti,ab,de 533 PULSED/TI,AB,DE

754 ELECTROMAGNETIC/TI,AB,DE

6784 FIELD/TI,AB,DE

43530 THERAPY/TI,AB,DE

S66 7 PULSED (W) ELECTROMAGNETIC (W) FIELD (W) THERAPY/TI,AB,DE

? s (electromagnetic? or magnetic?) (3W) field?/ti,ab,de

787 ELECTROMAGNETIC?/TI,AB,DE

6966 MAGNETIC?/TI,AB,DE

8099 FIELD?/TI,AB,DE

S67 572 (ELECTROMAGNETIC? OR MAGNETIC?) (3W) FIELD?/TI,AB,DE

? s biomagnetic? or bio $(\mathrm{W})$ magnetic? or pulsed signal/ti,ab,de

10 BIOMAGNETIC?

$392 \mathrm{BIO}$

6966 MAGNETIC?

2 BIO(W)MAGNETIC?

0 PULSED SIGNAL/TI,AB,DE

S68 12 BIOMAGNETIC? OR BIO (W) MAGNETIC? OR PULSED

SIGNAL/TI,AB,DE

? s PEMF/ti,ab,de

S69 $42 \mathrm{PEMF} / \mathrm{TI}, \mathrm{AB}, \mathrm{DE}$

? s s66:s69

S70 578 S66:S69

? s s 10 and s70

$1532 \mathrm{~S} 10$

$578 \mathrm{~S} 70$

S71 2 S10 AND S70

? s neuromuscular (W) blockade/ti,ab,de

3130 NEUROMUSCULAR/TI,AB,DE

802 BLOCKADE/TI,AB,DE

S72 81 NEUROMUSCULAR (W) BLOCKADE/TI,AB,DE

? s nerve $(2 \mathrm{~W})$ block?/ti,ab,de

13003 NERVE/TI,AB,DE

6218 BLOCK?/TI,AB,DE

S73 453 NERVE (2W) BLOCK?/TI,AB,DE

? s s72:s73

S74 533 S72:S73

? s s 10 and s 74

1532 S10

$533 \mathrm{~S} 74$

S75 9 S10 AND S74 
? s acupuncture?/ti,ab, de

S76 7224 ACUPUNCTURE?/TI,AB,DE

? s electroacupuncture? or electro (W) acupuncture?/ti,ab,de

996 ELECTROACUPUNCTURE?

565 ELECTRO/TI,AB,DE

7224 ACUPUNCTURE?/TI,AB,DE

303 ELECTRO/TI,AB,DE(W)ACUPUNCTURE?/TI,AB,DE

S77 1223 ELECTROACUPUNCTURE? OR ELECTRO (W) ACUPUNCTURE?/TI,AB,DE

? s osteopuncture? or osteo (W) puncture?/ti,ab,de

0 OSTEOPUNCTURE?

86 OSTEO/TI,AB,DE

587 PUNCTURE?/TI,AB,DE

0 OSTEO/TI,AB,DE(W)PUNCTURE?/TI,AB,DE

S78 0 OSTEOPUNCTURE? OR OSTEO (W) PUNCTURE?/TI,AB,DE

? s perioste? (3W) (stimulat? or therap? or needling)/ti,ab, de

455 PERIOSTE?/TI,AB,DE

13078 STIMULAT?/TI,AB,DE

58031 THERAP?/TI,AB,DE

657 NEEDLING/TI,AB,DE

S79 7 PERIOSTE? (3W) (STIMULAT? OR THERAP? OR

NEEDLING)/TI,AB,DE

? s s76:s79

S80 7685 S76:S79

? s s 10 and s 80

$1532 S 10$

$7685 \mathrm{~S} 80$

S81 88 S10 AND S80

? s massag? or acupressure or shiatsu or shiatzu or zhi (W) ya or chih (W) ya/ti,ab,de

1535 MASSAG?

201 ACUPRESSURE

58 SHIATSU

0 SHIATZU

$105 \mathrm{ZHI}$

21 YA

$0 \mathrm{ZHI}(\mathrm{W}) \mathrm{YA}$

$10 \mathrm{CHIH} / \mathrm{TI}, \mathrm{AB}, \mathrm{DE}$

$21 \mathrm{YA} / \mathrm{TI}, \mathrm{AB}, \mathrm{DE}$

0 CHIH/TI,AB,DE(W)YA/TI,AB,DE

S82 1692 MASSAG? OR ACUPRESSURE OR SHIATSU OR SHIATZU OR ZHI (W)

YA OR CHIH (W) YA/TI,AB,DE

? s s 10 and s82

$1532 \mathrm{~S} 10$

$1692 \mathrm{~S} 82$

S83 43 S10 AND S82 
? s rehabilitat? (2W) (program? or protocol?)/ti,ab,de

12934 REHABILITAT?/TI,AB,DE

21676 PROGRAM?/TI,AB,DE

6815 PROTOCOL?/TI,AB,DE

S84 2040 REHABILITAT? (2W) (PROGRAM? OR PROTOCOL?)/TI,AB,DE

? s s 10 and s 84

1532 S10

2040 S 84

S85 53 S10 AND S84

? s watch? (2W) wait? or conservative $(2 \mathrm{~W})$ treat?/ti,ab,de

$500 \mathrm{WATCH}$ ?

875 WAIT?

18 WATCH?(2W)WAIT?

3381 CONSERVATIVE/TI,AB,DE

79900 TREAT?/TI,AB,DE

1533 CONSERVATIVE/TI,AB,DE(2W)TREAT?/TI,AB,DE

S86 1551 WATCH? (2W) WAIT? OR CONSERVATIVE (2W) TREAT?/TI,AB,DE

? s s 10 and s 86

$1532 S 10$

$1551 \mathrm{~S} 86$

S87 51 S10 AND S86

? s management ( $2 \mathrm{~W})$ (decision? or option? or choice?)/ti,ab,de

18593 MANAGEMENT/TI,AB,DE

5371 DECISION?/TI,AB,DE

3950 OPTION?/TI,AB,DE

4823 CHOICE?/TI,AB,DE

S88 219 MANAGEMENT (2W) (DECISION? OR OPTION? OR

CHOICE?)/TI,AB,DE

? s s 10 and $\mathrm{s} 88$

$1532 S 10$

$219 \mathrm{~S} 88$

S89 1 S10 AND S88

? s s 85 or $\mathrm{s} 87$ or $\mathrm{s} 89$

$53 \mathrm{~S} 85$

$51 \mathrm{~S} 87$

$1 S 89$

S90 101 S85 OR S87 OR S89

? s s 16 or s 20 or $s 27$ or s 39 or $s 45$ or $s 52$ or s 54 or $s 58$ or $s 65$ or s 71 or s 75 or $s 81$ or s83 or s 90 $11 \mathrm{~S} 16$

32 S20

113 S27

313 S39

168 S45

44 S52

2 S54 
22 S58

$61 \mathrm{~S} 65$

2 S71

$9 \mathrm{~S} 75$

88 S81

$43 \mathrm{~S} 83$

$101 \mathrm{~S} 90$

S91 685 S16 OR S20 OR S27 OR S39 OR S45 OR S52 OR S54 OR S58 OR

S65 OR S71 OR S75 OR S81 OR S83 OR S90

? s py $=1966: 2010$

S92 89245 PY=1966:2010

? s s91 and s92

685 S 91

89245 S92

S93 189 S91 AND S92

\section{NHS Economic Evaluation Database (NHS EED)}

Interface: Cochrane Library Issue 32010

Search date: 23 March 2010

Strategy: as below

\begin{tabular}{|c|c|c|}
\hline ID & Search & Hits \\
\hline$\# 1$ & (frozen NEAR/6 shoulder $\left.{ }^{\star}\right): \mathrm{i}, \mathrm{ab}, \mathrm{kw}$ & 65 \\
\hline \#2 & (stiff* NEAR/3 shoulder*):ti,ab,kw & 63 \\
\hline \#3 & MeSH descriptor Bursitis explode all trees & 127 \\
\hline \#4 & (adhesive NEXT (capsulitis or capsulitides)):ti,ab,kw & 44 \\
\hline \#5 & (bursitis or bursitides) NEAR/6 shoulder*ti,ab,kw & 11 \\
\hline \#6 & (capsulitis or capsulitides) NEAR/6 shoulder* & 39 \\
\hline \#7 & $\begin{array}{l}\text { (periarthritis or peri-arthritis or periarthritides or peri-arthritides or peri-capsulitis or pericapsulitis) NEAR/6 } \\
\text { shoulder*:ti,ab,kw }\end{array}$ & 77 \\
\hline \#8 & MeSH descriptor Shoulder Pain explode all trees & 240 \\
\hline$\# 9$ & (shoulder* NEAR/3 (pain or pains or painful or complain*)):ti,ab,kw & 682 \\
\hline \#10 & MeSH descriptor Shoulder Impingement Syndrome explode all trees & 84 \\
\hline$\# 11$ & (shoulder* NEAR/6 impinge*):ti,ab,kw & 107 \\
\hline$\# 12$ & "subacromial impingement syndrome":ti,ab,kw & 20 \\
\hline$\# 13$ & (\#1 OR \#2 OR \#3 OR \#4 OR \#5 OR \#6 OR \#7 OR \#8 OR \#9 OR \#10 OR \#11 OR \#12) & 964 \\
\hline \#14 & MeSH descriptor Arthrography, this term only & 148 \\
\hline \#15 & (arthrograph* NEAR/6 (distension* or distention*)):ti,ab,kw & 10 \\
\hline \#16 & $\begin{array}{l}(\text { arthrogram* NEAR/6 (distension* or distention*)):ti,ab,kw or (glenohumeral NEAR/6 (distension* or } \\
\text { distention*)):ti,ab,kw }\end{array}$ & 3 \\
\hline$\# 17$ & MeSH descriptor Dilatation, this term only & 281 \\
\hline \#18 & (dilatation or hydrodilat*):ti,ab,kw & 2541 \\
\hline \#19 & (\#14 OR \#15 OR \#16 OR \#17 OR\#18) & 2695 \\
\hline \#20 & (\#13 AND \#19) & 25 \\
\hline \#21 & MeSH descriptor Arthroscopy explode all trees & 835 \\
\hline \#22 & (arthroscop* NEAR/6 (releas ${ }^{*}$ or decompress ${ }^{*}$ or capsulotom $)$ ):ti,ab,kw & 64 \\
\hline \#23 & (capsular NEAR/2 releas*):ti,ab,kw or "interventional microadhesiolysis":ti,ab,kw or (capsulotomy):ti,ab,kw & 197 \\
\hline
\end{tabular}




\begin{tabular}{|c|c|c|}
\hline \#24 & (\#21 OR \#22 0R \#23) & 1048 \\
\hline \#25 & (\#13 AND \#24) & 51 \\
\hline \#26 & MeSH descriptor Injections, Intra-Articular explode all trees & 628 \\
\hline \#27 & (\#13 AND \#26) & 62 \\
\hline \#28 & MeSH descriptor Injections explode all trees & 15,394 \\
\hline \#29 & (\#13 AND \#28) & 115 \\
\hline \#30 & (bursa* or intrabursa* or intra bursa* or periartic ${ }^{\star}$ or peri artic ${ }^{*}$ or intraartic ${ }^{\star}$ or intra artic $)$ NEAR/3 inject*:ti,ab,kw & 813 \\
\hline \#31 & (\#13 AND \#30) & 79 \\
\hline \#32 & 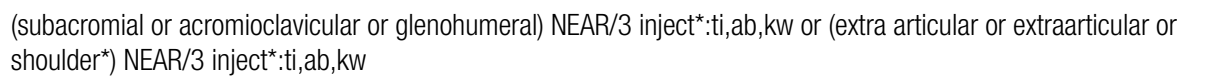 & 72 \\
\hline \#33 & (\#27 OR \#29 OR \#31 OR \#32) & 169 \\
\hline \#34 & MeSH descriptor Physical Therapy Modalities explode all trees & 9583 \\
\hline \#35 & $\begin{array}{l}\text { (physiotherapy or physiotherapies or (physical therap*) or (manual therap*)):ti,ab,kw or (passive NEXT (motion or } \\
\text { movement)):ti,ab, kw or (CPM):ti,ab,kw }\end{array}$ & 10,737 \\
\hline \#36 & MeSH descriptor Muscle Stretching Exercises explode all trees & 82 \\
\hline \#37 & $\begin{array}{l}\text { (stretching or stretches):ti,ab,kw or (mobilisation or mobilization):ti,ab,kw or (exercise* NEAR/2 (program or } \\
\text { strength* or intervention* or training or prescription* or prescrib*)):ti,ab,kw or (exercise* NEAR/2 (therap* or } \\
\text { therapeutic)):ti,ab,kw }\end{array}$ & 10,643 \\
\hline \#38 & 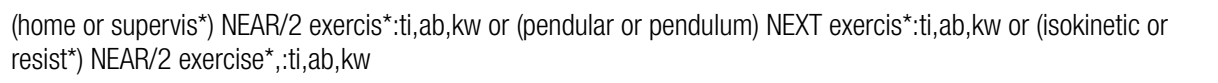 & 1658 \\
\hline \#39 & (\#34 OR \#35 OR \#36 OR \#37 OR \#38) & 23,617 \\
\hline \#40 & (\#13 AND \#39) & 300 \\
\hline \#41 & MeSH descriptor Musculoskeletal Manipulations explode all trees & 4745 \\
\hline \#42 & $\begin{array}{l}\text { (chiropractic*):ti,ab,kw or (osteopath»):ti,ab, kw or (manipulat* NEAR/3 (anesthesia or anaesthesia or anesthetic }{ }^{*} \text { or } \\
\text { anaesthetic*)):ti,ab,kw or (MUA):ti,ab,kw }\end{array}$ & 728 \\
\hline \#43 & (\#41 OR \#42) & 5242 \\
\hline \#44 & (\#13 AND \#43) & 119 \\
\hline \#45 & $\begin{array}{l}\text { (TENS or ALTENS):ti,ab,kw or (electric* NEAR/2 stimulat*) or (transcutaneous NEAR/2 stimulat*) or (transdermal } \\
\text { NEAR/2 electrostimulat*) or (cutaneous NEAR/2 electrostimulat*) or electroanalgesia or (electro analgesia):ti,ab,kw } \\
\text { or (muscle NEAR/2 stimulat*):ti,ab,kw or (neuromodulation or (neuro modulation) or neurostimulation or (neuro } \\
\text { stimulation)):ti,ab,kw or (interferential):ti,ab,kw }\end{array}$ & 17,526 \\
\hline \#46 & (\#13 AND \#45) & 76 \\
\hline \#47 & MeSH descriptor Biofeedback (Psychology) explode all trees & 710 \\
\hline \#48 & (biofeedback):ti,ab,kw & 1348 \\
\hline \#49 & (\#47 0R \#48) & 1348 \\
\hline$\# 50$ & (\#13 AND \#49) & 6 \\
\hline \#51 & MeSH descriptor Cryotherapy explode all trees & 866 \\
\hline \#52 & MeSH descriptor Ice explode all trees & 63 \\
\hline \#53 & MeSH descriptor Diathermy explode all trees & 620 \\
\hline \#54 & MeSH descriptor Hyperthermia, Induced explode all trees & 981 \\
\hline \#55 & MeSH descriptor Hot Temperature explode all trees & 1079 \\
\hline \#56 & $\begin{array}{l}\text { (cold or ice or heat or hot) NEXT (pack* or therap* or treat*):ti,ab,kw or (thermograph* or thermotherap* or (thermo } \\
\text { therap*) or hypertherm* or (hyper therm*)):ti,ab,kw or (diatherm* or cryotherap* or (cryo therap*)):ti,ab, kw }\end{array}$ & 2278 \\
\hline$\# 57$ & (\#51 OR \#52 OR \#53 OR \#54 OR \#55 OR \#56) & 4273 \\
\hline \#58 & (\#13 AND \#57) & 51 \\
\hline \#59 & MeSH descriptor Laser Therapy explode all trees & 2372 \\
\hline \#60 & MeSH descriptor Ultrasonic Therapy explode all trees & 523 \\
\hline \#61 & MeSH descriptor Ultrasonography, Interventional explode all trees & 529 \\
\hline \#62 & (ultrasound):ti,ab,kw or (electrotherapeutic NEAXT (intervention* or treat*)):ti,ab,kw & 5557 \\
\hline \#63 & (\#59 OR \#60 OR \#61 OR \#62) & 8267 \\
\hline \#64 & (\#13 AND \#63) & 58 \\
\hline \#65 & MeSH descriptor Magnetic Field Therapy explode all trees & 369 \\
\hline
\end{tabular}




\begin{tabular}{|c|c|c|}
\hline \#66 & $\begin{array}{l}\text { "pulsed electromagnetic field therapy":ti,ab,kw or (electromagnetic* or magnetic*) NEAR/3 field:ti,ab,kw or } \\
\text { (biomagnetic* or (bio magnetic*) or (pulsed signal)):ti,ab,kw or (PEMF):ti,ab,kw }\end{array}$ & 793 \\
\hline \#67 & (\#65 0R \#66) & 1121 \\
\hline \#68 & (\#13 AND \#67) & 12 \\
\hline \#69 & MeSH descriptor Nerve Block explode all trees & 1994 \\
\hline \#70 & MeSH descriptor Neuromuscular Blockade explode all trees & 266 \\
\hline$\# 71$ & (nerve NEAR/2 block*):ti,ab,kw & 2595 \\
\hline \#72 & (\#69 OR \#70 OR \#71) & 2854 \\
\hline \#73 & (\#13 AND \#72) & 49 \\
\hline$\# 74$ & MeSH descriptor Acupuncture Therapy explode all trees & 1830 \\
\hline \#75 & 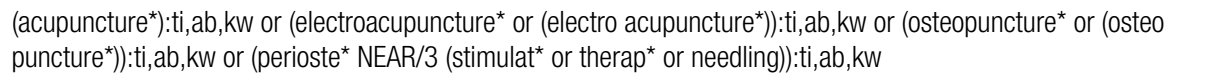 & 4213 \\
\hline \#76 & (\#74 OR \#75) & 4239 \\
\hline \#77 & (\#13 AND \#76) & 58 \\
\hline \#78 & MeSH descriptor Massage explode all trees & 595 \\
\hline \#79 & (massag* or acupressure or shiatsu or shiatzu or (zhi ya) or (chih ya)):ti,ab,kw & 1277 \\
\hline \#80 & (\#78 OR \#79) & 1277 \\
\hline \#81 & (\#13 AND \#80) & 20 \\
\hline \#82 & $\begin{array}{l}\left.\left(\text { rehabilitat }^{*} \text { NEAR/2 (program* or protocol }{ }^{*}\right)\right): t i, a b, k w \text { or }\left(\text { watch }^{*} \text { NEAR/2 wait*) or (conservative NEAR/2 }\right. \\
\text { treat*):ti,ab,kw or (management NEAR/2 (decision* or option* or choice*)):ti,ab,kw }\end{array}$ & 2974 \\
\hline \#83 & (\#13 AND \#82) & 34 \\
\hline \#84 & (\#20 OR \#25 OR \#33 OR \#40 OR \#44 OR \#46 OR \#50 OR \#58 OR \#64 OR \#68 OR \#73 OR \#77 OR \#81 OR \#83) & 588 \\
\hline
\end{tabular}

\section{National Technical Information Service (NTIS)}

Interface: Dialog Classic

Search date: 30 March 2010

Strategy: as below

Records identified: 8

File 6:NTIS 1964-2010/Mar W4

(c) 2010 NTIS, Intl Cpyrght All Rights Res

? s frozen (6W) shoulder?/ti,ab,de

4683 FROZEN/TI,AB,DE

1917 SHOULDER?/TI,AB,DE

S1 5 FROZEN (6W) SHOULDER?/TI,AB,DE

? s stiff? (3W) shoulder?/ti,ab,de

11654 STIFF?/TI,AB,DE

1917 SHOULDER?/TI,AB,DE

S2 1 STIFF? (3W) SHOULDER?/TI,AB,DE

? s adhesive (W) (capsulitis or capsulitides)/ti,ab,de

4903 ADHESIVE/TI,AB,DE

1 CAPSULITIS/TI,AB,DE

0 CAPSULITIDES/TI,AB,DE

S3 1 ADHESIVE (W) (CAPSULITIS OR CAPSULITIDES)/TI,AB,DE

? s (bursitis or bursitides) (6W) shoulder?/ti,ab,de

10 BURSITIS/TI,AB,DE 


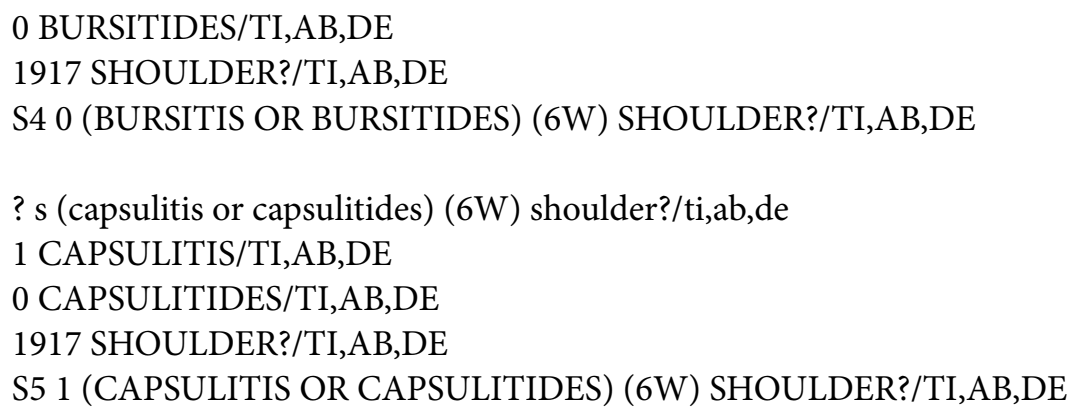

? s (periarthritis or peri-arthritis or periarthritides or peri-arthritides or peri-capsulitis or pericapsulitis) (6W) shoulder?/ti,ab,de 3 PERIARTHRITIS/TI,AB,DE 0 PERI-ARTHRITIS/TI,AB,DE 0 PERIARTHRITIDES/TI,AB,DE 0 PERI-ARTHRITIDES/TI,AB,DE 0 PERI-CAPSULITIS/TI,AB,DE 0 PERICAPSULITIS/TI,AB,DE 1917 SHOULDER?/TI,AB,DE S6 0 (PERIARTHRITIS OR PERI-ARTHRITIS OR PERIARTHRITIDES OR PERIARTHRITIDES OR PERI-CAPSULITIS OR PERICAPSULITIS) (6W) SHOULDER?/TI,AB,DE

? s shoulder? (3W) (pain or pains or painful or complain?)/ti,ab,de 1917 SHOULDER?/TI,AB,DE 1751 PAIN/TI,AB,DE 121 PAINS/TI,AB,DE 245 PAINFUL/TI,AB,DE 2900 COMPLAIN?/TI,AB,DE S7 19 SHOULDER? (3W) (PAIN OR PAINS OR PAINFUL OR COMPLAIN?)/TI,AB,DE

? s shoulder? (6W) impinge?/ti,ab,de 1917 SHOULDER?/TI,AB,DE 2858 IMPINGE?/TI,AB,DE S8 1 SHOULDER? (6W) IMPINGE?/TI,AB,DE

? s subacromial (W) impingement (W) syndrome/ti,ab,de 0 SUBACROMIAL/TI,AB,DE 1922 IMPINGEMENT/TI,AB,DE 3547 SYNDROME/TI,AB,DE S9 0 SUBACROMIAL (W) IMPINGEMENT (W) SYNDROME/TI,AB,DE

? s s1:s9

S10 26 S1:S9

? s arthrograph? (6W) (distension? or distention?)/ti,ab,de 4 ARTHROGRAPH?/TI,AB,DE 50 DISTENSION?/TI,AB,DE 39 DISTENTION?/TI,AB,DE S11 0 ARTHROGRAPH? (6W) (DISTENSION? OR DISTENTION?)/TI,AB,DE

? s arthrogram? (6W) (distension? or distention?)/ti,ab,de 0 ARTHROGRAM?/TI,AB,DE 
50 DISTENSION?/TI,AB,DE 39 DISTENTION?/TI,AB,DE

S12 0 ARTHROGRAM? (6W) (DISTENSION? OR DISTENTION?)/TI,AB,DE

? s glenohumeral (6W) (distension? or distention?)/ti,ab,de

5 GLENOHUMERAL/TI,AB,DE

50 DISTENSION?/TI,AB,DE

39 DISTENTION?/TI,AB,DE

S13 0 GLENOHUMERAL (6W) (DISTENSION? OR DISTENTION?)/TI,AB,DE

? s dilatation or hydrodilat?/ti,ab,de

336 DILATATION

0 HYDRODILAT?/TI,AB,DE

S14 336 DILATATION OR HYDRODILAT?/TI,AB,DE

? s s11:s14

S15 336 S11:S14

? s s 10 and s15

$26 \mathrm{~S} 10$

$336 \mathrm{~S} 15$

S16 0 S10 AND S15

? s arthroscop? (6W) (releas? or decompress? or capsulotom?)/ti,ab,de

11 ARTHROSCOP?/TI,AB,DE

45473 RELEAS?/TI,AB,DE

1628 DECOMPRESS?/TI,AB,DE

8 CAPSULOTOM?/TI,AB,DE

S17 0 ARTHROSCOP? (6W) (RELEAS? OR DECOMPRESS? OR CAPSULOTOM?)/TI,AB,DE

? s capsular (2W) releas? or interventional (W) microadhesiolysis or capsulotomy/ti,ab,de

121 CAPSULAR

46080 RELEAS?

0 CAPSULAR(2W)RELEAS?

72 INTERVENTIONAL

0 MICROADHESIOLYSIS

0 INTERVENTIONAL(W)MICROADHESIOLYSIS

5 CAPSULOTOMY/TI,AB,DE

S18 5 CAPSULAR (2W) RELEAS? OR INTERVENTIONAL (W) MICROADHESIOLYSIS OR

CAPSULOTOMY/TI,AB,DE

? s s17:s 18

S19 5 S17:S18

? s s 10 and s19

$26 \mathrm{~S} 10$

$5 \mathrm{~S} 19$

S20 0 S10 AND S19

? s injection?/ti,ab,de

$>>$ Term "DE.." is not defined in file 6 and is ignored

S21 22468 INJECTION?/TI,AB,DE.. 


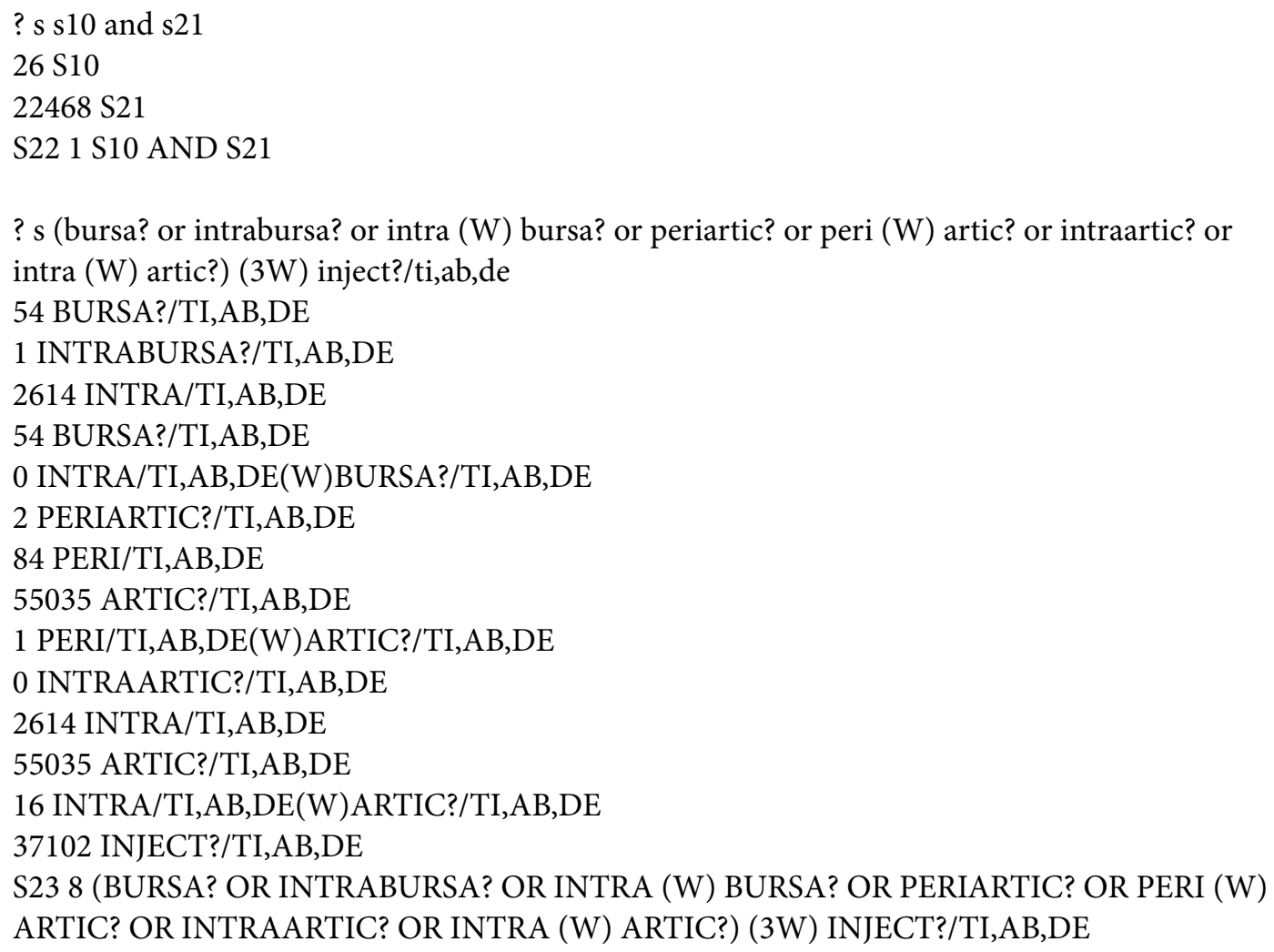


? s physiotherapy or physiotherapies or physical (W) therap? or manual(W) therap?/ti,ab,de 38 PHYSIOTHERAPY

0 PHYSIOTHERAPIES

226243 PHYSICAL

19307 THERAP?

1223 PHYSICAL(W)THERAP?

37866 MANUAL/TI,AB,DE

18488 THERAP?/TI,AB,DE

1 MANUAL/TI,AB,DE(W)THERAP?/TI,AB,DE

S28 1253 PHYSIOTHERAPY OR PHYSIOTHERAPIES OR PHYSICAL (W) THERAP? OR

MANUAL(W) THERAP?/TI,AB,DE

? s passive (W) (motion or movement)/ti,ab, de

13922 PASSIVE/TI,AB,DE

$56426 \mathrm{MOTION} / \mathrm{TI}, \mathrm{AB}, \mathrm{DE}$

19107 MOVEMENT/TI,AB,DE

S29 11 PASSIVE (W) (MOTION OR MOVEMENT)/TI,AB,DE

? s CPM/ti,ab,de

S30 $356 \mathrm{CPM} / \mathrm{TI}, \mathrm{AB}, \mathrm{DE}$

? s (stretching or stretches)/ti,ab,de

2022 STRETCHING/TI,AB,DE

319 STRETCHES/TI,AB,DE

S31 2331 (STRETCHING OR STRETCHES)/TI,AB,DE

? s (mobilisation or mobilization)/ti,ab,de

41 MOBILISATION/TI,AB,DE

2774 MOBILIZATION/TI,AB,DE

S32 2812 (MOBILISATION OR MOBILIZATION)/TI,AB,DE

? s exercise? $(2 \mathrm{~W})$ (program? or strength? or intervention? or training or prescription? or prescrib?)/ti,ab,de

14367 EXERCISE?/TI,AB,DE

480068 PROGRAM?/TI,AB,DE

72882 STRENGTH?/TI,AB,DE

8460 INTERVENTION?/TI,AB,DE

71866 TRAINING/TI,AB,DE

2399 PRESCRIPTION?/TI,AB,DE

8170 PRESCRIB?/TI,AB,DE

S33 651 EXERCISE? (2W) (PROGRAM? OR STRENGTH? OR INTERVENTION? OR

TRAINING OR PRESCRIPTION? OR PRESCRIB?)/TI,AB,DE

? s exercise? (2W) (therap? or therapeutic)/ti,ab,de

14367 EXERCISE?/TI,AB,DE

18488 THERAP?/TI,AB,DE

5073 THERAPEUTIC/TI,AB,DE

S34 37 EXERCISE? (2W) (THERAP? OR THERAPEUTIC)/TI,AB,DE

? s (home or supervis?) (2W) exercis?/ti,ab,de

14925 HOME/TI,AB,DE

10621 SUPERVIS?/TI,AB,DE 
14861 EXERCIS?/TI,AB,DE

S35 17 (HOME OR SUPERVIS?) (2W) EXERCIS?/TI,AB,DE

? s (pendular or pendulum) (W) exercis?/ti,ab,de

39 PENDULAR/TI,AB,DE

952 PENDULUM/TI,AB,DE

14861 EXERCIS?/TI,AB,DE

S36 0 (PENDULAR OR PENDULUM) (W) EXERCIS?/TI,AB,DE

? s (isokinetic or resist?) (2W) exercise?/ti,ab,de

223 ISOKINETIC/TI,AB,DE

74785 RESIST?/TI,AB,DE

14367 EXERCISE?/TI,AB,DE

S37 68 (ISOKINETIC OR RESIST?) (2W) EXERCISE?/TI,AB,DE

? s s28:s37

S38 7465 S28:S37

? s s 10 and s 38

$26 \mathrm{~S} 10$

7465 S38

S39 1 S10 AND S38

? s chiropractic?/ti,ab,de

S40 39 CHIROPRACTIC?/TI,AB,DE

? s osteopath?/ti,ab,de

S41 199 OSTEOPATH?/TI,AB,DE

? s manipulat? (3W) (anesthesia or anaesthesia or anesthetic? or anaesthetic? or musculoskeletal)/

ti,ab,de

13446 MANIPULAT?/TI,AB,DE

1006 ANESTHESIA/TI,AB,DE

47 ANAESTHESIA/TI,AB,DE

723 ANESTHETIC?/TI,AB,DE

41 ANAESTHETIC?/TI,AB,DE

1646 MUSCULOSKELETAL/TI,AB,DE

S42 1 MANIPULAT? (3W) (ANESTHESIA OR ANAESTHESIA OR ANESTHETIC? OR

ANAESTHETIC? OR MUSCULOSKELETAL)/TI,AB,DE

? s MUA/ti,ab,de

S43 $27 \mathrm{MUA} / \mathrm{TI}, \mathrm{AB}, \mathrm{DE}$

? s s40:s43

S44 257 S40:S43

? s s 10 and s 44

$26 \mathrm{~S} 10$

257 S44

S45 0 S10 AND S44 


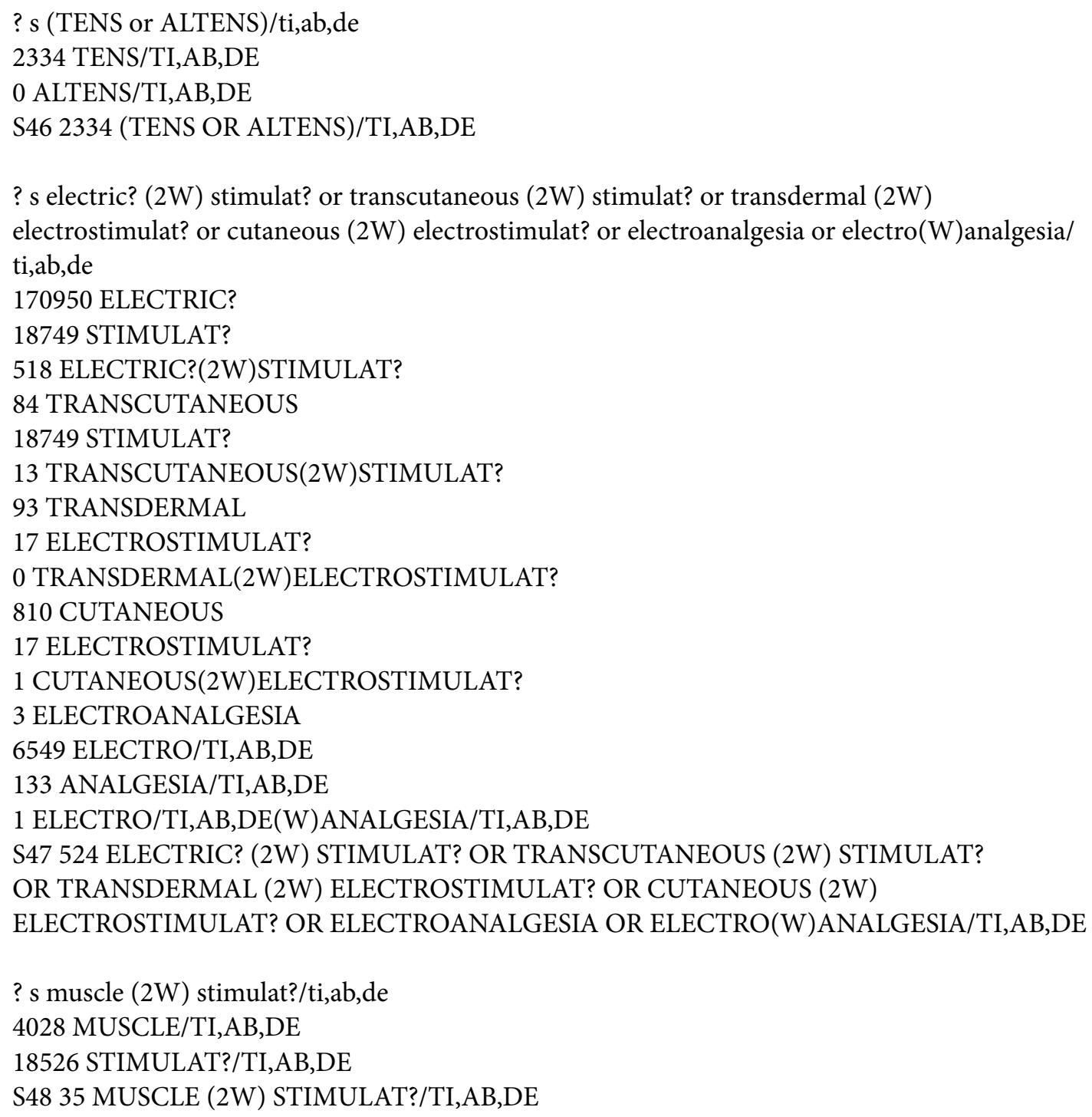




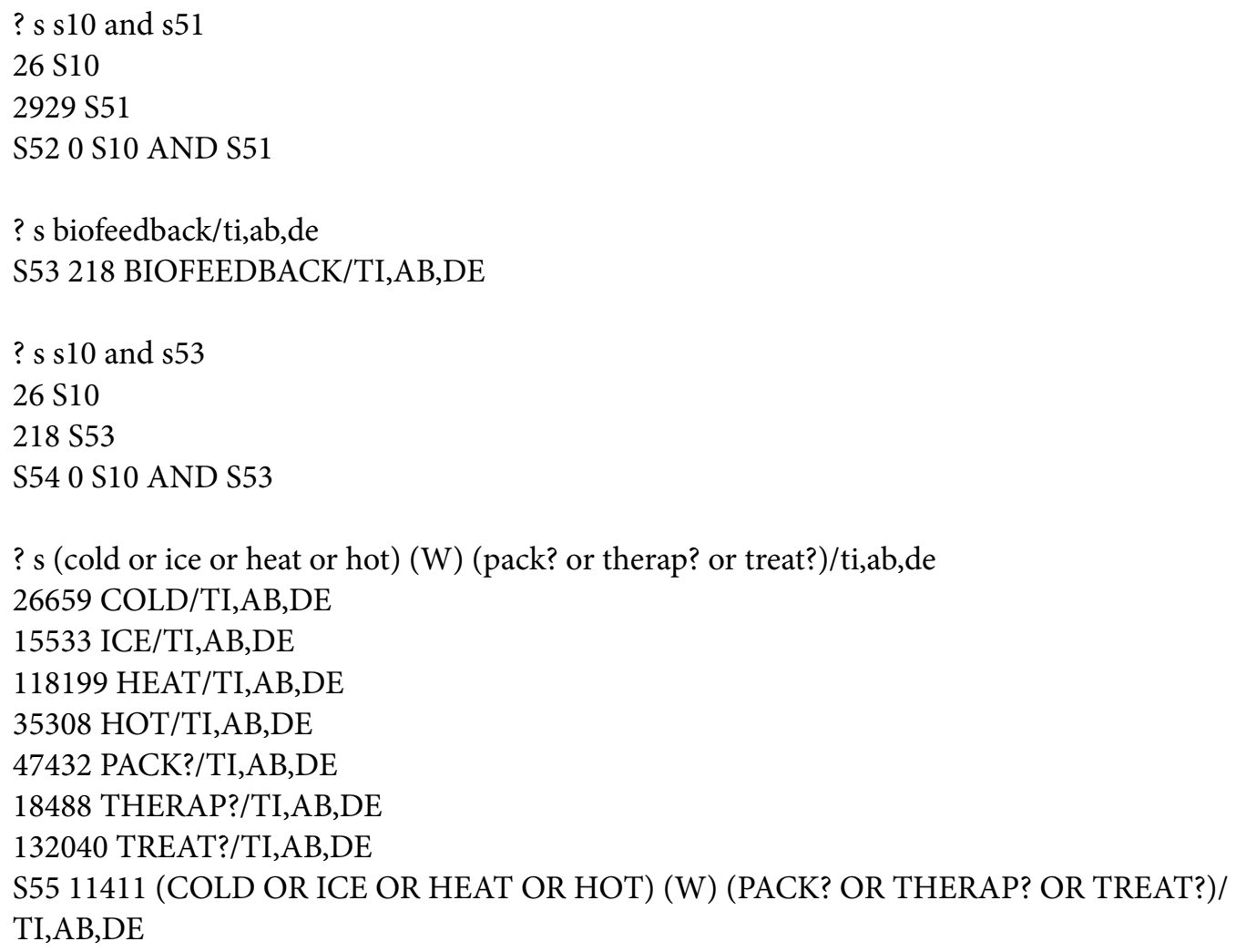




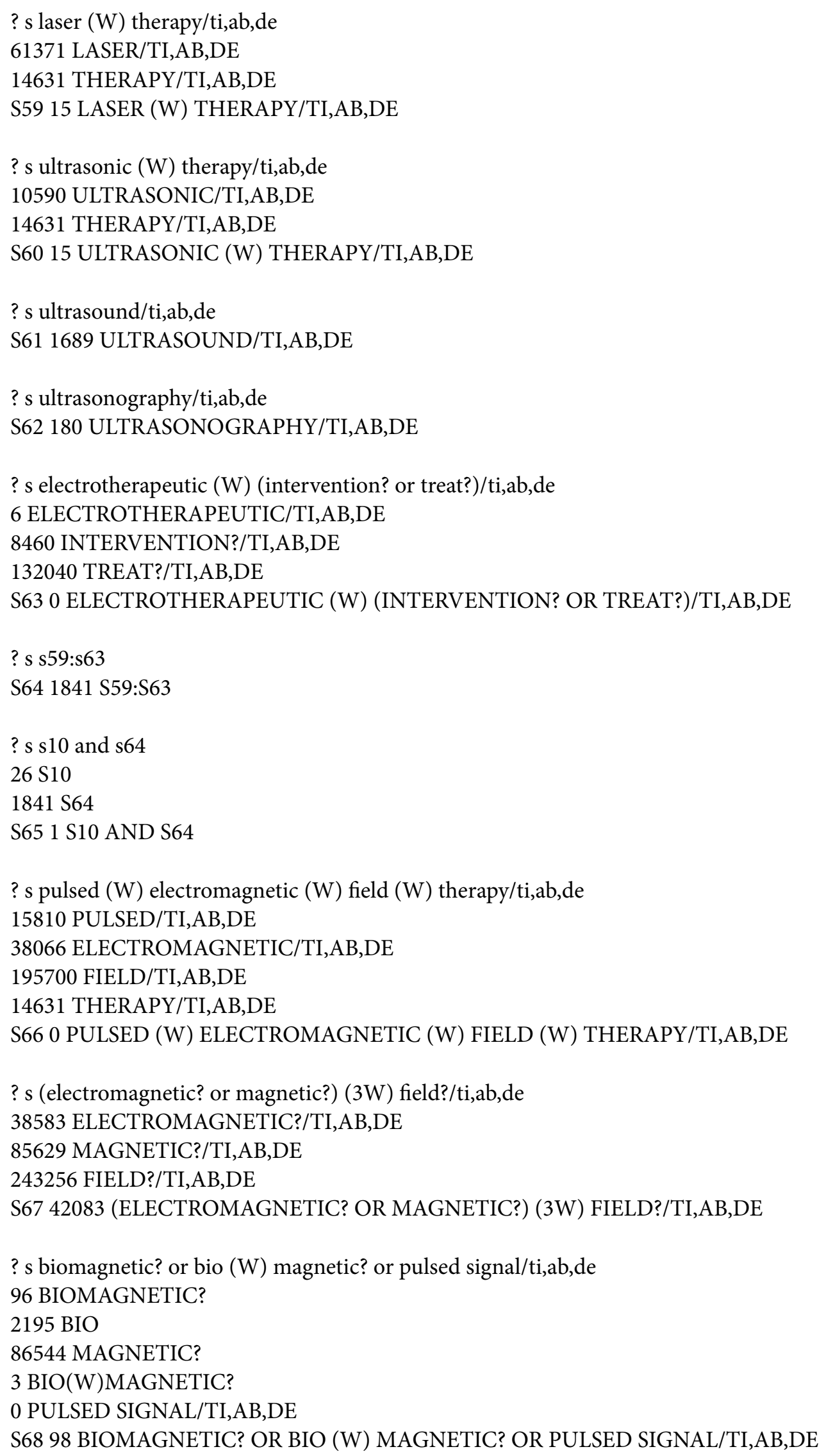


? s PEMF/ti,ab,de

S69 5 PEMF/TI,AB,DE

? s s66:s69

S70 42137 S66:S69

? s s 10 and $\mathrm{s} 70$

$26 \mathrm{~S} 10$

42137 S70

S71 0 S10 AND S70

? s neuromuscular (W) blockade/ti,ab,de

839 NEUROMUSCULAR/TI,AB,DE

$582 \mathrm{BLOCKADE} / \mathrm{TI}, \mathrm{AB}, \mathrm{DE}$

S72 19 NEUROMUSCULAR (W) BLOCKADE/TI,AB,DE

? s nerve (2W) block?/ti,ab,de

6188 NERVE/TI,AB,DE

25659 BLOCK?/TI,AB,DE

S73 170 NERVE (2W) BLOCK?/TI,AB,DE

? s s72:s73

S74 180 S72:S73

? s s 10 and s74

$26 \mathrm{~S} 10$

$180 \mathrm{~S} 74$

S75 0 S10 AND S74

? s acupuncture?/ti,ab,de

S76 87 ACUPUNCTURE?/TI,AB,DE

? s electroacupuncture? or electro (W) acupuncture?/ti,ab,de 6 ELECTROACUPUNCTURE?

6549 ELECTRO/TI,AB,DE

87 ACUPUNCTURE?/TI,AB,DE

4 ELECTRO/TI,AB,DE(W)ACUPUNCTURE?/TI,AB,DE

S77 8 ELECTROACUPUNCTURE? OR ELECTRO (W) ACUPUNCTURE?/TI,AB,DE

? s osteopuncture? or osteo (W) puncture?/ti,ab,de

0 OSTEOPUNCTURE?

6 OSTEO/TI,AB,DE

602 PUNCTURE?/TI,AB,DE

0 OSTEO/TI,AB,DE(W)PUNCTURE?/TI,AB,DE

S78 0 OSTEOPUNCTURE? OR OSTEO (W) PUNCTURE?/TI,AB,DE

? s perioste? (3W) (stimulat? or therap? or needling)/ti,ab,de

38 PERIOSTE?/TI,AB,DE

18526 STIMULAT?/TI,AB,DE

18488 THERAP?/TI,AB,DE

14 NEEDLING/TI,AB,DE

S79 1 PERIOSTE? (3W) (STIMULAT? OR THERAP? OR NEEDLING)/TI,AB,DE 
? s s76:s79

S80 89 S76:S79

? s $s 10$ and s 80

$26 \mathrm{~S} 10$

$89 \mathrm{~S} 80$

S81 0 S10 AND S80

? s massag? or acupressure or shiatsu or shiatzu or zhi (W) ya or chih (W) ya/ti,ab,de

69 MASSAG?

2 ACUPRESSURE

0 SHIATSU

0 SHIATZU

$2 \mathrm{ZHI}$

125 YA

$0 \mathrm{ZHI}(\mathrm{W}) \mathrm{YA}$

$12 \mathrm{CHIH} / \mathrm{TI}, \mathrm{AB}, \mathrm{DE}$

$122 \mathrm{YA} / \mathrm{TI}, \mathrm{AB}, \mathrm{DE}$

$0 \mathrm{CHIH} / \mathrm{TI}, \mathrm{AB}, \mathrm{DE}(\mathrm{W}) \mathrm{YA} / \mathrm{TI}, \mathrm{AB}, \mathrm{DE}$

S82 71 MASSAG? OR ACUPRESSURE OR SHIATSU OR SHIATZU OR ZHI (W) YA OR CHIH (W) YA/TI,AB,DE

? s s 10 and s82

$26 \mathrm{~S} 10$

$71 \mathrm{~S} 82$

S83 0 S10 AND S82

? s rehabilitat? (2W) (program? or protocol?)/ti,ab,de

8675 REHABILITAT?/TI,AB,DE

480068 PROGRAM?/TI,AB,DE

14132 PROTOCOL?/TI,AB,DE

S84 864 REHABILITAT? (2W) (PROGRAM? OR PROTOCOL?)/TI,AB,DE

? s s 10 and s84

$26 \mathrm{~S} 10$

$864 \mathrm{~S} 84$

S85 1 S10 AND S84

? s watch? (2W) wait? or conservative $(2 \mathrm{~W})$ treat?/ti,ab,de

2243 WATCH?

2348 WAIT?

18 WATCH?(2W)WAIT?

5006 CONSERVATIVE/TI,AB,DE

132040 TREAT?/TI,AB,DE

28 CONSERVATIVE/TI,AB,DE(2W)TREAT?/TI,AB,DE

S86 46 WATCH? (2W) WAIT? OR CONSERVATIVE (2W) TREAT?/TI,AB,DE

? s s 10 and s 86

$26 \mathrm{~S} 10$

$46 \mathrm{~S} 86$

S87 0 S10 AND S86 


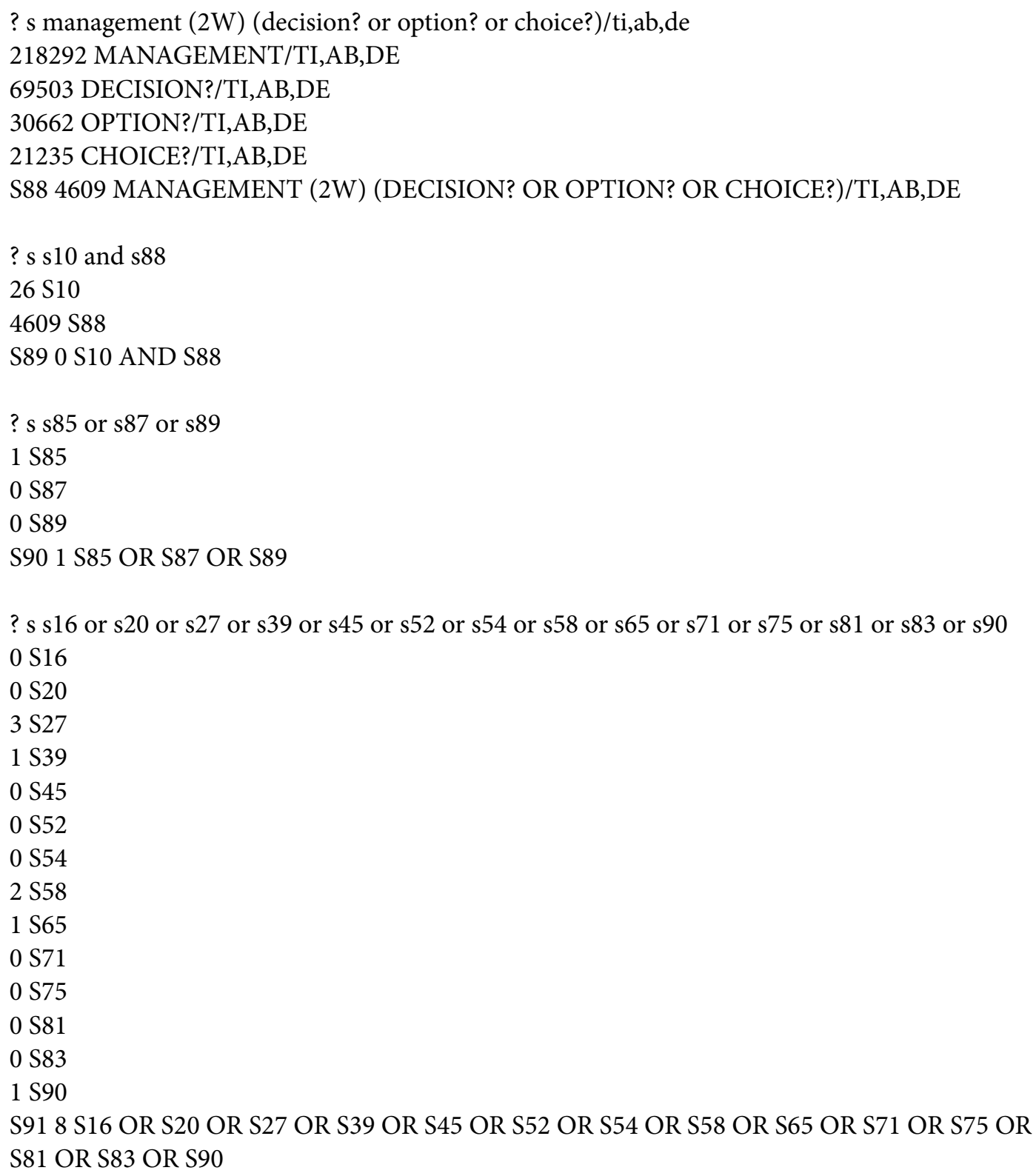

\section{PASCAL}

Interface: Dialog Classic

Search date: 30 March 2010

Strategy: as below

Records identified: 631

File 144:Pascal 1973-2010/Mar W1

(c) 2010 INIST/CNRS

? s frozen $(6 \mathrm{~W})$ shoulder?/ti,ab,de

29523 FROZEN/TI,AB,DE

18440 SHOULDER?/TI,AB,DE

S1 265 FROZEN (6W) SHOULDER?/TI,AB,DE 


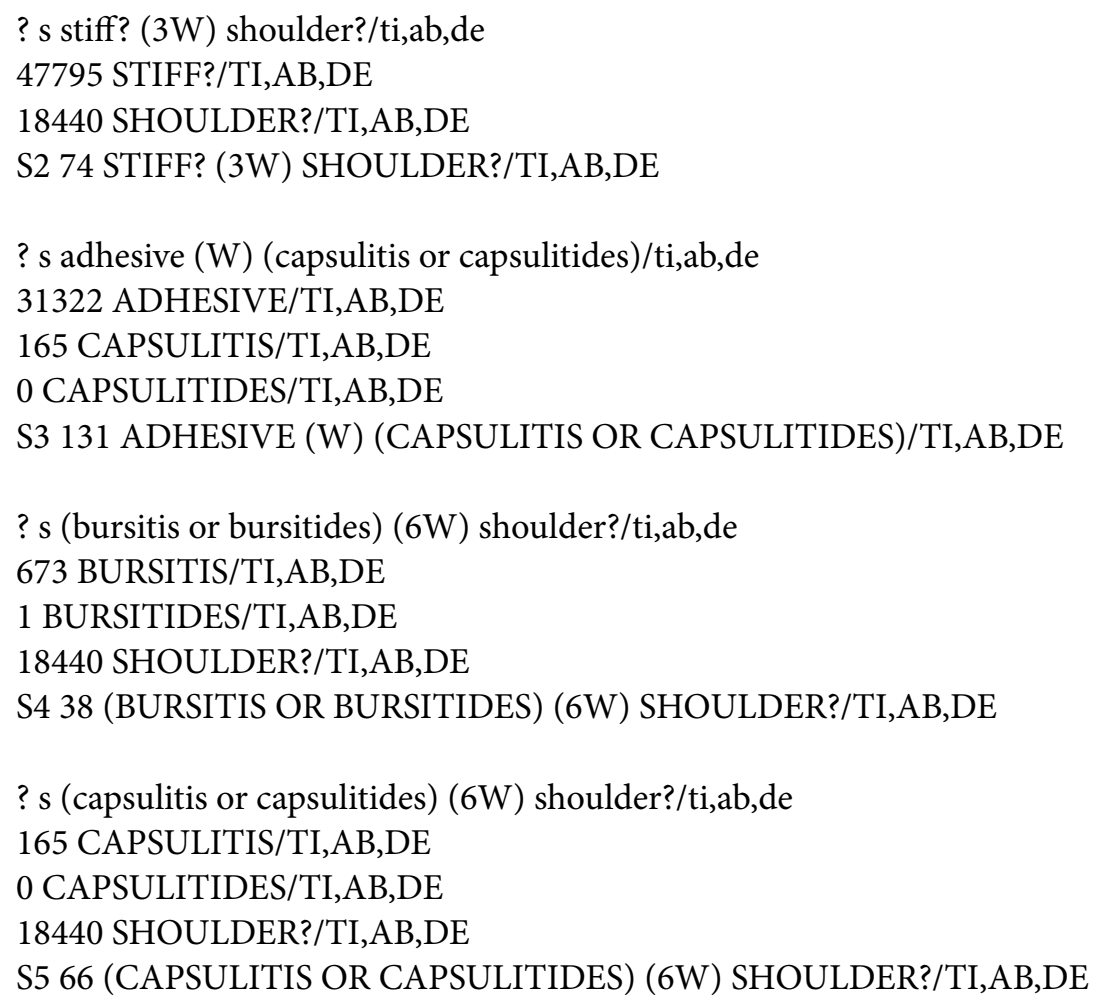


S9 147 SUBACROMIAL (W) IMPINGEMENT (W) SYNDROME/TI,AB,DE

? s s1:s9
S10 2177 S1:S9

? s arthrograph? (6W) (distension? or distention?)/ti,ab,de

2195 ARTHROGRAPH?/TI,AB,DE

5134 DISTENSION?/TI,AB,DE

1532 DISTENTION?/TI,AB,DE

S11 8 ARTHROGRAPH? (6W) (DISTENSION? OR DISTENTION?)/TI,AB,DE

? s arthrogram? (6W) (distension? or distention?)/ti,ab,de

228 ARTHROGRAM?/TI,AB,DE

5134 DISTENSION?/TI,AB,DE

1532 DISTENTION?/TI,AB,DE

S12 0 ARTHROGRAM? (6W) (DISTENSION? OR DISTENTION?)/TI,AB,DE

? s glenohumeral (6W) (distension? or distention?)/ti,ab,de

1197 GLENOHUMERAL/TI,AB,DE

5134 DISTENSION?/TI,AB,DE

1532 DISTENTION?/TI,AB,DE

S13 1 GLENOHUMERAL (6W) (DISTENSION? OR DISTENTION?)/TI,AB,DE

? s dilatation or hydrodilat?/ti,ab,de

56041 DILATATION

7 HYDRODILAT?/TI,AB,DE

S14 56047 DILATATION OR HYDRODILAT?/TI,AB,DE

? s s11:s14

S15 56055 S11:S14

? s s 10 and s15

2177 S10

$56055 \mathrm{~S} 15$

S16 10 S10 AND S15

? s arthroscop? (6W) (releas? or decompress? or capsulotom?)/ti,ab,de

8274 ARTHROSCOP?/TI,AB,DE

261595 RELEAS?/TI,AB,DE

13807 DECOMPRESS?/TI,AB,DE

1046 CAPSULOTOM?/TI,AB,DE

S17 306 ARTHROSCOP? (6W) (RELEAS? OR DECOMPRESS? OR CAPSULOTOM?)/TI,AB,DE

? s capsular (2W) releas? or interventional (W) microadhesiolysis or capsulotomy/ti,ab,de

6434 CAPSULAR

261595 RELEAS?

92 CAPSULAR(2W)RELEAS?

9302 INTERVENTIONAL

0 MICROADHESIOLYSIS

0 INTERVENTIONAL(W)MICROADHESIOLYSIS

925 CAPSULOTOMY/TI,AB,DE 
S18 1012 CAPSULAR (2W) RELEAS? OR INTERVENTIONAL (W) MICROADHESIOLYSIS OR CAPSULOTOMY/TI,AB,DE

? s s17:s 18

S19 1264 S17:S18

? s s 10 and s19

$2177 \mathrm{~S} 10$

1264 S19

S20 99 S10 AND S19

? s injection?/ti,ab,de

>> Term "DE.." is not defined in file 144 and is ignored

S21 225170 INJECTION?/TI,AB,DE.

? s s 10 and s 21

$2177 \mathrm{~S} 10$

225170 S21

S22 161 S10 AND S21

? s (bursa? or intrabursa? or intra (W) bursa? or periartic? or peri (W) artic? or intraartic? or intra (W) artic?) (3W) inject?/ti,ab,de

3240 BURSA?/TI,AB,DE

54 INTRABURSA?/TI,AB,DE

66732 INTRA/TI,AB,DE

3240 BURSA?/TI,AB,DE

3 INTRA/TI,AB,DE(W)BURSA?/TI,AB,DE

1029 PERIARTIC?/TI,AB,DE

9053 PERI/TI,AB,DE

705828 ARTIC?/TI,AB,DE

162 PERI/TI,AB,DE(W)ARTIC?/TI,AB,DE

2544 INTRAARTIC?/TI,AB,DE

66732 INTRA/TI,AB,DE

705828 ARTIC?/TI,AB,DE

2857 INTRA/TI,AB,DE(W)ARTIC?/TI,AB,DE

309289 INJECT?/TI,AB,DE

S23 1060 (BURSA? OR INTRABURSA? OR INTRA (W) BURSA? OR PERIARTIC? OR PERI

(W) ARTIC? OR INTRAARTIC? OR INTRA (W) ARTIC?) (3W) INJECT?/TI,AB,DE

? s s 10 and s 23

2177 S10

1060 S23

S24 34 S10 AND S23

? s (subacromial or acromioclavicular or glenohumeral) (3W) inject?/ti,ab,de

598 SUBACROMIAL/TI,AB,DE

667 ACROMIOCLAVICULAR/TI,AB,DE

1197 GLENOHUMERAL/TI,AB,DE

309289 INJECT?/TI,AB,DE

S25 62 (SUBACROMIAL OR ACROMIOCLAVICULAR OR GLENOHUMERAL) (3W)

INJECT?/TI,AB,DE 
? s (extra (W) articular or extraarticular or shoulder?) (3W) inject?/ti,ab,de

40008 EXTRA/TI,AB,DE

15525 ARTICULAR/TI,AB,DE

608 EXTRA/TI,AB,DE(W)ARTICULAR/TI,AB,DE

321 EXTRAARTICULAR/TI,AB,DE

18440 SHOULDER?/TI,AB,DE

309289 INJECT?/TI,AB,DE

S26 54 (EXTRA (W) ARTICULAR OR EXTRAARTICULAR OR SHOULDER?) (3W) INJECT?/

TI,AB,DE

? s s 22 or s 24 or s 25 or $\mathrm{s} 26$

$161 \mathrm{~S} 22$

$34 \mathrm{~S} 24$

62 S25

$54 \mathrm{~S} 26$

S27 229 S22 OR S24 OR S25 OR S26

? s physiotherapy or physiotherapies or physical (W) therap? or manual(W) therap?/ti,ab,de 5062 PHYSIOTHERAPY

2 PHYSIOTHERAPIES

540408 PHYSICAL

661276 THERAP?

1884 PHYSICAL(W)THERAP?

35077 MANUAL/TI,AB,DE

661276 THERAP?/TI,AB,DE

120 MANUAL/TI,AB,DE(W)THERAP?/TI,AB,DE

S28 6839 PHYSIOTHERAPY OR PHYSIOTHERAPIES OR PHYSICAL (W) THERAP? OR

MANUAL(W) THERAP?/TI,AB,DE

? s passive $(\mathrm{W})$ (motion or movement)/ti,ab,de

62904 PASSIVE/TI,AB,DE

$175972 \mathrm{MOTION} / \mathrm{TI}, \mathrm{AB}, \mathrm{DE}$

109985 MOVEMENT/TI,AB,DE

S29 511 PASSIVE (W) (MOTION OR MOVEMENT)/TI,AB,DE

? s CPM/ti,ab,de

S30 2329 CPM/TI,AB,DE

? s (stretching or stretches)/ti,ab,de

18320 STRETCHING/TI,AB,DE

1979 STRETCHES/TI,AB,DE

S31 20093 (STRETCHING OR STRETCHES)/TI,AB,DE

? s (mobilisation or mobilization)/ti,ab,de

10059 MOBILISATION/TI,AB,DE

14519 MOBILIZATION/TI,AB,DE

S32 19880 (MOBILISATION OR MOBILIZATION)/TI,AB,DE

? s exercise? (2W) (program? or strength? or intervention? or training or prescription? or prescrib?)/ti,ab,de 83485 EXERCISE?/TI,AB,DE

496453 PROGRAM?/TI,AB,DE 


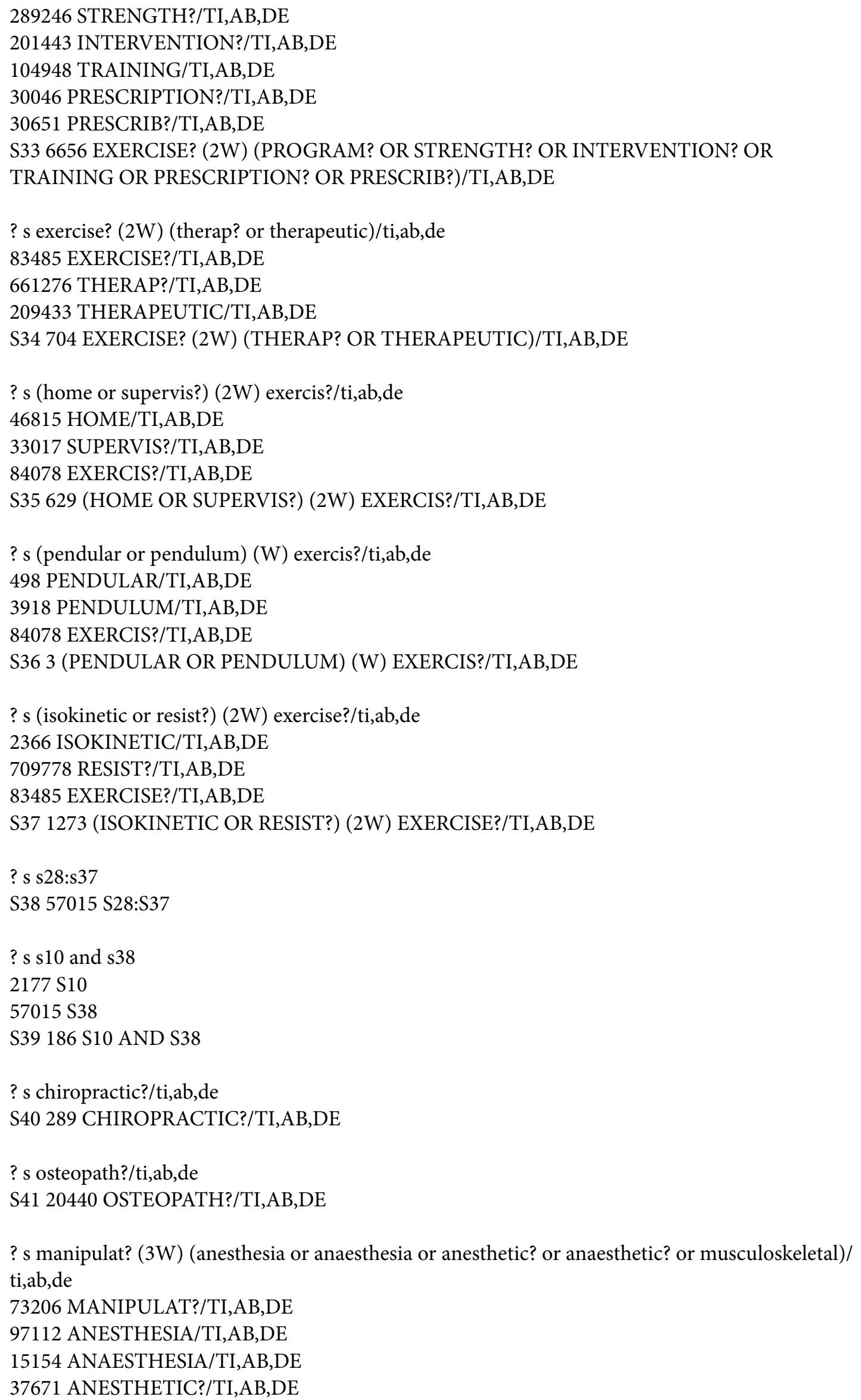


7809 ANAESTHETIC?/TI,AB,DE

7339 MUSCULOSKELETAL/TI,AB,DE

S42 130 MANIPULAT? (3W) (ANESTHESIA OR ANAESTHESIA OR ANESTHETIC? OR ANAESTHETIC? OR MUSCULOSKELETAL)/TI,AB,DE

? s MUA/ti,ab,de

S43 258 MUA/TI,AB,DE

? s s40:s43

S44 21093 S40:S43

? s s 10 and s 44

2177 S 10

21093 S44

S45 48 S10 AND S44

? s (TENS or ALTENS)/ti,ab,de

9868 TENS/TI,AB,DE

1 ALTENS/TI,AB,DE

S46 9869 (TENS OR ALTENS)/TI,AB,DE

? s electric? $(2 \mathrm{~W})$ stimulat? or transcutaneous $(2 \mathrm{~W})$ stimulat? or transdermal $(2 \mathrm{~W})$

electrostimulat? or cutaneous (2W) electrostimulat? or electroanalgesia or electro(W)analgesia/

ti,ab,de

640281 ELECTRIC?

347681 STIMULAT?

15569 ELECTRIC?(2W)STIMULAT?

3402 TRANSCUTANEOUS

347681 STIMULAT?

843 TRANSCUTANEOUS(2W)STIMULAT?

4939 TRANSDERMAL

1104 ELECTROSTIMULAT?

6 TRANSDERMAL(2W)ELECTROSTIMULAT?

40793 CUTANEOUS

1104 ELECTROSTIMULAT?

0 CUTANEOUS(2W)ELECTROSTIMULAT?

22 ELECTROANALGESIA

31673 ELECTRO/TI,AB,DE

20137 ANALGESIA/TI,AB,DE

4 ELECTRO/TI,AB,DE(W)ANALGESIA/TI,AB,DE

S47 15738 ELECTRIC? (2W) STIMULAT? OR TRANSCUTANEOUS (2W) STIMULAT?

OR TRANSDERMAL (2W) ELECTROSTIMULAT? OR CUTANEOUS (2W)

ELECTROSTIMULAT? OR ELECTROANALGESIA OR ELECTRO(W)ANALGESIA/TI,AB,DE

? s muscle (2W) stimulat?/ti,ab,de

248852 MUSCLE/TI,AB,DE

347681 STIMULAT?/TI,AB,DE

S48 1748 MUSCLE (2W) STIMULAT?/TI,AB,DE

? s neuromodulation or neuro (W) modulation or neurostimulation or neuro (W) stimulation/

ti,ab,de

1804 NEUROMODULATION 
11977 NEURO
134029 MODULATION
11 NEURO(W)MODULATION
428 NEUROSTIMULATION
11977 NEURO/TI,AB,DE
187063 STIMULATION/TI,AB,DE
17 NEURO/TI,AB,DE(W)STIMULATION/TI,AB,DE

S49 2211 NEUROMODULATION OR NEURO (W) MODULATION OR

NEUROSTIMULATION OR NEURO (W) STIMULATION/TI,AB,DE

? s interferential/ti,ab,de

S50 264 INTERFERENTIAL/TI,AB,DE

? s s46:550

S51 28886 S46:S50

? s s 10 and s51

$2177 \mathrm{~S} 10$

28886 S51

S52 26 S10 AND S51

? s biofeedback/ti,ab,de

S53 2966 BIOFEEDBACK/TI,AB,DE

? s s 10 and s53

$2177 \mathrm{~S} 10$

2966 S53

S54 0 S10 AND S53

? s (cold or ice or heat or hot) (W) (pack? or therap? or treat?)/ti,ab,de $95776 \mathrm{COLD} / \mathrm{TI}, \mathrm{AB}, \mathrm{DE}$

$45475 \mathrm{ICE} / \mathrm{TI}, \mathrm{AB}, \mathrm{DE}$

$392650 \mathrm{HEAT} / \mathrm{TI}, \mathrm{AB}, \mathrm{DE}$

$106420 \mathrm{HOT} / \mathrm{TI}, \mathrm{AB}, \mathrm{DE}$

138939 PACK?/TI,AB,DE

661276 THERAP?/TI,AB,DE

1969126 TREAT?/TI,AB,DE

S55 87567 (COLD OR ICE OR HEAT OR HOT) (W) (PACK? OR THERAP? OR TREAT?)/

TI,AB,DE

? s thermograph? or thermotherap? or thermo (W) therap? or hypertherm? or hyper (W) therm? or diatherm? or cryotherap? or cryo(W) therap?/ti,ab,de

7907 THERMOGRAPH?

1946 THERMOTHERAP?

16853 THERMO

661276 THERAP?

20 THERMO(W)THERAP?

15178 HYPERTHERM?

9780 HYPER

1038992 THERM?

48 HYPER(W)THERM?

1188 DIATHERM? 


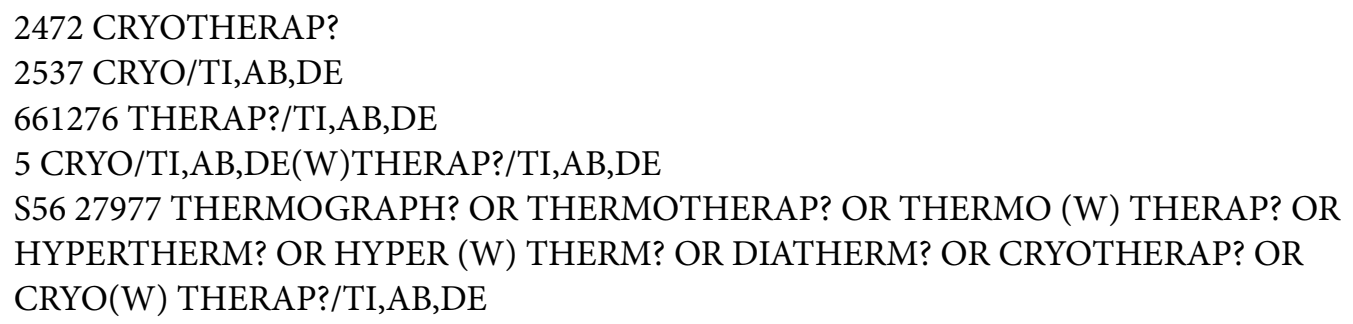




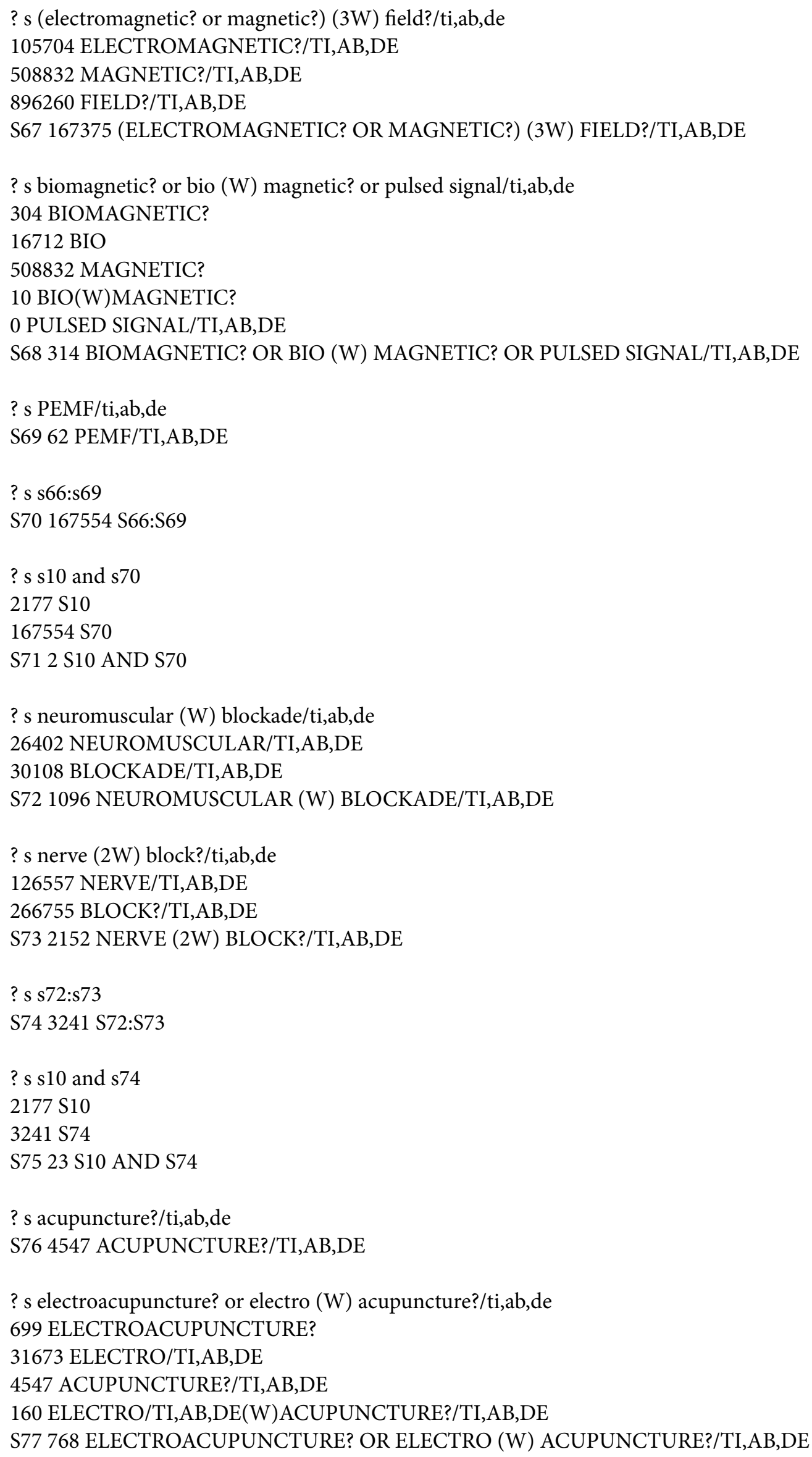




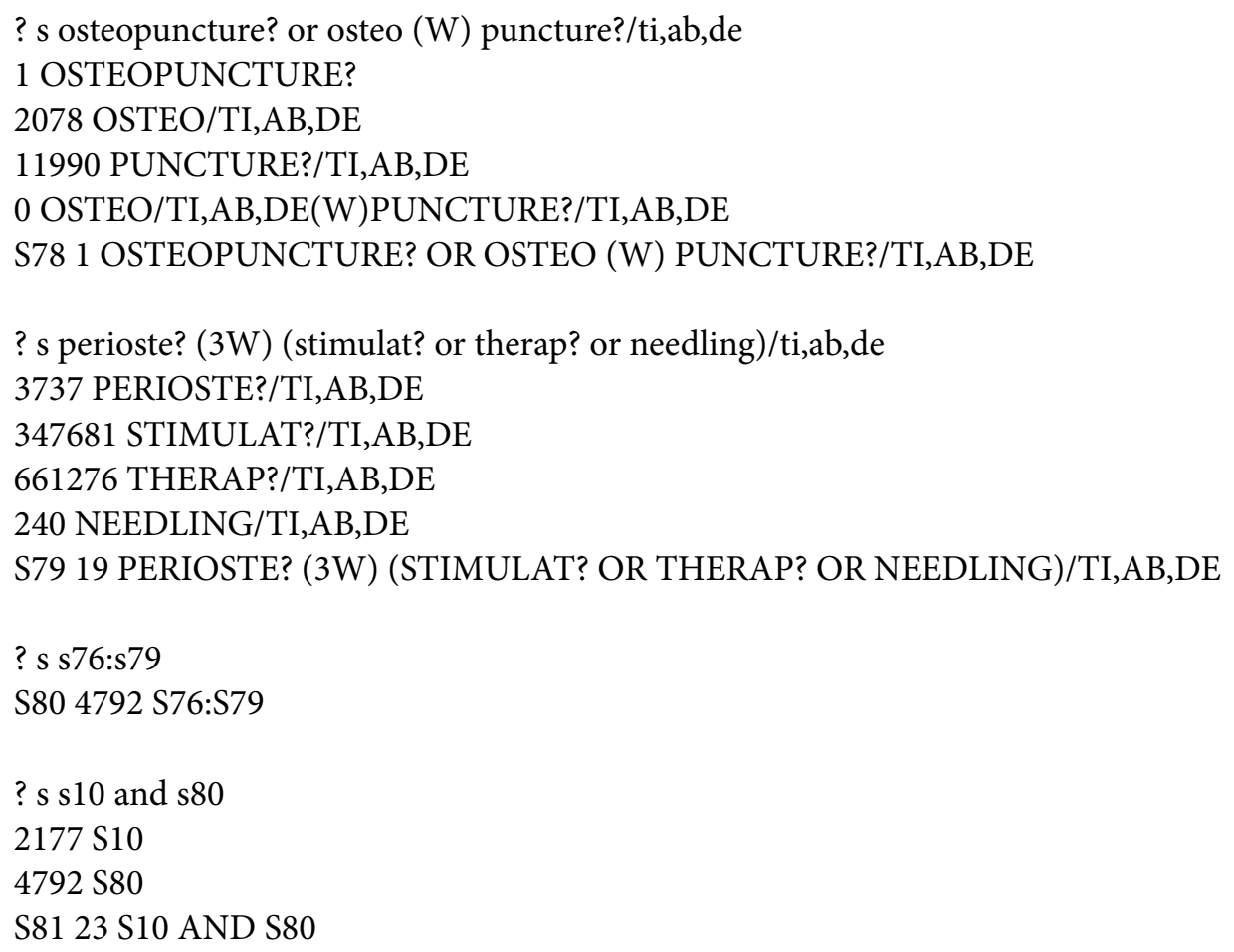




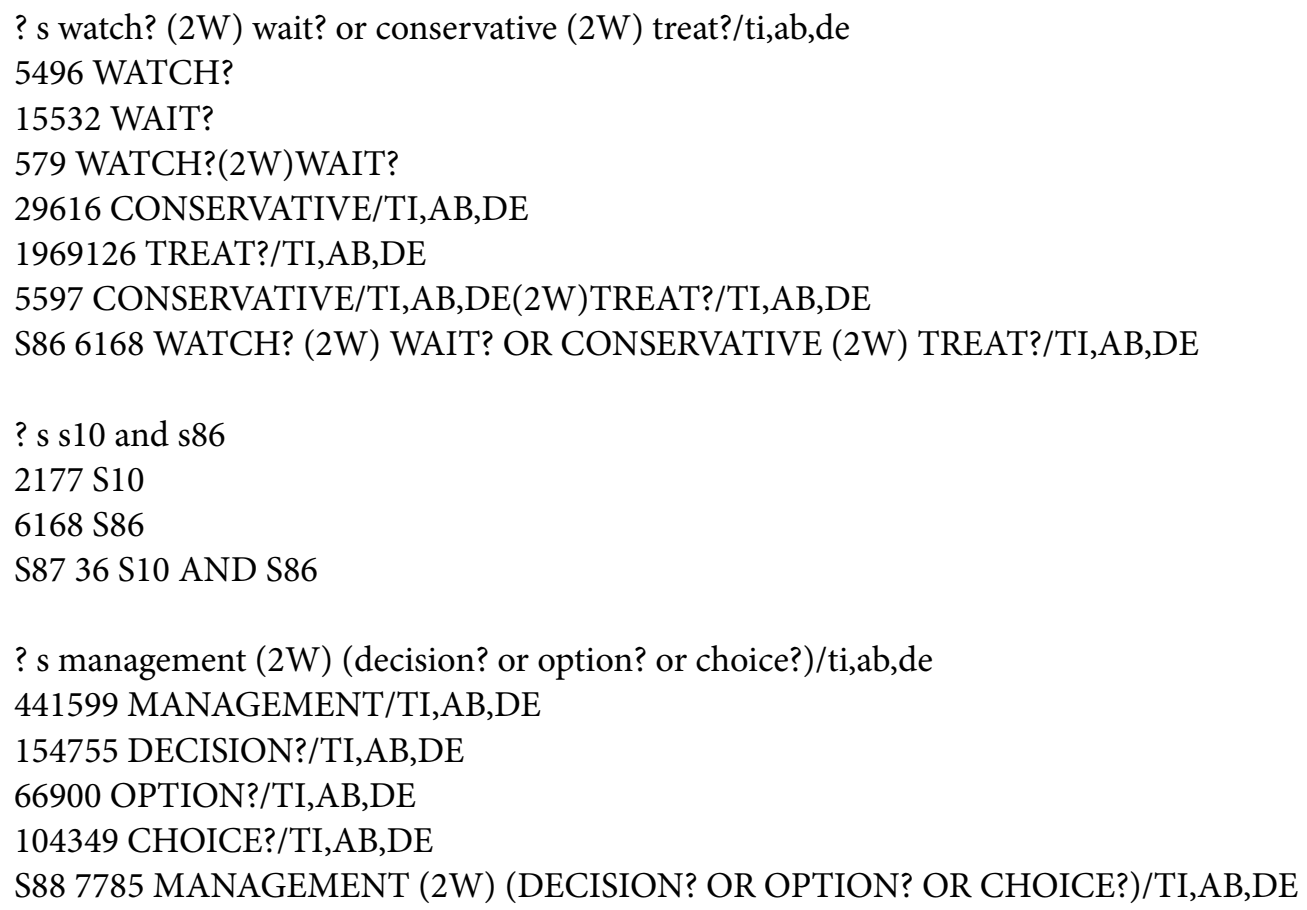




\section{Physiotherapy Evidence Database (PEDro)}

Interface: http://www.pedro.org.au/

Search date: 19 April 2010

Strategy: The database interface does not allow sophisticated searches to be undertaken so a broad search was carried out using the advanced search option. The body part 'upper arm, shoulder or shoulder girdle' was selected from the dropdown list provided and all the records identified from this search were downloaded.

Records identified: 718

\section{PREMEDLINE}

Interface: OvidSP

Search date: 25 March 2010

Strategy: as below

Records identified: 82

Database: Ovid MEDLINE(R) In-Process \& Other Non-Indexed Citations < March 24, 2010>

1. (frozen adj6 shoulder\$).ti,ab. (22)

2. (stiff\$adj3 shoulder\$).ti,ab. (16)

3. (adhesive adj (capsulitis or capsulitides)).ti,ab. (19)

4. ((bursitis or bursitides) adj6 shoulder\$).ti,ab. (0)

5. ((capsulitis or capsulitides) adj6 shoulder\$).ti,ab. (9)

6. ((periarthritis or peri-arthritis or periarthritides or peri-arthritides or peri-capsulitis or pericapsulitis) adj6 shoulder\$).ti,ab. (7)

7. (shoulder\$adj3 (pain or pains or painful or complain\$)).ti,ab. (191)

8. (shoulder\$adj6 impinge\$).ti,ab. (23)

9. subacromial impingement syndrome.ti,ab. (11)

10. 1 or 2 or 3 or 4 or 5 or 6 or 7 or 8 or $9(245)$

11. (arthrograph\$adj6 (distension\$or distention\$)).ti,ab. (1)

12. (arthrogram $\$$ adj6 (distension\$or distention\$)).ti,ab. (0)

13. (glenohumeral adj6 (distension\$or distention\$)).ti,ab. (0)

14. (dilatation or hydrodilat\$).ti,ab. (780)

15. (arthroscop\$adj6 (releas\$or decompress\$or capsulotom\$)).ti,ab. (25)

16. ((capsular adj2 releas\$) or interventional microadhesiolysis or capsulotomy).ti,ab. (43)

17. ((bursa\$or intrabursa\$or intra bursa\$or periartic\$or peri artic\$or intraartic\$or intra artic\$) adj3 inject\$).ti,ab. (92)

18. ((subacromial or acromioclavicular or glenohumeral) adj3 inject\$).ti,ab. (8)

19. ((extra articular or extraarticular or shoulder\$) adj3 inject\$).ti,ab. (1)

20. (physiotherapy or physiotherapies or physical therap\$or manual therap\$).ti,ab. (929)

21. (passive adj (motion or movement)).ti,ab. (51)

22. CPM.ti,ab. (129)

23. (stretching or stretches).ti,ab. (2719)

24. (mobilisation or mobilization).ti,ab. (806)

25. (exercise\$adj2 (program\$or strength\$or intervention\$or training or prescription\$or prescrib\$)).ti,ab. (641) 
26. (exercise\$adj2 (therap\$or therapeutic)).ti,ab. (112)

27. ((home or supervis\$) adj2 exercis\$).ti,ab. (89)

28. ((pendular or pendulum) adj exercis\$).ti,ab. (1)

29. ((isokinetic or resist\$) adj2 exercise\$).ti,ab. (126)

30. chiropractic $\$ . t i, a b .(454)$

31. osteopath\$.ti,ab. (136)

32. (manipulat\$adj3 (anesthesia or anaesthesia or anesthetic\$or anaesthetic\$)).ti,ab. (13)

33. MUA.ti,ab. (75)

34. (TENS or ALTENS).ti,ab. (1018)

35. ((electric $\$$ adj2 stimulat $\$$ ) or (transcutaneous adj2 stimulat\$) or (transdermal adj2 electrostimulat\$) or (cutaneous adj2 electrostimulat\$) or electroanalgesia or electro analgesia).ti,ab. (759)

36. (muscle adj2 stimulat\$).ti,ab. (93)

37. (neuromodulation or neuro modulation or neurostimulation or neuro stimulation).ti,ab. (93)

38. interferential.ti,ab. (41)

39. biofeedback.ti,ab. (119)

40. ((cold or ice or heat or hot) adj (pack\$or therap\$or treat\$)).ti,ab. (661)

41. (thermograph\$or thermotherap\$or thermo therap\$or hypertherm\$or hyper therm\$or diatherm\$or cryotherap\$or cryo therap\$).ti,ab. (821)

42. ((laser or ultrasonic) adj2 (therap\$or treat\$)).ti,ab. (384)

43. ultrasound.ti,ab. (4964)

44. (electrotherapeutic adj (intervention\$or treat\$)).ti,ab. (2)

45. pulsed electromagnetic field therapy.ti,ab. (1)

46. ((electromagnetic\$or magnetic\$) adj3 field\$).ti,ab. (6171)

47. (biomagnetic $\$$ or bio magnetic\$or pulsed signal).ti,ab. (29)

48. PEMF.ti,ab. (9)

49. (nerve adj2 block\$).ti,ab. (233)

50. neuromuscular block\$.ti,ab. (117)

51. acupuncture\$.ti,ab. (676)

52. (electroacupuncture $\$$ or electro acupuncture $\$$ ).ti,ab. (130)

53. (osteopuncture $\$$ or osteo puncture $\$$ ).ti,ab. (0)

54. (perioste\$adj3 (stimulat\$or therap\$or needling)).ti,ab. (4)

55. (massag\$or acupressure or shiatsu or shiatzu or zhi ya or chih ya).ti,ab. (223)

56. (rehabilitat\$adj2 (program\$or protocol\$)).ti,ab. (373)

57. ((watch\$adj2 wait\$) or (conservative adj2 treat\$)).ti,ab. (690)

58. (management adj2 (decision\$or option\$or choice\$)).ti,ab. (328)

59. or/11-58 (23503)

60. 10 and $59(82)$

61. limit 60 to yr="1966 -Current" (82)

62. from 61 keep $1-82(82)$

63. from 62 keep 1-82 (82) 


\section{Science Citation Index}

\section{Interface: Web of Science (ISI) via Web of Knowledge}

Search date: 30 March 2010

Strategy: as below

Records identified: 3140

\begin{tabular}{|c|c|c|}
\hline \multirow[t]{2}{*}{ \# 20} & 3140 & \#19 OR \#17 OR \#15 OR \#13 OR \#11 OR \#9 OR \#5 OR \#3 \\
\hline & & Timespan=All Years \\
\hline \multirow[t]{2}{*}{ \# 19} & 729 & \#18 AND \#1 \\
\hline & & Timespan=All Years \\
\hline \multirow[t]{2}{*}{ \# 18} & $>100,000$ & 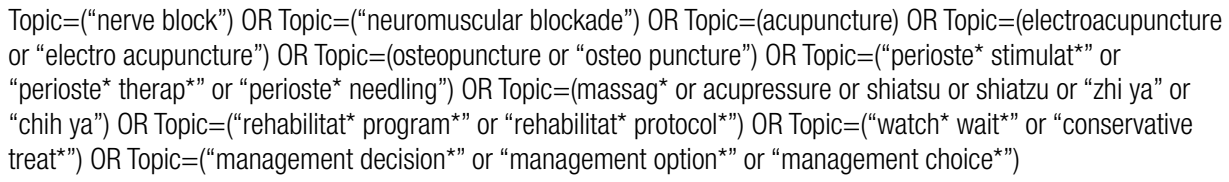 \\
\hline & & Timespan=All Years \\
\hline \multirow[t]{2}{*}{ \# 17} & 221 & \#16 AND \#1 \\
\hline & & Timespan=All Years \\
\hline \multirow[t]{2}{*}{ \# 16} & $>100,000$ & 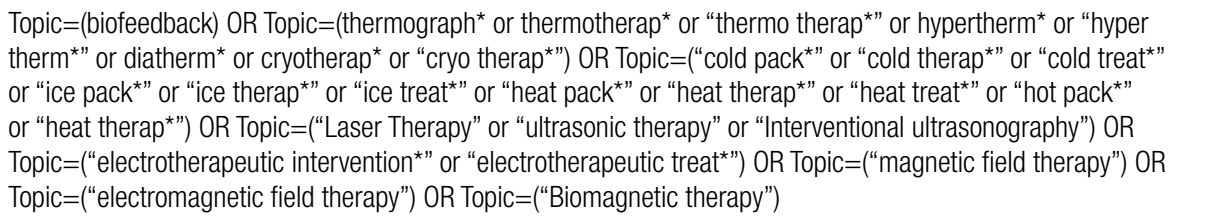 \\
\hline & & Timespan=All Years \\
\hline \multirow[t]{2}{*}{ \# 15} & 162 & \#14 AND \#1 \\
\hline & & Timespan=All Years \\
\hline \multirow[t]{2}{*}{ \# 14} & $>100,000$ & $\begin{array}{l}\text { Topic=(TENS or ALTENS) OR Topic=("electric* stimulat*" or "transcutaneous stimulat*"or "transdermal } \\
\text { electrostimulat”"or "cutaneous electrostimulat"or electroanalgesia or "electro analgesia") OR Topic=(“muscle } \\
\text { stimulat”") OR Topic=(neuromodulation or "neuro modulation" or neurostimulation or "neuro stimulation") OR } \\
\text { Topic=(interferential) }\end{array}$ \\
\hline & & Timespan=All Years \\
\hline \multirow[t]{2}{*}{ \# 13} & 184 & \#12 AND \#1 \\
\hline & & Timespan=All Years \\
\hline \multirow[t]{2}{*}{ \# 12} & 19,962 & $\begin{array}{l}\text { Topic }=\left(\text { chiropractic }^{\star}\right) \text { OR Topic }=\left(\text { osteopath }^{\star}\right) \text { OR Topic }=\left(\text { manipulat }{ }^{\star} \text { and }\left(\text { anesthesia or anaesthesia or anesthetic }{ }^{*} \text { or }\right.\right. \\
\left.\left.\text { anaesthetic }{ }^{\star}\right)\right) \text { OR Topic }=(\mathrm{MUA})\end{array}$ \\
\hline & & Timespan=All Years \\
\hline \multirow[t]{2}{*}{ \# 11} & 1502 & \#10 AND \#1 \\
\hline & & Timespan=All Years \\
\hline \multirow[t]{2}{*}{ \# 10} & $>100,000$ & 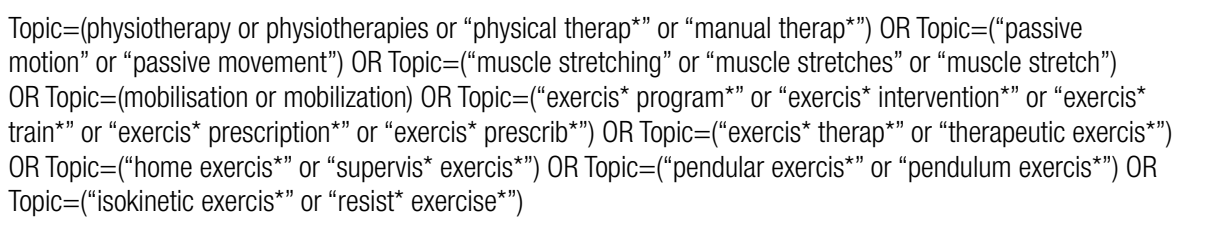 \\
\hline & & Timespan=All Years \\
\hline \multirow[t]{2}{*}{ \# 9} & 609 & \#8 OR \#7 \\
\hline & & Timespan=All Years \\
\hline \multirow[t]{2}{*}{ \# 8} & 121 & $\begin{array}{l}\text { Topic=("subacromial inject*” or "acromioclavicular inject*” or "glenohumeral inject*”) OR Topic=("extra articular } \\
\text { inject*” or "extraarticular inject*”) OR Topic=(“shoulder* inject*") }\end{array}$ \\
\hline & & Timespan=All Years \\
\hline \multirow[t]{2}{*}{$\# 7$} & 533 & \#6 AND \#1 \\
\hline & & Timespan=All Years \\
\hline
\end{tabular}




\begin{tabular}{|c|c|c|}
\hline \multirow[t]{2}{*}{$\# 6$} & $>100,000$ & 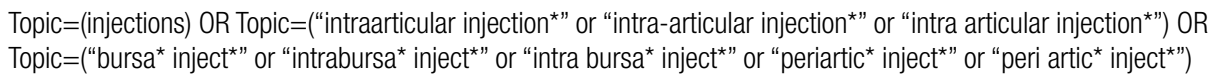 \\
\hline & & Timespan=All Years \\
\hline \multirow[t]{2}{*}{ \# 5} & 800 & \#4 AND \#1 \\
\hline & & Timespan=All Years \\
\hline \multirow[t]{2}{*}{$\# 4$} & 20,598 & $\begin{array}{l}\text { Topic=(Arthroscopy) OR Topic=("arthroscop* releas" or "arthroscop* decompress" or "arthroscop* capsulotom*”) } \\
\text { OR Topic=("capsular releas" or "interventional microadhesiolysis" or capsulotomy) }\end{array}$ \\
\hline & & Timespan=All Years \\
\hline \multirow[t]{2}{*}{ \# 3} & 12 & \#2 AND \#1 \\
\hline & & Timespan=All Years \\
\hline \multirow[t]{2}{*}{ \# 2} & 67 & $\begin{array}{l}\text { Topic=("arthrograph* distension*" or "arthograph* disten") OR Topic=("arthrogram* distension*" or "arthogram* } \\
\text { distention*") OR Topic=("glenohumeral distension*" or "glenohumeral distention*") OR Topic=(arthography) }\end{array}$ \\
\hline & & Timespan=All Years \\
\hline \multirow[t]{2}{*}{$\# 1$} & 8973 & $\begin{array}{l}\text { Topic=("frozen shoulde*”") OR Topic=("stiff* shoulder*") OR Topic=("adhesive capsulitis") OR Topic=("shoulder } \\
\text { bursitis") OR Topic=(periarthritis or peri-arthritis or periarthritides or peri-arthritides or peri-capsulitis or } \\
\text { pericapsulitis) OR Topic=("shoulder pain*" or "pain* shoulder" or "shoulder complaint"”) OR Topic=("shoulder" } \\
\text { impinge*") OR Topic=("subacromial impingement syndrome") }\end{array}$ \\
\hline & & Timespan=All Years \\
\hline
\end{tabular}

\section{Additional quality of life search strategy for information to inform the decision-analytic model}

Database: Ovid MEDLINE(R) <1950 to October Week 4 2010>

1. (sf36 or sf 36).tw. (9054)

2. (eq5d or eq $5 \mathrm{~d}$ or euroqol or euro qol).tw. (2024)

3. (short form 36 or shortform 36 or sf thirtysix or sf thirty six or shortform thirtysix or shortform thirty six or short form thirtysix or short form thirty six).tw. (4194)

4. (hrql or hrqol or h qol or hql or hqol).tw. (5810)

5. (hye or hyes or health\$year\$equivalent\$or health utilit\$).tw. (733)

6. health related quality of life.tw. (13228)

7. rosser.tw. (67)

8. (standard gamble\$or time trade off or time tradeoff or tto or willingness to pay).tw. (2460)

9. (disutilities or disutility or daly or disability adjusted life).tw. (1022)

10. "Quality of Life"/(87323)

11. health status indicators/(15760)

12. quality adjusted life year/(4741)

13. (qaly\$or quality adjusted life or quality of life or life quality).tw. (98027)

14. qwb\$.tw. (157)

15. (quality of wellbeing or quality of well being).tw. (275)

16. factor analysis.tw. (15866)

17. preference based.tw. (418)

18. health status.tw. (27567)

19. (state adj2 (value or values or valuing or valued)).tw. (1507)

20. hspv.tw. (2)

21. 1 or 2 or 3 or 4 or 5 or 6 or 7 or 8 or 9 or 10 or 11 or 12 or 13 or 14 or 15 or 16 or 17 or 18 or 19 or $20(185879)$

22. "VAS 0-100mm".mp. (4)

23. "VAS 0-10cm".mp. (1)

24. (VAS and pain).mp. (7387)

25. 10 point numeric scale.mp. (5) 
26. "4 point category rating scale".mp. (0)

27. Category rating scale.mp. (24)

28. Likert scale $0-10 . \mathrm{mp}$. (2)

29. (likert scale and pain).mp. (295)

30. SPADI 5-item pain subscale.mp. (0)

31. SPADI.mp. (76)

32. 22 or 23 or 24 or 25 or 26 or 27 or 28 or 29 or 30 or 31 (7739)

33. 21 and 32 (1173)

34. American Shoulder \& Elbow Surgeons score.mp. (2)

35. Constant score.mp. (869)

36. Flexilevel Scale of Shoulder Function.mp. (6)

37. Oxford shoulder scale.mp. (1)

38. Problem Elicitation Technique score.mp. (1)

39. Shoulder Disability score.mp. (1)

40. SPADI total score.mp. (3)

41. UCLA shoulder score.mp. (24)

42. Shoulder Disability Questionnaire.mp. (28)

43. Croft score.mp. (1)

44. DASH score.mp. (420)

45. (shoulder and Telephone questionnaire).mp. (4)

46. 34 or 35 or 36 or 37 or 38 or 39 or 40 or 41 or 42 or 43 or 44 or 45 (1313)

47. 21 and $46(131)$

48. 33 or $47(1282)$ 


\section{Appendix 2}

\section{Search strategy for review of views of people with frozen shoulder}

Three databases (CINAHL, MEDLINE and PsycINFO) were searched to identify qualitative 1 studies, with searches being restricted to English-language studies published from 1980 onwards. The search strategies used are given in full below.

\section{Cumulative Index to Nursing and Allied Health Literature (CINAHL)}

Interface: EBSCO

Search date: 3 June 2010, date coverage 1980-2010

Strategy: as below

Records identified: 311

\begin{tabular}{|c|c|}
\hline S24 & (S21 and S22 and S23) \\
\hline S23 & 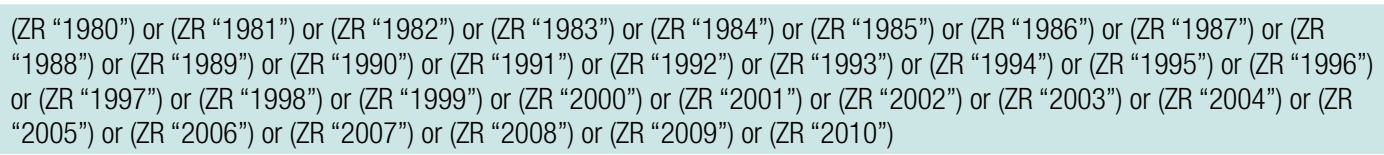 \\
\hline S22 & (ZL "english") \\
\hline S21 & $(\mathrm{S} 15$ or $\mathrm{S} 16$ or $\mathrm{S} 17$ or $\mathrm{S} 18$ or $\mathrm{S} 19)$ and (S14) \\
\hline S20 & $\mathrm{S} 15$ or $\mathrm{S} 16$ or $\mathrm{S} 17$ or $\mathrm{S} 18$ or $\mathrm{S} 19$ \\
\hline S19 & TX qualitative \\
\hline S18 & (ZU “interviews”) \\
\hline S17 & TX interview* \\
\hline S16 & TX findings \\
\hline S15 & TX findings \\
\hline S14 & $\mathrm{S} 1$ or $\mathrm{S} 2$ or $\mathrm{S} 3$ or $\mathrm{S} 4$ or $\mathrm{S} 5$ or $\mathrm{S} 6$ or $\mathrm{S} 7$ or $\mathrm{S} 8$ or $\mathrm{S} 9$ or $\mathrm{S} 10$ or $\mathrm{S} 11$ or $\mathrm{S} 12$ or $\mathrm{S} 13$ \\
\hline S13 & subacromial impingement syndrome \\
\hline S12 & shoulder* N6 impinge* \\
\hline S11 & (MH “Shoulder Impingement Syndrome”) \\
\hline S10 & shoulder* N3 pain or shoulder* N3 pains or shoulder* N3 painful or shoulder* N3 complain* \\
\hline S9 & (MH “Shoulder Pain") \\
\hline S8 & $\begin{array}{l}\text { periarthritis N6 shoulder* or peri-arthritis N6 shoulder* or periarthritides N6 shoulder* or peri-arthritides N6 shoulder* or } \\
\text { pericapsulitis N6 shoulder* or peri-capsulitis N6 shoulder* }\end{array}$ \\
\hline S7 & capsulitis N6 shoulder* \\
\hline S6 & bursitides N6 shoulder* \\
\hline S5 & bursitis N6 shoulder* \\
\hline S4 & adhesive N3 capsulitis \\
\hline S3 & (MH “Adhesive Capsulitis") \\
\hline S2 & stiff* N3 shoulder* \\
\hline S1 & frozen N6 shoulder* \\
\hline
\end{tabular}




\section{MEDLINE}

Interface: OvidSP

Search date: 3 June 2010

Strategy: as below

Records identified: 915

Database: Ovid MEDLINE(R) <1950 to May Week 4 2010>

1. (frozen adj6 shoulder\$).ti,ab. (599)

2. (stiff\$adj3 shoulder\$).ti,ab. (361)

3. exp bursitis/(3130)

4. (adhesive adj (capsulitis or capsulitides)).ti,ab. (336)

5. ((bursitis or bursitides) adj6 shoulder\$).ti,ab. (65)

6. ((capsulitis or capsulitides) adj6 shoulder\$).ti,ab. (195)

7. ((periarthritis or peri-arthritis or periarthritides or peri-arthritides or peri-capsulitis or pericapsulitis) adj6 shoulder\$).ti,ab. (176)

8. shoulder pain/(1861)

9. (shoulder\$adj3 (pain or pains or painful or complain\$)).ti,ab. (4029)

10. Shoulder Impingement Syndrome/(953)

11. (shoulder\$adj6 impinge\$).ti,ab. (505)

12. subacromial impingement syndrome.ti,ab. (117)

13. 1 or 2 or 3 or 4 or 5 or 6 or 7 or 8 or 9 or 10 or 11 or $12(9213)$

14. findings.af. (930557)

15. interview\$.af. (174135)

16. Interview/(20706)

17. qualitative.af. (81067)

18. 14 or 15 or 16 or 17 (1132990)

19. 13 and 18 (1085)

20. limit 19 to english language (928)

21. limit 20 to $\mathrm{yr}=$ "1980 -Current" (915)

\section{Psycinfo}

Interface: OvidSP

Search date: 3 June 2011

Strategy: as below

Records identified: 79

Database: PsycINFO <1967 to May Week 4 2010>

1. (frozen adj6 shoulder\$).ti,ab. (6)

2. (stiff\$adj3 shoulder\$).ti,ab. (22)

3. (adhesive adj (capsulitis or capsulitides)).ti,ab. (2)

4. ((bursitis or bursitides) adj6 shoulder\$).ti,ab. (2)

5. ((capsulitis or capsulitides) adj6 shoulder\$).ti,ab. (2)

6. ((periarthritis or peri-arthritis or periarthritides or peri-arthritides or peri-capsulitis or pericapsulitis) adj6 shoulder\$).ti,ab. (0)

7. (shoulder\$adj3 (pain or pains or painful or complain\$)).ti,ab. (234)

8. (shoulder\$adj6 impinge\$).ti,ab. (7)

9. subacromial impingement syndrome.ti,ab. (1) 
10. 1 or 2 or 3 or 4 or 5 or 6 or 7 or 8 or $9(263)$

11. findings.af. (407232)

12. interview\$.af. (228353)

13. interviews/(4877)

14. qualitative.af. (117407)

15. 11 or 12 or 13 or $14(613112)$

16. 10 and $15(81)$

17. limit 16 to (english language and $\mathrm{yr}=$ "1980 -Current") (79)

18. from 17 keep $1-79$ (79) 



\title{
Appendix 3
}

\section{$\mathrm{R}$ code for standard deviation}

\author{
$\mathrm{M}<-10000000$ \\ $\mathrm{N}<-15$ \\ omean $<-3.47$ \\ thesds $<-\operatorname{rep}(-1,100000)$ \\ $\mathrm{j}<-1$ \\ for (i in 1:M) \\ $\mathrm{x}<-$ sample $(0: 5, \mathrm{~N}$, replace $=\mathrm{T})$ \\ $\mathrm{mu}<-\operatorname{mean}(\mathrm{x})$ \\ if $($ signif $(\mathrm{mu}, 3)==$ omean $)\{$ \\ thesds $[\mathrm{j}]<-\operatorname{sd}(\mathrm{x})$ \\ $\mathrm{j}<-\mathrm{j}+1$ \\ \} \\ \} \\ thesds $<-$ thesds[thesds $!=-1]$ \\ mean(thesds)
}





\section{Health Technology Assessment programme}

\author{
Director, \\ Professor Tom Walley, CBE, \\ Director, NIHR HTA programme, \\ Professor of Clinical Pharmacology, \\ University of Liverpool
}

\author{
Deputy Director, \\ Professor Hywel Williams, \\ Professor of Dermato-Epidemiology, \\ Centre of Evidence-Based Dermatology, \\ University of Nottingham
}

\section{Prioritisation Group}

Members

\section{Chair,}

Professor Tom Walley, CBE, Director, NIHR HTA programme, Professor of Clinical Pharmacology, University of Liverpool

Professor Imti Choonara, Professor in Child Health, Academic Division of Child Health, University of Nottingham Chair - Pharmaceuticals Panel

Dr Bob Coates,

Consultant Advisor - Disease Prevention Panel

Dr Andrew Cook

Consultant Advisor - Intervention Procedures Panel

Dr Peter Davidson, Director of NETSCC, Health Technology Assessment
Dr Nick Hicks,
Consultant Adviser - Diagnostic

Technologies and Screening Panel, Consultant Advisor-Psychological and Community Therapies Panel

Ms Susan Hird,

Consultant Advisor, External

Devices and Physical Therapies Panel

Professor Sallie Lamb, Director, Warwick Clinical Trials Unit, Warwick Medical School, University of Warwick

Chair - HTA Clinical Evaluation and Trials Board

Professor Jonathan Michaels, Professor of Vascular Surgery, Sheffield Vascular Institute, University of Sheffield Chair - Interventional Procedures Panel
Professor Ruairidh Milne, Director - External Relations

Dr John Pounsford, Consultant Physician, Directorate of Medical Services, North Bristol NHS Trust

Chair - External Devices and Physical Therapies Panel

Dr Vaughan Thomas, Consultant Advisor -

Pharmaceuticals Panel, Clinical Lead - Clinical Evaluation Trials Prioritisation Group

Professor Margaret Thorogood, Professor of Epidemiology, Health Sciences Research Institute,

University of Warwick Chair - Disease Prevention Panel
Professor Lindsay Turnbull, Professor of Radiology, Centre for the MR Investigations, University of Hull

Chair - Diagnostic Technologies and Screening Panel

Professor Scott Weich, Professor of Psychiatry, Health Sciences Research Institute, University of Warwick Chair - Psychological and Community Therapies Panel

Professor Hywel Williams, Director of Nottingham Clinical Trials Unit, Centre of EvidenceBased Dermatology, University of Nottingham

Chair - HTA Commissioning

Board

Deputy HTA Programme Director

\section{HTA Commissioning Board}

\section{Chair,}

Professor Hywel Williams,

Professor of Dermato-Epidemiology, Centre of Evidence-Based Dermatology,

University of Nottingham
Deputy Chair,

Professor Jon Deeks,

Department of Public Health and

Epidemiology,

University of Birmingham
Professor Tom Walley, CBE,

Professor of Clinical Pharmacology,

Director, NIHR HTA programme,

University of Liverpoo

\section{Members}

Professor Ann Ashburn,

Professor of Rehabilitation and

Head of Research, Southampton

General Hospital

Professor Judith Bliss,

Director of ICR-Clinical Trials

and Statistics Unit, The Institute of

Cancer Research

Professor Peter Brocklehurst, Professor of Women's Health, Institute for Women's Health, University College London

Professor David Fitzmaurice, Professor of Primary Care Research, Department of Primary Care Clinical Sciences, University of Birmingham
Professor John W Gregory,

Professor in Paediatric

Endocrinology, Department of Child Health, Wales School of Medicine, Cardiff University

Professor Steve Halligan, Professor of Gastrointestinal Radiology, University College Hospital, London

Professor Angela Harden, Professor of Community and Family Health, Institute for Health and Human Development, University of East London

Dr Martin J Landray, Reader in Epidemiology, Honorary Consultant Physician, Clinical Trial Service Unit, University of Oxford
Dr Joanne Lord,

Reader, Health Economics

Research Group, Brunel University

Professor Stephen Morris, Professor of Health Economics, University College London Research Department of Epidemiology and Public Health, University College London

Professor Dion Morton,

Professor of Surgery, Academic Department of Surgery, University of Birmingham

Professor Gail Mountain Professor of Health Services Research, Rehabilitation and Assistive Technologies Group, University of Sheffield
Professor Irwin Nazareth, Professor of Primary Care and Head of Department, Department of Primary Care and Population Sciences, University College London

Professor E Andrea Nelson, Professor of Wound Healing and Director of Research, School of Healthcare, University of Leeds

Professor John David Norrie, Chair in Clinical Trials and Biostatistics, Robertson Centre for Biostatistics, University of Glasgow

Dr Rafael Perera,

Lecturer in Medical Statisitics, Department of Primary Health Care, University of Oxford 


\section{HTA Commissioning Board (continued)}

Professor Barney Reeves, Professorial Research Fellow in Health Services Research, Department of Clinical Science, University of Bristol
Professor Peter Tyrer, Professor of Community Psychiatry, Centre for Mental Health, Imperial College London

Professor Martin Underwood, Professor of Primary Care Research, Warwick Medical School, University of Warwick
Professor Caroline Watkins,

Professor of Stroke and Older People's Care, Chair of UK Forum for Stroke Training, Stroke Practice Research Unit, University of Central Lancashire
Dr Duncan Young,

Senior Clinical Lecturer and Consultant, Nuffield Department of Anaesthetics, University of Oxford

\section{Observers}

Dr Tom Foulks,

Medical Research Council
Dr Kay Pattison,

Senior NIHR Programme

Manager, Department of Health

\section{HTA Clinical Evaluation and Trials Board}

Chair,

Professor Sallie Lamb,

Director,

Warwick Clinical Trials Unit,

Warwick Medical School,

University of Warwick and Professor of

Rehabilitation,

Nuffield Department of Orthopaedic,

Rheumatology and Musculoskeletal Sciences,

University of Oxford

\section{Deputy Chair, \\ Professor Jenny Hewison,}

Professor of the Psychology of Health Care, Leeds Institute of Health Sciences,

University of Leeds

\section{Programme Director,}

Professor Tom Walley, CBE,

Director, NIHR HTA programme,

Professor of Clinical Pharmacology,

University of Liverpool

\section{Members}

Professor Keith Abrams,

Professor of Medical Statistics,

Department of Health Sciences,

University of Leicester

Professor Martin Bland,

Professor of Health Statistics,

Department of Health Sciences,

University of York

Professor Jane Blazeby,

Professor of Surgery and

Consultant Upper GI Surgeon,

Department of Social Medicine,

University of Bristo

Professor Julia M Brown,

Director, Clinical Trials Research

Unit, University of Leeds

Professor Alistair Burns, Professor of Old Age Psychiatry, Psychiatry Research Group, Schoo of Community-Based Medicine, The University of Manchester \& National Clinical Director for Dementia, Department of Health
Dr Jennifer Burr,

Director, Centre for Healthcare Randomised trials (CHART), University of Aberdeen

Professor Linda Davies, Professor of Health Economics, Health Sciences Research Group, University of Manchester

Professor Simon Gilbody, Prof of Psych Medicine and Health Services Research, Department of Health Sciences, University of York

Professor Steven Goodacre Professor and Consultant in Emergency Medicine, School of Health and Related Research, University of Sheffield

Professor Dyfrig Hughes, Professor of Pharmacoeconomics, Centre for Economics and Policy in Health, Institute of Medical and Social Care Research, Bangor University
Professor Paul Jones,

Professor of Respiratory Medicine,

Department of Cardiac and

Vascular Science, St George's

Hospital Medical School,

University of London

Professor Khalid Khan, Professor of Women's Health and Clinical Epidemiology, Barts and the London School of Medicine, Queen Mary, University of London

Professor Richard J McManus, Professor of Primary Care Cardiovascular Research, Primary Care Clinical Sciences Building, University of Birmingham

Professor Helen Rodgers, Professor of Stroke Care, Institute for Ageing and Health, Newcastle University

Professor Ken Stein,

Professor of Public Health, Peninsula Technology Assessment Group, Peninsula College of Medicine and Dentistry, Universities of Exeter and Plymouth
Professor Jonathan Sterne, Professor of Medical Statistics and Epidemiology, Department of Social Medicine, University of Bristol

Mr Andy Vail,

Senior Lecturer, Health Sciences Research Group, University of Manchester

Professor Clare Wilkinson, Professor of General Practice and Director of Research North Wales Clinical School, Department of Primary Care and Public Health, Cardiff University

Dr Ian B Wilkinson, Senior Lecturer and Honorary Consultant, Clinical Pharmacology Unit, Department of Medicine, University of Cambridge

\section{Observers}

Ms Kate Law,

Director of Clinical Trials, Cancer Research UK
Dr Morven Roberts,

Clinical Trials Manager, Health

Services and Public Health

Services Board, Medical Research

Council 


\section{Diagnostic Technologies and Screening Panel}

Members

\section{Chair,}

Professor Lindsay Wilson

Turnbull,

Scientific Director of the

Centre for Magnetic Resonance

Investigations and YCR Professor

of Radiology, Hull Royal Infirmary

Professor Judith E Adams,

Consultant Radiologist,

Manchester Royal Infirmary,

Central Manchester \& Manchester

Children's University Hospitals

NHS Trust, and Professor of

Diagnostic Radiology, University

of Manchester

Mr Angus S Arunkalaivanan,

Honorary Senior Lecturer,

University of Birmingham and

Consultant Urogynaecologist

and Obstetrician, City Hospital,

Birmingham

Dr Diana Baralle,

Consultant and Senior Lecturer

in Clinical Genetics, University of

Southampton

\section{Dr Stephanie Dancer,}

Consultant Microbiologist,

Hairmyres Hospital, East Kilbride

Dr Diane Eccles,

Professor of Cancer Genetics,

Wessex Clinical Genetics Service,

Princess Anne Hospital

Dr Trevor Friedman,

Consultant Liason Psychiatrist,

Brandon Unit, Leicester General Hospital

Dr Ron Gray,

Consultant, National Perinatal Epidemiology Unit, Institute of Health Sciences, University of Oxford

Professor Paul D Griffiths, Professor of Radiology, Academic Unit of Radiology, University of Sheffield

Mr Martin Hooper,

Public contributor
Professor Anthony Robert

Kendrick,

Associate Dean for Clinical

Research and Professor of Primary

Medical Care, University of

Southampton

Dr Nicola Lennard,

Senior Medical Officer, MHRA

Dr Anne Mackie,

Director of Programmes, UK

National Screening Committee, London

Mr David Mathew,

Public contributor

Dr Michael Millar,

Consultant Senior Lecturer in

Microbiology, Department of

Pathology \& Microbiology, Barts

and The London NHS Trust, Royal

London Hospital

Mrs Una Rennard,

Public contributor
Dr Stuart Smellie,

Consultant in Clinical Pathology, Bishop Auckland General Hospital

Ms Jane Smith,

Consultant Ultrasound

Practitioner, Leeds Teaching

Hospital NHS Trust, Leeds

Dr Allison Streetly,

Programme Director, NHS Sickle

Cell and Thalassaemia Screening

Programme, King's College School of Medicine

Dr Matthew Thompson,

Senior Clinical Scientist and GP

Department of Primary Health

Care, University of Oxford

Dr Alan J Williams,

Consultant Physician, General and

Respiratory Medicine, The Royal

Bournemouth Hospital

\section{Observers}

\section{Dr Tim Elliott,}

Team Leader, Cancer Screening,

Department of Health

Dr Joanna Jenkinson,

Board Secretary, Neurosciences

and Mental Health Board

(NMHB), Medical Research

Council
Professor Julietta Patnick,

Director, NHS Cancer Screening

Programme, Sheffield

Dr Kay Pattison,

Senior NIHR Programme

Manager, Department of Health
Professor Tom Walley, CBE, Director, NIHR HTA

programme, Professor of Clinical Pharmacology, University of Liverpool
Dr Ursula Wells,

Principal Research Officer, Policy Research Programme, Department of Health

\section{Disease Prevention Panel}

Members

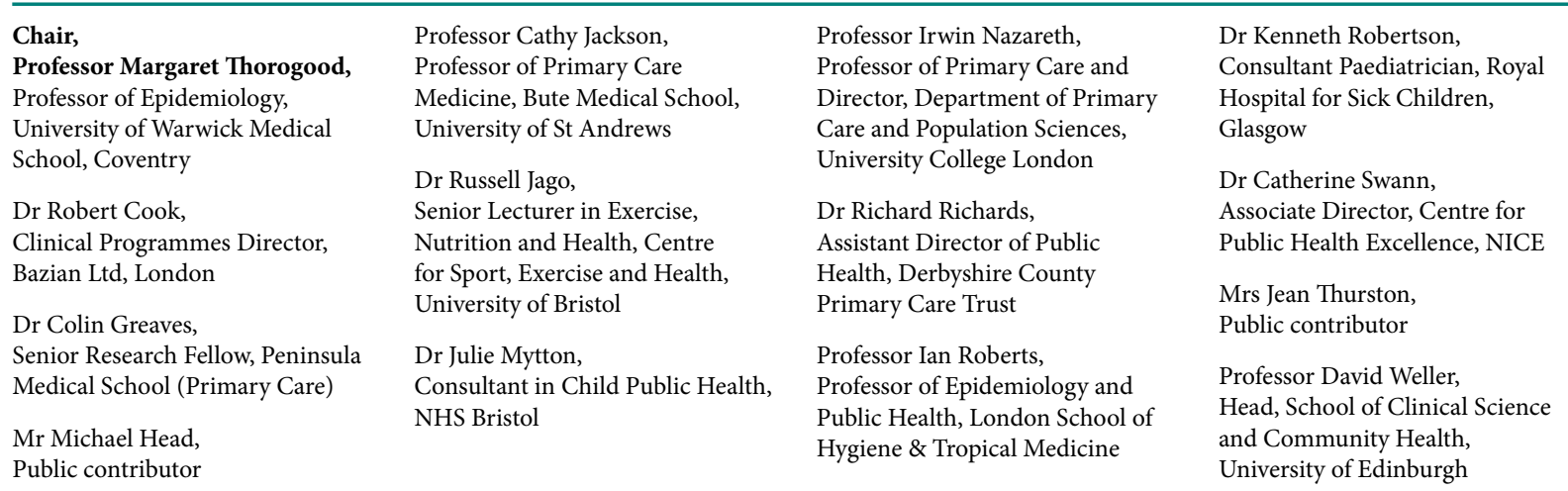

Observers

Ms Christine McGuire,

Research \& Development,

Department of Health
Dr Kay Pattison,

Senior NIHR Programme

Manager, Department of Health
Professor Tom Walley, CBE,

Director, NIHR HTA

programme, Professor of Clinical

Pharmacology, University of

Liverpool 


\section{External Devices and Physical Therapies Panel}

Members

\section{Chair,}

Dr John Pounsford,

Consultant Physician North Bristol

NHS Trust

Deputy Chair,

Professor E Andrea Nelson,

Reader in Wound Healing and

Director of Research, University

of Leeds

Professor Bipin Bhakta,

Charterhouse Professor in

Rehabilitation Medicine,

University of Leeds

Mrs Penny Calder,

Public contributor
Dr Dawn Carnes,

Senior Research Fellow, Barts and the London School of Medicine and Dentistry

Dr Emma Clark,

Clinician Scientist Fellow \& Cons.

Rheumatologist, University of Bristol

Mrs Anthea De Barton-Watson,

Public contributor

Professor Nadine Foster, Professor of Musculoskeletal Health in Primary Care Arthritis Research, Keele University
Dr Shaheen Hamdy,

Clinical Senior Lecturer and

Consultant Physician, University

of Manchester

Professor Christine Norton,

Professor of Clinical Nursing

Innovation, Bucks New University

and Imperial College Healthcare

NHS Trust

Dr Lorraine Pinnigton,

Associate Professor in

Rehabilitation, University of

Nottingham

Dr Kate Radford,

Senior Lecturer (Research),

University of Central Lancashire
Mr Jim Reece,

Public contributor

Professor Maria Stokes

Professor of Neuromusculoskeletal

Rehabilitation, University of

Southampton

Dr Pippa Tyrrell,

Senior Lecturer/Consultant

Salford Royal Foundation

Hospitals' Trust and University of Manchester

Dr Nefyn Williams,

Clinical Senior Lecturer, Cardiff

University

\section{Observers}

Dr Kay Pattison,

Senior NIHR Programme

Manager, Department of Health
Dr Morven Roberts,

Clinical Trials Manager, Health

Services and Public Health

Services Board, Medical Research

Council
Professor Tom Walley, CBE,

Director, NIHR HTA

programme, Professor of Clinical

Pharmacology, University of

Liverpool
Dr Ursula Wells,

Principal Research Officer, Policy

Research Programme, Department of Health

\section{Interventional Procedures Panel}

Members

\section{Chair, \\ Professor Jonathan Michaels, Professor of Vascular Surgery,}

University of Sheffield

\section{Deputy Chair,}

Mr Michael Thomas,

Consultant Colorectal Surgeon, Bristol Royal Infirmary

Mrs Isabel Boyer,

Public contributor

Mr Sankaran Chandra Sekharan, Consultant Surgeon, Breast Surgery, Colchester Hospital University NHS Foundation Trust

Professor Nicholas Clarke,

Consultant Orthopaedic Surgeon,

Southampton University Hospitals NHS Trust

Ms Leonie Cooke Public contributor

\section{Mr Seumas Eckford,}

Consultant in Obstetrics \&

Gynaecology, North Devon

District Hospital

Professor Sam Eljamel,

Consultant Neurosurgeon

Ninewells Hospital and Medical

School, Dundee

Dr Adele Fielding,

Senior Lecturer and Honorary

Consultant in Haematology,

University College London

Medical School

Dr Matthew Hatton,

Consultant in Clinical Oncology,

Sheffield Teaching Hospital

Foundation Trust

Dr John Holden,

General Practitioner, Garswood

Surgery, Wigan
Dr Fiona Lecky

Senior Lecturer/Honorary

Consultant in Emergency

Medicine, University of

Manchester/Salford Royal

Hospitals NHS Foundation Trust

Dr Nadim Malik,

Consultant Cardiologist/Honorary

Lecturer, University of Manchester

Mr Hisham Mehanna,

Consultant \& Honorary Associate

Professor, University Hospitals

Coventry \& Warwickshire NHS

Trust

Dr Jane Montgomery,

Consultant in Anaesthetics and

Critical Care, South Devon

Healthcare NHS Foundation Trust

Professor Yit Chiun Yang,

Consultant Ophthalmologist,

Royal Wolverhampton Hospitals

NHS Trust

\section{Observers}

Dr Kay Pattison,

Senior NIHR Programme

Manager, Department of Health
Dr Morven Roberts,

Clinical Trials Manager, Health

Services and Public Health

Services Board, Medical Research

Council
Professor Tom Walley, CBE,

Director, NIHR HTA

programme, Professor of Clinical

Pharmacology, University of

Liverpool
Dr Ursula Wells,

Principal Research Officer, Policy

Research Programme, Departmen of Health 


\section{Pharmaceuticals Panel}

Members

\section{Chair,}

Professor Imti Choonara

Professor in Child Health,

University of Nottingham

\section{Deputy Chair,}

Dr Yoon K Loke,

Senior Lecturer in Clinical

Pharmacology, University of East

Anglia

Dr Martin Ashton-Key,

Medical Advisor, National

Commissioning Group, NHS

London

Dr Peter Elton,

Director of Public Health, Bury

Primary Care Trust

Dr Ben Goldacre,

Research Fellow, Epidemiology

London School of Hygiene and

Tropical Medicine
Dr James Gray,

Consultant Microbiologist,

Department of Microbiology,

Birmingham Children's Hospital

NHS Foundation Trust

Dr Jurjees Hasan,

Consultant in Medical Oncology,

The Christie, Manchester

Dr Carl Heneghan,

Deputy Director Centre for

Evidence-Based Medicine and

Clinical Lecturer, Department of

Primary Health Care, University of Oxford

\section{Dr Dyfrig Hughes,}

Reader in Pharmacoeconomics and Deputy Director, Centre for Economics and Policy in Health, IMSCaR, Bangor University
Dr Maria Kouimtzi,

Pharmacy and Informatics

Director, Global Clinical Solutions,

Wiley-Blackwell

Professor Femi Oyebode,

Consultant Psychiatrist and Head

of Department, University of

Birmingham

Dr Andrew Prentice,

Senior Lecturer and Consultant

Obstetrician and Gynaecologist,

The Rosie Hospital, University of

Cambridge

Ms Amanda Roberts,

Public contributor

Dr Gillian Shepherd,

Director, Health and Clinical

Excellence, Merck Serono Ltd
Mrs Katrina Simister

Assistant Director New Medicines,

National Prescribing Centre,

Liverpool

Professor Donald Singer,

Professor of Clinical

Pharmacology and Therapeutics,

Clinical Sciences Research

Institute, CSB, University of

Warwick Medical School

Mr David Symes,

Public contributor

Dr Arnold Zermansky,

General Practitioner, Senior

Research Fellow, Pharmacy

Practice and Medicines

Management Group, Leeds

University

\section{Observers}

\section{Dr Kay Pattison,}

Senior NIHR Programme

Manager, Department of Health

Mr Simon Reeve,

Head of Clinical and Cost-

Effectiveness, Medicines,

Pharmacy and Industry Group,

Department of Health
Dr Heike Weber,

Programme Manager, Medical

Research Council

Professor Tom Walley, CBE,

Director, NIHR HTA

programme, Professor of Clinical

Pharmacology, University of

Liverpool
Dr Ursula Wells,

Principal Research Officer, Policy Research Programme, Department of Health

\section{Psychological and Community Therapies Panel}

\section{Members}

\begin{tabular}{ll}
\hline Chair, & Mrs Val Carlill, \\
Professor Scott Weich, & Public contribut
\end{tabular}

Professor of Psychiatry, University

of Warwick, Coventry

\section{Deputy Chair,}

\section{Dr Howard Ring}

Consultant \& University Lecture in Psychiatry, University of

Cambridge

Professor Jane Barlow, Professor of Public Health in the Early Years, Health Sciences Research Institute, Warwick Medical Schoo

Dr Sabyasachi Bhaumik Consultant Psychiatrist Leicestershire Partnership NHS Trust

\section{Public contributor}

Dr Steve Cunningham,

Consultant Respiratory

Paediatrician, Lothian Health

Board

Dr Anne Hesketh,

Senior Clinical Lecturer in Speech and Language Therapy, University of Manchester

Dr Peter Langdon,

Senior Clinical Lecturer, Schoo of Medicine, Health Policy and Practice, University of East Anglia

Dr Yann Lefeuvre,

GP Partner, Burrage Road Surgery, London
Dr Jeremy J Murphy,

Consultant Physician and

Cardiologist, County Durham and

Darlington Foundation Trust

Dr Richard Neal,

Clinical Senior Lecturer in General Practice, Cardiff University

Mr John Needham,

Public contributor

Ms Mary Nettle,

Mental Health User Consultant

Professor John Potter,

Professor of Ageing and Stroke Medicine, University of East

Anglia

Dr Greta Rait,

Senior Clinical Lecturer and

General Practitioner, University

College London
Dr Paul Ramchandani,

Senior Research Fellow/Cons.

Child Psychiatrist, University of Oxford

Dr Karen Roberts,

Nurse/Consultant, Dunston Hill Hospital, Tyne and Wear

Dr Karim Saad,

Consultant in Old Age Psychiatry, Coventry and Warwickshire Partnership Trust

Dr Lesley Stockton,

Lecturer, School of Health

Sciences, University of Liverpool

Dr Simon Wright,

GP Partner, Walkden Medical

Centre, Manchester

\section{Observers}

\section{Dr Kay Pattison,}

Senior NIHR Programme

Manager, Department of Health
Dr Morven Roberts,

Clinical Trials Manager, Health

Services and Public Health

Services Board, Medical Research

Council
Professor Tom Walley, CBE, Director, NIHR HTA programme, Professor of Clinical Pharmacology, University of Liverpool
Dr Ursula Wells,

Principal Research Officer, Policy Research Programme, Department of Health 

This version of the monograph does not include some of the appendices. This is to save download time from the HTA website.

The printed version also excludes some of the appendices.

View/download the appendices 


\section{Feedback}

The HTA programme and the authors would like to know your views about this report.

The Correspondence Page on the HTA website (www.hta.ac.uk) is a convenient way to publish your comments. If you prefer, you can send your comments to the address below, telling us whether you would like us to transfer them to the website.

We look forward to hearing from you.

NETSCC, Health Technology Assessment

Alpha House

University of Southampton Science Park

Southampton SO16 7NS, UK

Email: hta@hta.ac.uk

www.hta.ac.uk 